Please cite this article as:

O. Marinov, M. J. Deen, J. A. Jiménez-Tejada, C. H. Chen, Variable-range hopping charge transport in organic thin-film transistors, Physics Reports, (2020), 844, 1-105.

(C2020. This manuscript version is made available under the CC-BY-NC-

ND 4.0 license

http://creativecommons.org/licenses/by-nc-nd/4.0/

Digital Object Identifier:

10.1016/j.physrep.2019.12.002

Source:

https://www.sciencedirect.com/science/article/pii/S0370157319304028?via\%3Dihub 


\title{
Variable-Range Hopping Charge Transport in Organic Thin-Film Transistors
}

\author{
O. Marinov ${ }^{1}$, M. J. Deen ${ }^{1}$, J. A. Jiménez-Tejada ${ }^{2}$ and C. H. Chen ${ }^{1}$
}

${ }^{1}$ Department of Electrical and Computer Engineering, McMaster University, 1280 Main Street West, Hamilton, Ontario L8S 4K1, Canada

${ }^{2}$ Departamento de Electrónica y Tecnología de los Computadores, CITIC-UGR, Universidad de Granada, Granada 18071, Spain

\begin{abstract}
The charge transport in organic thin-film transistors (OTFTs) is assessed in terms of variable range hopping (VRH), by numerical simulations, analytical analyses and comparisons to published experimental results. A numerical simulator built on the fundamental relations for VRH, without approximations, provides a simple key dependence that the sum of hopping energy and energy bending under bias is equal to the hopping energy in the bulk material, the latter a bias-independent function of the absolute temperature. This relation binds electrostatics and VRH in OTFTs, at various assumptions for density of states (exponential, double-exponential and normal distributions). It generates and confirms many analytical expressions accumulated over the years for mobility, conductance, potential profiles in the depth of the organic semiconducting film and their relation to bias, film-thickness, also explaining the performance of OTFTs at elevated temperatures. The relations between charges, mobility and bias in OTFTs adhere from the above key dependence. We provide a method to obtain the distribution of the hopping time, which establishes explanations to non-stationary effects in OTFTs, such as dispersive transport, non-reciprocal transitions between on and off-states of the OTFT (usually attributed to gate bias stress and charge build-up), and low-frequency noise in the OTFT channel current.
\end{abstract}

Keywords: organic thin-film transistor (OTFT), variable range hopping (VRH), numerical simulations

Word count: 49,957 (excludes Extended abstract, Tables, Figure captions and Abbreviation nomenclature) 


\section{Nomenclature of abbreviations and notations}

(Note that the notations in Appendix 4 and Figure 45 in it are not given in the list, and deviate from this nomenclature)

\begin{tabular}{|c|c|c|}
\hline $\begin{array}{l}\text { Symbol } \\
\text { [primary unit] } \\
\text { (secondary units) } \\
\end{array}$ & Meaning and comments & see \\
\hline 1D, 2D, 3D & $\begin{array}{l}\text { One- dimensional, two- dimensional, three-dimensional. Mostly used for } \\
\text { vectors and matrices of numbers. }\end{array}$ & \\
\hline${ }_{2}$ HypGeom $_{1} \equiv_{2} \mathrm{~F}_{1}$ & Gauss hyper-geometric function & $\begin{array}{l}\text { eq. (103) and Appendix } \\
4\end{array}$ \\
\hline A, a [various] & $\begin{array}{l}\text { Supplementary notation for several quantities that have constant value as } \\
\text { explained in particular contexts }\end{array}$ & \\
\hline an [subscript] & $\begin{array}{l}\text { Supplementary subscript, which explicitly denotes that the quantity is after } \\
\text { analytical approximation, e.g., } N_{C, \text { an }} \text { is calculated by eq. (49), whereas } N_{C} \text { is } \\
\text { taken from the numerical simulation. }\end{array}$ & $\begin{array}{l}\text { text to Figure } 24 \text {, eq. } \\
\text { (71) and Figure } 28\end{array}$ \\
\hline $\operatorname{avgV_{B}[V]}$ & $\begin{array}{l}\text { Expected potential bending representative for the sheet conductance } \sigma_{\mathrm{sq}} \text {. The } \\
\text { value of avgV } V_{\mathrm{B}} \text { is an average of bending profile } \mathrm{V}_{\mathrm{B}}(\mathrm{D}) \text { weighted with the } \\
\text { profile } \sigma(\mathrm{D}) \text { of the specific conductance } \sigma \text {. }\end{array}$ & eq. (70) \\
\hline $\mathrm{b}$ [various] & $\begin{array}{l}\text { Supplementary notation for several quantities that have constant value as } \\
\text { explained in particular contexts }\end{array}$ & \\
\hline $\mathrm{B}$ & $\begin{array}{l}\text { Supplementary notation for values calculated during iterations that have to } \\
\text { meet a target value. In general, } \mathrm{B} \text { denotes the bonds per site, or proportional } \\
\text { quantity, so that } \mathrm{B}\left(\Gamma \geq \Gamma_{\mathrm{c}}\right)=\mathrm{B}\left(\tau \leq \tau_{\mathrm{c}}\right)=\mathrm{B}_{\mathrm{c}} \text {. }\end{array}$ & $\begin{array}{l}\text { eqs. (14), (20), (114)- } \\
(118)\end{array}$ \\
\hline $\mathrm{B}_{\mathrm{c}}$ [number] & $\begin{array}{l}B_{c} \sim 9 / \pi \approx 2.86 \text { is critical number for three-dimensional percolation network } \\
\left(B_{c} \sim 4 \text { after other assumptions, see eq. }(4.14) \text { in }[20]\right),\end{array}$ & eqs. (3), (5) \\
\hline BPS [number] & Supplementary notation for number of hopping bonds per site & eq. (17) \\
\hline CDM & Correlation disorder model & after eq. (127) \\
\hline CFDOS $\left[1 / \mathrm{cm}^{3}\right]$ & $\begin{array}{l}\text { Supplementary notation for the cumulative sum of the product } \mathrm{F} \times \mathrm{DOS} \times \mathrm{dE} \text { of } \\
\text { Fermi occupation factor, DOS and energy integration step } \mathrm{dE} \text { at given energy } \\
\mathrm{E}\end{array}$ & eq. (27) \\
\hline $\mathrm{C}_{\mathrm{I}} \equiv \mathrm{C}_{\mathrm{OX}}\left[\mathrm{F} / \mathrm{cm}^{2}\right]$ & Gate dielectric capacitance per unit area & \\
\hline $\mathrm{CS}_{\mathrm{c}}\left[1 / \mathrm{cm}^{3}\right]$ & Supplementary notation for concentration of critical sites & eq. (8) \\
\hline $\mathrm{dt}[\mathrm{cm}]$ & $\begin{array}{l}\text { Variable step for distance by numerical integration of Poisson equation. } \mathrm{dt}>0 \text {, } \\
\text { although } \mathrm{t}<0 \text {. }\end{array}$ & eq. (32) \\
\hline $\begin{array}{l}\mathrm{dE}, \mathrm{d} \Delta \mathrm{E}, \mathrm{dE}_{\mathrm{i}}, \mathrm{dE}_{\mathrm{j}} \\
{[\mathrm{eV}](\mathrm{meV})}\end{array}$ & Integration step for energy by calculations of Riemann sums & eqs. (8), (14), (17)-(20) \\
\hline $\mathrm{D}[\mathrm{cm}](\mathrm{nm})$ & $\begin{array}{l}\text { Depth in the semiconducting film with a reference }(D=0) \text { at the gate dielectric } \\
\text { interface and within the film thickness } t_{f}\left(D=t_{f}=\text { film back }\right)\end{array}$ & $\begin{array}{l}\text { before eq. (29) to eq. } \\
\text { (37) }\end{array}$ \\
\hline $\begin{array}{l}\mathrm{D}^{\prime \prime \prime} \\
{[\mathrm{cm}],(\mathrm{nm})}\end{array}$ & $\begin{array}{l}\mathrm{D}^{\prime \prime \prime}=\left\{\mathrm{L}_{\mathrm{A}}, \mathrm{D}_{95 \%} \% \text { sq }\right. \\
\text { characteristic electrostatic distances, defined below }\end{array}$ & sec. 4.4 .3 and Figure 27 \\
\hline $\begin{array}{l}\mathrm{D}_{95 \% \sigma \mathrm{sq}} \equiv \mathrm{D}_{95 \% \sigma} \\
{[\mathrm{cm}],(\mathrm{nm})}\end{array}$ & $\begin{array}{l}\text { Characteristic depth in the semiconducting film, which corresponds to } 95 \% \text { of } \\
\text { the sheet conductance } \sigma_{\mathrm{sq}}\end{array}$ & eq. (69) \\
\hline $\begin{array}{l}\mathrm{D}_{\text {avgVB }} \\
{[\mathrm{cm}],(\mathrm{nm})}\end{array}$ & $\begin{array}{l}\text { Characteristic depth in the semiconducting film, at which the potential bending } \\
\text { is with magnitude } \operatorname{avgV}_{\mathrm{B}} \text {. See also avgV } \mathrm{V}_{\mathrm{B}} \text {. }\end{array}$ & eq. (70) \\
\hline $\mathrm{dD}[\mathrm{cm}](\mathrm{nm})$ & Non-uniform step in the depth D of the OTFT semiconducting film & eq. (32) to after eq. (38) \\
\hline $\mathrm{DC}[\mathrm{u}]$ & Static value for a quantity in unit $[\mathrm{u}] . \mathrm{DC} \equiv \mathrm{X}_{\mathrm{STAT}}$. & eq. (144) \\
\hline $\operatorname{DOS}\left[\mathrm{cm}^{-3} \mathrm{eV}^{-1}\right]$ & $\begin{array}{l}\text { Density of states in semiconductor. DOS(E) is arbitrary, but predetermined } \\
\text { function of energy E, and DOS is spatially uniform. }\end{array}$ & eqs. (3), (8) \\
\hline $\mathrm{DOS}_{\mathrm{DE}}\left[\mathrm{cm}^{-3} \mathrm{eV}^{-1}\right]$ & Double-side exponential DOS & eq. (45) \\
\hline $\mathrm{DOS}_{\mathrm{ND}}\left[\mathrm{cm}^{-3} \mathrm{eV}^{-1}\right]$ & Normally distributed DOS & eq. (58) \\
\hline $\operatorname{DOS}_{\mathrm{SE}}\left[\mathrm{cm}^{-3} \mathrm{eV}^{-1}\right]$ & Single-side exponential DOS & eq. (22) \\
\hline $\mathrm{E}, \mathrm{E}_{\mathrm{i}}, \mathrm{E}_{\mathrm{j}}[\mathrm{eV}]$ & Energy, energy of states $\mathrm{i}$ and $\mathrm{j}$. See also $\Delta \mathrm{E}$. & eq. (6) \\
\hline $\mathrm{E}_{\mathrm{A} \sigma}[\mathrm{eV}]$ & Bias-dependent thermal activation energy of the VRH sheet conductance $\sigma_{\mathrm{sq}}$. & $\begin{array}{l}\text { eq. (56); } \\
\text { Figure } 19\end{array}$ \\
\hline
\end{tabular}




\begin{tabular}{|c|c|c|}
\hline $\begin{array}{l}\text { Symbol } \\
\text { [primary unit] } \\
\text { (secondary units) }\end{array}$ & Meaning and comments & see \\
\hline $\mathrm{E}_{\mathrm{A \mu \nu}}[\mathrm{eV}]$ & 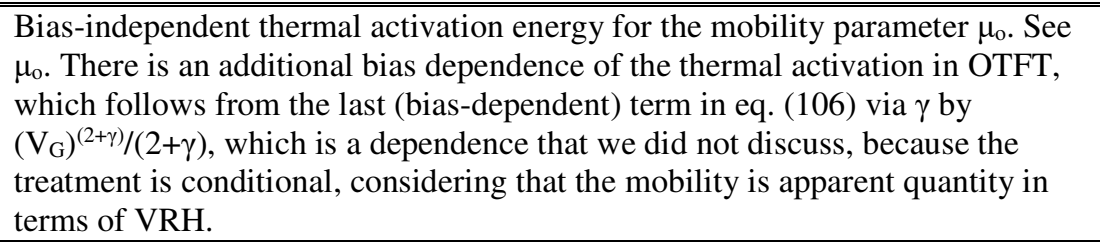 & eq. (113) \\
\hline $\mathrm{E}_{\mathrm{F}}[\mathrm{eV}]$ & Fermi energy level in bulk semiconductor. See also IMREF and FB. & eq. (6) \\
\hline $\mathrm{E}_{\mathrm{el}}[\mathrm{V} / \mathrm{cm}]$ & $\begin{array}{l}\text { Electric field induced by } \mathrm{V}_{\mathrm{G}} \text { in the film of the OTFT in the direction } \\
\text { perpendicular to the plane of the film }\end{array}$ & $\begin{array}{l}\text { before eq. (29) to eq. } \\
(36)\end{array}$ \\
\hline $\mathrm{E}_{\mathrm{bottom}}[\mathrm{eV}]$ & Lower limit for energy integrations, see also $\mathrm{E}_{\text {top }}$ & eq. (39) \\
\hline $\mathrm{E}_{\mathrm{H}}[\mathrm{eV}]$ & $\begin{array}{l}\text { Hopping energy: the maximum energy difference of successful charge hopping } \\
\text { (average value for the critical path in the percolation network) }\end{array}$ & eq. (2) \\
\hline $\mathrm{E}_{\mathrm{HFB}}[\mathrm{eV}]$ & Hopping energy in bulk semiconductor (no bias, bending $\mathrm{V}_{\mathrm{B}}=0$ ) & eqs. (30), (59) \\
\hline $\mathrm{E}_{\mathrm{o}}[\mathrm{eV}]$ & $\begin{array}{l}\text { Energy position parameter of DOS (For the single-mode DOS considered in } \\
\text { this work, } E_{0}=\text { HOMO for p-type OTFT and } E_{0}=\text { LUMO for n-type OTFT) }\end{array}$ & eqs. (22), (45), (58) \\
\hline $\mathrm{E}_{\text {top }}[\mathrm{eV}]$ & Upper limit for energy integrations, see also $\mathrm{E}_{\text {bottom }}$ & eq. (39) \\
\hline $\mathrm{E}_{\mathrm{T}}[\mathrm{eV}]$ & Supplementary notation by eq. (50). Later is shown that $\mathrm{E}_{\mathrm{T}} \equiv \mathrm{E}_{\mathrm{HFB}}$. & eqs. (50), (59) \\
\hline $\operatorname{errQ}_{\mathrm{G}}[\%]$ & $\begin{array}{l}\text { Relative error of unbalance between gate } \mathrm{Q}_{\mathrm{G}} \text { and film } \mathrm{Q}_{\mathrm{F}} \text { charges due to finite } \\
\text { step } \mathrm{dE} \text { in the numerical integrations. } \\
\operatorname{errQ}_{\mathrm{G}}=\left|\mathrm{Q}_{\mathrm{F}} / \mathrm{Q}_{\mathrm{G}}-1\right| \times 100 \% \propto \exp (\mathrm{dE} / \mathrm{kT})-1 \approx \mathrm{dE} / \mathrm{kT} \text { when } \mathrm{dE}<\mathrm{kT}\end{array}$ & Figure 34 \\
\hline$f(\ldots)$ & Supplementary notation for function & \\
\hline $\mathrm{f}[\mathrm{Hz}]$ & Frequency & From eq. (144) \\
\hline $\begin{array}{l}\mathrm{F}, \mathrm{F}_{\mathrm{n}}, \mathrm{F}_{\mathrm{p}} \\
{[\text { number }<1]}\end{array}$ & Fermi occupation factor, for electrons and holes & Before eq. (25) \\
\hline $\mathrm{FB}[\mathrm{eV}]$ & $\begin{array}{l}\text { Energy level, which satisfies the median condition for equal concentrations of } \\
\text { occupied states below and above } F B \text {. Note that } F B \text { varies with bias when using } \\
\text { quasi-Fermi IMREF }=E_{F}+q V_{B} \text {, instead of Fermi level } E_{F} \text {, to include the bending } \\
V_{B} \text { due to gate bias. FB is flat-band energy only if } V_{B}=0 \text {, thus in bulk } \\
\text { semiconductor. }\end{array}$ & eq. (26) \\
\hline $\mathrm{FB}_{\mathrm{AVG}}[\mathrm{eV}]$ & Another definition for FB as weighted average. Not used in the simulator. & eq. (28) \\
\hline FLOP & $\begin{array}{l}\text { Floating-point operation of multiplication. For other operations: summation = } \\
\text { 1/4FLOP; division=2FLOPs; exponentiation=17FLOPs }\end{array}$ & \\
\hline HOMO [eV] & Highest occupied molecular orbital in organic material (centroid level) & \\
\hline GDM & Gaussian disorder model & after eq. (127) \\
\hline $\mathrm{g}_{\mathrm{m}}[\mathrm{S} \equiv \mathrm{A} / \mathrm{V}]$ & Transconductance of OTFT (differential). $\mathrm{g}_{\mathrm{m}}=\partial \mathrm{I}_{\mathrm{D}} / \partial \mathrm{V}_{\mathrm{G}} \propto \mu$ & \\
\hline IMREF [eV] & $\begin{array}{l}\text { Quasi-Fermi energy level by potential bending } V_{B} \text {, see FB. IMREF }=\left(E_{F}+q V_{B}\right) \text {. } \\
\text { Note that other definitions for IMREF exist in the literature, e.g., quasi-Fermi } \\
\text { levels for electrons and holes, while we do not use those definitions. }\end{array}$ & eq. (23) \\
\hline $\begin{array}{l}\text { IFB [numerical } \\
\text { index] }\end{array}$ & $\begin{array}{l}\text { Index in the energy mesh } \mathrm{E} \text {, which corresponds to median condition of charge } \\
\text { occupation, that is, } \mathrm{FB}=\mathrm{E}_{\mathrm{IFB}} \text {. See FB. }\end{array}$ & eq. (27) \\
\hline $\mathrm{I}_{\mathrm{D}}[\mathrm{A}]$ & Channel or drain DC current in OTFT & \\
\hline $\mathrm{k}[\mathrm{eV} / \mathrm{K}]$ & Boltzmann constant $\left(\mathrm{k} \approx 8.62 \times 10^{-5} \mathrm{eV} / \mathrm{K}\right)$ & eq. (2) \\
\hline $\mathrm{kT}, \mathrm{kT} / \mathrm{q}[\mathrm{eV}, \mathrm{V}]$ & Thermal energy in electron-Volts, same as thermal voltage in Volts & eqs. (2), (4) \\
\hline $\mathrm{L}[\mathrm{cm}]$ & Channel length of the OTFT & \\
\hline LUMO [eV] & Lowest unoccupied molecular orbital in organic material (centroid level) & \\
\hline $\mathrm{L}_{\mathrm{A}}[\mathrm{cm}](\mathrm{nm})$ & $\begin{array}{l}\text { Electrostatic length of bias induced charge. } \mathrm{L}_{\mathrm{A}} \text { is the depth in the } \\
\text { semiconductor film from gate dielectric interface, which corresponds to } \\
{ }_{\mathrm{D}=0}^{\mathrm{L}_{\mathrm{A}}} \mathrm{N}_{\mathrm{C}}(\mathrm{D}) \mathrm{dD}=1 / 2 \mathrm{Q}_{\mathrm{G}} \text { of the gate charge, } \mathrm{Q}_{\mathrm{G}} \approx \mathrm{C}_{\mathrm{OX}} \mathrm{V}_{\mathrm{G}} \text {. }\end{array}$ & eqs. (47), (81) \\
\hline LFN & Low-frequency noise & \\
\hline LSB [number] & Least significant bit & \\
\hline $\mathrm{m}$ [various] & $\begin{array}{l}\text { Supplementary notation for multiplicative factor for several numerical } \\
\text { quantities, as always explained in particular contexts }\end{array}$ & \\
\hline $\mathrm{MC}$ & Monte Carlo method for numerical calculations & after eq. (127) \\
\hline MTR & Multiple trapping and release & after eq. (127) \\
\hline
\end{tabular}




\begin{tabular}{|c|c|c|}
\hline $\begin{array}{l}\text { Symbol } \\
\text { [primary unit] } \\
\text { (secondary units) }\end{array}$ & Meaning and comments & see \\
\hline n [number] & $\begin{array}{l}\text { Supplementary notation for several numerical quantities, as always explained } \\
\text { in particular contexts }\end{array}$ & \\
\hline $\mathrm{nE}_{\mathrm{H}}[\mathrm{eV}]$ & $\begin{array}{l}\text { A guess for new value of } E_{H} \text { in the next cycle of iteration procedure with } \\
\text { gradual variation of } E_{H}\end{array}$ & eq. (21) \\
\hline $\mathrm{N}_{\mathrm{C}}\left[1 / \mathrm{cm}^{3}\right]$ & $\begin{array}{l}\text { Concentration of occupied charge states in DOS and carrier concentration for } \\
\text { VRH mobility. Use quasi-Fermi IMREF }=E_{F}+q V_{B} \text {, instead of } E_{F} \text {, to include the } \\
\text { bending } V_{B} \text { due to gate bias. }\end{array}$ & eq. (25) \\
\hline $\begin{array}{l}\mathrm{N}_{\mathrm{CDO}} \equiv \mathrm{N}_{\mathrm{C}}(\mathrm{D}=0) \\
{\left[1 / \mathrm{cm}^{3}\right]}\end{array}$ & $\begin{array}{l}\text { Same as } N_{C} \text {, but for the semiconductor at the gate dielectric interface, thus, } \\
N_{C D O} \text { is } N_{C} \text { at depth } D=0 \text {. }\end{array}$ & eq. (154) \\
\hline $\mathrm{N}_{\mathrm{CFB}}\left[1 / \mathrm{cm}^{3}\right]$ & $\begin{array}{l}\text { Same as } N_{C} \text {, but for bulk semiconductor (no gate bias, thus, the electric field is } \\
\left.\text { zero, } E_{\mathrm{el}}=0 \text {, and the potential bending is zero, } V_{B}=0\right) \\
\text { For an exponential DOS, } N_{C F B}=N_{S} \exp \left(-\mid E_{0}-E_{F} / k T_{0}\right) \text {. }\end{array}$ & eq. (29), (155) \\
\hline $\mathrm{N}_{\mathrm{S}}\left[1 / \mathrm{cm}^{3}\right]$ & Concentration of charge states for all energies, $N_{S}=\int_{-\infty}^{+\infty} \operatorname{DOS}(E) d E$ & eqs. (22), (45), (58) \\
\hline $\mathrm{N}_{\mathrm{SEF}}\left[1 / \mathrm{cm}^{3}\right]$ & $\begin{array}{l}\text { Effective value for } \mathrm{N}_{S} \text {, which varies with temperature, as deduced in [11] after } \\
\text { deconvolution of the integral } \int \mathrm{dx} /\left(1+\mathrm{x}^{\mathrm{a}}\right) \text { with exponential DOS }\end{array}$ & $\begin{array}{l}\text { between eqs. (79) and } \\
(80)\end{array}$ \\
\hline $\mathrm{N}_{\mathrm{VG}}\left[1 / \mathrm{cm}^{3}\right]$ & $\begin{array}{l}\text { Gate bias induced charge concentration at } V_{G} \neq V_{F B}\left(N_{V G} \text { is difference between }\right. \\
\text { the non-equilibrium } N_{C} \text { at } V_{B} \neq 0 \text { and equilibrium } N_{C F B} \text { at } V_{B}=0 \text { for bulk } \\
\text { semiconductor) }\end{array}$ & eq. (34) \\
\hline $\mathrm{N}_{\text {ТОт }}$ [numeric] & Total number of charge carriers in the OTFT channel. See $S_{\text {NORM. }}$ & eqs. (144), (145) \\
\hline $\mathrm{P}, \mathrm{P}_{\mathrm{AVB}}, \mathrm{P}_{\sigma}, \mathrm{P}_{\mathrm{LA}} \equiv 1$ & $\begin{array}{l}\text { Bias independent proportions of characteristic depths. } \mathrm{P} \text { denotes any of } \\
\mathrm{P}_{\mathrm{AVB}}=\mathrm{D}_{\mathrm{avgVB}} / \mathrm{L}_{\mathrm{A}}, \mathrm{P}_{\sigma}=\mathrm{D}_{95 \% \sigma s q} / \mathrm{L}_{\mathrm{A}} \text { or } \mathrm{P}_{\mathrm{LA}}=\mathrm{L}_{\mathrm{A}} / \mathrm{L}_{\mathrm{A}} \equiv 1 \text {. }\end{array}$ & $\begin{array}{l}\text { between eqs. (76)-(77); } \\
\text { Figure } 27\end{array}$ \\
\hline PEAKING ${ }_{D O S}$ & "Peaking" of DOS. Characteristic constant for the DOS type & see after eq. (74) \\
\hline $\mathrm{PSD}\left[\mathrm{u}^{2} / \mathrm{Hz}\right]$ & $\begin{array}{l}\text { Power-spectrum density. For noise, } S(f) \text { denotes PSD. [u] is the unit of the } \\
\text { quantity, for which PSD is given, e.g., }[\mathrm{u}] \equiv[\mathrm{A}] \text { for current - see } \mathrm{S}_{\mathrm{ID}}\end{array}$ & \\
\hline $\mathrm{q}[\mathrm{C}]$ & $\mathrm{q}=1.602 \times 10^{-19} \mathrm{C}$ is the magnitude of the electron charge & eq. (3) \\
\hline $\mathrm{Q}_{\mathrm{F}}\left[\mathrm{C} / \mathrm{cm}^{2}\right]$ & Charge per unit area in the semiconducting film $\left(\mathrm{Q}_{\mathrm{F}} \approx \mathrm{Q}_{\mathrm{G}}\right)$ & eq. (93) \\
\hline $\mathrm{Q}_{\mathrm{G}}\left[\mathrm{C} / \mathrm{cm}^{2}\right]$ & $\begin{array}{l}\text { Charge per unit area of the gate dielectric } \mathrm{Q}_{\mathrm{G}}=\left[\mathrm{V}_{\mathrm{G}}-\left(\mathrm{V}_{\mathrm{FB}}+\mathrm{V}_{\mathrm{BS}}\right)\right] \mathrm{C}_{\mathrm{OX}} \text {, with } \\
\mathrm{V}_{\mathrm{BS}}=\mathrm{V}_{\mathrm{B}}(\mathrm{D}=0)\end{array}$ & eq. (31) \\
\hline$Q_{G}^{\prime}\left[\mathrm{C} / \mathrm{cm}^{2}\right]$ & $\begin{array}{l}\text { Supplementary variable for } Q_{G} \text { during integration in the depth } D \geq 0 \text { of the } \\
\text { semiconducting film } Q_{G}^{\prime}=\left[V_{G}-\left(V_{F B}+V_{B}\right)\right] C_{O X} \text {, with } V_{B} \text { for } D \geq 0\end{array}$ & eq. (31) \\
\hline $\mathrm{R}, \mathrm{R}_{\mathrm{ij}}[\mathrm{cm}](\mathrm{nm})$ & Hopping distance, distance between hopping sites $\mathrm{i}$ and $\mathrm{j}$ & eqs. (6), (7) \\
\hline $\mathrm{R}_{\mathrm{H}}[\mathrm{cm}](\mathrm{nm})$ & $\begin{array}{l}\text { Maximum distance of successful charge hopping (average value for the critical } \\
\text { path in the percolation network) }\end{array}$ & eq. (2) \\
\hline $\mathrm{R}_{\mathrm{HFB}}[\mathrm{cm}](\mathrm{nm})$ & Hopping distance $\mathrm{R}_{\mathrm{H}}$ for bulk semiconductor (no bias, $\mathrm{V}_{\mathrm{B}}=0$ ) & eq. (60) \\
\hline $\mathrm{R}_{\mathrm{o}} \equiv \Lambda_{\mathrm{o}}$ & Decay distance for the hopping rate, see $\Lambda_{o}$ & \\
\hline $\mathrm{REC}_{\mathrm{DOS}}$ & "Rectangularity" of DOS. Characteristic constant for the DOS type & eq. (74) \\
\hline $\mathrm{s}_{\mathrm{c}}$ [number] & $\begin{array}{l}\text { Hopping critical factor (attenuation factor of VRH conductivity in the critical } \\
\text { path of the hopping percolation network) }\end{array}$ & eq. (1) \\
\hline $\mathrm{S}_{\mathrm{cFB}}[$ number] & Hopping critical factor $\mathrm{s}_{\mathrm{c}}$ for bulk semiconductor (no bias, $\mathrm{V}_{\mathrm{B}}=0$ ) & eq. (60) \\
\hline $\mathrm{S}_{\mathrm{cT} \infty}$ [number] & $\mathrm{s}_{\mathrm{cT} \infty} \approx 10 \pm 4$ is extrapolated value for $\mathrm{s}_{\mathrm{cFB}}$ at infinite temperature $\mathrm{T}=\infty$ & eq. (61) \\
\hline $\mathrm{sE}_{\mathrm{H}}[\mathrm{eV}]$ & $\begin{array}{l}\text { Step prefactor for } E_{H} \text { by calculation of the guess } n E_{H} \text { for the next cycle of } \\
\text { iteration procedure with gradual variation of } E_{H} \text {. See } \mathrm{nE}_{\mathrm{H}} \text {. }\end{array}$ & eq. (21) \\
\hline $\mathrm{S}_{\mu}$ [number $]$ & $\begin{array}{l}\text { Parameter in the exponent of the power-law trend for mobility deduced in [82]. } \\
S_{\mu} \text { is material dependent. }\end{array}$ & eq. (82) \\
\hline SM & Scher and Montroll (dispersive transport formalism in [95]) & eq. (126) \\
\hline $\mathrm{S}(\mathrm{f})\left[\mathrm{u}^{2} / \mathrm{Hz}\right]$ & PSD of noise for quantity with unit [u]. See PSD, $S_{\text {ID }}$ and $S_{N O R M}$. & eq. (144) \\
\hline $\mathrm{S}_{\mathrm{ID}}\left[\mathrm{A}^{2} / \mathrm{Hz}\right]$ & PSD of the noise in the OTFT channel/drain current $I_{D}$ & eq. (150) \\
\hline $\mathrm{S}_{\mathrm{NORM}}[1 / \mathrm{Hz}]$ & Normalized power-spectrum density (PSD). $\mathrm{S}_{\mathrm{NORM}}=\mathrm{S}(\mathrm{f}) / \mathrm{DC}^{2}$ & eq. (144) \\
\hline $\mathrm{S}_{\mathrm{n} 1}[1 / \mathrm{Hz}]$ & Normalized PSD of the noise of single carrier. $S_{n 1} \approx \alpha_{H} / f^{\beta}$. See $\alpha_{H}$. & eq. (146) \\
\hline $\mathrm{T}[\mathrm{K}]$ & Absolute temperature (in unit of Kelvin) & eq. (2) \\
\hline $\mathrm{T}_{\mathrm{A}}[\mathrm{K}]$ & $\begin{array}{l}\text { Characteristic "temperature" proportional to the electrostatic effective depth } \\
\mathrm{L}_{\mathrm{A}} \text { of the conduction channel at gate bias voltage } \mathrm{V}_{\mathrm{G}} \neq 0 . \mathrm{T}_{\mathrm{A}} \approx \mathrm{T}_{\mathrm{o}} \text { for an } \\
\text { exponential DOS. See also } \varphi_{\mathrm{A}} \equiv \mathrm{kT} \mathrm{T}_{\mathrm{A}} / \mathrm{q} \text {. }\end{array}$ & eq. (47) \\
\hline
\end{tabular}




\begin{tabular}{|c|c|c|}
\hline $\begin{array}{l}\text { Symbol } \\
\text { [primary unit] } \\
\text { (secondary units) }\end{array}$ & Meaning and comments & see \\
\hline 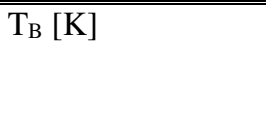 & $\begin{array}{l}\text { Characteristic "temperature" for the logarithmic decay of the potential bending } \\
\mathrm{V}_{\mathrm{B}} \text { in the depth of the film at gate bias voltage } \mathrm{V}_{\mathrm{G}} \neq 0 . \mathrm{T}_{\mathrm{B}} \approx \mathrm{T}_{\mathrm{o}} \text { for an exponential } \\
\text { DOS. See also } \varphi_{\mathrm{B}} \equiv \mathrm{k} T_{\mathrm{B}} / \mathrm{q} \text {. }\end{array}$ & eq. (48) \\
\hline $\mathrm{T}_{\mathrm{C}}[\mathrm{K}]$ & $\begin{array}{l}\text { Characteristic "temperature" proportional to the carrier concentration } N_{C} \text { at } \\
\text { gate bias voltage } V_{G} \neq 0 . T_{C} \approx T_{o} \text { for an exponential DOS. See also } \varphi_{C} \equiv k T_{C} / q \text {. }\end{array}$ & eq. (49) \\
\hline $\mathrm{T}_{\text {cold }}[\mathrm{K}]$ & $\begin{array}{l}\text { Effective characteristic "temperature" at absolute zero temperature. See } \\
\varphi_{\text {cold }} \equiv \mathrm{kT}_{\text {cold }} / \mathrm{q} \text {. }\end{array}$ & \\
\hline $\mathrm{T}_{\mathrm{G}}[\mathrm{K}]$ & $\begin{array}{l}\text { Characteristic temperature } \mathrm{T}_{\mathrm{G}} \approx 3830 \mathrm{~K} \text { in eq. }(80) \text {, which we observe to fit the } \\
\text { data from numerical simulations of Fig. } 6 \text { in [11] }\end{array}$ & eq. (80) \\
\hline $\mathrm{T}_{\mathrm{o}}[\mathrm{K}]$ & $\begin{array}{l}\text { Characteristic "temperature", which describes the energy width }\left(\mathrm{kT}_{\mathrm{o}}\right) \text { of the } \\
\text { distribution of DOS }\end{array}$ & eqs. (22), (45), (58) \\
\hline $\mathrm{T}_{\mathrm{OEF}}[\mathrm{K}]$ & $\begin{array}{l}\text { Effective characteristic "temperature", originating from convolution between } \\
\text { Fermi and } \mathrm{DOS} \text { distributions. For exponential } \mathrm{DOS}_{\mathrm{SE}}, \mathrm{T}_{\mathrm{OEF}} \approx \mathrm{T}_{\mathrm{o}} \text { at low } \\
\text { temperature } \mathrm{T}<\mathrm{T}_{\mathrm{o}} \text { and } \mathrm{T}_{\mathrm{OEF}} \approx \mathrm{T} \text { at high } \mathrm{T}>\mathrm{T}_{\mathrm{o}} \text {. In many instances, } \mathrm{T}_{\mathrm{OEF}} \text { can } \\
\text { replace } \mathrm{T}_{\mathrm{o}} \text {. See also } \varphi_{\mathrm{OEF}} \equiv \mathrm{kT} \mathrm{T}_{\mathrm{OFF}} / \mathrm{q} \text {. }\end{array}$ & eqs. (79), (80) \\
\hline $\mathrm{T}_{\sigma \mathrm{x}}[\mathrm{K}]$ & $\begin{array}{l}\text { Characteristic temperature parameter for VRH sheet conductance, which is } \\
\text { proportional to the thermal activation energy } E_{\mathrm{A} \sigma} \text { of } \sigma_{\mathrm{sq}} \text {. }\end{array}$ & $\begin{array}{l}\text { eqs. (55), (56); } \\
\text { Figure } 19\end{array}$ \\
\hline $\mathrm{T}^{\prime}[\mathrm{K}]$ & See $\Lambda^{\prime}$ & eq. (3) \\
\hline ToF & $\begin{array}{l}\text { Time-of-Flight, a method for transient current measurement after injection of } \\
\text { charge. The injection is usually by short illumination. }\end{array}$ & before eq. (126) \\
\hline TSF [numeric] & $\begin{array}{l}\text { Temperature shaping function in the TFT compact mobility model, introduced } \\
\text { in [6] }\end{array}$ & eqs. (108), (110) \\
\hline $\mathrm{t}[\mathrm{cm}]$ & $\begin{array}{l}\text { Supplementary variable for distance with direction opposite to the film depth } \\
\mathrm{D} \text {, by numerical integration of Poisson equation with step } \mathrm{dt}>0\end{array}$ & eq. (32) \\
\hline $\mathrm{t}[\mathrm{s}]$ & time of observation & after eq. (126) \\
\hline $\begin{array}{l}\mathrm{t}_{\mathrm{f}} \equiv \mathrm{t}_{\mathrm{FLM}} \\
{[\mathrm{cm}](\mathrm{nm})}\end{array}$ & Semiconducting film thickness in OTFT & \\
\hline $\mathrm{V}_{\mathrm{B}}[\mathrm{V}]$ & $\begin{array}{l}\text { Potential bending induced by the gate bias } \mathrm{V}_{\mathrm{G}} \text { causing non-equilibrium charge } \\
\text { in the semiconducting film of the OTFT. See also IMREF. The energy band } \\
\text { bending in semiconductors is }\left(-\mathrm{q}_{\mathrm{B}}\right) \text {, since the semiconductor band diagrams } \\
\text { are given for electron potential energy (by convention). }\end{array}$ & $\begin{array}{l}\text { before eq. (22) to after } \\
\text { eq. (23) }\end{array}$ \\
\hline $\mathrm{V}_{\mathrm{BS}}[\mathrm{V}]$ & $\begin{array}{l}V_{B S}=V_{B}(D=0) \text { is the potential bending in the semiconductor induced by the } \\
\text { gate bias } V_{G} \text { at the semiconductor-insulator interface }(D=0)\end{array}$ & eqs. (31) and (48) \\
\hline $\mathrm{V}_{\mathrm{D}}[\mathrm{V}]$ & $\begin{array}{l}\text { Drain bias voltage. The source terminal of the OTFT is assumed at zero } \\
\text { potential, that is, } V_{D} \equiv V_{D S} \text {. }\end{array}$ & \\
\hline $\mathrm{V}_{\mathrm{FB}}[\mathrm{V}]$ & $\begin{array}{l}\text { Flat-band voltage potential in the bulk semiconductor (no gate bias); } \mathrm{V}_{\mathrm{FB}}=\mathrm{FB} \\
{\left[\mathrm{eV} \text { ] by eq. }(26) \text { when } \mathrm{V}_{\mathrm{B}}=0 \text {, since } \mathrm{IMREF}=\mathrm{E}_{\mathrm{F}}\right.}\end{array}$ & eq. (29) \\
\hline $\mathrm{V}_{\mathrm{G}}[\mathrm{V}]$ & $\begin{array}{l}\text { Gate bias voltage. The source terminal of the OTFT is assumed at zero } \\
\text { potential, that is, } V_{\mathrm{G}} \equiv \mathrm{V}_{\mathrm{GS}} \text {. }\end{array}$ & \\
\hline $\mathrm{V}_{\mathrm{Gx}}[\mathrm{V}]$ & $\begin{array}{l}\text { Characteristic value that corresponds to extrapolated value of high gate bias, at } \\
\text { which the temperature dependence in the VRH sheet conductivity is cancelled }\end{array}$ & $\begin{array}{l}\text { eq. (54); } \\
\text { Figure } 19\end{array}$ \\
\hline $\mathrm{V}_{\text {on }}[\mathrm{V}]$ & $\begin{array}{l}V_{\text {on }} \text { is the gate voltage } V_{G} \text {, at which the gate bias induces conductivity larger } \\
\text { than the bulk conductivity, and } V_{\text {on }} \sim V_{F B} \text { corresponds roughly to the flat-band } \\
\text { potential } V_{F B}\end{array}$ & $\begin{array}{l}\text { eq. }(53),(54) \text {; } \\
\text { Figure } 18\end{array}$ \\
\hline VRH & Variable Range Hopping & \\
\hline $\mathrm{V}_{\mathrm{S}}[\mathrm{V}]$ & $\begin{array}{l}\text { Source bias voltage. The source terminal of the OTFT is assumed at zero } \\
\text { potential, that is, } V_{S}=0 \text {. }\end{array}$ & \\
\hline $\mathrm{V}_{\mathrm{T}}[\mathrm{V}]$ & $\begin{array}{l}\text { Threshold voltage for the gate bias voltage } \mathrm{V}_{\mathrm{G}} \text { of the OTFT. The value is } \\
\text { extrapolated from above threshold regime }- \text { see } \gamma \text {. }\end{array}$ & eq. (43) \\
\hline $\mathrm{W}[\mathrm{cm}]$ & $\begin{array}{l}\text { Channel width of the OTFT. Occasionally, W(x) also denotes the Lambert } \\
\text { function. }\end{array}$ & \\
\hline $\mathrm{X}, \mathrm{x}$ [various] & $\begin{array}{l}\text { Supplementary variable for denoting different quantities in several equations, } \\
\text { as explained in particular contexts }\end{array}$ & \\
\hline $\mathrm{X}_{\mathrm{AVG}}$ [various] & Averaged quantity, $\mathrm{X}_{\mathrm{AVG}}=\int \mathrm{XYdz} / \mathrm{Ydz}, \mathrm{Y}$ is weighting function & eq. (130) \\
\hline $\mathrm{X}_{\mathrm{NEW}}(\mathrm{t})$ & Equilibrium (thermalized) fraction of $\mathrm{X}$ during transient process & eq. (133) \\
\hline $\mathrm{X}_{\mathrm{OLD}}(\mathrm{t})$ & Non-equilibrium fraction of $\mathrm{X}$ during transient process & eq. (134) \\
\hline
\end{tabular}




\begin{tabular}{|c|c|c|}
\hline $\begin{array}{l}\text { Symbol } \\
\text { [primary unit] } \\
\text { (secondary units) }\end{array}$ & Meaning and comments & see \\
\hline $\mathrm{X}_{\mathrm{sq}}$ [various] & Sheet quantity (X per square-shaped area of the OTFT channel) & eq. (130) \\
\hline $\mathrm{X}_{\mathrm{STAT}}[$ various] & Static (DC) value of $X$ & eq. (132) \\
\hline $\mathrm{X}_{0}, \mathrm{X}_{\infty}$ & $\begin{array}{l}\text { States of } \mathrm{X} \text { before and after a transient process (at time } \mathrm{t}=0_{-} \text {and } \mathrm{t}=\infty \text {, } \\
\text { respectively) }\end{array}$ & eqs. (133), (134) \\
\hline Y, y [various] & Supplementary variable for different quantities. See $\mathrm{X}_{\mathrm{AVG}}$ & eq. (131), (147) \\
\hline$\alpha=1 / \Lambda_{\mathrm{o}}[1 / \mathrm{cm}]$ & Orbital overlap parameter, see $\Lambda_{\mathrm{o}}$ & eq. (3) \\
\hline$\alpha$ [numeric] & Dispersive parameter by Scher-Montroll (SM) formalism in [95] & eqs. (126), (127) \\
\hline$\alpha_{\mathrm{H}}$ & Hooge parameter for magnitude of the flicker noise of one carrier & eqs. (144)-(146) \\
\hline$\beta \approx 1$ & Frequency exponent of flicker $(1 / f)$ noise. $\mathrm{PSD}=\mathrm{S}(\mathrm{f})=\mathrm{constant} / \mathrm{f}^{\beta}$ & eqs. (144)-(146) \\
\hline$\gamma$ [number] & $\begin{array}{l}\text { Mobility enhancement factor, } \mu \propto\left(\mathrm{V}_{\mathrm{G}}-\mathrm{V}_{\mathrm{T}}\right)^{\gamma} \text { at } \mathrm{V}_{\mathrm{G}}>\mathrm{V}_{\mathrm{T}} \text {. (If OTFT is a p-type } \\
\text { FET, then invert polarity of } \mathrm{V}_{\mathrm{G}} \text { and } \mathrm{V}_{\mathrm{T}} \text {.) }\end{array}$ & eqs. (43), (105) \\
\hline$\Gamma, \Gamma_{\mathrm{ij}}[\mathrm{Hz}](1 / \mathrm{s})$ & Hopping rate, rate of charge hopping between sites $\mathrm{i}$ and $\mathrm{j}$ & eqs. (2), (6) \\
\hline$\Gamma_{\mathrm{c}}[\mathrm{Hz}](1 / \mathrm{s})$ & $\begin{array}{l}\text { Hopping critical rate (average rate of continuous hopping in the critical path of } \\
\text { the percolation network) }\end{array}$ & eq. (2) \\
\hline$\Gamma_{\mathrm{cFB}}[\mathrm{Hz}](1 / \mathrm{s})$ & Hopping critical rate for bulk semiconductor (no bias, $V_{B}=0$ ) & \\
\hline$\Gamma_{\mathrm{o}}[\mathrm{Hz}](1 / \mathrm{s})$ & Hopping attempt rate & eq. (2) \\
\hline$\delta_{\mu \mathrm{ff}}[\%]$ & $\begin{array}{l}\text { Relative variation of the effective mobility } \mu \text { in OTFT with finite film } \\
\text { thickness, compared with the mobility } \mu_{\mathrm{tf}=\infty} \text { of infinitely-thick film OTFT, } \\
\delta_{\mu \mathrm{tf}}=\left(\mu / \mu_{\mathrm{tf}=\infty}-1\right) \times 100 \%\end{array}$ & eqs. (96), (97), (98) \\
\hline$\delta_{\varphi}[\%]$ & Relative difference of $\varphi_{\mathrm{OEF}}$ from $\varphi_{\mathrm{T}}, \delta_{\varphi}=\left(\varphi_{\mathrm{OEF}}-\varphi_{\mathrm{T}}\right) / \varphi_{\mathrm{T}} \times 100 \%$ & eqs. (112), (113) \\
\hline$\Delta \mathrm{E}, \Delta \mathrm{E}_{\mathrm{ij}}[\mathrm{eV}]$ & $\begin{array}{l}\text { Maximum of the magnitudes of the energy jumps by hopping between states } i \\
\text { and } \mathrm{j} \text { and the Fermi level }\end{array}$ & eqs. (6), (7) \\
\hline$\Delta \mathrm{E}, \Delta \mathrm{T}$ & $\begin{array}{l}\text { Supplementary notations for energy and temperature differences in TSF, } \\
\text { obtained in [6] by assumption of Boltzmann statistics }\end{array}$ & $\begin{array}{l}\text { between eqs. (110) and } \\
\text { (111); Figure } 36\end{array}$ \\
\hline$\Delta \mathrm{E}_{\mathrm{H}}[\mathrm{eV}]$ & $\begin{array}{l}\text { Parametric variable for hopping energy by evaluation of distribution of } \\
\text { hopping time. } \Delta \mathrm{E}_{\mathrm{H}}=0 \ldots \mathrm{E}_{\mathrm{H}} \ldots\end{array}$ & eqs. (115), (116) \\
\hline$\Delta \mathrm{N}-\Delta \mu-\Delta \sigma-\Delta \tau$ & Notations for different hypotheses (assumptions) for noise origin & $\begin{array}{l}\text { Figure 41, Figure } 42 \text { eq. } \\
(147)\end{array}$ \\
\hline$\varepsilon_{\mathrm{o}}[\mathrm{F} / \mathrm{cm}]$ & Permittivity of vacuum, $\varepsilon_{0}=8.85 \times 10^{-14} \mathrm{~F} / \mathrm{cm}=85.5 \mathrm{fF} / \mathrm{cm}$ & \\
\hline $\begin{array}{l}\varepsilon_{\mathrm{f}} \equiv \varepsilon_{\mathrm{F}}[\mathrm{F} / \mathrm{cm}] \\
(\mathrm{fF} / \mathrm{cm})\end{array}$ & $\begin{array}{l}\text { Permittivity of the organic semiconducting film. In this work, we use average } \\
\text { value of } \varepsilon_{\mathrm{f}}=3 \varepsilon_{\mathrm{o}} \approx 2.66 \times 10^{-13} \mathrm{~F} / \mathrm{cm}=266 \mathrm{fF} / \mathrm{cm} \text {. }\end{array}$ & \\
\hline$\varepsilon_{\mathrm{z}}[\mathrm{F} / \mathrm{cm}]$ & $\begin{array}{l}\text { Parameter in the power-law trend for mobility deduced in }[82], \varepsilon_{z} \approx 5 \times 10^{-16} \\
\text { F/cm }( \pm 30 \%)\end{array}$ & eq. (82) \\
\hline$\eta\left[\mathrm{S}^{\left(\varphi_{\mathrm{T}} / \varphi_{\mathrm{OEF}} / \mathrm{C}\right]}\right.$ & $\begin{array}{l}\text { Parameter in the relation between charge } \mathrm{qN}_{\mathrm{C}} \text { and VRH specific conductivity } \\
\sigma . \text { In an ideal semiconductor } \varphi_{\mathrm{OEF}}=\varphi_{\mathrm{T}} \text {, and } \eta=\mu \text { becomes the bias- and } \\
\text { temperature-independent mobility. For an exponential DOS, } \eta \text { is also bias- and } \\
\text { spatially independent, but it is temperature dependent. }\end{array}$ & eq. (83) \\
\hline$\Lambda_{\mathrm{o}}[\mathrm{cm}](\mathrm{nm})$ & Decay distance for the hopping rate $\Gamma, \Gamma\left(\mathrm{R}+\Lambda_{\mathrm{o}}\right)=\Gamma(\mathrm{R}) / \exp (1)$ & eq. (2) \\
\hline$\Lambda^{\prime} \approx \Lambda_{\mathrm{o}}[\mathrm{cm}](\mathrm{nm})$ & $\begin{array}{l}\text { Supplementary notation for uncertain value of } \Lambda_{0} . \Lambda^{\prime} \propto\left(T^{\prime} / T\right)^{n} \text {, with n unity, } \\
\mathrm{kT}^{\prime} \approx 4 \mathrm{~B}_{\mathrm{c}} /\left[\Lambda_{0}{ }^{3} \times \operatorname{DOS}\left(\mathrm{E}_{\mathrm{F}}\right)\right] \text {. }\end{array}$ & eq. (3) \\
\hline$\mu\left[\mathrm{cm}^{2} / \mathrm{Vs}\right]$ & $\begin{array}{l}\text { Effective carrier mobility in OTFT. } \mu=\sigma_{\mathrm{sq}} / \mathrm{Q}_{\mathrm{G}} \approx \sigma_{\mathrm{sq}} / \mathrm{Q}_{\mathrm{F}} \text { is ratio of sheet } \\
\text { conductance to sheet charge, thus, } \mu \text { is sheet-type quantity. }\end{array}$ & eq. (42) \\
\hline$\mu_{\mathrm{D}}(\mathrm{D})\left[\mathrm{cm}^{2} / \mathrm{Vs}\right]$ & $\begin{array}{l}\text { Carrier mobility at given spot in the depth } \mathrm{D} \text { of the semiconductor (spot } \\
\text { mobility). } \mu_{\mathrm{D}}(\mathrm{D})=\sigma(\mathrm{D}) / \mathrm{qN}_{\mathrm{C}}(\mathrm{D}) \text { is ratio of VRH specific conductivity } \sigma(\mathrm{D}) \text { to } \\
\text { the charge concentration }\left[\mathrm{qN} \mathrm{N}_{\mathrm{C}}(\mathrm{D})\right] \text { at the spot with spatial coordinate } \mathrm{D} \text {, thus, } \\
\mu_{\mathrm{D}} \text { is volumetric quantity. Distinguish from the effective mobility } \mu \text { in OTFT, } \\
\text { which is a sheet-type quantity. }\end{array}$ & eq. (89) \\
\hline $\begin{array}{l}\mu_{\mathrm{DO}}=\mu(\mathrm{D}=0) \\
{\left[\mathrm{cm}^{2} / \mathrm{Vs}\right]}\end{array}$ & $\begin{array}{l}\text { Spot mobility in the semiconductor at the gate dielectric interface (depth } D=0) \text {. } \\
\text { See } \mu_{D}(D) \text { above. }\end{array}$ & eqs. (91), (92) \\
\hline$\mu_{\mathrm{FB}}\left[\mathrm{cm}^{2} / \mathrm{Vs}\right]$ & $\begin{array}{l}\text { Mobility in bulk semiconductor (no bias, } \mathrm{V}_{\mathrm{B}}=0 \text { ). } \mu_{\mathrm{FB}}(\mathrm{T})=\sigma_{\mathrm{FB}}(\mathrm{T}) / \mathrm{qN} \mathrm{N}_{\mathrm{CFB}}(\mathrm{T}) \text { is } \\
\text { temperature dependent, but spatially and bias independent. }\end{array}$ & eq. (89) \\
\hline$\mu_{\mathrm{o}}\left[\mathrm{V}^{-\gamma} \mathrm{cm}^{2} / \mathrm{Vs}\right]$ & Mobility in OTFT at gate voltage overdrive $\left|V_{\mathrm{G}}-\mathrm{V}_{\mathrm{T}}\right|=1 \mathrm{~V}$ & eqs. (106), (107), (108) \\
\hline$\mu_{\mathrm{oo}}\left[\mathrm{cm}^{2} / \mathrm{Vs}\right]$ & Mobility prefactor in the TFT compact mobility model [6] & eq. (108) \\
\hline$\mu_{\mathrm{tf}=\infty}\left[\mathrm{cm}^{2} / \mathrm{Vs}\right]$ & Effective carrier mobility in OTFT with infinitely-thick film, $\mathrm{t}_{\mathrm{f}}=\infty$ & eq. (96) \\
\hline$\mu_{\mathrm{z}}\left[\mathrm{cm}^{2} / \mathrm{Vs}\right]$ & $\begin{array}{l}\text { Parameter in power-law trend for mobility deduced in [82], } \mu_{\mathrm{z}} \approx 10^{5} \mathrm{~cm}^{2} / \mathrm{Vs} \\
( \pm 50 \%)\end{array}$ & eq. (82) \\
\hline
\end{tabular}




\begin{tabular}{|c|c|c|}
\hline $\begin{array}{l}\text { Symbol } \\
\text { [primary unit] } \\
\text { (secondary units) }\end{array}$ & Meaning and comments & see \\
\hline$\sigma[\mathrm{S} / \mathrm{cm}](\mathrm{S} / \mathrm{m})$ & Specific conductivity $(\mathrm{S} / \mathrm{m}=\mathrm{A} / \mathrm{Vm}=100 \mathrm{~S} / \mathrm{cm})$ & eq. (1), (63) \\
\hline$\sigma_{o}[\mathrm{~S} / \mathrm{cm}](\mathrm{S} / \mathrm{m})$ & Prefactor for specific conductivity $(\mathrm{S} / \mathrm{m}=\mathrm{A} / \mathrm{Vm}=100 \mathrm{~S} / \mathrm{cm})$ & eq. (1) \\
\hline $\begin{array}{l}\sigma_{\mathrm{DO}} \equiv \sigma(\mathrm{D}=0) \\
{[\mathrm{S} / \mathrm{cm}](\mathrm{S} / \mathrm{m})}\end{array}$ & $\begin{array}{l}\text { Specific conductivity in the semiconductor at the gate dielectric interface } \\
\text { (depth } \mathrm{D}=0 \text { ) }\end{array}$ & eqs. (87), (88) \\
\hline$\sigma_{\mathrm{FB}}[\mathrm{S} / \mathrm{cm}](\mathrm{S} / \mathrm{m})$ & $\begin{array}{l}\text { Specific conductivity } \sigma \text { for bulk semiconductor (no bias, no potential bending } \\
\mathrm{V}_{\mathrm{B}}=0 \text { ) }\end{array}$ & eqs. (30), (64) \\
\hline$\sigma_{\mathrm{sq}}[\mathrm{S}](\mathrm{S} / \square)$ & Sheet conductance of the semiconducting film under gate bias $\mathrm{V}_{\mathrm{G}}$ & eq. (41) \\
\hline$\sigma_{\mathrm{sqB}}[\mathrm{S}](\mathrm{S} / \square)$ & Sheet conductance of the semiconducting film at equilibrium (no bias, $V_{B}=0$ ) & eq. (40) \\
\hline$\sigma_{\mathrm{sqx}}[\mathrm{S}](\mathrm{S} / \square)$ & $\begin{array}{l}\text { Characteristic value for sheet conductance obtained by extrapolation of } V_{G} \text { to } \\
V_{\mathrm{Gx}} \text {, at which the temperature dependence of } \sigma_{\mathrm{sq}} \text { is cancelled. See } \mathrm{V}_{\mathrm{Gx}} \text {. }\end{array}$ & $\begin{array}{l}\text { eq. }(54) ; \\
\text { Figure } 19\end{array}$ \\
\hline$\tau[\mathrm{s}]$ & Hopping time, $\tau=1 / \Gamma$ & eqs. (114), (116) \\
\hline$\tau_{\mathrm{c}}[\mathrm{s}]$ & $\begin{array}{l}\text { Hopping critical time (average time for continuous hopping in the critical path } \\
\text { of the percolation network), } \tau_{\mathrm{c}}=1 / \Gamma_{\mathrm{c}}\end{array}$ & eq. (114) \\
\hline$\tau_{\min }, \tau_{\max }[\mathrm{s}]$ & Range of the "plateau" in the distribution of hopping times & $\begin{array}{l}\text { Figure } 37, \\
\text { eq. (128) }\end{array}$ \\
\hline$\tau_{\mathrm{o}}[\mathrm{s}]$ & Hopping attempt time, $\tau_{\mathrm{o}}=1 / \Gamma_{\mathrm{o}}$ & after eq. (117) \\
\hline$\varphi_{\mathrm{A}} \equiv \mathrm{kT} \mathrm{T}_{\mathrm{A}} / \mathrm{q}[\mathrm{V}]$ & $\begin{array}{l}\text { Characteristic "thermal-like voltage" proportional to the effective depth } \mathrm{L}_{\mathrm{A}} \text { of } \\
\text { the conduction channel. See also } \mathrm{T}_{\mathrm{A}} \text {. }\end{array}$ & eq. (47) \\
\hline$\varphi_{\mathrm{ABC}}[\mathrm{V}]$ & Supplementary notation for $\varphi_{\mathrm{A}}, \varphi_{\mathrm{B}}, \varphi_{\mathrm{C}}$ and $\varphi_{\mathrm{OEF}}$ & eq. (79) \\
\hline$\varphi_{\mathrm{B}} \equiv \mathrm{kT} \mathrm{T}_{\mathrm{B}} / \mathrm{q}[\mathrm{V}]$ & $\begin{array}{l}\text { Characteristic "thermal-like voltage" for the logarithmic decay of the potential } \\
\text { bending } V_{B} \text { in the depth of the film. See also } T_{B} \text {. }\end{array}$ & eq. (48) \\
\hline$\varphi_{\mathrm{C}} \equiv \mathrm{kT} \mathrm{C} / \mathrm{q}[\mathrm{V}]$ & $\begin{array}{l}\text { Characteristic "thermal-like voltage" proportional to the carrier concentration } \\
\mathrm{N}_{\mathrm{C}} \text {. See also } \mathrm{T}_{\mathrm{C}} \text {. }\end{array}$ & eq. (49) \\
\hline$\varphi_{\text {cold }} \equiv \mathrm{kT}_{\text {cold }} / \mathrm{q}[\mathrm{V}]$ & $\begin{array}{l}\text { Characteristic "thermal-like voltage" corresponding to the value of } \varphi_{\mathrm{OEF}} \\
\text { extrapolated to absolute zero temperature } \mathrm{T}=0 \text {. For an exponential } \mathrm{DOS}_{\mathrm{SE}} \text {, } \\
\varphi_{\text {cold }}=\varphi_{\mathrm{o}} . \text { See also } \varphi_{\mathrm{OEF}}=\mathrm{kT}_{\mathrm{OFF}} / \mathrm{q} \text {. }\end{array}$ & eq. (79) \\
\hline$\varphi_{\text {DOS }}[\mathrm{V}]$ & $\begin{array}{l}\mathrm{q} \varphi_{\mathrm{DOS}}=[\partial \ln (\mathrm{DOS}) / \partial \mathrm{E}]^{-1} \text { is the reciprocal of the logarithmic slope of } \mathrm{DOS} \text { at } \\
\text { condition for average bending } \mathrm{V}_{\mathrm{B}}=\mathrm{avg} \mathrm{V}_{\mathrm{B}} \text {, and } \varphi_{\mathrm{DOS}} \text { is compared with } \varphi_{\mathrm{B}, \text { an }} \text { of } \\
\text { exponential DOS approximation }\end{array}$ & eq. (71) \\
\hline$\varphi_{\mathrm{o}} \equiv \mathrm{kT}_{\mathrm{o}} / \mathrm{q}[\mathrm{V}]$ & $\begin{array}{l}\text { Characteristic "thermal-like voltage" proportional to the reciprocal of the } \\
\text { logarithmic slope of the single-side exponential DOS } \\
\text { from } \varphi_{\mathrm{To}}, \varphi_{\mathrm{o}}=\varphi_{\mathrm{DOS}} \text {. Distinguish }\end{array}$ & eq. (72) \\
\hline$\varphi_{\mathrm{OEF}} \equiv \mathrm{kT} \mathrm{TEF}_{\mathrm{OF}}[\mathrm{V}]$ & $\begin{array}{l}\text { Effective characteristic "thermal-like voltage", originating from convolution } \\
\text { between Fermi and DOS distributions. For the single-side exponential DOS } \\
\varphi_{\mathrm{SE}}, \\
\text { instances, } \varphi_{\mathrm{OEF}} \text { can replace } \varphi_{\mathrm{o}} \text {. See also } \mathrm{T}_{\mathrm{OEF}} \text {. } \\
\text { in }\end{array}$ & eqs. (79), (80) \\
\hline$\varphi_{\mathrm{T}}[\mathrm{V}](\mathrm{mV})$ & Thermal voltage given by definition as $\varphi_{\mathrm{T}} \equiv \mathrm{kT} / \mathrm{q}$. See also kT. & eq. (60) \\
\hline$\varphi_{\text {To }}[\mathrm{V}](\mathrm{mV})$ & $\begin{array}{l}\varphi_{\mathrm{To}} \approx(0.8 \mathrm{~V} \pm 0.1) \mathrm{V} \text { is voltage parameter, determining the slope in the } \mathrm{s}_{\mathrm{cFB}} \propto 1 / \mathrm{T} \\
\text { temperature dependence. Distinguish from } \varphi_{\mathrm{o}} \equiv \mathrm{kT}_{\mathrm{o}} / \mathrm{q} \text {. }\end{array}$ & eq. (61) \\
\hline$\varphi_{\mathrm{TFT}}[\mathrm{V}]$ & Bias-independent TFT specific voltage in the TFT compact mobility model [6] & eqs. (108), (111) \\
\hline$\psi(\mathrm{t})$ & Distribution density in SM formalism for dispersive transport [95] & eq. (126) \\
\hline $\int \mathrm{dE}, \iint \mathrm{dEdE}$ & $\begin{array}{l}\text { Methods or approaches for calculation of VRH specific conductivity by single } \\
\int \mathrm{dE} \text { and multiple } \iint \mathrm{dEdE} \text { integrations }\end{array}$ & $\begin{array}{l}\text { eqs. (13),(14); } \\
(17),(18),(19),(20)\end{array}$ \\
\hline$\partial\left(\mathrm{B} / \mathrm{B}_{\mathrm{c}}\right) / \partial \tau[1 / \mathrm{s}]$ & Distribution density of hopping times. & Eq. (117) \\
\hline
\end{tabular}




\section{Introduction}

Organic thin-film transistors (OTFTs) are essential building blocks for the organic electronics, which are actively pursued for low-cost, large-area applications. Intended applications are wearable and disposable electronic devices and flexible displays. The OTFT is normally an isolated-gate field-effect transistor (FET), with characteristics similar to a MOSFET. However, compared with the crystalline-semiconductor devices, the charge transport in OTFT is poor. To explain this poor charge transport, and in order to replicate the assumptions in semiconductors, the classical semiconductor theory was significantly modified in the so-called mobility edge models, separating the induced charge in two categories of trapped and mobile charges. The trapped charge, being the larger and with zero mobility, is localized at certain energy levels [1] or distributed in energy tails of states [2] inside an energy band-gap, whereas the mobile charge is considered with a constant characteristic value for the mobility like in a band-like charge transport model. With these assumptions in the mobility edge models, analytical models are developed and confirmed by numerical simulations $[1,2,3,4]$. Within the mobility edge framework, commercial numerical simulators are also available, e.g., Atlas from Silvaco [5] and Sentaurus from Synopsys [4]. On the other hand, the charge localization in organic materials implies that these materials should be understood in terms of semiinsulators, rather than considered as poor semiconductors, since the charge is well localized in the organic materials and spatially continuous transport bands are unlikely [6].

There are several approaches to explain the charge transport in materials with localized charges, assuming, e.g., localization barrier lowering, metallic transitions (Mott-Hubbard), polaron excitation-relaxation, or field emission. A common assumption in these approaches is that the charge is spatially and energetically localized in states, wells, or grains of the amorphous material, and the charge hops between the states with some probability and rate, according to the particular approach. Thus, one generally assumes the hopping charge transport in insulators, but not the freely moving at the thermal velocity charge carriers in the transport band of a semiconductor. One class of charge hopping theories considers that the charge propagates through the material, overcoming various distances and energy barrier heights, with various 
probabilities for successful hops, which is generally the situation in amorphous materials to which the OTFT normally belongs. Owing to the assumption for non-constant distance-energy ranges of hopping, this class of theories is known as variable range hopping (VRH). In this work, we address the numerical simulation of VRH for OTFT to verify many analytical relations for these devices, from different perspectives to complement the numerical simulations of mobility edge models. Surprisingly, reports of fully numerical simulations of VRH for OTFT are lacking in the literature, perhaps due to the large computational volume of these numerical simulations, although similar simulations of VRH have been carried out for other cases, e.g., organic diodes and dispersive transport in amorphous layers. All these numerical simulations, including the most recent (per March 2014), e.g., in [7], consider quasi-equilibrium of spatially-uniform charge concentrations, while it is known that the charge concentration profiles in OTFT are not uniform under gate biasing. Attempts to consider non spatially-uniform charge carrier density in the channel of OTFTs can be seen in later dc compact-model proposals $[8,9]$. The gradients in the OTFT accumulation layers possibly affect the charge transport in OTFT [10]. Note that VRH does not assume a transport band or distinguish between different types of charges, a concept more reasonable for semiinsulators to which the organic materials belong, although it is possible to derive expressions and calculate a "transport energy level" for VRH that is equivalent to an edge of the charge transport band [11, 12]. However, the discussions in [12] indicate several problems in determining the value of the transport energy, e.g., it exists only for Gaussian density of states by consideration of low carrier concentration, and it changes with a carrier-dependent Fermi level, the latter quite difficult to justify for a given material by the established semiconductor and electrochemical theories. The overall impression from the literature is that the band edge is not needed for hopping charge transport, in principle, and the band edge is introduced in the simulation programs for convenience [4] to relate to experimental data for the effective mobility in OTFT.

Recent studies on novel topologies of OTFTs or OTFTs working in different environments [13] use closed-form analytical expressions for the drain current based on analytical VRH expressions with assumed 
VRH parameters $[13,14,15]$. One of the advantages of numerical VRH simulation is that it can be extended to transistors based on 2D systems in which VRH transport is also present [16]. To the best of our knowledge, 2D analytical VRH models are not available or they may not have closed form solutions. Also, at present, numerical VRH simulation is still limited to bulk materials [17]. In addition, prior to analyzing OTFTs or OTFTS working in different environments, , it is important to understand their charge transport characteristics. Therefore, one the main objectives of this work is to build a numerical simulator from the basics of the VRH transport.

After several preceding works of Hung and Gliessman, Conwell, Mott, Anderson, Abrahams and other researchers in the period between 1950 and 1970 (please see references in [18]) it was established around 1970 , e.g. in $[19,20]$, that the VRH conduction is a hopping of localized charges in a random percolation network, and it is widely accepted over the years $[21,22,23]$ that the VRH conductivity is given in principle by

$$
\sigma=\sigma_{\mathrm{o}} \exp \left(-\mathrm{s}_{\mathrm{c}}\right)
$$

where $\sigma$ is the specific conductivity in $\mathrm{S} / \mathrm{m}$ or $\mathrm{S} / \mathrm{cm}$, and $\sigma_{\mathrm{o}}$ is the conductivity prefactor in the same unit. The prefactor $\sigma_{\mathrm{o}}$ is a differential limit for the charge displacement in infinitely small distance $\mathrm{dR} \rightarrow 1 / \infty$. The dimensionless factor $s_{c}>0$ reflects the reduction of charge hoping in the critical path of the percolation network of the amorphous material. The factor $s_{c}$ is a logarithmic measure of the difficulty, which the localized charge meets when propagating through the percolation network, owing to finite distances, energy and other barriers, or lack of empty site to move into, etc.

Conceptually, the critical path allows the localized charges to traverse a material in which there is not a transport band for this charge. The critical path spans the material from end to end (micrometer scale or larger), connecting nanometer-small conductive spots or clusters that may have $s<s_{c}$, but these conductive clusters stay separated from each other owing to the surrounding insulating media with $\mathrm{s}>\mathrm{s}_{\mathrm{c}}$. Thus, the critical paths with $s=s_{c}$ determine the overall hopping conduction, because these paths provide connections (called "bonds") between the conductive spots with $s<s_{c}$ (called "sites") in an otherwise insulating media 
with $\mathrm{s}>\mathrm{s}_{\mathrm{c}}$. The debate was and continues to be, what the factors and the expressions behind $\sigma_{\mathrm{o}}$ and $\mathrm{s}_{\mathrm{c}}$ are, what the particular formats of eq. (1) for different materials and structures are, and how to include temperature, bias (and eventually light illumination or emission) in these equations. To find these, several approaches were taken over the years, a variety of assumptions were made, and a vast amount of integrals were solved in order to obtain analytical expressions for specific or more general cases. However, the origin of the debate is that the VRH is a mathematical theory for a percolation network, in which the rules can be introduced by different ways, and the physics is determined by the type of the rules and parameters embedded in the otherwise purely mathematical template. Consequently, the equations for VRH and some parameters in them are different for "similar" cases, owing to different physically sound assumptions, sequences and techniques of substitutions (e.g., of semi-equalities or proportionalities), limits of integrations and neglecting terms by various considerations. Unfortunately, examining at the literature, one finds the final expressions, but not the detailed derivations, perhaps, because the derivations are bulky, and some steps in these derivations may be questionable. The problem with evaluating VRH models and derivations is evident at present, and it was addressed by critical discussions, such as in [7, 10].

In the next Sec. 2, we present the VRH expressions and methods for calculations, which we have used in our numerical simulator. We are also aware that there is not a unique treatment of charge hopping and many details in past works are missing. Since omissions of details may cause misinterpretations, we took exceptional care to spell in which way we use these VRH expressions. Then, in Sec. 3, we will address the issues for parameter assignment and will present the results from the numerical simulations compared with experimental data. In Sec. 4, a detailed discussion will demonstrate the consistency of the results from the numerical simulations, also outlining essential relations for VRH in OTFT. Building on these, in Sec. 5, we will attempt to give an outlook for feasibility of numerical VRH simulations and a scenario for bridging between analytical and numerical models for OTFT. While we are mainly focused on the stationary (DC) behavior of the OTFT, we shall also provide in Sec. 6 links to non-stationary behaviors of OTFT, such as 
dispersion of hopping time, long-living tails by switching the OTFT and noise. Finally, we will summarize in Sec. 7 our findings in the conclusions. Also, many details in the derivations are provided in appendices.

\section{Theoretical background of the simulator and implementation}

The general eq. (1) for VRH can be treated by several physical approaches, as can be deduced from the references in [18]. Nevertheless, it seems that the most productive approach for VRH became feasible after relating the critical factor $s_{c}$ with the hopping rate $\Gamma$, by

$$
\mathrm{s}_{\mathrm{c}}=\ln \left(\frac{\Gamma_{\mathrm{o}}}{\Gamma_{\mathrm{c}}}\right)=2 \frac{\mathrm{R}_{\mathrm{H}}}{\Lambda_{\mathrm{o}}}=\frac{\mathrm{E}_{\mathrm{H}}}{\mathrm{kT}},
$$

where $\Gamma_{\mathrm{o}}$ is the hopping attempt rate, $\Lambda_{\mathrm{o}}$ is a characteristic length which the charges overcome with probability $\exp (-1)$, and the thermal energy $\mathrm{kT}$ is the product of Boltzmann constant $\left(\mathrm{k} \approx 8.62 \times 10^{-5} \mathrm{eV} / \mathrm{K}\right)$ and absolute temperature T. It is seen in eq. (2) that only $\mathrm{kT}$ is providing the energy in VRH, which means that all other sources of energy are neglected, the system is in a thermal equilibrium. The diffusion is omitted, since no concentration gradient is present in eq. (2). Also, $\Lambda_{\mathrm{o}}$ is a decay distance for the hopping rate $\Gamma$, which is in a format similar to attenuation distance by tunneling, but one interprets $\Lambda_{\mathrm{o}}$ as a localization distance or an effective molecular orbital overlap in organic materials, rather than as the electron wave attenuation by tunneling. Corresponding to the percolation critical path, $\Gamma_{\mathrm{c}}$ is the average rate of continuous hopping in the critical path, $\mathrm{R}_{\mathrm{H}}$ is the maximum distance of successful charge hopping between the spatially distributed charge-localizing sites (from here, we usually use "states" instead of "sites", since "charge localization states" and "density of states, DOS" are widely used terms in semiconductor theories), and $\mathrm{E}_{\mathrm{H}}$ is the maximum energy difference that the carriers can overcome when hopping between states with different energy. Note that $\mathrm{R}_{\mathrm{H}}$ and $\mathrm{E}_{\mathrm{H}}$ are effective values for maximum distance and energy of hopping, thus, they are statistical expectations in distributions, and are not boundaries of the distributions. To obtain expectations, one uses averaging; and we specifically use $\mathrm{R}_{\mathrm{H}}$ and $E_{H}$ in the context of arithmetic averages. (In contrast, for example, the random walk in networks uses quadratic averaging and variances.) Eq. (2) was introduced by eqs. (4.9) and (4.10) in [20] with $\alpha=1 / \Lambda_{0}$, and 
thoroughly analyzed there. Also by eq. (6.1) in [20], when relating the critical factor $s_{c}$ with the hopping rate $\Gamma$, it was given that the conductivity prefactor $\sigma_{\mathrm{o}}$ in eq. (1) is

$$
\sigma_{\mathrm{o}}=\frac{\mathrm{q}^{2}}{\mathrm{kT}} \frac{\Gamma_{\mathrm{o}}}{\Lambda^{\prime}}=\frac{\mathrm{q}}{\left(\frac{\mathrm{kT}}{\mathrm{q}}\right)} \frac{\Gamma_{\mathrm{o}}}{\Lambda_{\mathrm{o}}},
$$

where $\mathrm{q}$ is the magnitude of the electron charge, $(\mathrm{kT} / \mathrm{q})$ is the thermal energy in unit $\mathrm{eV}$, or thermal voltage in $\mathrm{V}$, as used in the simulator, and $\Lambda^{\prime} \approx \Lambda_{0}$. Note that there is a theoretical uncertainty in eq. (3), since it is mentioned in relation to eq. (4.5) in [20] that $\Lambda^{\prime}$ might be not a constant, but a function of the temperature, e.g. $\Lambda^{\prime} \propto\left(\mathrm{T}^{\prime} / \mathrm{T}\right)^{\mathrm{n}}$, with $\mathrm{n} \sim$ unity, $\mathrm{kT} \mathrm{T}^{\prime} \approx 4 \mathrm{~B}_{\mathrm{c}} /\left[\Lambda_{\mathrm{o}}{ }^{3} \times \mathrm{DOS}\left(\mathrm{E}_{\mathrm{F}}\right)\right]$, where $\mathrm{B}_{\mathrm{c}} \sim 9 / \pi \approx 2.86$ is the critical number for threedimensional percolation network ( $\mathrm{B}_{\mathrm{c}} \sim 4$ after other assumptions, see eq. (4.14) in [20]), and $\mathrm{DOS}\left(\mathrm{E}_{\mathrm{F}}\right)$ is the density of states (in unit, e.g., $\mathrm{cm}^{-3} \mathrm{eV}^{-1}$ ) "slowly varying" at the Fermi energy level EF. For example, $\sigma_{0}=1 / 3 \mathrm{q} \Gamma_{\mathrm{o}} /\left[\Lambda_{\mathrm{o}}\left(\mathrm{k} \mathrm{T}_{\mathrm{o}} / \mathrm{q}\right)\right]$ was deduced in [21] after an assumption for random-walk hopping in exponential $\operatorname{DOS} \propto \exp \left[\left(\mathrm{E}-\mathrm{E}_{\mathrm{o}}\right) /\left(\mathrm{kT}_{\mathrm{o}}\right)\right]$, with energy $\mathrm{E}$ being between $\mathrm{E}_{\mathrm{F}}$ and a boundary energy $\mathrm{E}_{\mathrm{o}}$ of the exponential DOS, $E_{F} \leq E<E_{o}$, thus, $n=1$ and the temperature dependence in $\sigma_{o}$ effectively cancelled. One can find also other expressions for VRH conductivity, e.g. in [24] for bulk material based on earlier works of Mott and Davis and simple derivations in [23] that lead essentially to similar analytical results.

Approximate relations and uncertain values for several quantities are regularly observed in the literature on VRH. Numerical simulators, on the other hand, require exact equalities and values of parameters. Therefore, we state the following equation, which we think is the principal equation for the VRH specific conductivity

$$
\sigma=\left\{\frac{\mathrm{q}}{\left(\frac{\mathrm{kT}}{\mathrm{q}}\right)} \frac{\Gamma_{\mathrm{o}}}{\Lambda_{\mathrm{o}}} \exp \left(-\mathrm{s}_{\mathrm{c}}\right)\right\}_{\mathrm{k}=\left[\frac{\mathrm{J}}{\mathrm{K}}\right]} \equiv\left\{\frac{\mathrm{q}}{\mathrm{kT}} \frac{\Gamma_{\mathrm{o}}}{\Lambda_{\mathrm{O}}} \exp \left(-\mathrm{s}_{\mathrm{c}}\right)\right\}_{\mathrm{k}=\left[\frac{\mathrm{eV}}{\mathrm{K}}\right]} .
$$

The right-hand expression of eq. (4) is built in the numerical simulator, using $\mathrm{k} \approx 8.62 \times 10^{-5} \mathrm{eV} / \mathrm{K}$ for the Boltzmann constant, since the energies are in $\mathrm{eV}$ in the simulator. For the other quantities in eq. (4): $\sigma$ is in 
$\mathrm{S} / \mathrm{cm} \equiv \mathrm{A} /(\mathrm{Vcm}) \equiv(\mathrm{S} / \mathrm{m}) / 100 ; \mathrm{s}_{\mathrm{c}}$ is as defined in eq. (1) and obeys the relations in eq. (2), having the meaning of dimensionless natural-logarithm measure for reduction of the hopping rate $\Gamma_{\mathrm{c}}=\Gamma_{\mathrm{o}} \exp \left(-\mathrm{s}_{\mathrm{c}}\right)$ in the critical path of the percolation network, as compared to the hopping attempt rate $\Gamma_{\mathrm{o}} ; \Gamma_{\mathrm{c}}$ and $\Gamma_{\mathrm{o}}$ are in $\mathrm{Hz} \equiv 1 / \mathrm{s} ; \Lambda_{\mathrm{o}}$ is in $\mathrm{cm}$, being a characteristic length, which the charges overcome with probability $\exp (-1) ; \mathrm{q} \approx 1.602 \times 10^{-19} \mathrm{C}$ is the magnitude of the electron charge; and $\mathrm{T}$ is the absolute temperature in $\mathrm{K}$ (Kelvin).

With values depending on theoretical treatment of VRH and assumptions for the DOS, $\Gamma_{\mathrm{o}}$ and $\Lambda_{\mathrm{o}}$ are implemented as constants in the simulator (their values will be defined later for specific cases), leaving for the prefactor $\sigma_{\mathrm{o}}=\mathrm{q} \Gamma_{\mathrm{o}} /\left.\left(\mathrm{kT} \Lambda_{\mathrm{o}}\right)\right|_{\mathrm{k}=[\mathrm{eV} / \mathrm{K}]}$ a multiplicative uncertainty in the order of $\left[\mathrm{A} \times\left(\mathrm{T} / \mathrm{T}^{\prime}\right)^{\mathrm{n}}\right] \sim 0.05 \pm 2.2$ decades for temperatures $\mathrm{T}=100-300 \mathrm{~K}$, with $\mathrm{A} \sim 0.3 \pm 1$ decade, $\mathrm{n} \sim 0-2$ and $\mathrm{T}^{\prime} \sim 200-1000 \mathrm{~K}$. The hopping models use the concentration of charge states $\mathrm{N}_{\mathrm{S}}$ as the concentration of hoping sites [10]. Owing to a relation $\left(\mathrm{b} \Lambda_{\mathrm{o}}\right)^{3}=1 / \mathrm{N}_{\mathrm{S}}, \mathrm{b} \sim 2-10$, both ways of using $\Lambda_{\mathrm{o}}$ or $1 / \mathrm{N}_{\mathrm{S}}{ }^{1 / 3}$ are identically applicable in expressions related to hopping. The parameter $\mathrm{b}$ is normally embedded in another parameter, e.g. regarding eq. (4), as a divider of $\Gamma_{\mathrm{o}}$, or $\ln (\mathrm{b})$ is added to $\mathrm{s}_{\mathrm{c}}$, as in [10].

Eq. (4) is computationally efficient, no iteration loops or large matrices, requiring 3 multiplications ( $\sim$ FLOP each), one division ( 2 FLOPs) and one exponentiation ( 17 FLOPs), altogether about 20FLOPs (floating point operations) and less than 100 bytes of memory. However, the calculation of the value of $s_{c}$ that is used in eq. (4) is computationally extensive, as we discuss below. Therefore, we also keep track in Table VI of the computational volumes related to VRH calculations.

The determination of $s_{c}$ is not trivial, because eq. (2) does not provide a method for its calculation and includes three unknowns, namely $\mathrm{s}_{\mathrm{c}}, \mathrm{E}_{\mathrm{H}}$ and $\mathrm{R}_{\mathrm{H}}$. Thus, some assumptions are required, as detailed in [20], and summarized below.

i) Geometrical assumption. This first assumption provides that the hopping site has sufficient number of bonds to other sites in the critical path of the percolation network. This critical number of bonds per site for hopping in three-dimensional percolation is denoted with $B_{c}$, and satisfies the following relations [20, 21, 22], 
$\mathrm{B}_{\mathrm{c}}=($ Critical number of bonds per site $)$

$=($ Concentration of critical sites $) \times(\text { Mean hopping distance between critical sites })^{3}$,

$=\frac{\text { Concentration of critical bonds }}{\text { Concentration of critical sites }}$

where the first line of this equation is as per the appendix of [21], the second line is given by eq. (4.12) in [20], and the third line is given by eq. (5) in [22]. Any of the approaches in eq. (5) to calculate the critical number of bonds per site results in similar integrals. Also, as mentioned above, $B_{c} \sim 9 / \pi \approx 2.86$ is estimated for uniform DOS from eq. (4.13) in [20], but immediately in eq. (4.14) in [20], the value was revised to $B_{c} \sim 4$ after additional assumptions. In addition, different geometrically-only approach was undertaken in [7], using different formulation of the critical path (overlapping spheres with radii larger than $L^{*}=1 / 2 \Lambda_{0} T_{o} / T$ and distance between the spheres' centers less than $\mathrm{L}^{*}$ ), resulting in another number, 0.219 , for concentration of these spheres in a volume $\left(\mathrm{L}^{*}\right)^{3}$. Comparison of different hopping models in [10] also implies that $\mathrm{B}_{\mathrm{c}}$ is not a unique number and varies between different hopping models derived by different assumptions and techniques. Nevertheless, following the classical formulation for critical path as the ratio of bonds to sites, we have adopted $B_{c} \sim 9 / \pi \approx 2.86$ from $[21,22]$, and therefore, we use this value in the numerical simulator. However, it should be noted that the uncertainty for the value of $\mathrm{B}_{\mathrm{c}}$ is about $30 \%$.

ii) Sum of difficulties assumption. The second assumption for the calculation of $\mathrm{s}_{\mathrm{c}}$ is that the hopping rate $\Gamma$ depends on the "sum of difficulties", that is, the hopping between sites i and $\mathrm{j}$ is with lower rate $\Gamma_{\mathrm{ij}}$ for larger distances $\mathrm{R}_{\mathrm{ij}}$ and larger energy differences $\left|\mathrm{E}_{\mathrm{i}}-\mathrm{E}_{\mathrm{j}}\right|$ between the sites. From a detailed balance of charge around the Fermi level $\mathrm{E}_{\mathrm{F}}$, and at quasi-equilibrium, it is derived in [20] that the measure $\mathrm{s}_{\mathrm{ij}}$ of the difficulty for hopping between sites $\mathrm{i}$ and $\mathrm{j}$ is

$$
\begin{aligned}
& s_{i j}=\ln \left(\frac{\Gamma_{o}}{\Gamma_{i j}}\right) \\
& =2 \frac{R_{i j}}{\Lambda_{o}}+\frac{\left|E_{i}-E_{j}\right|+\left|E_{i}-E_{F}\right|+\left|E_{j}-E_{F}\right|}{2 k T} \\
& =2 \frac{R_{i j}}{\Lambda_{o}}+\frac{\max \left(\left|E_{i}-E_{j}\right|,\left|E_{i}-E_{F}\right|,\left|E_{j}-E_{F}\right|\right)}{k T}=2 \frac{R_{i j}}{\Lambda_{o}}+\frac{\Delta E_{i j}}{k T}
\end{aligned}
$$


where $\Delta \mathrm{E}_{\mathrm{ij}}$ is the maximum of the magnitudes of the energy jumps by hopping between states $\mathrm{i}$ and $\mathrm{j}$ and the Fermi level. When considering the critical path with $\mathrm{s}_{\mathrm{c}}$, then one obtains

$$
\mathrm{s}_{\mathrm{c}}=\ln \left(\frac{\Gamma_{\mathrm{o}}}{\Gamma_{\mathrm{c}}}\right)=2 \frac{\mathrm{R}}{\Lambda_{\mathrm{o}}}+\frac{\Delta \mathrm{E}}{\mathrm{kT}}>0 \text {, with } \mathrm{R}_{\mathrm{H}} \geq \mathrm{R} \geq 0 \text { for } 0 \leq \Delta \mathrm{E} \leq \mathrm{E}_{\mathrm{H}} \text {, }
$$

where the hopping distance $\mathrm{R}$ and energy difference $\Delta \mathrm{E}$ can vary between different hopping states, but their sum weighted by $2 / \Lambda_{0}$ and $1 / \mathrm{kT}$ in eq. (7) is equal to $\mathrm{s}_{\mathrm{c}}$; and the sum is (on average) independent of the particular pair of values for $\mathrm{R}$ and $\Delta \mathrm{E}$.

iii) DOS assumption. The above "geometrical" and "sum of difficulties" assumptions for VRH provide two relations for the three unknowns, namely $\mathrm{s}_{\mathrm{c}}, \mathrm{E}_{\mathrm{H}}$ and $\mathrm{R}_{\mathrm{H}}$. The third relation is from their link to the DOS. Various approaches to combine these three relations can be found in [7, 10]. We have implemented two approaches of single $\int \mathrm{dE}$ and multiple $\iint \mathrm{dEdE}$ integrations in the numerical simulator. In these approaches, one gradually increases the hopping energy $E_{H}$ in iterative calculations with predetermined and, thus, known DOS, until both the "geometrical" and "sum of difficulties" assumptions are satisfied. The details for single $\int \mathrm{dE}$ and multiple $\iint \mathrm{dEdE}$ integrations are given in Secs. 2.1 and 2.2.

\subsection{Determination of energy $E_{H}$, hopping critical factor $s_{c}$, and distance $R_{H}$ by single $\int d E$}

The simplest approach to determine $s_{c}$ is indirectly proposed in [20] by taking a nearly constant DOS. Consider the second line in eq. (5). Guess a value for the maximum hopping energy $\mathrm{E}_{\mathrm{H}}$ and, corresponding to that guess, obtain from eq. (2) the maximum hopping distance $R_{H}=\left(\Lambda_{0} / 2\right)\left(E_{H} / k T\right)$. The concentration of critical sites is

$$
\mathrm{CS}_{\mathrm{c}}\left(\mathrm{E}_{\mathrm{H}}\right)=\int_{-\mathrm{E}_{\mathrm{H}}}^{+\mathrm{E}_{\mathrm{H}}} \operatorname{DOS}\left(\mathrm{E}_{\mathrm{F}}+\Delta \mathrm{E}\right) \mathrm{d} \Delta \mathrm{E}
$$

Consider that the spatial distribution of the states is uniform. Then, the mean hopping distance can be obtained as the average of the normalized spherical volume, because $\mathrm{R}_{\mathrm{H}}$ is a constant, and 


$$
\langle\mathrm{R}\rangle^{3}=\mathrm{R}_{\mathrm{H}}^{3}\left\langle\frac{\mathrm{R}}{\mathrm{R}_{\mathrm{H}}}\right\rangle^{3}
$$

The normalized radius $r$ of the sphere is the ratio $R / R_{H}$, thus $r$ is between 0 and 1 , and the volume of the sphere is $4 \pi r^{3} / 3$. From eq. (7), $R$ is a linear function of $\Delta E$, and $R=0$ when $\Delta E=E_{H}$. Conversely, $R=R_{H}$ when $\Delta \mathrm{E}=0$. Therefore, in normalized form

$$
r(\Delta E)=\frac{R}{R_{H}}=1-\frac{|\Delta E|}{E_{H}} \geq 0 \text { for }|\Delta E| \leq E_{H}
$$

and the average volume of hopping around a state becomes

$$
\begin{aligned}
& \langle\mathrm{R}\rangle^{3}=\mathrm{R}_{\mathrm{H}}^{3}\left\langle\frac{\mathrm{R}}{\mathrm{R}_{\mathrm{H}}}\right\rangle^{3} \\
& =\mathrm{R}_{\mathrm{H}}^{3} \int_{-\mathrm{E}_{\mathrm{H}}}^{+\mathrm{E}_{\mathrm{H}}} \frac{4}{3} \pi\left(1-\frac{|\Delta \mathrm{E}|}{\mathrm{E}_{\mathrm{H}}}\right)^{3}\left[\frac{\mathrm{DOS}\left(\mathrm{E}_{\mathrm{F}}+\Delta \mathrm{E}\right)}{\mathrm{CS}_{\mathrm{c}}\left(\mathrm{E}_{\mathrm{H}}\right)}\right] \mathrm{d} \Delta \mathrm{E}
\end{aligned}
$$

where the averaging is weighted with the term $\left[\mathrm{DOS} / \mathrm{CS}_{\mathrm{c}}\right]$ in the square brackets. The term $\left[\mathrm{DOS} / \mathrm{CS}_{\mathrm{c}}\right]$ is the probability density to have the state with energy $\left(\mathrm{E}_{\mathrm{F}}+\Delta \mathrm{E}\right)$ within the population $\mathrm{CS}_{\mathrm{c}}\left(\mathrm{E}_{\mathrm{H}}\right)$ of critical sites with energy $|\Delta E| \leq E_{H}$; and $C_{c}$ is given by eq. (8). Note that $C_{c}$ is a constant in eq. (11), and can be moved outside the integral together with $4 \pi / 3$. Therefore, substituting eqs. (8) and (11) in the second line of eq. (5), $\mathrm{CS}_{\mathrm{c}}$ is cancelled, and using $\mathrm{R}_{\mathrm{H}}=\left(\Lambda_{\mathrm{o}} / 2\right)\left(\mathrm{E}_{\mathrm{H}} / \mathrm{kT}\right)$ from eq. (2), one gets

$$
\mathrm{B}_{\mathrm{c}}=\frac{4}{3} \pi\left[\left(\frac{\Lambda_{\mathrm{O}}}{2} \frac{\mathrm{E}_{\mathrm{H}}}{\mathrm{kT}}\right)^{3}\right]_{-\mathrm{E}_{\mathrm{H}}}^{+\mathrm{E}_{\mathrm{H}}}\left(1-\frac{|\Delta \mathrm{E}|}{\mathrm{E}_{\mathrm{H}}}\right)^{3} \operatorname{DOS}\left(\mathrm{E}_{\mathrm{F}}+\Delta \mathrm{E}\right) \mathrm{d} \Delta \mathrm{E}
$$

One sees in this equation that the left-hand side is a constant and the right-hand expression is a function only of one unknown, the hopping energy $\mathrm{E}_{\mathrm{H}}$. Thus, this equation can be solved to find $\mathrm{E}_{\mathrm{H}}$. In the numerical simulator, the equation is arranged as

$$
\operatorname{target}=\frac{6 \mathrm{~B}_{\mathrm{c}}}{\pi}\left(\frac{\mathrm{kT}}{\Lambda_{\mathrm{O}}}\right)^{3} \stackrel{?}{=} \int_{-\mathrm{E}_{\mathrm{H}}}^{0}\left(\mathrm{E}_{\mathrm{H}}+\mathrm{E}\right)^{3} \operatorname{DOS}\left(\mathrm{E}_{\mathrm{F}}+\mathrm{E}\right) \mathrm{dE}+\int_{0}^{+\mathrm{E}_{\mathrm{H}}}\left(\mathrm{E}_{\mathrm{H}}-\mathrm{E}\right)^{3} \mathrm{DOS}\left(\mathrm{E}_{\mathrm{F}}+\mathrm{E}\right) \mathrm{dE},
$$


with $\Delta \mathrm{E}$ simplified as $\mathrm{E}$ in the notations. Since $\mathrm{E}_{\mathrm{H}}$ is determined by one integration, then we denote this approach as "single $\int \mathrm{dE}$ ". The simulator gradually increases the value of $\mathrm{E}_{\mathrm{H}}$ in an iterative procedure until the integration reaches the target value of $\left(6 \mathrm{~B}_{\mathrm{c}} / \pi\right)\left(\mathrm{kT} / \Lambda_{\mathrm{o}}\right)^{3}$, as indicated by the question mark above the second equality sign. The integration is implemented by simple Riemann sums. In particular,

$$
\text { target }=\frac{6 \mathrm{~B}_{\mathrm{c}}}{\pi}\left(\frac{\mathrm{kT}}{\Lambda_{\mathrm{O}}}\right)^{3} \stackrel{?}{=} \mathrm{B}\left(\mathrm{E}_{\mathrm{H}}\right) \equiv \mathrm{dE} \sum_{\mathrm{i}=-\mathrm{E}_{\mathrm{H}} / \mathrm{dE}}^{+\mathrm{E}_{\mathrm{H}} / \mathrm{dE}} \operatorname{DOS}\left(\mathrm{E}_{\mathrm{F}}+\mathrm{E}_{\mathrm{i}}\right) \times\left\{\begin{array}{l}
\left(\mathrm{E}_{\mathrm{H}}-\mathrm{E}_{\mathrm{i}}\right)^{3}, \text { if } \mathrm{i}>0 \\
1, \text { if } \mathrm{i}=0 \\
\left(\mathrm{E}_{\mathrm{H}}+\mathrm{E}\right)^{3}, \text { if } \mathrm{i}<0
\end{array}\right.
$$

where $\mathrm{B}\left(\mathrm{E}_{\mathrm{H}}\right)$ is the value calculated by the right-hand expression. The computational volume of eq. (14) is moderate and the required memory is also not large - see Table VI. After the iterations, the value of the hopping energy $E_{H}$ is known, and the critical factor $\mathrm{s}_{c}=\mathrm{E}_{\mathrm{H}} / \mathrm{kT}$ is calculated from eq. (2). From the right-hand equality of the same equation, although $s_{c}$ is already known, the hopping distance $R_{H}=\left(\Lambda_{0} / 2\right)\left(E_{H} / k T\right)$ is also calculated at the assumed values for $\Lambda_{\mathrm{o}}$ and $\mathrm{kT}$.

\subsection{Determination of energy $E_{H}$, hopping critical factor $s_{c}$, and distance $R_{H}$ by multiple $\iint d E d E$}

The second approach implemented in the simulator for combining the "geometrical" and "sum of difficulties" assumptions for VRH is based on eq. (6) with detailed energy differences between hopping states and Fermi level. The calculation follows the procedure proposed in the appendix of [21] for cases when the DOS is not slowly varying. In contrast to the approach of single $\int \mathrm{dE}$, one needs to scan the entire $2 \mathrm{D}$ mesh $\left(\mathrm{E}_{\mathrm{i}}, \mathrm{E}_{\mathrm{j}}\right)$ of energies to find the differences $\Delta \mathrm{E}_{\mathrm{ij}}$ and eliminate those with magnitude larger than $\mathrm{E}_{\mathrm{H}}$, and then, to calculate hopping distances $\mathrm{R}_{\mathrm{ij}}$ and to perform $2 \mathrm{D}$ averaging. Thus, the calculation is $2 \mathrm{D}$; it is in several steps and with multiple integrations. Therefore, we denote this approach as "multiple $\iint \mathrm{dEdE}$ ", detailing below the calculation steps in this approach. Note that $E_{i}$ and $E_{j}$ are independent variables that also

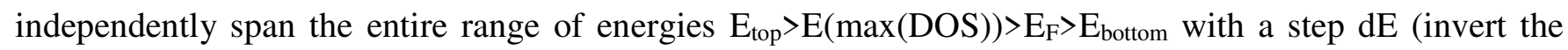
inequalities for p-type devices, as are the OTFTs normally). 


\subsubsection{Determination of the range of hopping distances in multiple $\iint \mathrm{dEdE}$}

The hopping distances $R_{i j}$ is a $2 D$ matrix for each pair $\left(E_{i}, E_{j}\right)$, and $R_{i j}$ also depend on the guess for maximum hopping energy $E_{H}$. When considering the critical path with $s_{c}$, then $s_{i j}=s_{c}=E_{H} / k T$ from eq. (2), and eq. (6) is rewritten as

$$
\begin{aligned}
& \mathrm{s}_{\mathrm{c}}=\ln \left(\frac{\Gamma_{\mathrm{o}}}{\Gamma_{\mathrm{c}}}\right)=\frac{\mathrm{E}_{\mathrm{H}}}{\mathrm{kT}} \\
& =2 \frac{\mathrm{R}_{\mathrm{ij}}}{\Lambda_{\mathrm{o}}}+\frac{\max \left(\left|\mathrm{E}_{\mathrm{i}}-\mathrm{E}_{\mathrm{j}}\right|,\left|\mathrm{E}_{\mathrm{i}}-\mathrm{E}_{\mathrm{F}}\right|,\left|\mathrm{E}_{\mathrm{j}}-\mathrm{E}_{\mathrm{F}}\right|\right)}{\mathrm{kT}}
\end{aligned}
$$

Solving for $\mathrm{R}_{\mathrm{ij}}$, one gets the hopping distances at given $\mathrm{E}_{\mathrm{H}}$ as function of $\mathrm{E}_{\mathrm{i}}$ and $\mathrm{E}_{\mathrm{j}}$ from

$$
\mathrm{R}_{\mathrm{ij}}\left(\mathrm{E}_{\mathrm{i}}, \mathrm{E}_{\mathrm{j}}, \mathrm{E}_{\mathrm{H}}\right)=\frac{\Lambda_{\mathrm{O}}}{2 \mathrm{kT}} \max \left\{0, \mathrm{E}_{\mathrm{H}}-\max \left(\left|\mathrm{E}_{\mathrm{i}}-\mathrm{E}_{\mathrm{j}}\right|,\left|\mathrm{E}_{\mathrm{i}}-\mathrm{E}_{\mathrm{F}}\right|,\left|\mathrm{E}_{\mathrm{j}}-\mathrm{E}_{\mathrm{F}}\right|\right)\right\}
$$

The two functions $\max (\ldots)$ choose the maximum of the energy jump and eliminate bonds that require a jump larger than $\mathrm{E}_{\mathrm{H}}$. Eq. (16) is simple for coding, but it is computationally extensive because it is $2 \mathrm{D}$ and requires large memory, as seen in Table VI and explained in Appendix 1.

\subsubsection{Determination of average hopping bonds for each $E$ in multiple $\iint \mathrm{dEdE}$}

This is the first integration along energies $E_{j}$. It calculates volumetrically the average number of hopping bonds per site (BPS) with energy $\mathrm{E}_{\mathrm{i}}$, from

$$
\operatorname{BPS}_{\mathrm{i}}\left(\mathrm{E}_{\mathrm{i}}, \mathrm{E}_{\mathrm{H}}\right)=\int_{-\mathrm{E}_{\mathrm{H}}}^{+\mathrm{E}_{\mathrm{H}}} \frac{4}{3} \pi\left[\mathrm{R}_{\mathrm{ij}}\left(\mathrm{E}_{\mathrm{i}}, \mathrm{E}_{\mathrm{j}}, \mathrm{E}_{\mathrm{H}}\right)\right]^{3} \operatorname{DOS}\left(\mathrm{E}_{\mathrm{F}}+\mathrm{E}_{\mathrm{j}}\right) d \mathrm{E}_{\mathrm{j}},
$$

having the averaging weighted by $\operatorname{DOS}_{j}$ for the states with energy $E_{j}$ receiving the charge from states with energy $E_{i}$. BPS $S_{i}$ is a vector of size $n=\left(E_{t o p}-E_{\text {bottom }}\right) / d E \sim 2000$. The integration is implemented in the simulator by a simple Riemann sum, as

$$
\operatorname{BPS}_{\mathrm{i}}\left(\mathrm{E}_{\mathrm{i}}, \mathrm{E}_{\mathrm{H}}\right)=\frac{4}{3} \pi \mathrm{dE} \sum_{\mathrm{j}=\mathrm{E}_{\text {bottom }} / \mathrm{dE}}^{+\mathrm{E}_{\text {top }} / \mathrm{dE}}\left[\mathrm{R}_{\mathrm{ij}}\left(\mathrm{E}_{\mathrm{i}}, \mathrm{E}_{\mathrm{j}}, \mathrm{E}_{\mathrm{H}}\right)\right]^{3} \operatorname{DOS}\left(\mathrm{E}_{\mathrm{F}}+\mathrm{E}_{\mathrm{j}}\right) .
$$


While simple for coding, one should be very careful with this equation, because it is computationally the most extensive - please see Appendix 1 and Table VI for eq. (18). One must convert the exponentiation $\left(\mathrm{R}_{\mathrm{ij}}\right)^{3}$ into multiplication $\left(\mathrm{R}_{\mathrm{ij}} \mathrm{R}_{\mathrm{ij}} \mathrm{R}_{\mathrm{ij}}\right)$ in order to have acceptable computational throughput. Sparse multiplication, omitting $\mathrm{R}_{\mathrm{ij}}=0$, would greatly reduce the computational volume. However, we did not use sparse matrices, in order to minimize the probability for human errors by coding, since the sparse matrix operations require code overhead for checking of omitted elements, changing the manner of the computation. The consequence was that we needed to run parallel computing, which we could afford in the particular investigation, but it is not desirable for commercial simulators in general, since the conductivity or mobility calculation is just one of the many tasks to be performed for simulation of the current in OTFTs.

\subsubsection{Determination of the overall average of hopping bonds per site in multiple $\iint \mathrm{dEdE}$}

This step determines the value that has to be compared with the "geometrical" assumption for VRH, the critical number $\mathrm{B}_{\mathrm{c}}$ of bonds per site, according to the first line of eq. (5) and comprises weighted averaging of $\mathrm{BPS}_{\mathrm{i}}$ along $\mathrm{E}_{\mathrm{i}}$. The weighting is with the density of bonds, and mathematically corresponds to

$$
\operatorname{target}=\mathrm{B}_{\mathrm{c}} \stackrel{?}{=} \int_{-\mathrm{E}_{\mathrm{H}}}^{+\mathrm{E}_{\mathrm{H}}} \mathrm{BPS}_{\mathrm{i}}\left(\mathrm{E}_{\mathrm{i}}, \mathrm{E}_{\mathrm{H}}\right)\left[\frac{\mathrm{BPS}_{\mathrm{i}}\left(\mathrm{E}_{\mathrm{i}}, \mathrm{E}_{\mathrm{H}}\right) \operatorname{DOS}\left(\mathrm{E}_{\mathrm{F}}+\mathrm{E}_{\mathrm{i}}\right)}{\int_{-\mathrm{E}_{\mathrm{H}}}^{+\mathrm{E}_{\mathrm{H}}} \mathrm{BPS}_{\mathrm{i}}\left(\mathrm{E}_{\mathrm{i}}, \mathrm{E}_{\mathrm{H}}\right) \operatorname{DOS}\left(\mathrm{E}_{\mathrm{F}}+\mathrm{E}_{\mathrm{i}}\right) \mathrm{dE}_{\mathrm{i}}}\right] \mathrm{dE}_{\mathrm{i}} \text {, }
$$

where the weighting function is the product of hopping bonds and hopping sites for each energy $E_{i}$, in the numerator in the square brackets, divided on the integral of this product for all energies in the denominator. As in eqs. (13) and (14), the question mark above the second equality sign indicates that $\mathrm{E}_{\mathrm{H}}$ is gradually increased in this and preceding steps of calculation by multiple $\iint \mathrm{dEdE}$, until the target value of $\mathrm{B}_{\mathrm{c}}$ is reached. Converting the integrals into Riemann sums, one also rationalizes the expressions, since the integral in the square brackets is a constant for the outer integral, and by cancelling the same uniform integration step $\mathrm{dE}_{\mathrm{i}}$ in the numerator and denominator, achieving 


$$
\operatorname{target}=\mathrm{B}_{\mathrm{c}} \stackrel{?}{=} \mathrm{B}\left(\mathrm{E}_{\mathrm{H}}\right) \equiv \frac{\sum_{\mathrm{i}=\mathrm{E}_{\text {bottom }} / \mathrm{dE}}^{+\mathrm{E}_{\text {top }} / \mathrm{dE}} \sum_{\mathrm{i}=\mathrm{E}_{\text {bottom }} / \mathrm{dE}} \operatorname{BPS}_{\mathrm{i}}\left(\mathrm{E}_{\mathrm{i}}, \mathrm{E}_{\mathrm{H}}\right) \mathrm{BPS}_{\mathrm{i}}\left(\mathrm{E}_{\mathrm{i}}, \mathrm{E}_{\mathrm{H}}\right) \operatorname{DOS}\left(\mathrm{E}_{\mathrm{F}}+\mathrm{E}_{\mathrm{i}}\right)}{\left.\mathrm{dE}_{\mathrm{i}}, \mathrm{E}_{\mathrm{H}}\right) \operatorname{DOS}\left(\mathrm{E}_{\mathrm{F}}+\mathrm{E}_{\mathrm{i}}\right)},
$$

where $\mathrm{B}\left(\mathrm{E}_{\mathrm{H}}\right)$ is the value calculated by the right-hand expression. The computational volume of the righthand expression is moderate, since BPS $\mathrm{i}_{\mathrm{i}}$ and DOS are vectors; see Table VI and Appendix 1.

Thus, the multiple $\iint \mathrm{dEdE}$ is an iteration loop of three steps of calculations with eqs. (16), (18) and (20), in which the value of $E_{H}$ is gradually increased, until $B\left(E_{H}\right)=B_{c}$ is reached and the iteration is terminated. After the iteration, the value of $\mathrm{E}_{\mathrm{H}}$ is known, and the critical factor $\mathrm{s}_{\mathrm{c}}=\mathrm{E}_{\mathrm{H}} / \mathrm{kT}$ is calculated from eq. (2). As in the single $\int \mathrm{dE}$, the hopping distance $\mathrm{R}_{\mathrm{H}}=\left(\Lambda_{0} / 2\right)\left(\mathrm{E}_{\mathrm{H}} / \mathrm{kT}\right)$ is also calculated at the assumed constant values for $\Lambda_{\mathrm{o}}$ and $\mathrm{kT}$.

\subsection{Summarizing comparison between the single $\int \mathrm{dE}$ and multiple $\iint \mathrm{dEdE}$}

To summarize, both methods of calculation use a given range $\left(\mathrm{E}_{\mathrm{top}}-\mathrm{E}_{\mathrm{bottom}}\right)$ of energies $\mathrm{E}$, arbitrary but predetermined DOS(E), Fermi level $E_{F}$ and a guess for the hopping energy $E_{H}$. The guess for $E_{H}$ is ramped gradually in the iteration loop until the calculations reach a target value that corresponds to the "geometrical" assumption for the critical number of bonds per hopping site. The last used value of $E_{H}$ is then the hopping energy, from which the critical factor $\mathrm{s}_{\mathrm{c}}=\mathrm{E}_{\mathrm{H}} / \mathrm{kT}$ and the hopping distance $\mathrm{R}_{\mathrm{H}}=\left(\Lambda_{\mathrm{o}} / 2\right)\left(\mathrm{E}_{\mathrm{H}} / \mathrm{kT}\right)$ are calculated using eq. (2).

The calculation approaches of single $\int \mathrm{dE}$ and multiple $\iint \mathrm{dEdE}$ integration are fully numerical. They are based on the principles for calculation of the VRH conductivity in a 3D percolation network, but not on analytical expressions that are valid only for particular type of DOS, e.g., exponential, or other various assumptions for DOS in analytical derivations.

The approach of single $\int \mathrm{dE}$ integration is a one-step calculation, which uses eq. (14) to calculate the value $\mathrm{B}\left(\mathrm{E}_{\mathrm{H}}\right)$ with a target value of $\left(6 \mathrm{~B}_{\mathrm{c}} / \pi\right)\left(\mathrm{kT} / \Lambda_{\mathrm{o}}\right)^{3}$, and the computational volume with this approach is small to moderate, scaling nearly linearly with the size $n=E_{H} / d E$ of the energy mesh. For 
$\mathrm{n}=\mathrm{E}_{\mathrm{H}} / \mathrm{dE} \approx 1 \mathrm{eV} / 1 \mathrm{meV} \sim 1000$, the computational volume and occupied memory are given in Appendix 1 and in line "sum 1" of Table VI.

The approach of multiple $\iint \mathrm{dEdE}$ integrations is three-step calculation, which uses eqs. (16), (18) and (20) with a target value of $B_{c}$ for the result $B\left(E_{H}\right)$ from the latter equation. The multiple $\iint d E d E$ is computationally extensive, because it requires spanning the $2 \mathrm{D}$ mesh of size $\mathrm{n}^{2}$ for the entire range of energies. Consequently, the computation scales as $n^{2}$ in the approach of multiple $\iint d E d E$ integrations. For $\mathrm{n}=\left(\mathrm{E}_{\text {top }}-\mathrm{E}_{\mathrm{bottom}}\right) / \mathrm{dE} \approx 2 \mathrm{eV} / 1 \mathrm{meV} \sim 2000$, the computational volume and occupied memory are given in Appendix 1 and in the line "sum 2" of Table VI.

Both approaches follow a similar iteration procedure for determining $\mathrm{E}_{\mathrm{H}}$ by a gradual variation of the values of $\mathrm{E}_{\mathrm{H}}$, until the calculations match the abovementioned target values, as explained below. One can also utilize other approaches for VRH calculations. However, we shall restrict the numerical simulations to the above two approaches of single $\int \mathrm{dE}$ and multiple $\iint \mathrm{dEdE}$ integrations, because these two approaches are the most basic methods for the determination of the VRH conduction for any type of DOS or combination of types. We have noted in the literature $[7,10,12,20,21,25]$ a vulnerability of the analytical expressions with respect to assumptions and derivations of VRH models, overlaying VRH with assumptions for DOS type $[10,12,21,25]$ or changing the rules of the percolation network calculation $[7,20,21]$.

\subsection{VRH calculation module}

Figure 1 depicts the iteration procedure of the VRH calculation, which first determines the hopping energy $\mathrm{E}_{\mathrm{H}}$ and then the other VRH quantities mentioned in the previous Sec. 2.3. The input variables for the VRH calculation module are the Fermi level $\mathrm{E}_{\mathrm{F}} \sim 1 \mathrm{eV}(\max )$, the uniform energy 1D mesh $\mathrm{E}$, and the 1D vector of DOS values for the same energy mesh. The energy mesh is with range $\left(E_{t o p}-E_{b o t t o m}\right) \sim 2 \mathrm{eV}$ and step $\mathrm{dE} \sim 1 \mathrm{meV}$. The energy mesh $\mathrm{E}$ and $\mathrm{DOS}(\mathrm{E})$ are generated in the electrostatic part of the simulator, which will be presented later. A selector chooses the branch either of single $\int \mathrm{dE}$ or multiple $\iint \mathrm{dEdE}$ integration. 
The selected branch calculates the value $\mathrm{B}\left(\mathrm{E}_{\mathrm{H}}\right)$ corresponding to the guess for $\mathrm{E}_{\mathrm{H}}$, the latter initially $\mathrm{E}_{\mathrm{H}}=\mathrm{s} \mathrm{E}_{\mathrm{H}} \approx \mathrm{kT}$. After the calculation, a new guess $\mathrm{nE}_{\mathrm{H}}$ of gradual variation of the hopping energy is made, by

$$
\mathrm{nE}_{\mathrm{H}}=\mathrm{E}_{\mathrm{H}}+\mathrm{s} \mathrm{E}_{\mathrm{H}} \times \ln \left[\frac{\mathrm{B}\left(\mathrm{E}_{\mathrm{H}}\right)}{\text { target }}\right] \text {, with } \mathrm{nE}_{\mathrm{H}}>\min \left(\mathrm{E}_{\mathrm{H}}\right) \sim \mathrm{kT} / 100 \text {, }
$$

where $\mathrm{nE}_{\mathrm{H}}$ is used instead of $\mathrm{E}_{\mathrm{H}}$ in the next iteration. The variation of $\mathrm{E}_{\mathrm{H}}$ is gradual, owing to the logarithmic function, and one must also take care setting $\mathrm{nE}_{\mathrm{H}}$ to a minimum positive value $\sim \mathrm{kT} / 100$, if the new guess $\mathrm{nE}_{\mathrm{H}}$ is wrong, e.g., $\mathrm{nE}_{\mathrm{H}}<0$. The variation of $\mathrm{E}_{\mathrm{H}}$ should be chosen gradual between iterations, since the integrals are steep functions of $\mathrm{E}_{\mathrm{H}}$. Then, one repeats the iteration procedure until the difference of $E_{\mathrm{H}}$ obtained after two consecutive iterations is small e.g. $\mathrm{nE}_{\mathrm{H}}-\mathrm{E}_{\mathrm{H}} \leq \pm 10^{-16}=4 \mathrm{LSB}$ (least significant bits) by double precision. With the value for $\mathrm{E}_{\mathrm{H}}$ from the last iteration, one calculates the critical factor $\mathrm{s}_{\mathrm{c}}=\mathrm{E}_{\mathrm{H}} / \mathrm{kT}$ by eq. (2) and from it, the final result for VRH specific conductivity $\sigma=\sigma_{0} \exp \left(-\mathrm{s}_{c}\right)$ by eq. (1) with $\sigma_{\mathrm{o}}=\mathrm{q} \Gamma_{\mathrm{o}} /\left[\Lambda_{\mathrm{o}}\left(\mathrm{kT}_{\mathrm{o}} / \mathrm{q}\right)\right]$ from eq. (3). It is sufficient to return $\sigma$ and $\mathrm{E}_{\mathrm{H}}$ from the calculation, and if necessary, one can also obtain the value for the hopping distance $R_{H}=\left(\Lambda_{0} / 2\right)\left(E_{H} / k T\right)$ from eq. (2).

The convergence of the iteration procedure is illustrated in Figure 2 for the surface and the back of the pentacene TFT using the parameters given in Table IV later. One observes that the rate of convergence is about one decimal digit for $\mathrm{E}_{\mathrm{H}}$ per 3-5 iterations. Thus, for 15 digits, one has 50-80 iterations, but rarely more than 100 iterations (at low temperature). Therefore, "100 max" is stated in column "iterations" in Table VI, and the computational volumes in rows "sum1" and "sum 2" correspond the computational volumes of the VRH calculation module operated in the branches of single $\int \mathrm{dE}$ and of multiple $\iint \mathrm{dEdE}$ integrations, respectively. The computational volume of the gradual stepping of $E_{H}$ by eq. (21) is small, as seen Table VI and explained in Appendix 1, compared to the computational volumes in the branches.

So far, we have presented the VRH numerical simulator for equilibrium, that is, for bulk material, without non-equilibrium charge and potential bending $V_{B}$ induced by the gate bias $V_{G}$ in the semiconducting film of the OTFT. Reviewing eqs. (11)-(14) and (17)-(20), one observes that the potential bending has to be included in the argument for the expressions for DOS, replacing the Fermi level with a quasi-Fermi level, known also as IMREF. The gate bias $V_{G}$ induces the bending voltage $V_{B}$ so that the DOS 
becomes "closer" to the Fermi level $\mathrm{E}_{\mathrm{F}}$ at higher bias, as illustrated in Figure 3. The bending voltage represents the bending of the semiconductor energy bands through $\left(-\mathrm{qV}_{\mathrm{B}}\right)$. The polarity inversion is due to the convention that the semiconductor energy diagrams are given for the potential energy of electrons. The Fermi level crosses the DOS tail, although $\mathrm{E}_{\mathrm{F}}$ might be not able to cross the HOMO centroid level even at a high bias, since this is equivalent to ionizing almost every single molecule in the organic material, which corresponds to very high carrier concentrations above $10^{21} \mathrm{~cm}^{-3}$. To clarify the following definition of IMREF, consider an exponential DOS for holes, given by

$$
\begin{aligned}
& \operatorname{DOS}\left(E \geq E_{o}, V_{B}=0\right)=\operatorname{DOS}_{S E}=\frac{N_{S}}{k T_{o}} \exp \left(\frac{E_{o}-E}{k T_{O}}\right) \\
& =f\left[E_{o}-E_{F}-\left(E-E_{F}\right)\right]=f\left[\left(E_{o}-E_{F}\right) \pm \Delta E\right] \equiv \operatorname{DOS}\left(E_{F} \pm \Delta E\right)
\end{aligned}
$$

where $\mathrm{N}_{\mathrm{S}}$ is the total concentration of states in $1 / \mathrm{cm}^{3}, \mathrm{~T}_{\mathrm{o}}$ is a characteristic "temperature" that describes the width and steepness of the exponential DOS, and $\mathrm{E}_{\mathrm{o}}$ is the HOMO level for bulk material with no bending $V_{B}=0$. As shown in the second line of eq. (22), the DOS can be rewritten as function of the difference $\left(E_{0}-E_{F}\right)$ and a span of energies $( \pm \Delta E)$, and thus, as a function $\operatorname{DOS}(E) \equiv D O S\left(E_{F} \pm \Delta E\right)$, as used in the VRH calculations by eqs. (11)-(14) and (17)-(20) above.

When the gate bias voltage $\mathrm{V}_{\mathrm{G}}$ is applied, then the HOMO levels near the gate oxide bend with $\left(-q V_{B}\right)$, as illustrated in Figure 3, and $\mathrm{HOMO}=\mathrm{E}_{\mathrm{o}}-\mathrm{q} \mathrm{V}_{\mathrm{B}}$. Consequently, since HOMO is shifted at $\mathrm{V}_{\mathrm{B}} \neq 0$, then the DOS shifts with the HOMO level, and the DOS becomes

$$
\begin{aligned}
& \operatorname{DOS}\left(E \geq E_{O}-q V_{B}\right)=\frac{N_{S}}{k T_{O}} \exp \left(\frac{E_{O}-q V_{B}-E}{k T_{O}}\right) \\
& =f\left[E_{O}-E_{F}-q V_{B}-\left(E-E_{F}\right)\right]=f\left\{\left[E_{O}-\left(E_{F}+q V_{B}\right)\right] \pm \Delta E\right\} \\
& =f\left[\left(E_{o}-I M R E F\right) \pm \Delta E\right] \equiv \operatorname{DOS}(I M R E F \pm \Delta E) \text {, with IMREF }=E_{F}+q V_{B}
\end{aligned}
$$

Note that defining the quasi-Fermi level as IMREF $=\left(E_{F}+q V_{B}\right)$, then one embeds in IMREF the potential bending $V_{B}$ in the semiconducting film, and IMREF plays the same role in eq. (23) at $V_{B} \neq 0$, as the Fermi level $E_{F}$ plays in eq. (22) at $V_{B}=0$. Note also that there are also other definitions for IMREF in semiconductors, e.g., quasi-Fermi levels for electrons and holes, while we do not use those definitions. 
The interplay between $\mathrm{E}_{\mathrm{F}}$ and IMREF is perhaps the reason why in many publications $[4,7,10,25]$ IMREF is termed as Fermi level, and varied with charge concentrations in order to relate charge concentrations and mobility in cases between no bias and with bias applied to the OTFT, or between OTFT and organic diodes. Observe in eqs. (22) and (23) that the DOS is not a function of $\mathrm{E}_{\mathrm{F}}$ or IMREF. However, compare the first and last lines in eq. (23). In the first line, the DOS is bent "down" with $\left(-q V_{B}\right)$ to pick a value at $\mathrm{E}=\left(\mathrm{E}_{\mathrm{F}} \pm \Delta \mathrm{E}\right)$, with $\mathrm{E}_{\mathrm{F}}=$ constant. Conversely, IMREF in the last line of eq. (23) is bent "up" in the opposite direction by the same amount $\mathrm{qV}_{\mathrm{B}}$ so that a un-bended DOS picks the same value (IMREF $\pm \Delta \mathrm{E}$ ), as the bended DOS picks from $\left(\mathrm{E}_{\mathrm{F}} \pm \Delta \mathrm{E}\right)$ in the first line. Therefore, to account for the potential bending $\mathrm{V}_{\mathrm{B}}$ due to a gate bias, in the numerical simulator, one simply passes IMREF to the VRH calculation, instead of $E_{F}$; please see again Figure 1 and the call-list in it. The potential bending voltage $V_{B}$ and IMREF $=\left(E_{F}+q V_{B}\right)$ are determined from the electrostatic calculations described below.

\subsection{Electrostatic calculation module}

The electrostatic computation consists of charge-energy calculations inside an iterative loop of chargedistance calculations, the latter solving the 1D Poisson equation.

\subsubsection{Charge-energy calculation module}

The module for charge-energy calculations first generates the vector of the $1 \mathrm{D}$ mesh of energies $\mathrm{E}$ with a size $(n \times 1)$ from $E_{\text {bottom }}$ to $E_{\text {top }}$ in steps $d E$. Thus, $E_{\text {bottom, }} E_{\text {top }}$ and $d E$ are single value input parameters for the charge-energy calculation module. The size of the vector $\mathrm{E}$ is $\mathrm{n}=\left(\mathrm{E}_{\mathrm{top}}-\mathrm{E}_{\mathrm{bottom}}\right) / \mathrm{dE} \sim 2 \mathrm{eV} / 1 \mathrm{meV} \approx 2000$. Then, the module generates several other vectors of size $(n \times 1)$ with values for each element in this energy mesh $E$. One vector is DOS, given by

$$
\operatorname{DOS}(E)=f(E, D O S p a r a m) \text {, in unit, e.g., } \mathrm{cm}^{-3} \mathrm{eV}^{-1} \text {, }
$$

where $\mathrm{f}(\ldots)$ is the function of the particularly selected DOS with specific parameters DOSparam, and $\mathrm{f}(\ldots)$ and DOS are functions of the energy E. For example, DOSparam contains $\mathrm{N}_{S}, \mathrm{~T}_{\mathrm{o}}$ and $\mathrm{E}_{\mathrm{o}}$ for exponential 
DOS, with $\mathrm{f}(\ldots)$ given either by eq. (22) above for single-side exponential DOS $\mathrm{SE}$, or $\mathrm{f}(\ldots)$ given by eq. (45) later for double-side exponential DOS $\mathrm{DE}$. The function $\mathrm{f}(\ldots)$ can be changed in the charge-energy calculation module, e.g., uniformly or normally distributed DOS, or combination of them, providing also the values of the DOSparam corresponding to the particular $\mathrm{f}(\ldots)$.

The vectors $\mathrm{E}$ and $\mathrm{DOS}(\mathrm{E})$ are return results from the charge-energy calculation module, because $\mathrm{E}$ and DOS(E) are used in the VRH calculation module. The computational volume for generation of the 1D energy mesh E and the DOS vector are shown in row "eq. (24)" of Table VI and explained in Appendix 1. The remaining vectors generated by the charge-energy calculation module are temporary and used only within the electrostatic calculation module and for visualization of the computation in this module, c.f. Figure 11.

Another $(\mathrm{n} \times 1)$ vector generated at the energy mesh by the charge-energy calculation module is the Fermi occupation factor $\mathrm{F}\left(\mathrm{E}-\mathrm{E}_{\mathrm{F}}\right)=\mathrm{F}_{\mathrm{n}}\left(\mathrm{E}-\mathrm{E}_{\mathrm{F}}\right)=1 /\left\{1+\exp \left[\left(\mathrm{E}-\mathrm{E}_{\mathrm{F}}\right) / \mathrm{kT}\right]\right\}$ for electrons, or its complementary $\mathrm{F}\left(\mathrm{E}-\mathrm{E}_{\mathrm{F}}\right)=\mathrm{F}_{\mathrm{p}}\left(\mathrm{E}-\mathrm{E}_{\mathrm{F}}\right)=1 /\left\{1+\exp \left[-\left(\mathrm{E}_{\mathrm{F}}-\mathrm{E}\right) / \mathrm{kT}\right]\right\}$ for holes, the latter applicable for an OTFT, which is normally a p-type field-effect transistor. The Fermi level $\mathrm{E}_{\mathrm{F}}$ is single-value input parameter for the charge-energy calculation module. The computation volume for the calculation of the Fermi occupation factor $\mathrm{F}$ is included in row "eq. (27)" of Table VI and explained in Appendix 1, since F(E) appears only in product with $\operatorname{DOS}(\mathrm{E})$.

Having the $\mathrm{F}\left(\mathrm{E}-\mathrm{E}_{\mathrm{F}}\right)$ and $\operatorname{DOS}(\mathrm{E})$ vectors, the concentration of occupied states $\mathrm{N}_{\mathrm{C}}$ (in unit, e.g., $\mathrm{cm}^{-3}$ ), being also assumed as carrier concentration in VRH, can be calculated from

$$
\begin{aligned}
\mathrm{N}_{\mathrm{C}} & =\int_{-\infty}^{+\infty} \mathrm{F}\left(\mathrm{E}-\mathrm{E}_{\mathrm{F}}\right) \operatorname{DOS}(\mathrm{E}) \mathrm{dE} \approx \int_{\mathrm{E}_{\mathrm{bottom}}}^{\mathrm{E}_{\text {top }}} \mathrm{F}\left(\mathrm{E}-\mathrm{E}_{\mathrm{F}}\right) \operatorname{DOS}(\mathrm{E}) \mathrm{dE} \\
& \approx \mathrm{dE} \sum_{\mathrm{i}=1}^{\mathrm{n}} \mathrm{F}_{1} \mathrm{DOS}_{\mathrm{i}}
\end{aligned}
$$

where $F_{i}=F\left(E_{i}-E_{F}\right)$ and $\operatorname{DOS}_{i}=D O S\left(E_{i}\right)$ are the elements of the vectors $F$ and DOS for the elements $E_{i}$ in the energy mesh $\mathrm{E}$, and $\mathrm{n}=\left(\mathrm{E}_{\mathrm{top}}-\mathrm{E}_{\text {bottom }}\right) / \mathrm{dE}$, as mentioned above before eq. (24). The integration is implemented in the charge-energy calculation module as a simple Riemann sum, given by the last expression in eq. (25). 
The concentration of occupied states $\mathrm{N}_{\mathrm{C}}$ is a single-number return result from the charge-energy calculation module, because $\mathrm{N}_{C}$ is used in the other electrostatic module for charge-distance calculations. Since $\mathrm{N}_{C}$ is assumed as a carrier concentration, then it can be also used for a calculation of the effective spot mobility (dividing the VRH conductance by $\mathrm{qN}_{C}$, as shown in eq. (89) later), although $\mathrm{N}_{\mathrm{C}}$ is unnecessary for the calculation of the VRH conductance; and $\mathrm{N}_{\mathrm{C}}$ is not used in the VRH calculation module.

The last quantity calculated and returned from the charge-energy calculation module is the flat-band (FB) level, which is the energy E satisfying the median condition for equal concentrations of occupied states below and above FB. Rearranging eq. (25),

$$
\begin{aligned}
& \int_{-\infty}^{F B} F\left(E-E_{F}\right) \operatorname{DOS}(E) d E=\int_{F B}^{+\infty} F\left(E-E_{F}\right) \operatorname{DOS}(E) d E \\
& =\frac{N_{C}}{2}=\frac{1}{2} \int_{-\infty}^{+\infty} F\left(E-E_{F}\right) \operatorname{DOS}(E) d E
\end{aligned}
$$

The implementation of the search for FB is made by first calculating a vector of the cumulative sum of the product $\mathrm{F}_{\mathrm{i}} \times \mathrm{DOS}_{\mathrm{i}}$, mathematically given by

$$
\operatorname{CFDOS}_{\mathrm{i}}=\mathrm{dE} \sum_{\mathrm{j}=1}^{\mathrm{i}=1,2, \ldots, \mathrm{n}} \mathrm{F}_{\mathrm{j}} \text { DOS }_{\mathrm{j}}
$$

that is effectively calculated by a loop, in which $\mathrm{CFDOS}_{\mathrm{i}}=\mathrm{F}_{\mathrm{i}} \times \mathrm{DOS}_{\mathrm{i}}+\mathrm{CFDOS}_{\mathrm{i}-1}$, with $\mathrm{CFDOS}_{1}=\mathrm{F}_{1} \times \mathrm{DOS}_{1}$ and $\mathrm{i}=2,3, \ldots, n$. Then, the last element $\operatorname{CFDOS}_{\mathrm{i}=\mathrm{n}}$ of the cumulative sum is $\mathrm{N}_{\mathrm{C}}$, (thus, eq. (25) is redundant and commented in the code of the simulator, replacing with $\mathrm{N}_{\mathrm{C}}=\mathrm{CFDOS}_{\mathrm{n}}$ ), and one finds the index IFB for which $\left|\mathrm{CFDOS}_{\mathrm{IFB}}-\mathrm{N}_{\mathrm{C}} / 2\right|=$ min. Consequently, $\mathrm{FB}=\mathrm{E}_{\mathrm{IFB}}$, taking the energy $\mathrm{E}_{\mathrm{IFB}}$ with index IFB from the energy mesh $\mathrm{E}$. The computational volume for $\mathrm{N}_{\mathrm{C}}$ and $\mathrm{FB}$ (combined with the computation volume for $\mathrm{F}$, as mentioned above) is shown in row "eq. (27)" of Table VI and explained in Appendix 1.

One could determine FB also as a weighted average, e.g., 


$$
\mathrm{FB}_{\mathrm{AVG}}=\frac{\int_{-\infty}^{+\infty} \mathrm{E} \times \mathrm{F}\left(\mathrm{E}-\mathrm{E}_{\mathrm{F}}\right) \operatorname{DOS}(\mathrm{E}) \mathrm{dE}}{\int_{-\infty}^{+\infty} \mathrm{F}\left(\mathrm{E}-\mathrm{E}_{\mathrm{F}}\right) \operatorname{DOS}(\mathrm{E}) \mathrm{dE}},
$$

but we did not implement this in the charge-energy calculation module, because the median FB of eq. (26) is more consistent with the concept for equal probability of charge occupation above and below FB. Other reasons to use eq. (27) are that the coding of the search for a median is simple, finding the value $\mathrm{FB}=\mathrm{E}_{\mathrm{IFB}}$ at a point of the energy mesh (avoiding any unforeseen problem, e.g., due to numeric rounding and truncation, when having $\mathrm{FB}_{\mathrm{AVG}}$ with value not at the mesh points), and also, the calculation volume of eq. (27) is one half of the volume of eq. (28), which is an important consideration, since the charge-energy calculation module is in the body of the loop with many cycles in the charge-distance calculation module, which is presented next. Note that the Fermi level $\mathrm{E}_{\mathrm{F}}$ is used only in the calculation of Fermi occupation factor $F\left(E-E_{F}\right)$, and one can use quasi-Fermi IMREF $=\left(E_{F}+q V_{B}\right)$, instead of $E_{F}$, to include the bending $V_{B}$ due to gate bias in the charge-energy calculation module, following to the same reasons discussed with eqs. (22) and (23) for the VRH calculation (taking $\mathrm{E}_{\mathrm{o}}=0$ ).

Overall, the computational volume of the charge-energy calculation module is moderate and scales with the size $n=\left(E_{\text {top }}-E_{\text {bottom }}\right) / \mathrm{dE} \sim 2 \mathrm{eV} / 1 \mathrm{meV} \approx 2000$ of the energy mesh $\mathrm{E}$, as shown in row "sum 3" of Table VI and explained in Appendix 1.

\subsubsection{Charge-distance calculation module}

The main purpose of this electrostatic module is to solve the 1D Poisson equation in the depth D of the OTFT semiconducting film, from $\mathrm{D}=0$ at gate dielectric interface to $\mathrm{D}=\mathrm{t}_{\mathrm{f}}$ in the back of the semiconducting film of thickness $t_{f}$ (see Figure $2 b$ earlier). This is to find the profile for the bending voltage $V_{B}$ in the film at given gate bias $V_{G}$, which is needed for the $V R H$ calculations, since $I M R E F=\left(E_{F}+q V_{B}\right)$ in eq. (23) is a linear function of $\mathrm{V}_{\mathrm{B}}$. Other profiles for the electrostatic quantities, such as volume charge concentrations $\mathrm{N}_{\mathrm{VG}}$ and electric field $\mathrm{E}_{\mathrm{el}}$ induced by $\mathrm{V}_{\mathrm{G}}$ in the film, are also obtained from the charge-distance calculation 
module. Along with the calculations, the module also generates the depth mesh $\mathrm{D}$ for the profiles of the electrostatic and VRH quantities.

A simplified flow of the algorithm of the charge-distance calculation module is shown in Figure 4. It is comprised of three parts.

(i) The first part is for bulk material in equilibrium, calculating the flat-band voltage potential $V_{\mathrm{FB}}$ and carrier concentration $\mathrm{N}_{\mathrm{CFB}}$ in the semiconducting film that is generated thermally when no gate bias is applied to the OTFT. These are obtained by calling the charge-energy calculation module with zero bending. From eqs. (25) and (26), it follows that

$$
\left.\begin{array}{l}
\mathrm{V}_{\mathrm{FB}}=\mathrm{FB} /\left.\left.\mathrm{q}\right|_{\mathrm{FB} \text { in }[\mathrm{J}]} \equiv \mathrm{FB}\right|_{[\mathrm{eV}]} \\
\mathrm{N}_{\mathrm{CFB}}=\mathrm{N}_{\mathrm{C}}
\end{array}\right\} \text {, when } \mathrm{V}_{\mathrm{B}}=0 \text {, thus IMREF=EF, }
$$

according to the definition of $\operatorname{IMREF}=\left(\mathrm{E}_{\mathrm{F}}+\mathrm{qV}_{\mathrm{B}}\right)$ by eq. (23) and the discussions after this eq. (23) and eq. (28). It is important to note that $\mathrm{N}_{\mathrm{CFB}}$ corresponds to zero electric field, $\mathrm{E}_{\mathrm{el}}=0$, in the entire semiconducting film. Therefore, $V_{B}=-\int E_{e l}(D) d D=0$. Thus, $N_{C F B}$ is not a net charge, but compensated with the opposite charge, since $\mathrm{N}_{\mathrm{CFB}}$ is not bias induced, but thermally (and could also be optically) generated in the material in equilibrium. We consider in the simulator that $\mathrm{N}_{\mathrm{CFB}}$ is bias independent, neglecting effects such as barrier lowering or impact ionization at high electric fields. Since $V_{B}=0$ for the semiconductor bulk, then a VRH calculation is also embedded in the first part of the charge-distance calculation module to yield the conductivity $\sigma_{\mathrm{FB}}$ and hopping energy $\mathrm{E}_{\mathrm{HFB}}$ for bulk semiconductor, as

$$
\left.\begin{array}{l}
\sigma_{\mathrm{FB}}=\sigma \\
\mathrm{E}_{\mathrm{HFB}}=\mathrm{E}_{\mathrm{H}}
\end{array}\right\} \text {, when } \mathrm{V}_{\mathrm{B}}=0 \text {, thus IMREF }=\mathrm{E}_{\mathrm{F}} .
$$

(ii) The second part in the charge-distance calculation module solves numerically Poisson's equation in the depth D of the OTFT semiconducting film, when the gate bias $V_{G}$ is not equal to the flat-band voltage $\mathrm{V}_{\mathrm{FB}}$. If $\mathrm{V}_{\mathrm{G}}=\mathrm{V}_{\mathrm{FB}}$, then there is no potential bending in the film. Thus, the film is in equilibrium and there is no need to have profiles, since the values in these profiles are identical to the values for the bulk semiconductor. When $\mathrm{V}_{\mathrm{G}} \neq \mathrm{V}_{\mathrm{FB}}$, then the calculation generates simultaneously the depth mesh $\mathrm{D}$ and the 
profiles for electrostatic quantities in this mesh. The calculation scheme is close to the schemes used in analytical derivations for OTFTs [1, 26], not overlooking the concerns [7, 10, 27] for zero field and zero charge in the derivations and interpretations of the results obtained by these schemes. The basic assumptions are that the potential is $\mathrm{V}_{\mathrm{FB}}$ in the "far depth $\mathrm{D} \rightarrow \infty$ " of the film, where the electric field is zero, and moving backward to the gate dielectric, the bending $V_{B}$ increases toward $V_{G}$. Thus, a charge is induced in the film due to $\mathrm{V}_{\mathrm{G}} \neq \mathrm{V}_{\mathrm{FB}}$ and the electric field increases in magnitude, so that at the film-dielectric interface $(\mathrm{D}=0)$, Gauss' law is satisfied, that is

$$
\varepsilon_{\mathrm{F}} \mathrm{E}_{\mathrm{el}}(\mathrm{D}=0)=\mathrm{Q}_{\mathrm{G}} \Rightarrow \text { integrate until } \frac{\mathrm{Q}_{\mathrm{G}}^{\prime}}{\varepsilon_{\mathrm{F}} \mathrm{E}_{\mathrm{el}}} \leq 1,
$$

which is the criterion to exit from the loop of numerical integrations, and where $Q_{G}=\left[V_{G}-\left(V_{F B}+V_{B S}\right)\right] C_{O X}$ is the charge per unit area of the gate dielectric, $V_{B S}=V_{B}(D=0)$ is the potential bending in the semiconductor at the gate dielectric interface $(\mathrm{D}=0)$, and $\mathrm{Q}_{\mathrm{G}}^{\prime}=\left[\mathrm{V}_{\mathrm{G}}-\left(\mathrm{V}_{\mathrm{FB}}+\mathrm{V}_{\mathrm{B}}\right)\right] \mathrm{C}_{\mathrm{OX}}$ is a supplementary variable for gate charge during the integration in the depth of the semiconducting film $(D \geq 0)$. Once the criterion for exit from the loop of numerical integration is reached, then $\mathrm{Q}_{\mathrm{G}} \approx \mathrm{Q}_{\mathrm{G}}$ and $\mathrm{V}_{\mathrm{B}} \approx \mathrm{V}_{\mathrm{BS}}$.

In many analytical derivations, it is assumed that the "far depth" is still inside the thin film of the OTFT. Therefore, the charge induced in the film is assumed equal to $\mathrm{Q}_{\mathrm{G}}$, allowing for the replacement of the distance integration with an integration over $V_{B}$ in the interval from zero to the surface potential at the gate dielectric interface. In contrast, we strictly follow the basic assumptions when building the Poisson solver, not restricting the bending $\mathrm{V}_{\mathrm{B}}$ and electric field $\mathrm{E}_{\mathrm{el}}$ to reach zero within the thin film of the OTFT. In our case, the electric field may, or may not, decay completely within the thin film of the OTFT; thus, there might be a fringing electric field at the back of the film. To cope with these different situations, we use the supplementary integration variable $\mathrm{t}$ for distance which decrements when moving the integration from the depth of the film toward gate oxide interface in a direction opposite to the film depth D. Since the "far depth" is unknown, then we arbitrary set $\mathrm{t}=0$ by initializing the numerical integration loop, decrementing $\mathrm{t}$ with variable step $\mathrm{dt}>0$, according to 


$$
\mathrm{t}=\mathrm{t}(\text { previous })-\mathrm{dt}, \mathrm{t}=0 \text { and } \mathrm{dt}=\mathrm{dD} \text { initially at "far depth", }
$$

where $\mathrm{dD} \sim 0.5 \mathrm{~nm}$ is parameter for nominal step in the distance mesh $\mathrm{D}$. Thus, in the "far depth" $\mathrm{t}=0, \mathrm{t} \neq \mathrm{t}_{\mathrm{f}}$, and we initialize the Poisson solver with zero electric field $\mathrm{E}_{\mathrm{el}}=0$ and guide the solver to do the first step for bending $V_{B}$ with a small magnitude equal to the energy step $d E$ in $e V$ in the direction $\operatorname{sign}\left(V_{G}-V_{F B}\right)$ of the gate bias overdrive $\left(\mathrm{V}_{\mathrm{G}}-\mathrm{V}_{\mathrm{FB}}\right)$, where the function $\operatorname{sign}(\mathrm{x})=1$, if $\mathrm{x}>0$ and $\operatorname{sign}(\mathrm{x})=-1$, when $\mathrm{x}<0$. Consequently, the charge-energy calculation module is called with the quasi-Fermi $\mathrm{IMREF}=\left(\mathrm{E}_{\mathrm{F}}+\mathrm{qV} \mathrm{V}_{\mathrm{B}}\right)$ to include the bending $\mathrm{V}_{\mathrm{B}}$, as noted after eq. (28). Bearing this in mind, the concentration of occupied states $\mathrm{N}_{\mathrm{C}}$ at $\mathrm{V}_{\mathrm{B}} \neq 0$ can be obtained from (25):

$$
\left.\begin{array}{l}
\mathrm{V}_{\mathrm{BFB}}=\mathrm{V}_{\mathrm{B}}+\mathrm{FB} /\left.\mathrm{q}\right|_{\mathrm{FB} \text { in [J] }} \equiv \mathrm{V}_{\mathrm{B}}+\left.\mathrm{FB}\right|_{[\mathrm{eV}]} \\
\mathrm{N}_{\mathrm{C}}=\mathrm{N}_{\mathrm{C}}(\mathrm{IMREF})
\end{array}\right\} \text {, when } \mathrm{V}_{\mathrm{B}} \neq 0 \text {, thus IMREF }=\left(\mathrm{E}_{\mathrm{F}}+\mathrm{q} \mathrm{V}_{\mathrm{B}}\right) \text {, }
$$

Subtracting the equilibrium charge concentration $\mathrm{N}_{\mathrm{CFB}}$, one obtains the bias induced charge concentration $\mathrm{N}_{\mathrm{VG}}$ as

$$
\mathrm{N}_{\mathrm{VG}}=\mathrm{N}_{\mathrm{C}}(\mathrm{IMREF})-\mathrm{N}_{\mathrm{CFB}}, \text { for } \mathrm{t}<0, \mathrm{IMREF}=\left(\mathrm{E}_{\mathrm{F}}+\mathrm{q} \mathrm{V}_{\mathrm{B}}\right), \mathrm{V}_{\mathrm{B}} \neq 0
$$

$\mathrm{N}_{\mathrm{VG}}$ is the non-equilibrium portion of $\mathrm{N}_{\mathrm{C}}$ at gate bias voltage $\mathrm{V}_{\mathrm{G}}$. Since $\mathrm{N}_{\mathrm{VG}}$ is not generated thermally, but by the bending $\mathrm{V}_{\mathrm{B}}$, then $\mathrm{N}_{\mathrm{VG}}$ is not compensated by opposite charge in the semiconductor, and $\mathrm{N}_{\mathrm{VG}}$ causes increments of the electric field $\mathrm{E}_{\mathrm{el}}$, so that

$$
\mathrm{E}_{\mathrm{el}}=\mathrm{E}_{\mathrm{el}}(\text { previous } \mathrm{t})+\mathrm{q} \frac{\mathrm{N}_{\mathrm{VG}}}{\varepsilon_{\mathrm{F}}} \mathrm{dt}, \text { for } \mathrm{t}<0, \mathrm{IMREF}=\left(\mathrm{E}_{\mathrm{F}}+\mathrm{q} \mathrm{V}_{\mathrm{B}}\right), \mathrm{V}_{\mathrm{B}} \neq 0
$$

which comprises the first integration of the Poisson equation. The second integration yields the value of the bending voltage

$$
\mathrm{V}_{\mathrm{B}}=\mathrm{V}_{\mathrm{B}}(\text { previous } \mathrm{t})-\mathrm{dt} \times \mathrm{E}_{\mathrm{el}}, \text { for } \mathrm{t}<0, \mathrm{IMREF}=\left(\mathrm{E}_{\mathrm{F}}+\mathrm{q} \mathrm{V}_{\mathrm{B}}\right), \mathrm{V}_{\mathrm{B}} \neq 0
$$

Then, the value of gate charge $\mathrm{Q}_{\mathrm{G}}^{\prime}=\left[\mathrm{V}_{\mathrm{G}}-\left(\mathrm{V}_{\mathrm{FB}}+\mathrm{V}_{\mathrm{B}}\right)\right] \mathrm{C}_{\mathrm{OX}}$ is updated to account for low biases around or below threshold voltage $\mathrm{V}_{\mathrm{T}}$ of the OTFT, although Q'G does not change significantly, when the OTFT operates well above $\mathrm{V}_{\mathrm{T}}$. Repeating iteratively eqs. (32)-(36), the supplementary integration variable $\mathrm{t}$ accumulates the distance mesh, for which the values of $\mathrm{N}_{\mathrm{VG}}, \mathrm{E}_{\mathrm{el}}$ and $\mathrm{V}_{\mathrm{B}}$ are stored and, thus, one obtains 
profiles for these quantities. Also, the magnitudes of $\mathrm{N}_{\mathrm{VG}}, \mathrm{E}_{\mathrm{el}}$ and $\mathrm{V}_{\mathrm{B}}$ increase at every next step of the loop, while Q'G gradually decreases. Therefore, the ratio $\mathrm{Q}_{\mathrm{G}} /\left(\varepsilon_{\mathrm{F}} \mathrm{E}_{\mathrm{el}}\right)$ decreases from a large value toward zero, and after a sufficient number of iterations, reaches the condition $\mathrm{Q}_{\mathrm{G}} \leq\left(\varepsilon_{\mathrm{F}} \mathrm{E}_{\mathrm{el}}\right)$ in eq. (31), which indicates that the integration has finished at the gate oxide interface, for which the last negative value of $t$ reaches a minimum. At this point, the profiles of charge, electric field and potential versus distance t have been calculated.

(iii) Finally, the third part in charge-distance calculation module reverts the integration variable for distance $t$ into the depth variable $\mathrm{D}$ in the semiconducting film as

$$
\mathrm{D}=\mathrm{t}-\min (\mathrm{t}), \text { interface }=0 \leq \mathrm{D} \leq \mathrm{t}_{\mathrm{f}}=\text { film back, }
$$

where the interface is at $\mathrm{D}=0$, the film thickness is at $t_{f}$, and the mesh points with $\mathrm{D}>\mathrm{t}_{\mathrm{f}}$ are deleted. Accordingly, the data in the profiles for $\mathrm{N}_{\mathrm{C}}$, the electric field and the potential, corresponding to the deleted mesh points, are also removed, since they are not in the semiconducting film of the OTFT. Thus, the chargedistance calculation module exits with return results for the bulk semiconductor and distance mesh and profiles at a given gate bias, as indicated in the bottom-right corner of Figure 4.

As described above, the algorithm of the charge-distance calculation module is straightforward and it strictly follows the basic assumptions for electrostatic calculation in a semiconducting thin film, not adding uncertain boundary conditions. However, looking closer at the rate of convergence, the number of mesh points and other computational issues, we note that the algorithm must be optimized for throughput and suitability for VRH numerical simulations. Omitting the error handling, several details of such optimization are outlined in Figure 4.

One problem is that the algorithm requires non-zero increments that are larger than the numerical truncations. For example, $V_{B}$ is low in the first iteration, and after calling the charge-energy calculation module with $\mathrm{IMREF}=\left(\mathrm{E}_{\mathrm{F}}+\mathrm{q} \mathrm{V}_{\mathrm{B}}\right)$ in the loop, the value for $\mathrm{N}_{\mathrm{C}}$ might be numerically identical to $\mathrm{N}_{\mathrm{CFB}}$. Thus, there will be no increment for the electric field, and $V_{B}$ will not change in the next iteration, causing the algorithm to fall in an infinite loop. Therefore, one "pushes gently" $\mathrm{N}_{\mathrm{C}}$ with a small step of, e.g., 
$\mathrm{N}_{\mathrm{C}}=\left(10^{-9} / \mathrm{cm}\right) \times \mathrm{Q}_{\mathrm{G}}^{\prime} / \mathrm{q}+\mathrm{N}_{\mathrm{CFB}}$ to have a distinguishable non-zero value for $\mathrm{N}_{\mathrm{VG}}=\left(\mathrm{N}_{\mathrm{C}}-\mathrm{N}_{\mathrm{CFB}}\right)$ in eq. (34) and non-zero increment of the electric field $E_{\text {el }}$ and potential bending $V_{B}$ in eqs. (35) and (36), respectively. The value $10^{-9} / \mathrm{cm}$ is empirically determined to be small enough so that the "push is gentle", and does not affect the number of iterations in the charge-distance module. The determination is after monitoring of the number of iterations in parallel computing of about one million trials and using error handling for a maximum number of iterations of 20000 (not shown in Figure 4).

Another problem is the distance step dt. A uniform distance mesh with a constant step is not suitable, since one has to have fine steps when the integration is close to the oxide interface, e.g. dt 0.01nm, but this small step may cause many millions of iterations in the integration at the "far depth", which can be $1 \mathrm{~mm}$ or occasionally even thicker. Therefore, we have implemented a control of the size of the step dt, as depicted in the bottom-left corner in Figure 4. If the bias-induced charge concentration $\mathrm{N}_{\mathrm{VG}}$ is much less than the equilibrium charge concentration $\mathrm{N}_{\mathrm{CFB}}$, e.g., $\left(\mathrm{N}_{\mathrm{VG}} / \mathrm{N}_{\mathrm{CFB}}\right)<0.5 \%$, or the gate charge $\mathrm{Q}_{\mathrm{G}}$ is much larger than the field flux $\varepsilon_{\mathrm{F}} \mathrm{E}_{\mathrm{el}}$, then the mesh point is far from the oxide interface (most probably beyond the film thickness $t_{f}$ ) and the integration is accelerated exponentially, doubling the integration step, to move quicker from the "far depth" toward the oxide interface. On the other hand, when the integration is within 1-2 nm from the gate oxide interface, the nominal step $\mathrm{dt}=\mathrm{dD} \sim 0.5 \mathrm{~nm}$ is coarse, because the electric field is large and $V_{B}$ changes rapidly, owing to the term $d t \times E_{e l}$ in eq. (36). Therefore, the mesh is refined, decreasing the step dt. In the particular implementation of the charge-distance calculation module, the criterion for refinement of the distance mesh is for the change of $V_{B}$ to be not greater than the step $d E$ (in $e V$ ) of the energy mesh, which is the same as $\mathrm{dE}>\left|\mathrm{E}_{\mathrm{el}}\right| \mathrm{dt}$. The decrease is done first by setting $\mathrm{dt}=\mathrm{dD}$ to the nominal step $\mathrm{dD} \sim 0.5 \mathrm{~nm}$, and if necessary, further exponential decrease of $\mathrm{dt}$ by division on 4 . In either case of enlargement or refinement of $\mathrm{dt}$, one recalculates $\mathrm{V}_{\mathrm{B}}$ for the next iteration from

$$
V_{B}(\text { next } t)=V_{B}(\text { current } t)-d t \times q \frac{N_{V G}}{\varepsilon_{F}} d t \text {, for the next iteration. }
$$

By the above management of the step $\mathrm{dt}$, the second part of the charge-distance calculation module is usually completed in about two thousands iterations (not more than ten thousands iterations after about one 
million trials), matching the gate charge $\mathrm{Q}_{\mathrm{G}}$ with inaccuracy less than 5\%, and providing distance mesh with about 20 points logarithmically spaced at $\mathrm{D}<2 \mathrm{~nm}$, about 200 points uniformly spaced at $\mathrm{dD}=0.5 \mathrm{~nm}$ for $2 \mathrm{~nm}<\mathrm{D}<100 \mathrm{~nm}$, and about 2000 points again nearly logarithmically spaced with larger mesh step for depths D> 100nm. Thus, the number of iterations is approximately equal to the number $n \sim 2000$ of energy points in the charge-energy calculation module, and the computational volume for electrostatics of the chargedistance calculation module is mostly determined by the calls of the charge-energy calculation module at every iteration. The details for the computation volume are explained in Appendix 1 and shown in Table VI in row "eSt" without and in rows "eSt+vrh1" and "eSt+vrh2" with the VRH calculations in the first part for bulk material in equilibrium.

It is possible, in principle, to include the VRH calculation in the loop of the electrostatic calculation, but the VRH calculation should be postponed to be performed on sub-sampled depth mesh D, as indicated in Figure 4, because, if it is included in the loop of the charge-distance calculation module, then the computational volume would be unnecessary large, as explained in Appendix 1, while the majority of the calculated data will be also deleted, since they correspond to film depths larger than the thickness of the semiconducting film of OTFT. Therefore, in the third part in charge-distance calculation module, the distance mesh D is logarithmically sub-sampled to about 50 points (to have many points near the oxide interface and also enough points in the depth of the semiconducting film) and the profiles are reduced only to the points of the sub-sampled mesh. The VRH calculation module is then called in pass 2 of the VRH simulator (presented in the next Sec. 2.6) only for the reduced distance mesh D. The reduction of the distance mesh for VRH calculation results in acceptable computational volume of the VRH simulator.

\subsection{VRH numerical simulator}

The overall flow of the VRH simulations is outlined in Figure 5. Running the simulator, it first completes the lists of parameters and settings with default values. Next, the parameters and settings are modified according to desired values for materials and layout properties of the OTFT and requirements for 
simulation, monitoring of the simulation, saving of results and selection of experimental results for comparisons. Then, the simulations are executed for different temperatures $\mathrm{T}$ and gate bias voltages $\mathrm{V}_{\mathrm{G}}$ in independent calculations for each pair of bias and temperature conditions $\left(\mathrm{V}_{\mathrm{G}}-\mathrm{T}\right.$ point). The simulator monitors the execution and once results for a $\mathrm{V}_{\mathrm{G}}-\mathrm{T}$ point are available, then the results are plotted. In Figure 5, for example, it is indicated when the mobility vs. reciprocal of the temperature is plotted. When the simulations for all temperatures and bias voltages are completed, then the simulator gathers the logged results and organizes the results in a merged file, which is stored. This arrangement allows the simulations for different $\mathrm{V}_{\mathrm{G}}-\mathrm{T}$ points to be executed sequentially by nesting loops for $\mathrm{T}$ and $\mathrm{V}_{\mathrm{G}}$, or in parallel, when a grid of computers is available. The parallel computing is highly desired when choosing the method of multiple $\iint \mathrm{dEdE}$ for VRH, since the computation is extensive and it may take half a day to calculate 20-30 $\mathrm{V}_{\mathrm{G}}-\mathrm{T}$ points, if the computation is sequential.

The core in the simulator is the VRH simulation for one $\mathrm{V}_{\mathrm{G}}-\mathrm{T}$ point. It is executed in three passes. Pass 1 is for electrostatic calculations, which are performed by the charge-distance calculation module presented Sec. 2.5.2 above. Therefore, the computation volume of pass 1 is the computation volume of the charge-distance calculation module (explained in Appendix 1) and it is shown in rows "eSt+vrh1" and "eSt+vrh2" of Table VI for the single $\int \mathrm{dE}$ and multiple $\iint \mathrm{dEdE}$ integrations, respectively. The output from this pass are electrostatic and VRH quantities for the bulk semiconductor $\left(\mathrm{V}_{\mathrm{FB}}, \mathrm{N}_{\mathrm{CFB}}, \sigma_{\mathrm{FB}}\right.$ and $\left.\mathrm{E}_{\mathrm{HFB}}\right)$ and for the given $V_{G}$, electrostatic profiles $\left(N_{V G}, E_{e l}\right.$ and $\left.V_{B}\right)$ in the semiconductor film-depth mesh $D$ reduced to about 50 points. The reduction is due to the large computational volume of the VRH calculations in pass 2. Pass 1 allocates the most of the memory.

Pass 2 performs the VRH calculations for the points in the reduced mesh D of about $\mathrm{m}=50$ points and creates the profiles for VRH specific conductivity $\sigma$ and hopping energy $E_{H}$ in this mesh. The calculation in pass 2 uses the values for the potential bending $V_{B}$ stored in the corresponding profile and performs the VRH calculation with quasi-Fermi level $I M R E F=\left(E_{F}+q V_{B}\right)$, instead of the Fermi level $E_{F}$ in bulk semiconductor, to account for the potential bending $V_{B}$ due to gate bias voltage $V_{G}$ of OTFT, and by the 
reasons explained after eq. (23) earlier. Thus, for each $\mathrm{V}_{\mathrm{B}}$ in the profile, two calculation steps are performed. The first step in pass 2 is to regenerate the energy mesh E and DOS(E) in this mesh by adjustment of $\mathrm{E}_{\text {top }}$ and $\mathrm{E}_{\text {bottom }}$ so that IMREF and the modes of DOS are well inside the mesh E, e.g.,

$$
\begin{aligned}
& \mathrm{E}_{\text {top }}=\max \left\{\mathrm{E}_{\mathrm{F}}, \mathrm{IMREF}, \mathrm{E}[\max (\mathrm{DOS})]\right\}+\text { reserveE } \\
& \mathrm{E}_{\text {bottom }}=\min \left\{\mathrm{E}_{\mathrm{F}}, \mathrm{IMREF}, \mathrm{E}[\max (\mathrm{DOS})]\right\}-\text { reserveE }
\end{aligned}
$$

where $E[\max (D O S)]$ is the mode of DOS, for example $E_{o}$ in an exponential DOS, c.f. eq. (22) earlier for single-side or eq. (45) later for double-side DOS, and the default value for the reserveE is $0.5 \mathrm{eV}$ in the VRH simulator. The reserveE can be increased, but we have observed that a larger reserveE does not change significantly the values of the results from simulations, while a larger reserveE increases the computation volume, since $n \approx\left(E_{\text {top }}-E_{\text {bottom }}\right) / d E$. As shown in row "pass 2" of Table VI and explained in Appendix 1, the computational volume scales up with $n$, either $\propto n$ or $\propto n^{2}$. Upon completing of pass 2 , all the essential data are available, and the VRH simulator proceeds to the next pass 3 for saving the results from the VRH simulation for one $\mathrm{V}_{\mathrm{G}}-\mathrm{T}$ point.

Pass 3 is logging the results from VRH simulation for one $V_{G}-T$ point. One should be careful with this pass, since the numerical simulators generate large volume of numbers and the proper organization of these numbers is essential for accessing the simulation results. While it is difficult to determine a universal format for the output from numerical simulators, there are several recommendations that must be followed. One recommendation is to have delimiters for beginning and end of the data. A second recommendation is for the data to be in a table format with separators between different tables and identifiers for different quantities, having also 2-3 columns in all tables with common key information for search and filtering. A third recommendation is for the data to be self-consistent and "normalized", that is, the data are minimized to only essential quantities, from which all other quantities can be recalculated later on, if necessary, without having to re-run the simulation. Some additional quantities can be also included, if it is expected that these quantities are often required and essential for the particular type of devices. For example, the sheet conductance $\sigma_{\mathrm{sq}}$ is handy to be readily available for OTFTs, although it can be calculated later from 
the profile of the specific conductance $\sigma$ within the film thicknesses. Therefore, as indicated in Figure 5, pass 3 is logging first the parameters and conditions for simulation for the particular $\mathrm{V}_{\mathrm{G}}-\mathrm{T}$ point, and the information for temperature $\mathrm{T}$ and bias $\mathrm{V}_{\mathrm{G}}$ is repeated in the beginning of each line, serving as filter keys. Then, the results for bulk semiconductor (at equilibrium, no gate bias) are logged, adding also the sheet conductance of the film in equilibrium, which is same as the conductance of film in square-shaped area, calculated according to

$$
\sigma_{\mathrm{sqB}}=\sigma_{\mathrm{FB}} \times \mathrm{t}_{\mathrm{f}}
$$

Next, the results for square-shaped OTFT under gate bias are logged, including the gate charge $\mathrm{Q}_{\mathrm{G}}$ obtained from the electrostatic simulation and adding the sheet conductance $\sigma_{\mathrm{sq}}$ of the film at $\mathrm{V}_{\mathrm{G}}$, calculated according to

$$
\begin{aligned}
\sigma_{\mathrm{sq}} & =\int_{0}^{\mathrm{t}_{\mathrm{f}}} \sigma(\mathrm{D}) \mathrm{dD} \\
& =\sum_{\mathrm{i}=1}^{\mathrm{m}-1} \sigma\left(\mathrm{D}_{\mathrm{i}}\right) \times \mathrm{dD}_{\mathrm{i}}=\sum_{\mathrm{i}=1}^{\mathrm{m}-1} \sigma\left(\mathrm{D}_{\mathrm{i}}\right) \times\left(\mathrm{D}_{\mathrm{i}+1}-\mathrm{D}_{\mathrm{i}}\right)
\end{aligned}
$$

where the Riemann sum corresponds to integration of the profile for specific conductivity $\sigma$ along the depth mesh $\mathrm{D}$ and $\mathrm{dD}_{\mathrm{i}}$ is the difference vector of D. Shown in row "eq. (41)" of Table VI, the computational volume of pass 3 is negligible, just for calculation of supplementary quantities, such as $\sigma_{\text {sq. }}$ Therefore, the row "total for a $\mathrm{V}_{\mathrm{G}}-\mathrm{T}$ point" replicates the row "pass 2" in Table VI, as explained at the end of Appendix 1. Finally, the reduced profiles of size $\mathrm{m}$ and the corresponding depth mesh D are logged as columns in a table for profiles. This completes pass 3 and the VRH simulation for one $\mathrm{V}_{\mathrm{G}}-\mathrm{T}$ point.

One sees that the mobility $\mu$ is not calculated or stored by the VRH simulator, because VRH provides values for conductance, but not for mobility, as mentioned before. On the other hand, $\mu$ is an essential parameter for OTFT, and most of the publications for OTFT report mobility, rarely conductance. In the literature, the comparisons between simulations and experiments are normally in terms of mobility, as indicated by dashed arrows in Figure 5 for the plot of mobility $\mu$ vs. the reciprocal of the absolute 
temperature 1/T. Considering the gate sheet charge $\mathrm{Q}_{\mathrm{G}}$ in field-effect transistors with isolated gates, the class of electronic devices to which the OTFTs belong, the mobility, in principle, is given by

$$
\mu=\frac{\sigma_{\mathrm{sq}}}{\mathrm{Q}_{\mathrm{G}}}=\frac{\int_{0}^{\mathrm{t}_{\mathrm{f}}} \sigma(\mathrm{D}) \mathrm{dD}}{\mathrm{Q}_{\mathrm{G}}}
$$

Thus, the mobility is easily obtained by a general relation from the results logged by the VRH simulator.

To summarize, we have built fully numerical one-dimensional simulator based on the main relations in VRH theory and electrostatics, but not on analytical expressions for specific cases. Therefore, the simulator can be used as independent tool for verification of the analytical models, the later derived by diverse techniques and with additional a priori assumptions. However, we have also observed pronounced theoretical uncertainties in the main relations, e.g., four decades for the conductivity prefactor $\sigma_{\mathrm{o}}$ by eq. (3), and variation in the approaches for use of the main relations. Therefore, we have built in the simulator two methods for VRH calculation, denoted as "single $\int \mathrm{dE}$ " and "multiple $\iint \mathrm{dEdE}$ " integrations, the latter being computationally extensive, in order to estimate to which extent the variations in derivations can affect the final prediction of analytical models. Consequently, we have used simple numerical techniques of forward integration by Riemann sum, instead of sophisticated integration techniques, to achieve reasonable calculation time, although we still need to run parallel computation for the multiple $\iint \mathrm{dEdE}$. Another reason for using the simple numerical techniques is the reduced probability of human errors by coding the simulator. Overall, we expect the simulator to reliably capture the behavior of VRH in OTFT, although it is noted that we will meet with challenging problems related to the amount of numerical information generated by the simulator, uncertainty of parameter values and perhaps numerical errors. These are addressed in the following sections, in which we present the results from simulations, comparisons to experimental data and predictions of analytical models, along with discussions for unexplored correlations between quantities and impact of parameters, intervals and coarseness of numerical integration, variations in assumptions for DOS, predictability of profiles and other issues. 
We would like to emphasize here that the simulator is flexible for research, since virtually every single relation can be modified (even for curiosity), but the simulator is computationally demanding, and it does not have figure-of-merit for accuracy or protection against improper use or wrong parameter and value assignments. Thus, the simulator should not be considered as a circuit simulator. It does not have user interface or tools for visualization and analysis of the simulation results, nor organization of data from multiple runs of the simulator.

Nevertheless, we will provide some insights on what should be improved and how one should properly guide the numerical simulator so that the numerical results are adequate, but not only columns of numbers. Finally, perhaps one has also observed that the charge carrier concentration $\mathrm{N}_{\mathrm{C}}$ (from Sec. 2.5 "Electrostatic calculation module") does not participate in the calculations of the VRH conductivity (Secs. 2.1 - 2.4), which indicates that the relation between charge and VRH is indirect. Considering the literature, the observation is counterintuitive, but it is correct, actually. A common quantity for electrostatic and VRH calculations is $\mathrm{IMREF}=\left(\mathrm{E}_{\mathrm{F}}+\mathrm{q} \mathrm{V}_{\mathrm{B}}\right)$, and in particular, beginning from Secs. 3.6 and 3.7, we will show and discuss in details that the relation between electrostatics and VRH conductance is the potential bending $\mathrm{V}_{\mathrm{B}}$, but not through a correlation between the charge concentration and the mobility. The latter correlation is affected by many factors and assumptions, and it can be a very complex correlation, as one can see in the literature that considers normally distributed DOS. However, we do not rule out the relation between charge and mobility in OTFT, especially the power-law dependence between them, which is well established experimentally, and also, very useful for compact modeling of OTFTs. Instead, we will show and extensively use in the next sections that this power-law correlation follows from the relation between electrostatics and VRH through $\mathrm{V}_{\mathrm{B}}$.

\section{Parameter assignment and results}

Numerical simulators have the advantage that layout design and material parameters can be used for simulation. Such parameters are the gate dielectric capacitance and thicknesses of layers, whereas the 
compact models usually do not consider the thickness of the OTFT semiconducting films. However, and in contrast to compact models, the numerical simulators do not provide for underlying functions of some quantities and for extraction techniques of related parameters. For example, the type of the distribution of the density of states (DOS) must be chosen and the values of the associated parameters must be determined prior to the numerical simulation. Then, the parameter values can be varied by external rules so that the fit between simulated and experimental data becomes acceptable. Thus, the parameter assignment, the numerical simulation and the results from simulations are in an iterative loop that has to be guided carefully for consistency in each instance, e.g., by a sequence that is presented in this section.

\subsection{Characteristic "temperature" $T_{0}$ of DOS}

The initial values for some of the parameters can be determined from experimental characteristics. The set of parameters and their values depend on the assumed type of DOS. If the DOS is of exponential type, then one provides for the characteristic width of the DOS by the parameter characteristic temperature $T_{0}$. Values around $\mathrm{T}_{\mathrm{o}} \approx 400 \mathrm{~K}$ are typical for OTFT. The value of $\mathrm{T}_{\mathrm{o}}$ can be determined experimentally from [6]

$$
\mathrm{T}_{\mathrm{O}}=\frac{\mathrm{T}}{2}(2+\gamma)
$$

where $\mathrm{T}$ is the absolute temperature ( $\mathrm{T} \sim 300 \mathrm{~K}$ at room ambient) and $\gamma$ is the mobility enhancement factor deduced from the I-V curves of the OTFT. For example, one can determine the value of $\gamma$ by a linear regression in the plot of the function $\mathrm{HVG}_{\mathrm{VG}}=\mathrm{I}_{\mathrm{Dsat}} \mathrm{VV}_{\mathrm{G}} / \mathrm{I}_{\mathrm{Dsat}}=\left(\mathrm{V}_{\mathrm{G}}-\mathrm{V}_{\mathrm{T}}\right) /(3+\gamma)$ obtained from the transfer characteristic $\mathrm{I}_{\mathrm{Dsat}}-\mathrm{V}_{\mathrm{G}}$ in the saturation regime of operation of the OTFT $[6,28,29]$, or simply to plot this transfer characteristic, $\mathrm{I}_{\text {Dsat }} \mathrm{Vs}$. $\left(\mathrm{V}_{\mathrm{G}}-\mathrm{V}_{\mathrm{T}}\right)$, in a log-log plot and the slope of this plot is $(2+\gamma)$. In a case when the dependence of the mobility $(\mu)$ on the gate bias $\left(V_{G}-V_{T}\right)$ is known, then the slope of the log-log plot of the characteristic $\mu$ vs. $\left(V_{G}-V_{T}\right)$ is the value of $\gamma$. A precise value for $\gamma$ (as well as for $V_{T}$, and consequently for $\mu$ and the contact resistance $R_{C}$ ) can be also obtained from the transfer characteristics $I_{D l i n}-V_{G}$ in the linear regime of operation of the OTFT [30] at $\mathrm{V}_{\mathrm{D}}<<\left(\mathrm{V}_{\mathrm{G}}-\mathrm{V}_{\mathrm{T}}\right)$ by means of linear regression in the plot of

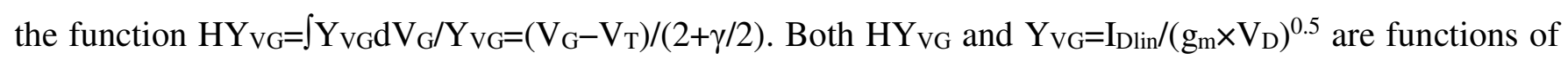


$\mathrm{V}_{\mathrm{G}}$, and $\mathrm{g}_{\mathrm{m}}=\partial \mathrm{I}_{\mathrm{Dlin}} / \partial \mathrm{V}_{\mathrm{G}}$ is the transconductance also function of $\mathrm{V}_{\mathrm{G}}$. A recent evolutionary parameter extraction method can also provide precise values for $\gamma, \mathrm{V}_{\mathrm{T}}, \mu$ and the contact resistance $\mathrm{R}_{\mathrm{C}}$, by an optimization procedure directly from the output characteristics of OTFTs [31].

In cases when $\gamma$ varies with the bias of OTFT, one has to consider that the DOS is not exponential. For normally distributed DOS (Gaussian DOS), there are bias-dependent crossovers [4, 12, 25].

\subsection{Concentration of states $\left(\mathrm{N}_{\mathrm{S}}\right)$ and orbital overlap $\left(\Lambda_{0}\right)$}

While the values for some parameters can be deduced from experimental characteristics, the initial values for many other parameters, however, might be unknown. One does not know a priori the total concentration of charge states $\left(\mathrm{N}_{\mathrm{S}}\right)$. Therefore, one needs to guess a value in the range $10^{21}-10^{22} \mathrm{~cm}^{-3}$, which corresponds to the molecular density of the organic material, e.g., for pentacene $[6,32,33](1.25-1.93) \times 10^{21} \mathrm{~cm}^{-3}$, multiplied by a factor 1-10, since there are multiple locations of $\pi$-bonds in the organic molecule (or monomers in polymers) that contribute to HOMO and LUMO levels [32, 34], as illustrated in Figure 6 for the HOMO in a pentacene molecule.

Another a priori unknown parameter is the orbital overlap $\left(\Lambda_{\mathrm{o}} \approx \mathrm{R}_{\mathrm{o}}\right.$ in hopping models $)$ and an appropriate guess can be made by assuming an exponential DOS, for which

$$
\begin{aligned}
\Lambda_{\mathrm{o}} \times \sqrt[3]{\mathrm{N}_{\mathrm{S}}} & \approx \frac{\sqrt[3]{7}}{\exp (1)}\left(\frac{2}{2+\gamma}\right) \exp \left(-\frac{4 / 3}{2+\gamma}\right) \\
& \approx \frac{1.4}{2+\gamma} \exp \left(-\frac{1.33}{2+\gamma}\right) \approx 0.3
\end{aligned}
$$

The value 0.3 corresponds to a mobility enhancement factor $\gamma=1$, as explained in [6], and used in numerical simulations by other authors [7]. In the literature $[4,10,12,25]$, values in the range $\Lambda_{0} \times\left(\mathrm{N}_{\mathrm{S}}\right)^{1 / 3} \approx 0.05-0.2$ are usually assumed. The uncertainty for the value is evident and it accumulates significant uncertainties for $\mathrm{N}_{S}$ and $\Lambda_{\mathrm{o}}$, as seen in Table IV later, and discussed in Sec. 4.1 with the help of Figure 22. The consequences of these inaccuracies are detailed in Sec. 5 . 


\subsection{Energy levels in materials and interfaces}

A third group of unknown a priori parameters are the relevant energies of the materials. The choice of values for the gate electrode work function, HOMO, LUMO and Fermi levels is not straightforward, because the reported values in the literature vary, as one can see in Table I for conductive materials [35, 36, $37,38,39,40,41,42,43,44,45,46,47,48,49,50]$, Table II for gate insulators $[38,47,48,51,52,53,54,55]$ and Table III for organic semiconductors $[3,32,34,35,39,40,42,44,45,46,48,50,56]$, owing to methods of material characterization or calculation, surface properties, chemically created dipoles at material interfaces and reference points. In addition, there is large discrepancy between molecular calculations and experiments, e.g. for the band gap of pentacene, the molecular crystal cell calculation has estimated $\sim 1 \mathrm{eV}$ in [32], whereas the experimental values suggest a gap of $2.2 \mathrm{eV}$. Therefore, we have summarized the most reasonable values to use in the VRH numerical simulator in columns "Recommended value" in Table I, Table II and Table III. These recommended values are also visualized in Figure 7 and in Figure 8. From the comparison of the metal work functions on the left-hand side of the figures ( $\mathrm{Pt}, \mathrm{p}^{+} \mathrm{Si}, \mathrm{Au}, \mathrm{PEDT} / \mathrm{PSS}$ ) with the organic semiconductors on the right-hand side of Figure 8 (P3HT, pentacene, PPV), these are usually used for OTFT fabrication, one can guess that the Fermi level $(\approx-4 \mathrm{eV}$, shifted down by about $0.5-1 \mathrm{eV}$ due to interface dipoles) in the organic semiconductor is about $0.3 \mathrm{eV}$ above the level corresponding to the work function of the gate electrode $(\approx-5 \mathrm{eV})$, and that the $\mathrm{HOMO}$ is about $0.9 \mathrm{eV}$ below the Fermi level. Therefore, we use these values in the following simulations, choosing also the gate electrode level as the zero reference level. Certainly, the choice for energy alignment is very approximate, and has to be researched further. Nevertheless, we believe that the choice is reasonable, and in agreement with the approaches for band alignment reported in [40, 46, 48].

The electrostatic simulation with the above choice resulted in energy diagrams as shown in Figure 9 for one case at low gate bias $\left(V_{G}=-5 V\right)$ and high temperature $\left(T=400 K=T_{0}\right)$ that corresponds to the energy width $\mathrm{kT}_{\mathrm{o}}$ of the DOS. Even at the large scale of $18 \mathrm{eV}$ in the figure, one can make several observations which require the following clarifications. One observation is the discontinuity of the vacuum level at the 
insulator-semiconductor interface, owing the abovementioned dipole shift. In "silicon" simulators, this shift is attributed to interface fixed charge, thus, it is not so unobvious, although it is interpreted in a different manner for organic TFTs. The second and the third observations in Figure 9 are that the potentials in the back of the semiconducting film do not reach the levels corresponding to bulk material, and there is a misalignment between Fermi level and flat-band (FB) level. We discuss these with the help of Figure 10, where the energy axis is zoomed in for a better view.

The closer look from left to right in Figure 10 for the energy diagrams at the insulator-semiconductor interface indicates that the variation of gate bias voltage $V_{G}$ does not cause unexpected changes in the diagram at any temperature, from low temperature of $\mathrm{T}=100 \mathrm{~K}$, through room temperature $\mathrm{T}=300 \mathrm{~K}$, elevated temperature $\mathrm{T}=400 \mathrm{~K}=\mathrm{T}_{\mathrm{o}}$ equal to the characteristic temperature of a double-sided exponential DOS, and even at high temperature $\mathrm{T}=500 \mathrm{~K}$, at which the pentacene film might easily degrade. However, the levels in the film-back do not reach the LUMO, FB and HOMO levels of the bulk material, which is contrary to the assumption for zero potential in the derivation of many models for OTFTs $[1,22,26,57]$. Thus, the film back is not a bulk material in an OTFT, and there is considerable potential bending in the entire depth of the TFT film [27], especially at low temperatures. The elevated temperatures reduce the difference between the film-back and the bulk material, but do not completely remove this "discrepancy".

The misalignment between Fermi level and flat-band (FB) level is small at low and room temperatures, but the "discrepancy" is considerable when the temperature is elevated close to or above the characteristic temperature $T_{0}$ of the exponential DOS. The reason for the misalignment is that the Fermi level $\left(E_{F}\right)$ is given for the Fermi occupation factor, $F_{n}(E)=1 /\left\{1+\exp \left[\left(E-E_{F}\right) / k T\right]\right\}$ for electrons, and its complementary $\mathrm{F}_{\mathrm{p}}(\mathrm{E})=1 /\left\{1+\exp \left[\left(\mathrm{E}_{\mathrm{F}}-\mathrm{E}\right) / \mathrm{kT}\right]\right\}$ for holes, whereas the $\mathrm{FB}$ depends also on the shape of the DOS, satisfying the (median) condition for equal concentrations of occupied states below and above FB, in particular given by eq. (26). 


\subsection{Flat band (FB) shift at temperature $T \geq T_{0}$}

For non-degenerated crystalline semiconductors, the flat band energy coincides well with the Fermi energy level, $\mathrm{FB} \approx \mathrm{E}_{\mathrm{F}}$, since the valence and conduction bands are well defined, having "sharp" edges, and one has dominant concentration of one type of dopant, either donors or acceptors of electrons. In amorphous semiconductors, however, there are tails of states asymmetrically placed around $E_{F}$ instead of bands with "sharp" edges. Consequently, FB becomes a strong function of the temperature, even if $\mathrm{E}_{\mathrm{F}}$ is the same. In the special case of an exponential DOS, it was discussed in detail in $[2,6]$ that the mode of the product $\mathrm{F}(\mathrm{E}) \mathrm{DOS}(\mathrm{E})$ moves from close to the Fermi level at low temperatures $\left(\mathrm{T}<\mathrm{T}_{\mathrm{o}}\right)$ toward the level $\mathrm{E}_{\mathrm{o}}$ of the DOS centroid at high temperatures $\left(\mathrm{T}>\mathrm{T}_{\mathrm{o}}\right)$. Therefore, one can infer that the quasi-Fermi level moves with the ratio $T_{0} / T$, but to avoid misinterpretations, we have the correct statement that the $F B$ varies with temperature, where FB is according to eq. (26). The evolution of the DOS occupancy and the FB shift with the temperature are illustrated in Figure 11 for a double-sided exponential DOS, defined by

$$
\begin{aligned}
\operatorname{DOS}(\mathrm{E}) & =\operatorname{DOS}_{\mathrm{DE}}(\mathrm{E}) \equiv \frac{\mathrm{N}_{\mathrm{S}}}{4 \mathrm{kT}_{\mathrm{o}} \mathrm{ch}^{2}\left(\frac{\mathrm{E}-\mathrm{E}_{\mathrm{O}}}{2 \mathrm{kT}_{\mathrm{o}}}\right)} \\
& =\mathrm{N}_{\mathrm{S}} \frac{\partial}{\partial \mathrm{E}}\left[1+\exp \left(\frac{\mathrm{E}_{\mathrm{O}}-\mathrm{E}}{\mathrm{kT}_{\mathrm{o}}} \text { polarity }\right)\right]^{-1},
\end{aligned}
$$

with the square of the hyperbolic cosine

$$
\begin{aligned}
& \operatorname{ch}^{2}(\mathrm{x})=\frac{1}{4}\left(\mathrm{e}^{\mathrm{x}}+\mathrm{e}^{-\mathrm{x}}\right)^{2} \\
& \text { polarity }=\left\{\begin{array}{l}
+1 \text { for electrons, } \mathrm{E}_{\mathrm{O}}=\mathrm{LUMO} \\
-1 \text { for holes, } \mathrm{E}_{\mathrm{O}}=\text { HOMO }
\end{array}\right. \\
& \text { and } \mathrm{N}_{\mathrm{S}}=\int_{-\infty}^{+\infty} \operatorname{DOS}(\mathrm{E}) \mathrm{dE}
\end{aligned}
$$

where $\mathrm{N}_{\mathrm{S}} \sim 10^{21} \mathrm{~cm}^{-3}$ is the (total) concentration of charge states in the organic semiconducting material. By this definition, the normalized double-exponential DOS/ $\mathrm{N}_{S}$ is similar to the Fermi distribution, with characteristic width $\mathrm{kT}_{\mathrm{o}}$ instead of thermal energy $\mathrm{kT}, \mathrm{E}_{\mathrm{o}}$ instead of $\mathrm{E}_{\mathrm{F}}$ and opposite polarity of variation with respect to the energy $(\mathrm{E})$. 
It is assumed in Figure 11 that the DOS does not change with temperature (solid gray line), and the peak value $\mathrm{N}_{\mathrm{S}} /\left(4 \mathrm{kT}_{\mathrm{o}}\right)$ of the double-exponential DOS (a circle) corresponds to the HOMO level $\mathrm{E}_{\mathrm{o}}$. The figure is for the bulk material pentacene. Therefore, the difference between the HOMO and the Fermi level $\left(\mathrm{E}_{\mathrm{F}}\right)$ is $0.9 \mathrm{eV}$, as discussed above, and these energy levels are depicted by gray-color vertical dashed lines. Since the pentacene is a p-type organic semiconductor, then the Fermi occupation factor for the majority carriers $(\mathrm{F})$ is close to unity at levels above the Fermi level $\left(\mathrm{E}_{\mathrm{F}}\right)$ at the right-hand side of the figure. Tracing the plot to the left, one observes that $F\left(E_{F}\right)=1 / 2$ at the Fermi level, shown by the other circle, and then $F$ decreases exponentially at lower energy levels $\left(\mathrm{E}<\mathrm{E}_{\mathrm{F}}\right)$ with a slope reciprocal to the absolute temperature, $\partial \ln (\mathrm{F}) / \partial \mathrm{E}=1 / \mathrm{kT}$. Therefore, the steepness of the slope decreases, increasing the temperature $\mathrm{T}$ from $\mathrm{T}<\mathrm{T}_{\mathrm{o}}$ to $\mathrm{T}>\mathrm{T}_{\mathrm{o}}$, as seen from the thick, thin and dotted lines intersecting in the circle labeled by " $1 / 2$ ".

The filling of the charge states is the DOS occupation with a density given by the product (F $\times$ DOS). The normalized DOS occupation is then $\left(\mathrm{F} \times \mathrm{DOS} / \mathrm{N}_{\mathrm{S}}\right)$, and it is shown by the polygon-like curves in Figure 11 for the three temperatures, for which the Fermi occupation factor (F) was given - thick, thin and dotted lines for low temperature $\left(T=300 K<T_{0}\right), T=T_{0}=400 K$ and high temperature $\left(T=500 K>T_{0}\right)$, respectively. Since both F and the double-sided exponential DOS have variable slopes with respect to E, then the slopes in the DOS occupancy also vary, and there are three regions in the curve for the DOS occupancy, because for pentacene or other organic semiconductor with hole conduction,

$$
\frac{\partial \ln (\mathrm{F} \times \mathrm{DOS})}{\partial \mathrm{E}}=\frac{\partial \ln (\mathrm{F})}{\partial \mathrm{E}}+\frac{\partial \ln (\mathrm{DOS})}{\partial \mathrm{E}}=\left\{\begin{array}{l}
\frac{1}{\mathrm{kT}}+\frac{1}{\mathrm{kT}_{\mathrm{o}}}, \text { when } \mathrm{E}<\mathrm{E}_{\mathrm{O}}<\mathrm{E}_{\mathrm{F}} \\
\frac{1}{\mathrm{kT}}-\frac{1}{\mathrm{kT}_{\mathrm{o}}}, \text { when } \mathrm{E}_{\mathrm{o}}<\mathrm{E}<\mathrm{E}_{\mathrm{F}} \\
-\frac{1}{\mathrm{kT}_{\mathrm{O}}}, \text { when } \mathrm{E}_{\mathrm{o}}<\mathrm{E}_{\mathrm{F}}<\mathrm{E}
\end{array}\right.
$$

Thus, having $\mathrm{T}_{0}=$ constant (DOS does not change with temperature), then the mode of the DOS occupancy varies with the temperature being at the energy level where the slope $\partial \ln (\mathrm{F} \times \mathrm{DOS}) / \partial \mathrm{E}=0$ and changes its sign. Consequently, the median flat-band (FB) energy follows the mode of the DOS occupancy. From eq. (46) for a p-type organic semiconductor, the slope is positive in the first region $\left(\mathrm{E}<\mathrm{E}_{\mathrm{o}}<\mathrm{E}_{\mathrm{F}}\right)$ and 
negative in the third region $\left(\mathrm{E}_{\mathrm{o}}<\mathrm{E}_{\mathrm{F}}<\mathrm{E}\right)$. However, the sign of slope in the middle region depends on the difference $\left(\mathrm{T}_{\mathrm{o}}-\mathrm{T}\right)$. At low temperature $\left(\mathrm{T}<\mathrm{T}_{\mathrm{o}}\right)$, the slope is positive in the middle region and the mode of the DOS occupancy is near the Fermi level $\left(\mathrm{E}_{\mathrm{F}}\right)$, as seen by the thick polygon-like line for $\left(\mathrm{F} \times \mathrm{DOS} / \mathrm{N}_{\mathrm{S}}\right)$ in Figure 11 for $\mathrm{T}=300 \mathrm{~K}<\mathrm{T}_{\mathrm{o}}$. Consequently, the median $\mathrm{FB}$ is also near $\mathrm{E}_{\mathrm{F}}$ for $\mathrm{T}=300 \mathrm{~K}<\mathrm{T}_{\mathrm{o}}$. Increasing the temperature to $\mathrm{T}=\mathrm{T}_{\mathrm{O}}=400 \mathrm{~K}$, then the DOS occupancy in the middle region is nearly constant, as shown with thin line in Figure 11, it is zero at $1 / 2\left(\mathrm{E}_{0}+\mathrm{E}_{\mathrm{F}}\right) \approx \mathrm{FB}$, since the DOS occupancy becomes almost symmetric around this energy level (neglecting the asymmetry in regions on the left and right when $\mathrm{E}_{\mathrm{o}}<<\mathrm{E}_{\mathrm{F}}$ for the bulk material, but not accurate for the OTFT operating well above the threshold, when large bending exists and $E_{o}$ is much closer to $E_{F}$, especially at the gate insulator interface, see again Figure 10). Further increase of the temperature, $\mathrm{T}>\mathrm{T}_{\mathrm{o}}$, results in a negative slope for the DOS occupancy in the middle region, as depicted with the dotted line in Figure 11, and the mode becomes close to the energy $\mathrm{E}_{\mathrm{o}}$ of the DOS centroid. Consequently, the median FB also shifts toward $E_{o}$ at high temperatures $\left(T=500 \mathrm{~K}>\mathrm{T}_{\mathrm{o}}\right)$. Thus, one should see considerable temperature dependence of the threshold voltage when the temperature $\mathrm{T} \approx \mathrm{T}_{\mathrm{o}}$ is near the characteristic temperature $\left(\mathrm{T}_{\mathrm{o}}\right)$ of the exponential DOS, because the change of FB in eV is replicated as a change of the threshold voltage $\mathrm{V}_{\mathrm{T}}$ in volts.

Overall, the increase of the temperature shifts $F B$ from $E_{F}$ to $E_{0}$, and the occupancy around $E_{o}$ changes several decades, depending exponentially on the ratio $\left|E_{0}-E_{F}-q V_{B}\right|\left(1 / k T-1 / k T_{o}\right)$, where $V_{B}$ is the potential bending due the gate bias of OTFT. The consequences for the mobility prefactor in compact models are discussed in [6] in terms of modification of a temperature shaping function (TSF). However, the impact of the temperature on the charge concentration $1 / 2 \int \mathrm{F} \times \mathrm{DOSdE}$ associated with FB from eq. (26) is less, as seen by the squares in Figure 11, and this impact is further reduced when the gate bias voltage $\mathrm{V}_{\mathrm{G}}$ is increased, since the DOS occupancy increases, the polygon-like curves in Figure 11 move up, and the width $\left|\mathrm{E}_{\mathrm{F}}-\mathrm{E}_{\mathrm{o}}\right|$ of middle region decreases, owing to the shift of $\mathrm{E}_{\mathrm{o}}$ toward $\mathrm{E}_{\mathrm{F}}$ due to potential bending at high $\mathrm{V}_{\mathrm{G}}$. An illustration for the bias dependence of FxDOS for normally distributed DOS in an n-type OTFT and fixed temperature can be found in Fig. 2 in [10] through changing the quasi-Fermi level $\left(E_{F}+q V_{B}\right) / k T_{o}$. In that 
figure, $E_{0}=0, E_{F}=-5 k T_{0}$, the mode of $F \times D O S$ is at $E_{F}$ at $q V_{B}<0$, and the mode shifts to $E_{0}=0$, increasing $\mathrm{qV}_{\mathrm{B}}$ to $+5 \mathrm{kT}_{\mathrm{o}}$. Further increase of $\mathrm{qV}_{\mathrm{B}}$ does not cause a shift of the mode, and the temperature does not significantly increase the median $\mathrm{FB}$. Thus, $\mathrm{FB} \approx \mathrm{E}_{\mathrm{F}}$ at low bias, and $\mathrm{FB}$ shifts to $\mathrm{FB} \approx \mathrm{E}_{\mathrm{o}}$ at high bias. (Please, inspect the definitions and notation in [10], since there, the reference level is $E_{o}$ of DOS, the quantities are normalized, $\mathrm{T}$ is fixed in that figure, and the quasi-Fermi level is denoted with $\mathrm{E}_{\mathrm{F}}$ and varied.)

\subsection{Potential bending and charge in the OTFT film}

Several profiles of the potential bending $V_{B}$ in the depth D of the OTFT semiconducting film at different temperatures and gate bias voltages $\mathrm{V}_{\mathrm{G}}$ are shown in Figure 12. It was suggested in [26] that the profile depends on the quantity $\left(\mathrm{D}+\mathrm{L}_{\mathrm{A}}\right)$, where the electrostatic depth $\mathrm{L}_{\mathrm{A}}$ is regarded as an effective depth of the conduction channel, and $\mathrm{L}_{\mathrm{A}}$ is given by

$$
\mathrm{L}_{\mathrm{A}}=\frac{2 \mathrm{kT}_{\mathrm{A}} \varepsilon_{\mathrm{f}}}{\mathrm{qC}_{\mathrm{ox}} \mathrm{V}_{\mathrm{G}}}=\frac{2 \phi_{\mathrm{A}} \varepsilon_{\mathrm{f}}}{\mathrm{C}_{\mathrm{ox}} \mathrm{V}_{\mathrm{G}}} \text {, with } \varphi_{\mathrm{A}} \equiv \mathrm{kT} \mathrm{T}_{\mathrm{A}} / \mathrm{q} \text { and } \mathrm{T}_{\mathrm{A}} \approx \mathrm{T}_{\mathrm{o}}
$$

considering the details in the reassessed derivation in [27] and the assumption in [22] that the bending affects the DOS occupancy as $\exp \left[\mathrm{V}_{\mathrm{B}} /\left(\mathrm{kT}_{\mathrm{o}}\right)\right]$ in the case of exponential DOS. The permittivity $\varepsilon_{\mathrm{f}}$ of organic materials is in the range $(2-4) \varepsilon_{\mathrm{o}}$, and one usually uses $\varepsilon_{\mathrm{f}}=3 \varepsilon_{\mathrm{o}} \approx 2.66 \times 10^{-13} \mathrm{~F} / \mathrm{cm}$. Then, following [27], one gets an analytical expression for the bending profile, given by

$$
\mathrm{V}_{\mathrm{B}}(\mathrm{D})=\mathrm{V}_{\mathrm{BS}}-2 \frac{\mathrm{kT}_{\mathrm{B}}}{\mathrm{q}} \ln \left(1+\frac{\mathrm{D}}{\mathrm{L}_{\mathrm{A}}}\right)=\mathrm{V}_{\mathrm{BS}}-2 \phi_{\mathrm{B}} \ln \left(1+\frac{\mathrm{D}}{\mathrm{L}_{\mathrm{A}}}\right) \text {, with } \varphi_{\mathrm{B}} \equiv \mathrm{kT}_{\mathrm{B}} / \mathrm{q} \text { and } \mathrm{T}_{\mathrm{B}} \approx \mathrm{T}_{\mathrm{o}} \text {, }
$$

where $V_{B S}=V_{B}(0)$ is the bending at the semiconductor-insulator interface $(D=0)$. Note that no temperature dependence is disclosed explicitly in eqs. (47) and (48), owing to the assumptions that $\mathrm{T}_{\mathrm{A}} \approx \mathrm{T}_{\mathrm{B}} \approx \mathrm{T}_{\mathrm{o}}$.

The dashed lines with solid circles in Figure 12 represent eq. (48) for two cases. The top one is for a high $\mathrm{V}_{\mathrm{G}}=-20 \mathrm{~V}$ and a low temperature $\mathrm{T}=100 \mathrm{~K}<\mathrm{T}_{\mathrm{o}}=400 \mathrm{~K}$, showing a good agreement (in shape and horizontal position) between eq. (48) and the profiles of $V_{B}$ at low temperature. However, the bottom dashed line, which is for eq. (48) at a low $V_{G}=-5 V$ and a high temperature $T=500 K>T_{0}$, has a slope different from the slope of the triplet of curves (without symbols) from simulation at $\mathrm{D}>\mathrm{L}_{\mathrm{A}}$, indicating that 
eq. (48) is a poor estimation for the bending at high temperatures $\mathrm{T}>\mathrm{T}_{\mathrm{o}}$. Thus, analytical models that consider $\exp \left[\mathrm{V}_{\mathrm{B}} /\left(\mathrm{kT}_{\mathrm{o}}\right)\right]$ in the derivations will be not accurate at $\mathrm{T}>1 / 2 \mathrm{~T}_{\mathrm{o}}$. Unfortunately, $\mathrm{T}_{\mathrm{o}}$ is in the range $350-450 \mathrm{~K}$ for organic materials [6], and $1 / 2 \mathrm{~T}_{\mathrm{o}}$ is normally below room temperature. Approaches to remedy this problem are given in $[6,11]$ and further discussions will be given in the next section with eqs. (79) and (80) in relation to Figure 28.

Profiles of the carrier concentration $\mathrm{N}_{\mathrm{C}}$ in the pentacene film are shown in Figure 13 with solid lines. These profiles correspond to the potential bending profiles shown in Figure 12, and similarly, the triplets of profiles at every given temperature for different $V_{G}$ 's coincide in the depth of the film, e.g., at $D>30 \mathrm{~nm}$. Again, taking the suggestion in [26], the reassessed derivation in [27] and the assumption in [22], one gets an analytical expression for $\mathrm{N}_{\mathrm{C}}$, which (for an exponential DOS) is given by

$$
\mathrm{N}_{C}(\mathrm{D})=\frac{2 \varepsilon_{\mathrm{f}} \mathrm{kT}_{\mathrm{C}}}{\mathrm{q}^{2}\left(\mathrm{D}+\mathrm{L}_{\mathrm{A}}\right)^{2}}=\frac{2 \varepsilon_{\mathrm{f}} \phi_{\mathrm{C}}}{\mathrm{q}\left(\mathrm{D}+\mathrm{L}_{\mathrm{A}}\right)^{2}} \text {, with } \varphi_{\mathrm{C}} \equiv \mathrm{k} \mathrm{T}_{\mathrm{C}} / \mathrm{q} \text { and } \mathrm{T}_{\mathrm{C}} \approx \mathrm{T}_{\mathrm{o}} \text {. }
$$

The circles in Figure 13 represent the calculations with eq. (49), showing very good overlap with the profiles at low temperature $\mathrm{T}<\mathrm{T}_{\mathrm{o}}=400 \mathrm{~K}$, especially for higher gate bias voltages. However, note again that there is no temperature dependence in eq. (49), owing to the assumption that $T_{C} \approx T_{0}$, whereas the profiles at shallow depths $\mathrm{D}<\mathrm{L}_{\mathrm{A}}$ decrease about $50 \%-100 \%$ at a high temperature $\mathrm{T}=500 \mathrm{~K}>\mathrm{T}_{\mathrm{o}}$. Nevertheless, although not exact at high temperatures $\mathrm{T}>\mathrm{T}_{\mathrm{o}}$, the quadratic decay of $\mathrm{N}_{C}$ predicted by eq. (49) at depths $\mathrm{D}>\mathrm{L}_{\mathrm{A}}$ is evident in the figure.

\subsection{Correlations between quantities in the OTFT film}

With the numerical simulator, we can investigate the evolution of the quantities in the depth of the organic film. The profiles of several quantities are shown in Figure 14. Similar to the previous two figures, the triplets of curves at each temperature in the plots of Figure 14 correspond to the three values of $\mathrm{V}_{\mathrm{G}}$, and the curves in each triplet coincide in the depth of the film, e.g., for D>30nm. The common point in all profiles is that the quantities are functions of $\left(D+L_{A}\right)$. For example, the profile of the electric field is $E_{e l}(D) \propto\left(D+L_{A}\right)^{-1}$, which is easy to deduce from eq. (49), since $E_{e l}(D) \propto \int N_{C}(D) d D$, and one observes in 
Figure $14 \mathrm{a}$ the reciprocal relation between $\mathrm{E}_{\mathrm{el}}$ and $\mathrm{D}$ at film depths $\mathrm{D}>10 \mathrm{~nm}>3 \mathrm{~L}_{\mathrm{A}}$. Consequently, from Figure $14 b$, the critical energy for hopping is a logarithmic function $\ln \left(D+L_{A}\right)$. From Figure $14 c$, the conductance $\sigma$ of the film is a very strong power-law function $\sigma \propto\left(D+L_{A}\right)^{-n}$. Also, and from Figure 14d, the carrier mobility $\mu$ in the film is also a strong power-law function $\mu \propto\left(D+L_{A}\right)^{-(n-2)}$, but with an exponent reduced by a factor of two, owing to the quadratic dependence in eq. (49) for the carrier concentration $\mathrm{N}_{\mathrm{C}}$, since $\mu=\sigma / q N_{C}$. This reduction in the exponent is explained and discussed in more details later with eq. (91). We will now clarify the functions behind profiles in Figure 12, Figure 13 and Figure 14 with the help of some additional plots.

The correlations between several quantities in the semiconducting film of an OTFT are summarized in Figure 15, organized as a matrix of plots. We denote the cells in this figure with $(r, c)$, where $r$ is the row and $\mathrm{c}$ is the column in the figure. Then, the correlation shown in cell $(\mathrm{c}, \mathrm{r})$ is the inverse of the correlation in cell $(r, c)$. The effect of increasing temperature is indicated by arrows. Note that, an up-shift in a cell below the diagonal with labels for quantities, c.f., cell (4,3), corresponds to right-shift in the above-diagonal cell $(3,4)$, and a clockwise rotation in cell $(5,4)$ corresponds to counter-clockwise rotation in cell $(4,5)$. The figure is deliberately arranged with a large number of cells, with the purpose to build the impression for the existence of correlations and dependences between the different quantities in the OTFT film. Observe the virtually straight lines in each cell, then intuitively, the relations perhaps are simple, thus, manageable analytically in practice. There are redundant relations (at least half, in the transposed cells) and we will not analyze each cell in great detail, but will comment on several interesting features.

The first observation in Figure 15 is that the correlations are independent of the gate bias voltage, since the lines for different $\mathrm{V}_{\mathrm{G}}=\{-5 \mathrm{~V},-10 \mathrm{~V},-20 \mathrm{~V}\}$ overlap, just spanning different intervals in the correlations. This indicates that there is an inherent relation between electrostatics and charge hopping, which is not explicitly explored in the literature. Bias-independent correlations are expected, once the material properties are independent of the electric field, which is one of the assumptions for the VRH simulator - please see the paragraph before eq. (30) earlier 
In contrast to the bias independence of the correlations in Figure 15, temperature affects the majority of the correlations, but not all of them. For example, the electrostatics is temperature independent (assuming a temperature independent permittivity) and there is no temperature dependence between carrier concentration and electric field in cells $(2,1)$ and $(1,2)$. However, observe that there is no temperature dependence between hopping distance and specific conductivity in cells $(6,5)$ and $(5,6)$, and this is not obvious at first glance. Observe also that there is a pronounced temperature dependence between specific conductance and mobility in cells $(7,6)$ and $(6,7)$. Therefore, the first-glance assumption for proportionality between mobility and conductance is probably an oversimplification, if overlooking that the carrier concentration might be temperature dependent [11]. However, inspecting the relation between carrier concentration and specific conductance cell $(6,1)$ or $(1,6)$ in conjunction with the relation between carrier concentration and mobility cell $(7,1)$ or $(1,7)$, one observes temperature dependences of different rates. Thus, the carrier concentration is temperature dependent, although the relation between electric field and carrier concentration is temperature independent, as mentioned above. Therefore, the mobility and conductivity are not really related by a temperature independent coefficient of proportionality. Consequently, one should expect different activation energies for mobility and conductance or current, and such comparisons $[58,59]$ are occasionally reported in the literature for OTFTs. Instead, semi-empirical observations for constant or bias-dependent thermal activation of mobility, conductance and current in OTFT are routinely reported $[1,11,22,24,26,34,41,44,60,61,62,63,64,65,66,67,68,69,70,71,72]$, also "successfully" fitting to the Meyer-Neldel rule [73, 74] and Gaussian disorder models for possible explanation of the thermal activation. Simple relations have been also deduced, such as (band bending + activation energy + Fermi level $)=($ transport band edge $)$ in Ref. [71], $\ln ($ charge $)=\ln ($ conductance $/$ mobility $) \propto($ mobility activation - conductance activation $) / k T \quad$ in $\quad$ ref. [59], proceeding to the next observation in Figure 15 that confirms the existence of simple relations. Alternative explanations for the activation energy in OTFT are also available, e.g., in terms of electrochemical description of the pentacene-oxide interface in Ref. [75]. 
The second observation is that the correlations resulted in straight lines in Figure 15 (with a small exception of a minute curvature for the correlation of the mobility at high bias and temperature, which cannot be seen in the figure). Since the axes in the figure are combinations of linear and logarithmic scales, then one can deduce four simple correlations between quantities in OTFTs.

- Linear correlation, when both axes are linear, e.g., cells $(4,3)$ and $(5,3)$ for the relations between hopping critical energy and distance to potential bending, respectively.

- Power-law correlation, when both axes are logarithmic, e.g., cell $(7,1)$ for the relation between mobility and carrier concentration.

- Exponential correlation, when $\mathrm{x}$-axis is linear and $\mathrm{y}$-axis is logarithmic, e.g., cell $(1,3)$ showing exponential dependence of the carrier concentration on potential bending, $\mathrm{N}_{\mathrm{C}} \propto \exp \left(\mathrm{V}_{\mathrm{B}}\right)$, for example.

- Logarithmic correlation, when $\mathrm{x}$-axis is logarithmic and $\mathrm{y}$-axis is linear, e.g., cell $(3,1)$ for the inverse relation $\mathrm{V}_{\mathrm{B}} \propto \ln \left(\mathrm{N}_{\mathrm{C}}\right)$.

Some of these correlations have been used in the derivation of physical and compact models. For example, eq. (10) in [22] uses $N_{C} \propto \exp \left(V_{B}\right)$ from cell $(1,3)$ in the normalized form $N_{C} / N_{S} \propto \exp \left(q V_{B} / k T_{o}\right)$ for the derivation of the widely-accepted VRH mobility model for OTFT. Another example is the correlation $\mu \propto\left(\mathrm{N}_{\mathrm{C}}\right)^{\gamma}$ is cell $(7,1)$ between mobility $\mu$ and charge $\mathrm{qN}$, which was experimentally established a while ago [33], and used in [28] to derive a TFT generic charge drift model.

The third observation in Figure 15, however, is that there are several simple correlations, which appear to be hidden behind the multiple assumptions and integrations required in the analytical derivations based on VRH in OTFTs with an exponential DOS. One often mentioned [22] but rarely discussed correlation is in cells $(6,5)$ and $(5,6)$ for the exponential dependence $\sigma \propto \exp \left(-R_{H}\right)$ of the conductivity $\sigma$ on the hopping critical distance $\mathrm{R}_{\mathrm{H}}$. Actually, this correlation is the basic assumption in VRH for the critical path that determines the percolation conductivity $\sigma \propto \exp \left(-\mathrm{s}_{\mathrm{c}}\right)$, eq. (1), and the equivalence of distance and energy for the factor $s_{c}$ of the hoping critical rate $\Gamma_{c}, s_{c}=\ln \left(\Gamma_{o} / \Gamma_{c}\right)=2 R_{H} / \Lambda_{o}=E_{H} / k T$, eq. (2), which was introduced in 
[20]. In fact, one must observe overlap of the curves in cells $(6,5)$ and $(5,6)$ at any temperature and bias, and the overlap is an indication of the accuracy of the numerical integrations in the simulator. A lack of overlap means a problem in the simulator, either the integration step (dE) for energy is coarse, or the limits of the integrations are narrow (or the numerical method failed because of another reason, e.g., error in code or lack of convergence). The other indicator for proper operation of the numerical simulator is in cells $(2,1)$ and $(1,2)$, where the electrostatic (Gauss) law for the electric field $\mathrm{E}_{\mathrm{el}} \propto \int \mathrm{N}_{\mathrm{C}} \mathrm{dD}$ must hold the same power-law function $\mathrm{N}_{\mathrm{C}} \propto\left(\mathrm{E}_{\mathrm{el}}\right)^{2}$ at any temperature and bias.

\subsection{Correlation between potential bending $\left(V_{B}\right)$ and hopping energy $\left(E_{H}\right)$}

An interesting "hidden" correlation is in cells $(4,3)$ and $(3,4)$ of Figure 15 for the linear dependence between potential bending $V_{B}$ and hopping critical energy $E_{H}$. The coefficient of proportionality is (-1), implying that the correlation is given by

$$
\mathrm{E}_{\mathrm{H}}+\mathrm{qV} \mathrm{V}_{\mathrm{B}}=\mathrm{E}_{\mathrm{T}}(\mathrm{T})
$$

where $\mathrm{E}_{\mathrm{T}}$ is a function only of the temperature. We did not to find this correlation in the literature. It is either fortunate or well hidden after the large equations with multiple integrals. However, this is a simple and handy relation between electrostatics and variable-range hopping, since the electrostatic calculation is quick (of order $\mathrm{n}^{2} \times 10^{5}$ FLOPs $\sim 0.4$ GFLOPs, see row "eSt" in Table VI and Appendix 1), while the hopping calculation is computationally extensive (of order $m \times n^{2} \times(750 F L O P s) ~ 150$ GFLOPs even for reduced depth mesh with m 50 points, see row "pass 2" for multiple $\iint \mathrm{dEdE}$ integrations in Table VI and Appendix 1). Therefore, we plot in Figure 16 the results for $\mathrm{E}_{\mathrm{T}}$ from simulation of several OTFTs, including the deviation from proportionality $(-1)$, and step by step, we will show that the relation is not fortunate, but is a consistent and basic bridge between hopping and electrostatics.

The results from the analyses of the $\mathrm{V}_{\mathrm{B}}-\mathrm{E}_{\mathrm{H}}$ correlation in eq. (50) are summarized in Figure 16. The results are obtained after fitting of the mobility at different temperatures $(\mathrm{T})$ and gate bias voltages $\left(\mathrm{V}_{\mathrm{G}}\right)$ in three OTFTs. These are from 108 simulations at different temperatures, using both single $\int \mathrm{dE}$ integration 
according to eq. (14) (gray color in Figure 16) and multiple $\iint \mathrm{dEdE}$ integrations according to eqs. (16), (18) and (20) (black color in Figure 16) for the calculation of the hopping conduction. The double-sided exponential DOS $\mathrm{DE}$ in eq. (45) was considered in the simulations. The fitting of the mobility will be shown shortly in Figure 17.

In Figure 16a, the values for $\mathrm{E}_{\mathrm{T}}$ are denoted with circle symbols $(O)$ for a pentacene OTFT from [73] for $\mathrm{V}_{\mathrm{G}}=\{-5 \mathrm{~V},-10 \mathrm{~V},-20 \mathrm{~V}\}$, with square symbols $(\square)$ for another pentacene OTFT from [22] for the same gate bias voltages, and with diamond symbols $(\diamond)$ for an annealed PQT-12 film OTFT from [60] at two times larger gate overdrive voltages $\left(\mathrm{V}_{\mathrm{G}}-\mathrm{V}_{\mathrm{T}}\right)=\{-10 \mathrm{~V},-20 \mathrm{~V},-40 \mathrm{~V}\}$. For each device, simulation method and temperature, the three symbols overlap for different gate bias voltages, confirming the bias-independent $\mathrm{E}_{\mathrm{T}}$ in eq. (50). The magnitudes of the proportionality coefficient between $\mathrm{E}_{\mathrm{H}}$ and $\mathrm{q} \mathrm{V}_{\mathrm{B}}$ are obtained from the slope $\left|\partial \mathrm{E}_{\mathrm{H}} / \partial \mathrm{V}_{\mathrm{B}}\right|$ of the regression between these quantities. The values of the slopes are shown with dash symbols (-) in Figure 16a, which are close to the ideal value of unity (dashed line in the figure), especially at high temperatures, but they deviate from unity at low temperatures.

Since the deviations from unity of the slope $\left|\partial \mathrm{E}_{\mathrm{H}} / \partial \mathrm{V}_{\mathrm{B}}\right|$ are small, we have performed a statistical analysis of the values (108 points), as summarized in Figure $16 \mathrm{~b}$. While values for $\left|\partial \mathrm{E}_{\mathrm{H}} / \partial \mathrm{V}_{\mathrm{B}}\right|$ are shown in the horizontal axis, note that the horizontal axis is reversed logarithmic axis of $\left(1-\left|\partial \mathrm{E}_{\mathrm{H}} / \partial \mathrm{V}_{\mathrm{B}}\right|\right)$, which is the deviation of the slope from the ideal value of unity. Therefore, the nearly uniform histogram (dotted bars) indicates an exponential distribution of the slope $\left|\partial \mathrm{E}_{\mathrm{H}} / \partial \mathrm{V}_{\mathrm{B}}\right|$ values and provides that $\left|\partial \mathrm{E}_{\mathrm{H}} / \partial \mathrm{V}_{\mathrm{B}}\right|=1 \pm 1 \%$ with $90 \%$ confidence. The curves denoted as "mean" represent the average values for the slope $\left|\partial \mathrm{E}_{\mathrm{H}} / \partial \mathrm{V}_{\mathrm{B}}\right|$ vs. the energy step $\mathrm{dE}=2 \mathrm{meV}$, used in the numerical integrations, normalized to the thermal energy $\mathrm{kT}$. Therefore, the fixed-value energy step is coarse at low temperature (dE/kT is large for low $\mathrm{T}$ ), and the energy step becomes fine at high temperature (dE/kT reduces at high $\mathrm{T})$. These curves in Figure 16b clearly show that the deviation from unity of the slope $\left|\partial \mathrm{E}_{\mathrm{H}} / \partial \mathrm{V}_{\mathrm{B}}\right|$ is an artifact of the numerical integration due to a coarse integration step at low temperatures $(\mathrm{dE} / \mathrm{kT}>10 \%$ at $\mathrm{T} \leq 200 \mathrm{~K})$, because a larger step $\mathrm{dE} / \mathrm{kT}$ causes larger deviation from unity of $\left|\partial \mathrm{E}_{\mathrm{H}} / \partial \mathrm{V}_{\mathrm{B}}\right|$. Also, less integration by the method of single $\int \mathrm{dE}$ integration (gray color) 
causes larger deviations, compared to the method of multiple $\iint \mathrm{dEdE}$ integrations (black lines). These observations are repeated proportionally for the standard and greatest deviations from unity of $\left|\partial \mathrm{E}_{\mathrm{H}} / \partial \mathrm{V}_{\mathrm{B}}\right|$, shown with horizontal error bars and denoted with "mean- $\sigma$ " and "min" in Figure 16b. Thus, the numerical simulations of variable-range hoping have justified the correlation in eq. (50) between potential bending $V_{B}$ and hopping critical energy $E_{H}$, although we cannot find in the literature an analytical derivation for this correlation. The consequences from eq. (50) will be given later in Sec. 4 "Discussion". The immediate deduction is that $\mathrm{E}_{\mathrm{T}}$ should be equal to the hopping critical energy in the bulk material, since the potential bending in bulk material is zero $\left(\mathrm{V}_{\mathrm{B}}=0\right)$.

\subsection{OTFT mobility $(\mu)$ and sheet conductance $\left(\sigma_{\mathrm{sq}}\right)$ from VRH conductivity $(\sigma)$}

The most critical OTFT performance parameter is the magnitude of the effective mobility $(\mu)$, since $\mu$ in OTFT is low and it depends on many factors - type and uniformity of organic semiconductors (e.g. grains), materials and layers at interfaces and contacts, layout, fabrication, encapsulation, temperature, bias, light, time, characterization techniques, etc., as reported in many publications and discussed in length in several review articles $[27,33,76]$. Therefore, the benchmark assessment for models and simulators is the prediction of $\mu$ in OTFT. On the other hand, the VRH theories provide for the specific conductivity ( $\sigma$, in unit, e.g. $\mathrm{A} / \mathrm{Vcm} \equiv \mathrm{S} / \mathrm{cm}$ ) of the materials $[20,21]$, but not directly for the mobility of charge carriers in OTFT. Therefore, one has used in [22] the following supplementary relations to obtain analytical expression for the mobility from conductivity in OTFT operating in the linear (Ohmic) regime.

$$
I_{D}=\frac{W}{L} V_{D} \int_{0}^{t_{f} \rightarrow \infty} \sigma(D) d D=\frac{W}{L} V_{D} \sigma_{s q},
$$

and

$$
\mu=\left[\frac{\mathrm{W}}{\mathrm{L}} \mathrm{V}_{\mathrm{D}}\right]^{-1} \frac{1}{\mathrm{C}_{\mathrm{I}}} \frac{\partial \mathrm{I}_{\mathrm{D}}}{\partial \mathrm{V}_{\mathrm{G}}}=\frac{1}{\mathrm{C}_{\mathrm{I}}} \frac{\partial \sigma_{\mathrm{sq}}}{\partial \mathrm{V}_{\mathrm{G}}}
$$


where $C_{I}$ is the gate insulator capacitance per unit area, $I_{D}$ is the drain current at bias voltages at the drain $\mathrm{V}_{\mathrm{D}}$ and gate $\mathrm{V}_{\mathrm{G}}$ with threshold voltage $\mathrm{V}_{\mathrm{T}}$ omitted. $\mathrm{W}$ and $\mathrm{L}$ are the width and length of the OTFT channel, respectively, and $\sigma_{\mathrm{sq}}$ is the sheet conductivity of the semiconducting film in unit Siemens per square area of the channel. This derivation sequence of using proportionalities, integrations and differentiations is vulnerable to cancelling of constant multipliers and omission of constants in the final expression for the mobility. Indeed, integration limits and details for approximations and neglecting term in several steps and substitutions by the derivations were omitted in [22]. Therefore, we use directly the results from the numerical simulator for the profiles of $\sigma$ (cf. Figure 14c) for the finite thickness $t_{f}$ of the OTFT film and obtain the effective mobility from the general relation in eq. (42).

The results of our calculations for the mobility $\mu$ are depicted in Figure 17 with lines, and compared with the experimental data shown as symbols. Plots (a), (b) and (c) in this figure correspond to the symbols in Figure 16a, in particular, to the circle symbols $(\bigcirc)$ for a pentacene OTFT from [73], square symbols $(\square)$ for the other pentacene OTFT from [22] and diamond symbols $(\diamond)$ for the annealed-PQT-12-film OTFT from [60], respectively. Also in correspondence with Figure 16, the thick gray lines are from simulation of the hopping conduction by the method of single $\int \mathrm{dE}$ integration according to eq. (14), and the thin black lines are after multiple $\iint \mathrm{dEdE}$ integrations according to eqs. (16), (18) and (20). The parameters used in the simulations are given in Table IV and are also compared with the values reported in the literature for these samples. In Figure 17b, we observe a good overlap between numerical simulation and experimental data, the latter used in [22] for verification of the analytical VRH model for OTFTs, and recently, for the generic and compact models [6] for the mobility in OTFT. The deviations observed in the figure and the scatter of the values of the parameters in Table IV will be addressed later in Sec. 4 "Discussion".

As mentioned above, the VRH theories and derivations, and the numerical simulators, consequently, provide for the specific conductivity $\sigma$. Using eq. (41), one obtains the sheet conductivity $\sigma_{\mathrm{sq}}=\int \sigma \mathrm{dD}$ in the channel of the OTFT by integration of the profile for $\sigma$ from the gate insulator interface $(\mathrm{D}=0)$ into the depth $\mathrm{D}$ of the organic semiconductor $\left(0 \leq \mathrm{D} \leq \mathrm{t}_{\mathrm{f}}\right)$, up to the thickness $\mathrm{t}_{\mathrm{f}}$ of the semiconducting film. Typical 
results for $\sigma_{\mathrm{sq}}$ are shown in Figure 18 at several temperatures and as function of the gate overdrive $\left(\mathrm{V}_{\mathrm{G}}-\mathrm{V}_{\text {on }}\right)$, where the turn-on voltage $\mathrm{V}_{\mathrm{on}}$ is the gate voltage $\mathrm{V}_{\mathrm{G}}$, at which the gate bias induces a conductivity larger than the bulk conductivity, and $\mathrm{V}_{\mathrm{on}} \sim \mathrm{V}_{\mathrm{FB}}$ corresponds roughly to the flat-band potential $\mathrm{V}_{\mathrm{FB}}$ referred to the gate conductor work function in our simulator. Note that $\mathrm{V}_{\text {on }}$ is "below" the threshold voltage $\mathrm{V}_{\mathrm{T}}$, and the range $\mathrm{V}_{\text {on }}<\mathrm{V}_{\mathrm{G}}<\mathrm{V}_{\mathrm{T}}$ is usually regarded as the sub-threshold regime of operation of the OTFT. OTFTs are typically p-type field-effect transistors, for which one uses inverted values for the voltages, e.g., $\left(-\mathrm{V}_{\mathrm{on}}\right)<\left(-\mathrm{V}_{\mathrm{G}}\right)<\left(-\mathrm{V}_{\mathrm{T}}\right)$, as in Figure 18.

The simulation results for $\sigma_{\mathrm{sq}}$ are shown with lines in Figure 18 with colors corresponding to the colors in Figure 16a and Figure 17c. The thick gray lines are from simulation of the hopping conduction by the method of single $\int \mathrm{dE}$ integration according to eq. (14), and the thin black lines are after multiple $\iint \mathrm{dEdE}$ integrations according to eqs. (16), (18) and (20). The parameter values are the same as for Figure 17c and are given in Table IV. We present in Figure 18 the same results in three plots: linear, semi-logarithmic and logarithmic, since the values are over several decades, and the different plot formats provide different insights. The linear plot in Figure 18a illustrates that the two methods of single $\int \mathrm{dE}$ and multiple $\iint \mathrm{dEdE}$ integrations predict different magnitudes for $\sigma_{\mathrm{sq}}$, with differences up to about $20 \%$ at a given bias and temperature, which is an estimate for how accurate the numerical simulations of VRH can be in practice. The semi-logarithmic plot in Figure 18b illustrates, however, that both methods predict in very similar manner the behaviors of $\sigma_{\mathrm{sq}}$ as a function of bias and temperature. The higher temperatures increase the OTFT's conductivity at given gate bias (especially at low bias), but the higher bias reduces the temperature effect. Conversely, higher temperatures reduce (in relative units) the dependence of the conductance on the bias, since the steepness of the curves in Figure $18 \mathrm{~b}$ is reduced at higher temperatures. The semi-logarithmic plot in Figure 18b shows the dependences qualitatively, and the actual form of these dependences becomes clear from the logarithmic plot in Figure 18c. Here, the lines are almost straight, illustrating the power-law dependence of the conductance on the bias, $\sigma_{\mathrm{sq}} \propto\left|\mathrm{V}_{\mathrm{G}}-\mathrm{V}_{\mathrm{on}}\right|^{\mathrm{n}}$, with the exponent factor (n) increasing, when the temperature decreases, $\mathrm{n} \propto 1 / \mathrm{T}$, and lines intersecting at some high overdrive $\left|\mathrm{V}_{\mathrm{G}}-\mathrm{V}_{\text {on }}\right| \sim 240 \mathrm{~V}$, at which 
the temperature dependence is virtually cancelled. These details from simulation will be further discussed shortly, after justifying the consistency of the numerical simulation with experiments.

The open and filled symbols in Figure 18 are recalculated data for two PQT-12 OTFTs. Drain current $I_{D}-V_{G}$ transfer curves at a low drain voltage $V_{D}=-1 V$ are reported in Fig. 2a at lower $\left|V_{G}\right|$ and in Fig. 8a at higher $\left|V_{G}\right|$ in [60]. Therefore, we assume that the data correspond to the linear regime of operation of OTFT, and from eq. (51), one can estimate the sheet conductance by

$$
\sigma_{\mathrm{sq}} \approx\left|\frac{\mathrm{I}_{\mathrm{D}}}{\mathrm{V}_{\mathrm{D}}}\right| \frac{\mathrm{L}}{\mathrm{W}} \text {, for linear regime }\left|\mathrm{V}_{\mathrm{D}}\right|<\left|\mathrm{V}_{\mathrm{G}}-\mathrm{V}_{\mathrm{T}}\right| \sim\left|\mathrm{V}_{\mathrm{G}}-\mathrm{V}_{\mathrm{on}}\right|,
$$

as far as $\left(-\mathrm{V}_{\mathrm{G}}\right)$ is several volts above the threshold voltage $\left(-\mathrm{V}_{\mathrm{T}}\right)$, the later reported around 10V for the annealed-PQT-12-film OTFTs and $\mathrm{V}_{\mathrm{on}}= \pm 1 \mathrm{~V}$ at room temperature. The information for the samples and their parameters is aggregated in ranges in [60], but unfortunately, not sample by sample at different temperatures. Nevertheless, I-V transfer curves at different temperatures are provided in [60], which is a much better situation than not reporting any temperature-dependent I-V characteristic in [22, 73], but only the mobility for the pentacene samples, c.f. Figure $17 \mathrm{a}$ and $\mathrm{b}$. We tried to recover the missing information for the PQT-12 OTFTs, using the procedure described in the next paragraph.

It is provided in [60] that the samples had $\mathrm{W}=0.5-1 \mathrm{~mm}$ and $\mathrm{L}=40-100 \mu \mathrm{m}$, and the threshold voltage in Fig. $2 \mathrm{~b}$ in this publication increased from $\left(-\mathrm{V}_{\mathrm{T}}\right)=5 \mathrm{~V}$ at room temperature to $13-14 \mathrm{~V}$ when the temperature was decreased to $\mathrm{T}=200-150 \mathrm{~K}$. Thus, considering also the aforementioned room-temperature $\mathrm{V}_{\mathrm{T}}$ and $\mathrm{V}_{\text {on, }}$, we let $\mathrm{W} / \mathrm{L}$ to vary between 5 and $25 \pm 20 \%$ and $\left(-\mathrm{V}_{\text {on }}\right)$ to vary between $(-2 \mathrm{~V})$ and $(+15 \mathrm{~V})$ until a good match between simulated and recalculated by eq. (53) values occurs at higher gate overdrive $\left|V_{G}-V_{\text {on }}\right|$ at all temperatures, as shown in Figure 18 for matching of the recalculated $\sigma_{\mathrm{sq}}$ from $\mathrm{I}_{\mathrm{D}}-\mathrm{V}_{\mathrm{G}}$ transfer curves to simulation of VRH with multiple $\iint \mathrm{dEdE}$ integrations. Similar good match was obtained also to the other method with single $\int \mathrm{dE}$ integration, but with different values for $\mathrm{W} / \mathrm{L}$ and $\mathrm{V}_{\text {on }}$, and the symbols for this latter match are omitted only for clarity in the figure. The values for $\mathrm{W} / \mathrm{L}$ and $\mathrm{V}_{\text {on }}$ are given in Table $\mathrm{V}$ for both fittings. Both $\mathrm{W} / \mathrm{L}$ and $\mathrm{V}_{\text {on }}$ vary with the temperature even for the same sample, which signifies that that there is a large uncertainty in the recalculation. Therefore, the comparison between simulations and 
experiments in Figure 18 should be taken qualitatively, not quantitatively. However, particular sample information in [60] is missing, as we have mentioned, and we cannot discriminate which values are correct and which are wrong, since all values for $\mathrm{W} / \mathrm{L}$ and $\mathrm{V}_{\text {on }}$ in Table $\mathrm{V}$ are within the intervals stated in [60]. Also, lowering the bias below $\left|\mathrm{V}_{\mathrm{G}}-\mathrm{V}_{\mathrm{on}}\right|<5 \mathrm{~V}$, one observes discrepancies between simulation and recalculated experimental data; e.g., in Figure 18c, the experimental data level off ( $\square$ and $\bigcirc$ for $\mathrm{T}=200 \mathrm{~K}$ and $\mathrm{T}=150 \mathrm{~K}$ ), while the simulations bend down (clearly seen by the lines for $\mathrm{T}=300 \mathrm{~K}$ ). These discrepancies are because the OTFT moves in the saturation and subthreshold regimes when $\left|\mathrm{V}_{\mathrm{G}}-\mathrm{V}_{\text {on }}\right|<5 \mathrm{~V}$, and the recalculation by eq. (53) is incorrect, since the condition for linear regime and validity of this equation are violated. Nevertheless, the qualitative comparison at sufficiently high gate bias, e.g. $\left|\mathrm{V}_{\mathrm{G}}-\mathrm{V}_{\text {on }}\right|>10 \mathrm{~V}$, indicates that the aforementioned power-law and 1/T behaviors deduced by numerical simulations of VRH are reproduced in the experiments. Also, note that the numerical evaluation of eqs. (41) and (42) is independent of the value of (W/L). Next, we closely inspect these relations.

\subsection{Bias dependence and thermal activation of the OTFT sheet conductance $\left(\sigma_{\mathrm{sq}}\right)$ by VRH}

A close look at the predictions from numerical simulation of the sheet conductance $\sigma_{\mathrm{sq}}$ in OTFT is given in Figure 19 for an expanded biasing range up to $\left(\mathrm{V}_{\mathrm{G}}-\mathrm{V}_{\mathrm{on}}\right)=-700 \mathrm{~V}$ for clarity, although the $100 \mathrm{~nm} \mathrm{SiO}_{2}$ gate dielectric (see Table IV again) in the real PQT-12 devices in [60] may break down at a lower bias. The square symbols in this figure are the results for $\sigma_{\mathrm{sq}}$ from simulation, and correspond to the lines in Figure $18 \mathrm{c}$, from which we have deduced above that the sheet conductance $\sigma_{\mathrm{sq}}\left(\mathrm{V}_{\mathrm{G}}, \mathrm{T}\right)$ should be a temperaturedependent power-law function of the bias, given by

$$
\frac{\sigma_{\mathrm{sq}}\left(\mathrm{V}_{\mathrm{G}}, \mathrm{T}\right)}{\sigma_{\mathrm{sqx}}}=\left(\frac{\mathrm{V}_{\mathrm{G}}-\mathrm{V}_{\mathrm{on}}}{\mathrm{V}_{\mathrm{Gx}}-\mathrm{V}_{\mathrm{on}}}\right)^{\mathrm{n}(\mathrm{T})} \approx\left(\frac{\mathrm{V}_{\mathrm{G}}}{\mathrm{V}_{\mathrm{Gx}}}\right)^{\mathrm{n}(\mathrm{T})} \text {, since } \mathrm{V}_{\mathrm{Gx}}>\mathrm{V}_{\mathrm{G}}>\mathrm{V}_{\mathrm{on}} \sim \pm 1 \mathrm{~V} \text {, }
$$

where $\sigma_{\mathrm{sqx}}$ and $\mathrm{V}_{\mathrm{Gx}}$ are some characteristic parameters, and the temperature dependence of $\sigma_{\mathrm{sq}}$ is due to variation of the exponential factor (n) in the power-law function as reciprocal of the temperature, e.g., 


$$
\mathrm{n}(\mathrm{T})=\frac{\mathrm{T}_{\sigma \mathrm{x}}}{\mathrm{T}}=\frac{\partial \ln \left(\sigma_{\mathrm{sq}}\right)}{\partial \ln \left(\left|\mathrm{V}_{\mathrm{G}}-\mathrm{V}_{\mathrm{on}}\right|\right)}
$$

where $T_{\sigma x}$ is also a characteristic temperature parameter for $\sigma_{\mathrm{sq}}$. Thus, we fit the power-law trend lines in Figure 19 to the simulation data points that align in straight lines in the logarithmic plot. These data points are mostly in the bias range $-\left(\mathrm{V}_{\mathrm{G}}-\mathrm{V}_{\mathrm{on}}\right)=10-100 \mathrm{~V}$, and are denoted with filled squares. The trend lines intersect at points $\left(\mathrm{V}_{\mathrm{Gx}}, \sigma_{\mathrm{sqx}}\right)$ denoted with circles in the upper-right corner of the figure, indicating almost constant values for $\mathrm{V}_{\mathrm{Gx}} \approx-240 \mathrm{~V}$ and $\sigma_{\mathrm{sqx}} \approx 0.35 \mu \mathrm{S} /$ square after both methods of VRH simulation by single $\int \mathrm{dE}$ integration according to eq. (14), and multiple $\iint \mathrm{dEdE}$ integrations according to eqs. (16), (18) and (20). Therefore, we conclude that eq. (54) holds for moderate biases. Furthermore, the slopes of the power-law trend lines in the logarithmic plot are the exponential factor (n) in the power-law function, as indicated by the last term in eq. (55). The values for $\mathrm{n}$ are shown in the inset of Figure 19 with circles as function of the reciprocal of the temperature $1 / \mathrm{T}$. These values also align with the straight lines in the linear plot of the inset, validating eq. (55), with $\mathrm{T}_{\sigma \mathrm{x}} \approx 400 \mathrm{~K}$. Therefore, we conclude that the conductance in the OTFT at moderate bias has a thermal activation energy in the form of

$$
\mathrm{E}_{\mathrm{A} \sigma} \approx \mathrm{kT}_{\sigma \mathrm{x}} \ln \left(\frac{\mathrm{V}_{\mathrm{Gx}}}{\mathrm{V}_{\mathrm{G}}}\right) \text {, at moderate bias }\left|\mathrm{V}_{\mathrm{Gx}}-\mathrm{V}_{\mathrm{on}}\right| 2>\left|\mathrm{V}_{\mathrm{G}}-\mathrm{V}_{\mathrm{on}}\right|>10 \mathrm{~V} \text {, }
$$

since from eqs. (54) and (55), it follows that

$$
\frac{\sigma_{\mathrm{sq}}\left(\mathrm{V}_{\mathrm{G}}, \mathrm{T}\right)}{\sigma_{\mathrm{sqx}}} \approx\left(\frac{\mathrm{V}_{\mathrm{G}}}{\mathrm{V}_{\mathrm{Gx}}}\right)^{\mathrm{n}(\mathrm{T})}=\left(\frac{\mathrm{V}_{\mathrm{G}}}{\mathrm{V}_{\mathrm{Gx}}}\right)^{\frac{\mathrm{T}_{\sigma \mathrm{x}}}{\mathrm{T}}}=\exp \left\{-\frac{\mathrm{T}_{\sigma \mathrm{x}}}{\mathrm{T}} \ln \left(\frac{\mathrm{V}_{\mathrm{Gx}}}{\mathrm{V}_{\mathrm{G}}}\right)\right\}
$$

Note the conditions in eq. (56), which were used as selection criteria of the points for fitting of the powerlaw trend lines. At lower or higher gate bias, the deviations from the power-law dependence are evident, as seen by the data points denoted with open squares in Figure 19. At low bias, the deviation is due to the uncertain value of $\mathrm{V}_{\text {on. }}$. At high bias, the potential bending in the organic semiconductor crosses and is above the DOS centroid level $\mathrm{E}_{\mathrm{o}}$, and the power-law is violated, since the charge hopping is no longer in the exponential tail of the DOS, and the DOS occupation tends to saturate. In this case, we have observed that 
the inaccuracy of numerical simulator is also larger, owing to the larger differences in the results from the two methods of single $\int \mathrm{dE}$ integration and multiple $\iint \mathrm{dEdE}$ integrations, although both methods predict saturation in the VRH conductivity.

\section{Discussion}

The previous section addressed the essential properties and behaviors that the VRH predicts for OTFTs. In this section, we address several other cases that occur in the application of VRH, such as the type of DOS in the next sub-section.

\subsection{Effects due to the assumption for the type of DOS}

The simulation results shown in the previous section are with the assumption that the DOS is a double-sided exponential DOS $\mathrm{DE}$, according to eq. (45). On the other hand, VRH was analyzed in the literature as either a single-sided exponential DOS $\mathrm{SE}$, eq. (22), or a normally distributed $\mathrm{DOS}_{\mathrm{ND}}$, that can be given in the form

$$
\operatorname{DOS}(\mathrm{E})=\operatorname{DOS}_{\mathrm{ND}}(\mathrm{E}) \equiv \frac{\mathrm{N}_{\mathrm{S}}}{\mathrm{kT}_{\mathrm{o}} \sqrt{2 \pi}} \exp \left[-\left(\frac{\mathrm{E}-\mathrm{E}_{\mathrm{O}}}{\mathrm{kT}_{\mathrm{o}} \sqrt{2}}\right)^{2}\right]
$$

where $\mathrm{E}_{\mathrm{o}}$ is the energy position of $\mathrm{DOS}_{\mathrm{ND}}$, e.g. HOMO for p-type OTFT, and $\left(\mathrm{kT} \mathrm{T}_{\mathrm{o}}\right)$ defines the width of the distribution, similarly as for the exponential types of DOS. Comparing eqs. (22), (45) and (58), one sees that the assumptions for DOS might be quite different. Therefore, it is reasonable to investigate the impact of the DOS type on VRH.

The effect of the assumption for DOS on the mobility in OTFT is illustrated in Figure 20 for both methods of VRH numerical calculation with single $\int \mathrm{dE}$ and multiple $\iint \mathrm{dEdE}$ integrations. One observes in the figure that the experimental data (open circles $\bigcirc$ ) for the OTFT mobility can be fitted well by any of the assumptions for DOS type in an interval of $\pm(50-100) \mathrm{K}$ around room temperature $(\mathrm{T} \sim 300 \mathrm{~K})$, when choosing appropriate values for the parameters. Thus, one might be unable to determine the DOS type from the Arrhenius plots, $\log (\mu)$ vs. $1 / \mathrm{T}$, of the experimental data for mobility. The trend in the figure is that the 
DOS type affects the behavior at very low and very high temperatures, and the effects are in correspondence with the "rectangularity" of DOS. A possible definition of DOS "rectangularity" is given later in eq. (74). The trend and the "rectangularity" are illustrated in the cartoon of Figure 21. The single-sided exponential $\mathrm{DOS}_{\mathrm{SE}}$ is a "peaking" function of the energy with low "rectangularity". For DOS $\mathrm{SE}_{\mathrm{SE}}$, the behavior of the mobility is $\mu \propto \exp \left(-\mathrm{T}_{\mathrm{o}} / \mathrm{T}\right)$ at low temperatures, leveling off at high temperatures. The increased "rectangularity" of the double-sided exponential DOS ${ }_{\mathrm{DE}}$ and the normally distributed DOS $\mathrm{ND}_{\mathrm{N}}$ bends up the $\log (\mu)-1 / T$ dependence at low and high temperatures, without major changes at moderate temperatures around room temperature.

The type of DOS has a moderate effect on the behavior of the VRH mobility. However, the different assumptions for the type of DOS and the different methods of VRH calculations have significant impacts on the values of the parameters. As mentioned earlier, when discussing the deviations between simulation and experimental data in Figure 17, the values of the parameters are collected in Table IV. These parameters have been used in the simulations shown in the preceding figures, e.g., Figure 20 above for mobility. Column "comment" in Table IV discloses which data set where was used. The scatter of values is addressed here. The values of the parameters from Table IV are visualized in Figure 22.

The top-left plot in Figure 22 is for the "characteristic temperature" $\mathrm{T}_{\mathrm{o}}$, which describes the energy width $\left(\mathrm{kT}_{\mathrm{o}}\right)$ of the distribution of the DOS. One observes that $\mathrm{T}_{\mathrm{o}}$ for exponential types of DOS is about 2-3 times smaller than $\mathrm{T}_{\mathrm{o}}$ for normally distributed $\mathrm{DOS}_{\mathrm{ND}}$ [6]. Also, the values for $\mathrm{T}_{\mathrm{o}}$ are very similar for both the single-sided exponential DOS $\mathrm{SE}$ and the double-sided exponential DOS $\mathrm{DE}_{\mathrm{DE}}$. Further, there is a good match to values for $\mathrm{T}_{\mathrm{o}}$ from analytical VRH calculations with exponential DOS reported in the literature for these samples. Unfortunately, this coherent situation for $\mathrm{T}_{\mathrm{o}}$ is not the case for the other parameters related to VRH in OTFTs.

The middle-left plot in Figure 22 is for the total concentration of states $\mathrm{N}_{\mathrm{S}}$ for all energies, $\mathrm{N}_{S}=\int \mathrm{DOS}(\mathrm{E}) \mathrm{dE}$ with $(-\infty \leq \mathrm{E} \leq+\infty)$. Two trends are observed in this plot. One trend is that the method of single $\int \mathrm{dE}$ integration for VRH requires 2-3 times higher value for $\mathrm{N}_{\mathrm{S}}$, as compared with the method of 
multiple $\iint \mathrm{dEdE}$ integrations, in order to calculate similar VRH mobility for any given type of DOS in an OTFT. This trend indicates that at present, the VRH theories do not provide a mature and unique method for calculation. The second trend is that $\mathrm{N}_{\mathrm{S}}$ is higher for OTFTs with lower mobility, comparing from left to right for the two pentacene OTFTs, and $\mathrm{N}_{\mathrm{S}}$ is even higher for the PQT-12 OTFT. We note that the values for $\mathrm{N}_{\mathrm{S}}$ are larger than the molecular density of pentacene and PQT-12. The unit cell sizes of pentacene molecular crystals are reported as $1.603 \mathrm{~nm} \times 0.793 \mathrm{~nm} \times 0.614 \mathrm{~nm}$ in [32] and $1.6 \mathrm{~nm} \times 0.79 \mathrm{~nm} \times 0.606 \mathrm{~nm}$ in [33], resulting in molecular density $\sim 1.3 \mathrm{~nm}^{-3}=1.3 \times 10^{21} \mathrm{~cm}^{-3}$ with $11 \pi$ bonds per molecule, that is, the $\pi$ orbital density is in order of $2 \times 10^{22} \mathrm{~cm}^{-3}$. The crystallographic studies of PQT-12 films in [77, 78] reveal that the unit cell of this polymer is of size $1.64 \mathrm{~nm} \times 1.55 \mathrm{~nm} \times 0.38 \mathrm{~nm}$, having four rings with two $\pi$ bonds per ring, resulting in molecular density $\sim 1.04 \mathrm{~nm}^{-3}=1.04 \times 10^{21} \mathrm{~cm}^{-3}$ and $\pi$-orbital density of $1.7 \times 10^{22} \mathrm{~cm}^{-3}$. Comparing with $\mathrm{N}_{\mathrm{S}}=(2.5-70) \times 10^{21} \mathrm{~cm}^{-3}$, on average $1.7 \times 10^{22} \mathrm{~cm}^{-3}$ in the middle-left plot in Figure 22 , it seems that $\mathrm{N}_{\mathrm{S}}$ corresponds to the $\pi$-orbital density, rather than to molecular density, which was deduced in earlier investigations on conductivity in polymers [79]. Certainly, values for $\mathrm{N}_{\mathrm{S}}>2 \times 10^{22} \mathrm{~cm}^{-3}$ are questionable, indicating again the non-mature methods for VRH calculations that are vulnerable to arbitrary procedures and subjective assessments by fitting of experimental data. Unfortunately, this is the state-ofthe-art at present.

The bottom-left plot in Figure 22 is for the decay distance $\Lambda_{o}$ of the hopping rate. Usually, $\Lambda_{o}$ is attributed to overlap of the $\pi$ orbitals in organic materials. Again, two trends are observed in this plot. One trend is that $\Lambda_{0}$ is lower when $N_{S}$ is higher, compared with the plot above. Interestingly, while $\Lambda_{0}$ varies about 5 times and $\mathrm{N}_{\mathrm{S}}$ varies about 30 times, the product $\Lambda_{\mathrm{o}} \times\left(\mathrm{N}_{\mathrm{S}}\right)^{1 / 3}$ varies less than 4 times between $12 \%$ and $42 \%$ among all samples, despite assumptions for different DOS and methods of VRH calculation, being on average $\sim 25 \%$. Since the product is related to the enhancement factor $\gamma$ (see eq. (44) earlier), then $\gamma \sim 2$ is expected for the OTFTs, as explained in [6]. However, the second trend in the bottom-left plot in Figure 22 is that the values for $\Lambda_{o}$ from numerical simulations (symbols in the plot) are about two times smaller than the values reported in the literature (horizontal dashed lines in the plot) and deduced by using of the 
analytical model [22]. This discrepancy again questions the absolute precision by the derivation of analytical models, although the analytical models have been proven $[6,11]$ to have consistent behaviors with temperature and bias of the OTFT.

Consider now the top-right and middle-right plots in Figure 22. The top-right plot is for the conductance prefactor $\sigma_{\mathrm{o}}$ usually taken as a constant parameter in the analytical VRH model for OTFTs [22], whereas the middle-right plot is for the hopping attempt rate $\Gamma_{\mathrm{o}}$ in the principal VRH physical model of eq. (2). The relation between $\sigma_{\mathrm{o}}$ and $\Gamma_{\mathrm{o}}$ is given by eq. (3), showing proportionality to the first order of approximation, and the proportionality between the two plots is evident. However, there are again problems with the values. The numerical simulations suggest 2-3 orders of magnitude lower values for $\sigma_{\mathrm{o}}$, compared to those reported in the literature by fitting of an analytical model (dashed lines in the top-right plot). Even so, some of the corresponding values for $\Gamma_{\mathrm{o}}$ are unrealistically large, being in the range of $\mathrm{PHz}$ $\left(\mathrm{PHz}=10{ }^{15} \mathrm{~Hz}\right)$, especially with the assumption of normally distributed DOS, and well above the limit of $1 \mathrm{PHz}$ derived in [21] by assuming random walk in the percolation network. In fact, frequencies above $300 \mathrm{PHz}$ are improbable for $\pi$ orbitals in organic materials with radius $\sim 0.15 \mathrm{~nm}$ or larger, since $\mathrm{c} /(2 \pi \times 0.15 \mathrm{~nm})=318 \mathrm{PHz}$, where $\mathrm{c} \approx 3 \times 10^{10} \mathrm{~cm} / \mathrm{s}$ is the speed of the light. Thus, any value for $\Gamma_{\mathrm{o}}>10 \mathrm{PHz}$, and consequently from eq. (3) $\sigma_{\mathrm{o}}>1.6 \times 10^{-19} \times 10 \mathrm{PHz} /(26 \mathrm{mV} \times 0.5 \mathrm{~nm}) \sim 10^{7} \mathrm{~S} / \mathrm{cm}=10^{9} \mathrm{~S} / \mathrm{m}$, are just extrapolated model parameters without physical validity. Note that many points from the numerical simulations in the top-right and middle-right plots in Figure 22 are above the limits for physical significance, and we are afraid that the values for $\sigma_{\mathrm{o}}$ reported in the literature correspond to an even higher $\Gamma_{\mathrm{o}}>100 \mathrm{PHz}$, thus is physically improbable. We do not delete the apparently wrong values and strongly emphasize that one meets with disappointing results fairly easily after lengthy derivations and simulations based on VRH. Therefore, the VRH theory seems again vulnerable to mistakes, owing to overlooked issues when using proportionalities and sequences of integrations and differentiations, and not paying attention to absolute values and magnitudes of quantities. 
Nevertheless, in the bottom-right plot of Figure 22, we show the hopping energy $\mathrm{E}_{\mathrm{HFB}}$ in the bulk organic semiconductor, although $\mathrm{E}_{\mathrm{HFB}}$ is a quantity, and not a parameter. The data scatter about $0.3 \mathrm{eV}$ around $1 \mathrm{eV}$, but considering that $\mathrm{E}_{\mathrm{HFB}}$ accumulates much larger variations for $\mathrm{T}_{\mathrm{o}}, \Lambda_{\mathrm{o}}$ and $\Gamma_{\mathrm{o}}$ by different assumptions for DOS and methods of calculation, we think that the variations in $\mathrm{E}_{\mathrm{HFB}}$ are reasonable. Therefore, as we have mentioned in the previous section, the consequences from eq. (50) for the linear relation between potential bending $\mathrm{V}_{\mathrm{B}}$ and hopping critical energy $\mathrm{E}_{\mathrm{H}}$ are discussed next.

\subsection{The linear correlation between potential bending $\left(V_{B}\right)$ and hopping energy $\left(E_{H}\right)$ revisited}

The immediate deduction from eq. (50) is that the hopping energy $E_{H}\left(D, V_{G}, T\right)$ and the potential bending energy $\mathrm{qV}_{\mathrm{B}}\left(\mathrm{D}, \mathrm{V}_{\mathrm{G}}, \mathrm{T}\right)$ at any depth $\mathrm{D}, 0 \leq \mathrm{D} \leq \mathrm{t}_{\mathrm{f}}$, in the organic semiconducting film of thickness $\mathrm{t}_{\mathrm{f}}$ and any gate bias voltage $V_{G}$ (but at given temperature $T$ ) is equal to the hopping energy in the bulk semiconductor

$$
\mathrm{E}_{\mathrm{H}}\left(\mathrm{D}, \mathrm{V}_{\mathrm{G}}, \mathrm{T}\right)+\mathrm{qV}_{\mathrm{B}}\left(\mathrm{D}, \mathrm{V}_{\mathrm{G}}, \mathrm{T}\right)=\mathrm{E}_{\mathrm{T}}(\mathrm{T})=\mathrm{E}_{\mathrm{H}}\left(\mathrm{D}=\infty \text { or } \mathrm{V}_{\mathrm{B}}=0, \mathrm{~T}\right) \equiv \mathrm{E}_{\mathrm{HFB}}(\mathrm{T}),
$$

where $\mathrm{E}_{\mathrm{HFB}}(\mathrm{T})$ is the hopping energy in the bulk semiconductor at the flat-band condition of zero bending $\left(V_{B}=0\right)$, see eq. (30), therefore $E_{H F B}$ is not function of depth or bias, and $E_{H F B}(T)=E_{H}\left(D=\infty\right.$ or $\left.V_{B}=0, T\right)$ is function only of the temperature T. Considering the relation between hopping energy $E_{H}$ and distance $R_{H}$ in eq. (2) for the hopping critical factor $\mathrm{s}_{\mathrm{c}}$, then one divides eq. (59) by $\mathrm{kT}$, and obtains the corresponding relations for the hoping distances and hopping attenuation factors

$$
\begin{aligned}
& \mathrm{s}_{\mathrm{cFB}}(\mathrm{T})= 2 \frac{\mathrm{R}_{\mathrm{HFB}}(\mathrm{T})}{\Lambda_{\mathrm{O}}}=\frac{\mathrm{E}_{\mathrm{HFB}}(\mathrm{T})}{\mathrm{kT}} \\
&= \frac{\mathrm{E}_{\mathrm{H}}\left(\mathrm{D}, \mathrm{V}_{\mathrm{G}}, \mathrm{T}\right)}{\mathrm{kT}}+\frac{\mathrm{qV}_{\mathrm{B}}\left(\mathrm{D}, \mathrm{V}_{\mathrm{G}}, \mathrm{T}\right)}{\mathrm{kT}} \\
&= 2 \frac{\mathrm{R}_{\mathrm{H}}\left(\mathrm{D}, \mathrm{V}_{\mathrm{G}}, \mathrm{T}\right)}{\Lambda_{\mathrm{o}}}+\frac{\mathrm{V}_{\mathrm{B}}\left(\mathrm{D}, \mathrm{V}_{\mathrm{G}}, \mathrm{T}\right)}{\mathrm{kT} / \mathrm{q}} \\
&=\mathrm{s}_{\mathrm{c}}\left(\mathrm{D}, \mathrm{V}_{\mathrm{G}}, \mathrm{T}\right)+\frac{\mathrm{V}_{\mathrm{B}}\left(\mathrm{D}, \mathrm{V}_{\mathrm{G}}, \mathrm{T}\right)}{\phi_{\mathrm{T}}}
\end{aligned}
$$

where $\mathrm{R}_{\mathrm{HFB}}(\mathrm{T})$ and $\mathrm{S}_{\mathrm{cFB}}(\mathrm{T})$ are the bias-independent hopping distance and attenuation factor in bulk semiconductor, respectively, and $\varphi_{\mathrm{T}}=\mathrm{kT} / \mathrm{q}$ is the thermal voltage. 
Interestingly, despite the variations in experimental data, assumptions for DOS, parameter values, methods of VRH calculation and levels of gate bias (via $V_{B}$ ), the ratio $2 R_{H F B} / \Lambda_{0}=s_{c F B}$ is apparently a well defined linear function of the reciprocal temperature, as shown in Figure 23. The linear trend implies that VRH in OTFT produces simple and relatively stable relation with electrostatics, despite the large uncertainties for parameters, various assumptions for DOS and bulky integrations. The trend is summarized in the following two equations. The hopping attenuation critical factor in bulk semiconductor is

$$
\mathrm{s}_{\mathrm{cFB}}(\mathrm{T})=\mathrm{s}_{\mathrm{cT} \infty}+\frac{\phi_{\mathrm{To}}}{\phi_{\mathrm{T}}(\mathrm{T})}
$$

where $\mathrm{s}_{\mathrm{cT} \infty} \approx 10 \pm 4$ is an extrapolated value for $\mathrm{s}_{\mathrm{cFB}}$ at infinite temperature $\mathrm{T}=\infty$ and $\varphi_{\mathrm{To}} \approx(0.8 \mathrm{~V} \pm 0.1) \mathrm{V}$ is a voltage that determines the slope in the $\mathrm{S}_{\mathrm{cFB}} \propto 1 / \mathrm{T}$ temperature dependence, thus, $\mathrm{q} \varphi \mathrm{To}_{\mathrm{s}}$ is the activation energy for the hopping attenuation critical factor $\mathrm{s}_{\mathrm{cFB}}$ in bulk semiconductor. Note that $\mathrm{s}_{\mathrm{c} T \infty}$ and $\varphi_{\text {To }}$ are constant parameters, which vary a little by different assumptions for DOS and methods for VRH calculation, and the temperature dependence in $\mathrm{s}_{\mathrm{cFB}}$ is due to the thermal voltage $\varphi_{\mathrm{T}}=\mathrm{kT} / \mathrm{q}$.

The second equation related to the trend in Figure 23 is

$$
\begin{aligned}
\mathrm{s}_{\mathrm{c}}\left(\mathrm{D}, \mathrm{V}_{\mathrm{G}}, \mathrm{T}\right) & =\mathrm{s}_{\mathrm{cFB}}(\mathrm{T})-\frac{\mathrm{V}_{\mathrm{B}}\left(\mathrm{D}, \mathrm{V}_{\mathrm{G}}, \mathrm{T}\right)}{\phi_{\mathrm{T}}(\mathrm{T})} \\
& =\mathrm{s}_{\mathrm{cT} \infty}+\frac{\phi_{\mathrm{To}}-\mathrm{V}_{\mathrm{B}}\left(\mathrm{D}, \mathrm{V}_{\mathrm{G}}, \mathrm{T}\right)}{\phi_{\mathrm{T}}(\mathrm{T})},
\end{aligned}
$$

indicating that the gate bias dependence in the hopping critical factor $s_{c}$ is due to the potential bending voltage $V_{B}$ caused by the gate bias voltage $V_{G}$ at a given depth $D$ in the film. Higher bias causes higher $V_{B}$, which "withdraws" from thermal activation $\varphi_{\text {To. }}$ Conversely, higher temperature increases $\varphi_{\mathrm{T}}$ and decreases the sensitivity of the critical factor $s_{c}$ (and conductivity) to bias, since $V_{B} / \varphi_{T}$ decreases. These effects have been discussed in the previous section in Figure 18b. Thus, the electrostatics enters VRH in OTFT by means of a simple linear algebraic relation between $s_{c}$ and $V_{B}$. Therefore, precise knowledge for $V_{B}(D)$ in the depth D of the film of the OTFT at given temperature and bias would guarantee reliable calculation of the profile 
$\sigma(D)=\sigma\left[V_{B}(D)\right]$ of the VRH conduction, considering the principal equation (4) for the VRH specific conductivity $\sigma$, which becomes

$$
\begin{aligned}
& \sigma\left(\mathrm{D}, \mathrm{V}_{\mathrm{G}}, \mathrm{T}\right)=\frac{\mathrm{q}}{\phi_{\mathrm{T}}} \frac{\Gamma_{\mathrm{O}}}{\Lambda_{\mathrm{o}}} \exp \left(-\mathrm{s}_{\mathrm{cT} \infty}\right) \exp \left[\frac{\mathrm{V}_{\mathrm{B}}\left(\mathrm{D}, \mathrm{V}_{\mathrm{G}}, \mathrm{T}\right)-\phi_{\mathrm{To}}}{\phi_{\mathrm{T}}}\right] \\
& =\sigma_{\mathrm{FB}}(\mathrm{T}) \exp \left[\frac{\mathrm{V}_{\mathrm{B}}\left(\mathrm{D}, \mathrm{V}_{\mathrm{G}}, \mathrm{T}\right)}{\phi_{\mathrm{T}}}\right] \text { under } \mathrm{V}_{\mathrm{G}} \text { so that } \mathrm{V}_{\mathrm{B}} \neq 0
\end{aligned}
$$

where $\sigma_{\mathrm{FB}}$ for the bulk semiconductor is

$$
\sigma_{\mathrm{FB}}(\mathrm{T})=\frac{\mathrm{q}}{\phi_{\mathrm{T}}} \frac{\Gamma_{\mathrm{o}}}{\Lambda_{\mathrm{O}}} \exp \left(-\mathrm{s}_{\mathrm{cT} \infty}\right) \exp \left(-\frac{\phi_{\mathrm{To}}}{\phi_{\mathrm{T}}}\right) \text { without bias, thus } \mathrm{V}_{\mathrm{B}}=0 \text {. }
$$

Note that the last two equations are valid for any DOS and method of VRH calculation. The assumption for DOS can affect $V_{B}$ in the electrostatic calculations, but only modifies the constant values for $\mathrm{s}_{\mathrm{c}} \mathrm{s}_{\infty}$ and $\varphi_{\text {To. }}$ The method of VRH calculation has no effect on $V_{B}$ and slightly changes the values for $\mathrm{s}_{\mathrm{c}} \mathrm{T}_{\infty}$ and $\varphi_{\text {To. }}$.

To obtain the sheet conductivity $\sigma_{\mathrm{sq}}$, consider the integration in eq. (41) along the depth $\mathrm{D}$ of the semiconductor film of thickness $t_{f}, 0 \leq \mathrm{D} \leq \mathrm{t}_{\mathrm{f}}$, using the expression for $\sigma(\mathrm{D})$ from eq. (63), in which $\sigma_{\mathrm{FB}}$ is constant in respect to D. Performing the substitutions and the integration, we get

$$
\sigma_{\mathrm{sq}}=\int_{0}^{\mathrm{t}_{\mathrm{f}}} \sigma(\mathrm{D}) \mathrm{dD}=\sigma_{\mathrm{FB}} \int_{0}^{\mathrm{t}_{\mathrm{f}}} \exp \left[\frac{\mathrm{V}_{\mathrm{B}}(\mathrm{D})}{\phi_{\mathrm{T}}}\right] \mathrm{dD}
$$

Then, one can obtain the OTFT mobility by dividing $\sigma_{\mathrm{sq}}$ by the gate charge $\mathrm{Q}_{\mathrm{G}}$, as given by eq. (42). Again, precise knowledge for the profile $\mathrm{V}_{\mathrm{B}}(\mathrm{D})$ is required to calculate $\sigma_{\mathrm{sq}}$ from eq. (65), because the profile depends on the type of DOS. However, an approximate analytical solution of the integral is also suitable in practice. This is because the shape of the bending profile $V_{B}(D)$ does not deviate significantly from the functional form given by eq. (48), if small adjustments of the values of the parameters $L_{\mathrm{A}}$ and $\varphi_{\mathrm{B}}$ are allowed. One adjustment is the use of effective temperature, $\mathrm{T}_{\mathrm{OEF}}$ in $\mathrm{Sec}$. 4.4.4 later.

To obtain the analytical solutions, substitute eq. (48) in eq. (65), and perform the integration with respect to the depth $\mathrm{D}$, assuming $\mathrm{L}_{\mathrm{A}}$ and $\varphi_{\mathrm{B}}$ as constants. The derivations, given in Appendix 2, show that the sheet conductivity of the OTFT film becomes 


$$
\sigma_{\mathrm{sq}} \sim \frac{\mathrm{q} \varepsilon_{\mathrm{f}}}{\mathrm{C}_{\mathrm{OX}} \mathrm{V}_{\mathrm{G}}} \frac{\Gamma_{\mathrm{o}}}{\Lambda_{\mathrm{o}}} \exp \left(-\mathrm{s}_{\mathrm{cT} \infty}\right) \exp \left(-\frac{\phi_{\mathrm{To}}}{\phi_{\mathrm{T}}}\right)\left[\frac{\left(\mathrm{C}_{\mathrm{ox}} \mathrm{V}_{\mathrm{G}}\right)^{2}}{2 \mathrm{q} \varepsilon_{\mathrm{f}} \phi_{\mathrm{A}} \mathrm{N}_{\mathrm{S}}} \exp \left(\frac{\left|\mathrm{E}_{\mathrm{o}}-\mathrm{E}_{\mathrm{F}}\right|}{\mathrm{kT}_{\mathrm{o}}}\right)\right]^{\mathrm{T}_{\mathrm{o}} / \mathrm{T}}
$$

with $\varphi_{\mathrm{A}} \equiv \mathrm{kT} \mathrm{T}_{\mathrm{A}} / \mathrm{q}$ and $\mathrm{T}_{\mathrm{A}} \approx \mathrm{T}_{\mathrm{o}}$ and at conditions $\mathrm{V}_{\mathrm{BS}}>2 \varphi_{\mathrm{o}}>\varphi_{\mathrm{T}}$ for the bending voltage $\mathrm{V}_{\mathrm{BS}}$ at the gate dielectricsemiconductor interface. From eq. (42), one more division on the gate charge $\mathrm{Q}_{\mathrm{G}}=\mathrm{C}_{\mathrm{OX}} \mathrm{V}_{\mathrm{G}}$ yields also a formula for mobility

$$
\mu=\frac{\sigma_{\mathrm{sq}}}{\mathrm{Q}_{\mathrm{G}}} \sim \mathrm{q} \varepsilon_{\mathrm{f}} \frac{\Gamma_{\mathrm{o}}}{\Lambda_{\mathrm{o}}} \exp \left(-\mathrm{s}_{\mathrm{cT} \infty}\right) \exp \left(-\frac{\phi_{\mathrm{To}}}{\phi_{\mathrm{T}}}\right)\left[\frac{\exp \left(\left|\mathrm{E}_{\mathrm{o}}-\mathrm{E}_{\mathrm{F}}\right| / \mathrm{kT}_{\mathrm{o}}\right)}{2 \mathrm{q} \varepsilon_{\mathrm{f}} \phi_{\mathrm{A}} \mathrm{N}_{\mathrm{S}}}\right]^{\mathrm{T}_{\mathrm{o}} / \mathrm{T}}\left(\mathrm{C}_{\mathrm{ox}} \mathrm{V}_{\mathrm{G}}\right)^{2\left(\mathrm{~T}_{\mathrm{o}} / \mathrm{T}-1\right)}
$$

The above format of the equations is compact, but does not show the actual terms related to thermal activation. Since $\mathrm{T}_{\mathrm{A}} \approx \mathrm{T}_{\mathrm{o}}$, then $\varphi_{\mathrm{A}} \equiv \mathrm{kT} \mathrm{T}_{\mathrm{A}} / \mathrm{q} \approx \mathrm{kT} \mathrm{T}_{\mathrm{o}} / \mathrm{q}$, and these equations can be rewritten together as

$$
\begin{aligned}
& \sigma_{\mathrm{sq}} \mathrm{Q}_{\mathrm{G}} \approx \mu \mathrm{Q}_{\mathrm{G}}^{2}=\mathrm{q} \varepsilon_{\mathrm{f}} \frac{\Gamma_{\mathrm{o}}}{\Lambda_{\mathrm{o}}} \exp \left(-\mathrm{s}_{\mathrm{cT} \infty}\right) \exp \left\{-\frac{\mathrm{E}_{\mathrm{A}}}{\mathrm{kT}}\right\}, \text { with } \mathrm{Q}_{\mathrm{G}} \approx \mathrm{C}_{\mathrm{ox}} \mathrm{V}_{\mathrm{G}} \text { and } \\
& \text { activation energy } \mathrm{E}_{\mathrm{A}}=\mathrm{kT}_{\mathrm{o}}\left\{1+\ln \left[\frac{2 \mathrm{q}_{\mathrm{f}} \mathrm{N}_{\mathrm{S}}}{\mathrm{Q}_{\mathrm{G}}^{2}} \times \frac{\mathrm{kT}_{\mathrm{o}}}{\mathrm{q}}\right]\right\}-\left|\mathrm{E}_{\mathrm{o}}-\mathrm{E}_{\mathrm{F}}\right|
\end{aligned}
$$

The logarithmic function will be explained later, just before eq. (75). In the last three equations, one clearly sees the terms, which outline the available freedoms in VRH for tuning magnitude by $\left(\Gamma_{\mathrm{o}} / \Lambda_{\mathrm{o}}\right) \exp \left(-\mathrm{S}_{\mathrm{c} T \infty}\right)$ and thermal activation by $\exp \left(-\mathrm{T}_{\mathrm{o}} / \mathrm{T}\right)$, the latter logarithmically bias dependent through $-2 \mathrm{k} \mathrm{T}_{\mathrm{o}} \times \ln \left(\mathrm{V}_{\mathrm{G}}\right)$. These are the main consequence from eq. (50) for the linear relation between potential bending $V_{B}$ and hopping critical energy $\mathrm{E}_{\mathrm{H}}$. The other terms in the equations for conductance and mobility of the OTFT, including the bias dependence of the thermal activation, are either material constants or follow from electrostatics through the integral $\int \exp \left[V_{B}(D) / \varphi_{T}\right] d D$ of the potential bending profile $V_{B}(D)$, but are not due to VRH.

\subsection{Verification of the correlation between potential bending $\left(V_{B}\right)$ and hopping energy $\left(E_{H}\right)$}

To support the above derivation of analytical formulas based on linear dependence between $V_{B}$ and $E_{H}$, we have inspected the profiles from numerical simulations and the analytical approximations. Examples are shown in Figure 24 for a pentacene OTFT. The mobility of this OTFT was reported in [73] and fitted by VRH calculations with multiple $\iint \mathrm{dEdE}$ integrations as shown earlier in the bottom-left plot of Figure 20. 
The three rows of plots in Figure 24 are corresponding to assumptions for single-side exponential DOS (top row of plots), double-side exponential DOS (middle row of plots) and normally distributed DOS (bottom row of plots). The open circles in Figure 24 are after the numerical calculation. The lines through the circles are after approximations and analytical calculations. The three columns of plots in Figure 24 are respectively for the profiles of the carrier concentration $\mathrm{N}_{\mathrm{C}}$ (left-hand column), potential bending $\mathrm{V}_{\mathrm{B}}=\left(\mathrm{IMREF}-\mathrm{E}_{\mathrm{F}}\right) / \mathrm{q}$ (middle column) and specific conductivity $\sigma$ (right-hand column). The middle column of plots also includes the DOS, as assumed in the numerical calculations (thick gray lines) and the exponential approximations of DOS (thin black lines that coincide with the thick gray lines) as deduced from analytical calculations. Note that the values for DOS are in the horizontal axes and given as function of the bending in the vertical axes. The examples in Figure 24 are for two temperatures ( $\mathrm{T}=100 \mathrm{~K}$ and $300 \mathrm{~K})$ and two gate bias voltages $\left(\mathrm{V}_{\mathrm{G}}=-5 \mathrm{~V}\right.$ and $\left.-20 \mathrm{~V}\right)$. The other temperatures and the intermediate bias $\mathrm{V}_{\mathrm{G}}=-10 \mathrm{~V}$ are omitted only for clarity in the figure.

The procedure of extraction of the parameter values in the analytical equations is now given. This procedure was repeated for every pair of conditions for temperature and bias, and every type of DOS. The profiles are obtained from the numerical calculations. The analytical expressions are fitted to the numerical data. The data from analytical calculations are denoted below with additional subscript "an" to the notations of the quantities from the numerical simulations. The values for the carrier concentration $N_{C, a n}(0)=N_{C}(D=0)$ and the potential bending $\mathrm{V}_{\mathrm{BS} \text {,an }}=\mathrm{V}_{\mathrm{BS}}$ in the semiconductor at the gate dielectric interface $(\mathrm{D}=0)$ are taken from the numerical simulation. The bulk semiconductor specific conductivity $\sigma_{\mathrm{FB}, \mathrm{an}}(\mathrm{T})$ is calculated with eq. (64) by adopting the values for $s_{\mathrm{c} T \infty}$ and $\varphi_{\text {To }}$ obtained by evaluation of the $1 / \mathrm{T}$ dependence for the critical factor $\mathrm{s}_{\mathrm{cBB}}$, as indicated in the left-hand column of the small plots in Figure 23.

Owing to the expected $\left(D+L_{A}\right)^{-2}$ dependence from eq. (49) for the profile of the carrier concentration $N_{C}(D)$, then the quantity $D \times N_{C}(D)$ is a peaking function at $D=L_{A}$, from which an initial value for $L_{A}$ is obtained, and then, the numerical data for $\mathrm{N}_{\mathrm{C}}$ are fitted by adjustment of the value for $\mathrm{L}_{\mathrm{A}, \text { an }}$, in the analytical 
expression $\mathrm{N}_{C}(\mathrm{D}) \approx \mathrm{N}_{\mathrm{C} \text {,an }}(\mathrm{D})=\mathrm{N}_{\mathrm{C} \text {,an }}(0)\left[\mathrm{L}_{\mathrm{A}, \text { an }} /\left(\mathrm{D}+\mathrm{L}_{\mathrm{A}, \text { an }}\right)\right]^{2}$, until good fit is obtained, as illustrated in the left-hand plots of Figure 24.

Next, having the value for $\mathrm{L}_{\mathrm{A}, \mathrm{an}}$, the value of the characteristic voltage $\varphi_{\mathrm{B}, \mathrm{an}} \equiv \mathrm{kT} \mathrm{B}_{\mathrm{B}, \mathrm{an}} / \mathrm{q}$ for the logarithmic decay of the potential bending $V_{B}$ in the depth of the film is determined by varying $\varphi_{B \text {,an }}$ in the analytical expression $V_{B, a n}(D)=V_{B S, a n}-2 \varphi_{B}, a n=\ln \left(1+D / L_{A, a n}\right)$ of eq. (48). As illustrated in the middle column of plots in Figure 24, a good fit to the numerical data for bending profile $V_{B}(D)$ is obtained. We gather the values for $\mathrm{V}_{\mathrm{B}, \mathrm{an}}(\mathrm{D})$ from the analytical calculation for the same depth mesh $\mathrm{D}$, as by the numerical calculation. With these values for $\mathrm{V}_{\mathrm{B}, \mathrm{an}}(\mathrm{D})$, the profile for the VRH specific conductivity $\sigma_{\mathrm{an}}(\mathrm{D})=\sigma_{\mathrm{FB}, \text { an }}(T) \times \exp \left[\mathrm{V}_{\mathrm{B}, \text { an }}(\mathrm{D}) / \varphi_{\mathrm{T}}\right]$ is calculated by the last expression of eq. (63), as depicted by the lines in the right-hand column of plots in Figure 24. The comparison between circles and lines in these plots indicates that the match for $\sigma$ from numerical and $\sigma_{\text {an }}$ from analytical calculations is good, which validates the above derivation of the analytical formulas to be suitable for approximate calculation of VRH by any type of DOS. Furthermore, since the analytical formulas are based on the linear correlation between potential bending $\left(V_{B}\right)$ and hopping energy $\left(E_{H}\right)$, then the good match in Figure 24 also verifies the correlation.

\subsection{More quantitative comparison between numerical and analytical calculations of VRH}

Looking closer at Figure 24, one observes several details, which have been discussed in the literature. One observation in the top row of plots in this figure is that the match between analytical and numerical calculations is almost perfect for single-sided exponential DOS, which is not surprising considering that the equations of the analytical calculations are based on assumption for this type of DOS. However, deviating from an exponential DOS, the analytical approximations become less accurate (not distinguished at first glance, but comparing the actual numbers, as follows). Consider the low bias $\mathrm{V}_{\mathrm{G}}=-5 \mathrm{~V}$ in the left-hand column of plots in Figure 24. There is virtually no temperature dependence for $\mathrm{N}_{\mathrm{C}}$ by the single-sided exponential DOS, and the analytical approximation overlaps the numerical simulation. However, there is a 
small difference between numerical simulation and analytical approximation for double-exponential DOS and a visible difference by normally distributed DOS, the latter accompanied with some deviation from the $\left(\mathrm{D}+\mathrm{L}_{\mathrm{A}}\right)^{-2}$ dependence. The differences between numerical simulation and analytical approximations are reinforced in the profiles for conductivity in the right-hand column of plots in Figure 24; note these plots are over many decades. Nevertheless, one observes that the magnitudes and slopes of DOS are similar for the ranges to where the semiconductor is bent, irrespective of the type of DOS [61, 62]. In an attempt to quantify the magnitudes and variations, we compare several parameters and quantities from numerical and analytical calculations.

\subsubsection{Selection of quantities for comparison}

To choose the quantities for comparison, one should review the situation after numerical VRH calculations. The situation is illustrated in Figure 25 for the pentacene OTFT, which mobility was reported in [73] and fitted by VRH calculations with multiple $\iint \mathrm{dEdE}$ integrations as shown earlier in the bottom-left plot of Figure 20. For clarity, Figure 25 is only for one set of temperature-bias conditions $(T=100 \mathrm{~K}$ and gate bias $\mathrm{V}_{\mathrm{G}}=-20 \mathrm{~V}$ ), and for one assumption for DOS (normally distributed DOS). The bending profile and DOS have been also shown in the middle plot at the bottom row of plots in Figure 24. These are repeated in Figure 25 for immediate reference, following the same styles for symbols and lines, and accordingly enhanced to view the situation after VRH calculation and to thoroughly define the quantities for comparison.

One enhancement in Figure 25 is the gray color error bars for hopping energy $E_{H}$ and distance $R_{H}$, as obtained by the numerical calculations. The height of vertical error bars indicates the value of $\mathrm{E}_{\mathrm{H}}$, at any depth D. All the vertical error bars end at the dotted horizontal blue line, fulfilling the aforementioned relation by eqs. (50) and (59) for the constant energy level $\left(\mathrm{E}_{\mathrm{H}}+\mathrm{qV}_{\mathrm{B}}\right)=\mathrm{E}_{\mathrm{HFB}}$, which the carriers reach by VRH (above the Fermi level $\mathrm{E}_{\mathrm{F}}$ ), depending on temperature, but irrespectively of bias. The value $\mathrm{E}_{\mathrm{HFB}}=0.889 \mathrm{eV} \approx 103 \times \mathrm{kT}$ for $\mathrm{T}=100 \mathrm{~K}$ was indicated by the trend equation in the upper-left corner in the 
bottom plot in the left-hand column of small plots in Figure 23 by the evaluation of the 1/T dependence for the critical factor $\mathrm{S}_{\mathrm{cFB}}$. (The substitution in the trend equation gives $\left.\mathrm{E}_{\mathrm{HFB}} / \mathrm{kT}=0.79 / \mathrm{kT}+11.86 \approx 0.79 \mathrm{eV} / 8.62 \mathrm{meV}+11.86 \approx 103\right)$. Note in Figure 25 that $\mathrm{E}_{\mathrm{HFB}}$ reaches deep in the DOS, even at low temperature, although IMREF in the organic semiconductor is more than $0.2 \mathrm{eV}$ below the DOS centroid level $\mathrm{E}_{\mathrm{o}}$, where $\left|\mathrm{E}_{\mathrm{o}}-\mathrm{E}_{\mathrm{F}}\right|=0.9 \mathrm{eV}$ was taken in the simulations. At the gate dielectric interface, the difference $\left|\mathrm{E}_{\mathrm{o}}-\operatorname{IMREF}(0)\right|=\left|\mathrm{E}_{\mathrm{O}}-\left(\mathrm{q} \mathrm{V}_{\mathrm{BS}}+\mathrm{E}_{\mathrm{F}}\right)\right| \sim 0.22 \mathrm{eV}$ is the smallest, while the difference increases to $\left|\mathrm{E}_{\mathrm{o}}-\mathrm{IMREF}(\mathrm{D})\right| \sim 0.4 \mathrm{eV}$ in the film depth, being $\sim 0.5 \mathrm{eV}$ at the back of the film at $\mathrm{D}=\mathrm{t}_{\mathrm{f}}=50 \mathrm{~nm}$, since the potential bending $\mathrm{V}_{\mathrm{B}}(\mathrm{D})$ decreases with $\mathrm{D}$. Thus, we will monitor $\left(\mathrm{E}_{\mathrm{F}}+\mathrm{E}_{\mathrm{HFB}}\right)$ and $\operatorname{IMREF}(\mathrm{D}=0)=\left(q \mathrm{~V}_{\mathrm{BS}}+\mathrm{E}_{\mathrm{F}}\right)$ at the gate dielectric interface $(\mathrm{D}=0)$ in respect to the energy position $\mathrm{E}_{\mathrm{o}}$ of DOS by comparison of quantities.

Regarding the horizontal error bars in Figure 25, their length indicates the hopping distance $\mathrm{R}_{\mathrm{H}}$. All the horizontal bars end at the dotted curve $\left(\mathrm{R}_{\mathrm{H}}+\mathrm{D}\right)$. One observes in the left-hand plot that the carriers reach depths $\left(\mathrm{R}_{\mathrm{H}}+\mathrm{D}\right)$, which are significantly larger than the depth $\mathrm{D}$ of the profile close to gate dielectric interface, while the ratio $\left(\mathrm{R}_{\mathrm{H}}+\mathrm{D}\right) / \mathrm{D}$ decreases when moving toward the back of the semiconducting film.

A better perspective for $\mathrm{R}_{\mathrm{H}}$ is given in the right-hand plot of Figure 25 with linear scale for the distance axis at the bottom. In this plot, one observes a linear dependence between $\mathrm{R}_{H}$ and $\mathrm{V}_{\mathrm{B}}$ by the line (green color) labeled with $\mathrm{R}_{\mathrm{H}}$, and $\mathrm{R}_{\mathrm{H}}$ is larger at lower bending $\mathrm{V}_{\mathrm{B}}$, whereas $\mathrm{D}$ is nearly an exponential function of $V_{B}$, since $V_{B}$ is nearly a logarithmic function of $D$, as discussed just above. The linear dependence between bending voltage $V_{B}$ and hopping distance $R_{H}$ is expected, considering $E_{H}+q V_{B}=E_{H F B}=$ constant at a given temperature and the proportionality between $E_{H}$ and $R_{H}$ by eq. (2), from which follows the linear dependence after a division on kT, as given earlier by eq. (60). The interesting observation for $R_{H}$ in Figure 25 is that $R_{H}$ can be an order of magnitude larger than three characteristic depths: $\mathrm{D}_{\text {avgVB }}, \mathrm{D}_{95 \% \text { \%sq }}$ and $\mathrm{L}_{\mathrm{A}}$. These characteristic depths are indicated with the three large symbols $(\bigcirc \diamond \square)$, respectively, on the bending profile (thin red line). The triad of these characteristic depths we 
denote with $\mathrm{D}^{\prime \prime \prime} \equiv\left\{\mathrm{L}_{\mathrm{A}}, \mathrm{D}_{95 \% \sigma \mathrm{sq}}, \mathrm{D}_{\mathrm{avgVB}}\right\}$. The significances of these characteristic depths, in reverse order, are the following.

$\mathrm{L}_{\mathrm{A}}$ denotes an electrostatic condition for the depth in which the majority of the carriers are induced by the gate bias $\mathrm{V}_{\mathrm{G}}$. Considering the profile of the carrier concentration $\mathrm{N}_{\mathrm{C}}(\mathrm{D})$ given in eq. (49), the following relation between $\mathrm{N}_{C}(0)$ evaluated at the interface, and $\mathrm{N}_{C}\left(\mathrm{~L}_{\mathrm{A}}\right)$ evaluated at $\mathrm{L}_{\mathrm{A}}$ is $\mathrm{N}_{C}\left(\mathrm{~L}_{\mathrm{A}}\right)=1 / 4 \mathrm{~N}_{C}(0)$. Also, integrating eq. (49) from the gate dielectric interface $\mathrm{D}=0$ to $\mathrm{D}=\mathrm{L}_{\mathrm{A}}$, the induced charge in the film up to this depth $\mathrm{L}_{\mathrm{A}}$ is $\mathrm{q}_{0} \int^{\mathrm{L}_{\mathrm{A}}} \mathrm{N}_{\mathrm{CdD}}=1 / 2 \mathrm{q}_{0} \int^{\infty} \mathrm{N}_{\mathrm{CdD}} \approx 1 / 2 \mathrm{Q}_{\mathrm{G}}$, being one half of the gate charge $\mathrm{Q}_{\mathrm{G}} \approx \mathrm{C}_{\mathrm{OXX}} \mathrm{V}_{\mathrm{G}}$. Thus, the majority of the carriers induced by the gate bias $\mathrm{V}_{\mathrm{G}}$, are within the distance $\mathrm{L}_{\mathrm{A}}$, since $\int \mathrm{N}_{\mathrm{CdE}} \sim 1 / \mathrm{D}$ at $\mathrm{D}>>\mathrm{L}_{\mathrm{A}}$.

The second characteristic depth $\mathrm{D}_{95 \% \text { \%sq }}$ denotes the condition for almost full sheet conductivity of the film, according to

$$
\int_{0}^{\mathrm{D}_{95 \% \sigma \mathrm{sq}}} \sigma(\mathrm{D}) \mathrm{dE}=0.95 \int_{0}^{\mathrm{t}_{\mathrm{f}}} \sigma(\mathrm{D}) \mathrm{dE}=95 \% \sigma_{\mathrm{sq}} \approx \sigma_{\mathrm{sq}}
$$

Considering the analytical approximation in eq. (151) for $\varphi_{\mathrm{T}} \neq 2 \varphi_{\mathrm{B}}$, and assuming $1.05 \approx 1 / 0.95, \varphi_{\mathrm{T}} \sim \varphi_{\mathrm{B}}$ at room temperature and $t_{f}>>L_{A}$, the order of magnitude for $D_{95 \% \sigma s q}$ is roughly $D_{95 \% \sigma s q} \sim 20 L_{A}$, but the multiplier 20 is different at low temperature, when $\varphi_{T}<\varphi_{B}$, which is the case in Figure 25.

The third characteristic depth $\mathrm{D}_{\mathrm{avgVB}}$ corresponds to the condition for the expected bending avg $\mathrm{V}_{\mathrm{B}}$, which is mostly representative for the sheet conductance $\sigma_{\mathrm{sq}}$, and $\operatorname{avg} \mathrm{V}_{\mathrm{B}}$ is an average of bending profile $V_{B}(D)$ weighted with the profile $\sigma(D)$ of the specific conductance $\sigma$, according to

$$
\operatorname{avg} V_{B}=\frac{\int_{0}^{t_{f}} V_{B}(D) \sigma(D) d D}{\int_{0}^{t_{f}} \sigma(D) d D}=\frac{\int_{0}^{t_{f}} V_{B}(D) \sigma(D) d D}{\sigma_{s q}} .
$$

Both profiles are known from the numerical calculations. Consequently, $\mathrm{D}_{\mathrm{avgvB}}$ can be calculated by interpolation of the bending profile $\mathrm{V}_{\mathrm{B}}(\mathrm{D})$ between points neighboring avg $\mathrm{V}_{\mathrm{B}}$, or alternatively, by inverting 
the analytical approximation of eq. (48), e.g., as $D_{a v g v B} \approx L_{A} \times\left\{\exp \left[\left(V_{B S}-a v g V_{B}\right) / 2 \varphi_{B}\right]-1\right\}$. We have used the former approach of interpolation for calculation of $\mathrm{D}_{\text {avgVB. }}$.

Once these characteristics depths for average $\mathrm{V}_{\mathrm{B}}, 95 \%$ of $\sigma_{\mathrm{sq}}$ and $\mathrm{L}_{\mathrm{A}}$ are defined, we will also monitor quantities at these three conditions. The values at these conditions for the following quantities are indicated in Figure 25. The large circles $(O)$ in the left-hand plot denote values at $\operatorname{avg} \mathrm{V}_{\mathrm{B}}$, in particular, from left to right, values for $\mathrm{D}_{\mathrm{avgVB}}, \%$ of $\sigma_{\mathrm{sq}}$ and DOS; and in addition in the right-hand plot, $\mathrm{R}_{\mathrm{H}}$ at avgV $\mathrm{V}_{\mathrm{B}}$. In a similar way, at the condition of $95 \%$ of $\sigma_{\mathrm{sq}}$, from left to right in the left-hand plot, the large diamonds $(\diamond)$ denote values for depth and bending, $\%$ of $\sigma_{\mathrm{sq}}$ and DOS; and $\mathrm{R}_{\mathrm{H}}$ at $95 \%$ of $\sigma_{\mathrm{sq}}$ in the right-hand plot. Consequently, large squares $(\square)$ denote the values for $\mathrm{V}_{\mathrm{B}}, \%$ of $\sigma_{\mathrm{sq}}$ and DOS at $\mathrm{L}_{\mathrm{A}}$ in the left-hand plot; and $\mathrm{R}_{\mathrm{H}}$ at $\mathrm{L}_{\mathrm{A}}$ in the right-hand plot. The approximation with exponential DOS is shown with dashed lines in Figure 25. The magnitude of the DOS approximation is taken equal to the magnitude of the assumed by numerical simulations DOS (normally distributed DOS in Figure 25) at the condition for average $\mathrm{V}_{\mathrm{B}}$, $\operatorname{avgV}$. Therefore, DOS and its approximation intersect at the large circles in the two plots in the figure. The slope of the exponential DOS approximation is $\partial \ln (\mathrm{DOS}) / \partial \mathrm{E}=1 / \mathrm{q} \varphi_{\mathrm{B}, \mathrm{an}}$, with value of $\varphi_{\mathrm{B}, \text { an }}$ as deduced after a fit of the potential bending with the analytical expression $V_{B}(D) \approx V_{B, a n}(D)=V_{B S}-2 \varphi B, a n \times \ln \left(1+D / L_{A}\right.$,an $)$ of eq. (48). Therefore, when comparing to $\varphi_{\mathrm{B}, \text { an, }}$ which is the reciprocal of the logarithmic slope of the exponential DOS approximation, for convenience we will monitor the reciprocal of the logarithmic slope of the DOS, which is

$$
\varphi_{\text {DOS }}\left(\operatorname{avg} V_{B}\right)=\frac{\partial(E / q)}{\partial[\ln (D O S)]} \mid \text { at } \operatorname{avgV}_{B} .
$$

Note that for single-sided exponential $\mathrm{DOS}_{\mathrm{SE}}$ of eq. (22), the logarithmic slope of $\mathrm{DOS}_{\mathrm{SE}}$ is constant in the energy range where $\mathrm{DOS}_{\mathrm{SE}}>0$, and the reciprocal of $\mathrm{DOS}_{\mathrm{SE}}$ logarithmic slope is

$$
\varphi_{\mathrm{o}}=\varphi_{\mathrm{DOS}_{\mathrm{SE}}}=\frac{\partial(\mathrm{E} / \mathrm{q})}{\partial\left\{\ln \left[\exp \left(\left|\mathrm{E}_{\mathrm{o}}-\mathrm{E}\right| / \mathrm{kT}_{\mathrm{o}}\right)\right]\right\}} \equiv \frac{\mathrm{kT}_{\mathrm{o}}}{\mathrm{q}}
$$


For non-exponential types of DOS, the value of $\varphi_{\text {DOS }}$ varies with the bending $V_{B}$, via IMREF $=\left(E_{F}+q V_{B}\right)$. For the normally distributed DOS, eq. (58) for example, the reciprocal of the logarithmic DOS slope at $\operatorname{avg} V_{B}$ is

$$
\varphi_{\mathrm{DOS}_{\mathrm{ND}}}=\frac{\partial(\mathrm{E} / \mathrm{q})}{\partial\left\{\ln \left[\mathrm{DOS}_{\mathrm{ND}}\left(\left|\mathrm{E}_{\mathrm{o}}-\mathrm{IMREF}\right| / \mathrm{kT}_{\mathrm{o}}\right)\right]\right\}}=\frac{\left(\mathrm{kT}_{\mathrm{o}}\right)^{2}}{\mathrm{E}_{\mathrm{o}} / \mathrm{q}-\mathrm{E}_{\mathrm{F}} / \mathrm{q}-\operatorname{avgV}_{\mathrm{B}}}
$$

Among many other quantities, $\varphi_{\mathrm{o}}$ is also related with the energy width $\left(\mathrm{q} \varphi_{\mathrm{o}}\right)$ and "rectangularity" of DOS, the latter defined, for example, as

$$
\operatorname{REC}_{\text {DOS }}=q \varphi_{\mathrm{o}} \frac{\operatorname{DOS}\left(\mathrm{E}_{\mathrm{O}}+\mathrm{q} \varphi_{\mathrm{o}}\right)+\operatorname{DOS}\left(\mathrm{E}_{\mathrm{o}}-\mathrm{q} \varphi_{\mathrm{o}}\right)}{\int_{-\infty}^{+\infty} \operatorname{DOS}(\mathrm{E}) \mathrm{dE}}, \text { with } \mathrm{N}_{\mathrm{S}}=\int_{-\infty}^{+\infty} \operatorname{DOS}(\mathrm{E}) \mathrm{dE}
$$

The values of DOS "rectangularity" are $\mathrm{REC}_{\mathrm{DOS}}=\{36.8 \%, 39.3 \%, 48.4 \%, 50 \%$ and $100 \%\}$ for $\mathrm{DOS}_{\mathrm{SE}}$, DOS $_{\mathrm{DE}}, \mathrm{DOS}_{\mathrm{ND}}$, triangular DOS and uniform DOS, respectively. A complementary definition is the DOS peaking, e.g., PEAKING $_{\mathrm{DOS}}=\left(1 / \mathrm{REC}_{\mathrm{DOS}}\right)-1$.

We proceed now to the comparison of the monitored quantities described above. Since the data is large, we shall present the comparisons only for one device and one case of VRH numerical calculations. To preserve relation to the preceding figures, we choose the pentacene OTFT, the experimental data of which were reported in [73], and the simulation case is after fitting the experimental data for mobility by assumption for normally distributed DOS and by multiple $\iint \mathrm{dEdE}$ integrations in the VRH numerical calculations, as shown earlier in the bottom-left plot of Figure 20. The actual reason for the particular choice is that the results are representative to what we have observed in comparisons for other devices and methods of integrations, and the differences between numerical and analytical calculations were the largest for normally-distributed DOS, as mentioned in the introduction paragraph of Sec. 4.4 above. 


\subsubsection{Comparison of energies}

The first comparison of the monitored quantities is illustrated in Figure 26 for the bending $q V_{B}$, DOS and hopping energy at the various conditions for extraction of the values for the potential bending $\mathrm{V}_{\mathrm{B}}$. The conditions for value extractions of $\mathrm{V}_{\mathrm{B}}$ and the corresponding notations in Figure 26 (a) and (b) are: solid lines (-) for the semiconductor-gate dielectric interface (depth $\left.\mathrm{D}=0, \mathrm{~V}_{\mathrm{B}}(0) \equiv \mathrm{V}_{\mathrm{BS}}\right)$; circles $(\bigcirc$, filled in red color) for the average bending $\operatorname{avg} \mathrm{V}_{\mathrm{B}}$ - see eq. (70); diamonds ( $\diamond$, filled in green color) for the bending at depth $\mathrm{D}_{95 \% \sigma s q}$ in the semiconducting film corresponding to sheet conductance $95 \%$ of $\sigma_{\mathrm{sq}}-$ see eq. (69); and the squares ( $\square$, filled in yellow color) are for the bending at depth $\mathrm{D}=\mathrm{L}_{\mathrm{A}}$, where $\mathrm{L}_{\mathrm{A}}=\mathrm{L}_{\mathrm{A}}$,an is obtained after fitting the charge profile $N_{C}(D)$ from numerical calculations with $N_{C}(D) \propto\left(D+L_{A}, \text { an }\right)^{-2}$, see eq. (49) and the bottom of the left-hand plots in Figure 24.

Three observations can be made in Figure 26 (a) for a given gate bias $\mathrm{V}_{\mathrm{G}}=-20 \mathrm{~V}$. The first observation is that the bending is high at low temperature, and it is about $0.2 \mathrm{eV}$ below the DOS centroid $\mathrm{E}_{\mathrm{o}}$ [61, 62], but the bending decreases at high temperature. The second observation is that always $V_{B S}>a v g V_{B}>V_{B}(95 \% \sigma s q)$, having the opposite temperature sensitivity $\partial \mathrm{V}_{\mathrm{BS}} / \partial \mathrm{T}<\partial \mathrm{avg} \mathrm{V}_{\mathrm{B}} / \partial \mathrm{T}<\partial \mathrm{V}_{\mathrm{B}}\left(95 \% \sigma_{\mathrm{sq}}\right) / \partial \mathrm{T}$. The bending $\mathrm{V}_{\mathrm{B}}\left(\mathrm{L}_{\mathrm{A}}\right)$ at the electrostatic depth $\mathrm{L}_{\mathrm{A}}$ is the lowest at low temperature, but $\mathrm{V}_{\mathrm{B}}\left(\mathrm{L}_{\mathrm{A}}\right)>\operatorname{avg} \mathrm{V}_{\mathrm{B}}$ at high temperature, since the temperature sensitivity $\partial \mathrm{V}_{\mathrm{B}}\left(\mathrm{L}_{\mathrm{A}}\right) / \partial \mathrm{T} \sim \partial \mathrm{V}_{\mathrm{BS}} / \partial \mathrm{T}$ is low, as expected for electrostatic quantities that are independent of hopping in principle. From eq. (49), the electrostatic depth $\mathrm{L}_{\mathrm{A}}$ corresponds to $1 / 2 \mathrm{Q}_{\mathrm{G}}$ of the gate charge $\mathrm{Q}_{\mathrm{G}} \approx \mathrm{C}_{\mathrm{OX}} \mathrm{V}_{\mathrm{G}}$, see before eq. (69). The third observation in Figure 26 (a) is that the scatter in values between $V_{B}$ at different conditions for value extraction is minimal at low temperature and the scatter increases at high temperature, owing to different $\partial \mathrm{V}_{\mathrm{B}} / \partial \mathrm{T}$. The trend of decreasing bending and increasing scatter of values by increasing the temperature is illustrated with small dashes surrounded with dotted ellipses in Figure 26 (c), aggregating the data for bending for all cases of biasing and conditions for extraction.

Nevertheless, although varying with temperature, the slopes in Figure 26 (b) indicate that $V_{B}$ at different conditions for value extraction have the same logarithmic bias dependence at a given temperature, 
with a slope $\partial \mathrm{V}_{\mathrm{B}} / \partial\left[\log \left(\mathrm{V}_{\mathrm{G}}\right)\right]$ increasing at higher temperature. The trend in the evolution of the bending and hopping energy as function of temperature is shown in Figure 26 (c), and compared to DOS, the latter depicted with solid parabolic curve (blue color), having centroid level $\mathrm{E}_{\mathrm{o}}$ (dashed horizontal line). The blank bars illustrate the decrease of the bending. The solid straight line (green color) is the hopping energy $\mathrm{E}_{\mathrm{HFB}}$ for the bulk semiconductor, and $\mathrm{E}_{\mathrm{HFB}}$ increases with temperature. From eq. (59), the difference between $E_{H F B}$ and bending is the hopping energy $E_{H}=\left(E_{H F B}-q V_{B}\right)$ (shown with shaded gray color bars in the figure), which also increases with the temperature. The interesting observation is that the bias-independent quantity $E_{H F B}=\left(E_{H}+q V_{B}\right)$ reaches levels deep in DOS. Therefore, VRH scans the DOS almost entirely, at least half of DOS even at low temperatures, which questions the assumption in the analytical derivations $[21,22]$ that the hopping is in the DOS tail, where the bending is. Comparing $\mathrm{qV}_{\mathrm{B}}$ and $\mathrm{E}_{\mathrm{H}}$, blank and shaded bars in Figure 26 (c), one sees that the proportion is in favor of bending at low temperatures (thus, electrostatics in DOS tail), but the proportion becomes reciprocal at high temperature, with dominating hopping around the centroid level $\mathrm{E}_{\mathrm{o}}$ of the DOS. At intermediate temperatures, normally around room temperature for OTFTs, the bending and hopping have similar contributions to $\mathrm{E}_{\mathrm{HFB}}$. The consequence of the variation of the proportion between bending and hopping is that the steepness of DOS tail is important at low temperatures, whereas the DOS itself is important at high temperature, as noted in [6]. Therefore, analytical expressions for VRH derived from the condition for low temperature, require corrections for intermediate and high temperature. For example, the analytical expression for mobility derived in [22] contains a term $\operatorname{sinc}\left(\pi \mathrm{T} / \mathrm{T}_{\mathrm{o}}\right)$, where $\operatorname{sinc}(\mathrm{x})=\sin (\mathrm{x}) / \mathrm{x}, \mathrm{x}=\pi \mathrm{T} / \mathrm{T}_{\mathrm{o}}$ and $\mathrm{kT} \mathrm{T}_{\mathrm{o}} \equiv \mathrm{q} \varphi_{\mathrm{o}}$ is the energy width of exponential DOS, but this term causes problem at $T \rightarrow T_{0}$, since $\operatorname{sinc}(2 \pi \geq x \geq \pi) \leq 0$ causes an unrealistic drop to zero and negative mobility, and the term should be replaced with other function, e.g. with $\left(\mathrm{T}_{\mathrm{o}} / \mathrm{T}-1\right) /\left\{1-\exp \left[\left(1-\mathrm{T}_{\mathrm{o}} / \mathrm{T}\right)\left(\mathrm{E}_{\mathrm{o}}-\mathrm{E}_{\mathrm{F}}\right) /\left(\mathrm{k} \mathrm{T}_{\mathrm{o}}\right)\right]\right\}$ suggested in [6] for the temperature "shaping" function $\left(\mathrm{TSF}^{2}\right)$, or an effective value $\mathrm{T}_{\mathrm{OEF}}$ for the parameter $\mathrm{T}_{\mathrm{o}}$ should be assumed to be an increasing function of temperature $\mathrm{T}$, as suggested in the appendix of [11] and discussed later by eq. (79). Analytical approximations related to the problem at $\mathrm{T} \rightarrow \mathrm{T}_{\mathrm{o}}$ are also given in [2] for amorphous silicon TFTs. 


\subsubsection{Comparison of distances}

The second comparison of the monitored quantities is illustrated in Figure 27 for the set of three characteristic electrostatic distances $D^{\prime \prime \prime}=\left\{L_{A}, D_{95 \% \sigma s q}, D_{\text {avgVB }}\right\}$ and hopping distances $R_{H}$ at various conditions for extraction of the values for $\mathrm{D}^{\prime \prime \prime}$ and $\mathrm{R}_{\mathrm{H}}$. (The conditions and biasing are the same as in Figure 26.) The aggregated data in the top-left plot of Figure 27 indicate large scattering of values for the set of electrostatic distances $\mathrm{D}^{\prime \prime \prime}$ ( $\square$, black color), variations over 1.5 decades with bias and extraction conditions at a given temperature, and with a power-law trend of decreasing D at lower temperatures $\mathrm{T}$ (higher 1000/T) of slope steeper than $T^{1.3}$. In contrast, the hopping distances $R_{H}(O$, red color) vary little, less than a factor of 2 with the bias and extraction conditions at a given temperature, between $1 \mathrm{~nm}$ and $3 \mathrm{~nm}$ for all temperatures, and with a power-law trend of increasing $\mathrm{R}_{\mathrm{H}}$ at lower temperatures $\mathrm{T}$ (higher 1000/T) of gradual slope of $(1 / T)^{0.3}$. Interestingly, the product $\mathrm{D}^{\prime \prime \prime} \times \mathrm{R}_{\mathrm{H}}=\left\{\mathrm{L}_{\mathrm{A}} \times \mathrm{R}_{\mathrm{H}}, \mathrm{D}_{95 \% \sigma s q} \times \mathrm{R}_{\mathrm{H}}, \mathrm{D}_{\mathrm{avg} \text { v }} \times \mathrm{R}_{\mathrm{H}}\right\}(-$, bluecolor dashes in the top left figure) has a power law trend with slope $(T)^{1}$, therefore, the trend is that $\mathrm{D}^{\prime \prime \prime} \times \mathrm{R}_{\mathrm{H}}$ is proportional to the absolute temperature $T$, being a counter part of $\left(E_{H}+q V_{B}\right)=$ constant, although $D^{\prime \prime \prime} \times R_{H}$ is strongly varying with gate bias.

To get more insights in the details for the temperature dependences of the characteristic depths $\mathrm{D}^{\prime \prime \prime}$ and $\mathrm{R}_{\mathrm{H}}$, consider the bottom-left plot in Figure 27 at given gate bias voltage $\mathrm{V}_{\mathrm{G}}=-20 \mathrm{~V}$. The small symbols connected with dashed lines are for $\mathrm{R}_{\mathrm{H}}$, by triangles $(\boldsymbol{\Delta})$ for the semiconductor-gate dielectric interface (depth $\mathrm{D}=0$ ), circles $(\bigcirc)$ at condition for average bending $\operatorname{avg} \mathrm{V}_{\mathrm{B}}-$ see eq. $(70)$, diamonds $(\diamond)$ at depth D95\% \%sq corresponding to sheet conductance $95 \%$ of $\sigma_{\text {sq }}-$ see eq. (69), and squares $(\square)$ are for depth $\mathrm{L}_{\mathrm{A}}$, where $L_{A}=L_{A, \text { an }}$ is obtained after fitting the charge profile $\mathrm{N}_{C}(\mathrm{D})$ from numerical calculations with $\mathrm{N}_{C}(\mathrm{D}) \propto\left(\mathrm{D}+\mathrm{L}_{\mathrm{A}, \mathrm{an}}\right)^{-2}$, see eq. (49), and $\mathrm{L}_{\mathrm{A}}$ corresponds to ${ }^{1 / 2} \mathrm{Q}_{\mathrm{G}}$ of the gate charge $\mathrm{Q}_{\mathrm{G}} \approx \mathrm{C}_{\mathrm{OX}} \mathrm{V}_{\mathrm{G}}$, see before eq. (69). Since $V_{B}(D=0)=V_{B S}$ is the largest, then $R_{H}(D=0)$ is the smallest, and the triangles are always below other symbols for $\mathrm{R}_{\mathrm{H}}$. Consequently, a larger depth corresponds to a larger $\mathrm{R}_{\mathrm{H}}$, and the vertical order of small symbols for $\mathrm{R}_{\mathrm{H}}$ follows the vertical order of the large symbols for characteristic depths at every given 
temperature, but at different proportion of magnitude, and in narrow range of $1.2-2 \mathrm{~nm}$ for $\mathrm{R}_{\mathrm{H}}$. Looking closer at the dashed lines for $\mathrm{R}_{\mathrm{H}}$ as function of the reciprocal of the temperature, one observes that $\mathrm{R}_{\mathrm{H}}$ increases at low temperature, but the dependence is weak and not exactly a power-law function. In contrast (looking at the large symbols connected with solid lines), the temperature dependences for $\mathrm{D}_{95 \% \text { ssq }}(\diamond)$ and $\mathrm{D}\left(\operatorname{avg} \mathrm{V}_{\mathrm{B}}\right)(\mathrm{O})$ are well-pronounced power-law functions of high steepness, $>\mathrm{T}^{2}$ and $>\mathrm{T}^{1.5}$, whereas the purely electrostatic depth $\mathrm{L}_{\mathrm{A}}(\square)$ is constant at low temperature, but increases at high temperature. Thus, $\mathrm{D}^{\prime \prime \prime} \times \mathrm{R}_{H} \propto \mathrm{T}$ is just a trend, but not a relation. Note again in the upper-left plot that the set $\mathrm{D}^{\prime \prime \prime}$ is a collection of characteristic distances with large dispersion.

The bias dependences of the characteristic distances $\mathrm{D}^{\prime \prime \prime}$ and $\mathrm{R}_{\mathrm{H}}$ are given in the middle and right-hand columns of plots in Figure 27 at low and high temperatures, $\mathrm{T}=100 \mathrm{~K}$ and $\mathrm{T}=500 \mathrm{~K}$ on top and bottom plots, respectively. In the middle column, one observes linear dependences between $\mathrm{D}^{\prime \prime \prime}$ and $\left(1 / \mathrm{V}_{\mathrm{G}}\right)$, which confirms the reciprocal dependence $\mathrm{D}^{\prime \prime \prime} \propto 1 / \mathrm{V}_{\mathrm{G}}$ between electrostatic distances and bias voltages, e.g. for $\mathrm{L}_{\mathrm{A}}$ in eq. (47). Note that $\mathrm{L}_{\mathrm{A}}$ is the largest in the triad $\left\{\mathrm{L}_{\mathrm{A}}, \mathrm{D}_{95 \% \sigma s q}, \mathrm{D}_{\mathrm{avgvB}}\right\}$ at low temperature, but is the smallest at high temperature, which implies that $\mathrm{L}_{\mathrm{A}}$ is not a definite measure for the effective channel depth regarding conductance and mobility in OTFT, although $\mathrm{L}_{\mathrm{A}}$ is representative for the induced charge $\mathrm{Q}_{\mathrm{G}}$. Nevertheless, in the right-hand column of plots, one clearly observes the logarithmic bias dependence $\mathrm{R}_{\mathrm{H}} \propto($ constant $)-\log \left(\mathrm{V}_{\mathrm{G}}\right)$, which is expected, considering the following simplified derivations valid for low temperature and exponential DOS approximation.

After taking natural logarithm of eq. (157) in Appendix 2, followed by multiplication by the characteristic "thermal-like" voltage $\varphi_{\mathrm{o}} \equiv \mathrm{kT} \mathrm{T}_{\mathrm{o}} / \mathrm{q}$ of the exponential DOS approximation, one gets the bending voltage $\mathrm{V}_{\mathrm{BS}}$ at the gate dielectric-semiconductor interface, as

$$
\mathrm{V}_{\mathrm{BS}}=\frac{\left|\mathrm{E}_{\mathrm{O}}-\mathrm{E}_{\mathrm{F}}\right|}{\mathrm{q}}+\varphi_{\mathrm{o}} \ln \left[\frac{\left(\mathrm{C}_{\mathrm{ox}} \mathrm{V}_{\mathrm{G}}\right)^{2}}{2 \mathrm{q} \varepsilon_{\mathrm{f}} \varphi_{\mathrm{A}} \mathrm{N}_{\mathrm{S}}}\right] \text {, with } \varphi_{\mathrm{A}} \equiv \mathrm{kT} \mathrm{T}_{\mathrm{A}} / \mathrm{q} \text { and } \mathrm{T}_{\mathrm{A}} \approx \mathrm{T}_{\mathrm{o}}
$$

Substituting in eq. (48), the bending at depth $\mathrm{D}$ in the film becomes 


$$
\mathrm{V}_{\mathrm{B}}(\mathrm{D})=\frac{\left|\mathrm{E}_{\mathrm{O}}-\mathrm{E}_{\mathrm{F}}\right|}{\mathrm{q}}+\varphi_{\mathrm{o}} \ln \left[\frac{\left(\mathrm{C}_{\mathrm{OX}} \mathrm{V}_{\mathrm{G}}\right)^{2}}{2 \mathrm{q} \varepsilon_{\mathrm{f}} \varphi_{\mathrm{A}} \mathrm{N}_{\mathrm{S}}}\right]-2 \varphi_{\mathrm{B}} \ln \left(1+\frac{\mathrm{D}}{\mathrm{L}_{\mathrm{A}}}\right) \text {, with } \varphi_{\mathrm{B}} \equiv \mathrm{k} \mathrm{T}_{\mathrm{B}} / \mathrm{q} \text { and } \mathrm{T}_{\mathrm{B}} \approx \mathrm{T}_{\mathrm{o}} \text {. }
$$

From eq. (47), $1 / \mathrm{L}_{A}=\mathrm{C}_{\mathrm{OX}} \mathrm{V}_{\mathrm{G}} /\left(2 \varepsilon_{\mathrm{f}} \varphi_{\mathrm{A}}\right)$, and substituting in the last eq. (76) above one can exclude $\mathrm{L}_{\mathrm{A}}$ from the expression, but looking closer at middle column of the plots in Figure 27, one observes that the characteristic depths stay in proportions (each to other), since they are linear functions of $1 / \mathrm{V}_{\mathrm{G}}$ at any temperature. Thus, the proportions are bias independent, although changing with temperature, and the proportions in ratio to $\mathrm{L}_{\mathrm{A}}$ are in narrow interval of values. Let us denote the proportions with $\mathrm{P}=\mathrm{P}_{\mathrm{AVB}}=\mathrm{D}_{\text {avgVB }} / \mathrm{L}_{\mathrm{A}}, \mathrm{P}=\mathrm{P}_{\sigma}=\mathrm{D}_{95 \% \sigma s q} / \mathrm{L}_{\mathrm{A}}$ and $\mathrm{P}=\mathrm{P}_{\mathrm{LA}}=\mathrm{L}_{\mathrm{A}} / \mathrm{L}_{\mathrm{A}} \equiv 1$. Then, from the slope coefficients in the linear approximations in the plots, $\mathrm{P}_{\mathrm{AVB}} \sim 0.25-1.3$ and $\mathrm{P}_{\sigma} \sim 0.8-7$, resulting in negligible magnitudes of $2 \varphi_{\mathrm{B}} \ln (1+\mathrm{P})<2 \times 50 \mathrm{mV} \times(0.2-2)=0.02-0.2 \mathrm{~V}$ in eq. (76), as compared to the first term $\left|\mathrm{E}_{\mathrm{O}}-\mathrm{E}_{\mathrm{F}}\right| \mathrm{q}=0.9 \mathrm{~V}$. Thus, $2 \varphi_{\mathrm{B}} \ln (1+\mathrm{P})$ is omitted below, since it is also bias independent. So, at given temperature $\mathrm{T}$, and at any depth $D$ and bias $V_{G}$, the sum of hopping energy $E_{H}$ and bending $q V_{B}$ is the hopping energy $E_{H F B}$ in bulk semiconductor, as given by eq. (59), which is rewritten for $E_{H}(D)=\left[E_{H F B}-q V_{B}(D)\right]$. Substituting the last eq. (76) above in this relation, omitting $2 \varphi_{\mathrm{B}} \ln \left(1+\mathrm{D} / \mathrm{L}_{\mathrm{A}}\right)$ and taking $\varphi_{\mathrm{A}} \approx \varphi_{\mathrm{B}} \approx \varphi_{\mathrm{o}}$, the hopping energy $\mathrm{E}_{\mathrm{H}}(\mathrm{D})$ as function of bias and depth becomes

$$
\mathrm{E}_{\mathrm{H}}(\mathrm{D}) \approx \mathrm{E}_{\mathrm{HFB}}-\left|\mathrm{E}_{\mathrm{o}}-\mathrm{E}_{\mathrm{F}}\right|+\mathrm{q} \varphi_{\mathrm{o}} \ln \left(\frac{2 \mathrm{q} \varepsilon_{\mathrm{f}} \varphi_{\mathrm{o}} \mathrm{N}_{\mathrm{S}}}{\mathrm{C}_{\mathrm{ox}}}\right)+2 \mathrm{q} \varphi_{\mathrm{o}} \ln \left(\frac{1}{\mathrm{~V}_{\mathrm{G}}}\right)
$$

Note that the bias dependence is due to the last term under the logarithm. Subsequent substitution in the principal eq. (2) between hopping energy and distance yields

$$
\begin{aligned}
& \frac{2 \mathrm{R}_{\mathrm{H}}}{\Lambda_{\mathrm{o}}}=\frac{\mathrm{E}_{\mathrm{H}}}{\mathrm{kT}}=\frac{\mathrm{E}_{\mathrm{H}} / \mathrm{q}}{\varphi_{\mathrm{T}}} \\
& \quad=\frac{\left(\mathrm{E}_{\mathrm{HFB}}-\left|\mathrm{E}_{\mathrm{o}}-\mathrm{E}_{\mathrm{F}}\right|\right) / \mathrm{q}+2 \varphi_{\mathrm{o}} \ln \left(2 \mathrm{q} \varepsilon_{\mathrm{f}} \varphi_{\mathrm{o}} \mathrm{N}_{\mathrm{S}} / \mathrm{C}_{\mathrm{oX}}\right)}{\varphi_{\mathrm{T}}}+2 \frac{\varphi_{\mathrm{o}}}{\varphi_{\mathrm{T}}} \ln \left(\frac{1}{\mathrm{~V}_{\mathrm{G}}}\right) \\
& \Rightarrow \mathrm{R}_{\mathrm{H}}\left(\mathrm{V}_{\mathrm{G}}\right) \approx \Lambda_{\mathrm{o}} \frac{\varphi_{\mathrm{o}}}{\varphi_{\mathrm{T}}} \ln \left(\frac{1}{\mathrm{~V}_{\mathrm{G}}}\right)+\text { constant, when } \mathrm{T}=\text { constant. }
\end{aligned}
$$

Therefore, $R_{H}$ is expected to be a linear function of the logarithm of $1 / V_{G}$, with the slope coefficient $\Lambda_{0} \varphi_{\mathrm{o}} / \varphi_{\mathrm{T}} \propto 1 / \mathrm{T}$ lower at high temperature, and the slope coefficient independent of the depth to the first order 
of approximation, when $\mathrm{D} / \mathrm{L}_{\mathrm{A}}<10$, which covers almost all realistic cases for characteristic depths with plausible significance for OTFT. These features are clearly observed in the right-hand plots of Figure 27. One detail in these plots is that the slope coefficients are in ratio 3:1 for temperatures in ratio 1:5. Thus, $\varphi_{\mathrm{o}}$ appears to be temperature dependent, as discussed in the next subsection.

\subsubsection{Increase of $T_{0}$ at high $T$ ?}

The third comparison of the monitored quantities is illustrated in Figure 28 for characteristic "thermal-like" voltages, which participate in analytical expressions or define the energy width and slope of the assumed DOS: $\varphi_{\mathrm{A}}$ in (47), $\varphi_{\mathrm{B}}$ in (48), $\varphi_{\mathrm{C}}$ in (49) and $\varphi_{\mathrm{DOS}_{\mathrm{ND}}}$ in (73). The device and the temperature and biasing conditions are identical with the preceding two figures, including the assumption for normally distributed DOS $_{\mathrm{ND}}$, to which the left-hand plots in Figure 28 correspond. Since the values scatter in these plots, and also to inspect closer the trends, we have added in the right-hand plots of Figure 28 the same type of information and for the same device, but after inspecting the data from numerical calculations with the different assumption for single-sided exponential $\mathrm{DOS}_{\mathrm{SE}}$. The values denoted with symbols in Figure 28 are extracted from the profiles and quantities obtained after the numerical simulation, by fitting the different analytical expressions to the different profiles with a freedom of independent variation of the values of the different characteristic voltages. Therefore, the values of the characteristic voltages correspond to analytical approximations and have the additional subscript "an" in the notations, as mentioned earlier. The procedure of evaluation of these characteristic voltages is now explicitly given.

Eq. (47) is rewritten for $\varphi_{\mathrm{A}, \mathrm{an}}=\mathrm{L}_{\mathrm{A}, \mathrm{an}} \mathrm{Q}_{\mathrm{G}} / 2 \varepsilon_{\mathrm{f}}$, where the value of gate dielectric charge $\mathrm{Q}_{\mathrm{G}}$ is taken from the numerical calculations according to eq. (31), but not from the approximate $\mathrm{Q}_{\mathrm{G}} \approx \mathrm{C}_{\mathrm{OX}} \mathrm{V}_{\mathrm{G}}$, the value of $\mathrm{L}_{\mathrm{A}, \mathrm{an}}$ is determined by fitting of the charge profile $\mathrm{N}_{\mathrm{C}}(\mathrm{D})$ in the film, from the peak of $\mathrm{D} \times \mathrm{N}_{\mathrm{C}}(\mathrm{D})$, as explained earlier in relation to the left-hand plots of Figure 24, and the value for permittivity of the organic material is taken $\varepsilon_{\mathrm{f}}=3 \varepsilon_{\mathrm{o}} \approx 2.66 \times 10^{-13} \mathrm{~F} / \mathrm{cm}$, as set by the numerical simulations. The values for $\varphi_{\mathrm{A} \text {,an }}$ are shown with squares $(\square)$ in Figure 28. Also as explained earlier in relation to the plots in the middle column 
of Figure 24, the bending profiles $\mathrm{V}_{\mathrm{B}}(\mathrm{D})$ from numerical simulation have been fitted by independently varying the value of $\varphi_{B, \text { an }}$ in the analytical expression $V_{B, a n}(D)=V_{B S, a n}-2 \varphi_{B}, a n=\ln \left(1+D_{1} / L_{A, a n}\right)$ of eq. (48), and the values for $\varphi_{\mathrm{B}, \text { an }}$ are shown with diamonds $(\diamond)$ in Figure 28. Next, eq. (49) is rewritten as $\varphi_{\mathrm{C}, \text { an }}=\left(\mathrm{qL}_{\mathrm{A}, \mathrm{an}}\right)^{2} \mathrm{~N}_{\mathrm{C}}(\mathrm{D}=0) / 2 \varepsilon_{\mathrm{f}}$ for the carrier concentration $\mathrm{N}_{\mathrm{C}}(\mathrm{D}=0)$ at the gate dielectric-semiconductor interface $(D=0)$, and using the values for $\mathrm{N}_{C}(D=0)$ directly as obtained by the numerical simulator, the corresponding values for $\varphi_{\mathrm{C} \text {,an }}$ are calculated and shown with triangles $(\triangle)$ in Figure 28.

The values shown with circles $(\bigcirc)$ in Figure 28 are for $\varphi_{\text {DOS, }}$, the reciprocal logarithmic slope of DOS. For the normally distributed $\mathrm{DOS}_{\mathrm{ND}}$ in the left-hand plots, the values for $\varphi_{\mathrm{DOS}}$ are calculated by eq. (73) at the condition for average bending $\left(\mathrm{IMREF}=\mathrm{E}_{\mathrm{F}}+\mathrm{q} \times \operatorname{avg} \mathrm{V}_{\mathrm{B}}\right)$, where $\operatorname{avg} \mathrm{V}_{\mathrm{B}}$ is determined by eq. (70) from averaging of the bending profile with the conductance profile. Since the bending decreases at higher temperatures and increases at higher bias (see Figure 26 (a) and (b)), then $\varphi_{\text {DOs }}$ for the normally distributed DOS $_{\mathrm{ND}}$ decreases with temperature and increases with bias in the left-hand plots of Figure 28. In contrast, since $\varphi_{\mathrm{DOS}}=\mathrm{kT} / \mathrm{q}=\varphi_{\mathrm{o}}$ EXP is a constant for exponential DOSSE, see eq. (72), then the circles for $\varphi_{\mathrm{DOS}}$ overlap

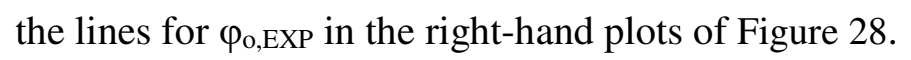

The other observations, which can be made in Figure 28 for the characteristic "thermal-like" voltages, are the following. Although being in the same order of magnitude, the values of the characteristic voltages scatter by assumption for normally distributed $\mathrm{DOS}_{\mathrm{ND}}$, while the values coincide either with $\varphi_{\mathrm{o}}$ or $\varphi_{\mathrm{T}}$ by assumption for exponential DOS. Therefore, the analytical expressions are only approximations by nonexponential types of DOS, and the observed similarity in $[61,62]$ for equal slopes at different types of DOS is not precise, although the slopes look similar when plotting in graphs (c.f. Figure 24 earlier). The values in the group $\varphi_{\mathrm{ABC}}=\left\{\varphi_{\mathrm{A}, \mathrm{an}}, \varphi_{\mathrm{B}, \mathrm{an}}, \varphi_{\mathrm{C}, \mathrm{an}}\right\}$ of characteristic voltages for profiles vary with temperature, but the values are nearly independent of bias. The trend in the variation of $\varphi_{\mathrm{ABC}}=\varphi_{\mathrm{A}, \mathrm{an}}, \varphi_{\mathrm{B} \text {,an }}$ or $\varphi_{\mathrm{C} \text {,an }}$ with the temperature is that at low temperature, $\varphi_{\mathrm{ABC}}$ tends to a constant value $\varphi_{\text {cold }}=\varphi_{\mathrm{ABC}}(\mathrm{T}=0)$, whereas at high temperature $\varphi_{\mathrm{ABC}} \approx \varphi_{\mathrm{T}}$ tends to follow the increasingly larger thermal voltage $\varphi_{\mathrm{T}}=\mathrm{kT} / \mathrm{q}$. This behavior leads 
to expressions for "dominance of the larger". For example, one suitable for $\varphi_{\mathrm{ABC}}$ form for "dominance of the larger" is

$$
\left(\varphi_{\mathrm{ABC}}\right)^{\mathrm{n}}=\left(\varphi_{\text {cold }}\right)^{\mathrm{n}}+\left(\varphi_{\mathrm{T}}\right)^{\mathrm{n}} \approx\left(\varphi_{\mathrm{OEF}}\right)^{\mathrm{n}} \approx\left\{\begin{array}{l}
\left(\varphi_{\text {cold }}\right)^{\mathrm{n}} \text { for } \varphi_{\mathrm{T}}<\varphi_{\text {cold }} \\
\left(\varphi_{\mathrm{T}}\right)^{\mathrm{n}} \text { for } \varphi_{\mathrm{T}}>\varphi_{\text {cold }}
\end{array}\right.
$$

in which the numerical parameter (n) in the exponents controls the "sharpness" of the transition between $\varphi_{\text {cold }}$ and $\varphi_{\mathrm{T}}$ around the point $\varphi_{\text {cold }}=\varphi_{\mathrm{T}}$, at which $\varphi_{\mathrm{ABC}}=\varphi_{\text {cold }} \times(\mathrm{n} \sqrt{2})$. The resulting effective voltage is denoted with بOEF. The larger is $\mathrm{n}$, the sharper is the transition and with smaller deviation from $\varphi_{\text {cold }}$ at the transition point, since $(\mathrm{n} \sqrt{2}) \rightarrow 1$ when $n>>1$. The limiting case of $n=\infty$ was written for temperatures as $T_{O E F}=\max \left(T_{0}\right.$, T), and used as a rule by derivations of conductance and current of amorphous-silicon TFTs [80, 81]. For OTFT, however, neither experimental data nor theoretical elaboration is available at present for variations of the characteristic temperature $T_{0}$ with the ambient temperature $T$, while such variations are deduced in [11] by numerical simulations of trapped charge in TFTs with exponential DOS. The results from [11] will be addressed immediately after observing rule for the "dominance of the larger" in the results from our simulations in Figure 28.

The dashed lines shown in Figure 28 are calculated from eq. (79) with $n=5$ and $\varphi_{\text {cold }} \approx 1 / 4 \varphi_{o}, N D$ for the normally distributed $\mathrm{DOS}_{\mathrm{ND}}$ in the left-hand plots, and with $\mathrm{n}=10$ and $\varphi_{\text {cold }}=\varphi_{\mathrm{o}, \mathrm{EXP}}$ for the exponential DOSSE $_{\mathrm{SE}}$ the right-hand plots, which indicates sharper temperature transitions by assumption for exponential DOS $\mathrm{SE}$ and sustainable definition of $\varphi_{\text {cold }}$ for this $\mathrm{DOS}_{\mathrm{SE}}$. The values for $\varphi_{\text {cold }}$ are slightly lower for the normally-distributed DOS $\mathrm{ND}$, since, compared to exponential DOSSE, $\varphi_{o, N D}$ is about 2.5-3 times larger for DOS $\mathrm{ND}_{\mathrm{N}}$, see again the plot for $\mathrm{T}_{\mathrm{o}}=\varphi_{\mathrm{o}} \mathrm{q} / \mathrm{k}$ in Figure 22.

The solid black lines Figure 29 show data from [11] for several characteristic temperatures $T_{0}$ of the DOS width and various ambient temperatures $T$. In the main plot $\mathrm{T}_{\mathrm{OEF}} / \mathrm{T}_{\mathrm{o}}$ vs. $\mathrm{T}_{\mathrm{o}} / \mathrm{T}$, the data have been obtained after deconvolution of the integral $\int d x /\left(1+x^{a}\right)$, followed by fitting with exponential DOS of temperature-varying effective concentration of states $\mathrm{N}_{\mathrm{SEF}}$ and effective characteristic temperature $\mathrm{T}_{\mathrm{OEF}}$. We show in the figure the sub-set of data for values of $\mathrm{T}_{0}=\{250 \mathrm{~K}, 350 \mathrm{~K}, 425 \mathrm{~K}$ and $500 \mathrm{~K}\}$, which cover 
practically all cases for OTFT. The inset in this figure shows the same data, recalculated as "thermal-like" voltages $\varphi_{\mathrm{OEF}}=\mathrm{kT} \mathrm{OEF}_{\mathrm{q}} / \mathrm{q}$ and $\varphi_{\mathrm{o}} \equiv \mathrm{kT} \mathrm{T}_{\mathrm{o}} / \mathrm{q}$, and vs. the thermal voltage $\varphi_{\mathrm{T}}=\mathrm{kT} / \mathrm{q}$. In both plots, the symbols denote values, which are calculated by eq. (79) with $\varphi_{\text {cold }}=\varphi_{\mathrm{o}}$ and $n=10$, as deduced for exponential DOS $\mathrm{SE}$ just above. Noticeably, in both plots of Figure 29, the symbols are fitting the lines well for the order of magnitude and in the behavior, confirming the correctness of the "dominance of the larger" rule in eq. (79). However, the lines with data from the numerical calculations in [11] for different $T_{o}$ are different in the main plot, whereas the symbols with data from the analytical expression above are aligned on the same curve for all values of $\mathrm{T}_{\mathrm{o}}$, which indicates that the rule is not precise in the simple form of eq. (79), and also, the value $n=10$ we have obtained empirically. Therefore, we have refined the analytical expression, in order to be valid also for higher values of $\mathrm{T}_{0}$, for which the discrepancy between eq. (79) and the numerical simulations is more evident. After researching several possible variants, we arrived to the following modified expression, which is rewritten for characteristic temperatures as

$$
\begin{aligned}
\mathrm{T}_{\mathrm{OEF}}^{\mathrm{n}} & =\mathrm{T}_{\mathrm{o}}^{\mathrm{n}}+\left\{\mathrm{T}\left[1+\left(\frac{\mathrm{T}_{\mathrm{O}}}{\mathrm{T}_{\mathrm{G}}}\right)^{\mathrm{m}}\right]^{\frac{1}{\mathrm{~m}}}\right\}^{\mathrm{n}} \\
& \approx \mathrm{T}_{\mathrm{O}}^{\mathrm{n}}+\mathrm{T}^{\mathrm{n}}\left[1+\sqrt{\left(\frac{\mathrm{T}_{\mathrm{O}}}{\mathrm{T}_{\mathrm{G}}}\right)^{\mathrm{n}}}\right]^{2}, \text { with } \mathrm{m}=\frac{\mathrm{n}}{2}, \mathrm{n}=\frac{\mathrm{T}_{\mathrm{G}}}{2}\left(\frac{1}{\mathrm{~T}_{\mathrm{o}}}+\frac{1}{\mathrm{~T}}\right) \\
& \text { and } \mathrm{T}_{\mathrm{G}} \approx \frac{0.33 \mathrm{eV}}{\mathrm{k}} \approx 3830 \mathrm{~K} \approx \frac{1}{6} \frac{\text { band gap }}{\mathrm{k}} .
\end{aligned}
$$

The modification is basically in the term $\left[1+\left(\mathrm{T}_{0} / \mathrm{T}_{\mathrm{G}}\right)^{\mathrm{n} / 2}\right]^{2}$ and also provides a rule for calculation of (n) from a characteristic temperature $\mathrm{T}_{\mathrm{G}}$, which we observe to be corresponding to a fraction of the energy band gap of the organic semiconductor. The modified expression for the "dominance of the larger" rule fits very well the data from numerical simulations, as illustrated in Figure 30, with standard deviation error 5.7K or $0.71 \%$, and maximum error $17 \mathrm{~K}$ or $2.08 \%$ over the whole set of values for $\mathrm{T}_{\mathrm{o}}=\{250 \mathrm{~K}, 350 \mathrm{~K}, 425 \mathrm{~K}, 500 \mathrm{~K}$, $600 \mathrm{~K}, 800 \mathrm{~K}$ and $1000 \mathrm{~K}$ \} and temperatures up to $\mathrm{T} \leq 1173 \mathrm{~K}=900^{\circ} \mathrm{C}$. These errors are comparable with the errors of digitizing the plot of Fig. 6 in [11], from where the data of the numerical calculation are adopted. 
Since the modified expression is valid well beyond the realistic operation conditions for OTFT, it might be helpful as a guideline for the expected format of analytical solutions of the integral $\int \mathrm{dx} /\left(1+\mathrm{x}^{\mathrm{a}}\right)$, which are unknown at present. Another format for approximate solution of this integral is given in [2].

\subsubsection{Charge concentrations and different DOS types from electrostatics and for VRH}

The fourth comparison of the monitored quantities is illustrated in Figure 31, in which with characteristic values for the DOS and carrier concentration $\mathrm{N}_{\mathrm{C}}$ (precisely, concentration of occupied charge states and carriers in VRH) are evaluated at the characteristic distances $\mathrm{D}=0, \mathrm{~L}_{\mathrm{A}}, \mathrm{D}_{95 \% \text { osq }}$ and $\mathrm{D}_{\mathrm{avgVB}}$ from the gate dielectric into the semiconducting film. This fourth comparison is an attempt to verify deductions made in $[61,62]$ that the magnitudes of DOS and charge are similar at different assumptions for the type of DOS, considering these also for VRH.

The device, the temperature and the biasing conditions in Figure 31 are identical with those in the figures of the preceding three comparisons. The solid lines in Figure 31 are at assumption for normally distributed $\mathrm{DOS}_{\mathrm{ND}}$. The dashed lines in Figure 31 are for single-sided exponential DOS $\mathrm{SE}$. The styles of notation symbols are as in Figure 28. For the purely electrostatic quantities: $D O S(D=0)$ and $N_{C}(D=0)$, corresponding to the semiconductor-gate dielectric interface (depth $\mathrm{D}=0$ ), are denoted with triangles $(\triangle$ ); and the squares $(\square)$ are for $\operatorname{DOS}\left(\mathrm{L}_{\mathrm{A}}\right)$ and $\mathrm{N}_{\mathrm{C}}\left(\mathrm{L}_{\mathrm{A}}\right)$ at depth equal to the electrostatic length $\mathrm{L}_{\mathrm{A}}$, where $\mathrm{L}_{\mathrm{A}}$ corresponds to $1 / 2 \mathrm{Q}_{\mathrm{G}}$ of the gate charge $\mathrm{Q}_{\mathrm{G}} \approx \mathrm{C}_{\mathrm{Ox}} \mathrm{V}_{\mathrm{G}}$ - see before eq. (69). As expected form eq. (49), $\mathrm{N}_{\mathrm{C}}\left(\mathrm{L}_{\mathrm{A}}\right) / \mathrm{N}_{\mathrm{C}}(0) \approx \operatorname{DOS}\left(\mathrm{L}_{\mathrm{A}}\right) / \operatorname{DOS}(0) \approx 1 / 4$ in all plots of Figure 31 , irrespectively of temperature and biasing conditions. Furthermore, looking at the upper-right plot of Figure $31, \mathrm{~N}_{C}(\mathrm{D}=0)$ and $\mathrm{N}_{\mathrm{C}}\left(\mathrm{L}_{\mathrm{A}}\right)$ are weakly affected by the temperature at the given gate bias voltage $V_{G}=-20 \mathrm{~V}$, which is expected, since the substitution of the expression for $\mathrm{L}_{\mathrm{A}}$ from eq. (47) into eq. (49) for $\mathrm{N}_{\mathrm{C}}$, with $\varphi_{\mathrm{OEF}}$ instead of $\varphi_{\mathrm{o}}, \varphi_{\mathrm{A}}$ or $\varphi_{C}$, yields

$$
\left.\begin{array}{r}
\mathrm{N}_{\mathrm{C}}(\mathrm{D}=0) \\
\mathrm{N}_{\mathrm{C}}\left(\mathrm{D}=\mathrm{L}_{\mathrm{A}}\right)
\end{array}\right\}=\frac{\mathrm{V}_{\mathrm{G}}^{2}}{\varphi_{\mathrm{OEF}}}\left[\frac{\mathrm{C}_{\mathrm{OX}}^{2}}{\mathrm{q} \varepsilon_{\mathrm{f}}} \times\left\{\begin{array}{l}
1 / 2, \text { for } \mathrm{D}=0 \\
1 / 8, \text { for } \mathrm{D}=\mathrm{L}_{\mathrm{A}}
\end{array}\right]\right.
$$


where the term in the square brackets is bias and temperature independent. The bias dependence is due to the gate voltage $V_{G}$, and a temperature dependence can arise from the temperature variations of poEF discussed just above. At temperatures $\mathrm{T}>\mathrm{T}_{\mathrm{o}} / 2 \sim 200 \mathrm{~K}$ for this OTFT, $\varphi$ OEF begins increasing, according to eq. (79), and therefore, $\mathrm{N}_{C}(\mathrm{D}=0)$ and $\mathrm{N}_{\mathrm{C}}\left(\mathrm{L}_{\mathrm{A}}\right)$ begin decreasing, e.g., at $\mathrm{T}=500 \mathrm{~K}$ down to about $80 \%$ of the lowtemperature values, since $\mathrm{T}_{\mathrm{o}} / \mathrm{T}=400 \mathrm{~K} / 500 \mathrm{~K}=80 \%$. The other expected consequence from eq. (81) is the quadratic dependence of $\mathrm{N}_{\mathrm{C}}$ on the gate bias voltage $\mathrm{V}_{\mathrm{G}}$. The quadratic dependence is clearly observed by the almost identical slopes in the bottom-right plot of Figure 31 for $\mathrm{N}_{\mathrm{C}}$ at all characteristic conditions, $\mathrm{D}=0$, $\mathrm{L}_{\mathrm{A}}, \operatorname{avg} \mathrm{V}_{\mathrm{B}}$ and $95 \%$ of $\sigma_{\mathrm{sq}}$, and at any given temperature. The trend in the data in this plot is with slope $\partial \ln \left(\mathrm{N}_{\mathrm{C}}\right) / \partial \ln \left(\mathrm{V}_{\mathrm{G}}\right)=2.04( \pm 0.102$ standard deviation $)$, which is $2 \%$ average deviation and $5 \%$ standard deviation from the quadratic dependence. For comparison, the charge unbalance between the gate dielectric capacitor charge and the charge in the semiconducting film is in the range of $5 \%$ by the numerical simulation, as mentioned after eq. (38), and it will be addressed again in Sec. 5.1 with the help of Figure 34 in relation to computational volume. The other observations in Figure 31 are the following.

The values of DOS are different at different characteristic conditions. In the upper-left corner of the upper left plot of Figure 31, the values of DOS are in the range $10^{20}-10^{21} \mathrm{~cm}^{-3} \mathrm{eV}^{-1}$, being similar for different conditions and assumptions for DOS type. However, significant differences emerge increasing the temperature, as seen in the right-hand side of this plot, where the values spread over many decades from $10^{16}-10^{20} \mathrm{~cm}^{-3} \mathrm{eV}^{-1}$. The overall trend in this plot is that the values of DOS decrease with temperature for each of the characteristic conditions, and the steepness of the decrease of $\operatorname{DOS}(\mathrm{D}=0)$ and $\operatorname{DOS}\left(\mathrm{L}_{\mathrm{A}}\right)$ for the purely electrostatic conditions $(\mathrm{D}=0)$ and $\mathrm{L}_{\mathrm{A}}$ are similar and smaller than the steepness of $\mathrm{DOS}\left(\operatorname{avg} \mathrm{V}_{\mathrm{B}}\right)$ and $\operatorname{DOS}\left(95 \% \sigma_{\mathrm{sq}}\right)$, the latter being with the steepest temperature dependence. Therefore, the values for DOS from electrostatic calculations are approximately representative for values of DOS for VRH only at low temperature, but these values are different and should be not mixed each with other at high temperature. Emphasizing, only qualitatively, the type of DOS does not affect dramatically the magnitude of DOS at given characteristic condition, because the solid and dashed lines for given symbol $(\triangle, \bigcirc, \diamond$, or $\square)$ 
indicate similar behaviors and ranges, both by changing the temperature $\mathrm{T}$ in the upper-left plot of Figure 31 and gate bias voltage $V_{G}$ in the bottom-left plot of Figure 31. Thus, the approximations with exponential DOS deduced in $[61,62]$ are reasonable for low temperature. However, the approximations are inaccurate for high temperature, because the temperature dependences of the values of DOS at given characteristic condition by normally distributed $\mathrm{DOS}_{\mathrm{ND}}$ are weaker, whereas the bias dependences are stronger, when comparing to exponential DOS $\mathrm{SE}$, as seen by the pairs of solid and dashed lines for each symbol $(\triangle, \bigcirc, \diamond$, or $\square$ ) in the upper-left and bottom-left plots of Figure 31, respectively.

The different temperature behaviors of characteristic values at electrostatic and VRH conditions are clearly evident for the characteristic values of the carrier concentration $\mathrm{N}_{\mathrm{C}}$ in the upper-right plot of Figure 31. As for other electrostatic quantities addressed above, the electrostatic $N_{C}(D=0)$ and $N_{C}\left(L_{A}\right)$ are weakly affected by the temperature, resulting in $(\triangle)$ and $(\square)$ aligned around almost horizontal lines in the upper half of this plot. In contrast, the characteristic values $\mathrm{N}_{C}\left(\operatorname{avgV} \mathrm{V}_{\mathrm{B}}\right)$ and $\mathrm{N}_{\mathrm{C}}\left(95 \% \sigma_{\mathrm{sq}}\right)$ for VRH replicate the $\exp \left[-\left|\operatorname{avg} V_{B}-V_{B S}\right| / \varphi O E F\right]$ and $\exp \left[-\left|V_{B}\left(D_{95 \% \sigma s q}\right)-V_{B S}\right| / \varphi\right.$ OEF $]$ dependences, where $V_{B S}=V_{B}(D=0)$ is the bending at the gate dielectric-semiconductor interface, with always $\left|\mathrm{V}_{\mathrm{BS}}\right|>\left|\operatorname{avg} \mathrm{V}_{\mathrm{B}}\right|>\left|\mathrm{V}_{\mathrm{B}}\left(\mathrm{D}_{95 \% \sigma s q}\right)\right|$, and increasing in magnitude differences $0<\left|\operatorname{avg} \mathrm{V}_{\mathrm{B}}-\mathrm{V}_{\mathrm{BS}}\right|<\left|\mathrm{V}_{\mathrm{B}}\left(\mathrm{D}_{95 \% \sigma s q}\right)-\mathrm{V}_{\mathrm{BS}}\right|$ at higher temperature. The bending voltage $V_{B}$ was shown in Figure 26a. Therefore, at given bias, e.g. $V_{G}=-20 V$, the representative for VRH carrier concentrations $\mathrm{N}_{\mathrm{C}}\left(\operatorname{avg} \mathrm{V}_{\mathrm{B}}\right)$ and $\mathrm{N}_{\mathrm{C}}\left(95 \% \sigma_{\mathrm{sq}}\right)$ decrease with temperature significantly stronger, $\propto\left[\varphi_{\mathrm{OEF}} \times \exp \left(\Delta \mathrm{V}_{\mathrm{B}} / \varphi_{\mathrm{OEF}}\right)\right]^{-1}$, than the representative for electrostatics $\mathrm{N}_{\mathrm{C}}(\mathrm{D}=0)$ and $\mathrm{N}_{\mathrm{C}}\left(\mathrm{L}_{\mathrm{A}}\right)$, only $\propto\left[\varphi_{\mathrm{OEF}}\right]^{-1}$, as one can see in the upper-right plot of Figure 31. In the bottom-right plot of this figure, all characteristic values for $\mathrm{N}_{\mathrm{C}}$ are proportional to the square of the gate bias $\mathrm{V}_{\mathrm{G}}$, as discussed just above by eq. (81). Again, and similarly to DOS characteristic values, the electrostatic $\mathrm{N}_{C}(\mathrm{D}=0)$ and $\mathrm{N}_{C}\left(\mathrm{~L}_{\mathrm{A}}\right)$ can be taken as representative also for $\mathrm{VRH}$ at low temperature, since the $\mathrm{VRH}$ representative values $\mathrm{N}_{\mathrm{C}}\left(\operatorname{avg} \mathrm{V}_{\mathrm{B}}\right)$ and $\mathrm{N}_{C}\left(95 \% \sigma_{\mathrm{sq}}\right)$ are between the electrostatic $\mathrm{N}_{C}(\mathrm{D}=0)$ and $\mathrm{N}_{\mathrm{C}}\left(\mathrm{L}_{\mathrm{A}}\right)$ at $\mathrm{T}=100 \mathrm{~K}$. However, this assumption becomes gradually incorrect by increasing the temperature, because first $\mathrm{N}_{C}(95 \% \sigma s q)$ and then $\mathrm{N}_{C}\left(\operatorname{avg} \mathrm{V}_{\mathrm{B}}\right)$ leave the interval $N_{C}(D=0)-N_{C}\left(L_{A}\right)$, as seen in the upper-right plot of Figure 31. At high temperature 
$\mathrm{T}=500 \mathrm{~K}$, both $\mathrm{N}_{C}\left(\operatorname{avg} \mathrm{V}_{\mathrm{B}}\right)$ and $\mathrm{N}_{C}(95 \% \sigma s q)$ are smaller than $\mathrm{N}_{C}\left(\mathrm{~L}_{\mathrm{A}}\right)$, with $\mathrm{N}_{\mathrm{C}}\left(95 \% \sigma_{\mathrm{sq}}\right)$ being more than a decade below $\mathrm{N}_{\mathrm{C}}\left(\mathrm{L}_{\mathrm{A}}\right)$ at any bias, as seen in the bottom-right plot of Figure 31.

To summarize briefly the observations in Figure 31, the values for DOS and carrier concentrations $\mathrm{N}_{\mathrm{C}}$ evaluated at characteristic electrostatic distances is possible to be assumed valid also for VRH at low temperatures, but this assumption will be incorrect at high temperatures and for temperature dependences. The quadratic bias dependence $\mathrm{N}_{\mathrm{C}} \approx \mathrm{m} \times\left(\mathrm{V}_{\mathrm{G}}\right)^{2}$ is valid for both electrostatic and VRH conditions by determination of the values for $\mathrm{N}_{\mathrm{C}}$. This relation $\mathrm{N}_{\mathrm{C}} \approx \mathrm{m} \times\left(\mathrm{V}_{\mathrm{G}}\right)^{2}$ is independent of DOS type and temperature up to a multiplicative factor $\mathrm{m}$. The factor $\mathrm{m}$ is temperature and DOS dependent, but $\mathrm{m}$ is nearly biasindependent. Thus, the relation between electrostatics and VRH is not through linear scaling (multiplication) of charge concentrations, but by a power-law function, as shown later by eq. (83). The linear relation between electrostatics and VRH is through the linear correlation between VRH energy $\mathrm{E}_{\mathrm{H}}$ and potential bending $\mathrm{V}_{\mathrm{B}}$, as discussed in preceding sections, e.g., in Sec. 4.2.

\subsection{Film thickness and mobility in OTFT}

An interesting outcome from the numerical VRH simulations is that the mobility $\mu$ in OTFTs increases with very thin semiconducting films. The film thickness dependence of the mobility was observed experimentally many times, and was discussed in [82], where a power-law trend was deduced as

$$
\mu \sim \mu_{\mathrm{z}}\left(\frac{\varepsilon_{\mathrm{z}}}{\mathrm{t}_{\mathrm{f}} \mathrm{C}_{\mathrm{ox}}}\right)^{\mathrm{S}_{\mu}}
$$

Here, $\mu_{\mathrm{z}} \approx 10^{5} \mathrm{~cm}^{2} / \mathrm{Vs}( \pm 50 \%), \varepsilon_{\mathrm{z}} \approx 5 \times 10^{-16} \mathrm{~F} / \mathrm{cm}( \pm 30 \%)$, and the slope $S_{\mu}$ is a material-dependent parameter with values of $1.6 \pm 0.2$ for pentacene and $2.6 \pm 0.4$ for solution-processed polymers, such as PQT-12 and P3HT. The trend implies that the OTFT with thinner film exhibits higher effective mobility, which is counterintuitive and in contrast to the analytical formula of eq. (151) in Appendix 2. Therefore, one usually attributes the film thickness dependence of the effective mobility to contact effects, since it is shown, e.g., by the injection-drift limited model in [27], that the injection limit can strongly interfere with the transfer 
characteristics $I_{D}-V_{G}$ of the OTFT, effectively reducing the current $I_{D}$ and equivalently observing values of mobility, which are a decade below the mobility in the organic film itself. Recent investigations attribute the thicker film with higher values of the contact resistance, which causes a degenerating current feedback at the source terminal of the OTFT, reducing the transconductance $\mathrm{g}_{\mathrm{m}}=\partial \mathrm{I}_{\mathrm{D}} / \partial \mathrm{V}_{\mathrm{G}}$. Consequently, a lower value for $g_{m}$ implies a lower value of the mobility, since $g_{m} \propto \mu$ is a widely used relation in the experimental characterization of mobility, e.g., eq. (52) for the linear regime of operation of the OTFT. We shall not address here the contact effects in OTFT, because this topic is broad and under intensive research at present, since contact effects are very prominent in OTFT. Instead, we are interested in whether VRH itself causes a thickness dependence of the effective mobility in OTFT. Certainly, the numerical simulator in this work does not consider contacts with the drain or the source terminals, but only the stack of the gate-insulatorsemiconductor at the idealized condition of free space at the back of the film. This idealized condition provides the opportunity to observe the mobility solely in the film, which is experimentally not feasible. Again, the numerical VRH simulations show that the mobility $\mu$ increases in OTFT of very thin semiconducting film, as illustrated in Figure 32.

The data in Figure 32 are after numerical VRH simulations of virtual devices of different film thicknesses $t_{f}$ over a wide range from $t_{f}=2 n m$ to $t_{f}=500 \mathrm{~nm}$. To preserve a link to real devices, we have used the double-exponential $\mathrm{DOS}_{\mathrm{DE}}$ given by eq. (45) and the corresponding parameter values from Table IV, which we have proven to represent real OTFTs of single film thickness by the good fit in Figure 17 (b) and (c) between numerical simulation and the experimental data for mobility reported in [22] and [60]. Depending on the method of integration, we switch between the values of the parameters corresponding to VRH calculation with single $\int \mathrm{dE}$ and multiple $\iint \mathrm{dEdE}$ integrations, but not changing the type of DOS $\mathrm{DE}_{\text {. The }}$ only device parameter, which we vary in the virtual OTFTs, is the film thickness $t_{\mathrm{f}}$. To ensure further consistency with Figure 17, we have used the same biasing and temperature conditions and the same settings of the numerical simulator, e.g. energy integration step $\mathrm{dE}=2 \mathrm{meV}$, the latter only changed to $1 \mathrm{meV}$ and $5 \mathrm{meV}$, in order to observe variations that are caused by numerical integration. Furthermore, we have 
inspected the profiles for unforeseen discrepancies, which have not been observed. For example, at given temperature $T$ and bias voltage $V_{G}$, the charge profiles $N_{C}(D)$ have overlapped for the virtual OTFTs with different $t_{f}$, and from the peak of the products $D \times N_{C}(D)$, as explained earlier in relation to the left-hand plots of Figure 24, we have determined identical values for the electrostatic length $\mathrm{L}_{\mathrm{A}}$, irrespective of the values of $t_{f}$. The values of $L_{A}$ are shown in the horizontal axis by the symbols connected with dashed lines in Figure 32(a) vs. $\mu$ (in the vertical axis) of the virtual pentacene device with $\mathrm{t}_{\mathrm{f}}=50 \mathrm{~nm}$, and the many symbols overlap at a given temperature $\mathrm{T}$ and bias voltage $\mathrm{V}_{\mathrm{G}}$. The black and gray color solid lines in this plot show the values of the mobility, obtained from $\mu=\sigma_{\mathrm{sq}} / \mathrm{Q}_{\mathrm{G}}$, as explained by eq. (42), after using the two methods of VRH calculation with single $\int \mathrm{dE}$ and multiple $\iint \mathrm{dEdE}$ integrations for the determination of the sheet conductance $\sigma_{\mathrm{sq}}$. Evidently, both calculation methods also give almost overlapping values for mobility. Noticeably, the mobility increases for very thin films, $\mathrm{t}_{\mathrm{f}}<10 \mathrm{~nm}$ at $\mathrm{T}=100 \mathrm{~K}$ and $\mathrm{t}_{\mathrm{f}}<20 \mathrm{~nm}$ at $\mathrm{T}=300 \mathrm{~K}$, but this increase cannot be related to the electrostatic length $\mathrm{L}_{\mathrm{A}}$, which has significantly lower values between $0.56 \mathrm{~nm}$ and $2.4 \mathrm{~nm}$, and almost no temperature variation in this temperature interval, see again the squares for 1000/T>3 [1000/K] in the bottom-left plot of Figure 27.

Suspecting that the observed film-thickness dependence of the mobility can be an accidental artifact of parameter values, we have repeated the simulations for the PQT-12 OTFT, which has very different values for $\mathrm{N}_{\mathrm{S}}, \mathrm{T}_{\mathrm{o}}, \Lambda_{\mathrm{o}}, \Gamma_{\mathrm{o}}$ and $\mathrm{C}_{\mathrm{OX}}$ (compare the parameter values in Table IV) and also different biasing conditions. The simulation results for the mobility in the virtual PQT-12 OTFTs of different film thicknesses are shown in Figure 32(b), where we observe very similar behavior as for the pentacene virtual OTFTs in Figure 32(a). The overlap of the results is good by the two methods of VRH calculations. Just the increase of $\mu$ is relatively more pronounced in the PQT-12 OTFT, when decreasing $t_{f}$ in the same intervals. This is consistent with the higher value of the slope $S_{\mu}$ for solution-processed polymer semiconductors in eq. (82).

Another doubt for accidental outcome from numerical simulation could be the size of the integration step. Therefore, we have rerun the simulations for the PQT-12 virtual devices at finer and coarser energy integrations steps $\mathrm{dE}=1 \mathrm{meV}$ and $5 \mathrm{meV}$, respectively, and have kept the device parameters and simulator 
settings identical to those in Figure 32(b). The effect of the variation of the energy integration step size is illustrated in Figure 32(c). The thin black lines in Figure 32(c) are the same as the black lines in Figure 32(b), corresponding to multiple $\iint \mathrm{dEdE}$ integrations with $\mathrm{dE}=2 \mathrm{meV}$. The gray-color solid lines in Figure 32(c) show that the coarse integration step of $\mathrm{dE}=5 \mathrm{meV}$ suppresses the increase of the mobility, whereas the dashed lines show that the fine integration step of $\mathrm{dE}=1 \mathrm{meV}$ enhances the increase of the mobility. Looking closer at the dashed lines for room temperature $\mathrm{T}=300 \mathrm{~K}$, one observes an interesting non-monotonic behavior. Decreasing the film thickness of OTFT below 30nm, the mobility increases, as reported in [83, 84, 85], for example. However, thinning the film below few nm, e.g. below 10-15nm, the mobility begins dropping, which was also reported in $[84,85,86,87]$ and attributed to a degradation of the film uniformity in few mono-layer films (or weak contact of the thin film to the metal of the source terminal in a bottomcontact configuration of OTFT). However, this drop can be also explained by the analytical formula of eq. (151) in Appendix 2, adjusting the values of some parameters in it. In any case, owing to fabrication convenience, most of the actual OTFTs have 30-50nm thick films, range in which the mobility results are independent on the integration step.

While the variations of the magnitudes with the integration step indicate issues with the accuracy of the numerical calculations, these calculations clearly indicate that VRH also predicts mobility enhancement in OTFTs of very thin films. Note that we have not made additional adjustments in the simulator, such as two-dimensional VRH or quantum effects, space quantization with inter-layer and atomic distances, etc. Therefore, we conclude that the numerical VRH simulator captures the thickness dependence of the mobility, which is a useful feature of the simulator from the practical perspective. We believe that the prediction of the numerical calculation for the increase of the mobility in very thin film OTFTs is qualitatively correct, since the calculations are based on principles, and not on formulas. In addition, the data from simulations coincide well with the trend of eq. (82) for the film-thickness dependence of mobility, as seen in Figure 33 with an example for the virtual PQT-12 OTFTs of different film thicknesses (filled circles $\bigcirc$, blue color) at room temperature $\mathrm{T}=300 \mathrm{~K}$, when compared with the experimental data collected in 
[88] for PQT-12 OTFTs of film thicknesses $t_{\mathrm{f}}=35-40 \mathrm{~nm}$, the open triangle $(\triangle)$ and diamond $(\diamond)$, reported in $[60,77]$, respectively. In the next section, we will address the film-thickness dependence of the mobility by eq. (98) and Figure 35, showing that the results from the numerical simulations are expected.

\section{Outlook for feasibility of numerical VRH simulations}

In the previous two sections, we have presented a numerical simulator based on the VRH principles. The results from this simulator are consistent with both theoretical and experimental findings for OTFTs. It is, therefore, reasonable to outline the feasibility of the numerical VRH simulations, since many other models, mostly analytical, are proposed in the literature, and the analytical models are found to be suitable for device characterizations and circuit simulations. Thus, correspondence to existing and missing analytical models will be addressed. We will mostly deal with possible approaches for acceleration of the VRH numerical simulations, along with several other issues, e.g., dispersion of time constants, charge build-up, and noise. So, we begin with one of the problems of the numerical VRH calculations: the computational volume and the associated large amount of computational resources and simulation time, which place barriers in using the VRH numerical calculations in circuit simulators.

\subsection{Computational volume and uncertainty management in VRH numerical simulations}

The main advantage of the numerical VRH simulations presented here is that they are based on principles and material properties, but not on approximate analytical formulas, the latter valid with additional assumptions. However, as discussed in the previous sections, there are problems for the VRH numerical calculations. One of these problems is the computational volume demanded by the VRH numerical simulations, which is large, as summarized in Table VI and explained in Appendix 1. Reviewing these, the computational volume of the VRH numerical simulations for one temperature-bias point $\left(\mathrm{T}-\mathrm{V}_{\mathrm{G}}\right)$ scale as $\mathrm{m} \times \mathrm{n} \times(800 \mathrm{FLOPs}) \sim 50 \times 2000 \times(800 \mathrm{FLOPs}) \sim 0.1$ GFLOPs for the method of single $\int \mathrm{dE}$ integration and $\mathrm{m} \times \mathrm{n}^{2} \times(750$ FLOPs $) \sim 50 \times(2000)^{2} \times(750$ FLOPs $) \sim 150$ GFLOPs for multiple $\iint \mathrm{dEdE}$ integrations, where $\mathrm{m} \sim 50$ is 
the number of points in the depth of the organic film and n 2000 is the number of steps in the energy mesh. To minimize the computation volume, a dedicated procedure for reduction of the number of points in the spatial mesh was undertaken (Sec. 2.5.2) to keep $\mathrm{m}$ 50 during the VRH calculations, since $\mathrm{m}$ is large (m 2000) during the electrostatic calculations. In order to support various types of DOS, no reduction of the energy mesh is undertaken, and the energy mesh size is $n=\left(E_{t o p}-E_{b o t t o m}\right) / d E \sim 2 e V / 1 m e V \approx 2000$ for the method of single $\int \mathrm{dE}$ integration, while the energy mesh size is $\mathrm{n} \times \mathrm{n}$ in the $2 \mathrm{D}$ calculations for the multiple $\iint \mathrm{dEdE}$ integrations. Consequently, a refinement of the step $\mathrm{dE}<0.5 \mathrm{meV}$ in the VRH calculations with the method of multiple $\iint \mathrm{dEdE}$ integrations results in a very large computational volume in the range of TFLOPs.

Figure 34 summarizes the computational requirements above for the one-dimensional VRH numerical calculation at one bias-temperature point $\left(\mathrm{V}_{\mathrm{G}}-\mathrm{T}\right)$. In the upper half of the figure, the circles $(0)$ are for VRH calculation with multiple $\iint \mathrm{dEdE}$ integrations and the diamonds $(\bullet)$ are for VRH calculation with single $\int \mathrm{dE}$ integration, and they are fitted with quadratic and linear trend lines, respectively, showing the computational time when using parallel computing of 18 or more $\left(\mathrm{V}_{\mathrm{G}}-\mathrm{T}\right)$ points, and from left to right, for three values of the energy integration step $\mathrm{dE}=5 \mathrm{meV}, 2 \mathrm{meV}$ and $1 \mathrm{meV}$. The width of energy mesh was always $\left|\mathrm{E}_{\text {top }}-\mathrm{E}_{\text {bottom }}\right|=1.9 \mathrm{eV}$, having $\mathrm{n}=\left|\mathrm{E}_{\text {top }}-\mathrm{E}_{\text {bottom }}\right| \mathrm{dE}=380,950$ and 1900 points, respectively at the different dE. From the trend lines, one observes that there was about 100 seconds overhead in the computations. The VRH calculation with single $\int \mathrm{dE}$ integration scales linearly with the energy mesh size, requiring only about $3.5 \mathrm{~s}$ for $\mathrm{n}=1000$ energy mesh points. However, the VRH calculation with multiple $\iint \mathrm{dEdE}$ integrations is much more demanding. It takes about 5 minutes for $\mathrm{n}=1000$ energy mesh points, and it scales with the square of $\mathrm{n}$, as $5 \min \times(\mathrm{n} / 1000)^{2}$, which is in the range of 20-30 minutes, when the fine energy integration step of $\mathrm{dE}=1 \mathrm{mV}$ was chosen. Further refinement of $\mathrm{dE}=0.5 \mathrm{meV}$ would require 2 hours or more to calculate one bias-temperature point $\left(\mathrm{V}_{\mathrm{G}}-\mathrm{T}\right)$ by multiple $\iint \mathrm{dEdE}$ integrations, which is impractical. Aligning the expression for computational volume of the VRH calculation with multiple $\iint \mathrm{dEdE}$ integrations, denoted with dashed line without symbols, to the trend line of this calculation, we have 
estimated that the throughput of one computer in the grid of computers is approximately (0.1-0.2)GFLOP per second for our numerical simulator. This throughput is about 1/10 of LINPACK benchmark test [89], indicating that there is a room for optimization of the code for throughput. For comparison, scaling the throughput, the other two dashed lines in the figure show the much lower requirements to the computation of VRH with single $\int \mathrm{dE}$ integration $(\square)$ and for electrostatic calculations $(\triangle)$, the latter always executed prior any VRH calculation and with moderate computational volume of approximately $\mathrm{m} \times \mathrm{n} \times 105 \mathrm{FLOPs} \sim(2000)^{2} \times(105 \mathrm{FLOPs}) \sim 0.41$ MFLOPs, with $\mathrm{m} \approx \mathrm{n} \sim 2000$, as estimated in Appendix 1 and summarized in row "eSt" of Table VI. Thus, from perspective of the user time, one would try to reduce the computational time to be not too much higher than the overhead of 100 seconds.

One way for reduction of the computational time is to enlarge the integration step dE. However, the error of the numerical calculation will also increase. For example, the unbalance errQ $\mathrm{G}_{\mathrm{G}}=\left|\mathrm{Q}_{\mathrm{F}} / \mathrm{Q}_{\mathrm{G}}-1\right|$ of the gate and film charge is shown in Figure 34 by the circles with dotted trend line errQ $\mathrm{G}_{\mathrm{G}}=5 \% / \mathrm{n}^{0.99}$, errQ $\mathrm{G}_{\mathrm{G}} \approx(5 \pm 1.6) \% /(\mathrm{n} / 1000)$, including also the standard deviation error $\left(\sim \frac{1 / 3}{3} \mathrm{err}_{\mathrm{G}}\right)$ to the average error $\left(\operatorname{errQ}_{\mathrm{G}}\right)$. Note that $(\mathrm{n})$ is in thousands of points in the trend line expressions in Figure 34. The unbalance is due to the finite energy step $d E$ in the numerical integrations and $\operatorname{errQ}_{\mathrm{G}} \propto[\exp (\mathrm{dE} / \mathrm{kT})-1]$. Since $\mathrm{dE} / \mathrm{kT}<1$, then $[\exp (\mathrm{dE} / \mathrm{kT})-1] \sim \mathrm{dE} / \mathrm{kT} \propto \mathrm{dE} \propto 1 / \mathrm{n}$, as seen in the figure. A second suggestion for reduction of the computation time can be to use VRH calculation with single $\int \mathrm{dE}$ integration in preliminary simulation, followed by multiple $\iint \mathrm{dEdE}$ integrations for obtaining the final result, since we have shown in the previous sections that both calculations can fit experimental data almost equally well. However, this is also not a straightforward approach, because the parameter values are different for the two methods of integration. Compare again the values of the parameters in Table IV and the black with gray-color symbols in Figure 22. The remaining approach is to substitute the VRH calculation with an equivalent, but simple calculation that scales the electrostatic calculation, the latter unavoidable, but relatively fast and available in many electrostatic simulators. Fortunately, this is a reasonable approach, considering the consequences from eq. (59) for the linear relation $\left(\mathrm{E}_{\mathrm{H}}+\mathrm{q} \mathrm{V}_{\mathrm{B}}\right)=\mathrm{E}_{\mathrm{HFB}}$ between the hopping energy $\mathrm{E}_{\mathrm{H}}$ and the bending voltage $\mathrm{V}_{\mathrm{B}}$ at a 
given bias and spatial coordinate in the OTFT film. The hopping energy in bulk material $\mathrm{E}_{\mathrm{HFB}}$ is only temperature dependent. Therefore, in the outlook below, we will focus mainly on the feasibility to substitute the integrations of the VRH calculations with scaled electrostatic quantities.

\subsection{Substitution of integrations in VRH numerical simulations with analytical relations}

In principle, eq. (63) gives the relation between electrostatics and VRH, but the numerical simulators rarely gather the bending voltage $\mathrm{V}_{\mathrm{B}}$, and therefore, it is desired to have relations with the charge concentration $\mathrm{N}_{\mathrm{C}}$, eq. (25), which is always present in the output from electrostatic solvers. We have shown in the previous sections that several relations hold, and they are numerically precise by assuming an exponential DOS, when considering in the expressions the effective characteristic "thermal-like voltage" ( and (80), which varies with the temperature and originates from a convolution between the Fermi and DOS distributions. Therefore, we use QOEF, which varies with temperature, instead of the DOS-width characteristic voltage $\varphi_{\mathrm{o}}=\mathrm{kT} / \mathrm{q}=\varphi_{\mathrm{DOS}} \mathrm{SE}_{\mathrm{S}}$, eq. (72) that provides a proportionality of $\varphi_{\mathrm{o}}$ to the reciprocal of the logarithmic slope of the exponential DOS $\mathrm{SE}$, thus, $\varphi_{\mathrm{o}}$ has a constant value and $\varphi_{\mathrm{o}}$ does not apply for elevated

temperatures $\mathrm{T}>\mathrm{T}_{\mathrm{o}}$. We shall analyze the relations of $\mathrm{VRH}$ quantities to the charge $\mathrm{N}_{\mathrm{C}}$ in three aspects: (i) at a given spatial point in the OTFT film (Section 5.2.1), (ii) at a given cross-section for sheet quantities at position $0 \leq \mathrm{x} \leq \mathrm{L}$ along the channel length $\mathrm{L}$ of the OTFT (Section 5.2.2), and (iii) for the current of the entire OTFT of unit channel width $\mathrm{W}$ and length $\mathrm{L}$, that is, $\mathrm{W}=\mathrm{L}$ for a square-shaped OTFT (Section 5.2.3). The starting point is that the bias voltages $\mathrm{V}_{\mathrm{G}}, \mathrm{V}_{\mathrm{S}}=0$ and $\mathrm{V}_{\mathrm{D}}$ of the gate, source and drain terminals, respectively, the temperature $\mathrm{T}$ and the thermal voltage $\varphi_{\mathrm{T}}=\mathrm{kT} / \mathrm{q}$, and the bias-independent parameters $\mathrm{C}_{\mathrm{OX}}, \mathrm{t}_{\mathrm{f}}, \varepsilon_{\mathrm{f}}, \varphi_{\mathrm{OEF}}$, $\sigma_{F B}$ and $N_{C F B}$ are all known.

\subsubsection{Spatial point (spot) charge concentration, conductance and mobility, and profiles}

For a spatial point of depth D in the OTFT film, the relation between VRH specific conductivity $\sigma$ and the charge concentration $\mathrm{qN}_{\mathrm{C}}$ is obtained in Appendix 3, as 


$$
\frac{[\sigma(D)]^{\varphi_{T} / \varphi_{\mathrm{OEF}}}}{\mathrm{qN}_{\mathrm{C}}(\mathrm{D})}=\frac{\left[\sigma_{\mathrm{FB}}(\mathrm{T})\right]^{\varphi_{\mathrm{T}} / \varphi_{\mathrm{OEF}}}}{\mathrm{qN}_{\mathrm{CFB}}(\mathrm{T})}=\eta(\mathrm{T}) \text {, exact as long as } \frac{\mathrm{N}_{\mathrm{C}}(\mathrm{D})}{\mathrm{N}_{\mathrm{CFB}}}=\exp \left[\frac{\mathrm{V}_{\mathrm{B}}(\mathrm{D})}{\varphi_{\mathrm{OEF}}}\right] \text { is valid. }
$$

This is a basic relation between charge and VRH conductance, in which the parameter $\eta(T)$ can have temperature dependence, e.g. a power-law function with exponent $T / T_{0}$ at low temperature $T<T_{0}$, but $\eta$ is spatially and bias independent, thus $\eta$ is a constant for a given temperature, as long as the semiconductor is uniform and the exponential DOS approximation is valid. For an ideal semiconductor, since the transport energy band is sharp $\left(T_{0} \approx 0 \Rightarrow \varphi_{\mathrm{T}}>\varphi_{0} \rightarrow 0\right)$, then $\varphi_{\mathrm{OEF}}=\varphi_{\mathrm{T}}$, as follows from eqs. (79) and (80), and $\eta=\mu$ becomes the bias-independent mobility in the ideal semiconductor. For amorphous semiconductors, however, the distribution of the DOS is wide; in fact wider than the Fermi distribution at room temperature, thus $\varphi_{0}>\varphi_{\mathrm{T}}$, and $\varphi_{\mathrm{OEF}} \approx \varphi_{\mathrm{o}}$. The relation in eq. (83) is also handy for numerical simulations, since it scales the electrostatic charge $\left(\mathrm{qN}_{\mathrm{C}}\right.$ ) into the VRH specific conductance $\sigma$, resulting in

$$
\begin{aligned}
\sigma(\mathrm{D}) & =\sigma_{\mathrm{FB}}(\mathrm{T})\left[\frac{\mathrm{N}_{\mathrm{C}}(\mathrm{D})}{\mathrm{N}_{\mathrm{CFB}}(\mathrm{T})}\right]^{\varphi_{\mathrm{OEF}} / \varphi_{\mathrm{T}}}, \\
& =[\eta(\mathrm{T})]^{\varphi_{\mathrm{OEF}} / \varphi_{\mathrm{T}}} \times\left[\mathrm{qN}_{\mathrm{C}}(\mathrm{D})\right]^{\varphi_{\mathrm{OEF}} / \varphi_{\mathrm{T}}},
\end{aligned}
$$

showing that the scaling is for the bulk conductance $\sigma_{F B}$ by a power-law function $x^{n}$ with $x=N_{C} / N_{C F B}$ being ratio of induced to bulk charge and exponent $\mathrm{n}=\varphi_{\mathrm{OEF}} / \varphi_{\mathrm{T}} \geq 1$.

Using the above scaling rule, one can obtain an expression for the profile of the VRH specific conductivity $\sigma(D)$, as follows. From eq. (154) in Appendix 2, the charge concentration $q N_{C D O}=q_{C}(D=0)$ in the semiconductor at the gate dielectric interface (depth $\mathrm{D}=0$ ) is

$$
\mathrm{qN}_{\mathrm{CDO}} \equiv \mathrm{qN}_{\mathrm{C}}(\mathrm{D}=0)=\frac{2 \varepsilon_{\mathrm{f}} \varphi_{\mathrm{OEF}}}{\mathrm{L}_{\mathrm{A}}^{2}}=\frac{\mathrm{Q}_{\mathrm{G}}^{2}}{2 \varepsilon_{\mathrm{f}} \varphi_{\mathrm{OEF}}} \text {, with } \varphi \mathrm{OEF} \text { in place of } \varphi_{\mathrm{A}} \text { and } \varphi \mathrm{C} \text {, }
$$

recalling again from eq. (160) that $\mathrm{Q}_{\mathrm{G}} /\left(2 \varepsilon_{f} \varphi_{\mathrm{OEF}}\right)=1 / \mathrm{L}_{\mathrm{A}}$. Then, one can rewrite eq. (159) for $\mathrm{qN}_{\mathrm{C}}(\mathrm{D})$, using the expressions for $\mathrm{qN} \mathrm{NDO}_{\mathrm{CDO}}$ and $1 / \mathrm{L}_{\mathrm{A}}$, as

$$
\mathrm{qN}_{\mathrm{C}}(\mathrm{D})=\frac{\mathrm{qN}_{\mathrm{CDO}}}{\left(1+\frac{\mathrm{D}}{\mathrm{L}_{\mathrm{A}}}\right)^{2}}=\frac{\mathrm{Q}_{\mathrm{G}}^{2}}{2 \varepsilon_{\mathrm{f}} \varphi_{\mathrm{OEF}}\left(1+\mathrm{D} \frac{\mathrm{Q}_{\mathrm{G}}}{2 \varepsilon_{\mathrm{f}} \varphi_{\mathrm{OEF}}}\right)^{2}} .
$$


Thus, the charge profile $\mathrm{qN}_{\mathrm{C}}(\mathrm{D})$ is determined once the bias and temperature are known, because $\mathrm{Q}_{\mathrm{G}}=\mathrm{Cox}_{\mathrm{OX}} \mathrm{V}_{\mathrm{G}}$, and since $\varphi_{\mathrm{OEF}}$ is obtained from eqs. (79) or $(80)$, e.g. as $\varphi_{\mathrm{OEF}}=\left[\left(\varphi_{\mathrm{o}}\right)^{\mathrm{n}}+\left(\varphi_{\mathrm{T}}\right)^{\mathrm{n}}\right]^{(1 / \mathrm{n})}$ from eq. (79) with $\mathrm{n} \sim 10$. The substitution of $\left(\mathrm{qN}_{\mathrm{C}}\right.$ ) in eq. (84) yields the expression for the profile of the VRH specific conductivity $\sigma(\mathrm{D})$, as

$$
\sigma(\mathrm{D})=\frac{\sigma_{\mathrm{DO}}}{\left(1+\frac{\mathrm{D}}{\mathrm{L}_{\mathrm{A}}}\right)^{2 \varphi_{\mathrm{OEF}} / \varphi_{\mathrm{T}}}}=\frac{\sigma_{\mathrm{DO}}}{\left(1+\mathrm{D} \frac{\mathrm{Q}_{\mathrm{G}}}{2 \varepsilon_{\mathrm{f}} \varphi_{\mathrm{OEF}}}\right)^{2 \varphi_{\mathrm{OEF}} / \varphi_{\mathrm{T}}}}
$$

where the specific conductivity $\sigma_{\mathrm{DO}}=\sigma(\mathrm{D}=0)$ in the semiconductor at the gate-dielectric interface (depth $\mathrm{D}=0)$ is

$$
\begin{aligned}
\sigma_{\mathrm{DO}}=\sigma(\mathrm{D}=0) & =\sigma_{\mathrm{FB}}(\mathrm{T})\left[\frac{\mathrm{qN}}{\mathrm{qN} N_{\mathrm{CFB}}(\mathrm{T})}\right]^{\varphi_{\mathrm{OEF}} / \varphi_{\mathrm{T}}} \\
& =[\eta(\mathrm{T})]^{\varphi_{\mathrm{OEF}} / \varphi_{\mathrm{T}}} \times\left[\mathrm{qN}_{\mathrm{CDO}}\right]^{\varphi_{\mathrm{OEF}} / \varphi_{\mathrm{T}}} \\
& =[\eta(\mathrm{T})]^{\varphi_{\mathrm{OEF}} / \varphi_{\mathrm{T}}}\left(\frac{\mathrm{Q}_{\mathrm{G}}^{2}}{2 \varepsilon_{\mathrm{f}} \varphi_{\mathrm{OEF}}}\right)^{\varphi_{\mathrm{OEF}} / \varphi_{\mathrm{T}}}
\end{aligned}
$$

Denoting again $n=\varphi_{\mathrm{OEF}} / \varphi_{T} \geq 1$, the comparison of eqs. (86) and (87) clearly shows that the profiles for $\sigma \propto\left(1+\mathrm{D} / \mathrm{L}_{\mathrm{A}}\right)^{-2 \mathrm{n}}$ are $\mathrm{n}$-times steeper in logarithmic plots, compared with the charge profiles, $\mathrm{N}_{\mathrm{C}} \propto\left(1+\mathrm{D} / \mathrm{L}_{\mathrm{A}}\right)^{-2}$, see again Figure 13 and Figure 14(c).

As noted in the last paragraph of Sec. 4.4.5, the scaling between VRH conductance and charge is not multiplicative, that is, there is not a direct proportionality between charge and VRH conductance. Any search for a coefficient of proportionality between $\sigma$ and $\mathrm{N}_{\mathrm{C}}$ will be wrong in terms of VRH, whereas it is an obvious approach for mobility edge models, in which the induced charge is separated in two categories of trapped and mobile charges. The trapped charges are with zero mobility. The mobile charges are "freelike" carriers with a constant characteristic value for the mobility in a band-like charge transport model, which replicates the assumptions in semiconductors. Also, as mentioned several times earlier, beginning from Sec. 1, VRH does not require the assumption of transport band and there is no distinction between different types of charges, a concept more reasonable for semi-insulators to which the organic materials 
belong, since the charge is localized in the organic materials and spatially continuous transport bands are very unlikely [6]. Also mentioned earlier, the VRH mobility is a resulting quantity from division of conductance by charge concentration; and this division, using eq. (84) yields for the spot mobility $\mu_{\mathrm{D}}(\mathrm{D})$ that

$$
\begin{aligned}
\mu_{\mathrm{D}}(\mathrm{D}) & =\frac{\sigma(\mathrm{D})}{\mathrm{qN}_{\mathrm{C}}(\mathrm{D})} \\
& =\frac{\sigma_{\mathrm{FB}}(\mathrm{T})}{\mathrm{qN}_{\mathrm{CFB}}(\mathrm{T})}\left[\frac{\mathrm{qN}_{\mathrm{C}}(\mathrm{D})}{\mathrm{qN}_{\mathrm{CFB}}(\mathrm{T})}\right]^{\varphi_{\mathrm{OEF}} / \varphi_{\mathrm{T}}-1}=\mu_{\mathrm{FB}}(\mathrm{T})\left[\frac{\mathrm{N}_{\mathrm{C}}(\mathrm{D})}{\mathrm{N}_{\mathrm{CFB}}(\mathrm{T})}\right]^{\varphi_{\mathrm{OEF}} / \varphi_{\mathrm{T}}-1} \\
& =[\eta(\mathrm{T})]^{\varphi_{\mathrm{OEF}} / \varphi_{\mathrm{T}}} \times\left[\mathrm{qN}_{\mathrm{C}}(\mathrm{D})\right]^{\varphi_{\mathrm{OEF}} / \varphi_{\mathrm{T}}-1}
\end{aligned}
$$

Similar to the expression for the specific conductivity $\sigma(D)$ in eq. (87), the spot mobility $\mu_{D}(D)$ in eq. (89) is again a version of the mobility $\mu_{\mathrm{FB}}=\sigma_{\mathrm{FB}} /\left(\mathrm{qN} \mathrm{N}_{\mathrm{FB}}\right)$ in bulk material scaled by a power-law function $\mathrm{x}^{\mathrm{n}-1}$, with $\mathrm{x}=\mathrm{N}_{\mathrm{C}} / \mathrm{N}_{\mathrm{CFB}}$ being ratio of induced to bulk charge, and just the exponent $(\mathrm{n}-1)=\left(\varphi_{\mathrm{OEF}} / \varphi_{\mathrm{T}}-1\right) \geq 0$ has a value reduced by one, compared with the exponent for $\sigma(\mathrm{D})$. This reduction was elaborated several times for the mobility edge models, e.g. in [26]. However, note that the prefactor $\eta^{\varphi_{\mathrm{OEF}}}{ }^{/ \varphi_{\mathrm{T}}}$ is the same in the last expressions of eqs. (84) and (89), indicating a hard relation $\sigma_{\mathrm{FB}}=\mu_{\mathrm{FB}} \mathrm{NN}_{\mathrm{CFB}}$ in bulk semiconductor. However, the relation becomes different under biasing when $\mathrm{N}_{C} \neq \mathrm{N}_{\mathrm{CFB}}$, which is a direct consequence of the scaling with ratio of charges, but not with the charge magnitude itself, and follows from the power-law dependences. Therefore, in principle, since there is no proportionality between conductance and charge in VRH, then there is no principal proportional relation between VRH mobility and charge either. So, the search for a direct relation between charge and mobility usually results in complicated derivations and discussions, in which some authors speculate that the DOS in organic materials might not be of an exponential type at all [25]. Then, the same authors derive an analytical model for VRH based on exponential DOS in [7] along with the most recently reported in the literature (up to March 2014) MonteCarlo VRH numerical simulator that, however, considers a uniform profile of the charge concentration, which is not the case in OTFT under gate biasing. After extensive comparison of hopping models, an interesting approximation for a relation between mobility and charge is suggested in Ref. [10] in the form of 


$$
\begin{aligned}
& \mu\left(\frac{\mathrm{N}_{\mathrm{C}}}{\mathrm{N}_{\mathrm{S}}}\right)=\mu_{\mathrm{FB}}(\mathrm{T}) \times \exp \left[\mathrm{a}_{1,2} \times\left(2 \frac{\mathrm{N}_{\mathrm{C}}}{\mathrm{N}_{\mathrm{S}}}\right)^{\mathrm{b}_{1,2}}\right] \text {, with } \\
& \frac{\mathrm{N}_{\mathrm{C}}}{\mathrm{N}_{\mathrm{S}}}=\int_{-\infty}^{+\infty} \mathrm{F}(\mathrm{E}) \frac{\mathrm{DOS}_{\mathrm{ND}}(\mathrm{E})}{\mathrm{N}_{\mathrm{S}}} \mathrm{dE}<<1=\int_{-\infty}^{+\infty} \frac{\mathrm{DOS}_{\mathrm{ND}}(\mathrm{E})}{\mathrm{N}_{\mathrm{S}}} \mathrm{dE} \text {, and } \\
& \left\{\begin{array}{l}
\mathrm{a}_{1}=\frac{1}{2}\left(\mathrm{~T}_{\mathrm{o}} / \mathrm{T}\right)^{2}+\ln 2 \\
\mathrm{~b}_{1}=2 \frac{\ln \left[\left(\mathrm{T}_{\mathrm{o}} / \mathrm{T}\right)^{2}+\ln 4\right]-\ln (\ln 4)}{\left(\mathrm{T}_{\mathrm{o}} / \mathrm{T}\right)^{2}}
\end{array}\right\} \text { or }\left\{\begin{array}{l}
\mathrm{a}_{2}=\frac{1}{2}\left[\left(\mathrm{~T}_{\mathrm{o}} / \mathrm{T}\right)^{2}-\mathrm{T}_{\mathrm{o}} / \mathrm{T}\right] \\
\left.\mathrm{b}_{2}=2 \frac{\ln \left[\left(\mathrm{T}_{\mathrm{o}} / \mathrm{T}\right)^{2}-\mathrm{T}_{\mathrm{o}} / \mathrm{T}\right]-\ln (\ln 4)}{\left(\mathrm{T}_{\mathrm{o}} / \mathrm{T}\right)^{2}}\right\} .
\end{array}\right.
\end{aligned}
$$

where the use of the parameters $a_{1}, a_{2}, b_{1}$ and $b_{2}$ is explained in [10]. This approximation is derived for normally distributed $\mathrm{DOS}_{\mathrm{ND}}$ (eq. (58)) and sometimes is called Coehoorn-Pasveer mobility model in following publications $[4,50]$.

Nevertheless, substituting in eq. (89) the expressions for the non-uniform charge profile in the OTFT depth from eq. (86), one finds that the profile of the spot mobility is

$$
\mu_{\mathrm{D}}(\mathrm{D})=\frac{\mu_{\mathrm{DO}}}{\left(1+\frac{\mathrm{D}}{\mathrm{L}_{\mathrm{A}}}\right)^{2 \varphi_{\mathrm{OEF}} / \varphi_{\mathrm{T}}-2}}=\frac{\mu_{\mathrm{DO}}}{\left(1+\mathrm{D} \frac{\mathrm{Q}_{\mathrm{G}}}{2 \varepsilon_{\mathrm{f}} \varphi_{\mathrm{OEF}}}\right)^{2 \varphi_{\mathrm{OEF}} / \varphi_{\mathrm{T}}-2}}
$$

where the spot mobility $\mu_{\mathrm{DO}}=\mu(\mathrm{D}=0)$ in the semiconductor at the gate dielectric interface $($ depth $\mathrm{D}=0)$ is

$$
\begin{aligned}
\mu_{\mathrm{DO}} & =\mu_{\mathrm{D}}(\mathrm{D}=0)=\frac{\sigma_{\mathrm{DO}}(\mathrm{T})}{\mathrm{qN}_{\mathrm{CDO}}} \\
& =\frac{\sigma_{\mathrm{FB}}(\mathrm{T})}{\mathrm{qN}}\left[\frac{\mathrm{qN}_{\mathrm{CDB}}(\mathrm{T})}{\mathrm{qN}_{\mathrm{CFB}}(\mathrm{T})}\right]^{\varphi_{\mathrm{OEF}} / \varphi_{\mathrm{T}}-1}=\mu_{\mathrm{FB}}(\mathrm{T})\left[\frac{\mathrm{N}_{\mathrm{CDO}}}{\mathrm{N}_{\mathrm{CFB}}(\mathrm{T})}\right]^{\varphi_{\mathrm{OEF}} / \varphi_{\mathrm{T}}-1} \\
& =[\eta(\mathrm{T})]^{\varphi_{\mathrm{OEF}} / \varphi_{\mathrm{T}}} \times\left[\mathrm{qN}_{\mathrm{CDO}}\right]^{\varphi_{\mathrm{OEF}} / \varphi_{\mathrm{T}}-1} \\
& =[\eta(\mathrm{T})]^{\varphi_{\mathrm{OEF}} / \varphi_{\mathrm{T}}}\left(\frac{\mathrm{Q}_{\mathrm{G}}^{2}}{2 \varepsilon_{\mathrm{f}} \varphi_{\mathrm{OEF}}}\right)^{\varphi_{\mathrm{OEF}} / \varphi_{\mathrm{T}}-1}
\end{aligned}
$$

Denoting again $n=\varphi_{\mathrm{OEFF}} / \varphi_{\mathrm{T}} \geq 1$, one sees from eqs. (87) and (91) that the profiles for $\mu_{\mathrm{D}} \propto\left(1+\mathrm{D} / \mathrm{L}_{\mathrm{A}}\right)^{(2 \mathrm{n}-2)}$ in Figure 14(d) are less steep than the profiles for $\sigma \propto\left(1+\mathrm{D} / \mathrm{L}_{\mathrm{A}}\right)^{2 \mathrm{n}}$ in Figure 14(c). Furthermore, if $\mathrm{n}<2$, then the profile $\mu_{\mathrm{D}} \propto\left(1+\mathrm{D} / \mathrm{L}_{\mathrm{A}}\right)^{(2 \mathrm{n}-2)}$ in Figure $14(\mathrm{~d})$ can become less steep than the profile for the charge. Also, observe in Figure 13 that at high temperature $T>T_{0}$, the depth variation $\mu_{D} \propto\left(1+D / L_{A}\right)^{(2 n-2)}$ in eq. (91) tends 
to vanish, because $\varphi_{\mathrm{OEF}} \approx \varphi_{\mathrm{T}}$, according to eqs. (79) and (80). In summary, the charge and mobility need separate models, as seen in the equations provided in this section, and an explicit relation between charge and mobility might be not possible to establish in general.

\subsubsection{Areal (sheet) charge concentration, conductance and mobility, and film thickness}

We now turn to the second aspect for scaling between sheet quantities in VRH and electrostatics. This aspect is particularly significant for acceleration of numerical calculations and for analytical modeling of OTFTs, because the reduction of the depth coordinate $\mathrm{D}$ provides for the film sheet conductivity $\sigma_{\mathrm{sq}}(\mathrm{x})$ at position $0 \leq \mathrm{x} \leq \mathrm{L}$ along the channel length $\mathrm{L}$ of the OTFT. The reduction of the depth coordinate is by integration of a volumetric variable $X$ along $D$ from the gate dielectric interface $(D=0)$ to the back of the semiconducting film of thickness $t_{f}$. Thus, $X_{S Q}=\int X(D) d D$, in limits $0 \leq D \leq t_{f}$, where $X_{S Q}$ is the sheet representation of the quantity $\mathrm{X}$. One example for this integration is eq. (41) for the sheet conductance $\sigma_{\mathrm{sq}}$. So, the integration of the charge profile $\mathrm{qN}_{C}(\mathrm{D})$, eq. (86), yields that the sheet charge $\mathrm{QF}_{\mathrm{F}}$ of the semiconducting film is

$$
\begin{aligned}
\mathrm{Q}_{\mathrm{F}} & =\int_{0}^{\mathrm{t}_{\mathrm{f}}} \mathrm{qN}_{\mathrm{C}}(\mathrm{D}) \mathrm{dD}=\int_{0}^{\mathrm{t}_{\mathrm{f}}} \frac{\mathrm{qN}_{\mathrm{CDO}}}{\left(1+\mathrm{D} / \mathrm{L}_{\mathrm{A}}\right)^{2}} \mathrm{dD} \\
& =\mathrm{qN}_{\mathrm{CDO}} \mathrm{L}_{\mathrm{A}} \int_{\mathrm{D}=0}^{\mathrm{t}_{\mathrm{f}}} \frac{\mathrm{d}\left(\mathrm{D} / \mathrm{L}_{\mathrm{A}}\right)}{\left(1+\mathrm{D} / \mathrm{L}_{\mathrm{A}}\right)^{2}}=\left.\frac{\mathrm{qN}_{\mathrm{CDO}} \mathrm{L}_{\mathrm{A}}}{-2+1} \frac{1}{\left(1+\mathrm{D} / \mathrm{L}_{\mathrm{A}}\right)}\right|_{\mathrm{D}=0} ^{\mathrm{t}_{\mathrm{f}}} \\
& =\frac{\mathrm{qN}_{\mathrm{CDO}} \mathrm{L}_{\mathrm{A}}}{-1}\left[\frac{1}{\left(1+\mathrm{t}_{\mathrm{f}} / \mathrm{L}_{\mathrm{A}}\right)}-1\right]=\mathrm{Q}_{\mathrm{G}}\left[1-\frac{1}{\left(1+\mathrm{t}_{\mathrm{f}} / \mathrm{L}_{\mathrm{A}}\right)}\right] \\
& \approx \mathrm{Q}_{\mathrm{G}}, \text { if } \mathrm{t}_{\mathrm{f}} \gg \mathrm{L}_{\mathrm{A}}
\end{aligned}
$$

The quantity $\mathrm{qN}_{\mathrm{CDO}} \mathrm{L}_{\mathrm{A}}=\mathrm{Q}_{\mathrm{G}}$ is equal to the gate charge $\mathrm{Q}_{\mathrm{G}}$, since recalling eqs. (160) and (85)

$$
\mathrm{qN}_{\mathrm{CDO}} \mathrm{L}_{\mathrm{A}}=\frac{2 \varepsilon_{\mathrm{f}} \varphi_{\mathrm{OEF}}}{\mathrm{L}_{\mathrm{A}}^{2}} \mathrm{~L}_{\mathrm{A}}=\frac{2 \varepsilon_{\mathrm{f}} \varphi_{\mathrm{OEF}}}{\mathrm{L}_{\mathrm{A}}}=\mathrm{Q}_{\mathrm{G}}
$$

Consequently, the integration of the profile for VRH specific conductivity $\sigma(\mathrm{D})$, eq. (87), yields the sheet conductance $\sigma_{\mathrm{sq}}$ of the film, as 


$$
\begin{aligned}
\sigma_{\mathrm{sq}} & =\int_{0}^{\mathrm{t}_{\mathrm{f}}} \sigma(\mathrm{D}) \mathrm{dD}=\int_{0}^{\mathrm{t}_{\mathrm{f}}} \frac{\sigma_{\mathrm{DO}}}{\left(1+\mathrm{D} / \mathrm{L}_{\mathrm{A}}\right)^{2 \varphi_{\mathrm{OEF}} / \phi_{\mathrm{T}}} \mathrm{dD}} \\
& =\frac{\sigma_{\mathrm{DO}} \mathrm{L}_{\mathrm{A}}}{2 \varphi_{\mathrm{OEF}} / \varphi_{\mathrm{T}}-1}\left[1-\frac{1}{\left(1+\mathrm{t}_{\mathrm{f}} / \mathrm{L}_{\mathrm{A}}\right)^{2 \varphi_{\mathrm{OEF}} / \varphi_{\mathrm{T}}-1}}\right] \\
& \approx \frac{\sigma_{\mathrm{DO}} \mathrm{L}_{\mathrm{A}}}{2 \varphi_{\mathrm{OEF}} / \varphi_{\mathrm{T}}-1}, \text { if } \mathrm{t}_{\mathrm{f}}>>\mathrm{L}_{\mathrm{A}}, \text { since } \varphi_{\mathrm{OEF}}>\varphi_{\mathrm{T}}
\end{aligned}
$$

Therefore, as a ratio of conductance to charge, the effective mobility $\mu$ of the semiconducting film is

$$
\begin{aligned}
\mu & =\frac{\sigma_{\mathrm{sq}}}{\mathrm{Q}_{\mathrm{F}}}=\frac{\frac{\sigma_{\mathrm{DO}} \mathrm{L}_{\mathrm{A}}}{\mathrm{qN}_{\mathrm{CDO}} \mathrm{L}_{\mathrm{A}}}}{\left(2 \varphi_{\mathrm{OEF}} / \varphi_{\mathrm{T}}-1\right)}\left\{\frac{1-\frac{1}{\left(1+\mathrm{t}_{\mathrm{f}} / \mathrm{L}_{\mathrm{A}}\right)^{2 \varphi_{\mathrm{OEF}} / \varphi_{\mathrm{T}}-1}}}{1-\frac{1}{\left(1+\mathrm{t}_{\mathrm{f}} / \mathrm{L}_{\mathrm{A}}\right)}}\right\}=\left(\mu_{\mathrm{tf}=\infty}\right) \times\left\{1+\delta_{\mu \mathrm{tf}}\right\} \\
& \approx \frac{\mu_{\mathrm{DO}}}{\left(2 \varphi_{\mathrm{OEF}} / \varphi_{\mathrm{T}}-1\right)}=\mu_{\mathrm{tf}=\infty}<\mu_{\mathrm{DO}}, \text { if } \mathrm{t}_{\mathrm{f}}>\mathrm{L}_{\mathrm{A}} \\
& \approx \frac{[\eta(\mathrm{T})]^{\varphi_{\mathrm{OEF}} / \varphi_{\mathrm{T}}}}{\left(2 \varphi_{\mathrm{OEF}} / \varphi_{\mathrm{T}}-1\right)}\left(\frac{\mathrm{Q}_{\mathrm{G}}^{2}}{2 \varepsilon_{\mathrm{f}} \varphi_{\mathrm{OEF}}}\right)^{\varphi_{\mathrm{OEF}} / \varphi_{\mathrm{T}}-1}, \text { if } \mathrm{t}_{\mathrm{f}}>>\mathrm{L}_{\mathrm{A}}
\end{aligned}
$$

Here, $\mu_{\mathrm{tf}=\infty}$ is the effective mobility of an OTFT with an infinitely-thick semiconducting film, and $\delta_{\mu \mathrm{tf}}$ corresponds to the film thickness variations, originating from the terms in the large curly brackets \{\} .

Two observations can be made in eq. (96) for the effective mobility of the OTFT. One is that the effective mobility is $\left(2 \varphi_{\mathrm{OEF}} / \varphi_{\mathrm{T}}-1\right)$ times smaller than the mobility $\mu_{\mathrm{DO}}$ in the semiconductor at the gate dielectric interface. The reduction of the effective mobility $\mu$ in comparison to the interface mobility $\mu_{\mathrm{DO}}$ was addressed in [90] from a combination of analytical and numerical analyses. At room temperature, the reported reduction was $9 \%$ for a P3HT OTFT with a DOS characteristic temperature $\mathrm{T}_{0}=425 \mathrm{~K}$ and $15 \%$ for a PTV OTFT with $\mathrm{T}_{\mathrm{o}}=382 \mathrm{~K}$. The reduction is evident, but the magnitudes do not fit with eq. (96). The reason is that the charge profiles used in [90] have $\left(1+\mathrm{D} / \mathrm{L}_{\mathrm{A}}\right)^{-1}$ dependence, instead of the $\left(1+\mathrm{D} / \mathrm{L}_{\mathrm{A}}\right)^{-2}$ dependence given by eq. (86). Therefore [91], we cannot extend a quantitative comparison with [90].

The second observation in eq. (96) is that there is a film-thickness variation in the effective mobility, which we will inspect with the help of the supplementary quantity $\delta_{\mu t f}$ for the relative increase of the mobility from the value $\mu_{\mathrm{tf}=\infty}$ for an infinitely-thick film. When $\mathrm{t}_{\mathrm{f}} / \mathrm{L}_{\mathrm{A}}$ is large, then $\mu \approx \mu_{\mathrm{tf}=\infty}$, because the 
expression in the large curly brackets \{\} of eq. (96) becomes equal to one, since $\varphi_{\mathrm{OEF}} / \varphi_{\mathrm{T}}>1$ follows from eqs. (79) and (80). When $t_{f}$ decreases, then both the numerator and the denominator in these \{\} brackets decrease, resulting in a slightly complicated dependence. To get insight for the effect of the film thickness on the effective mobility, consider $t_{\mathrm{f}}>2 \mathrm{~L}_{\mathrm{A}}$, since $\mathrm{L}_{\mathrm{A}} \sim 1 \mathrm{~nm}$ at normal biases of the OTFT. Then, the subtracting terms in the curly brackets are smaller than one, and a logarithm of the expression in these \{\} brackets can be taken, in order to use $\ln (1 \pm x) \approx \pm x$ when $0 \leq x<<1$. In this way

$$
\begin{aligned}
& \ln \left\{1+\delta_{\mu \mathrm{tf}}\right\}=\ln \left[1-\frac{1}{\left(1+\mathrm{t}_{\mathrm{f}} / \mathrm{L}_{\mathrm{A}}\right)^{2 \varphi_{\mathrm{OEF}} / \varphi_{\mathrm{T}}-1}}\right]-\ln \left[1-\frac{1}{\left(1+\mathrm{t}_{\mathrm{f}} / \mathrm{L}_{\mathrm{A}}\right)}\right] \\
& \delta_{\mu \mathrm{tf}} \approx-\frac{1}{\left(1+\mathrm{t}_{\mathrm{f}} / \mathrm{L}_{\mathrm{A}}\right)^{2 \varphi_{\mathrm{OEF}} / \varphi_{\mathrm{T}}-1}+\frac{1}{\left(1+\mathrm{t}_{\mathrm{f}} / \mathrm{L}_{\mathrm{A}}\right)}}
\end{aligned}
$$

and the relative variation of the mobility as function of the film thickness becomes

$$
\delta_{\mu \mathrm{tf}}=\frac{\mu}{\mu_{\mathrm{tf}=\infty}}-1 \approx \frac{1}{\left(1+\mathrm{t}_{\mathrm{f}} / \mathrm{L}_{\mathrm{A}}\right)}\left[1-\frac{1}{\left(1+\mathrm{t}_{\mathrm{f}} / \mathrm{L}_{\mathrm{A}}\right)^{2 \varphi_{\mathrm{OEF}} / \varphi_{\mathrm{T}}-2}}\right] \text {, valid for } \mathrm{t}_{\mathrm{f}}>2 \mathrm{~L}_{\mathrm{A}} \text {. }
$$

The two multiplying terms have opposite effect on varying $t_{f}$. On decreasing $t_{f}$, the first term $\left(1+\mathrm{t}_{\mathrm{f}} / \mathrm{L}_{\mathrm{A}}\right)^{-1}$ increases, causing $\delta_{\mu \mathrm{tf}}$ to rise toward the value of the term in the square brackets [ ], which is normally of unity value at $t_{\mathrm{f}}>>\mathrm{L}_{\mathrm{A}}$, but this term in the square brackets decreases toward zero, when decreasing $t_{\mathrm{f}}$ toward $\mathrm{L}_{\mathrm{A}}$, owing to the subtraction. Note that the variation rates depend on $\varphi_{\mathrm{OEF}} / \varphi_{\mathrm{T}}$ and bias, since $\mathrm{Q}_{\mathrm{G}} /\left(2 \varepsilon_{\mathrm{f}} \varphi \mathrm{OEF}\right)=1 / \mathrm{L}_{\mathrm{A}}$ from eq. (160) in Appendix 3. When explicitly showing the gate bias $\mathrm{Q}_{\mathrm{G}}=\mathrm{C}_{\mathrm{Ox}} \mathrm{V}_{\mathrm{G}}$, then the relative variation of the mobility as function of the film thickness $t_{f}$, gate bias $V_{G}$ and temperature via $\varphi_{\mathrm{OEF}} / \varphi_{\mathrm{T}}$ is approximately

$$
\delta_{\mu t f}=\frac{\mu}{\mu_{\mathrm{tf}=\infty}}-1 \approx \frac{1}{\left(1+\frac{\mathrm{t}_{\mathrm{f}} \mathrm{C}_{\mathrm{OX}} \mathrm{V}_{\mathrm{G}}}{2 \varepsilon_{\mathrm{f}} \varphi_{\mathrm{OEF}}}\right)}\left[1-\left(1+\frac{\mathrm{t}_{\mathrm{f}} \mathrm{C}_{\mathrm{OX}} \mathrm{V}_{\mathrm{G}}}{2 \varepsilon_{\mathrm{f}} \varphi_{\mathrm{OEF}}}\right)^{2\left(1-\varphi_{\mathrm{OEF}} / \varphi_{\mathrm{T}}\right)}\right],
$$

which is valid when $\frac{t_{f}}{L_{A}}=\frac{t_{f} C_{O X} V_{G}}{2 \varepsilon_{f} \varphi_{O E F}}>2$. The behavior of this equation is illustrated in Figure 35 . 
In Figure 35(a), the relative variation $\delta_{\mu t f}$ of the mobility is shown as function of the film thickness $\mathrm{t}_{\mathrm{f}}$. It is clear from the plot that the mobility in OTFTs with thinner semiconducting films increases up to a thickness $t_{\mathrm{f}} \sim \mathrm{L}_{\mathrm{A}}$, after which, the mobility decreases. This non-monotonic behavior confirms the results from the numerical simulations shown earlier in Figure 32, although it is difficult to match the magnitudes of the variations in these two figures. Other observations in Figure 35(a) are that at a given film thickness, the mobility variations are larger at low temperature and low bias. These are detailed for $t_{\mathrm{f}}=20 \mathrm{~nm}$ in Figure 35(b) and (c). The temperature dependences in Figure 35(b) indicate that $\delta_{\mu \mathrm{tf}}$ is large at low temperature and low gate bias voltage $V_{G}$, and gradually vanishes at higher temperature $T>T_{0}$ and high bias. The bias dependence in Figure 35(c) implies nearly a reciprocal dependence between $\delta_{\mu t f}$ and $V_{G}$ at low temperature, but the dependence smears at high temperatures to nearly a power-law dependence with exponent of approximately -0.7 . Looking at the three plots in Figure 35, one observes a trend that the effective mobility relatively increases (as compared to the mobility $\mu_{\mathrm{tf}=\infty}$ of infinite-thick film OTFT), when the film thickness $\left(\mathrm{t}_{\mathrm{f}}\right)$, the temperature $(\mathrm{T})$ or the bias $\left(\mathrm{V}_{\mathrm{G}}\right)$ decrease, which is the opposite to the temperature and bias dependence of the absolute values for VRH conductance and mobility, e.g., $\mu$ and $\mu_{\mathrm{tf}=\infty}$ increase with the temperature and the gate bias of the OTFT.

\subsubsection{VRH and the OTFT current}

For the most practical purpose for relating to analytical models for the DC current of the OTFT [28], we address now the third aspect for the current of the entire OTFT of unit channel area with width W and length $\mathrm{L}$, that is, $\mathrm{W}=\mathrm{L}$ for a square shaped OTFT. Considering the sheet conductance $\sigma_{\mathrm{sq}}(\mathrm{X})$ of eq. (95) varying with the position $X$ along the channel length $L$ of the OTFT, $0 \leq X \leq L$ the magnitude of the drift current is

$$
\begin{aligned}
I_{D} \frac{L}{W} & =\int_{V_{S}=0}^{V_{D}} \sigma_{s q}(X) d V_{X} \\
& =\int_{V_{S}=0}^{V_{D}} \frac{\sigma_{D O} L_{A}}{2 \varphi_{\text {OEF }} / \varphi_{T}-1}\left[1-\frac{1}{\left(1+t_{f} / L_{A}\right)^{2 \varphi_{\text {OEF }} / \varphi_{T}-1}}\right] d_{X}
\end{aligned}
$$


where $V_{X}$ is the potential in the channel, changing from the source potential $V_{S}=0$ to the magnitude of the drain potential $V_{D}>0$. Varying $V_{X}$ with $X$, then the magnitude of the gate charge also varies with $X$ as $\mathrm{Q}_{\mathrm{G}}(\mathrm{X})=\left(\mathrm{V}_{\mathrm{G}}-\mathrm{V}_{\mathrm{X}}-\mathrm{V}_{\mathrm{T}}\right) \mathrm{C}_{\mathrm{OX}} \approx\left(\mathrm{V}_{\mathrm{G}}-\mathrm{V}_{\mathrm{X}}\right) \mathrm{C}_{\mathrm{OX}}$, since $\mathrm{Q}_{\mathrm{G}}$ is a product of the gate dielectric capacitance $\mathrm{C}_{\mathrm{OX}}$ (per unit area) and the magnitude of the gate overdrive voltage $\left(V_{G}-V_{X}\right)$, assuming that the OTFT has a small in magnitude threshold voltage $\mathrm{V}_{\mathrm{T}}$. The gate charge $\mathrm{Q}_{\mathrm{G}}(\mathrm{X})$ enters the equation through $\mathrm{L}_{\mathrm{A}}$ and $\sigma_{\mathrm{DO}}$, given by eqs. (85) and (88), respectively, which are substituted in eq. (99) to yield

$$
\begin{aligned}
& \mathrm{I}_{\mathrm{D}} \frac{\mathrm{L}}{\mathrm{W}}=\int_{\mathrm{V}_{\mathrm{S}}=0}^{\mathrm{V}_{\mathrm{D}}} \sigma_{\mathrm{Sq}}(\mathrm{X}) \mathrm{d} \mathrm{V}_{\mathrm{X}} \\
& =\int_{\mathrm{V}_{\mathrm{S}}=0}^{\mathrm{V}_{\mathrm{D}}} \frac{[\eta(\mathrm{T})]^{\varphi_{\mathrm{OEF}} / \varphi_{\mathrm{T}}}\left(\mathrm{Q}_{\mathrm{G}}\right)^{2 \varphi_{\mathrm{OEF}} / \varphi_{\mathrm{T}}-1}}{\left(2 \varphi_{\mathrm{OEF}} / \varphi_{\mathrm{T}}-1\right)\left(2 \varepsilon_{\mathrm{f}} \varphi_{\mathrm{OEF}}\right)^{\varphi_{\mathrm{OEF}} / \varphi_{\mathrm{T}}-1}}\left[1-\frac{1}{\left(1+\frac{\mathrm{t}_{\mathrm{f}} \mathrm{Q}_{\mathrm{G}}}{2 \varepsilon_{\mathrm{f}} \varphi_{\mathrm{OEF}}}\right)^{2 \varphi_{\mathrm{OEF}} / \varphi_{\mathrm{T}}-1}}\right] \mathrm{dV}_{\mathrm{X}}
\end{aligned}
$$

Let us denote several constants (bias-independent quantities), as

$$
\begin{aligned}
& \mathrm{n}=\frac{2 \varphi_{\mathrm{OEF}}}{\varphi_{\mathrm{T}}}-1>1 \\
& \mathrm{a}=\frac{[\eta(\mathrm{T})]^{\varphi_{\mathrm{OEF}} / \varphi_{\mathrm{T}}}\left(2 \varepsilon_{\mathrm{f}} \phi_{\mathrm{OEF}}\right)^{\varphi_{\mathrm{OEF}} / \varphi_{\mathrm{T}}}}{\left(2 \varphi_{\mathrm{OEF}} / \varphi_{\mathrm{T}}-1\right) \mathrm{t}_{\mathrm{f}}^{\mathrm{t}_{\mathrm{OEF}} / \varphi_{\mathrm{T}}-1}}=\frac{[\eta(\mathrm{T})]^{(\mathrm{n}+1) / 2}\left(2 \varepsilon_{\mathrm{f}} \varphi_{\mathrm{OEF}}\right)^{(\mathrm{n}+1) / 2}}{\mathrm{nt}_{\mathrm{f}}^{\mathrm{n}}} \\
& \mathrm{b}=\frac{\mathrm{t}_{\mathrm{f}} \mathrm{C}_{\mathrm{OX}}}{2 \varepsilon_{\mathrm{f}} \varphi_{\text {OEF }}} \sim \frac{50 \mathrm{~nm} \times 20 \mathrm{nF} / \mathrm{cm}^{2}}{2 \times 2.66 \times 10^{-13} \mathrm{~F} / \mathrm{cm} \times 35 \mathrm{mV}} \sim 5 \mathrm{~V}^{-1}
\end{aligned}
$$

and rewrite eq. (100) as

$$
I_{D} \frac{L}{W}=\left(\frac{a}{b}\right)\left\{\int_{V_{X}=V_{D}}^{V_{s} \approx 0}\left(b\left(V_{G}-V_{X}\right)\right)^{n}\left[1-\frac{1}{\left(1+b\left(V_{G}-V_{X}\right)\right)^{n}}\right] d\left[b\left(V_{G}-V_{X}\right)\right]\right\}
$$

Changing to variable $\mathrm{x}=\mathrm{b}\left(\mathrm{V}_{\mathrm{G}}-\mathrm{V}_{\mathrm{X}}\right)$, one gets

$$
I_{D} \frac{L}{W}=\left(\frac{a}{b}\right)\left\{\int_{x=b\left(V_{G}-V_{D}\right)}^{b\left(V_{G}-V_{S}\right) \approx b V_{G}} x^{n}\left[1-\frac{1}{(1+x)^{n}}\right] d x\right\}
$$

This integral consists of two parts. The first part is trivial, $\int \mathrm{X}^{\mathrm{n}} \mathrm{dx}=\mathrm{x}^{(\mathrm{n}+1)} /(\mathrm{n}+1)$, but the second part $\int X^{n} d x /(1+x)^{n}={ }_{2} H_{y p G e o m} \times x^{(n+1)} /(n+1)$ leads to the Gauss hyper-geometric function ${ }_{2}$ HypGeom $_{1}$ [92]. 
Details are given in the Appendix 4, where it is also shown that the contribution of the hyper-geometric function is small compared to unity. Therefore, one can approximately use only the trivial part for the OTFT. Thus, from the derivations in the Appendix 4, the current of the OTFT is given by

$$
\begin{aligned}
& \mathrm{I}_{\mathrm{D}} \frac{\mathrm{L}}{\mathrm{W}} \approx \frac{\mathrm{ab}}{\mathrm{n}+1}\left\{\mathrm{~V}_{\mathrm{G}}^{\mathrm{n}+1}-\left(\mathrm{V}_{\mathrm{G}}-\mathrm{V}_{\mathrm{D}}\right)^{\mathrm{n}+1}\right\} \\
& =\left[\frac{\varphi_{\mathrm{T}} / \varphi_{\mathrm{OEF}}}{\left(2 \varphi_{\mathrm{OEF}} / \varphi_{\mathrm{T}}-1\right)}\left(\frac{\eta(\mathrm{T}) \mathrm{C}_{\mathrm{OX}}^{2}}{2 \varepsilon_{\mathrm{f}} \varphi_{\mathrm{OEF}}}\right)^{\varphi_{\mathrm{OEF}} / \varphi_{\mathrm{T}}-1}\right]\left\{\eta(\mathrm{T}) \mathrm{C}_{\mathrm{Ox}} \frac{\mathrm{V}_{\mathrm{G}}^{2\left[\varphi_{\mathrm{OEF}} / \varphi_{\mathrm{T}}\right]}-\left(\mathrm{V}_{\mathrm{G}}-\mathrm{V}_{\mathrm{D}}\right)^{2\left[\varphi_{\mathrm{OEF}} / \varphi_{\mathrm{T}}\right]}}{2}\right\}
\end{aligned}
$$

Note that for an ideal semiconductor $\varphi_{\mathrm{OEF}} / \varphi_{\mathrm{T}}=1$, the expressions in the square brackets are equal to one, and the expression in the curly brackets is the generic equation for an ideal MOSFET [28], with biasindependent mobility $\mu=\eta$, as explained after eq. (83). Note also that there is no thickness dependence in eq. (104), because the thickness dependence is in the hyper-geometric part, which is neglected as it is small.

Equation (104) provides the links to several analytical models published in the literature. These models have been reviewed in $[6,28,29]$. From eq. (43), the terms containing the ratio rewritten in terms of the mobility enhancement factor $\gamma$ as

$$
\begin{aligned}
& 2 \frac{\varphi_{\mathrm{OEF}}}{\varphi_{\mathrm{T}}}=2 \frac{\mathrm{T}_{\mathrm{OEF}}}{\mathrm{T}}=2+\gamma \\
& 2 \frac{\varphi_{\mathrm{OEF}}}{\varphi_{\mathrm{T}}}-1=2 \frac{\mathrm{T}_{\mathrm{OEF}}}{\mathrm{T}}-1=1+\gamma \\
& 2\left(\frac{\varphi_{\mathrm{OEF}}}{\varphi_{\mathrm{T}}}-1\right)=2\left(\frac{\mathrm{T}_{\mathrm{OEF}}}{\mathrm{T}}-1\right)=\gamma \\
& \frac{\varphi_{\mathrm{OEF}}}{\varphi_{\mathrm{T}}}=\frac{\mathrm{T}_{\mathrm{OEF}}}{\mathrm{T}}=1+\frac{\gamma}{2} \Leftrightarrow \frac{\varphi_{\mathrm{T}}}{\varphi_{\mathrm{OEF}}}=\frac{2}{2+\gamma}
\end{aligned}
$$

where YOEF and TOEF are given by eqs. (79) and (80). Substituting in eq. (104), the current of the OTFT becomes

$$
\begin{aligned}
\mathrm{I}_{\mathrm{D}} \frac{\mathrm{L}}{\mathrm{W}} & \approx\left\{\frac{[\eta(\mathrm{T})]^{1+\gamma / 2}}{(1+\gamma)}\left(\frac{\mathrm{C}_{\mathrm{OX}}}{\sqrt{2 \varepsilon_{\mathrm{f}} \varphi_{\mathrm{OEF}}}}\right)^{\gamma}\right\} \mathrm{C}_{\mathrm{ox}} \frac{\mathrm{V}_{\mathrm{G}}^{2+\gamma}-\left(\mathrm{V}_{\mathrm{G}}-\mathrm{V}_{\mathrm{D}}\right)^{2+\gamma}}{2+\gamma} \\
& =\mu_{\mathrm{o}} \mathrm{C}_{\mathrm{ox}} \frac{\mathrm{V}_{\mathrm{G}}^{2+\gamma}-\left(\mathrm{V}_{\mathrm{G}}-\mathrm{V}_{\mathrm{D}}\right)^{2+\gamma}}{2+\gamma}
\end{aligned}
$$


which is the TFT generic charge drift model, eq. (8) in [28] with $\mathrm{V}_{\mathrm{S}}=\mathrm{V}_{\mathrm{T}}=0$ and

$$
\mu_{\mathrm{o}}=\frac{[\eta(\mathrm{T})]^{1+\gamma / 2}}{(1+\gamma)}\left(\frac{\mathrm{C}_{\mathrm{OX}}}{\sqrt{2 \varepsilon_{\mathrm{f}} \varphi_{\mathrm{OEF}}}}\right)^{\gamma}=\frac{\mu_{\mathrm{FB}}}{(1+\gamma)}\left(\frac{\mathrm{C}_{\mathrm{Ox}}}{\sqrt{2 \mathrm{qN} \mathrm{CFB}_{\mathrm{f}} \varphi_{\mathrm{OEF}}}}\right)^{\gamma}
$$

with $\mu_{\mathrm{FB}}$ and $\mathrm{qN}_{\mathrm{CFB}}$ being the mobility and charge concentration in bulk semiconductor (no bending, $\mathrm{V}_{\mathrm{B}}=0$ ) since, having $\varphi_{\mathrm{OEF}} / \varphi_{\mathrm{T}}=(2+\gamma) / 2$ by eq. (105), it follows from eqs. (83) and (89) that

$$
\eta(\mathrm{T})=\frac{\left[\sigma_{\mathrm{FB}}(\mathrm{T})\right]^{\varphi_{\mathrm{T}} / \varphi_{\mathrm{OEF}}}}{\mathrm{qN}_{\mathrm{CFB}}(\mathrm{T})}=\frac{\left[\sigma_{\mathrm{FB}}(\mathrm{T})\right]^{2 /(2+\gamma)}}{\mathrm{qN}_{\mathrm{CFB}}(\mathrm{T})}
$$

and

$$
\eta(\mathrm{T})^{(2+\gamma) / 2}=\left\{\frac{\left[\sigma_{\mathrm{FB}}(\mathrm{T})\right]^{2 /(2+\gamma)}}{\mathrm{qN}_{\mathrm{CFB}}(\mathrm{T})}\right\}^{(2+\gamma) / 2}=\frac{\sigma_{\mathrm{FB}} /\left(\mathrm{qN}_{\mathrm{CFB}}\right)}{\left(\mathrm{qN}_{\mathrm{CFB}}\right)^{\gamma / 2}}=\frac{\mu_{\mathrm{FB}}}{\left(\sqrt{\mathrm{qN}_{\mathrm{CFB}}}\right)^{\gamma}}
$$

Note that $\mu_{\mathrm{o}}$ is bias independent, but the temperature dependence in eq. (107) is complicated, because $\mu_{\mathrm{FB}}, \gamma$, $\mathrm{N}_{\mathrm{CFB}}$ and $\varphi \mathrm{OEF}$ are all functions of temperature.

One can expand eq. (107) in terms of other parameters by the following sequence. Firstly, obtain poEF from parameters $\varphi_{\mathrm{T}}=\mathrm{kT} / \mathrm{q}$ and $\varphi_{\mathrm{o}}=\mathrm{kT}_{\mathrm{o}} / \mathrm{q}, \mathrm{T}_{\mathrm{o}} \sim 400 \mathrm{~K}$, e.g., using eq. (79) with $\varphi_{\text {cold }} \equiv \varphi_{\mathrm{o}}$ and by choosing $\mathrm{n} \sim 5-10$ in this equation. Secondly, obtain $\mathrm{N}_{\mathrm{CFB}}=\mathrm{N}_{\mathrm{S}} \times \exp \left(-\mid \mathrm{E}_{\mathrm{o}}-\mathrm{E}_{\mathrm{F}} / \mathrm{q} \varphi \mathrm{OEF}\right)$ from parameters $\mathrm{E}_{\mathrm{o}}, \mathrm{E}_{\mathrm{F}}$ and $\mathrm{N}_{\mathrm{S}} \sim 10^{21}-10^{22} \mathrm{~cm}^{-3}$, as detailed by eq. (166). Thirdly, obtain $\sigma_{\mathrm{FB}}$ from eq. (64) with parameters $\Gamma_{\mathrm{o}} \sim 1000 \mathrm{THz}$ [21], $\Lambda_{\mathrm{o}} \sim 0.1 \mathrm{~nm}, \mathrm{~s}_{\mathrm{c} T \infty \sim} \sim 10$ and $\varphi_{\mathrm{To}} \sim 0.8 \mathrm{eV}$, and find $\mu_{\mathrm{FB}}=\sigma_{\mathrm{FB}} /\left(\mathrm{qN}_{\mathrm{CFB}}\right)$. Fourthly, obtain $\mu_{\mathrm{o}}$ by substituting in eq. (107) with additional parameters $C_{\mathrm{OX}}$ and $\varepsilon_{\mathrm{f}}$, considering also that $(1+\gamma)=\left(2 \varphi_{\mathrm{OEF}} / \varphi_{\mathrm{T}}-1\right)$ and $\gamma=2\left(\varphi_{\mathrm{OEF}} / \varphi_{\mathrm{T}}-1\right)$ from eq. (105). Lastly, rationalize the expression for $\mu_{\mathrm{o}}$, since it is large, to have it in a form, such as 


$$
\mu_{\mathrm{o}}=\left\{\begin{array}{c}
\frac{\varphi_{\mathrm{OEF}}}{2 \varphi_{\mathrm{OEF}}-\phi_{\mathrm{T}}}\left[\frac{\Gamma_{\mathrm{o}} \exp \left(-\mathrm{s}_{\mathrm{cT} \infty}\right)}{\Lambda_{\mathrm{o}} \mathrm{N}_{\mathrm{S}} \varphi_{\mathrm{o}}}\right] \\
\times\left[\exp \left(\frac{\left|\mathrm{E}_{\mathrm{o}}-\mathrm{E}_{\mathrm{F}}\right| / \mathrm{q}-\varphi_{\mathrm{To}}}{\varphi_{\mathrm{O}}}\right)\left(\frac{\varphi_{\mathrm{o}}}{\varphi_{\mathrm{OEF}}}\right)^{\frac{\varphi_{\mathrm{OEF}}}{\varphi_{\mathrm{o}}}}\right]^{\frac{\varphi_{\mathrm{o}}}{\varphi_{\mathrm{T}}}} \\
\left.\times\left[\frac{\mathrm{C}_{\mathrm{OX}}^{2}}{2 \mathrm{qN} \varepsilon_{\mathrm{S}} \varphi_{\mathrm{f}}}\right]^{\frac{\varphi_{\mathrm{OEF}}}{\varphi_{\mathrm{T}}}}\right]^{\frac{\varphi_{\mathrm{OEF}}}{2 \varphi_{\mathrm{OEF}}-\varphi_{\mathrm{T}}}\left[\mu_{\mathrm{Oo}}\right]} \\
\times\left[\mathrm{TSF}^{2}\right]^{\varphi_{\mathrm{o}} / \varphi_{\mathrm{T}}} \\
\times\left[\frac{1}{\varphi_{\mathrm{TFT}}^{2}}\right]^{\varphi_{\mathrm{OEF}} / \varphi_{\mathrm{T}}-1}
\end{array}\right\},
$$

obtaining the expression for $\mu_{\mathrm{o}}$ in the format of the TFT compact mobility model [6], in which $\mu_{\mathrm{oo}}$ is the mobility prefactor, $\mathrm{TSF}^{2}$ is the temperature shaping function and $\varphi_{T F T}$ is a bias-independent TFT specific voltage, with the definitions for $\mu_{\mathrm{oo}}, \mathrm{TSF}^{2}$ and $\varphi_{\mathrm{TFT}}$, as depicted by the corresponding square brackets in each line of eq. (108).

Consider from eqs. (79) and (80) that $\varphi_{\mathrm{OEF}} \approx \varphi_{\mathrm{o}}$ at low temperature $\mathrm{T}<0.5 \mathrm{~T}_{\mathrm{o}}$, while $\varphi_{\mathrm{OEF}} \approx \varphi_{\mathrm{T}}$ at high temperature $\mathrm{T}>2 \mathrm{~T}_{\mathrm{o}}$, in the following eqs. (109)-(113). Increasing the temperature from $\mathrm{T}<0.5 \mathrm{~T}_{\mathrm{o}}$ toward $\mathrm{T}>2 \mathrm{~T}_{\mathrm{o}}$, a small and gradual increase from 0.5 to 1 is encountered for the ratio

$$
\frac{\varphi_{\mathrm{OEF}}}{2 \varphi_{\mathrm{OEF}}-\varphi_{\mathrm{T}}} \approx(0.65 \pm 0.1) \approx\left\{\begin{array}{l}
\frac{\varphi_{\mathrm{o}}}{2 \varphi_{\mathrm{o}}-\varphi_{\mathrm{T}}} \approx 0.5 \ldots 0.67, \text { at low } \mathrm{T}<0.5 \mathrm{~T}_{\mathrm{o}} \Rightarrow \varphi_{\mathrm{OEF}} \approx \varphi_{\mathrm{o}} \\
\frac{\varphi_{\mathrm{T}}}{2 \varphi_{\mathrm{T}}-\varphi_{\mathrm{T}}} \approx 1, \text { at high } \mathrm{T}>2 \mathrm{~T}_{\mathrm{o}} \Rightarrow \varphi_{\mathrm{OEF}} \approx \varphi_{\mathrm{T}}
\end{array}\right.
$$

which can be neglected and accumulated in the value of $\mu_{\mathrm{oo}}$, since much larger uncertainties for $\Gamma_{\mathrm{o}}, \Lambda_{\mathrm{o}}$, $\exp \left(\mathrm{S}_{\mathrm{c}} \infty\right)$ and $\mathrm{N}_{\mathrm{S}}$ exist in the practice. However, the temperature shaping function $\mathrm{TSF}^{2}$ varies at high temperature, because

$$
\mathrm{TSF}^{2} \equiv \mathrm{A} \times\left(\frac{\varphi_{\mathrm{O}}}{\varphi_{\mathrm{OEF}}}\right)^{\frac{\varphi_{\mathrm{OEF}}}{\varphi_{\mathrm{o}}}} \approx\left\{\begin{array}{l}
\mathrm{A}=\exp \left(\frac{\left|\mathrm{E}_{\mathrm{O}}-\mathrm{E}_{\mathrm{F}}\right| / \mathrm{q}-\varphi_{\mathrm{To}}}{\varphi_{\mathrm{o}}}\right)=\text { constant } \sim \mathrm{e}^{3}, \text { at low } \mathrm{T}<0.5 \mathrm{~T}_{\mathrm{O}} \\
\mathrm{A} \times \exp \left[-\frac{\varphi_{\mathrm{T}}}{\varphi_{\mathrm{o}}} \ln \left(\frac{\varphi_{\mathrm{T}}}{\varphi_{\mathrm{O}}}\right)\right], \text { at high } \mathrm{T}>2 \mathrm{~T}_{\mathrm{O}}
\end{array}\right.
$$

Note that the power-law $\mathrm{TSF}^{2} / \mathrm{A}=\left(\mathrm{T}_{\mathrm{o}} / \mathrm{T}_{\mathrm{OEF}}\right)^{\left(\mathrm{TOEF} / \mathrm{T}_{0}\right)}$ in eq. (110) obeys the requirement stated in [6] for a monotonic increase with the reciprocal of the absolute temperature 1/T. However, the power-law $\mathrm{TSF}^{2}$ in 
eq. (110) is different from the discussed in [6], the "sinc"-function " $\sin \left(\pi \mathrm{T} / \mathrm{T}_{\mathrm{o}}\right)$ " $=\mathrm{e}^{-3} \sin \left(\pi \mathrm{T} / \mathrm{T}_{\mathrm{o}}\right) /\left(\pi \mathrm{T} / \mathrm{T}_{\mathrm{o}}\right)$, and the "difference" function " $\Delta \mathrm{T} /[\exp (\Delta \mathrm{T})-1] "=\mathrm{e}^{-3}\left(\mathrm{~T}_{\mathrm{o}} / \mathrm{T}-1\right) /\left\{1-\exp \left[\left(1-\mathrm{T}_{\mathrm{o}} / \mathrm{T}\right) \Delta \mathrm{E} /\left(\mathrm{kT} \mathrm{T}_{\mathrm{o}}\right)\right]\right\}$, the latter with $\Delta \mathrm{E} \sim\left|\mathrm{E}_{\mathrm{o}}-\mathrm{E}_{\mathrm{F}}\right|-\left|\mathrm{kT}+\mathrm{k} \mathrm{T}_{\mathrm{o}}\right|$ by assuming Boltzmann statistics and single-side exponential DOSSE. These three temperature shaping functions, normalized to their constant multiplier $\mathrm{e}^{ \pm 3}$, are compared in Figure 36, showing that $\mathrm{TSF}^{2}$ is not a unique function, but depends on and accumulates the assumptions and techniques of derivation of models. Consequently, the $\mathrm{TSF}^{2}$ in eq. (108) causes temperature variations in the prediction of the thermal activation of the mobility $\mu_{\mathrm{o}}$ in OTFT, to which the last term in eq. (108), also contributes, since

$$
\left[\frac{1}{\varphi_{\mathrm{TFT}}^{2}}\right]^{\frac{\varphi_{\mathrm{OEF}}}{\varphi_{\mathrm{T}}}-1}=\varphi_{\mathrm{TFT}}^{2} \exp \left(-\frac{\varphi_{\mathrm{OEF}}}{\varphi_{\mathrm{T}}} \ln \left(\varphi_{\mathrm{TFT}}^{2}\right)\right) \approx\left\{\begin{array}{l}
\varphi_{\mathrm{TFT}}^{2} \exp \left(-\frac{\text { constant }}{\mathrm{kT}}\right), \text { at low } \mathrm{T}<0.5 \mathrm{~T}_{\mathrm{o}} \\
\approx 1, \text { at high } \mathrm{T}>2 \mathrm{~T}_{\mathrm{o}}
\end{array}\right.
$$

The temperature-dependent POEF in eq. (108) seems to be the origin of variable thermal activation via the term with $\varphi$ TFT, because, as seen in Figure 36, the power-law $\mathrm{TSF}^{2}=\left(\mathrm{T}_{\mathrm{o}} / \mathrm{T}_{\mathrm{OEF}}\right)^{\left(\mathrm{TOEF}_{\mathrm{o}}\right)}$ causes a negligible modification of less than $2 \mathrm{kT}_{\mathrm{o}} \sim 60-80 \mathrm{meV}$ in the much larger activation energy for $\mu_{\mathrm{o}}$ in the range of hundreds meV for an OTFT, which can be inspected also by rearranging eq. (108), so that one gets 


$$
\begin{aligned}
& \ln \left(\frac{\mu_{\mathrm{O}}}{\mu_{\mathrm{oo}}}\right) \approx \frac{\left|\mathrm{E}_{\mathrm{O}}-\mathrm{E}_{\mathrm{F}}\right| / \mathrm{q}-\varphi_{\mathrm{To}}}{\varphi_{\mathrm{T}}}-\frac{\varphi_{\mathrm{OEF}}}{\varphi_{\mathrm{T}}} \ln \left(\frac{\varphi_{\mathrm{OEF}}}{\varphi_{\mathrm{T}}}\right)-\frac{\varphi_{\mathrm{OEF}}-\varphi_{\mathrm{T}}}{\varphi_{\mathrm{T}}} \ln \left(\varphi_{\mathrm{TFT}}^{2}\right) \\
& \approx \frac{\left|\mathrm{E}_{\mathrm{O}}-\mathrm{E}_{\mathrm{F}}\right| / \mathrm{q}-\varphi_{\mathrm{To}}}{\varphi_{\mathrm{T}}}-\left\{\begin{array}{r}
\frac{\varphi_{\mathrm{o}}}{\varphi_{\mathrm{T}}}\left[\ln \left(\frac{\varphi_{\mathrm{o}}}{\varphi_{\mathrm{T}}}\right)+2\left(1-\frac{\varphi_{\mathrm{T}}}{\varphi_{\mathrm{O}}}\right) \ln \left(\varphi_{\mathrm{TFT}}\right)\right] \\
\text { at low } \mathrm{T}<0.5 \mathrm{~T}_{\mathrm{O}} \\
\left(1+\delta_{\varphi}\right) \ln \left(1+\delta_{\varphi}\right)+2 \delta_{\varphi} \ln \left(\varphi_{\mathrm{TFT}}\right) \\
\text { at } \operatorname{high} \mathrm{T}>2 \mathrm{~T}_{\mathrm{O}}
\end{array}\right. \\
& \sim \frac{100 \mathrm{mV} \pm 100 \mathrm{mV} ?}{\varphi_{\mathrm{T}}}-\left\{\begin{array}{r}
\frac{\varphi_{\mathrm{o}}}{\varphi_{\mathrm{T}}}\left[1+\ln \left(\varphi_{\mathrm{TFT}}\right)\right] \sim \frac{160 \mathrm{mV} \pm 150 \mathrm{mV} ?}{\varphi_{\mathrm{T}}}, \\
\text { at low } \mathrm{T}<0.5 \mathrm{~T}_{\mathrm{o}}, \text { since } \varphi_{\mathrm{OEF}} \approx \varphi_{\mathrm{o}} \\
1 \%+2 \% \ln \left(\varphi_{\mathrm{TFT}}\right) \sim \frac{5.9 \mathrm{mV} \pm 6.4 \mathrm{mV}}{\varphi_{\mathrm{T}}} \rightarrow 0, \\
\text { at high } \mathrm{T}>2 \mathrm{~T}_{\mathrm{o}}, \text { since } \varphi_{\mathrm{OEF}} \approx \varphi_{\mathrm{T}}
\end{array}\right.
\end{aligned}
$$

where, by using a conservative value for $\mathrm{n}=5$ in eq. (79), we estimate that

$$
\begin{aligned}
& \delta_{\varphi}=\frac{\varphi_{\mathrm{OEF}}-\varphi_{\mathrm{T}}}{\varphi_{\mathrm{T}}}=\sqrt[n]{1+\left(\frac{\varphi_{\mathrm{o}}}{\varphi_{\mathrm{T}}}\right)^{\mathrm{n}}}-1 \\
& \approx \frac{1}{\mathrm{n}}\left(\frac{\varphi_{\mathrm{o}}}{\varphi_{\mathrm{T}}}\right)^{\mathrm{n}} \leq \frac{1}{\mathrm{n} 2^{\mathrm{n}}} \leq \frac{1}{5 \times 2^{5}}<1 \%, \text { at high } \mathrm{T}>2 \mathrm{~T}_{\mathrm{o}}, \text { since } \varphi_{\mathrm{OEF}} \approx \varphi_{\mathrm{T}},
\end{aligned}
$$

and we have taken from the numerical simulations that $\left|\mathrm{E}_{\mathrm{o}}-\mathrm{E}_{\mathrm{F}}\right|=0.9 \mathrm{eV}$ and $\varphi_{\mathrm{To}}=0.8 \mathrm{~V} \pm 0.1 \mathrm{~V}$ from eq. (61) and Figure 23, and adopted from [6] characteristic values for $\mathrm{T}_{\mathrm{o}} \sim 400 \mathrm{~K}, \varphi_{\mathrm{o}}=34.5 \mathrm{mV}$ and $\varphi_{\mathrm{TFT}}=45 \mathrm{~V} \pm 2$ decades. The numerator of eq. (112) provides the following expression for the activation energy of $\mu_{\mathrm{o}}$ in eq. (106) for the OTFT current, as 


$$
\begin{aligned}
\mathrm{E}_{\mathrm{A \mu o}}= & \left(\left|\mathrm{E}_{\mathrm{O}}-\mathrm{E}_{\mathrm{F}}\right|-\mathrm{q} \varphi_{\mathrm{To}}\right)-\mathrm{q}\left[\varphi_{\mathrm{OEF}} \ln \left(\frac{\varphi_{\mathrm{OEF}}}{\varphi_{\mathrm{T}}}\right)+\left(\varphi_{\mathrm{OEF}}-\varphi_{\mathrm{T}}\right) \ln \left(\varphi_{\mathrm{TFT}}^{2}\right)\right] \\
& \approx\left(\left|\mathrm{E}_{\mathrm{O}}-\mathrm{E}_{\mathrm{F}}\right|-\mathrm{q} \varphi_{\mathrm{To}}\right)-\left\{\begin{array}{r}
\mathrm{q} \varphi_{\mathrm{O}}\left[1+\ln \left(\varphi_{\mathrm{TFT}}\right)\right], \\
\text { at low } \mathrm{T}<0.5 \mathrm{~T}_{\mathrm{o}}, \text { since } \varphi_{\mathrm{OEF}} \approx \varphi_{\mathrm{o}} \\
\mathrm{q} \varphi_{\mathrm{T}} \delta_{\varphi}\left[1+2 \ln \left(\varphi_{\mathrm{TFT}}\right)\right], \\
\text { at } \operatorname{high} \mathrm{T}>2 \mathrm{~T}_{\mathrm{o}}, \text { since } \varphi_{\mathrm{OEF}} \approx \varphi_{\mathrm{T}}
\end{array}\right. \\
& \sim(100 \mathrm{meV} \pm 100 \mathrm{meV} ?)-\left\{\begin{array}{r}
160 \mathrm{meV} \pm 150 \mathrm{meV} ?, \text { at low } \mathrm{T}<0.5 \mathrm{~T}_{\mathrm{o}} \\
5.9 \mathrm{mV} \pm 6.4 \mathrm{mV} \rightarrow 0, \text { at high } \mathrm{T}>2 \mathrm{~T}_{\mathrm{o}}, \text { with } \delta_{\varphi} \sim 1 \%
\end{array}\right.
\end{aligned}
$$

While eqs. (108), (112) and (113) are consistent in behavior and related firmly with the linear dependence between hopping energy and electrostatic bending, we should note that that the bottle neck is in the values of the parameters, which we have marked with question symbols (?) in eqs. (112) and (113). The problem is that the values of many parameters have to be determined. In particular, behind the equations, seven parameters are unknown, namely $\mathrm{N}_{\mathrm{S}}, \mathrm{E}_{\mathrm{o}}, \mathrm{E}_{\mathrm{F}}, \Gamma_{\mathrm{o}}, \Lambda_{\mathrm{o}}, \mathrm{s}_{\mathrm{c} \mathrm{T}_{\infty}}$ and $\varphi_{\mathrm{To}}$, even when $\varphi_{\mathrm{o}}$ for DOS and $\mathrm{n}$ in eq. (79) or $\mathrm{T}_{\mathrm{G}}$ in eq. (80) are determined from experimental data for the mobility enhancement factor $\gamma$ by the help of the relations in eq. (105). On the other hand, the independent relations are less, eq. (113) for activation energy $\mathrm{E}_{\mathrm{A \mu o}}$, from which $\left(\left|\mathrm{E}_{\mathrm{o}}-\mathrm{E}_{\mathrm{F}}\right|-\mathrm{q} \varphi_{\mathrm{To}}\right)$ can be found, and eq. (108) for $\mu_{\mathrm{o}}$, from which $\left(\Gamma_{\mathrm{o}} \exp \left(\mathrm{s}_{\mathrm{c}} \mathrm{T}_{\infty}\right) / \Lambda_{\mathrm{o}}\right)$ can be found, provided that these equations are coupled in an iterative procedure with proper variation of $\mathrm{N}_{\mathrm{S}}$ for determining $\mu_{\mathrm{oo}}$ and $\varphi_{\mathrm{TFT}}$. Thus, the relations are only three, requiring to assume values for $\left|\mathrm{E}_{\mathrm{O}}-\mathrm{E}_{\mathrm{F}}\right|$ and also for two of the three parameters $\Gamma_{\mathrm{o}}, \Lambda_{\mathrm{o}}$ and $\mathrm{s}_{\mathrm{c} T \infty}$. It is highly desired to have relations between $\mathrm{s}_{\mathrm{c} T \infty}, \varphi_{\mathrm{To}}$ and $\Lambda_{0}$, but we do not know these relations; and this is at the origin of our doubt for the immature relation between VRH for OTFT and material properties. We are not aware of a characterization procedure that leads to determination of unique values of the parameters, which, unfortunately, is the state-of-the-art VRH for OTFTs at present. What we certainly confirm and routinely observed in the numerical simulations is that the linear dependence between hopping energy and electrostatic bending leads to many of the models available in the literature for OTFT, as discussed above in this section. Thus, we expect that this dependence 
might be actually a principal relation for VRH, since it remains valid by changing various assumptions, e.g. type of DOS, values of VRH parameters, temperature and materials.

\section{VRH beyond the static (DC) characteristics of OTFT}

Up to this point, we have dealt with integrations and averages of the VRH that correspond to the static (DC) characteristics of OTFT and possible approaches for acceleration of the VRH numerical simulations. However, considering the wide range of the hopping energy up to $\mathrm{E}_{\mathrm{H}} \sim 1 \mathrm{eV}$, one expects prominent dispersion in the hopping time, and we would like also to address several issues related to this dispersion, e.g., transient current, charge build-up and noise. The concepts in this section have been presented previously in $[93,94]$. Here, we expand the details and discussions.

\subsection{Hopping time distribution}

To access the distribution of the hopping time $\tau$ from the numerical VRH simulations, consider the fraction of bonds per site $\mathrm{B}(\geq \Gamma)$ that have hopping rates larger than a given hopping rate $\Gamma$, and define the hopping time as $\tau=1 / \Gamma$. Then, this fraction of bonds $\mathrm{B}(\geq \Gamma) \equiv \mathrm{B}(\leq \tau)$ with hopping rates faster than $\Gamma$ becomes proportional to the cumulative distribution of the hopping time shorter than $\tau$. Since the cumulative distribution for the fraction of bonds $\mathrm{B}(\leq \tau)$ approaches the bonds $\mathrm{B}_{\mathrm{c}}$ in the critical path of the percolation network, then the cumulative distribution of hopping times $\tau$ is

$$
\frac{\mathrm{B}(>\Gamma)}{\mathrm{B}_{\mathrm{c}}\left(=\Gamma_{\mathrm{c}}\right)}=\frac{\mathrm{B}(<\tau)}{\mathrm{B}_{\mathrm{c}}\left(=\tau_{\mathrm{c}}=1 / \Gamma_{\mathrm{c}}\right)},
$$

where, for the critical percolation path, $\mathrm{B}_{\mathrm{c}} \sim 9 / \pi \approx 2.86$ is the constant that represents the "geometrical" assumption for sufficient bonds per site by continuous percolation, see eqs. (3) and (5), $\Gamma_{\mathrm{c}}$ is the hopping rate in the critical path, and $\tau_{\mathrm{c}}=1 / \Gamma_{\mathrm{c}}=\exp \left(\mathrm{E}_{\mathrm{H}} / \mathrm{kT}\right) / \Gamma_{\mathrm{o}}$ is the corresponding hopping time in the critical path, as follows from the principal eq. (2) for VRH. Accordingly, the derivative with respect to $\tau$ is both the distribution density of hopping bonds B and distribution density of hopping time, and it is 


$$
\frac{\partial \mathrm{B}(<\tau) / \mathrm{B}_{\mathrm{c}}}{\partial \tau}=\frac{\partial \mathrm{B} / \mathrm{B}_{\mathrm{c}}}{\partial \Delta \mathrm{E}_{\mathrm{H}}} \frac{\partial \Delta \mathrm{E}_{\mathrm{H}}}{\partial \tau}
$$

where $\Delta \mathrm{E}_{\mathrm{H}}$ corresponds to the particular value of $\tau$, for which the derivative is given, and the Greek symbol $\Delta$ is added to the notation $\Delta \mathrm{E}_{\mathrm{H}}$ to distinguish from the hopping energy $\mathrm{E}_{\mathrm{H}}$ for the critical path, since $\mathrm{E}_{\mathrm{H}}$ corresponds to $\mathrm{B}_{\mathrm{c}}, \Gamma_{\mathrm{c}}$ and $\tau_{\mathrm{c}}$. As follows from the principal eq. (2) for $\mathrm{VRH}$, the relation between $\Delta \mathrm{E}_{\mathrm{H}}$ and $\tau$ is

$$
\begin{aligned}
& \ln \left(\tau \Gamma_{\mathrm{o}}\right)=\ln \left(\frac{\Gamma_{\mathrm{o}}}{\Gamma}\right)=\frac{\Delta \mathrm{E}_{\mathrm{H}}}{\mathrm{kT}} \\
& \Rightarrow\left\{\begin{array}{c}
\Delta \mathrm{E}_{\mathrm{H}}=\mathrm{kT} \ln \left(\tau \Gamma_{\mathrm{o}}\right) \Rightarrow \frac{\partial \Delta \mathrm{E}_{\mathrm{H}}}{\partial \tau}=\frac{\mathrm{kT}}{\tau} \\
\tau=\frac{1}{\Gamma_{\mathrm{o}}} \exp \left(\frac{\Delta \mathrm{E}_{\mathrm{H}}}{\mathrm{kT}}\right), \text { substitute above }
\end{array}\right\}, \\
& \Rightarrow \frac{\partial \Delta \mathrm{E}_{\mathrm{H}}}{\partial \tau}=\mathrm{kT}_{\mathrm{o}} \exp \left(-\frac{\Delta \mathrm{E}_{\mathrm{H}}}{\mathrm{kT}}\right)
\end{aligned}
$$

Therefore, with respect to the hopping time $\tau$, the distribution density of the hopping bonds and the distribution density of the hopping time can be found from

$$
\frac{\partial \mathrm{B} / \mathrm{B}_{\mathrm{c}}}{\partial \tau}=\mathrm{kT} \Gamma_{\mathrm{o}} \exp \left(-\frac{\Delta \mathrm{E}_{\mathrm{H}}}{\mathrm{kT}}\right) \frac{\partial \mathrm{B}\left(\Delta \mathrm{E}_{\mathrm{H}}\right) / \mathrm{B}_{\mathrm{c}}}{\partial \Delta \mathrm{E}_{\mathrm{H}}} \text {, for } \tau=\tau_{\mathrm{o}} \exp \left(\frac{\Delta \mathrm{E}_{\mathrm{H}}}{\mathrm{kT}}\right)
$$

as a parametric function of hopping energy $\Delta \mathrm{E}_{\mathrm{H}}$, where $\tau_{\mathrm{o}}=1 / \Gamma_{\mathrm{o}}$ is the hopping attempt time. Since the hopping time is in unit of seconds, then the distribution density $\partial\left(\mathrm{B} / \mathrm{B}_{\mathrm{c}}\right) / \partial \tau$ is in the reciprocal unit $(1 / \mathrm{s}=\mathrm{Hz})$. The distribution of the hopping bonds and time is normalized to the critical path, so that

$$
\int_{0}^{\tau_{\mathrm{c}}} \frac{\partial \mathrm{B} / \mathrm{B}_{\mathrm{c}}}{\partial \tau} \mathrm{d} \tau=\int_{0}^{\mathrm{B}_{\mathrm{c}}} \frac{\mathrm{dB}}{\mathrm{B}_{\mathrm{c}}}=1, \text { with } \Delta \mathrm{E}_{\mathrm{H}}=\mathrm{E}_{\mathrm{H}} \text { for } \tau_{\mathrm{c}}=\tau_{\mathrm{o}} \exp \left(\frac{\mathrm{E}_{\mathrm{H}}}{\mathrm{kT}}\right)
$$

corresponding to the continuous (DC) hopping in the critical path, but note that the distribution is not limited by $\tau_{\mathrm{c}}, \mathrm{B}_{\mathrm{c}}$ or $\mathrm{E}_{\mathrm{H}}$; and $\tau>\tau_{\mathrm{c}}$ or $\Delta \mathrm{E}_{\mathrm{H}}>\mathrm{E}_{\mathrm{H}}$ correspond to non-propagating fluctuations, which may spatially occur, but do not contribute to the stationary (DC) VRH conductance.

After obtaining $E_{H}$ for the critical path by the VRH simulator, the values of the derivative $\partial\left(\mathrm{B} / \mathrm{B}_{\mathrm{c}}\right) \partial \Delta \mathrm{E}_{\mathrm{H}}$ and hopping time $\tau$ in eq. (117) are evaluated numerically for every triplet $\left\{\mathrm{V}_{\mathrm{G}}, \mathrm{T}, \mathrm{D}\right\}$ of gate 
bias $\mathrm{V}_{\mathrm{G}}$, temperature $\mathrm{T}$ and depth $\mathrm{D}$ in the profile of the OTFT semiconducting film, by stepping $\Delta \mathrm{E}_{\mathrm{Hi}}=10 \mathrm{meV}, 20 \mathrm{meV}, \ldots, \Delta \mathrm{E}_{\mathrm{Hi}}, \ldots, \mathrm{E}_{\mathrm{H}}, \ldots, 1.5 \mathrm{eV}, \mathrm{i}=1 \ldots 150$, and calculating the corresponding fraction $\mathrm{B}_{\mathrm{i}}\left(\leq \Delta \mathrm{E}_{\mathrm{H}}\right)$ by either single $\int \mathrm{dE}$, eq. (14) or multiple $\iint \mathrm{dEdE}$ integrations, eqs. (16)-(19). This calculation for the single $\int \mathrm{dE}$ integration, by rewriting eq. (12), is according to

$$
\mathrm{B}_{\mathrm{i}}\left(\leq \Delta \mathrm{E}_{\mathrm{Hi}}\right)=\frac{4}{3} \pi\left[\left(\frac{\Lambda_{\mathrm{O}}}{2} \frac{\Delta \mathrm{E}_{\mathrm{Hi}}}{\mathrm{kT}}\right)^{3}\right]_{-\Delta \mathrm{E}_{\mathrm{Hi}}}^{+\Delta \mathrm{E}_{\mathrm{Hi}}}\left(1-\frac{|\Delta \mathrm{E}|}{\mathrm{E}_{\mathrm{H}}}\right)^{3} \operatorname{DOS}\left(\mathrm{E}_{\mathrm{F}}+\Delta \mathrm{E}\right) \mathrm{d} \Delta \mathrm{E} .
$$

The corresponding hopping times $\tau_{\mathrm{i}}\left(\Delta \mathrm{E}_{\mathrm{Hi}}\right)$ are calculated by eq. (117), as

$$
\tau_{\mathrm{i}}=\frac{1}{\Gamma_{\mathrm{o}}} \exp \left(\frac{\Delta \mathrm{E}_{\mathrm{Hi}}}{\mathrm{kT}}\right)
$$

Both $\mathrm{B}_{\mathrm{i}}$ and $\tau_{\mathrm{i}}$ are numerical vectors of size of the vector $\Delta \mathrm{E}_{\mathrm{Hi}}$. The values of the derivative $\partial\left(\mathrm{B} / \mathrm{B}_{\mathrm{c}}\right) \partial \Delta \mathrm{E}_{\mathrm{H}}$ are then calculated as ratio of finite differences, as

$$
\left.\frac{\partial \mathrm{B}\left(\Delta \mathrm{E}_{\mathrm{Hi}}\right) / \mathrm{B}_{\mathrm{c}}}{\partial \Delta \mathrm{E}_{\mathrm{H}}}\right|_{\mathrm{i} \geq 2} \approx \frac{1}{\mathrm{~B}_{\mathrm{c}}} \frac{\mathrm{B}_{\mathrm{i}}-\mathrm{B}_{\mathrm{i}-1}}{\Delta \mathrm{E}_{\mathrm{Hi}}-\Delta \mathrm{E}_{\mathrm{H}(\mathrm{i}-1)}} \text {, completed with }\left.\frac{\partial \mathrm{B} / \mathrm{B}_{\mathrm{c}}}{\partial \Delta \mathrm{E}_{\mathrm{H}}}\right|_{\mathrm{i}=1}=\left.\frac{\partial \mathrm{B} / \mathrm{B}_{\mathrm{c}}}{\partial \Delta \mathrm{E}_{\mathrm{H}}}\right|_{\mathrm{i}=2} .
$$

The substitution of the values in eq. (117) yields the matrix of values for the distribution density of the hopping bonds and hopping time, which is of size $(\mathrm{n} \times \mathrm{m})$, with $\mathrm{n}$ equal to the length of the $\tau_{\mathrm{i}}$ vector and $\mathrm{m}$ equal to the size of the reduced depth mesh $\mathrm{D}$ at given $\mathrm{V}_{\mathrm{G}}$ and $\mathrm{T}$. Obviously, the computation volume is increased and the data became large. Therefore, the calculation of the hopping distributions is normally suppressed in the VRH simulator.

The distributions $\partial\left(\mathrm{B} / \mathrm{B}_{\mathrm{c}}\right) / \partial \tau$ of the hopping bonds and hopping time obtained by the above numerical procedure are illustrated in Figure 37 for several characteristic depths in the film of a pentacene OTFT at one bias-temperature point. These distributions are shown with solid polygon-like curves in the figure. There are characteristic features in these distributions.

One feature is that the distributions are different at different depths in the semiconducting film of the OTFT. The top solid curve is for the semiconductor-gate insulator interface (depth $\mathrm{D}=0$ ), and the bottom 
solid curve is for bulk material (depth $\mathrm{D}=\infty$ ). Moving from $\mathrm{D}=0$ into the depth of the film toward the back of the film, the distributions become wider and wider.

A second feature is that the distributions are bounded by the hopping attempt time $\tau_{\mathrm{o}}$ on left, which is obvious, but they are not bonded by the hopping time $\tau_{\mathrm{c}}$ in the critical path. The values for $\tau_{\mathrm{c}}$ are denoted with the circles $(O)$ aligned to the dotted trend line $0.4 / \tau_{\mathrm{c}}$. Instead, as indicated by dashed trend lines $\tau^{-0.9}$ and $\tau^{-1}$, the distributions are bounded on the right-hand side by nearly $1 / \tau$ distribution at hopping time $1-2$ decades larger than $\tau_{\mathrm{c}}$.

A third feature is that the distributions have ranges from $\tau_{\min } \approx \tau_{0} \exp (6) \approx 0.4 \mathrm{ps}$ to $\tau_{\max } \approx \tau_{\mathrm{c}} \exp (-6)$, in which the slope in the double-logarithmic plot is almost constant, and corresponds to the slope of $\tau^{\mathrm{n}}$ distribution, where $n=-1 / 4$ in the particular example, as indicated by the dashed trend line $\tau^{-1 / 4}$ in the bottom of the plot. The values for $\tau_{\min }$ and $\tau_{\max }$ are denoted with small diamonds $(\bullet)$ for each distribution, and respectively, are aligned to the dashed trend lines $\tau_{\min }=0.4 \mathrm{ps}$ and $0.02 / \tau_{\max }$, which together with the trend line $\tau^{-1 / 4}$ surround the region, in which the hopping time has $\tau^{\mathrm{n}}$ distribution.

A fourth feature is that distributions in OTFT are broad functions of a power-law type of the hopping time $\tau$, causing the so-called dispersive transport [95], which is continuously being elaborated for time-offlight measurements in sandwiched diode-like structures for more than 30 years, but fairly unexplored for thin-film structures.

We can summarize the observations in Figure 37. At given temperature $T$, gate bias $\mathrm{V}_{\mathrm{G}}$ and spot in the depth $\mathrm{D}$ of the amorphous semiconducting film of the OTFT, the VRH predicts three regions for the distribution $\partial\left(\mathrm{B} / \mathrm{B}_{\mathrm{c}}\right) / \partial \tau$ of the hopping bonds $\mathrm{B}$ and hopping time $\tau$, given by

$$
\begin{aligned}
& \frac{\partial \mathrm{B} / \mathrm{B}_{\mathrm{c}}}{\partial \tau} \approx 0, \text { for } \tau<\tau_{\mathrm{o}} \exp (3) \sim 10^{-12} \mathrm{~s},(\text { faster than the transport in OTFT), } \\
& \frac{\partial \mathrm{B} / \mathrm{B}_{\mathrm{c}}}{\partial \tau} \approx \frac{\mathrm{n}+1}{\tau_{\mathrm{c}}^{\mathrm{n}+1}} \tau^{\mathrm{n}} \propto \tau^{\mathrm{n}}, \text { for } \tau_{\mathrm{o}} \exp (3)<\tau<\tau_{\mathrm{c}}=\tau_{\mathrm{o}} \exp \left(\mathrm{s}_{\mathrm{c}}\right), \text { (dispersive transport in OTFT), }
\end{aligned}
$$

where $\mathrm{s}_{\mathrm{c}}=\mathrm{E}_{\mathrm{H}} / \mathrm{kT}=2 \mathrm{R}_{\mathrm{H}} / \Lambda_{\mathrm{o}}$, according to the principal VRH eq. (2), and 


$$
\frac{\partial \mathrm{B} / \mathrm{B}_{\mathrm{c}}}{\partial \tau} \approx \frac{\mathrm{n}+1}{\tau} \propto \frac{1}{\tau} \text {, for } \tau_{\mathrm{c}}<\tau \text {, (no transport, just fluctuations and charge trapping). }
$$

Note that the formulas are given at conditions $\tau_{\mathrm{o}}<\tau_{\min }<<\tau_{\mathrm{c}}=\tau_{\mathrm{o}} \exp \left(\mathrm{s}_{\mathrm{c}}\right)$ and $\mathrm{n}>(-1)$, so that eq. (118) is satisfied for the second region

$$
\begin{aligned}
& \int_{\tau_{\min }}^{\tau_{\mathrm{c}}} \frac{\partial \mathrm{B} / \mathrm{B}_{\mathrm{c}}}{\partial \tau} \mathrm{d} \tau=\int_{\tau_{\min }}^{\tau_{\mathrm{c}}} \frac{\mathrm{n}+1}{\tau_{\mathrm{c}}^{\mathrm{n}+1}} \tau^{\mathrm{n}} \mathrm{d} \tau=\frac{\mathrm{n}+1}{\tau_{\mathrm{c}}^{\mathrm{n}+1}} \int_{\tau_{\min }}^{\tau_{\mathrm{c}}} \tau^{\mathrm{n}} \mathrm{d} \tau \\
& =\frac{\mathrm{n}+1}{\tau_{\mathrm{c}}^{\mathrm{n}+1}}\left(\frac{\tau_{\mathrm{c}}^{\mathrm{n}+1}-\tau_{\min }^{\mathrm{n}+1}}{\mathrm{n}+1}\right)=1-\mathrm{O}\left[\left(\frac{\tau_{\min }}{\tau_{\mathrm{c}}}\right)^{\mathrm{n}+1}\right] \approx 1
\end{aligned}
$$

Also, the value $\partial\left(B / B_{c}\right) / \partial \tau=(n+1) / \tau_{c}$ is aligned at the boundary $\tau=\tau_{c}$ between the second and third regions.

The first region, described with eq. (122), implies that the organic material is unable to transfer carriers between charge states at rates close to $\Gamma_{\mathrm{o}}$. In the second region, eq. (123) describes the dynamics of the charge transport in OTFT, and the power-law distribution $\tau^{\mathrm{n}}$ suggests a link to dispersive transport, which we will address below. In the third region, eq. (124) implies a $1 / \tau$ distribution of the hopping bonds, providing for phenomena that do not contribute to the charge transport directly, but are superimposed and accompany the transport. Such phenomena are low-frequency noise and charge build-up, for example. One essential remark to eqs. (123) and (124) is that for an OTFT under bias, these equations are for a spot along the channel and in a slice of the semiconducting film at depth D; and proper weighting of contributions from different slices and spots is necessary in order to obtain the overall (effective) distribution in the entire OTFT.

\subsection{Hopping as dispersive charge transport and approach to transient behavior}

There are several theoretical approaches to the dispersive charge transport in solid amorphous materials.

These theories address the propagation of charge packets by time-of-flight (ToF) measurements. Chronologically, the first approach was for random walk of hopping charges; it was authored by Scher and Montroll (SM) in [95] in 1975, introducing the co-called dispersive parameter $\alpha>0$. Later, it was found that 
$\alpha$ depends on temperature, as $\alpha=\mathrm{T} / \mathrm{T}_{\mathrm{o}}[96,97,98,99]$. Below, we inspect the presence of the dispersive transport in our numerical simulations of VRH, and discuss the details.

The significance of the dispersive parameter $\alpha$ is that the charge transport is dispersive when $\alpha<1$, since the hopping times do not obey the exponential distribution for a system with single transition rate, but the hopping events are time-variant and are widely distributed as a power-law function $\psi(\mathrm{t})$ of the time of observation, given by

$$
\text { SM distribution density: } \psi(t)=\frac{\text { constant }}{t^{(1+\alpha)}}, 0<\alpha<1,0<t<\infty \text { is time of observation. }
$$

The smaller is $\alpha$, the wider is $\psi$, and the transport becomes more dispersive, compared to the transport with normal diffusion by exponential distribution. Note that $\mathrm{t}$ is time of observation, but not the hopping time $\tau$, and the distribution of hopping time and bonds $\partial\left(\mathrm{B} / \mathrm{B}_{\mathrm{c}}\right) / \partial \tau$ is time invariant, as calculated by the procedure above for VRH in OTFT at the thermal equilibrium, thus $\partial\left(\mathrm{B} / \mathrm{B}_{\mathrm{c}}\right) / \partial \tau$ is a limit corresponding to an observation time $\mathrm{t} \rightarrow \infty$. The relation between $\psi(\mathrm{t})$ and $\partial\left(\mathrm{B} / \mathrm{B}_{\mathrm{c}}\right) / \partial \tau$ is via the slopes of the distributions, and by the arguments discussed below, the relation is

$$
\alpha=\frac{\mathrm{T}}{\mathrm{T}_{\mathrm{o}}}=\mathrm{n}+1 \text {, for an exponential DOS of energy width } \mathrm{kT}_{\mathrm{o}} \text {. }
$$

The relation $T / T_{0}=(n+1) \leftrightarrow n=\left(T / T_{0}-1\right)=(300 K / 400 K-1)=-1 / 4$ is observed in Figure 37 for the ranges $\left(\tau_{\min }-\tau_{\max }\right)$, in which $\partial\left(\mathrm{B} / \mathrm{B}_{\mathrm{c}}\right) / \partial \tau \propto \tau^{-1 / 4}$, and corresponds to eq. (123). The observation was repeated for other temperatures, as shown in the following Figure 38. The other relation $\alpha=T / T_{0}$ was given for exponential DOS by several works $[96,97,98,99]$ in the period 1977-1982, which have also established that the multiple trapping and release (MTR) can explain the dispersive transport. The equivalence of SM random walk and MTR was elaborated theoretically in [100]. The investigation approaches in these works are two, either experiments furnished with analytical formulas [97, 98], or utilization of Monte Carlo (MC) simulations [96, 99]. Later development of MTR includes Gaussian DOS in the so-called Gaussian disorder model (GDM) [101] and spatial correlation in the so-called correlation disorder model (CDM) [102, 103, 104], as reviewed in $[105,106]$. Another approach to MTR is by addressing the charge propagation by means of diffusion of 
charges, instead of random-walk hopping, and by introduction of modifications in the regular differential equations for the charge transport, in particular, in the terms related to charge trapping, emission and diffusion. The anomalous diffusion paradigm in MTR was undertaken first by Rudenko and Arkhipov [107, 108, 109], and it is mathematically complex and diverse, as seen in $[107,108,109,110,111,112,113,114]$. Interestingly, these works describe the details of time-of-flight (ToF) measurements, but the emphasis is moved to the complicated mathematical treatments, e.g. fractional differential equations in later works, and the results are difficult to transfer to other cases, such as dispersive transport in OTFT, owing the many assumptions that accompany the derivations. For example, a constant electric field and relatively low (on average) concentration of the induced excessive (non-equilibrium) charge are assumed during the ToF experiments.

Nevertheless, the works on MTR, especially the earlier ones [97, 98, 99, 108, 110], have introduced the concept for the transient demarcation between charges in thermal quasi-equilibrium and charges in nonequilibrium, the latter gradually vanishing with time. The demarcation evolves with time, describing the gradual process of the so-called "thermalization" of the charge from non-equilibrium state into thermal quasi-equilibrium state, the latter described by the Fermi statistics. The idea behind the thermalization is that the thermodynamic relaxation process needs time $\mathrm{t} \approx \tau$ to perform a change $\Delta \mathrm{E}$ of the energy of a charge, and the time (either for climbing the DOS up by phonon absorption or relaxing down by phonon emission) is longer, if the two energy states are far apart each other or from the Fermi level EF. For VRH, mathematically this is equivalent to vary the Fermi energy with $\Delta \mathrm{E}$ in eq. (6), from which, in the form of eq. (120), one gets that

$$
\frac{\mathrm{t} \approx \tau}{\tau_{\min }}=\exp \left[\frac{\Delta \mathrm{E}(\mathrm{t})}{\mathrm{kT}}\right], \text { where } \tau_{\min } \sim \tau_{\mathrm{o}} \exp (6) \sim \frac{1}{\Gamma_{\mathrm{o}}} \exp \left(\frac{\mathrm{s}_{\mathrm{cT} \infty}}{2}\right) \sim \mathrm{ps}
$$

as one can see in Figure 37, recalling also from eq. (61) that $\mathrm{s}_{\mathrm{cT} \infty} \sim 10$ is solely for tunneling by VRH at very high temperature $\mathrm{T} \rightarrow \infty$. The charge with $\tau<\mathrm{t}$ is in thermal quasi-equilibrium, because there was enough time for this charge to relax into the new state, whereas the charge with $\tau>t$ is in non-equilibrium, still "being" in the preceding state before $\mathrm{t}=0$. The demarcation energy $\Delta \mathrm{E}$ describes the boundaries around the Fermi or 
quasi-Fermi levels, within which the charge is in thermal quasi-equilibrium at time $0<\mathrm{t}<\infty$ during a transient process. Thus, the demarcation energy $\Delta \mathrm{E}=\mathrm{kT} \times \ln \left(\mathrm{t} / \tau_{\min }\right)$ moves the quasi-Fermi level IMREF from the value at time $\mathrm{t}=0$ toward the value for $\mathrm{t}=\infty$ by a rate of magnitude

$$
\frac{\partial \mathrm{IMREF}}{\partial \mathrm{t}}=\frac{\partial \Delta \mathrm{E}(\mathrm{t})}{\partial \mathrm{t}}=\frac{\mathrm{kT}}{\mathrm{t}} \approx \frac{\mathrm{kT}}{\tau}
$$

The actual time variation of IMREF in OTFT is more complicated, considering the definition $\mathrm{IMREF}=\left(\mathrm{E}_{\mathrm{F}}+\mathrm{q} \mathrm{V}_{\mathrm{B}}\right)$ by eq. (23) with the potential bending $\mathrm{V}_{\mathrm{B}}$ included, because, in contrast to ToF in a sandwiched film, $V_{B}$ in OTFT is not constant by a step change of the OTFT bias at $t=0$. The complication is due to the discontinuity at $\mathrm{t}=\tau$ between thermalized and non-equilibrium charges, the former tending to the state at $\mathrm{t}=\infty$, whereas the latter still "memorizing" the state before $\mathrm{t}=0$, while the electrostatic balance of charge (by the Gauss law) at the semiconductor-gate dielectric interface is instantaneous. There is no suggestion in the literature for a closed-form formula that can describe the variation of $\mathrm{V}_{\mathrm{B}}$ simultaneously in time and in the semiconductor depth. Also, there is no clear guideline on how one can calculate VRH conductance by time-varying discontinuity of the quasi-Fermi level and when the charge is not in thermal equilibrium. Below, we give a procedure, based on simplified parameterization with respect to hopping time $\tau$ and demarcation at time $\mathrm{t}$, so that $\tau<\mathrm{t}$ corresponds to the emerging new state at thermal quasi-equilibrium, while $\mathrm{t}<\tau$ corresponds to the gradually vanishing old state of non-equilibrium.

The procedure for transient VRH calculations with demarcation considers the following.

- The states of the charge and VRH are known before $(t \leq 0$, past state) and after $(t=\infty$, future state) the transient process, as given by stationary (static, or DC) calculations, including distributions $\partial \mathrm{X} / \partial \tau$ for the quantities $\mathrm{X}$ related to the hopping in these states, i.e., $\mathrm{X}=\mathrm{X}(\tau)$ and $\partial \mathrm{X} / \partial \tau=\partial\left(\mathrm{B} / \mathrm{B}_{\mathrm{c}}\right) / \partial \tau$ for hopping time $\tau$ in Figure 37 and Figure 38 for the state under gate bias of the OTFT and bulk semiconductor (no-bias off-state of the OTFT). To distinguish a quantity $\mathrm{X}$ in the "past" and "future" states, we include in the notations additional subscripts 0 (zero) and $\infty$ (infinite), e.g., $\mathrm{X}_{0}$ and $\mathrm{X}_{\infty}$, respectively for $(\mathrm{t} \leq 0)$ and $(\mathrm{t}=\infty)$. 
- Depth dependences can be and are reduced by proper superposition or weighted averaging along the depth $\mathrm{D}$ in the semiconductor, $0 \leq \mathrm{D} \leq \mathrm{t}_{\mathrm{f}}$, with $\mathrm{D}=0$ being the semiconductor-gate insulator interface and $\mathrm{t}_{\mathrm{f}}$ being the thickness of the semiconductor film, thus $\mathrm{D}=\mathrm{t}_{\mathrm{f}}$ corresponds to the back of the film. For quantities $X$, which have meaning in sheet representation $X_{s q}=\int X(D) d D$, e.g., sheet conductance $\sigma_{\mathrm{sq}}$ for specific conductance $\sigma(\mathrm{D})$, the superposition is an integration along the depth $\mathrm{D}$ in the semiconductor. According to the Leibniz rule for differentiation under the integral, the superposition is also valid for derivatives. Therefore,

$$
\frac{\partial X_{s q}(\tau)}{\partial \tau}=\int_{D=0}^{D=t_{f}} \frac{\partial X(\tau, D)}{\partial \tau} d D \text {, distribution of sheet quantity } X_{s q}
$$

For other quantities (that do not have meaning in sheet representation), such as hopping time $\tau$, bonds per hopping site $\mathrm{B}$, or mobility, one uses the averaging with a weighting function $\mathrm{Y}(\mathrm{D})$. The weighting function $Y(D)$ is the profile of a quantity, which is assumed stationary, e.g., $Y(D)=N_{C}(D)$ can be the profile of the charge concentration $\mathrm{N}_{\mathrm{C}}$ at $\mathrm{t}=\infty$. Then, the weighted average is given as

$$
\frac{\partial X_{A V G}(\tau)}{\partial \tau}=\frac{\int_{D=0}^{D=t_{f}} \frac{\partial X(\tau, D)}{\partial \tau} Y(D) d D}{\int_{D=0}^{D=t_{f}} Y(D) d D} \text {, average distribution of } X
$$

The thick lines (red-colored) in Figure 38 illustrate calculations with eq. (131) for the average of the distribution $\partial\left(\mathrm{B} / \mathrm{B}_{\mathrm{c}}\right) / \partial \tau$ of the hopping time $\tau$ in a pentacene OTFT at different temperatures $\mathrm{T}$ and at a gate bias voltage $\mathrm{V}_{\mathrm{G}}=-20 \mathrm{~V}$. The profiles $\mathrm{N}_{\mathrm{C}}(\mathrm{D})$ of the charge concentration $\mathrm{N}_{\mathrm{C}}$ were used as the weighting function $\mathrm{Y}(\mathrm{D})=\mathrm{N}_{\mathrm{C}}(\mathrm{D})$ to calculate the weighted averages. The profiles are after static VRH calculations, and have been shown earlier in Figure 13.

- Any quantity $X(\tau)$ can be expressed explicitly as a function of hopping time $\tau$, or in a differential form as a distribution density function of the hopping time $\tau$, e.g., $\partial \mathrm{X} / \partial \tau=\partial \mathrm{X} / \partial\left(\mathrm{B} / \mathrm{B}_{\mathrm{c}}\right) \times \partial\left(\mathrm{B} / \mathrm{B}_{\mathrm{c}}\right) / \partial \tau$. With respect to transient VRH, both $\partial \mathrm{X} / \partial\left(\mathrm{B} / \mathrm{B}_{\mathrm{c}}\right)$ and $\partial\left(\mathrm{B} / \mathrm{B}_{\mathrm{c}}\right) / \partial \tau$ can be different in the "past" and "future" states, before and after the transient process, respectively, but the dependences in the "past" 
and "future" states as functions of the hopping time $\tau$ are identical with the known stationary dependences, which are deduced from the static VRH calculations for both the "past" and the "future" states. There is transient demarcation at time $t$, which switches the values of quantities and dependences from "past" state to "future" state, when $\tau=\mathrm{t}$ in a manner explained in the next bullet. Inferred from the concept for critical path by VRH, one constraint for the differential form $\partial \mathrm{X} / \partial \tau$ is the correspondence to the static value $\mathrm{X}_{\text {STAT }}$ obtained from the static VRH calculations. The static VRH calculations neglect the bonds with slow hopping rate $\Gamma<\Gamma_{\mathrm{c}}$ as non-contributing to the continuous hopping, thus $\tau>\tau_{\mathrm{c}}$ is not considered in the static VRH calculation of Xstat. Therefore, the correspondence between the distribution $\partial \mathrm{X} / \partial \tau$ and the static value $\mathrm{X}_{\mathrm{STAT}}$ of the quantity $\mathrm{X}$ is by an integration up to the finite hopping time $\tau_{\mathrm{c}}$ for the critical path, that is

$$
\mathrm{X}_{\mathrm{STAT}}(\mathrm{D})=\int_{\tau=0}^{\tau_{\mathrm{c}}} \frac{\partial \mathrm{X}(\tau, \mathrm{D})}{\partial \tau} \mathrm{d} \tau \text {, correspondence to static VRH at every depth D. }
$$

Note that the integration is not to an infinite limit of $\tau=\infty$, but to the finite limit $\tau_{\mathrm{c}}$ for the critical path, where $\tau_{\mathrm{c}}=1 / \Gamma_{\mathrm{c}}$ was defined earlier between eqs. (114) and (115) and the expression for $\tau_{\mathrm{c}}$ is also given in eq. (118) as a function of the hopping energy $\mathrm{E}_{\mathrm{H}}$ at every given spot in the semiconductor. Since the static VRH does not assume spatial correlations, then $\mathrm{X}_{\text {STAT }}$ is spatially independent (valid for every spot in the semiconductor independently of any other spot), and we extrapolate this assumption also to the transient VRH. The main usage of the finite-limit integral correspondence in eq. (132) is to obtain the value (or expression) for the multiplicative coefficient in the distribution $\partial \mathrm{X} / \partial \tau$ of the quantity $\mathrm{X}$, so that for the hopping time $\tau_{\mathrm{c}}$ in the critical path, the distribution matches $\mathrm{X}_{\mathrm{STAT}}$ from the static VRH calculation. In fact, the finite-limit integral correspondence by eq. (132) is a normalization to the value $\mathrm{X}_{\mathrm{STAT}}$ in the critical path; and with eqs. (118) and (125), we have already performed the normalization of the hopping bonds $\mathrm{B}$ to the bonds $\mathrm{B}_{\mathrm{c}}$ for the critical path, when obtaining the distribution $\partial\left(\mathrm{B} / \mathrm{B}_{\mathrm{c}}\right) / \partial \tau$ of the hopping time $\tau$ in eqs. (117) and (123). In addition to the correspondence to static VRH, an advantage of the normalization to the critical path is that one can define and deal with 
distributions, which can be unbounded for $\tau=\infty$, c.f. the $1 / \tau$ distribution in eq. (124). However, one should also be aware of some consequences, e.g., the unbounded at $\tau=\infty$ distribution implies that the static state $\mathrm{X}_{\infty}$ for the quantity $\mathrm{X}$ may not exist precisely, and furthermore, a distribution density normalized to finite limit is not a probability distribution, since $\left(1 / \mathrm{X}_{\mathrm{STAT}}\right) \times \int^{\infty} \partial \mathrm{X} / \partial \tau \mathrm{d} \tau \neq 1$.

- The transient process can be and it is sufficiently accurately described by the demarcation with time and the fractional superposition of state $t=0$ and state $t=\infty$, so that a window from 0 to $t$ corresponds to a sum of the thermalized "fraction" of the future state $t=\infty$ (with $\tau<t$ ) and the non-equilibrium "fraction" of the past state $t=0$ (with $\tau>t$ ), the former continuously expanding with $\mathbf{t}$, while the latter gradually vanishing with $\mathbf{t}$. From the static VRH calculations of a variable $\mathrm{X}$ in the "past" state and in the "future" state, one obtains $\mathrm{X}_{0}(\tau), \mathrm{X}_{\infty}(\tau)$ and the corresponding distribution densities $\partial \mathrm{X}_{0} / \partial \tau, \partial \mathrm{X}_{\infty} / \partial \tau$ with respect of the hopping time $\tau$ for the "past" and "future" states. Then one can find the "fractions" by

$\mathrm{X}_{\mathrm{NEW}}(\mathrm{t})=\int_{0}^{\mathrm{t}} \frac{\partial \mathrm{X}_{\infty}(\tau)}{\partial \tau} \mathrm{d} \tau=\mathrm{X}_{\infty}(\tau=\mathrm{t})$ for the expanding thermalized fraction with $\mathrm{X}_{\infty}(\tau=0)=0$

$\mathrm{X}_{\mathrm{OLD}}(\mathrm{t})=\int_{\mathrm{t}}^{\infty} \frac{\partial \mathrm{X}_{0}(\tau)}{\partial \tau} \mathrm{d} \tau=\mathrm{X}_{0}(\tau=\infty)-\mathrm{X}_{0}(\tau=\mathrm{t})$ for the vanishing non-equilibrium fraction.

Consequently, the superposition yields the transient value of the quantity, as

$$
\mathrm{X}(\mathrm{t})=\mathrm{X}_{\mathrm{NEW}}(\mathrm{t})+\mathrm{X}_{\mathrm{OLD}}(\mathrm{t}) \text { at time } \mathrm{t}
$$

Strictly speaking, the considerations in the above four bullets are not perfect, but are approximations. The first consideration can be obscured due to a divergence by integrating power-law functions, since $\int^{\infty} \mathrm{x}^{\mathrm{n}} \mathrm{dx}=\infty$, if $\mathrm{n} \geq(-1)$, thus, a perfect stationary state might not exist. The second consideration can also be questioned for precision, because one may use different weighting functions for different quantities, and the selection of the type of the weighting function requires further considerations. For example, one can choose the electrostatic charge profile $\mathrm{N}_{C}(\mathrm{D})$, the $\mathrm{VRH}$ specific conductance profile $\sigma(\mathrm{D})$ or their product as weighting functions by calculating the distribution of the hopping time $\tau$, when analyzing the low frequency 
noise in terms of number, mobility or correlated number-mobility fluctuations. We will show an attempt of such an analysis later. The third and fourth considerations assume that there is no correlation between the "past" and "future" states and the transition is a step function at the demarcation $\tau=\mathrm{t}$, whereas, the transition region around the demarcation is not sharp, but for about $2 \mathrm{kT}$ (twice the thermal energy $\mathrm{kT}$ ), which causes a transition for a time decade, since $\Delta \mathrm{t} / \mathrm{t} \sim \exp (2 \mathrm{kT} / \mathrm{kT}) \sim 7.4$ follows from eqs. (128) and (129). In addition, the third consideration neglects the instantaneous balance of the charge at the gate dielectric interface (by Gauss' law), assuming that the charge in the film at $\mathrm{t}=0_{+}$immediately after the step of the bias of the OTFT is the same as the charge at the quasi-equilibrium state at $t=\infty$. Thus the potential bending is constant in the entire interval from $t=0$ to $t=\infty$, and the new state of bending does not affect either the charge or the bending in the old state for $\mathrm{t}<0$, which is not exactly what occurs in an OTFT. Nevertheless, although the above four considerations are not perfect, we believe that the considerations and equations are sufficient to capture in mathematically simple way the behavior and the order of magnitudes during the transient processes after stepping the gate bias of the OTFT, which would be helpful to explain some experimental observations by the lack of theory for VRH dispersive transport in OTFT at present.

\subsection{Transient hopping transport}

We now proceed to the implementation of the procedure for transient VRH calculations with time demarcation, outlined in the above four bullets. The implementation addresses the operation of the OTFT in the linear (Ohmic) regime, in which, according to eq. (51), the drain current $I_{D}$ is proportional to the sheet conductance $\sigma_{\mathrm{sq}}$, and $\sigma_{\mathrm{sq}}$ is nearly a constant for any position along the channel width $\mathrm{W}$ and length $\mathrm{L}$. Thus, we will analyze the channel conductance as function of time after the step of the gate bias voltage $\mathrm{V}_{\mathrm{G}}$, and the current $I_{D}=(W / L) V_{D} \sigma_{s q}$ will be assumed always accessible from the results for $\sigma_{s q}$ by scaling $\sigma_{s q}$ with the product of the ratio $\mathrm{W} / \mathrm{L}$ and the drain bias voltage $\mathrm{V}_{\mathrm{D}}$. Therefore, we will present the results for the channel conductance and omit the scaled version of these results for the channel current in the OTFT. Furthermore, we shall analyze two transitions, from off-state to on-state followed by the opposite transition from on-state 
to off-state of the OTFT. The off-state corresponds to the gate bias $\mathrm{V}_{\mathrm{G}}=\mathrm{V}_{\mathrm{FB}} \approx 0$ equal to the flat-band $\mathrm{V}_{\mathrm{FB}}$, so that the off-state at $t=\infty$ is the same as the equilibrium state of the bulk semiconductor. The on-state corresponds to $\mathrm{V}_{\mathrm{G}}=-20 \mathrm{~V}$ for the pentacene OTFT from [73], which is chosen to preserve the correspondence to preceding figures, formulas for exponential type of DOS and the values of the parameters in Table IV for this device, specifically, for static VRH calculations with multiple $\iint \mathrm{dEdE}$ integrations by assumption for double-exponential DOS $\mathrm{DE}$.

One obtains the distribution of the VRH specific conductance from

$$
\frac{\partial \sigma(\tau, \mathrm{D})}{\partial \tau}=\frac{\partial \sigma}{\partial \mathrm{B}} \frac{\partial \mathrm{B}}{\partial \tau}=\frac{\partial \sigma}{\partial \mathrm{B}} \mathrm{B}_{\mathrm{c}} \frac{\partial \mathrm{B} / \mathrm{B}_{\mathrm{c}}}{\partial \tau}
$$

where $B_{c} \sim 9 / \pi \approx 2.86$ is the constant that represents the "geometrical" assumption for sufficient bonds per site by continuous hopping in the percolation network, see eqs. (3) and (5), and the distributions $\partial\left(\mathrm{B} / \mathrm{B}_{\mathrm{c}}\right) / \partial \tau$ for the hopping time $\tau$ are obtained as explained above by eqs. (114) to (121). The values for $\partial\left(\mathrm{B} / \mathrm{B}_{\mathrm{c}}\right) / \partial \tau$ were shown for different temperatures in Figure 38, both for bulk semiconductor (off-state) and for different depths $\mathrm{D}$ in the semiconducting film at gate bias $\mathrm{V}_{\mathrm{G}}=-20 \mathrm{~V}$ (on-state). One can obtain an expression for the derivative $\partial \sigma / \partial \mathrm{B}$, considering that in principle the fraction of conductivity $\partial \sigma=b \Gamma \partial \mathrm{B}$ is proportional to the fraction of the bonds $\partial \mathrm{B}$ that are associated with a hopping rate $\Gamma$ and $\mathrm{b}$ is a constant that will be determined by the normalization to $\sigma$ for the critical path by means of eq. (132). Since we have defined $\tau=1 / \Gamma$ before eq. (114), then

$$
\frac{\partial \sigma(\tau, \mathrm{D})}{\partial \tau}=\frac{\partial \sigma}{\partial \mathrm{B}} \mathrm{B}_{\mathrm{c}} \frac{\partial \mathrm{B} / \mathrm{B}_{\mathrm{c}}}{\partial \tau}=\mathrm{b} \Gamma \mathrm{B}_{\mathrm{c}} \frac{\partial \mathrm{B} / \mathrm{B}_{\mathrm{c}}}{\partial \tau}=\frac{\mathrm{bB}}{\tau} \frac{\partial \mathrm{B} / \mathrm{B}_{\mathrm{c}}}{\partial \tau}
$$

showing that the distribution of the VRH specific conductivity is proportional to the distribution $\partial\left(\mathrm{B} / \mathrm{B}_{\mathrm{c}}\right) / \partial \tau$ of the hopping time $\tau$, divided by the hopping time $\tau$ itself. Therefore, one can rewrite the approximations for $\partial\left(\mathrm{B} / \mathrm{B}_{\mathrm{c}}\right) / \partial \tau$ of eqs. (122), (123) and (124) also for $\partial \sigma / \partial \tau$, as

$$
\begin{aligned}
& \frac{\partial \sigma}{\partial \tau} \approx 0, \text { for } \tau<\tau_{\mathrm{o}},(\text { faster than the transport in OTFT), } \\
& \frac{\partial \sigma}{\partial \tau} \approx \mathrm{bB} \mathrm{B}_{\mathrm{c}} \frac{\mathrm{n}+1}{\tau_{\mathrm{c}}^{\mathrm{n}+1}} \tau^{\mathrm{n}-1}=\mathrm{A} \tau^{\mathrm{n}-1}, \text { for } \tau_{\mathrm{o}}<\tau<\tau_{\mathrm{c}}=\tau_{\mathrm{o}} \exp \left(\mathrm{s}_{\mathrm{c}}\right), \text { (dispersive transport), }
\end{aligned}
$$


where $\mathrm{s}_{\mathrm{c}}=\mathrm{E}_{\mathrm{H}} / \mathrm{kT}=2 \mathrm{R}_{\mathrm{H}} / \Lambda_{\mathrm{o}}>\mathrm{s}_{\mathrm{c} T \infty}$, according to the principal VRH eq. (2), and

$$
\frac{\partial \sigma}{\partial \tau} \approx \mathrm{bB}_{\mathrm{c}} \frac{\mathrm{n}+1}{\tau^{2}}=\frac{\mathrm{A} \tau_{\mathrm{c}}^{\mathrm{n}+1}}{\tau^{2}} \text {, for } \tau_{\mathrm{c}}<\tau \text {, (no transport, just fluctuations and charge trapping). }
$$

The value of the normalization coefficient $A$ is then obtained by substituting eq. (139) in eq. (132) for the correspondence to $\sigma$ from static VRH calculation for the critical path. After the substitution, one gets

$$
\sigma(D)=\int_{\tau_{\mathrm{o}}}^{\tau_{\mathrm{c}}} \mathrm{bB} \mathrm{B}_{\mathrm{c}} \frac{\mathrm{n}+1}{\tau_{\mathrm{c}}^{\mathrm{n}+1}} \tau^{\mathrm{n}-1} \mathrm{~d} \tau \approx \mathrm{A} \times\left\{\begin{array}{l}
\tau_{\mathrm{o}}^{\mathrm{n}} /(-\mathrm{n}), \text { if } 0>\mathrm{n}>-1 \\
\ln \left(\tau_{\mathrm{c}} / \tau_{\mathrm{o}}\right), \text { if } \mathrm{n} \approx 0 \\
\tau_{\mathrm{c}}^{\mathrm{n}} / \mathrm{n}, \text { if } \mathrm{n}>0
\end{array}\right.
$$

since $\tau_{\mathrm{c}}>>\tau_{\mathrm{o}}$, and, therefore, the normalization coefficient $\mathrm{A}$ is given by

$$
\mathrm{A} \approx \sigma(\mathrm{D}) \times\left\{\begin{array}{l}
(-\mathrm{n}) / \tau_{\mathrm{o}}^{\mathrm{n}}, \text { if } 0>\mathrm{n}>-1 \text { for } \mathrm{T}<\mathrm{T}_{\mathrm{o}} \\
1 / \mathrm{s}_{\mathrm{c}}, \text { if } \mathrm{n} \approx 0 \text { at } \mathrm{T} \approx \mathrm{T}_{\mathrm{o}}, \text { with } \mathrm{s}_{\mathrm{c}}=\ln \left(\tau_{\mathrm{c}} / \tau_{\mathrm{o}}\right)>\mathrm{s}_{\mathrm{cT} \infty} \sim 10 \\
\mathrm{n} / \tau_{\mathrm{c}}^{\mathrm{n}}, \text { if } \mathrm{n}>0 \text { at } \mathrm{T}>\mathrm{T}_{\mathrm{o}}
\end{array}\right.
$$

The different cases in eq. (142) imply that the normalization coefficient $\mathrm{A}$ in the expressions of the analytical approximation for the distribution $\partial \sigma / \partial \tau$ of the VRH conductance, eq. (139) and eq. (140), can be evaluated from the specific conductance $\sigma$ itself and the value of the hopping time $\tau$, for which the distribution $\partial\left(\mathrm{B} / \mathrm{B}_{\mathrm{c}}\right) / \partial \tau$ of the hopping time $\tau$ has maximum. From Figure 38 , the maximum in the distribution $\partial\left(\mathrm{B} / \mathrm{B}_{\mathrm{c}}\right) / \partial \tau$ is near $\tau_{\mathrm{o}} \exp (3)$ at low temperature $\left(\mathrm{T}<\mathrm{T}_{\mathrm{o}}\right)$, near $\tau_{\mathrm{c}} / \exp (3)$ at high temperature $\left(\mathrm{T}>\mathrm{T}_{\mathrm{o}}\right)$, and close to the geometric mean $\left(\tau_{\mathrm{o}} \tau_{\mathrm{c}}\right)^{1 / 2}$ at $\mathrm{T}=\mathrm{T}_{\mathrm{o}}$. Thus, once the VRH conductance $\sigma$ and the hopping time distribution $\partial\left(\mathrm{B} / \mathrm{B}_{\mathrm{c}}\right) / \partial \tau$ are known (e.g., from simulation), the normalization coefficient $\mathrm{A}$ can be calculated from eq. (142), because either $\tau_{\mathrm{o}}, \tau_{\mathrm{c}}$ or $\left(\tau_{\mathrm{o}} \tau_{\mathrm{c}}\right)$ can be found from the position of the maximum in the distribution $\partial\left(\mathrm{B} / \mathrm{B}_{\mathrm{c}}\right) / \partial \tau$, and $\mathrm{n}$ is the slope of the tilted "plateau" in the distribution $\partial\left(\mathrm{B} / \mathrm{B}_{\mathrm{c}}\right) / \partial \tau$ and $\mathrm{n}=\left(\mathrm{T} / \mathrm{T}_{\mathrm{o}}-1\right)$, according eq. $(127)$.

As given by eq. (127), $\mathrm{n}=\left(\mathrm{T} / \mathrm{T}_{\mathrm{o}}-1\right)$ for exponential DOS, $\tau_{\mathrm{o}}=1 / \Gamma_{\mathrm{o}}$ is a parameter and $\tau_{\mathrm{c}}=\tau_{\mathrm{o}} \exp \left(\mathrm{E}_{\mathrm{H}} / \mathrm{kT}\right)$ is easily obtained from eq. (118), because the values in the profile $E_{H}(D)$ of the hopping energy $E_{H}$ in the critical path in the depth D of the semiconductor film are gathered by the numerical VRH simulator. Thus, the values of normalization coefficients A are calculated using eq. (142) and substituted in eqs. (139) and 
(140) to yield approximations for the distributions $\partial \sigma / \partial \tau$ of the VRH specific conductance $\sigma$, as shown with dotted lines in Figure 39. The thin solid lines in this figure are calculated numerically according to eq. (137), reusing the data for the distributions $\partial\left(\mathrm{B} / \mathrm{B}_{\mathrm{c}}\right) / \partial \tau$ for the hopping time $\tau$, dividing the values for $\partial\left(\mathrm{B} / \mathrm{B}_{\mathrm{c}}\right) / \partial \tau$ on $\tau$, and performing the normalization numerically. The numerical normalization determines $b$ in eq. (137) so that the numerical integration of the last expression of eq. (137) from zero to $\tau=\tau_{\mathrm{c}}$ matches the value of the static conductance $\sigma(\mathrm{D})$ known from the numerical simulation. The thick lines are the distributions $\partial \sigma_{\mathrm{sq}} / \partial \tau$ of the sheet conductance $\sigma_{\mathrm{sq}}$, which are obtained after numerical integration by

$$
\frac{\partial \sigma_{\mathrm{sq}}(\tau)}{\partial \tau}=\int_{\mathrm{D}=0}^{\mathrm{D}=\mathrm{t}_{\mathrm{f}}} \frac{\partial \sigma(\tau, \mathrm{D})}{\partial \tau} \mathrm{dD}, \text { distribution of the sheet conductance } \sigma_{\mathrm{sq}}
$$

according to eq. (130) with $\mathrm{X}=\sigma$. The profiles $\sigma(\mathrm{D})$ of the VRH specific conductance in the depth $\mathrm{D}$ of the film have been shown earlier in Figure $14(\mathrm{c})$, and the sheet conductance is $\sigma_{\mathrm{sq}}=\int \sigma \mathrm{dD}$ (see eq. (41)). For the bulk material, equivalent to the off-state of the OTFT as mentioned above, the integration is replaced with multiplication by the thickness $\mathrm{t}_{\mathrm{f}}$ of the semiconductor film, $\partial \sigma_{\mathrm{sq}} / \partial \tau=\mathrm{t}_{\mathrm{f}} \times \partial \sigma / \partial \tau$, as in eq. (40).

By comparing the dotted with the thin lines in Figure 39, one observes that the approximations with eqs. (139) and (140) are consistent with the distributions $\partial \sigma / \partial \tau$ of the VRH specific conductance $\sigma$ obtained from the numerical simulations at different depths in the organic semiconductor of the OTFT. However, the approximations are not exact, there are deviations of several orders of magnitude at some places, and also, one needs to tune the values of $\tau_{\mathrm{o}}$ and $\tau_{\mathrm{c}}$, as stated in the caption of the figure, in order to obtain a good fitting between the numerical and analytical calculations. Therefore, we will use the data from the numerical calculation in the next step of calculation of the transient conductance. In particular, we will use the data shown by the labeled thick lines in Figure 39 for the distributions $\partial \sigma_{\mathrm{sq}} / \partial \tau$ of the sheet conductance $\sigma_{\mathrm{sq}}$ when switching between off- and on-states of the OTFT. The off-state corresponds to label " $\partial \sigma_{\mathrm{sq}} / \partial \tau$ of bulk". The on-state corresponds to label " $\partial \sigma_{\mathrm{sq}} / \partial \tau$ at $\mathrm{V}_{\mathrm{G}}$ ".

The transients of the sheet conductance $\sigma_{\mathrm{sq}}$ of an OTFT are shown in Figure 40, as calculated numerically using eqs. (133), (134) and (135) with demarcation between on- and off-states at time $t=\tau$. The 
steps of the gate bias voltage $V_{G}$ are depicted on the top of the plots for the sheet conductance $\sigma_{\text {sq. }}$. The lefthand side in the figure corresponds to the transition from off- to on-state, while the right-hand side corresponds to a following transition from on- to off-state. The lines in the left-hand plot are $\mathrm{X}_{\mathrm{NEW}}$ and $\mathrm{X}_{\mathrm{OLD}}$, calculated, respectively, using the distributions $\partial \sigma_{\mathrm{sq}} / \partial \tau$ of the sheet conductance $\sigma_{\mathrm{sq}}$ at $\mathrm{V}_{\mathrm{G}}=-20 \mathrm{~V}$ for $\partial \mathrm{X}_{\infty} / \partial \tau$ and the distribution for bulk semiconductor for $\partial \mathrm{X}_{0} / \partial \tau$. Thus, the circles in the left-hand plot represent the transition $\sigma_{\mathrm{sq}}(\mathrm{t})=\mathrm{X}(\mathrm{t})=\left[\mathrm{X}_{\mathrm{NEW}}\left(\mathrm{V}_{\mathrm{G}}\right)+\mathrm{X} \mathrm{OLD}(\mathrm{bulk})\right]$ of the sheet conductance to the on-state of the OTFT. We observe that the transition from off- to on-state is very fast and depends on the thermalization of the new state $\mathrm{X}_{\mathrm{NEW}}$ of the applied on-bias voltage $\mathrm{V}_{\mathrm{G}}=-20 \mathrm{~V}$.

Conversely, in the right-hand plot, the lines are after swapping the distributions used to calculate $\mathrm{X}_{\mathrm{NEW}}$ and $\mathrm{X}_{\mathrm{OLD}}$. The calculation for $\mathrm{X}_{\mathrm{NEW}}$ uses the distribution for bulk semiconductor as $\partial \mathrm{X}_{\infty} / \partial \tau$ in eq. (133). The calculation of $\mathrm{X}_{\mathrm{OLD}}$ uses the distribution at $\mathrm{V}_{\mathrm{G}}=-20 \mathrm{~V}$ as $\partial \mathrm{X}_{0} / \partial \tau$ in eq. (134). Thus, the circles in the right-hand plot represent the transition $\sigma_{\mathrm{sq}}(\mathrm{t})=\mathrm{X}(\mathrm{t})=\left[\mathrm{X}_{\mathrm{NEW}}(\mathrm{bulk})+\mathrm{X}_{\mathrm{OLD}}\left(\mathrm{V}_{\mathrm{G}}\right)\right]$ of the sheet conductance toward the off-state of the OTFT. These transitions toward the off-state are much slower, especially at low temperature, owing to the long time needed for the thermal relaxation of the previous conducting state. Looking closer at the values, one observes that the thermal relaxation of the OTFT conductance is in the range of a large fraction to several seconds at room temperature $\mathrm{T}=300 \mathrm{~K}$, and may take weeks for the lower temperature of $200 \mathrm{~K}$. Therefore, the transition of the OTFT from conductive to non-conductive state is a highly non-equilibrium process, which causes hysteresis and threshold shifts in the OTFT, as reported many times in the literature and summarized in [27] for the charge build-up in OTFT. Here, we deduce from Figure 40 that the dispersed hopping time causes long-lived tails in the VRH conductance, which are in addition with the charge build-up. 


\subsection{Random fluctuations (noise) in the hopping transport}

The last topic in this work is on the ability of VRH to predict the low-frequency noise (LFN) in OTFT. The approach to the problem considers two basic relations. The first is the phenomenological Hooge equation for the normalized flicker (1/f) noise $[115,116]$, given by

$$
\mathrm{S}_{\mathrm{NORM}}(\mathrm{f})=\frac{\mathrm{S}(\mathrm{f})}{\mathrm{DC}^{2}}=\frac{\alpha_{\mathrm{H}}}{\mathrm{N}_{\mathrm{TOT}} \times \mathrm{f}^{\beta}}, \quad \beta \approx 1, \mathrm{~N}_{\mathrm{TOT}}=\mathrm{WLQ}_{\mathrm{G}} / \mathrm{q} \approx \mathrm{WLC}_{\mathrm{OX}} \mathrm{V}_{\mathrm{G}} / \mathrm{q}
$$

where $\mathrm{S}_{\mathrm{NORM}}(\mathrm{f})$ is the normalized power-spectrum density $(\mathrm{PSD})$ of the flicker noise in unit $[1 / \mathrm{Hz}]$, being a ratio of the power-spectrum density $\mathrm{S}(\mathrm{f})$ of the LFN of a quantity, (e.g., current, voltage, mobility, conductance, resistance, etc.) to the square of the stationary (DC) value for this quantity. The other notations in eq. (144) are: $\mathrm{N}_{\text {TOт }}$ is the total number (on average) of moving entities, which experience fluctuation in their motion, that is, $\mathrm{N}_{\text {TOT }}$ is the total number of charge carriers in the electronic device (OTFT); $\alpha_{\mathrm{H}}$ is the Hooge parameter, which is a number that depends on materials, but is nearly independent of bias and temperature; $f$ is the frequency in $[\mathrm{Hz}]$ and $\beta \approx 1$ is the frequency exponent of the flicker LFN. If $D C$ is in unit $[u]$, then the noise power-spectrum density $S(f)$ is in unit $\left[\mathrm{u}^{2} / \mathrm{Hz}\right]$. Also, if $\beta=1$, then $\alpha_{\mathrm{H}}$ is purely numeric, otherwise, the unit of $\alpha_{\mathrm{H}}$ is $\left[(\mathrm{Hz})^{\beta-1}\right]$.

As follows from Hooge eq. (144), $\alpha_{\mathrm{H}}$ is the magnitude of the normalized noise $\mathrm{S}_{\mathrm{NORM}}$ for one carrier $\mathrm{N}_{\text {TOT }}=1$ at frequency $\mathrm{f}=1 \mathrm{~Hz}$, and for other frequency and number of carriers

$$
\frac{\alpha_{\mathrm{H}}}{\mathrm{f}^{\beta}}=\mathrm{N}_{\mathrm{TOT}} \times \mathrm{S}_{\mathrm{NORM}}(\mathrm{f})=\mathrm{N}_{\mathrm{TOT}} \frac{\mathrm{S}(\mathrm{f})}{\mathrm{DC}^{2}},
$$

which is the complementary characterization format of the Hooge eq. (144). Behind eq. (144) are the statistically uncorrelated and invariant random variables (fluctuations) of population with size $\mathrm{N}_{\text {TOT }}$, for which the total variance $S=\sum S_{K}=N_{T O T} \times\left\langle S_{K}\right\rangle$ is the sum of the variances $S_{K}$ of the different fluctuations, $\mathrm{K}=1,2, \ldots, \mathrm{N}_{\mathrm{TOT}}$, and $\left\langle\mathrm{S}_{\mathrm{K}}\right\rangle=\left(\sum \mathrm{S}_{\mathrm{K}}\right) / \mathrm{N}_{\mathrm{TOT}}$ denotes the average variance that is attributed to each individual fluctuation in the population. Accordingly, the square of the standard deviation (noise) for all carriers in the population is the sum of squares of the standard deviations (noise) from each individual carrier, considering the above assumption for statistically uncorrelated random variables. 
On the other hand, the average DC is also proportional to the number of carriers, $\mathrm{DC}=\mathrm{N}_{\mathrm{TOT}} \times\langle\mathrm{DC}\rangle$, where $\left\langle\mathrm{DC}_{\mathrm{K}}\right\rangle=\left(\sum \mathrm{DC}_{\mathrm{K}}\right) / \mathrm{N}_{\mathrm{TOT}}$ is the average contribution of a carrier to DC. Thus, the ratio $\mathrm{S} / \mathrm{DC}^{2} \propto 1 / \mathrm{N}_{\mathrm{TOT}}$, from which follows eq. (144) by assuming that each carrier has a normalized noise equal to $\alpha_{H} / f^{\beta}$. One remark is that the OTFT aspect ratio W/L of channel width $\mathrm{W}$ to channel length $\mathrm{L}$ affects linearly DC and quadratically $\mathrm{S}(\mathrm{f})$, so, W/L is cancelled in the expression for $\mathrm{S}_{\mathrm{NORM}}$. One should not be confused with apparent $\mathrm{L}^{2}$ dependences in equations derived from eq. (144) for field-effect transistors. After careful inspection of the publications [117], one can always obtain $\mathrm{S}_{\mathrm{NORM}} \propto 1 / \mathrm{Area}=1 /(\mathrm{WL}) \propto 1 / \mathrm{N}_{\mathrm{TOT}}$ from these equations.

The second basic relation for LFN is due to the time distribution in the fluctuation of carrier motion. As follows from the mathematical suggestions in $[118,119]$, the normalized noise for a single carrier is given by the integral in the left-hand expression in the following equation

$$
\mathrm{a} \int_{\tau_{\min }}^{\tau_{\max }} \frac{\partial X_{A V G}(\tau)}{\partial \tau} \frac{\tau}{1+(2 \pi f \tau)^{2}} d \tau=S_{n 1}(f) \approx \frac{\alpha_{H}}{f^{\beta}}, \text { single-carrier normalized noise } S_{n 1}(f)
$$

where $\mathrm{a}=4$ for the bistable move-wait process with $50 \%$ duty cycle, which is a condition at which the average is equal to the amplitude of the fluctuation. If the process is asymmetric, then $a<4$, and we will take a 1 for VRH. Since the left-hand expression is for a single carrier, then we equate it to the right hand expression $\alpha_{\mathrm{H}} / \mathrm{f}^{\beta}$ for the flicker noise of a single carrier from eq. (145). Note that $\partial \mathrm{X}_{\mathrm{AVG}} / \partial \tau$ is given by eq. (131) for normalized distribution, e.g., for hopping time $\tau$, that is $\partial \mathrm{X} / \partial \tau=\partial\left(\mathrm{B} / \mathrm{B}_{\mathrm{c}}\right) / \partial \tau$ in eq. (131), as explicitly rewritten in eq. (147) below.

Thus, one can access the flicker noise in OTFT operating in the linear regime from numerical VRH simulation at given gate bias $\mathrm{V}_{\mathrm{G}}$ and temperature $\mathrm{T}$ by the following sequence:

Step 1. Obtain the distribution $\partial\left(B / B_{c}\right) / \partial \tau$ of hopping time $\tau$ for different depths $D$ in the semiconducting film, according to the procedure explained earlier by eqs. (117) and (121). 
Step 2. Perform appropriate averaging of $\partial\left(B / B_{c}\right) / \partial \tau$ to reduce the film depth D. One can obtain several average distributions $\partial\left(\mathrm{B} / \mathrm{B}_{\mathrm{c}}\right)_{\mathrm{AVG}} / \partial \tau$ that address different hypotheses for the noise, depending on the weighting function Y(D) used in eq. (131). In particular,

$$
\begin{aligned}
& \frac{\partial\left(B / B_{c}\right)_{A V G}}{\partial \tau}=\frac{\int_{D=0}^{D=t_{f}} \frac{\partial\left(B / B_{c}\right)}{\partial \tau} Y(D) d D}{D=t_{f}}, \text { with } \\
& \int_{D=0} Y(D) d D \\
& Y(D)=\left\{\begin{array}{l}
1, \text { at hypothesis (1) for } \Delta \tau \text { noise from hopping bonds } \\
\sigma(D) / N_{C}(D) \propto \mu(D), \text { at hypothesis (2) for } \Delta \mu \text { noise } \\
N_{C}(D), \text { at hypothesis (3) for } \Delta N \text { noise } \\
\left.\sigma(D) \propto N_{C}(D) \times \mu(D), \text { at hypothesis (4) for } \Delta \sigma \text { noise ( correlated } \Delta \mu-\Delta N\right) \\
\sigma(D) \times N_{C}(D), \text { at hypothesis (5) for correlated } \Delta \sigma-\Delta \text { noise }
\end{array}\right.
\end{aligned}
$$

Step 3. Choose a logarithmic frequency mesh $f$ in the range $f_{\min }-f_{\max }$. Obtain normalized noise spectra $S_{\mathrm{n} 1}(\mathrm{f})$ for single carrier at the different hypotheses by the left-hand expression of eq. (146), taking $\mathrm{a}=1$.

Step 4. Fit power-law functions to the single-carrier normalized noise spectra $S_{\mathrm{n} 1}(\mathrm{f})$ for the different hypotheses, using the right-hand expression of eq. (146). The values of the frequency exponent $\beta$ are the average slopes

$$
\beta=-\left.\frac{\partial \ln \left(\mathrm{S}_{\mathrm{n} 1}\right)}{\partial \ln (\mathrm{f})}\right|_{\mathrm{AVG}},
$$

and the values of the Hooge parameter $\alpha_{\mathrm{H}}$ are the values of the fitted power-law functions at $\mathrm{f}=1 \mathrm{~Hz}$.

Step 5. Obtain the normalized flicker noise $S_{\text {NORM }}$ of the OTFT for different hypotheses. As follows from eq. (144),

$$
\mathrm{S}_{\mathrm{NORM}}(\mathrm{f})=\frac{\mathrm{S}_{\mathrm{n} 1}(\mathrm{f})}{\mathrm{N}_{\mathrm{TOT}}}=\frac{\mathrm{S}_{\mathrm{n} 1}(\mathrm{f})}{\mathrm{WLQ}_{\mathrm{G}} / \mathrm{q}}, \quad \mathrm{Q}_{\mathrm{G}} \approx \mathrm{C}_{\mathrm{OX}} \mathrm{V}_{\mathrm{G}} .
$$

Step 6. De-normalize DC and flicker noise at different hypotheses, e.g., in terms of channel current 


$$
\begin{aligned}
& \mathrm{I}_{D}=\frac{\mathrm{W}}{\mathrm{L}} \sigma_{\mathrm{sq}} \mathrm{V}_{\mathrm{D}} \\
& \mathrm{S}_{\mathrm{ID}}(\mathrm{f})=\mathrm{S}_{\text {NORM }}(\mathrm{f}) \times \mathrm{I}_{\mathrm{D}}^{2}
\end{aligned}
$$

Step 7. Compare with experimental data to validate the most reasonable hypothesis, and if necessary, multiply the results for $\alpha_{\mathrm{H}}, \mathrm{S}_{\mathrm{NORM}}$ and $\mathrm{S}_{\mathrm{ID}}$ by numeric factor of $0.25<\mathrm{a}<4$, since $\mathrm{a}=1$ was used in Step 3 . If the values for the frequency exponent $\beta$ of the simulated flicker noise are different from the experimental data, then one should reconsider the type of DOS and repeat the entire VRH simulation.

To preserve correspondence with previous DC and transient analyses, we illustrate now the above steps for calculation of LFN with examples for the same pentacene OTFT and VRH calculations, as given in the caption of Figure 37. The results from the calculations by Step 1 for the distribution $\partial\left(B / B_{c}\right) / \partial \tau$ of the hopping time $\tau$ at different depths $\mathrm{D}$ in the semiconducting film are shown with thin solid lines in this Figure 37 and in the following Figure 38. The thick lines (red color) in the latter figure also depict the reduction of the film depth $D$ in Step 2 by weighted averaging with the charge profile $N_{C}(D)$, which corresponds to hypothesis (3) for $\Delta \mathrm{N}$ noise in eq. (147). The variation of $\partial\left(\mathrm{B} / \mathrm{B}_{\mathrm{c}}\right)_{\mathrm{AvG}} / \partial \tau$ by the change of the hypothesis is illustrated in Figure 41. Among the several observations stated in the caption of the figure, the significant one for LFN are that irrespectively of the hypothesis, bias and temperature, the average distributions $\partial\left(\mathrm{B} / \mathrm{B}_{\mathrm{c}}\right)_{\mathrm{AVG}} / \partial \tau$ coincide with virtually unique $1 / \tau$ distribution at high values of the hoping time $\tau>1 \mathrm{~ms}$, while temperature and bias-dependent deviations from $1 / \tau$ occur at low $\tau<10 \mu$ s. The significance of the observation becomes clear when performing Steps 3 and 4.

For Step 3, we choose $f_{\min }=1 \mathrm{~Hz}, \mathrm{f}_{\max }>100 \mathrm{kHz}$ and 8 frequencies in each decade, having the logarithmic frequency mesh of $\mathrm{f} \approx\{1,1.3,1.8,2.4,3.2,4.2,5.6,7.5\} \times 10^{\{1,2,3,4,5\}}$. For each of these frequencies, and for each of the average distributions $\partial\left(\mathrm{B} / \mathrm{B}_{\mathrm{c}}\right)_{\mathrm{AVG}} / \partial \tau$ at different hypotheses by eq. (147), we have performed numerically the integration in the left-hand expression of eq. (146), obtaining the points in the single-carrier normalized noise spectra $S_{n 1}(f)$ at several temperatures $T$ and gate bias voltages $V_{G}$, as shown in the main plots of Figure 42. Several observations can be made in these plots.

The first observation in the main plots of Figure 42 is that $S_{\mathrm{n} 1}(\mathrm{f})$ is nearly $1 / \mathrm{f}$ noise. 
The second observation in Figure 42 is that the change of the weighting function in eq. (147), thus the hypothesis for the origin of the noise, does not "dramatically" change the noise level, except for hypothesis (1) for $\Delta \tau$ noise, which fails at low temperature due to neglecting the highly non-uniform profiles of conductance and carriers in the depth of the film by using equal weights $Y=1$ in eq. (147).

Further details for the numerically calculated single-carrier noise spectra $S_{n 1}$ are obtained after performing Step 4 for fitting $S_{n 1}$ with the approximation $\alpha_{\mathrm{H}} / \mathrm{f}^{\beta} \approx \mathrm{S}_{\mathrm{n} 1}$, see eq. (146), in which the Hooge parameter $\alpha_{H}$ and the frequency exponent $\beta$ of the flicker noise are assumed frequency independent. The fitting lines are also shown in the main plots of Figure 42, and the fitting lines overlap with the numerically calculated spectra, although careful inspection showed 10\%-20\% difference between the numerically calculated spectra $S_{\mathrm{n} 1}$ and the approximation with $\alpha_{\mathrm{H}} / \mathrm{f}^{\beta}$. The obtained values for $\alpha_{\mathrm{H}}$ and $\beta$ are shown in the insets of Figure 42.

As depicted by arrows in the insets of the figure, the third observation in Figure 42 is that the noise levels increase in the order of hypotheses numbering (1)(2) (3) (4) (5), since the values of the Hooge parameter $\alpha_{H}$ increase in the same order (1) (2) (3)(4) (5) and the values of the frequency exponent $\beta$ of flicker noise decrease simultaneously in this order (1) (2) (3) (4) (5).

The fourth observation in the insets of Figure 42 is that there are weak temperature and bias dependences in $\alpha_{H}$ and $\beta$. As seen in the insets of Figure 42(a), and excepted for hypothesis (2) for $\Delta \mu$ noise, $\alpha_{H}$ and $\beta$ are linear functions of the reciprocal temperature $1 / T$, being much weaker than the Arrhenius exponential activation $\exp \left(\mathrm{E}_{\mathrm{A}} / \mathrm{kT}\right)$.

Note the zigzag in the insets of Figure 42(a), especially in the bottom-left inset for $\alpha_{H} v s .1 / T$, in which at low temperature (2) $\approx(3)$ and (4) $\approx$ (5) at the right-hand side of the inset, while at high temperature (2) $\approx$ (1) and (4) $\approx(3)$ at the left-hand side of the inset. This zigzag indicates crossovers between different noise generating mechanisms when changing the temperature, but the crossovers are very difficult to discriminate experimentally, because the temperature dependences are weak, causing changes within only a factor of 2 (3dB) for $\alpha_{H}$ and $5 \%-10 \%$ for $\beta$ in a wide temperature range from $200 \mathrm{~K}$ to $500 \mathrm{~K}$, whereas the noise 
measurements have normally instrumental uncertainties of at least $\pm 3 \mathrm{~dB}$ for spectrum magnitudes and $\pm 0.5 \mathrm{~dB} \sim \pm 12 \%$ for spectrum flatness. Weak logarithmic bias dependences are observed in the insets of Figure 42(b). There is virtually no bias dependence for $\alpha_{H}$ and $\beta$ by the hypothesis (1) for $\Delta \tau$ noise, there are minute dependences of about $\pm 3 \%$ /decade by the hypothesis (2) for $\Delta \mu$ noise, and the dependences gradually rise to $50 \% / \mathrm{dec}$ for $\alpha_{\mathrm{H}}$ and (-5)\%/dec for $\beta$ by hypotheses (3) (4) (5) for $\Delta \mathrm{N}, \Delta \sigma$ and correlated $\Delta \sigma-\Delta \mathrm{N}$ noise. These weak bias dependences are also difficult to discriminate experimentally by the instrumental uncertainties mentioned above.

From the observations in Figure 42, we can summarize the VRH prediction for the low-frequency noise in OTFT. The single-carrier normalized noise $S_{n} \approx \alpha_{H} / f^{\beta}$ is the flicker-type noise, and it is almost bias and temperature independent. In addition, the single-carrier normalized noise $\mathrm{S}_{\mathrm{n} 1}$ can be calculated by eq. (146) with almost the same magnitude $\alpha_{H}$ and frequency slope $\beta$ with any of the hypotheses (2) (3) (4) for $\Delta \mu$, $\Delta \mathrm{N}$ or $\Delta \sigma$ origin of the noise and using the corresponding weighting function $\mathrm{Y}=\sigma / \mathrm{N}_{C}, \mathrm{Y}=\mathrm{N}_{\mathrm{C}}$ or $\mathrm{Y}=\sigma$ in eq. (147). Hypotheses (1) and (5) are redundant, because hypothesis (1) for $\Delta \tau$ noise is for uniform conductance in the OTFT's film depth, and fails at low temperature. There is a minimal contribution from the correlation between the conductance $\sigma$ and carrier concentration $\mathrm{N}_{\mathrm{C}}$ in hypothesis (5) for a correlated $\Delta \sigma-\Delta \mathrm{N}$ noise, compared to the hypothesis (4) for $\Delta \sigma$ noise only from conductance fluctuation or to hypothesis (3) for $\Delta \mathrm{N}$ noise only from carrier number $\mathrm{N}_{\mathrm{C}}$ fluctuation. So, since VRH is developed mainly for specific conductance $\sigma$, then we shall consider only hypothesis (4) for $\Delta \sigma$ noise in the following illustrations.

For Step 5, we use the additional information in [73] that the channel of the pentacene OTFT had width $\mathrm{W}=2 \mathrm{~cm}$ and length $\mathrm{L}=10 \mu \mathrm{m}$. Thus, as obtained from the values for the unit-area gate charge $\mathrm{Q}_{\mathrm{G}}$ by the numerical simulator, the total number of carriers $\mathrm{N}_{\mathrm{TOT}}=\mathrm{WLQ}_{\mathrm{G}} / \mathrm{q}$ in eq. (149) is $\mathrm{N}_{\mathrm{TOT}}=4.1 \times 10^{9} \pm 1.7 \%$ for $\mathrm{V}_{\mathrm{G}}=-20 \mathrm{~V}$ at temperatures $\mathrm{T}=\{200 \mathrm{~K}, 300 \mathrm{~K}, 400 \mathrm{~K}, 500 \mathrm{~K}\}$ and $\mathrm{N}_{\mathrm{TOT}}=\{0.99,2.04,4.15\} \times 10^{9}$ for $\mathrm{V}_{\mathrm{G}}=\{-5 \mathrm{~V}$, $-10 \mathrm{~V},-20 \mathrm{~V}\}$ at room temperature $\mathrm{T}=300 \mathrm{~K}$. Scaled version of the reciprocal $\left(1 / \mathrm{N}_{\mathrm{TOT}}\right)$ of these values for $\mathrm{N}_{\text {TOт }}$ are shown by diamonds $(\bullet)$ in the insets of Figure 43 , where $\left(1 / \mathrm{N}_{\mathrm{TOT}}\right)$ was multiplied by $3 \times 10^{10}$ to be plotted about a decade above the values for $\alpha_{H}$. Using $C_{0 x}=17 \mathrm{nF} / \mathrm{cm}^{2}$ from Table IV, the approximation 
$\mathrm{N}_{\mathrm{TOT}} \approx \mathrm{WLC} \mathrm{CXX}_{\mathrm{G}} / \mathrm{q}$ in eq. (149) gives slightly higher values, e.g., $4.24 \times 10^{9}$ for $\mathrm{V}_{\mathrm{G}}=-20 \mathrm{~V}$, since the approximation considers zero flat-band at any temperature and bias, but the difference is small and can be neglected, since the inaccuracy of the charge calculation is about $5 \%$ in the numerical simulator, c.f. Figure 34 for $\mathrm{dE}=2 \mathrm{meV}$.

Next, we take the numerically calculated values for the single-carrier normalized noise $S_{\mathrm{n} 1}$ and its approximation with $\alpha_{H} / f^{\beta} \approx S_{n 1}$. These have been shown in Figure 42. The substitutions in eq. (149) yielded the values for the power spectrum densities of the device normalized noise $S_{N O R M}$, as depicted in the main plots of Figure 43 with solid lines for the numerical calculation $S_{N O R M}=S_{n 1} / N_{T O T}$ and with dashed lines for the approximation $\mathrm{S}_{\mathrm{NORM}} \approx\left(\alpha_{\mathrm{H}} / \mathrm{f}^{\beta}\right) / \mathrm{N}_{\text {TOT. }}$. As in the previous figure, Figure $43(\mathrm{a})$ is for different temperatures $\mathrm{T}=\{200 \mathrm{~K}, 300 \mathrm{~K}, 400 \mathrm{~K}, 500 \mathrm{~K}\}$ at a gate bias voltage $\mathrm{V}_{\mathrm{G}}=-20 \mathrm{~V}$, and Figure $43(\mathrm{~b})$ is for different $\mathrm{V}_{\mathrm{G}}=\{-5 \mathrm{~V}$, $-10 \mathrm{~V},-20 \mathrm{~V}\}$ at room temperature $\mathrm{T}=300 \mathrm{~K}$. For clarity in the figure and the following discussion, we show only the results after hypothesis (4) for $\Delta \sigma$ origin of the noise. The observations are qualitatively similar after calculations from the other hypotheses (1) (2) (3) (5), but quantitatively $\mathrm{S}_{\mathrm{NORM}}$ varies about 1 decade between the different hypotheses, replicating the variations for $S_{n 1}, \alpha_{H}$ and $\beta$ shown in the previous Figure 42.

One can make the following observations in Figure 43 for the device normalized noise $\mathrm{S}_{\mathrm{NORM}}$, as deduced from VRH calculations and by eq. (149). First, the overlap is good between the solid lines for the numerically calculated spectra $\mathrm{S}_{\mathrm{NORM}}=\mathrm{S}_{\mathrm{n} 1} / \mathrm{N}_{\mathrm{TOT}}$ and the dashed lines for the approximation $\mathrm{S}_{\mathrm{NORM}} \approx\left(\alpha_{\mathrm{H}} / \mathrm{f}^{\beta}\right) / \mathrm{N}_{\mathrm{TOT}}$, indicating the applicability of both the numerical VRH calculation and the analytical approximation with Hooge eq. (144) for characterization of noise in OTFT. A second observation, however, is that the VRH numerical calculations suggest temperature, bias and frequency variable magnitudes and slopes for the flicker noise, that is, $\alpha_{H}$ and $\beta$ vary with these factors and are not constant device parameters. To get insights for the variations, we choose one frequency $\mathrm{f}=75 \mathrm{~Hz}$ and focus on the evolution of the values for $\mathrm{S}_{\mathrm{NORM}}(75 \mathrm{~Hz})$, denoted with open circles $(\mathrm{O})$ in all plots. In particular, the absolute values of $\mathrm{S}_{\mathrm{NORM}}(75 \mathrm{~Hz})$ are depicted on the noise spectra in the main plots, and scaled version of these values of 
$\mathrm{S}_{\mathrm{NORM}}(75 \mathrm{~Hz})$ are also given in the insets. The scaling in the insets is by multiplying $\mathrm{S}_{\mathrm{NORM}}(75 \mathrm{~Hz})$ with a constant number of $10^{12}$, so that the circles for $\mathrm{S}_{\mathrm{NORM}}(75 \mathrm{~Hz})$ are about $1 / 2$ decade above the squares $(\boldsymbol{\square})$, which depict the values of the Hooge parameter $\alpha_{H}$.

The purpose of the insets of Figure 43 is to split and examine the contributions from different factors in the variations of the device normalized noise $\mathrm{S}_{\mathrm{NORM}}$. Consider the approximation $\mathrm{S}_{\mathrm{NORM}} \approx\left(\alpha_{\mathrm{H}} / \mathrm{f}^{\beta}\right) / \mathrm{N}_{\mathrm{TOT}}$. The increase of the device normalized noise $\mathrm{S}_{\mathrm{NORM}}$ is proportional to the increase of the reciprocal $\left(1 / \mathrm{N}_{\mathrm{TOT}}\right)$ of the total number of carriers $\mathrm{N}_{\text {TOT. }}$. The increase of $\mathrm{S}_{\mathrm{NORM}}$ is also proportional to the increase of the Hooge parameter $\alpha_{H}$. On the other hand, the decrease of the frequency exponent $\beta$ of the flicker noise causes the noise level to be larger when the frequency $f>>1$, so that $f / f^{\beta}$ is larger when $\beta$ is smaller. Therefore, $f / f^{\beta}$ can be regarded as the third factor, to which the increase of $S_{\text {NORM }}$ is proportional. By this formulation of $\left(1 / \mathrm{N}_{\mathrm{TOT}}\right), \alpha_{\mathrm{H}}$ and $\mathrm{f} / \mathrm{f}^{\beta}$ as split factors, the factors are with multiplicative contribution to $\mathrm{S}_{\mathrm{NORM}}$, and therefore, can be plotted in logarithmic scales when examining the contributions by variations of temperature, bias and frequency. Thus, we plot in the insets of Figure 43 the scaled version of $\left(1 / \mathrm{N}_{\mathrm{TOT}}\right)$ with diamonds $(\bullet)$, the Hooge parameter $\alpha_{\mathrm{H}}$ with squares $(\boldsymbol{\square})$ and $\mathrm{f} / \mathrm{f}^{\beta}$ at $\mathrm{f}=75 \mathrm{~Hz}$ with triangles $(\boldsymbol{\Delta})$, together with the scaled version of $S_{\text {NORM }}(75 \mathrm{~Hz})$, open circles $(\mathrm{O})$; and we attribute the variation of $S_{\text {NORM }}$ to the factor, which has similar variation in the plot of the particular inset.

Looking at the inset of Figure 43(a), one observes that the device normalized noise $\mathrm{S}_{\mathrm{NORM}}$ increases with temperature $\mathrm{T}$ for $\mathrm{T}<\mathrm{T}_{0}=400 \mathrm{~K}$, but not much at $\mathrm{T}>\mathrm{T}_{\mathrm{o}}$. The temperature variation of $\mathrm{S}_{\mathrm{NORM}}$ follows the temperature variation of the Hooge parameter $\alpha_{\mathrm{H}}$. There is also a minute contribution to $\mathrm{S}_{\mathrm{NORM}}$ from a decrease of the frequency exponent $\beta$, via the gradual increase of $f / f^{\beta}$ with temperature, but this contribution is small, when compared with the larger contribution from $\alpha_{\mathrm{H}}$. There is no significant temperature dependence in $S_{\text {NORM }}$ contributed from the total number of carriers $\mathrm{N}_{T O T}$, since $\mathrm{N}_{\text {TOT }} \approx \mathrm{WLC} \mathrm{C}_{\mathrm{OX}} \mathrm{V}_{\mathrm{G}} / \mathrm{q}$ is fixed electrostatically by the constant gate bias $V_{G}=-20 \mathrm{~V}$ in Figure 43(a). The temperature variation of $\mathrm{N}_{\text {TOT }}$ is about $\pm 1.7 \%$ or less in Figure 43(a), with is negligible for the noise. 
Conversely, by changing the gate bias of the OTFT at a given temperature, one observes in the inset of Figure 43(b), that the significances of the contributions from the different factors are interchanged. One expects and observes that the device normalized noise $\mathrm{S}_{\text {NORM }}$ decreases at higher magnitude of the gate bias $\mathrm{V}_{\mathrm{G}}$, owing to the increase of the total number of carriers $\mathrm{N}_{\mathrm{TOT}} \propto \mathrm{V}_{\mathrm{G}}$. However, when comparing the rate of decrease of $\left(1 / \mathrm{N}_{\mathrm{TOT}}\right) \propto 1 / \mathrm{V}_{\mathrm{G}}$ with the lower rate for $\mathrm{S}_{\mathrm{NORM}}$, one sees in the inset of Figure $43(\mathrm{~b})$ that the bias-dependent decrease of $\mathrm{S}_{\mathrm{NORM}}$ is less steep than $1 / \mathrm{V}_{\mathrm{G}}$. The reduction of the bias dependence of $\mathrm{S}_{\mathrm{NORM}}$ is mainly due to the increase of the Hooge parameter $\alpha_{H}$ and to a lesser extent due to the decrease of the frequency exponent $\beta$, which causes a minute increase of $f / f^{\beta}$ at higher $V_{G}$.

Overall, although the insets of Figure 43 imply that VRH causes bias and temperature dependences in the Hooge parameter $\alpha_{H}$ and in the frequency exponent $\beta$, the variations of $\alpha_{H}$ and $\beta$ are not dramatically large; these variations are within the experimental inaccuracy of LFN measurements. Therefore, as mentioned above, the approximation $\mathrm{S}_{\mathrm{NORM}} \approx\left(\alpha_{\mathrm{H}} / \mathrm{f}^{\beta}\right) / \mathrm{N}_{\mathrm{TOT}}$ in terms of the Hooge eq. (144) is applicable for OTFT noise characterizations, and the numerical VRH calculations of $\mathrm{S}_{\mathrm{NORM}}$ confirm this applicability.

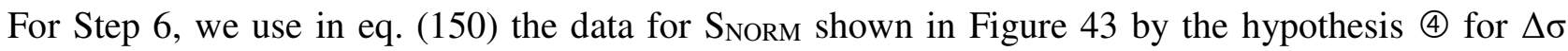
origin of the noise and choose several drain bias voltages $V_{D}$ to calculate the channel current $I_{D}$ and the power-spectrum density (PSD) of the channel noise current $\mathrm{S}_{\mathrm{ID}}$, according to eq. (150) for the Ohmic regime of operation of the OTFT $\left(\left|V_{D}\right| \leq\left|V_{G}\right|\right)$. The results in Figure 44(a) are for different temperatures $\mathrm{T}=\{200 \mathrm{~K}, 300 \mathrm{~K}, 400 \mathrm{~K}, 500 \mathrm{~K}\}$ at fixed biases $\left\{\mathrm{V}_{\mathrm{G}}=-20 \mathrm{~V}, \mathrm{~V}_{\mathrm{D}}=-2 \mathrm{~V}\right\}$, and in Figure $44(\mathrm{~b})$ for $\mathrm{V}_{\mathrm{G}}=\{-5 \mathrm{~V}$, $-10 \mathrm{~V},-20 \mathrm{~V}\}$ and two different values of $\mathrm{V}_{\mathrm{D}}=\{-0.2 \mathrm{~V},-5 \mathrm{~V}\}$ at room temperature $\mathrm{T}=300 \mathrm{~K}$. The solid lines in the main plots of Figure 44 are after numerical VRH calculations. The tilted dashed lines represent the approximations with the de-normalized Hooge equation $S_{I D}=I_{D}^{2} \alpha_{H} /\left(N_{T O T} f^{\beta}\right)$ for the flicker noise component, and the horizontal dashed lines depict the white noise due to the fundamental thermal or shot noise. The PSD of the white noise current is separately calculated either as $4 \mathrm{kT} \sigma_{\mathrm{sq}} \mathrm{W} / \mathrm{L}$ from the conductance $\sigma_{\mathrm{sq}} \mathrm{W} / \mathrm{L}$ of the OTFT channel for the thermal noise, or as $2 \mathrm{qI}_{\mathrm{D}}$ from the DC current for the shot noise. Then, the assumed white noise is added to the flicker current noise of the channel, and the results are shown with the 
solid lines. The assumption for the type of the white noise is arbitrary, because the white noise in an OTFT is not clearly observed experimentally, since the flicker noise dominates in noise measurements of OTFT, c.f. in the experimental data shown with gray lines and small gray squares in the main plot of Figure 44(b).

Eq. (150) suggests a quadratic dependence $S_{I D} \propto I_{D}{ }^{2}$ of the noise $S_{I D}$ on the DC current $I_{D}$. To inspect this dependence, we choose $\mathrm{S}_{\mathrm{ID}}(75 \mathrm{~Hz})$ at frequency $\mathrm{f}=75 \mathrm{~Hz}$, as in the previous figure. The circles on the solid lines in the main plots of Figure 44 and in the insets are $S_{I D}(75 \mathrm{~Hz})$ as obtained from the numerical calculation with the white noise added. The squares in the insets depict the values of the DC current. The comparison of the evolution of $\mathrm{S}_{\mathrm{ID}}(75 \mathrm{~Hz})$ and $\mathrm{I}_{\mathrm{D}}$ with the temperature in the inset of the left-hand Figure 44(a) suggests that $\mathrm{S}_{\mathrm{ID}}$ is not exactly a quadratic function of $\mathrm{I}_{\mathrm{D}}$. Since the gate and drain bias voltages are constants in Figure 44(a), then $\mathrm{N}_{\mathrm{TOT}}$ is also constant, and the "hyper"-quadratic dependence of $\mathrm{S}_{\mathrm{ID}}$ on $\mathrm{I}_{\mathrm{D}}$ is due to the temperature increase of the Hooge parameter $\alpha_{H}$, which has already been shown in the previous figure.

On the other hand, in the inset of the right-hand Figure 44(b), the temperature is constant and the bias is varied. Considering that the increase of the gate bias $\mathrm{V}_{\mathrm{G}}$ is accompanied with proportional increases of $\mathrm{N}_{\mathrm{TOT}}$ and $\mathrm{I}_{\mathrm{D}}$, then one would expect a linear dependence between $\mathrm{S}_{\mathrm{ID}}$ and $\mathrm{I}_{\mathrm{D}}$, e.g. the circles should be aligned to the slope of the dashed line in the inset, which is a scaled version of $\mathrm{I}_{\mathrm{D}}$ by drawing to the axis of $\mathrm{S}_{\mathrm{ID}}$. (The slope of the dashed line in the drawing becomes $1 / 2$ of the slope of $\mathrm{I}_{\mathrm{D}}$ by drawing on its axis.) However, $S_{I D}$ increases faster than $I_{D}$, owing to the increase of the Hooge parameter with the gate bias voltage $\mathrm{V}_{\mathrm{G}}$, as has been shown in the inset of the previous figure. The dependence of $\mathrm{S}_{\mathrm{ID}}$ becomes between a linear and a quadratic function of $\mathrm{I}_{\mathrm{D}}$, when varying $\mathrm{V}_{\mathrm{G}}$. Nevertheless, the drain bias scales equally $\mathrm{S}_{\mathrm{ID}}$ and $I_{D}$, so that the ratio $S_{I D} / I_{D}^{2}$ does not change with $V_{D}$, as seen by the comparison of the separation between circles and squares for the two cases of low $V_{D}=-0.2 \mathrm{~V}$ in the lower portion of the inset and for higher $\mathrm{V}_{\mathrm{D}}=-5 \mathrm{~V}$ in the upper portion of the inset. The reasons for the dependences between LFN, bias and geometry of the OTFT are discussed in $[120,121,122,123,124,125]$. These reasons are weakly related to the assumption for VRH charge transport; therefore, these reasons are not discussed here. 
For Step 7, one desires to compare the VRH prediction for LFN against experimental data. Unfortunately, no data for LFN were reported for the particular pentacene sample, for which the mobility data given in [73] were used to determine the VRH parameters in Table IV. Therefore, we have placed in the right-hand Figure 44(b) some data reported in the literature for several other OTFTs, showing that the calculation of LFN by means of VRH correctly spans the range of values that one normally observes in LFN experiments with OTFTs. The experimental data shown in Figure 44(b) are, as follows. The upper gray symbols are three sets of overlapping data. One set is from [120] for a bottom-contact pentacene OTFT $\left(\mathrm{W}=220 \mu \mathrm{m}, \mathrm{L}=10 \mu \mathrm{m}, \mathrm{V}_{\mathrm{D}}=-5 \mathrm{~V}, \mathrm{~V}_{\mathrm{G}} \approx-10 \mathrm{~V}, \mathrm{I}_{\mathrm{D}} \sim 1 \mu \mathrm{A}\right)$, and two sets of data from [121] for a p-channel DH$\alpha 5 \mathrm{~T}$ OTFT $\left(\mathrm{W}=250 \mu \mathrm{m}, \mathrm{L}=12 \mu \mathrm{m}, \mathrm{V}_{\mathrm{D}}=-15 \mathrm{~V}, \mathrm{~V}_{\mathrm{G}}=-15 \mathrm{~V}, \mathrm{I}_{\mathrm{D}} \sim 0.3 \mu \mathrm{A}\right)$ and $\mathrm{n}$-channel F-CuPC OTFT $\left(\mathrm{W}=250 \mu \mathrm{m}, \mathrm{L}=12 \mu \mathrm{m}, \mathrm{V}_{\mathrm{D}}=100 \mathrm{~V}, \mathrm{~V}_{\mathrm{G}}=100 \mathrm{~V}, \mathrm{I}_{\mathrm{D}} \sim 5 \mu \mathrm{A}\right)$. The gray lines are from our research on noise in poly(3-alkylthiophene) OTFTs [122, 123]. In particular, the data are for poly(3-hexadecylthiophene), P3HDT, OTFTs of sizes $\mathrm{W}=1.6 \mathrm{~cm}, \mathrm{~L}=10 \mu \mathrm{m}$, at $\mathrm{V}_{\mathrm{D}}=-5 \mathrm{~V}, \mathrm{~V}_{\mathrm{G}}=-27.6 \mathrm{~V}, \mathrm{I}_{\mathrm{D}}=0.78 \mu \mathrm{A}$ for the upper gray line and $\mathrm{V}_{\mathrm{D}}=-12 \mathrm{~V}, \mathrm{~V}_{\mathrm{G}}=-24 \mathrm{~V}, \mathrm{I}_{\mathrm{D}}=0.112 \mu \mathrm{A}$ for the lower gray line. The gray squares between the gray lines are from [124] for an OTFT with a slowly grown 10-nm thick pentacene film (W, L, $V_{D}, V_{G}$ and $I_{D}$ have not been reported). The gray squares in the bottom of the figure are from [125] for a PTV OTFT (W=500$\left.1000 \mu \mathrm{m}, \mathrm{L}=40 \mu \mathrm{m}, \mathrm{V}_{\mathrm{D}}=-3 \mathrm{~V}, \mathrm{~V}_{\mathrm{G}} \approx-16 \mathrm{~V}, \mathrm{I}_{\mathrm{D}} \sim 50 \mathrm{nA}\right)$. Some values are restored by our best guess, since the information for the samples, conditions or data processing are not fully disclosed in the publications. Again, the comparison between experiments and prediction of VRH for LFN is only qualitative, since the samples are very different. Overall, the comparison confirms that the procedure of the above seven steps of VRH numerical calculations correctly predicts both the magnitude and the shape of the low-frequency noise, as well as the bias and temperature dependences for LFN in OTFTs.

\section{Conclusions}

We have confirmed by fully numerical simulations that the VRH theory and corresponding analytical models correctly describe the behavior of the conductivity and mobility in OTFTs. One key reason causing 
difficulties of using VRH theory for OTFTs are problems with the values of parameters and magnitudes of quantities related to VRH. The origin of these problems is that a unique approach for derivations, calculations and characterization based on VRH is not currently available. Therefore, we have described our numerical simulator in detail, in order to anticipate the fitting and interpretation of experimental data by analytical VRH models, which are often subjective (e.g., "up to a constant multiplier" that might be in exponents of the analytical expressions).

While the above problems with values indicate that VRH is not mature for OTFTs at present, there are sustainable trends in VRH for OTFT. We have shown that there are simple relations behind the bulky integrals for VRH. For example, VRH is strongly tied to the electrostatics in OTFTs by the potential bending in the semiconducting film, and the steepness of the logarithmic slopes and the magnitude of DOS at the quasi-Fermi level play important roles for the VRH conductance, although the type of DOS has only marginal importance for the overall behavior of VRH. Therefore, the approximation with an exponential DOS works well in practice, especially at low temperatures, but the exponential DOS approximation does affect magnitudes and details in the behaviors at high temperatures.

Among the simple relations, we can confirm for VRH in OTFT that there is a linear dependence between hopping energy and electrostatic bending, which we have routinely observed in the numerical simulations by any of the variations of the assumptions, even for different types of DOS. This linear dependence binds VRH and electrostatics, leading to the currently available VRH analytical models. Both the VRH and electrostatics depend on the effective steepness $\mathrm{kT}_{\mathrm{OEF}}$ of the convolution between Fermi and DOS distributions (with exponential steepness of $\mathrm{kT}$ and $\mathrm{kT}_{\mathrm{o}}$, respectively), where $\mathrm{kT}_{\mathrm{OEF}}$ is nearly the larger of $\mathrm{kT}$ and $\mathrm{kT}_{\mathrm{o}}$. The linear dependence between hopping energy and electrostatic bending also holds when the effective steepness $\mathrm{kT}_{\mathrm{OEF}}$ is considered. It is worth noting that there is not a direct relation between charge and VRH, and the indirect relations follow from the linear dependence between hopping energy and potential bending. The linear dependence between hopping energy and electrostatic bending also suggests a convenient parameterization of VRH in terms of the hopping critical factor for bulk semiconductor at 
infinite temperature $\left(\mathrm{s}_{\mathrm{cT} \mathrm{T}_{\infty}} \approx 10 \pm 4\right)$ and the activation energy $\mathrm{q} \varphi_{\mathrm{To}} \approx(0.8 \pm 0.1) \mathrm{eV}$ of the critical factor. These parameters can replace the presently used and difficult for characterization parameters, such as orbital overlap and hopping attempt rate. The present-day VRH parameters require multiple integrations in order to be used, by also imposing assumptions for DOS.

We have also shown a method for calculation of the distribution of the hopping time. The distribution allows for establishing relations to non-stationary effects in OTFTs, such as dispersive transport, transitions between on and off-states of the OTFT accompanied with the so-called "gate bias stress" or charge buildup, and low-frequency noise in the channel current of the OTFT. Also, while we have demonstrated that VRH is sufficient to describe many effects in OTFT, we should note that VRH is not a necessary condition for the explanation of OTFTs characteristics since several of the relations, which we have discussed, have been addressed by other approaches in the literature.

\section{Acknowledgements}

The authors gratefully acknowledge support from the Canada Research Chair program and the natural Sciences and Engineering Research Council of Canada.

\section{Declaration of Interest Statement}

The authors declare no conflict of interest in this research work.

\section{Appendix 1. Volume of numerical computation of VRH for OTFT}

The volume of computations in the VRH simulator is summarized in Table VI. The VRH principal eq. (4) is computationally efficient, no iteration loops or large matrices, requiring 3 multiplications ( FLOP each), one division ( 2 FLOPs) and one exponentiation ( 17 FLOPs), altogether about 20FLOPs (floating point operations) and less than 100 bytes of memory. However, the calculation the value of $s_{c}$ that is used in eq. (4) is computationally extensive, as discussed below. 
The computational volume of eq. (14) for single $\int \mathrm{dE}$ integration is moderate and it is approximately (8FLOPs $\left.\times \mathrm{E}_{\mathrm{H}} / \mathrm{dE}\right)$, where $\mathrm{E}_{\mathrm{H}} \sim 1 \mathrm{eV}$ and $\mathrm{dE} \sim 1 \mathrm{meV}$, thus, $\sim 8 \mathrm{kFLOPs} /$ iteration for normally less than 100 iterations. The required memory is also not large, approximately 8 Bytes $\times 10$ vectors $\times 2 \mathrm{E}_{\mathrm{H}} / \mathrm{dE} \sim 160 \mathrm{kBytes}$ for double precision.

Eq. (16) is simple for coding, but it is computationally extensive because it is $2 \mathrm{D}$ and requires large memory. Taking $\left(\mathrm{E}_{\text {top }}-\mathrm{E}_{\text {bottom }}\right) 2 \mathrm{eV}$ and $\mathrm{dE} \sim 1 \mathrm{meV}$, then $\mathrm{n}=\left(\mathrm{E}_{\text {top }}-\mathrm{E}_{\text {bottom }}\right) / \mathrm{dE} \sim 2000$, and $\mathrm{R}_{\mathrm{ij}}$ is with a size of $\mathrm{n}^{2} \times(8 \mathrm{~B} y t e s) \sim 32$ Mbytes for double precision, requiring approximately $\mathrm{n}^{2} \times(9 / 4)$ FLOPs $\sim 9$ MFLOPs per iteration with $E_{H}$, since the summation and subtraction are usually $1 / 4$ FLOP, compared to one FLOP for multiplication. Since many elements in the $\mathrm{R}_{\mathrm{ij}}$ matrix are zero, then one may use sparse matrices. However, trading memory for speed, one should be careful when coding with sparse matrices. Depending on the compiler, the sparse matrices might be converted to complete matrices prior to operations; thus, the operation with sparse matrices might be slow and require large memory, contrary to the expectations. Sparse matrices are useful for saving data on disc, but this is not the case with eq. (16) during VRH simulations.

Eq. (18) is simple for coding, but one should be very careful with this equation, because it is computationally the most extensive. The compilers usually perform the exponentiation $y=x^{a}$ as $y=\exp (a \times \ln (x))$, which requires about 35-40FLOPs, consequently 40n²FLOPS 160MFLOPs per iteration (taking $\left.\mathrm{n}=\left(\mathrm{E}_{\text {top }}-\mathrm{E}_{\mathrm{bottom}}\right) / \mathrm{dE} 2000\right)$, and one needs to also resolve the case $\mathrm{y}(\mathrm{x}=0)=0$. The calculation, as done in the simulator, is much faster when coding $(4 \pi \mathrm{dE} / 3) \sum\left[\mathrm{R}_{\mathrm{ij}} \mathrm{R}_{\mathrm{ij}} \mathrm{R}_{\mathrm{ij}} D O S_{\mathrm{j}}\right]$, which requires approximately $(5 \mathrm{n}+1) \mathrm{FLOPs}$ per $\mathrm{E}_{\mathrm{i}}$, or $\mathrm{n}(5 \mathrm{n}+1)$ FLOPs $\approx 5 \mathrm{n}^{2}$ FLOPS 20 MFLOPs per iteration, without problem when $\mathrm{R}_{\mathrm{ij}}=0$. Sparse multiplication, omitting $\mathrm{R}_{\mathrm{ij}}=0$, would greatly reduce the computational volume, but we did not use sparse matrices, in order to minimize the probability for human errors when coding. The consequence was that we needed to run parallel computing. However, sparse multiplication is desirable for commercial simulators, since the conductivity or mobility calculation is just one of the many tasks to be performed for subsequent simulation of the current in OTFTs. The vector BPS $\mathrm{s}_{\mathrm{i}}$ that is generated by eq. $(18)$ is of small size, $n=\left(\mathrm{E}_{\mathrm{top}}-\mathrm{E}_{\mathrm{bottom}}\right) / \mathrm{dE} 2000$, occupying approximately $\mathrm{n} \times 8$ bytes $=16 \mathrm{kB}$ for double precision, which is small increase in the allocated memory. 
In eq. (20), the computational volume of the right-hand expression is moderate, since BPS $\mathrm{i}_{\mathrm{i}}$ and DOS are vectors of size $n=\left(E_{\text {top }}-E_{\text {bottom }}\right) / d E \sim 2000$, requiring approximately not more than $2 n \times 4 F L O P s \sim 16 k F L O P s$ per iteration and (8Bytes) $\times 4$ vectors $\times \mathrm{n} \sim 64 \mathrm{kBytes}$ memory for double precision.

Summarizing the approach of single $\int \mathrm{dE}$ integration for Sec. 2.3 in line "sum 1" in Table VI, the approach of single $\int \mathrm{dE}$ is a one-step calculation, which uses eq. (14), and the computational volume with this approach is small to moderate, approximately $\mathrm{n} \times 8 \mathrm{FLOPs} \sim 8 \mathrm{kFLOPs} /$ iteration (floating point operations of multiplication) and $\mathrm{n} \times 160 \mathrm{Bytes} \sim 160 \mathrm{kBytes}$ memory for double precision by $1 \mathrm{D}$ energy mesh of size $\mathrm{n}=\mathrm{E}_{\mathrm{H}} / \mathrm{dE} \approx 1 \mathrm{eV} / 1 \mathrm{meV} \sim 1000$.

Summarizing the approach of multiple $\iint \mathrm{dEdE}$ integrations for Sec. 2.3 in line "sum 2" in Table VI, the approach of multiple $\iint \mathrm{dEdE}$ is a three-step calculation, which uses eqs. (16), (18) and (20), the calculation is computationally extensive, because it is required to span the $2 \mathrm{D}$ mesh of size $\mathrm{n}^{2}$ for the entire range of energies, and $\mathrm{n}=\left(\mathrm{E}_{\text {top }}-\mathrm{E}_{\text {bottom }}\right) / \mathrm{dE} \approx 2 \mathrm{eV} / 1 \mathrm{meV} \sim 2000$. Consequently, the multiple $\iint \mathrm{dEdE}$ requires approximately $\mathrm{n}^{2} \times(7.5 \mathrm{FLOPs}) \sim 30 \mathrm{MFLOPs} /$ iteration and $\mathrm{n}^{2} \times(8$ Bytes $) \sim 32 \mathrm{MBytes}$.

Eq. (21) has a small contribution to the computational volume of the VRH calculation module. It requires 1/2 FPLOP for summation, 3 FLOPs for multiplication and division, and 17 FLOPs for logarithm; altogether, 20 FLOPs per iteration and not more than 100 bytes of memory. These are negligible, fractions of a percent, compared to rows "sum1" and "sum2 in Table VI.

Row "eq. (24)" in Table VI shows the combination of computational volumes for the generation of the 1D energy mesh $\mathrm{E}$ and the $\mathrm{DOS}(\mathrm{E})$ vectors. The computational volume for the generation of the vector $\mathrm{E}$ with double precision is small, roughly $\mathrm{n} \times 1 \mathrm{FLOP} 2 \mathrm{kFLOPs}$ and $\mathrm{n} \times 8 \mathrm{Bytes} \sim 16 \mathrm{kBytes}$ for $\mathrm{n}=\left(\mathrm{E}_{\mathrm{top}}-\mathrm{E}_{\mathrm{bottom}}\right) / \mathrm{dE} \sim 2 \mathrm{eV} / 1 \mathrm{meV} \approx 2000$. The computational volume of eq. (24) depends on the selected function $\mathrm{f}(\ldots)$, it is approximately $\mathrm{n} \times($ exponentiation + division + multiplication + subtraction $)=$ $\mathrm{n} \times(17+2+1+1 / 4)$ FLOPs $\approx \mathrm{n} \times 20$ FLOPs $\sim 40 \mathrm{kFLOPs}$ for single-side exponential DOS $\mathrm{SE}$ of eq. (22) and two times larger for the double-side exponential $\mathrm{DOS}_{\mathrm{DE}}$ of eq. (45). Thus, the computational volume is not more than 100kFLOPs for eq. (24) with $n=2000$. A small memory of $n \times 8$ Bytes $16 k B y t e s$ is required for eq. (24) for the DOS vector of double precision. The vectors E and DOS are regenerated every time when calling the charge- 
energy calculation module, since the computational volume of the regeneration is small, keeping also code compatibility in using both $\mathrm{E}_{\mathrm{F}}$ and IMREF.

Row "eq. (27)" in Table VI shows the combination of the computation volumes for the calculation of the Fermi occupation factor $\mathrm{F}(\mathrm{E})$, the concentration of occupied states $\mathrm{N}_{\mathrm{C}}$ and the flat-band level FB. For $\mathrm{n}=\left(\mathrm{E}_{\text {top }}-\mathrm{E}_{\text {bottom }}\right) / \mathrm{dE} \sim 2 \mathrm{eV} / 1 \mathrm{meV} \approx 2000$, the expression of the Fermi occupation factor $\mathrm{F}\left(\mathrm{E}-\mathrm{E}_{\mathrm{F}}\right)=1 /\left\{1+\exp \left[\left(\mathrm{E}-\mathrm{E}_{\mathrm{F}}\right) / \mathrm{kT}\right]\right\}$ results in a computation volume of $\mathrm{n} \times($ exponentiation + division $\times 2+$ sum + subtraction $) \approx \mathrm{n} \times(17+3 \times 2+1 / 2)$ FLOPs $\sim 57 \mathrm{kFLOPs}$ and memory $\mathrm{n} \times 8$ Bytes $\sim 16 \mathrm{kBytes}$ for double precision of the vector $\mathrm{F}(\mathrm{E})$. The computational volume for $\mathrm{N}_{\mathrm{C}}$ and $\mathrm{FB}$ by eq. (27) is approximately $\mathrm{n} \times($ multiplication + summation $\times 4)=n \times 2$ FLOPs $\sim 4 k F L O P s$ and $n \times 8$ Bytes for the vector CFDOS. The search for the index IFB uses roughly two subtractions per element, that is $n \times 1 \frac{1}{2}$ FLOPs $\sim 2 k F L O P s$.

Row "sum 3" of Table VI shows that the computational volume of the charge-energy calculation module is moderate and scales with the size $\mathrm{n}=\left(\mathrm{E}_{\mathrm{top}}-\mathrm{E}_{\mathrm{bottom}}\right) / \mathrm{dE} \sim 2 \mathrm{eV} / 1 \mathrm{meV} \approx 2000$ of energy mesh: $\mathrm{n} \times 1 \mathrm{FLOP}$ and $\mathrm{n} \times 8$ Bytes for calculation of the energy mesh $\mathrm{E}$; less than $\mathrm{n} \times 50 \mathrm{FLOPs}$ and $\mathrm{n} \times 8$ Bytes for the DOS vector in eq. (24); $\mathrm{n} \times 23.5 \mathrm{FLOPs}$ and $\mathrm{n} \times 8$ Bytes for the Fermi occupation factor $\mathrm{F} ; \mathrm{n} \times 2 \mathrm{FLOPs}$ for $\mathrm{N}_{\mathrm{C}}$ and $\mathrm{FB}$ and $\mathrm{n} \times 8$ Bytes for the vector CFDOS in eq. (27). Altogether, this is not more than $n \times 100$ FLOPs $200 \mathrm{kFLOPs}$ and $\mathrm{n} \times 8$ bytes $\times 10$ vectors $~ 160 \mathrm{kBytes}$, taking a conservative estimate for overhead due to for-loops, comparisons and branching and temporary variables in charge-energy calculation module.

In row "eSt" of Table VI, the computational volume of the charge-distance calculation module is shown only for the electrostatic calculations. The computational volume for electrostatics of the charge-distance calculation module is mostly determined by the calls of the charge-energy calculation module for different spatial points, the latter charge-energy calculation module having the computation volume shown in row "sum 3" of Table VI, as mentioned above. The spatial point is one (for bulk material) in the first part the chargedistance calculation module, while in the second part, the number of spatial iterations is approximately equal to the number $\mathrm{n} 2000$ of energy points in the charge-energy calculation module. The computational volume for electrostatics of the charge-distance calculation module is mostly determined by the calls of the charge-energy calculation module at every spatial iteration, eq. (33), including also the call for bulk material at far depth in the first part of the charge-distance calculation module, eq. (29). Therefore, the computational volume of the charge- 
distance calculation module becomes $\left(n^{2}+n\right) \times 100$ FLOPs 400MFLOPs and 160kBytes for calling charge-energy calculation, including the calculation for bulk semiconductor. Other calculations in the charge-distance calculation module add negligible $\mathrm{n} \times(50 \mathrm{FLOPs}) \sim 100 \mathrm{kFLOPs}$ for calculation of profiles and management of the step $\mathrm{dt}$ and iterations for $\mathrm{n} \times(8 \mathrm{~B} y \mathrm{tes} \times 12$ vectors $) \sim 200 \mathrm{kBytes}$ for distance mesh $\mathrm{t}$ and profiles with double precision. Altogether, a moderate computational volume of $n^{2} \times 105$ FLOPs $\sim 10$ MFLOPs and 0.4 Mbytes memory for electrostatic calculations is required.

In row "eSt+vrh1" of Table VI, the computational volume of the charge-distance calculation module is shown together with the VRH calculation for bulk conductivity that adds $\mathrm{n} \times(50 \mathrm{FLOPs}) \sim 1$ MFLOPs for the single $\int \mathrm{dE}$, using memory $\sim 0.2 \mathrm{MBytes}$, which is of little concern. As above, $\mathrm{n}=\left(\mathrm{E}_{\mathrm{top}}-\mathrm{E}_{\mathrm{bottom}}\right) / \mathrm{dE} \sim 2000$ is the size of energy mesh for VRH calculation. However, in row "eSt+vrh2" of Table VI, if the VRH bulk conductivity calculation uses multiple $\iint \mathrm{dEdE}$, then the VRH calculation dominates the computational volume of the chargedistance calculation module, requiring $\mathrm{n}^{2} \times(750 \mathrm{FLOPs}) \sim 3$ GFLOPs and $\sim 32 \mathrm{MBytes}$ memory. The computational volume of the charge-distance calculation module is also the computational volume of pass 1 of the VRH numerical simulator (Sec. 2.6 and Figure 5). Pass 1 allocates the most of the memory, which can be as large as 100MBytes for multiple $\iint \mathrm{dEdE}$, including overhead and temporary matrices. After return from the chargedistance calculation module, the memory is released and reused by the following pass 2 and pass 3 . Therefore, the memory allocation is not considered for further discussion in this Appendix 1.

It is possible, in principle, to include the VRH calculation in the loop of the electrostatic calculation, but the VRH calculation should be postponed to be performed on a sub-sampled depth mesh $\mathrm{D}$, as indicated in Figure 4, because, if it is included in the loop of the charge-distance calculation module, then the computational volume would be unnecessary large, e.g., $\mathrm{n}^{2} \times(50 \mathrm{FLOPs}) 200 \mathrm{MFLOPs}$ for the single $\int \mathrm{dE}$, which is acceptable, but $n^{3} \times(750$ FLOPs) 6TFLOPs, which is not a task for a regular desktop computer, while the majority of the calculated data will be also deleted, since they correspond to film depths larger than the thickness of the semiconducting film of the OTFT. Therefore, in the third part in charge-distance calculation module, the distance mesh D is logarithmically sub-sampled to about 50 points (to have many points near the oxide interface and also enough points in the depth of the semiconducting film) and the profiles are reduced only to the points of the sub-sampled mesh. The VRH calculation module is then called in pass 2 of the VRH simulator presented in 
Sec. 2.6 only for the reduced distance mesh D. The reduction of the distance mesh for VRH calculation results in acceptable computational volume of approximately $50 \mathrm{n}^{2} \times(750 \mathrm{FLOPs}) \sim 150 \mathrm{GFLOPs}$ per VRH simulation of one bias-temperature point $\left(\mathrm{V}_{\mathrm{G}}-\mathrm{T}\right.$ point) even when the approach of multiple $\iint \mathrm{dEdE}$ integrations for calculation of the VRH conductivity is used.

In row "pass 2" of Table VI, the computational volume of the VRH numerical simulator is shown. The energy mesh $\mathrm{E}$ and $\mathrm{DOS}(\mathrm{E})$ have not been stored in pass 1 in order to save memory, and because the regeneration of the energy mesh $\mathrm{E}$ is easy by calling the charge-energy calculation module, which is computationally efficient, only $\mathrm{m} \times \mathrm{n} \times 100 \mathrm{FLOPs} \sim 10$ MFLOPs for the reduced depth mesh $\mathrm{D}$ of about $\mathrm{m}=50$ points. After the regeneration, the second step in pass 2 is the call of the VRH calculation module with IMREF and DOS(E), which calculates the specific conductivity $\sigma$ and hopping energy $E_{H}$ for the particular point in the reduced mesh $\mathrm{D}$. Thus, the profiles for $\sigma$ and $\mathrm{E}_{\mathrm{H}}$ are obtained by $\mathrm{m}$ sequential calls of the VRH calculation module, which computational volume is the largest in the VRH simulator, approximately $\mathrm{m} \times \mathrm{n}^{2} \times(750 \mathrm{FLOPs}) \sim 50 \times(2000)^{2} \times(750 \mathrm{FLOPs}) \sim 150 \mathrm{GFLOPs}$ for multiple $\iint \mathrm{dEdE}$ integrations by calculation of the VRH conduction, and much smaller for single $\int \mathrm{dE}$ integration, approximately $\mathrm{m} \times \mathrm{n} \times(800$ FLOPs $) \sim 50 \times 2000 \times(800$ FLOPs $)<0.1$ GFLOPs.

In row "eq. (41)" of Table VI, the computational volume of pass 3 in the VRH numerical simulator is given for calculation of supplementary quantities, such as the sheet conductance $\sigma_{\mathrm{sq}}$ by eq. (41). The computational volume of pass 3 is negligible. For eq. (41), for example, it is $m \times($ multiplication + summation + subtraction $)=\mathrm{m} \times(\mathrm{FLOP}+1 / 2 \mathrm{FLOP})<100 \mathrm{FLOPs}$ for $\mathrm{m} \approx 50$ points in the reduced spatial mesh $\mathrm{D}$, using a memory of $\mathrm{m} \times 8 \mathrm{~B} y \mathrm{tes} \approx 500 \mathrm{Bytes}$ for storing the values of $\sigma_{\mathrm{sq}}$ with double precision. Even conservatively multiplying by 10 these computational volume and memory, as given in row "eq. (41)" of Table VI, the computational volume of pass 3 is still negligible, compared to those in row "pass 2" of Table VI. Therefore, the row "pass 2" of Table VI is essentially repeated in the row "total for a $V_{G}-T$ point" for the computational volume of the VRH numerical simulator for one $\mathrm{V}_{\mathrm{G}}-\mathrm{T}$ (bias-temperature) point. 


\section{Appendix 2. Derivation of analytical expression for sheet conductivity $\sigma_{\mathrm{sq}}$}

Several steps of the derivation of eq. (66) for the OTFT sheet conductivity $\sigma_{\mathrm{sq}}$ are given below. The substitution of eq. (48) in eq. (65) yields

$$
\sigma_{\mathrm{sq}}=\int_{0}^{\mathrm{t}_{\mathrm{f}}} \sigma(\mathrm{D}) \mathrm{dD}=\sigma_{\mathrm{FB}} \int_{0}^{\mathrm{t}_{\mathrm{f}}} \exp \left(\frac{\mathrm{V}_{\mathrm{BS}}}{\varphi_{\mathrm{T}}}\right) \exp \left[-\frac{2 \varphi_{\mathrm{B}}}{\varphi_{\mathrm{T}}} \ln \left(1+\frac{\mathrm{D}}{\mathrm{L}_{\mathrm{A}}}\right)\right] \mathrm{dD}
$$

Placing the constant multipliers in front of the integral, then

$$
\sigma_{\mathrm{sq}}=\sigma_{\mathrm{FB}} \exp \left(\frac{\mathrm{V}_{\mathrm{BS}}}{\varphi_{\mathrm{T}}}\right) \int_{\mathrm{D}=0}^{\mathrm{t}_{\mathrm{f}}} \exp \left\{\ln \left[\left(1+\frac{\mathrm{D}}{\mathrm{L}_{\mathrm{A}}}\right)^{-2 \varphi_{\mathrm{B}} / \varphi_{\mathrm{T}}}\right]\right\} \mathrm{dD}
$$

Since $\mathrm{L}_{\mathrm{A}}$ is assumed constant, then one can change the integration variable, as

$$
\sigma_{\mathrm{sq}}=\mathrm{L}_{\mathrm{A}} \sigma_{\mathrm{FB}} \exp \left(\frac{\mathrm{V}_{\mathrm{BS}}}{\varphi_{\mathrm{T}}}\right)_{\mathrm{D}=0}^{\mathrm{t}_{\mathrm{f}}}\left(1+\frac{\mathrm{D}}{\mathrm{L}_{\mathrm{A}}}\right)^{-2 \varphi_{\mathrm{B}} / \varphi_{\mathrm{T}}} \mathrm{d}\left(1+\frac{\mathrm{D}}{\mathrm{L}_{\mathrm{A}}}\right)
$$

The solution of the integral depends on whether $\varphi_{T}=2 \varphi_{B}$ or not, thus

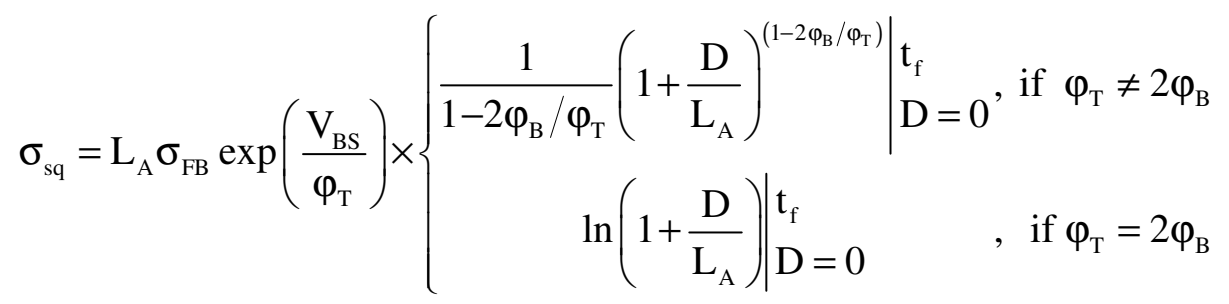

Substituting the limits for $\mathrm{D}$ from zero to $\mathrm{t}_{\mathrm{f}}$, then

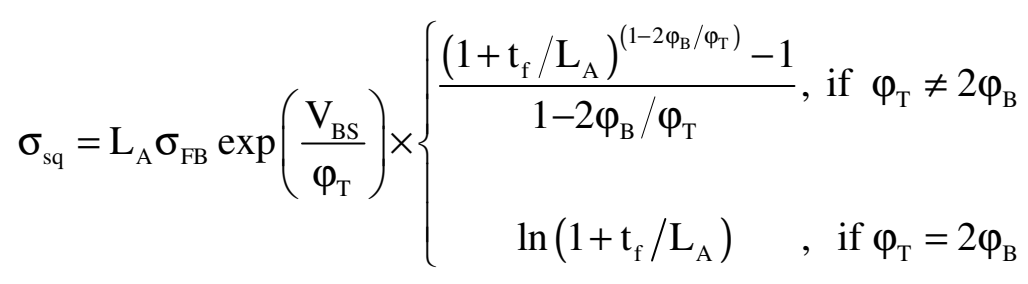

Thus, the analytical formula for the sheet conductivity $\sigma_{\mathrm{sq}}$ becomes

$$
\begin{aligned}
& \sigma_{\mathrm{sq}}=\int_{0}^{\mathrm{t}_{\mathrm{f}}} \sigma(\mathrm{D}) \mathrm{dD} \\
& =\mathrm{L}_{\mathrm{A}} \sigma_{\mathrm{FB}} \exp \left(\frac{\mathrm{V}_{\mathrm{BS}}}{\varphi_{\mathrm{T}}}\right) \times\left\{\begin{array}{cc}
\frac{1-\left(1+\mathrm{t}_{\mathrm{f}} / \mathrm{L}_{\mathrm{A}}\right)^{-\left(2 \varphi_{\mathrm{B}} / \varphi_{\mathrm{T}}-1\right)}}{\left(2 \varphi_{\mathrm{B}} / \varphi_{\mathrm{T}}-1\right)}, & \text { if } \varphi_{\mathrm{T}} \neq 2 \varphi_{\mathrm{B}} \\
\ln \left(1+\mathrm{t}_{\mathrm{f}} / \mathrm{L}_{\mathrm{A}}\right) \quad, \text { if } \varphi_{\mathrm{T}}=2 \varphi_{\mathrm{B}}
\end{array}\right.
\end{aligned}
$$


where the terms in the round brackets are usually larger than unity, $(\ldots)>1$, because normally the film thickness $\mathrm{t}_{\mathrm{f}}>>\mathrm{L}_{\mathrm{A}}$ and the potential bending $\mathrm{V}_{\mathrm{BS}}$ in the semiconductor at the gate dielectric interface is in the range $0.5-1 \mathrm{eV}$ (see Figure 24). Thus, $\mathrm{V}_{\mathrm{BS}}>2 \varphi_{\mathrm{B}}>\varphi_{\mathrm{T}}$, since $\varphi_{\mathrm{B}} \equiv \mathrm{k} \mathrm{T}_{\mathrm{B}} / \mathrm{q} \sim 0.02-0.05 \mathrm{~V}$ with $\mathrm{T}_{\mathrm{B}} \approx \mathrm{T}_{\mathrm{o}} \sim 240-600 \mathrm{~K}$ with the exponential representation of the DOS (see Table I in [6]), and OTFTs are mainly suitable for room-temperature applications $\mathrm{T}<273 \mathrm{~K}+100^{\circ} \mathrm{C}$, thus the thermal voltage is $\varphi_{\mathrm{T}}=\mathrm{kT} / \mathrm{q}<0.033 \mathrm{~V}$. Therefore, one can omit the case $\varphi_{\mathrm{T}}=2 \varphi_{\mathrm{B}}$ and some terms in eq. (151), and expanding $\mathrm{L}_{\mathrm{A}}$ from eq. (47), the order of magnitude for the sheet conductivity $\sigma_{\mathrm{sq}}$ can be estimated by

$$
\begin{aligned}
\sigma_{\mathrm{sq}} & =\int_{0}^{\mathrm{t}_{\mathrm{f}}} \sigma(\mathrm{D}) \mathrm{dD} \approx \frac{\varphi_{\mathrm{A}}}{\varphi_{\mathrm{B}}} \frac{\varphi_{\mathrm{T}} \varepsilon_{\mathrm{f}}}{\mathrm{C}_{\mathrm{ox}} \mathrm{V}_{\mathrm{G}}} \sigma_{\mathrm{FB}} \exp \left(\frac{\mathrm{V}_{\mathrm{BS}}}{\varphi_{\mathrm{T}}}\right) \\
& \sim \frac{\varphi_{\mathrm{T}} \varepsilon_{\mathrm{f}}}{\mathrm{C}_{\mathrm{ox}} \mathrm{V}_{\mathrm{G}}} \sigma_{\mathrm{FB}} \exp \left(\frac{\mathrm{V}_{\mathrm{BS}}}{\varphi_{\mathrm{T}}}\right), \varphi_{\mathrm{A}} \approx \varphi_{\mathrm{B}}>\varphi_{\mathrm{T}}, \mathrm{t}_{\mathrm{f}} / \mathrm{L}_{\mathrm{A}}>10 \mathrm{~nm} / 1 \mathrm{~nm}
\end{aligned}
$$

Expanding $\sigma_{\mathrm{FB}}$ with the expression from eq. (64), then the order of magnitude for the sheet conductivity $\sigma_{\mathrm{sq}}$ becomes as

$$
\sigma_{\mathrm{sq}} \sim \frac{\mathrm{q} \varepsilon_{\mathrm{f}}}{\mathrm{C}_{\mathrm{OX}} \mathrm{V}_{\mathrm{G}}} \frac{\Gamma_{\mathrm{o}}}{\Lambda_{\mathrm{o}}} \exp \left(-\mathrm{s}_{\mathrm{cT} \infty}\right) \exp \left(-\frac{\varphi_{\mathrm{To}}}{\varphi_{\mathrm{T}}}\right) \exp \left(\frac{\mathrm{V}_{\mathrm{BS}}}{\varphi_{\mathrm{T}}}\right)
$$

Note that $\varphi_{\mathrm{T}}$ can be cancelled from numerator and denominator. Thus, the term (kT/q) in the expression for the conductivity prefactor $\sigma_{\mathrm{o}}$ in eq. (3) and in the principal eq. (4) for VRH has little significance for an OTFT, when (kT/q) is outside the exponential terms. The potential bending $\mathrm{V}_{\mathrm{BS}}$ in the semiconductor at the gate dielectric interface $(D=0)$ can be estimated as follows.

Consider eq. (49) for $\mathrm{D}=0$ and substitute $\mathrm{L}_{\mathrm{A}}$ with the expression from eq. (47). The carrier concentration $\mathrm{N}_{\mathrm{C}}(\mathrm{D}=0) \equiv \mathrm{N}_{\mathrm{CDO}}$ in the semiconductor at the gate dielectric interface becomes approximately

$$
\mathrm{N}_{\mathrm{CDO}} \equiv \mathrm{N}_{\mathrm{C}}(\mathrm{D}=0)=\frac{2 \varepsilon_{\mathrm{f}} \varphi_{\mathrm{C}}}{\mathrm{qL} \mathrm{L}_{\mathrm{A}}^{2}}=\frac{2 \varepsilon_{\mathrm{f}} \varphi_{\mathrm{C}}}{\mathrm{q}\left(\frac{2 \phi_{\mathrm{A}} \varepsilon_{\mathrm{f}}}{\mathrm{C}_{\mathrm{ox}} \mathrm{V}_{\mathrm{G}}}\right)^{2}}=\frac{\left(\mathrm{C}_{\mathrm{ox}} \mathrm{V}_{\mathrm{G}}\right)^{2}}{2 \mathrm{q} \varepsilon_{\mathrm{f}} \varphi_{\mathrm{A}}} \text {, with } \varphi_{\mathrm{A}} \approx \varphi_{\mathrm{C}} \equiv \mathrm{k} \mathrm{T}_{\mathrm{C}} / \mathrm{q} \text { and } \mathrm{T}_{\mathrm{C}} \approx \mathrm{T}_{\mathrm{o}} \text {, }
$$

when also considering the single-sided exponential distribution $\mathrm{DOS}_{\mathrm{SE}}$ of eq. (23) with $\mathrm{V}_{\mathrm{B}}=\mathrm{V}_{\mathrm{BS}}$. Assume $\mathrm{kT}<\mathrm{kT}_{\mathrm{o}}$ and step approximation for Fermi occupancy factor $F$ at $I M R E F=\left(E_{F}+q V_{B S}\right)$ in eq. (25). Then 


$$
\begin{aligned}
\mathrm{N}_{\mathrm{C}}(\mathrm{D}=0) & =\int_{-\infty}^{+\infty} \mathrm{F}(\mathrm{E}-\mathrm{IMREF}) \operatorname{DOS}_{\mathrm{SE}}(\mathrm{E}) \mathrm{dE} \\
& \approx \mathrm{N}_{\mathrm{S}} \exp \left(-\frac{\left|\mathrm{E}_{\mathrm{o}}-\mathrm{E}_{\mathrm{F}}\right|}{\mathrm{kT}_{\mathrm{o}}}\right) \exp \left(\frac{\left|\mathrm{V}_{\mathrm{BS}}\right|}{\mathrm{kT}_{\mathrm{o}} / \mathrm{q}}\right)=\mathrm{N}_{\mathrm{CFB}} \exp \left(\frac{\left|\mathrm{V}_{\mathrm{BS}}\right|}{\mathrm{kT}_{\mathrm{o}} / \mathrm{q}}\right),
\end{aligned}
$$

where $N_{C F B}=N_{S} \exp \left(-\mid E_{0}-E_{F} / / k T_{o}\right)$ is the concentration of occupied charge states in the DOS and the carrier concentration for VRH in bulk semiconductor (no bending, $\mathrm{V}_{\mathrm{B}}=0$ ). The quantities in the left-hand sides of eqs. (154) and (155) are the same, $\mathrm{N}_{\mathrm{CDO}} \equiv \mathrm{N}_{\mathrm{C}}(\mathrm{D}=0)$, then

$$
\begin{aligned}
\mathrm{N}_{\mathrm{S}} \exp \left(-\frac{\left|\mathrm{E}_{\mathrm{o}}-\mathrm{E}_{\mathrm{F}}\right|}{\mathrm{kT} \mathrm{T}_{\mathrm{o}}}\right) \exp \left(\frac{\left|\mathrm{V}_{\mathrm{BS}}\right|}{\mathrm{kT}_{\mathrm{o}} / \mathrm{q}}\right)=\frac{\left(\mathrm{C}_{\mathrm{ox}} \mathrm{V}_{\mathrm{G}}\right)^{2}}{2 \mathrm{q} \varepsilon_{\mathrm{f}} \varphi_{\mathrm{A}}} \text {, with } \varphi_{\mathrm{A}} \equiv \mathrm{kT}_{\mathrm{A}} / \mathrm{q} \text { and } \mathrm{T}_{\mathrm{A}} \approx \mathrm{T}_{\mathrm{o}} \\
\exp \left(\frac{\mathrm{q}\left|\mathrm{V}_{\mathrm{BS}}\right|}{\mathrm{kT}}\right)=\frac{\left(\mathrm{C}_{\mathrm{o}} \mathrm{V}_{\mathrm{G}}\right)^{2}}{2 \mathrm{q} \varepsilon_{\mathrm{f}} \varphi_{\mathrm{A}} \mathrm{N}_{\mathrm{S}}} \exp \left(\frac{\left|\mathrm{E}_{\mathrm{o}}-\mathrm{E}_{\mathrm{F}}\right|}{\mathrm{kT}}\right) \text {, with } \varphi_{\mathrm{o}} \equiv \mathrm{kT}_{\mathrm{A}} / \mathrm{q} \text { and } \mathrm{T}_{\mathrm{A}} \approx \mathrm{T}_{\mathrm{o}}
\end{aligned}
$$

Since $\exp (\mathrm{x} / \mathrm{a})=\exp [(\mathrm{x} / \mathrm{b}) \times(\mathrm{b} / \mathrm{a})]=[\exp (\mathrm{x} / \mathrm{b})]^{(\mathrm{b} / \mathrm{a})}$, then $\exp (\mathrm{x} / \mathrm{b})=[\exp (\mathrm{x} / \mathrm{a})]^{(\mathrm{a} / \mathrm{b})}$, and having $\mathrm{a}=\mathrm{kT} \mathrm{T}_{\mathrm{o}}$ and $\mathrm{b}=\mathrm{kT}=\mathrm{q} \varphi_{\mathrm{T}}$, then

$$
\exp \left(\frac{\left|\mathrm{V}_{\mathrm{BS}}\right|}{\varphi_{\mathrm{T}}}\right)=\left[\frac{\left(\mathrm{C}_{\mathrm{ox}} \mathrm{V}_{\mathrm{G}}\right)^{2}}{2 \mathrm{q} \varepsilon_{\mathrm{f}} \varphi_{\mathrm{A}} \mathrm{N}_{\mathrm{S}}} \exp \left(\frac{\left|\mathrm{E}_{\mathrm{o}}-\mathrm{E}_{\mathrm{F}}\right|}{\mathrm{kT}_{\mathrm{o}}}\right)\right]^{\mathrm{T}_{\mathrm{o}} / \mathrm{T}}
$$

Substituting in eq. (153), one obtains the formula in eq. (66) for the sheet conductivity $\sigma_{\mathrm{sq}}$ in a format expected for VRH in OTFTs.

\section{Appendix 3. Derivation details for the relation between conductance and charge concentration, eq. (83)}

At given spatial point in the depth D of the OTFT semiconducting film, the relation between VRH specific conductivity $\sigma$ and the charge concentration $\mathrm{qN}_{\mathrm{C}}$ is obtained by the following derivations. Rewrite eq. (49) for $\left(1+\mathrm{D} / \mathrm{L}_{\mathrm{A}}\right)^{2}$, as

$$
\left(1+\frac{\mathrm{D}}{\mathrm{L}_{\mathrm{A}}}\right)^{2}=\frac{2 \varepsilon_{\mathrm{f}} \varphi_{\mathrm{OEF}}}{\mathrm{L}_{\mathrm{A}}^{2} \mathrm{qN}_{\mathrm{C}}(\mathrm{D})} \text {, with } \varphi \text { OEF in place of } \varphi \mathrm{C} \text {. }
$$

Note from eq. (47), that

$$
\mathrm{Q}_{\mathrm{G}}=\mathrm{C}_{\mathrm{Ox}} \mathrm{V}_{\mathrm{G}}=\frac{2 \varepsilon_{\mathrm{f}} \varphi_{\mathrm{OEF}}}{\mathrm{L}_{\mathrm{A}}}
$$

After substitution, take the natural logarithm of the previous eq. (159) to get 


$$
\ln \left(1+\frac{\mathrm{D}}{\mathrm{L}_{\mathrm{A}}}\right)^{2}=\ln \left(\frac{\mathrm{Q}_{\mathrm{G}}}{\mathrm{L}_{\mathrm{A}}}\right)-\ln \left[\mathrm{qN}_{\mathrm{C}}(\mathrm{D})\right]
$$

Rewrite now eq. (48) for the same quantity, as

$$
\ln \left(1+\frac{\mathrm{D}}{\mathrm{L}_{\mathrm{A}}}\right)^{2}=\frac{\mathrm{V}_{\mathrm{BS}}}{\varphi_{\mathrm{OEF}}}-\frac{\mathrm{V}_{\mathrm{B}}(\mathrm{D})}{\varphi_{\mathrm{OEF}}} \text {, with } \varphi_{\mathrm{OEF}} \text { in place of } \varphi_{\mathrm{B}}
$$

Since the left-hand sides of the last two equations are the same, then

$$
\ln \left(\frac{\mathrm{Q}_{\mathrm{G}}}{\mathrm{L}_{\mathrm{A}}}\right)-\ln \left[\mathrm{qN}_{\mathrm{C}}(\mathrm{D})\right]=\frac{\mathrm{V}_{\mathrm{BS}}}{\varphi_{\mathrm{OEF}}}-\frac{\mathrm{V}_{\mathrm{B}}(\mathrm{D})}{\varphi_{\mathrm{OEF}}} .
$$

Now, the key eq. (63) for the relation between VRH specific conductivity $\sigma(\mathrm{D})$ and electrostatics is rewritten for the bending voltage $V_{B}(D)$, as

$$
\mathrm{V}_{\mathrm{B}}(\mathrm{D})=\varphi_{\mathrm{T}} \ln \left[\frac{\sigma(\mathrm{D})}{\sigma_{\mathrm{FB}}(\mathrm{T})}\right]
$$

where $\sigma_{\mathrm{FB}}$ for the bulk semiconductor is due to eq. (64), $\sigma_{\mathrm{FB}}$ is bias-space independent, although it strongly depends on temperature, and $\sigma_{\mathrm{FB}}$ is known, as assumed above. Substitute $\mathrm{V}_{\mathrm{B}}$ in the preceding eq. (163), arrange the terms so that $\sigma$ and $\mathrm{qN}_{\mathrm{C}}$ are on the left-hand side, and take antilogarithm to obtain

$$
\frac{[\sigma(\mathrm{D})]^{\varphi_{\mathrm{T}} / \varphi_{\mathrm{OEF}}}}{\mathrm{qN}_{\mathrm{C}}(\mathrm{D})}=\exp \left(\frac{\mathrm{V}_{\mathrm{BS}}}{\varphi_{\mathrm{OEF}}}\right) \frac{\mathrm{L}_{\mathrm{A}}}{\mathrm{Q}_{\mathrm{G}}}\left[\sigma_{\mathrm{FB}}(\mathrm{T})\right]^{\varphi_{\mathrm{T}} / \varphi_{\mathrm{OEF}}} .
$$

With $\mathrm{Q}_{\mathrm{G}}=\mathrm{C}_{\mathrm{OX}} \mathrm{V}_{\mathrm{G}}$ and with $\varphi_{\mathrm{OEF}}$ in place of $\mathrm{k} \mathrm{T}_{\mathrm{o}} / \mathrm{q}$, one gets an expression for the term $\exp \left(\mathrm{V}_{\mathrm{BS}} / \varphi_{\mathrm{OEF}}\right)$ from eq. (157), as

$$
\exp \left(\frac{\mathrm{V}_{\mathrm{BS}}}{\varphi_{\mathrm{OEF}}}\right)=\frac{\mathrm{Q}_{\mathrm{G}}^{2}}{2 \mathrm{q} \varepsilon_{\mathrm{f}} \varphi_{\mathrm{OEF}}}\left[\mathrm{N}_{\mathrm{S}} \exp \left(-\frac{\left|\mathrm{E}_{\mathrm{o}}-\mathrm{E}_{\mathrm{F}}\right|}{\mathrm{q} \varphi_{\mathrm{OEF}}}\right)\right]^{-1}=\frac{\mathrm{Q}_{\mathrm{G}}}{\mathrm{L}_{\mathrm{A}} \mathrm{qN} \mathrm{N}_{\mathrm{CFB}}}
$$

The last expression is obtained by recalling from eq. (160) that $\mathrm{Q}_{\mathrm{G}} /\left(2 \varepsilon_{\mathrm{f}} \varphi_{\mathrm{OEF}}\right)=1 / \mathrm{L}_{\mathrm{A}}$, and noticing that the term in the square brackets is the concentration $\mathrm{N}_{\mathrm{CFB}}$ of occupied charge states in the DOS and the carrier concentration for VRH in the bulk semiconductor (no bending, $\mathrm{V}_{\mathrm{B}}=0$ ) in eq. (155) in Appendix 2 at the assumption for an exponential DOS. The substitution of the last eq. (166) in the previous eq. (165) is accompanied with cancelling of the bias-dependent $\mathrm{Q}_{\mathrm{G}}$ and $\mathrm{L}_{\mathrm{A}}$, and yields eq. (83). Indeed, there is a shortcut for deriving eq. (83), by 
expressing $\varphi_{\mathrm{T}} \ln \left(\sigma / \sigma_{\mathrm{FB}}\right)=\mathrm{V}_{\mathrm{B}}=\varphi_{\mathrm{OEF}} \ln \left(\mathrm{N}_{\mathrm{C}} / \mathrm{N}_{\mathrm{CFB}}\right)$ from eqs. (63) and (155), respectively, but this shortcut hides the reasons behind the scaling rule, and therefore, it is not discussed.

\section{Appendix 4. Contributions from the trivial and non-trivial integrals in eq. (103)}

In this appendix, we estimate the contributions from the non-trivial $\int \mathrm{X}^{\mathrm{n}} \mathrm{dx} /(1+\mathrm{x})^{\mathrm{n}}$ and the trivial $\int \mathrm{X}^{\mathrm{n}} \mathrm{dx}=\mathrm{x}^{(\mathrm{n}+1) /(\mathrm{n}+1)}$ integrals in eq. (103), repeated below with the constant multiplier omitted, and considering that the constant $b$ defined by eq. (101) is in the range $1 \mathrm{~V}^{-1}$ to $10 \mathrm{~V}^{-1}$, while $\mathrm{V}_{\mathrm{G}}>1 \mathrm{~V}$ and $\mathrm{V}_{\mathrm{D}}$ does not exceed $\mathrm{V}_{\mathrm{G}}$.

$$
\begin{aligned}
& I_{D} \frac{L}{W}=\left(\frac{a}{b}\right) \times I, \\
& I=\int_{x=b\left(V_{G}-V_{D}\right)}^{b\left(V_{G}-V_{S}\right) \approx b V_{G}} n\left[1-\frac{1}{(1+x)^{n}}\right] d x
\end{aligned}
$$

We also follow notations accepted in mathematics, which are different from the notation in the main text.

The solution of the trivial portion of the integral, $\mathrm{I}_{\mathrm{TR}}$, is

$$
I_{T R}=\int_{x=b\left(V_{G}-V_{D}\right)}^{b\left(V_{G}-V_{S}\right) \approx b V_{G}} x^{n} d x=\left.\frac{x^{n+1}}{n+1}\right|_{x=b\left(V_{G}-V_{D}\right)} ^{b V_{G}}=\frac{b^{n+1}}{n+1} V_{G}^{n+1}-\frac{b^{n+1}}{n+1}\left(V_{G}-V_{D}\right)^{n+1}
$$

The solution of the non-trivial portion of the integral ( $\mathrm{I}_{\mathrm{NTR}}$ ) can be found as a difference of definite integrals, by

$$
I_{\text {NTR }}=\int_{x=b\left(V_{G}-V_{D}\right)}^{b\left(V_{G}-V_{S}\right) \approx b V_{G}} \frac{x^{n}}{(1+x)^{n}} d x=\int_{0}^{b\left(V_{G}-V_{S}\right) \approx b V_{G}} \frac{x^{n}}{(1+x)^{n}} d x-\int_{0}^{b\left(V_{G}-V_{D}\right)} \frac{x^{n}}{(1+x)^{n}} d x
$$

As follows from integral number 3.194 .1 on page 313 in [92] with $\mu=n+1, \nu=n$ and $\beta=1$, or by using software for symbolic integration, such as Maple, the solution of the definite integrals is

$$
\mathrm{I}(\mathrm{u})=\int_{0}^{\mathrm{u}} \frac{\mathrm{x}^{\mathrm{n}}}{(1+\mathrm{x})^{\mathrm{n}}} \mathrm{dx}=\frac{\mathrm{u}^{\mathrm{n}+1}}{\mathrm{n}+1}{ }_{2} \mathrm{~F}_{1}(\mathrm{n}, \mathrm{n}+1 ; \mathrm{n}+2 ;-\mathrm{u}) ; \operatorname{Re}[\mathrm{n}+1]>0,|\arg (1+\mathrm{u})|<\pi,
$$

where the Gauss hyper-geometric function ${ }_{2} \mathrm{~F}_{1} \equiv_{2} \mathrm{HypGeom}$ is denoted with ${ }_{2} \mathrm{HypGeom}_{1}$ in the main text in order to distinguish it from Fermi occupation factor. The Gauss hyper-geometric function ${ }_{2} \mathrm{~F}_{1}$ with the particular parameters is given by 


$$
\begin{aligned}
{ }_{2} \mathrm{~F}_{1}(\mathrm{n}, \mathrm{n}+1 ; \mathrm{n}+2 ;-\mathrm{u})= & \sum_{\mathrm{k}=0}^{\infty}\left[\frac{(\mathrm{n})_{\mathrm{k}}(\mathrm{n}+1)_{\mathrm{k}}}{(\mathrm{n}+2)_{\mathrm{k}}} \frac{(-\mathrm{u})^{\mathrm{k}}}{\mathrm{k} !}\right] \\
= & 1+\frac{\mathrm{n} \times(\mathrm{n}+1)}{(\mathrm{n}+2)} \frac{(-\mathrm{u})}{1}+\frac{\mathrm{n}(\mathrm{n}+1) \times(\mathrm{n}+1)(\mathrm{n}+2)}{(\mathrm{n}+2)(\mathrm{n}+3)} \frac{(-\mathrm{u})^{2}}{1 \times 2} \\
& +\frac{\mathrm{n}(\mathrm{n}+1)(\mathrm{n}+2) \times(\mathrm{n}+1)(\mathrm{n}+2)(\mathrm{n}+3)}{(\mathrm{n}+2)(\mathrm{n}+3)(\mathrm{n}+4)} \frac{(-\mathrm{u})^{3}}{1 \times 2 \times 3}+\ldots,
\end{aligned}
$$

where the Pochhammer symbols can be expressed by the Gamma function $\Gamma(\mathrm{z})$, and denote

$$
\begin{aligned}
& (\mathrm{n})_{\mathrm{k}}=\frac{\Gamma(\mathrm{n}+\mathrm{k})}{\Gamma(\mathrm{n})}=\mathrm{n}(\mathrm{n}+1)(\mathrm{n}+2) \ldots(\mathrm{n}+\mathrm{k}-1)=\prod_{\mathrm{i}=0}^{\mathrm{k}-1}(\mathrm{n}+\mathrm{i}) \\
& (\mathrm{n}+1)_{\mathrm{k}}=\frac{\Gamma(\mathrm{n}+1+\mathrm{k})}{\Gamma(\mathrm{n}+1)}=(\mathrm{n}+1)(\mathrm{n}+2)(\mathrm{n}+3) \ldots(\mathrm{n}+\mathrm{k})=\prod_{\mathrm{i}=0}^{\mathrm{k}-1}(\mathrm{n}+1+\mathrm{i}) \\
& (\mathrm{n}+2)_{\mathrm{k}}=\frac{\Gamma(\mathrm{n}+2+\mathrm{k})}{\Gamma(\mathrm{n}+2)}=(\mathrm{n}+2)(\mathrm{n}+3)(\mathrm{n}+4) \ldots(\mathrm{n}+\mathrm{k}+1)=\prod_{\mathrm{i}=0}^{\mathrm{k}-1}(\mathrm{n}+2+\mathrm{i})
\end{aligned}
$$

Substituting in eq. (168), the non-trivial portion of the integral is

$$
\begin{aligned}
& I_{N T R}=\int_{x=b\left(V_{G}-V_{D}\right)}^{b\left(V_{G}-V_{S}\right) \approx b V_{G}} \frac{x^{n}}{(1+x)^{n}} d x=\int_{0}^{b\left(V_{G}-V_{S}\right) \approx b V_{G}} \frac{x^{n}}{(1+x)^{n}} d x-\int_{0}^{b\left(V_{G}-V_{D}\right)} \frac{x^{n}}{(1+x)^{n}} d x \\
& =\frac{\mathrm{b}^{\mathrm{n}+1} \mathrm{~V}_{\mathrm{G}}^{\mathrm{n}+1}}{\mathrm{n}+1}{ }_{2} \mathrm{~F}_{1}\left(\mathrm{n}, \mathrm{n}+1 ; \mathrm{n}+2 ;-\mathrm{bV} \mathrm{V}_{\mathrm{G}}\right)-\frac{\mathrm{b}^{\mathrm{n}+1}\left(\mathrm{~V}_{\mathrm{G}}-\mathrm{V}_{\mathrm{D}}\right)^{\mathrm{n}+1}}{\mathrm{n}+1}{ }_{2} \mathrm{~F}_{1}\left(\mathrm{n}, \mathrm{n}+1 ; \mathrm{n}+2 ;-\mathrm{b}\left(\mathrm{V}_{\mathrm{G}}-\mathrm{V}_{\mathrm{D}}\right)\right)
\end{aligned}
$$

Combining with the trivial portion $\mathrm{I}_{\mathrm{TR}}$, the current of the square-shaped OTFT becomes

$$
\begin{aligned}
I_{D, F U L L} & =I_{D} \frac{L}{W}=\left(\frac{a}{b}\right) \times\left(I_{T R}-I_{N T R}\right) \\
& =\frac{a b^{n}}{n+1}\left\{\begin{array}{c}
V_{G}^{n+1}\left[1-{ }_{2} F_{1}\left(n, n+1 ; n+2 ;-b V_{G}\right)\right] \\
-\left(V_{G}-V_{D}\right)^{n+1}\left[1-{ }_{2} F_{1}\left(n, n+1 ; n+2 ;-b\left(V_{G}-V_{D}\right)\right)\right]
\end{array}\right\}
\end{aligned}
$$

whereas, omitting the hyper-geometric part, the current is approximately

$$
\mathrm{I}_{\mathrm{D}, \mathrm{APPR}}=\mathrm{I}_{\mathrm{D}} \frac{\mathrm{L}}{\mathrm{W}}=\left(\frac{\mathrm{a}}{\mathrm{b}}\right) \times \mathrm{I}_{\mathrm{TR}}=\frac{\mathrm{ab}}{\mathrm{n}+1}\left\{\mathrm{~V}_{\mathrm{G}}^{\mathrm{n}+1}-\left(\mathrm{V}_{\mathrm{G}}-\mathrm{V}_{\mathrm{D}}\right)^{\mathrm{n}+1}\right\}
$$

We draw $\mathrm{I}_{\mathrm{D}, \mathrm{FULL}}, \mathrm{I}_{\mathrm{D}, \mathrm{APPR}}$ and the magnitude of the difference $\Delta_{\mathrm{ID}}=\left|\mathrm{I}_{\mathrm{D}, \mathrm{FULL}}-\mathrm{I}_{\mathrm{D}, \mathrm{APPR}}\right|$ in Figure 45 , using in eqs. (173) and (174) the interpolation for effective overdrive [28], given by $\mathrm{V}_{\mathrm{EODR}}\left(\mathrm{V}_{\mathrm{G}}, \mathrm{V}\right)=\mathrm{V}_{\mathrm{SS}} \times \ln \left\{1+\exp \left[\left(\mathrm{V}_{\mathrm{G}}-\mathrm{V}\right) / \mathrm{V}_{\mathrm{SS}}\right]\right\}$, instead of $\mathrm{V}_{\mathrm{G}}$ and $\left(\mathrm{V}_{\mathrm{G}}-\mathrm{V}_{\mathrm{D}}\right)$, where $\mathrm{V}_{\mathrm{SS}}$ is related to the 
subthreshold slope and $\mathrm{V}$ denotes either $\mathrm{V}_{\mathrm{S}}=0$ or $\mathrm{V}_{\mathrm{D}} \neq 0$. The interpolation resolves the problem with complex numbers from $\left(V_{G}-V_{D}\right)^{n+1}$ in the saturation regime of operation of the OTFT when $\left(V_{G}-V_{D}\right)<0$, preserving $\mathrm{V}_{\mathrm{EODR}} \approx\left(\mathrm{V}_{\mathrm{G}}-\mathrm{V}_{\mathrm{D}}\right)>0$ in the linear regime of operation of the OTFT. One observes in the figure that the difference between the currents is small, in the range of $0.01-0.3 \%$ at the reasonable gate bias $\left|\mathrm{V}_{\mathrm{G}}\right|>10 \mathrm{~V}$ for this virtual OTFT. Thus, the contribution of the hyper-geometric component can be neglected in practice. Reverting the notations for $\mathrm{a}, \mathrm{b}$ and $\mathrm{n}$ from eq. (101), then the magnitude of the drain current for a square-shaped OTFT can be given only with the trivial part, as

$$
\begin{aligned}
& \mathrm{I}_{\mathrm{D}, \mathrm{APPR}}=\mathrm{I}_{\mathrm{D}} \frac{\mathrm{L}}{\mathrm{W}}=\left(\frac{\mathrm{a}}{\mathrm{b}}\right) \times \mathrm{I}_{\mathrm{TR}} \\
& =\left[\frac{\varphi_{\mathrm{T}} / \varphi_{\mathrm{OEF}}}{\left(2 \varphi_{\mathrm{OEF}} / \varphi_{\mathrm{T}}-1\right)}\left(\frac{\eta(\mathrm{T}) \mathrm{C}_{\mathrm{OX}}^{2}}{2 \varepsilon_{\mathrm{f}} \varphi_{\mathrm{OEF}}}\right)^{\varphi_{\mathrm{OEF}} / \varphi_{\mathrm{T}}-1}\right]\left\{\eta(\mathrm{T}) \mathrm{C}_{\mathrm{OX}} \frac{\mathrm{V}_{\mathrm{G}}^{2\left[\varphi_{\mathrm{OEF}} / \varphi_{\mathrm{T}}\right]}-\left(\mathrm{V}_{\mathrm{G}}-\mathrm{V}_{\mathrm{D}}\right)^{2\left[\varphi_{\mathrm{OEF}} / \varphi_{\mathrm{T}}\right]}}{2}\right\}
\end{aligned}
$$

which is eq. (104) in the main text. 


\section{References}

1. G. Horowitz, P. Delannoy, "An Analytical Model for Organic-Based Thin-Film Transistors", Journal of Applied Physics, 70(1), 469-475, 1991.

2. M. Shur, M. Hack, "Physics of amorphous silicon based alloy field-effect transistors", Journal of Applied Physics, 55(10), 3831-3842, 1984.

3. A. Volkel, R. Street, D. Knipp, "Carrier Transport and Density of State Distributions in Pentacene Transistors", Physical Review B, 66(19), 195336, 2002.

4. S. Scheinert, G. Paasch, "Influence of the Carrier Density in Disordered Organics with Gaussian Density of States on Organic Field-Effect Transistors", Journal of Applied Physics, 115(4), 044507-1 to -7, 2014.

5. A. Rankov, E. Smith, J. Halls, T. Kugler, C. Newsome, S. Mijalkovic, D. Green, A. Nejim, "Modelling of Organic Thin Film Transistors for Technology and Circuit Design", IEEE 1st Int. Workshop on Compact TFT Modelling for Circuit Simulation, Cambridge, 2008.

6. O. Marinov, M. J. Deen, R. Datars, "Compact Modeling of Charge Carrier Mobility in Organic Thin-Film Transistors", Journal of Applied Physics, 106(6), 064501-1 to -13, 2009.

7. A. Nenashev, F. Jansson, J. Oelerich, D. Huemmer, A. Dvurechenskii, F. Gebhard, S. Baranovskii, “Advanced Percolation Solution for Hopping Conductivity”, Physical Review B, 87(23), 235204-1 to -9, 2013.

8. F. Torricelli, M. Ghittorelli, M. Rapisarda, A. Valletta, L. Mariucci, S. Jacob, R. Coppard, E. Cantatore, Z.M. Kovacs-Vajna, L. Colalongo, "Unified drain-current model of complementary p- and n-type OTFTs", Organic Electronics, 22, 5-11, 2015. DOI: https://doi.org/10.1016/j.orgel.2015.03.021

9. L. Colalongo, "SQM-OTFT: A compact model of organic thin-film transistors based on the symmetric quadrature of the accumulation charge considering both deep and tail states" Organic Electronics, 32, 70-77, 2016._DOI: https://doi.org/10.1016/j.orgel.2016.02.005

10. R. Coehoorn, W. Pasveer, P. Bobbert, M. Michels, "Charge-carrier Concentration Dependence of the Hopping Mobility in Organic Materials with Gaussian Disorder", Physical Review B, 72(15), 155206-1 to 20, 2005.

11. P. Servati, A. Nathan, G. Amaratunga, "Generalized Transport-Band Field-Effect Mobility in Disordered Organic and Inorganic Semiconductors”, Physical Review B, 74(24), 245210-1 to -7, 2006.

12. J. Oelerich, D. Huemmer, M. Weseloh, S. Baranovskii, "Concentration Dependence of the Transport Energy Level for Charge Carriers in Organic Semiconductors", Applied Physics Letters, 97(14), 143302-1 to -3, 2010.

13. A. Jouili, S. Mansouri, A. G. Al-Sehemi, A. A. Al-Ghamdi, L. E. Mir, F. Yakuphanoglu, "Numerical studies of surface potential, mobility and Seebeck coefficient of organic thin film transistor based on 2,3 benzanthracene: Light effect”, Synthetic Metals, 233, 119 - 126, 2017. 
14. L. Colalongo, "Charge-Based Model of Symmetric Double-Gate Organic Thin-Film Transistors" IEEE Trans. Electron Devices, 65, 1510-1515, 2018.

15. A. L. R. Melzi, A. J. Chiquito, "The interplay between Arrhenius and hopping conduction mechanisms in a percolating nanowire network", J. of Physics D: Applied Physics, 49, 315303, 2016.

16. R. Cheruku, D. S. Bhaskaram, G. Govindaraj, "Variable range hopping and relaxation mechanism in graphene oxide sheets containing sp3 hybridization induced localization”, J. of Materials Science: Materials in Electronics, 29, 9663-9672, 2018.

17. J. L. Roca-Gonzalez, M. Caravaca, A. Soto-Meca, "Extension of percolation theory to determine the three dimensional variable-range hopping conductivity in glassy chalcogenides", Chalcogenide Letters, 15(5), 261166, 2018.

18. M. Pollak, "Hopping - Past, Present and Future(?)", Physica Status Solidi (b), 230(1), 295-304, 2002.

19. N. F. Mott, "Charge Transport in Non-Crystalline Semiconductors", Advances in Solid State Physics, Festkörperprobleme 9, 22-45, 1969.

20. V. Ambegaokar, B. Halperin, J. Langer, "Hopping Conductivity in Disordered Systems", Physical Review B, 4(8), 2612-2620, 1971.

21. M. Grunewald, P. Thomas, "A Hopping Model for Activated Charge Transport in Amorphous Silicon", Physica Status Solidi (b), 94(1), 125-133, 1979.

22. M. Vissenberg, M. Matters, "Theory of the Field-Effect Mobility in Amorphous Organic Transistors", Physical Review B, 57(20), 12964-12967, 1998.

23. G. Paasch, T. Lindner, S. Scheinert, "Variable Range Hopping as Possible Origin of a Universal Relation between Conductivity and Mobility in Disordered Organic Semiconductors", Synthetic Metals, 132(1), 97104, 2002.

24. A. Brown, C. Jarrett, D. de Leeuw, M. Matters, "Field-Effect Transistors Made from Solution-Processed Organic Semiconductors", Synthetic Metals, 88(1), 37-55, 1997.

25. J. Oelerich, D. Huemmer, S. Baranovskii, "How to Find Out the Density of States in Disordered Organic Semiconductors", Physical Review Letters, 108(22), 226403-1 to -5, 2012.

26. G. Horowitz, M. Hajlaoui, R. Hajlaoui, "Temperature and Gate Voltage Dependence of Hole Mobility in Polycrystalline Oligothiophene Thin Film Transistors", Journal of Applied Physics, 87(9), 4456-4463, 2000.

27. O. Marinov, M. J. Deen, B. Iniguez, "Charge Transport in Organic and Polymer Thin-Film Transistors: Recent Issues", IEE Proc. - Circuits, Devices and Systems, 152(3), 189-209, 2005.

28. O. Marinov, M. J. Deen, U. Zschieschang, H. Klauk, "Organic Thin-Film Transistors: Part I-Compact DC Modeling”, IEEE Trans. Electron Devices, 56(12), 2952-2961, 2009.

29. M. J. Deen, O. Marinov, U. Zschieschang, H. Klauk, “Organic Thin-Film Transistors: Part II-Parameter Extraction", IEEE Trans. Electron Devices, 56(12), 2962-2968, 2009.

30. O. Marinov, M. J. Deen, C. Feng, Y. Wu, "Precise Parameter Extraction Technique for Organic Thin-Film Transistors Operating in the Linear Regime", Journal of Applied Physics, 115(3), 034506-1 to 10, 2014. 
31. A. Romero, J. Gonzalez, R. Picos, M. J. Deen, J. A. Jimenez-Tejada, "Evolutionary Parameter Extraction for an Organic TFT Compact Model Including Contact Effects”, Organic Electronics, 61, 242-253, 2018. DOI: https://doi.org/10.1016/j.orgel.2018.05.062

32. R. Endres, C. Fong, L. Yang, G. Witte, C. Woll, "Structural and Electronic Properties of Pentacene Molecule and Molecular Pentacene Solid", Computational Materials Science, 29, 362-370, 2004.

33. C. Dimitrakopoulos, A. Brown, A. Pomp, "Molecular Beam Deposited Thin Films of Pentacene for Organic Field Effect Transistor Applications", Journal of Applied Physics, 80(4), 2501-2508, 1996.

34. N. Martin, L. Sanchez, C. Seoane, E. Orti, P. Viruela, R. Viruela, "Synthesis, Properties and Theoretical Characterization of Largely $\pi$-Extended Tetrathiafulvalene Derivatives with Quinonoid Structures", J. Org. Chem., 63, 1268-1279, 1998.

35. P. Bullejos, J. Tejada, M. J. Deen, O. Marinov, W. Datars, "Unified Model for the Injection and Transport of Charge in Organic Diodes", Journal of Applied Physics, 103(6), 064504, 2008.

36. CRC, Handbook on Chemistry and Physics, 2008.

37. Y. Tsividis, "Operation and Modeling of the MOS Transistor", $2^{\text {nd }}$ ed., McGraw-Hill, 1999.

38. B. Van Zeghbroeck, "Principles of Semiconductor Devices", online book, 2009, http://ecewww.colorado.edu/ bart/book/

39. P. Bullejos, J. Tejada, S. Rodriguez-Bolivar, M. J. Deen, O. Marinov, "Model for the Injection of Charge Through the Contacts of Organic Transistors”, Journal of Applied Physics, 105(8), 084516, 2009.

40. N. Koch, A. Kahn, J. Ghijsen, J.-J. Pireaux, J. Schwartz, R. L. Johnson, A. Elschner, “Conjugated Organic Molecules on Metal versus Polymer Electrodes: Demonstration of a Key Energy Level Alignment Mechanism”, Applied Physics Letters, 82(1), 70, 2003.

41. L. Burgi, T. Richards, R. Friend, H. Sirringhaus, "Close Look at Charge Carrier Injection in Polymer FieldEffect Transistors", Journal of Applied Physics, 94(9), 6129, 2003.

42. C. Rost, D. Gundlach, S. Karg, Walter Ries, “Ambipolar Organic Field-Effect Transistor Based on an Organic Heterostructure”, Journal of Applied Physics, 95(10), 5782, 2004.

43. T. Sakanoue, E. Fujiwara, R. Yamada, H. Tada, "Visible Light Emission from Polymer-Based Field-Effect Transistors", Applied Physics Letters, 84(16), 3037, 2004.

44. B. Hamadani, "Electronic Charge Injection and Transport in Organic Field-Effect Transistors", Ph. D. Thesis, Rice University, Houston, Texas, 2007.

45. Z. Li, S. Yang, H. Meng, Y. Chen, Y. Yang, C. Liu, S. Horng, C. Hsu, L. Chen, J. Hu, R. Lee, "PatterningFree Integration of Polymer Light-Emitting Diode and Polymer Transistor”, Applied Physics Letters, 84(18), 3558, 2004.

46. M. Hamilton, S. Martin, J. Kanicki, “Thin-Film Organic Polymer Phototransistors”, IEEE Trans. Electron Devices, 51(6), 877, 2004.

47. J. Robertson, "Interfaces and Defects of High-K Oxides on Silicon”, Solid-State Electronics, 49(3), 283-293, 2005. 
48. I. Hill, J. Hwang, A. Kahn, C. Huang, J. McDermott, J. Schwartz, "Energy Level Alignment Between 9Phosphonoanthracene Self-Assembled Monolayers and Pentacene”, Applied Physics Letters, 90(1), 012109, 2007.

49. S. Scheinert, M. Grobosch, J. Sprogies, I. Horselmann, M. Knupfer, G. Paasch, "Organic [6,6]-phenyl-C61butyric-acid-methyl-ester Field Effect Transistors: Analysis of the Contact Properties by Combined Photoemission Spectroscopy and Electrical Measurements", Journal of Applied Physics, 113(17), 174504-1 to $-9,2013$.

50. S. Scheinert, M. Grobosch, G. Paasch, I. Horselmann, M. Knupfer, J. Bartsch, "Contact Characterization by Photoemission and Device Performance in P3HT Based Organic Transistors", Journal of Applied Physics, 111(6), 064502-1 to $-10,2012$.

51. C. Ng, T. Chen, Y. Liu, C. Sun, S. Fung, "Influence of Nitrogen on Tunneling Barrier Heights and Effective Masses of Electrons and Holes at Lightly-Nitrided $\mathrm{SiO}_{2} / \mathrm{Si}$ Interface”, Journal of Applied Physics, 96(10), 5912, 2004.

52. P. Srinivasan, E. Simoen, R. Singanamalla, H. Yu, C. Claeys, D. Misra, "Gate Electrode Effects on LowFrequency (1/f) Noise in p-MOSFETs with High-k Dielectrics”, Solid-State Electronics, 50(6) 992-998, 2006.

53. H. Kim, T. Kim, J. Lee, "Threshold Behavior in Kinetic Electron Emission from Oxide Insulators”, Physical Review B, 76(16), 165434, 2007.

54. S. Rangan, E. Bersch, R. Bartynski, E. Garfunkel, E. Vescovo, "Band Offsets of a Ruthenium Gate on Ultrathin High-k Oxide Films on Silicon”, Physical Review B, 79(7), 075106, 2009.

55. B. Min, S. Devireddy, Z. Celik-Butler, F. Wang, A. Zlotnicka, H.-H. Tseng, P. Tobin, "Low-Frequency Noise in Submicrometer MOSFETs with $\mathrm{HfO}_{2}, \mathrm{HfO}_{2} / \mathrm{Al}_{2} \mathrm{O}_{3}$ and $\mathrm{HfAlO}_{\mathrm{x}}$ Gate Stacks", IEEE Trans. Electron Devices, 51(10), 1679, 2004.

56. M. J. Deen, M. Kazemeini, Y. Haddara, J. Yu, G. Vamvounis, S. Holdcroft, W. Woods, "Electrical Characterization of Polymer-Based FETs Fabricated by Spin-Coating Poly(3-alkylthiophene)s", IEEE Trans. Electron Devices, 51(11), 1892, 2004.

57. E. Calvetti, L. Colalongo, Z. Kovacs-Vajna, "Organic Thin Film Transistors: a DC/Dynamic Analytical Model”, Solid-State Electronics, 49(4), 567-577, 2005.

58. F. Yan, Y. Hong, P. Migliorato, "Temperature Dependent Characteristics of all Polymer Thin-Film Transistors Based on Poly(9,9-dioctylfluorene-co-bithiophene)", Journal of Applied Physics, 101(6), 064501$1-4,2007$.

59. D. Guo, T. Miyadera, S. Ikeda, T. Shimada, K. Saiki, “Analysis of Charge Transport in a Polycrystalline Pentacene Thin Film Transistor by Temperature and Gate Bias Dependent Mobility and Conductance", Journal of Applied Physics, 102(2), 023706-1 to -8, 2007.

60. A. Salleo, T. Chen, A. Volkel, Y. Wu, P. Liu, B. Ong, R. Street, "Intrinsic Hole Mobility and Trapping in a Regioregular Poly(thiophene)", Physical Review B, 70(11), 115311, 2004.

61. E. von Hauff, J. Parisi, V. Dyakonov, "Investigations of Electron Injection in a Methanofullerene Thin Film Transistor", Journal of Applied Physics, 100(7), 073713-1 to -7, 2006. 
62. C. Tanase, E. Meijer, P. Blom, D. de Leeuw, "Unification of the Hole Transport in Polymeric Field-Effect Transistors and Light-Emitting Diodes”, Physical Review Letters, 91(21), 216601-1 to 4, 2003.

63. D. Knipp, R. Street, A. Volkel, "Morphology and Electronic Transport of Polycrystalline Pentacene ThinFilm Transistors", Applied Physics Letters, 82(22), 3907-3909, 2003.

64. D. Taylor, H. Gomes, A. Underhill, S. Edge, P. Clemenson, "Effect of Oxygen on the Electrical Characteristics of Field Effect Transistors Formed from Electrochemically Deposited Films of Poly(3methilthophene)", Journal of Physics D: Applied Physics, 24(11), 2032-2038, 1991.

65. J. Veres, S. Ogier, S. Leeming, D. Cupertino, S. Khaffaf, "Low-k Insulators as the Choice of Dielectrics in Organic Field-Effect Transistors", Adv. Funct. Mater., 13(3), 199-204, 2003.

66. B. Hamadani, D. Natelson, "Nonlinear Charge Injection in Organic Field-Effect Transistors", Journal of Applied Physics, 97(6), 064508-1 to -7, 2005.

67. M. Hamilton, S. Martin, J. Kanicki, "Field-Effect Mobility of Organic Polymer Thin-Film Transistors", Chem. Mater., 16(23), 4699-4704, 2004.

68. A. Salih, J. Marshall, "High-Mobility Low-Threshold-Voltage Pentacene Thin-Film Transistors Prepared at Rapid Growth Rates by Pulsed-Laser Deposition”, Philosophical Magazine Letters, 75(3), 169-177, 1997.

69. J. Takeya, C. Goldmann, S. Haas, K. Pernstich, B. Ketterer, B. Batlogg, “ Field-Induced Charge Transport at the Surface of Pentacene Single Crystals: A Method to Study Charge Dynamics of Two-Dimensional Electron Systems in Organic Crystals”, Journal of Applied Physics, 94(9), 5800-5804, 2003.

70. F. Zhu, H. Wang, D. Song, K. Lou, D. Yan, "Charge Transport in Accumulation Layers of Organic Heterojunctions", Applied Physics Letters, 93(10), 103308-1 to -3, 2008.

71. W. Kalb,K. Mattenberger, B. Batlogg, "Oxygen-Related Traps in Pentacene Thin Films: Energetic Position and Implications for Transistor Performance", Physical Review B, 78(3), 035334-1 to -11, 2008.

72. V. Butko, X. Chi, D. Lang, A. Ramirez, "Field-effect Transistor on Pentacene Single Crystal", Applied Physics Letters, 83(23), 4773-4775, 2003.

73. E. Meijer, M. Matters, P. Herwig, D. de Leeuw, T. Klapwijk, "The Meyer-Neldel Rule in Organic Thin-Film Transistors", Applied Physics Letters, 76(23), 3433-3435, 2000.

74. R. Chesterfield, J. McKeen, C. Newman, C. Frisbie, "Variable Temperature Film and Contact Resistance Measurements on Operating n-Channel Organic Thin Film Transistors", Journal of Applied Physics, 95(11), 6396-6405, 2004.

75. M. Daraktchiev, A. von Muhlenen, F. Nuesch, M. Schaer, M. Brinkmann, M.-N. Bussac, L. Zuppiroli ,“ Ultrathin Organic Transistors on Oxide Surfaces”, New Journal of Physics, 7(1), 133-1 to -8, 2005.

76. A. Lodha, R. Singh, "Prospects of Manufacturing Organic Semiconductor-Based Integrated Circuits", IEEE Trans. Semiconductor Manufacturing, 14(3), 281-296, 2001.

77. B. Ong, Y. Wu, P. Liu, S. Gardner, "High-Performance Semiconducting Polythiophenes for Organic ThinFilm Transistors", J. American Chemical Society, 126(11), 3378-3379, 2004.

78. N. Zhao, G. Botton, S. Zhu, A. Duft, B. Ong, Y. Wu, P. Liu, "Microscopic Studies on Liquid Crystal Poly(3,3"'-dialkylquaterthiophene) Semiconductor", Macromolecules, 37(22), 8307-8312, 2004. 
79. C. Chiang, C. Fincher, Y. Park, A. Heeger, H. Shirakawa, E. Louis, S. Gau, A. MacDiarmid, "Electrical Conductivity in Doped Polyacetylene”, Physical Review Letters, 39(17), 1098-1101, 1977.

80. S. Kishida, Y. Naruke, Y. Uchida, M. Matsumura, "Theoretical Analysis of Amorphous-Silicon Field-EffectTransistors", Japanese Journal of Applied Physics, 22(3), 511-517, 1983.

81. T. Leroux, "Static and Dynamic Analysis of Amorphous-Silicon Field-Effect Transistors", Solid-State Electronics, 29(1), 47-58, 1986.

82. O. Marinov, M. J. Deen, B. Iniguez, "Performance of Organic Thin-Film Transistors", Journal of Vacuum Science and Technology B, 24(4), 1728-1733, 2006. (Also in Virtual Journal of Nanoscale Science and Technology, 14(2), 2006)

83. R. Schroeder, L. Majewski, M. Grell, "Improving Organic Transistor Performance with Schottky Contacts", Applied Physics Letters, 84(6), 1004-1006, 2004.

84. W. Wang, J. Shi, W. Jiang, S. Guo, H. Zhang, B. Quan, D. Ma, "High-Mobility Pentacene Thin-Film Transistors with Copolymer-Gate Dielectric”, Microelectronics Journal, 38(1), 27-30, 2007.

85. L. Mariucci, D. Simeone, S. Cipolloni, L. Maiolo, A. Pecora, G. Fortunato, S. Brotherton, "Effect of Active Layer Thickness on Electrical Characteristics of Pentacene TFTs with PMMA Buffer Layer", Solid-State Electronics, 52(3), 412-416, 2008.

86. F. Dinelli, M. Murgia, P. Levy, M. Cavallini, F. Biscarini, D. de Leeuw, "Spatially Correlated Charge Transport in Organic Thin Film Transistors”, Physical Review Letters, 92(11), 116802-1 to -4, 2004.

87. L. Majewski, R. Schroeder, M. Voigt, M. Grell, "High Performance Organic Transistors on Cheap, Commercial Substrates”, Journal of Physics D: Applied Physics, 37(24), 3367-3372, 2004.

88. O. Marinov, "Organic and Polymeric Transistors - Data Collection for Mobility", Raw Data in Electronics, 1(2), 60, 2005 (www.RDIE.ca, online)

89. Intel, "Optimized MP LINPACK Benchmark for Clusters”, www.intel.com/software/products/mkl.

90. C. Tanase, E. Meijer, P. Blom, D. de Leeuw, "Local Charge Carrier Mobility in Disordered Organic FieldEffect Transistors", Organic Electronics, 4(1), 33-37, 2003.

91. We have inspected the charge profiles reported by Fig. 1 in [90], and observed that the shape of the profiles corresponds to $\mathrm{N}_{C} \propto\left(1+\mathrm{D} / \mathrm{L}_{\mathrm{A}}\right)^{-1}$ dependence, which contradicts with [26, 27], as well as with the results of the numerical simulations, c.f. Figure 13. The inspection plot is not included, since it presents erroneous information.

92. L. Gradsbteyn, I. Ryzbik, "Table of Integrals, Series, and Products", $6^{\text {th }}$ edition, Eds. A. Jeffrey and D. Zwillinger, Academic Press, 2000. ISBN 0-12-294757-6.

93. O. Marinov, M. J. Deen, "Transient Behavior of Variable Range Hopping”, Electrochemical Society, 219"th ECS Meeting, Abstract 1291, Montreal, May 2011.

94. O. Marinov, M. J. Deen, "Flicker Noise due to Variable Range Hopping in Organic Thin-Film Transistors", (IEEE) $21^{\text {st }}$ Int. Conf. on Noise and Fluctuations, ICNF 2011, 287-290, 2011.

95. H. Scher, E. Montroll, "Anomalous Transit-Time Dispersion in Amorphous Solids", Physical Review B, 12(6), 2455-2477, 1975. 
96. M. Silver, L. Cohen, "Monte Carlo Simulation of Anomalous Transit-Time Dispersion of Amorphous Solids", Physical Review B, 15(6), 3276-3278, 1977.

97. J. Orenstein, M. Kastner, "Photocurrent Transient Spectroscopy: Measurement of the Density of Localized States in a-As $\mathrm{Se}_{3}$ ”, Physical Review Letters, 46(21), 1421-1424, 1981.

98. E. A. Schiff, “Trap-Controlled Dispersive Transport and Exponential Band Tails in Amorphous Silicon”, Physical Review B, 24(10), 6189-6192, 1981.

99. M. Silver, G. Schonherr, H. Bassler, "Dispersive Hopping Transport from an Exponential Energy Distribution of Sites", Physical Review Letters, 48(5), 352-355, 1982.

100. J. Noolandi, "Multiple-trapping model of anomalous transit-time dispersion in a-Se", Physical Review B, 16(10), 4466-4473, 1977.

101. P. Borsenberger, L. Pautmeier, H. Bassler, "Charge Transport in Disordered Molecular Solids”, Journal of Chemical Physics, 94(8), 5447-5454, 1991.

102. Y. Gartstein, E. Conwell, "High-Field Hopping Mobility in Molecular Systems with Spatially Correlated Energetic Disorder”, Chemical Physics Letters, 245(4-5), 351-358, 1995.

103. S. Novikov, A. Vannikov, "Cluster Structure in the Distribution of the Electrostatic Potential in a Lattice of Randomly Oriented Dipoles”, Journal of Physical Chemistry, 99(40), 14573-14576, 1995.

104. D. Dunlap, P. Parris, V. Kenkre, "Charge-Dipole Model for the Universal Field Dependence of Mobilities in Molecularly Doped Polymers", Physical Review Letters, 77(3), 542-545, 1996.

105. S. Novikov, A. Vannikov, "Hopping Charge Transport in Disordered Organic Materials: Where Is the Disorder?", Journal of Chemical Physics C, 113(6), 2532-2540, 2009.

106. M. Parameswaran, G. Balaji, T. Jin, C. Vijila, S. Vadukumpully, Z. Furong, S. Valiyaveettil, "Charge Transport studies in Fluorene - Dithieno[3,2-b:2',3'-d]pyrrole Oligomer using Time-of-Flight Photoconductivity Method", Organic Electronics, 10(8), 1534-1540, 2009.

107. A. Rudenko, "Theory of Trap-Controlled Transient Current Injection", Journal of Non-Crystalline Solids, 22(1), 215-218, 1976.

108. V. Arkhipov, A. Rudenko, "On the Study of Amorphous Material Band Structure by Current Injection", Physics Letters A, 61(1), 55-57, 1977.

109. V. Arkhipov, M. Iovu, A. Rudenko, S. Shutov, "An Analysis of the Dispersive Charge Transport in Vitreous $0.55 \mathrm{As}_{2} \mathrm{~S}_{3}: 0.45 \mathrm{Sb}_{2} \mathrm{~S}_{3}$ ”, Physica Status Solidi A, 54(1), 67-77, 1979.

110. V. Arkhipov, V. Kolesnikov, A. Rudenko, "Dispersive Transport of Charge Carriers in Polycrystalline Pentacene Layers", Journal of Physics D: Applied Physics, 17(6), 1241-1254, 1984.

111. H. Nishizawa, A. Hirao, "Relationship between Mobility and Diffusion Coefficient in Molecularly Doped Polymers", Japanese Journal of Applied Physics, 45(8), L250-L252, 2006.

112. W. Tomaszewicz, "On Weakly Dispersive Multiple-Trapping Transport”, Journal of Non-Crystalline Solids, 355(24-27), 1414-1418, 2009.

113. R. Sibatov, V. Uchaikin, "Fractional differential approach to dispersive transport in semiconductors", Uspekhi Fizicheskikh Nauk, 179(10), 1079-1104, 2009 (in Russian) 
114. S. Chen, F. Liu, P. Zhuang, V. Anh, "Finite Difference Approximations for the Fractional Fokker-Planck Equation", Applied Mathematical Modelling, 33(1), 256-273, 2009.

115. F. Hooge, "1/f Noise is No Surface Effect", Physics Letters A, 29(3), 139-140, 1969.

116. F. Hooge, "1/f Noise Sources”, IEEE Trans. Electron Devices, 41(11), 1926-1935, 1994.

117. M. J. Deen, O. Marinov, "Low-Frequency Noise in Electronic Devices-Past, Present and Future”, AIP Conf. Proc., 1129 (ICNF-2009), 197-200, 2009.

118. M. Surdin, "Fluctuations de Courant Thermionique et le 'Flicker Effect"', J. de Physique et le Radium, Ser. 7, 10(4), 188-189, 1939.

119. F. Du Pre, “A Suggestion Regarding the Spectral Density of Flicker Noise”, Physical Review, 78(5), 615-615, 1950.

120. P. Necliudov, S. Rumyantsev, M. Shur, D. Gundlach, T. Jackson, "1/f Noise in Pentacene Organic Thin Film Transistors", Journal of Applied Physics, 88(9), 5395-5399, 2000.

121. S. Martin, A. Dodabalapur, Z. Bao, B. Crone, H. Katz, W. Li, A. Passner, J. Rogers, "Flicker Noise Properties of Organic Thin-Film Transistors", Journal of Applied Physics, 87(7), 3381-3385, 2000.

122. M. J. Deen, O. Marinov, J. Yu, S. Holdcroft, W. Woods, "Low-Frequency Noise in Polymer Transistors", IEEE Trans. Electron Devices, 48(8), 1688-1695, 2001.

123. O. Marinov, M. J. Deen, J. Yu, G. Vamvounis, S. Holdcroft, W. Woods, "Low-Frequency Noise in Polymer Thin-Film Transistors”, IEE Proc. - Circuits, Devices and Systems, 151(5), 466-472, 2004.

124. B. Conrad, W. Cullen, W. Yan, E. Williams, "Percolative Effects on Noise in Pentacene Transistors", Applied Physics Letters, 91(24), 242110-1 to -3, 2007.

125. L. Vandamme, R. Feyaerts, G. Trefan, C. Detcheverry, "1/f noise in Pentacene and Poly-Thienylene Vinylene Thin Film Transistors", Journal of Applied Physics, 91(2), 719-723, 2002. 
Table I. Energy parameters of materials for gate electrodes

\begin{tabular}{|c|c|c|c|c|c|}
\hline Material & Parameter & $\begin{array}{l}\text { Recommended } \\
\text { value }\end{array}$ & $\begin{array}{l}\min (\max ) \\
\text { values }\end{array}$ & $\begin{array}{l}\text { Average value } \\
\text { (st. deviation) }\end{array}$ & References \\
\hline $\mathrm{AgHSC}_{2} \mathrm{H}_{4} \mathrm{C}_{6} \mathrm{~F}_{17}$ & -work function & $-5.5 \mathrm{eV}$ & & & {$[35]$} \\
\hline Platinum $(\mathrm{Pt})$ & -work function & $-5.5 \pm 0.4 \mathrm{eV}$ & $-5.93(-5.12) \mathrm{eV}$ & $-5.525(0.573) \mathrm{eV}$ & {$[36]$} \\
\hline $\mathrm{p}^{+}$Silicon $(\mathrm{Si})$ & Fermi level & $-5.16 \mathrm{eV}$ & & & {$[37,38]$} \\
\hline Gold $(\mathrm{Au})$ & -work function & $-5.1 \pm 0.2 \mathrm{eV}$ & $-5.47(-4.8) \mathrm{eV}$ & $-5.172(0.222) \mathrm{eV}$ & $\begin{array}{l}{[35,36,37,38,39,} \\
40,41,42,43,44, \\
49,50]\end{array}$ \\
\hline Silver (Ag) & -work function & $-5 \pm 0.2 \mathrm{eV}$ & $-5.1(-4.26) \mathrm{eV}$ & $-4.69(0.31) \mathrm{eV}$ & {$[35,36,37,38,41]$} \\
\hline $\begin{array}{l}\text { PEDT/PSS } \\
\text { also PEDOT/PSS } \\
\text { poly(3,4- } \\
\text { ethylenedioxythiophene)/ } \\
\text { poly(styrenesulfonate) }\end{array}$ & -work function & $-5 \pm 0.2 \mathrm{eV}$ & $-5.2(-4.8) \mathrm{eV}$ & $-5(0.204) \mathrm{eV}$ & {$[40,45]$} \\
\hline Copper $(\mathrm{Cu})$ & -work function & $-4.9 \pm 0.25 \mathrm{eV}$ & $-5.1(-4.53) \mathrm{eV}$ & $-4.73(0.22) \mathrm{eV}$ & {$[36,37,41,44]$} \\
\hline Tungsten (W) & -work function & $-4.8 \pm 0.5 \mathrm{eV}$ & $-5.22(-4.32) \mathrm{eV}$ & $-4.77(0.64) \mathrm{eV}$ & [36] \\
\hline Chromium (Cr) & -work function & $-4.7 \pm 0.15 \mathrm{eV}$ & $-4.8(-4.25) \mathrm{eV}$ & $-4.57(0.166) \mathrm{eV}$ & $\begin{array}{l}{[36,38,39,41,43,} \\
44,46]\end{array}$ \\
\hline $\begin{array}{l}\text { ITO (Indium Tin } \\
\text { Oxide), } \mathrm{In}_{2} \mathrm{O}_{3} / \mathrm{SnO}_{2} \\
\text { typ. } 90 \% / 10 \% \text { ) }\end{array}$ & -work function & $-4.65 \pm 0.25 \mathrm{eV}$ & $-4.9(-4.3) \mathrm{eV}$ & $-4.65(0.25) \mathrm{eV}$ & {$[35,45,46]$} \\
\hline intrinsic Silicon $(\mathrm{Si})$ & Fermi level & $-4.6 \pm 0.15 \mathrm{eV}$ & $-5.1(-4.55) \mathrm{eV}$ & $-4.74(0.23) \mathrm{eV}$ & {$[36,38,47,48]$} \\
\hline Nickel (Ni) & -work function & $-4.5 \pm 0.1 \mathrm{eV}$ & $-4.55(-4.45) \mathrm{eV}$ & $-4.5(0.05) \mathrm{eV}$ & {$[37,38]$} \\
\hline $\operatorname{Tin}(\mathrm{Sn})$ & -work function & $-4.42 \mathrm{eV}$ & & & {$[35,36]$} \\
\hline Titanium (Ti) & -work function & $-4.33 \mathrm{eV}$ & & & {$[35,36]$} \\
\hline Aluminium (Al) & -work function & $-4.1 \pm 0.1 \mathrm{eV}$ & $-4.33(-4) \mathrm{eV}$ & $-4.16(0.118) \mathrm{eV}$ & $\begin{array}{l}{[35,36,37,38,41,} \\
43,49]\end{array}$ \\
\hline Indium (In) & -work function & $-4.1 \pm 0.03 \mathrm{eV}$ & $-4.12(-4.09) \mathrm{eV}$ & $-4.105(0.021) \mathrm{eV}$ & {$[35,36]$} \\
\hline $\mathrm{n}^{+}$Silicon $(\mathrm{Si})$ & Fermi level & $-4.04 \mathrm{eV}$ & & & {$[37,38]$} \\
\hline Magnesium (Mg) & -work function & $-3.5 \pm 0.2 \mathrm{eV}$ & $-3.7(-3.25) \mathrm{eV}$ & $-3.56(0.204) \mathrm{eV}$ & {$[35,36,37,38,42]$} \\
\hline Calcium $(\mathrm{Ca})$ & -work function & $-2.9 \pm 0.05 \mathrm{eV}$ & $-2.9(-2.87) \mathrm{eV}$ & $-2.885(0.021) \mathrm{eV}$ & {$[35,36]$} \\
\hline
\end{tabular}


Table II. Energy parameters of materials for gate insulators

\begin{tabular}{|c|c|c|c|c|c|}
\hline Material & Parameter & $\begin{array}{l}\text { Recommended } \\
\text { value }\end{array}$ & $\begin{array}{l}\min (\max ) \\
\text { values }\end{array}$ & $\begin{array}{l}\text { Average value } \\
\text { (st. deviation) }\end{array}$ & References \\
\hline \multirow{2}{*}{$\begin{array}{l}\text { Silicon Dioxide } \\
\left(\mathrm{SiO}_{2}\right)\end{array}$} & -affinity & $-0.9 \pm 0.2 \mathrm{eV}$ & $-1.3(-0.7) \mathrm{eV}$ & $-0.95(0.18) \mathrm{eV}$ & \multirow{2}{*}{$\begin{array}{l}{[38,47,48,51,52} \\
53,54]\end{array}$} \\
\hline & -ionization & $-9.9 \pm 0.2 \mathrm{eV}$ & $-10.2(-9.52) \mathrm{eV}$ & $-9.81(0.24) \mathrm{eV}$ & \\
\hline \multirow[t]{2}{*}{$\left(\mathrm{Al}_{2} \mathrm{O}_{3}\right)$} & -affinity & $-1.7 \pm 0.75 \mathrm{eV}$ & $-2.5(-1.2) \mathrm{eV}$ & $-1.6(0.75) \mathrm{eV}$ & \multirow[t]{2}{*}[47,54,55]{} \\
\hline & -ionization & $-9.7 \pm 0.3 \mathrm{eV}$ & $-10(-9.5) \mathrm{eV}$ & $-9.75(0.35) \mathrm{eV}$ & \\
\hline \multirow{2}{*}{$\begin{array}{l}\text { Nitrided } \mathrm{Si} \text { oxide } \\
\left(\mathrm{Si}_{\mathrm{x}} \mathrm{N}_{2 \%-5 \%} \mathrm{O}_{\mathrm{y}}\right)\end{array}$} & -affinity & $-1.15 \pm 0.1 \mathrm{eV}$ & $-1.2(-1.01) \mathrm{eV}$ & $-1.095(0.081) \mathrm{eV}$ & \multirow[t]{2}{*}{ [51] } \\
\hline & -ionization & $-9.25 \pm 0.1 \mathrm{eV}$ & $-9.38(-8.91) \mathrm{eV}$ & $-9.17(0.201) \mathrm{eV}$ & \\
\hline \multirow[t]{2}{*}{$\left(\mathrm{HfSiO}_{4}\right)$} & -affinity & $-2.5 \mathrm{eV}$ & & & \multirow[t]{2}{*}{ [47] } \\
\hline & -ionization & $-8.5 \mathrm{eV}$ & & & \\
\hline \multirow[t]{2}{*}{ (HfO2) } & -affinity & $-2.6 \pm 0.2 \mathrm{eV}$ & $-2.87(-2.5) \mathrm{eV}$ & $-2.62(0.21) \mathrm{eV}$ & \multirow[t]{2}{*}[47,52,54,55]{} \\
\hline & -ionization & $-8.4 \pm 0.15 \mathrm{eV}$ & $-8.5(-8.2) \mathrm{eV}$ & $-8.4(0.173) \mathrm{eV}$ & \\
\hline \multirow{2}{*}{$\begin{array}{l}\text { Silicon Nitride } \\
\left(\mathrm{Si}_{3} \mathrm{~N}_{4}\right)\end{array}$} & -affinity & $-1.6 \mathrm{eV}$ & & & \multirow[t]{2}{*}{ [47] } \\
\hline & -ionization & $-6.9 \mathrm{eV}$ & & & \\
\hline \multirow{2}{*}{$\begin{array}{l}\text { BCB } \\
\text { (benzocyclobutene) }\end{array}$} & -affinity & $-1.35 \mathrm{eV}$ & & & \multirow[t]{2}{*}{ [46] } \\
\hline & -ionization & $-6.45 \mathrm{eV}$ & & & \\
\hline \multirow{2}{*}{$\begin{array}{l}\text { SAM (9-phospho } \\
\text { anthracene) }\end{array}$} & LUMO & $-2 \mathrm{eV}$ & & & \multirow[t]{2}{*}[48]{} \\
\hline & HOMO & $-6.1 \mathrm{eV}$ & & & \\
\hline
\end{tabular}


Table III. Energy parameters of organic semiconducting materials

\begin{tabular}{|c|c|c|c|c|c|}
\hline Material & Parameter & $\begin{array}{l}\text { Recommended } \\
\text { value }\end{array}$ & $\begin{array}{l}\min (\max ) \\
\text { values }\end{array}$ & $\begin{array}{l}\text { Average value } \\
\text { (st. deviation) }\end{array}$ & References \\
\hline \multirow[t]{3}{*}{ pentacene derivatives } & LUMO & $-4.2 \pm 0.3 \mathrm{eV}$ & $-4.63(-4) \mathrm{eV}$ & $-4.23(0.286) \mathrm{eV}$ & \multirow[t]{3}{*}{ [34] } \\
\hline & Fermi level & $-5.7 \pm 0.3 \mathrm{eV}$ & $-5.92(-5.62) \mathrm{eV}$ & $-5.73(0.14) \mathrm{eV}$ & \\
\hline & HOMO & $-7.2 \pm 0.1 \mathrm{eV}$ & $-7.24(-7.22) \mathrm{eV}$ & $-7.23(0.012) \mathrm{eV}$ & \\
\hline \multirow[t]{3}{*}{ PTCDA } & LUMO & $-4.5 \pm 0.3 \mathrm{eV}$ & $-4.6(-4.1) \mathrm{eV}$ & $-4.35(0.354) \mathrm{eV}$ & \multirow[t]{3}{*}{ [35] } \\
\hline & Fermi level & $-5.55 \pm 0.3 \mathrm{eV}$ & $-5.7(-5.2) \mathrm{eV}$ & $-5.45(0.354) \mathrm{eV}$ & \\
\hline & HOMO & $-6.6 \pm 0.3 \mathrm{eV}$ & $-6.8(-6.3) \mathrm{eV}$ & $-6.55(0.354) \mathrm{eV}$ & \\
\hline \multirow{3}{*}{$\begin{array}{l}\text { 6P } \\
\text { (para-sexiphenyl) }\end{array}$} & vacuum level & $0 \mathrm{eV}$ & $-0.8 \mathrm{eV}$ on $\mathrm{Au}$ & $0.35 \mathrm{eV}$ on PD/PSS & \multirow[t]{3}{*}{ [40] } \\
\hline & Fermi level & $-4.9 \pm 0.3 \mathrm{eV}$ & $-5.15(-4.3) \mathrm{eV}$ & $-4.725(0.601) \mathrm{eV}$ & \\
\hline & HOMO & $-6 \pm 0.1 \mathrm{eV}$ & $-6.1(-5.9) \mathrm{eV}$ & $-6(0.14) \mathrm{eV}$ & \\
\hline \multirow{3}{*}{$\begin{array}{l}\text { Green-B (green } \\
\text { electrolum. conjug. } \\
\text { polyfluorene) }\end{array}$} & LUMO & $-3.2 \mathrm{eV}$ & & & \multirow[t]{3}{*}[45]{} \\
\hline & Fermi level & $-4.55 \mathrm{eV}$ & & & \\
\hline & HOMO & $-5.9 \mathrm{eV}$ & & & \\
\hline \multirow{3}{*}{$\begin{array}{l}\text { Alq3 } \\
\text { [aluminium tris( } 8- \\
\text { hydroxyquinoline)] }\end{array}$} & LUMO & $-3 \pm 0.2 \mathrm{eV}$ & $-3.2(-2.5) \mathrm{eV}$ & $-2.85(0.495) \mathrm{eV}$ & \multirow[t]{3}{*}[35]{} \\
\hline & \begin{tabular}{|l|} 
Fermi level \\
\end{tabular} & $-4.4 \mathrm{eV}$ & $-4.5(-4.05) \mathrm{eV}$ & $-4.275(0.32) \mathrm{eV}$ & \\
\hline & HOMO & $-5.8 \pm 0.1 \mathrm{eV}$ & $-5.8(-5.6) \mathrm{eV}$ & $-5.7(0.14) \mathrm{eV}$ & \\
\hline \multirow{3}{*}{$\begin{array}{l}\text { MEH-PPV [2-methoxy, 5- } \\
\text { (2'-ethyl-hexoxy)-1, 4- } \\
\text { phenylenevinylene] }\end{array}$} & LUMO & $-3.5 \pm 0.5 \mathrm{eV}$ & $-3.8(-2.8) \mathrm{eV}$ & $-3.3(0.71) \mathrm{eV}$ & \multirow[t]{3}{*}[35]{} \\
\hline & \begin{tabular}{|l|} 
Fermi level \\
\end{tabular} & $-4.5 \pm 0.3 \mathrm{eV}$ & $-4.7(-3.85) \mathrm{eV}$ & $-4.275(0.6) \mathrm{eV}$ & \\
\hline & HOMO & $-5.5 \pm 0.2 \mathrm{eV}$ & $-5.6(-4.9) \mathrm{eV}$ & $-5.25(0.495) \mathrm{eV}$ & \\
\hline \multirow{3}{*}{$\begin{array}{l}\text { F8T2 [poly }(9,9- \\
\text { dioctylfluorene-co- } \\
\text { bithiophene)] }\end{array}$} & LUMO & $-2.3 \pm 0.1 \mathrm{eV}$ & $-2.35 \mathrm{eV}$ & $-2.35 \mathrm{eV}$ & \multirow[t]{3}{*}[39,46]{} \\
\hline & Fermi level & $-3.9 \mathrm{eV}$ & & & \\
\hline & HOMO & $-5.5 \pm 0.1 \mathrm{eV}$ & $-5.5(-5.45) \mathrm{eV}$ & $-5.475(0.035) \mathrm{eV}$ & \\
\hline \multirow{3}{*}{$\begin{array}{l}\mathrm{PTCDI}_{-\mathrm{C}_{13} \mathrm{H}_{27}\left(\mathrm{~N}, \mathrm{~N}^{\prime}-\mathrm{di}\right.} \\
\text { tridecylperylene- } 3,4,9,10 \text { - } \\
\text { tetracarboxylic diimide) }\end{array}$} & LUMO & $-3.4 \mathrm{eV}$ & \multicolumn{2}{|c|}{ n-type conductance } & \multirow[t]{3}{*}[42]{} \\
\hline & \begin{tabular}{|l|} 
Fermi level \\
\end{tabular} & $-4.4 \mathrm{eV}$ & & & \\
\hline & HOMO & $-5.4 \mathrm{eV}$ & & & \\
\hline \multirow{3}{*}{$\begin{array}{l}\text { TPD [N,N'-diphenyl-N, } \\
\text { N'-bis(3-methylphenyl)- } \\
\text { [1,1'biphenyl]4,4'diamine] }\end{array}$} & LUMO & $-2 \pm 0.1 \mathrm{eV}$ & $-2.1(-1.8) \mathrm{eV}$ & $-1.95(0.21) \mathrm{eV}$ & \multirow[t]{3}{*}[35]{} \\
\hline & Fermi level & $-3.7 \pm 0.2 \mathrm{eV}$ & $-3.77(-3.45) \mathrm{eV}$ & $-3.61(0.226) \mathrm{eV}$ & \\
\hline & HOMO & $-5.4 \pm 0.25 \mathrm{eV}$ & $-5.44(-5.1) \mathrm{eV}$ & $-5.27(0.24) \mathrm{eV}$ & \\
\hline \multirow{4}{*}{$\begin{array}{l}\alpha-N P D \text { [N,N'-bis-(1- } \\
\text { naphthyl)-N,N'-diphenyl1- } \\
\text { 1,1-biphenyl1-4,4'- } \\
\text { diamine] }\end{array}$} & vacuum level & $0 \mathrm{eV}$ & $-1.15 \mathrm{eV}$ on $\mathrm{Au}$ & $-0.3 \mathrm{eV}$ on PD/PSS & \multirow[t]{4}{*}[35,40]{} \\
\hline & LUMO & $-0.8 \pm 0.5 \mathrm{eV}$ & $-2.3(-0.75) \mathrm{eV}$ & $-1.3(0.87) \mathrm{eV}$ & \\
\hline & Fermi level & $-3.05 \pm 0.5 \mathrm{eV}$ & $-4.85(-3.85) \mathrm{eV}$ & $-4.23(0.551) \mathrm{eV}$ & \\
\hline & HOMO & $-5.3 \pm 0.1 \mathrm{eV}$ & $-5.4(-5.25) \mathrm{eV}$ & $-5.333(0.076) \mathrm{eV}$ & \\
\hline P3HDT [poly(3- & \begin{tabular}{|l|} 
Fermi level \\
\end{tabular} & $-4.72 \mathrm{eV}$ & & & {$[35,56]$} \\
\hline hexadecylthiophene)] & HOMO & $-5.2 \mathrm{eV}$ & & & \\
\hline P3HT & vacuum level & $0 \mathrm{eV}$ & $-0.5 \mathrm{eV}$ on $\mathrm{Au}$ & & {$[35,44,45,50,56]$} \\
\hline [poly(3-hexylthiophene)] & LUMO & $-3 \pm 0.1 \mathrm{eV}$ & & & \\
\hline & Fermi level & $-4.1 \pm 0.2 \mathrm{eV}$ & $-4.92(-4.05) \mathrm{eV}$ & $-4.49(0.355) \mathrm{eV}$ & \\
\hline & HOMO & $-5.2 \pm 0.2 \mathrm{eV}$ & $-5.7(-5.1) \mathrm{eV}$ & $-5.243(0.207) \mathrm{eV}$ & \\
\hline NPB & LUMO & $-2.1 \mathrm{eV}$ & & & {$[35]$} \\
\hline (4,4-bis-1-naphtyl-N- & Fermi level & $-3.65 \mathrm{eV}$ & & & \\
\hline phenylaminobiphenyl) & HOMO & $-5.2 \mathrm{eV}$ & & & \\
\hline PDOT & LUMO & $-3.4 \mathrm{eV}$ & $-3.42 \mathrm{eV}$ & & [35] \\
\hline & Fermi level & $-4.2 \mathrm{eV}$ & $-4.22 \mathrm{eV}$ & & \\
\hline & HOMO & $-5 \mathrm{eV}$ & $-5.02 \mathrm{eV}$ & & \\
\hline pentacene & vacuum level & $0 \mathrm{eV}$ & $-1.05 \mathrm{eV}$ on $\mathrm{Au}$ & $-0.1 \mathrm{eV}$ on PD/PSS & {$[3,32,35,40,42$,} \\
\hline $\mathrm{C}_{22} \mathrm{H}_{14}$ & LUMO & $-2.8 \pm 0.3 \mathrm{eV}$ & $-5.27(-2.5) \mathrm{eV}$ & $-3.07(0.991) \mathrm{eV}$ & \\
\hline & Fermi level & $-3.9 \pm 0.3 \mathrm{eV}$ & $-5.79(-3.65) \mathrm{eV}$ & $-4.44(0.77) \mathrm{eV}$ & \\
\hline & HOMO & $-5 \pm 0.3 \mathrm{eV}$ & $-6.35(-4.8) \mathrm{eV}$ & $-5.128(0.474) \mathrm{eV}$ & \\
\hline PPV & LUMO & $-2.5 \mathrm{eV}$ & & & [35] \\
\hline & Fermi level & $-3.75 \mathrm{eV}$ & & & \\
\hline & HOMO & $-5 \mathrm{eV}$ & & & \\
\hline
\end{tabular}


Table IV. Parameters used in the majority of the numerical simulations, assuming a double-exponential DOS $_{D E}$ and values reported in the literature for the three OTFTs whose characteristics are shown in several figures. The values of $\mathrm{N}_{S}, \mathrm{~T}_{\mathrm{o}}, \Lambda_{\mathrm{o}}$ and $\Gamma_{\mathrm{o}}$ are different by the different assumptions for single-side exponential DOS $\mathrm{SE}$, eq. (22), and normally-distributed $\mathrm{DOS}_{\mathrm{ND}}$, eq. (58), as indicated in Figure 22.

\begin{tabular}{|c|c|c|c|c|}
\hline Parameter & single $\int \mathrm{dE}$ & multiple $\iint \mathrm{dEdE}$ & literature & comment \\
\hline \multicolumn{4}{|c|}{ pentacene OTFT from [73] } & \\
\hline $\mathrm{N}_{\mathrm{S}}, \mathrm{nm}^{-3}$ & 10 & 5 & & \multirow{6}{*}{$\begin{array}{l}\text { Figure } 2 \\
\bigcirc \text { in Figure } 16 \mathrm{a} \\
\text { Figure } 17 \mathrm{a} \\
(\ldots) \text { in Figure } 20 \\
\text { middle Figure } 24 \\
\text { Figure } 37 \text { and after }\end{array}$} \\
\hline $\mathrm{T}_{\mathrm{o}}, \mathrm{K}$ & 441 & 400 & Meyer-Neldel E=38meV [73] & \\
\hline$\Lambda_{\mathrm{o}}, \mathrm{nm}$ & 0.195 & 0.175 & & \\
\hline$\Gamma_{\mathrm{o}}, \mathrm{THz}$ & 150 & 800 & & \\
\hline $\mathrm{C}_{\mathrm{I}}, \mathrm{nF} / \mathrm{cm}^{2}$ & 17 & 17 & $200 \mathrm{~nm} \mathrm{SiO}_{2}[73]$ & \\
\hline DOS type & \multicolumn{2}{|c|}{ double-exponential, eq. (45) } & & \\
\hline \multicolumn{4}{|c|}{ pentacene OTFT from [22] } & \\
\hline $\mathrm{N}_{\mathrm{S}}, \mathrm{nm}^{-3}$ & 20 & 10 & Stated irrelevant in [22] & \multirow{6}{*}{$\begin{array}{l}\square \text { in Figure } 16 \mathrm{a} \\
\text { Figure } 17 \mathrm{~b} \\
(\ldots) \text { in Figure } 20 \\
\text { Figure } 32 \mathrm{a}\end{array}$} \\
\hline $\mathrm{T}_{\mathrm{o}}, \mathrm{K}$ & 385 & 385 & $385[22]$ & \\
\hline$\Lambda_{\mathrm{o}}, \mathrm{nm}$ & 0.107 & 0.139 & $0.22[22]$ & \\
\hline$\Gamma_{\mathrm{o}}, \mathrm{THz}$ & 250 & 330 & $\sigma_{o}=1.6 \times 10^{10} \mathrm{~S} / \mathrm{m}[22]$ & \\
\hline $\mathrm{C}_{\mathrm{I}}, \mathrm{nF} / \mathrm{cm}^{2}$ & 17 & 17 & $200 \mathrm{~nm} \mathrm{SiO}_{2},[24]$ & \\
\hline DOS type & \multicolumn{2}{|c|}{ double-exponential, eq. (45) } & & \\
\hline \multicolumn{4}{|c|}{ annealed PQT-12 film OTFT from [60] } & \multirow{7}{*}{$\begin{array}{l}\diamond \text { in Figure } 16 \mathrm{a} \\
\text { Figure } 17 \mathrm{c} \\
\text { Figure } 18 \\
\text { Figure } 19 \\
(\ldots) \text { in Figure } 20 \\
\text { Figure } 32 \mathrm{~b} \text { and c }\end{array}$} \\
\hline $\mathrm{N}_{\mathrm{S}}, \mathrm{nm}^{-3}$ & 70 & 15 & $0.65-1.5$ for mobility edge in [60] & \\
\hline $\mathrm{T}_{\mathrm{o}}, \mathrm{K}$ & 270 & 270 & $320-325,(\mathrm{kTo}=27.6-28 \mathrm{meV})[60]$ & \\
\hline$\Lambda_{\mathrm{o}}, \mathrm{nm}$ & 0.05 & 0.05 & $0.1-0.128[60]$ & \\
\hline$\Gamma_{\mathrm{o}}, \mathrm{THz}$ & 4500 & 700000 & $\sigma_{\mathrm{o}}=(0.35-1.9) \times 10^{12} \mathrm{~S} / \mathrm{m}[60]$ & \\
\hline $\mathrm{C}_{\mathrm{I}}, \mathrm{nF} / \mathrm{cm}^{2}$ & 30 & 30 & $100 \mathrm{~nm} \mathrm{SiO} \mathrm{S}_{2}$, with SAM [60] & \\
\hline DOS type & \multicolumn{2}{|c|}{ double-exponential, eq. (45) } & & \\
\hline \multicolumn{4}{|c|}{ All numerical simulations and all devices } & \\
\hline $\mathrm{E}_{\mathrm{F}}, \mathrm{eV}$ & 0.3 , referre & to gate conductor & & \multirow[t]{4}{*}{ All devices } \\
\hline $\mathrm{E}_{\mathrm{o}}, \mathrm{eV}$ & -0.6, referr & d to gate conductor & & \\
\hline$\varepsilon_{\mathrm{f}}=3 \varepsilon_{\mathrm{o}}, \mathrm{fF} / \mathrm{cm}$ & & 265.5 & & \\
\hline $\mathrm{t}_{\mathrm{f}}, \mathrm{nm}$ & & 50 & $30-50[22], 20-60[60]$ & \\
\hline $\mathrm{B}_{\mathrm{c}}$ & & $\pi \approx 2.86$ & approx., from [20], adopted in [22] & \multirow[t]{4}{*}{ All simulations } \\
\hline $\mathrm{dE}, \mathrm{meV}$ & & 2 & & \\
\hline $\mathrm{E}_{\mathrm{top}}, \mathrm{eV}$ & 0.8, referre & to gate conductor & & \\
\hline Ebottom, $\mathrm{eV}$ & -1.1, referr & d to gate conductor & & \\
\hline
\end{tabular}


Table V. Variation of non-reported (in [60]) values for sample parameters of two PQT-12 OTFTs, when matching by means of eq. (53), the experimental data for the drain current $\mathrm{I}_{\mathrm{D}}-\mathrm{V}_{\mathrm{G}}$ transfer curves at low drain voltage $\mathrm{V}_{\mathrm{D}}=-1 \mathrm{~V}$, to numerical VRH simulations of the OTFT channel sheet conductance $\sigma_{\mathrm{sq}}$. This is illustrated in Figure 18 .

\begin{tabular}{|c|c|c|c|c|c|}
\hline $\begin{array}{l}\text { For device with } \\
I_{D}-V_{G} \text { reported } \\
\text { in }\end{array}$ & $\begin{array}{l}\text { matching } \sigma_{\mathrm{sq}} \\
\text { simulation by } \\
\text { method of }\end{array}$ & $\begin{array}{l}\text { at absolute } \\
\text { temperature } \\
\mathrm{T}, \text { Kelvin }\end{array}$ & $\begin{array}{l}\text { using channel } \\
\text { width to length } \\
\text { ratio W/L }\end{array}$ & $\begin{array}{l}\text { and taking turn- } \\
\text { on voltage } \mathrm{V}_{\text {on }} \text {, } \\
\text { Volts }\end{array}$ & $\begin{array}{l}\text { Symbol in } \\
\text { Figure } 18\end{array}$ \\
\hline \multirow{6}{*}{ Fig. $2 \mathrm{a}$ in [60] } & $\int \mathrm{dE}$ & $150 \mathrm{~K}$ & 19.00 & $-4.3 \mathrm{~V}$ & \multirow{3}{*}{ Not shown } \\
\hline & $\int \mathrm{dE}$ & $200 \mathrm{~K}$ & 20.00 & $-6.5 \mathrm{~V}$ & \\
\hline & $\int \mathrm{dE}$ & $300 \mathrm{~K}$ & 30.00 & $-1.5 \mathrm{~V}$ & \\
\hline & $\iint \mathrm{dEdE}$ & $150 \mathrm{~K}$ & 25.00 & $-5.5 \mathrm{~V}$ & $\mathrm{O}$ \\
\hline & $\iint \mathrm{dEdE}$ & $200 \mathrm{~K}$ & 20.00 & $-6.5 \mathrm{~V}$ & $\square$ \\
\hline & $\iint \mathrm{dEdE}$ & $300 \mathrm{~K}$ & 30.00 & $-1.5 \mathrm{~V}$ & $\diamond$ \\
\hline \multirow{6}{*}{ Fig. $8 \mathrm{a}$ in [60] } & $\int \mathrm{dE}$ & $90 \mathrm{~K}$ & 5.00 & $+2.0 \mathrm{~V}$ & \multirow{3}{*}{ Not shown } \\
\hline & $\int \mathrm{dE}$ & $140 \mathrm{~K}$ & 14.00 & $-10.0 \mathrm{~V}$ & \\
\hline & $\int \mathrm{dE}$ & $180 \mathrm{~K}$ & 19.50 & $-13.0 \mathrm{~V}$ & \\
\hline & $\iint \mathrm{dEdE}$ & $90 \mathrm{~K}$ & 4.25 & $0 \mathrm{~V}$ & $\bar{\Delta}$ \\
\hline & $\iint \mathrm{dEdE}$ & $140 \mathrm{~K}$ & 10.50 & $-8.5 \mathrm{~V}$ & 0 \\
\hline & $\iint \mathrm{dEdE}$ & $180 \mathrm{~K}$ & 16.00 & $-12.0 \mathrm{~V}$ & $\square$ \\
\hline
\end{tabular}


This Table VI should be placed in Appendix 1

Table VI. Computational volume of the numerical simulations for VRH in one bias-temperature point (Details in Appendix 1)

\begin{tabular}{|c|c|c|c|c|c|c|}
\hline row label & simulation & FLOPs & memory & $\mathrm{E}_{\mathrm{H}}$ iterations & Energy steps, $\mathrm{n}$ & Spatial steps, m \\
\hline eq. (4) & $\int \mathrm{dE}$ and $\iint \mathrm{dEdE}$ & 20 & $<100$ Bytes & 1 & any & 1 \\
\hline eq. (14) & $\int \mathrm{dE}$ & $0.8 \times 10^{6}$ & $\sim 160 \mathrm{kB}$ & $100 \max$ & 1000 & 1 \\
\hline eq. (16) & $\iint \mathrm{dEdE}$ & $0.9 \times 10^{9}$ & $\sim 32 \mathrm{MB}$ & $100 \max$ & 2000 & 1 \\
\hline eq. (18) & $\iint \mathrm{dEdE}$ & $2 \times 10^{9}$ & $\sim 16 \mathrm{kB}$ & $100 \max$ & 2000 & 1 \\
\hline eq. $(20)$ & $\iint \mathrm{dEdE}$ & $1.6 \times 10^{6}$ & $\sim 64 \mathrm{kB}$ & $100 \max$ & 2000 & 1 \\
\hline sum 1 & $\propto \mathrm{n}$ for $\int \mathrm{dE}$ & $0.8 \times 10^{6}$ & $\sim 160 \mathrm{kB}$ & $100 \max$ & 1000 & 1 \\
\hline sum 2 & $\propto \mathrm{n}^{2}$ for $\iint \mathrm{dEdE}$ & $3 \times 10^{9}$ & $\sim 32 \mathrm{MB}$ & $100 \max$ & 2000 & 1 \\
\hline eq. (21) & $\int \mathrm{dE}$ and $\iint \mathrm{dEdE}$ & $2 \times 10^{3}$ & $<100$ Bytes & $100 \max$ & any & 1 \\
\hline eq. (24) & charge-energy & $0.1 \times 10^{6}$ & $\sim 32 \mathrm{kB}$ & any & 2000 & 1 \\
\hline eq. (27) & charge-energy & $63 \times 10^{3}$ & $\sim 32 \mathrm{kB}$ & any & 2000 & 1 \\
\hline sum 3 & $\propto \mathrm{n}$ for charge-energy & $0.2 \times 10^{6}$ & $\sim 160 \mathrm{kB}$ & any & 2000 & 1 \\
\hline $\mathrm{eSt}$ & $\propto \mathrm{m} \times \mathrm{n}$ for charge-distance & $0.41 \times 10^{9}$ & $\sim 400 \mathrm{kB}$ & any & 2000 & $2000 \approx n$ \\
\hline $\mathrm{eSt}+\mathrm{vrh} 1$ & $\int \mathrm{dE}$ for bulk & $0.41 \times 10^{9}$ & $\sim 600 \mathrm{kB}$ & $100 \max$ & 2000 & $2000 \approx n$ \\
\hline $\mathrm{eSt}+\mathrm{vrh} 2$ & JddEdE for bulk & $3 \times 10^{9}$ & $\sim 32 \mathrm{MB}$ & $100 \max$ & 2000 & $2000 \approx n$ \\
\hline \multirow[b]{2}{*}{ pass 2} & $\propto \mathrm{m} \times \mathrm{n}$ for $\int \mathrm{dE}$ & $0.11 \times 10^{9}$ & $\sim 600 \mathrm{kB}$ & \multirow[b]{2}{*}{$\mathrm{m} \times 100 \max$} & \multirow[b]{2}{*}{2000} & \multirow{2}{*}{50} \\
\hline & $\propto \mathrm{m} \times \mathrm{n}^{2}$ for $\iint \mathrm{dEdE}$ & $150 \times 10^{9}$ & $\sim 32 \mathrm{MB}$ & & & \\
\hline eq. (41) & $\propto \mathrm{m}$ for supplements & 1000 & $\sim 5 \mathrm{kB}$ & any & any & 50 \\
\hline \multirow{2}{*}{$\begin{array}{l}\text { total for a } \\
V_{G}-T \text { point }\end{array}$} & $\propto \mathrm{m} \times \mathrm{n}$ for $\int \mathrm{dE}$ & $0.11 \times 10^{9}$ & $\sim 605 \mathrm{kB}$ & \multirow{2}{*}{$\mathrm{m} \times 100 \max$} & \multirow{2}{*}{2000} & \multirow{2}{*}{50} \\
\hline & $\propto \mathrm{m} \times \mathrm{n}^{2}$ for $\iint \mathrm{dEdE}$ & $150 \times 10^{9}$ & $\sim 32 \mathrm{MB}$ & & & \\
\hline \multicolumn{7}{|c|}{$\begin{aligned} \text { FLOP }= & \text { floating point operation of multiplication of double precision. } \\
& \text { Comparison, summation and subtraction }=1 / 4 \text { FLOP } \\
& \text { Division }=2 \text { FLOPs } \\
& \text { Exponentiation, square rooting and logarithm }=17 \text { FLOPs }\end{aligned}$} \\
\hline
\end{tabular}




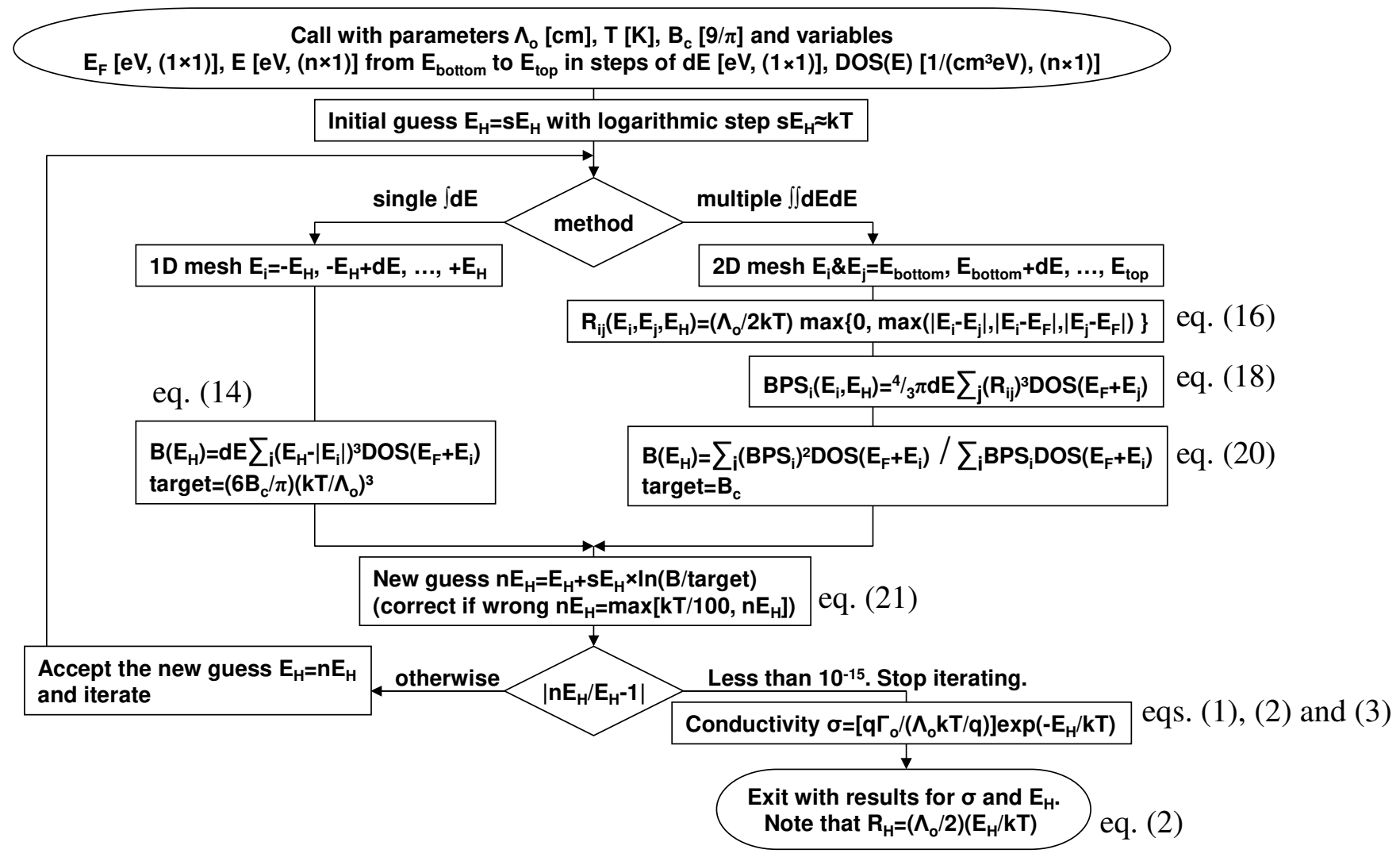

Figure 1. Algorithm of the VRH calculation module with iterative numerical calculation of hopping energy $E_{\mathrm{H}}$ by methods of single $\int \mathrm{dE}$ and multiple $\iint \mathrm{dEdE}$ integrations, and with a gradual variation of $\mathrm{E}_{\mathrm{H}}$. The output from the VRH calculation module includes $\mathrm{E}_{\mathrm{H}}$ and the specific conductivity $\sigma$. 


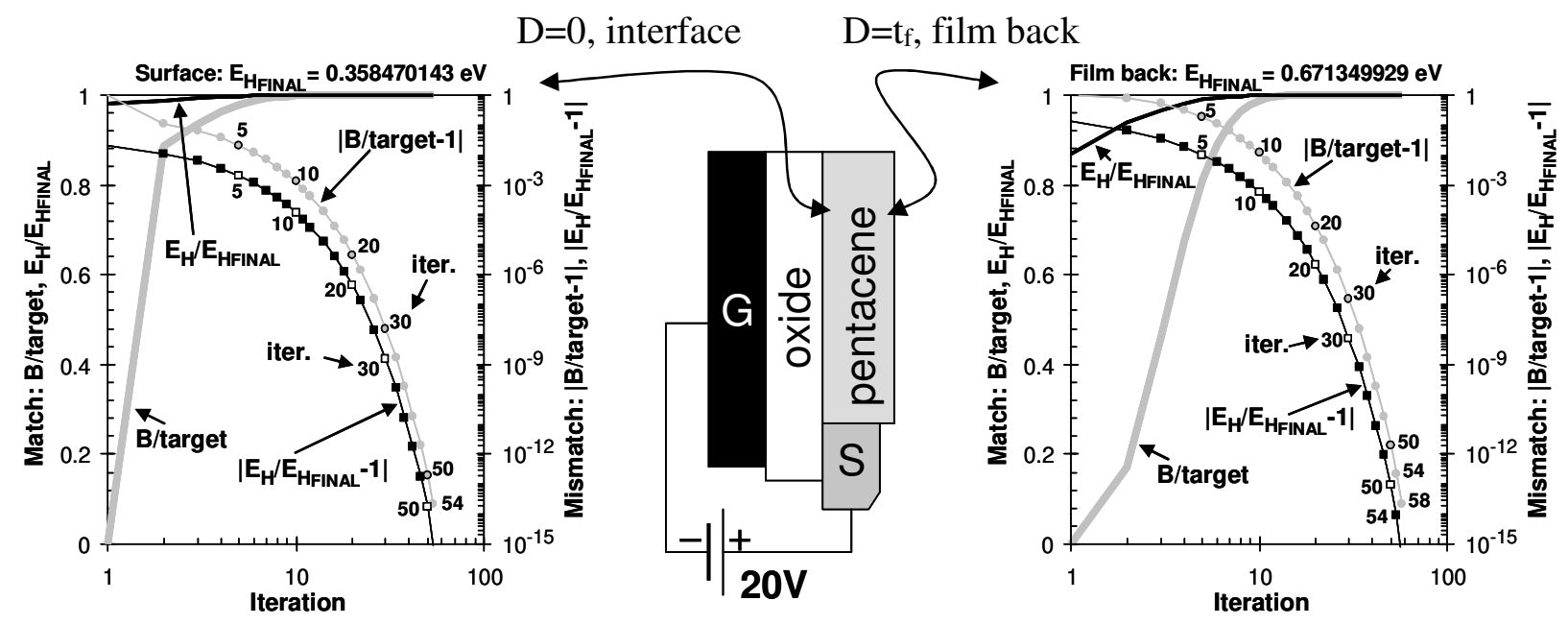

(a)

(b)

(c)

Figure 2. Convergence of the iterative numerical calculation of VRH by multiple $\iint \mathrm{dEdE}$ integrations with a gradual variation of $\mathrm{E}_{\mathrm{H}}$ at surface with gate oxide (left-hand plot) and at the pentacene film back (right-hand plot), and at room temperature $\mathrm{T}=300 \mathrm{~K}$. Increasing the iterations (horizontal axes), the lines without symbols show the improved matches $\mathrm{B}\left(\mathrm{E}_{\mathrm{H}}\right) / \mathrm{B}_{\mathrm{c}}$ and $\mathrm{E}_{\mathrm{H}}$ (iteration)/ $\mathrm{E}_{\mathrm{H}}$ (final) with thick gray and black lines, respectively. The lines with symbols show the decreasing mismatches $\left|\mathrm{B}\left(\mathrm{E}_{\mathrm{H}}\right) / \mathrm{B}_{\mathrm{c}}-1\right|$ and $\mid E_{\mathrm{H}}($ iteration $) / \mathrm{E}_{\mathrm{H}}(\mathrm{final})-1 \mid$ with gray and black colors, respectively. Open symbols with numbers close them highlight results after 5, 10, 20 and 50 iterations. 


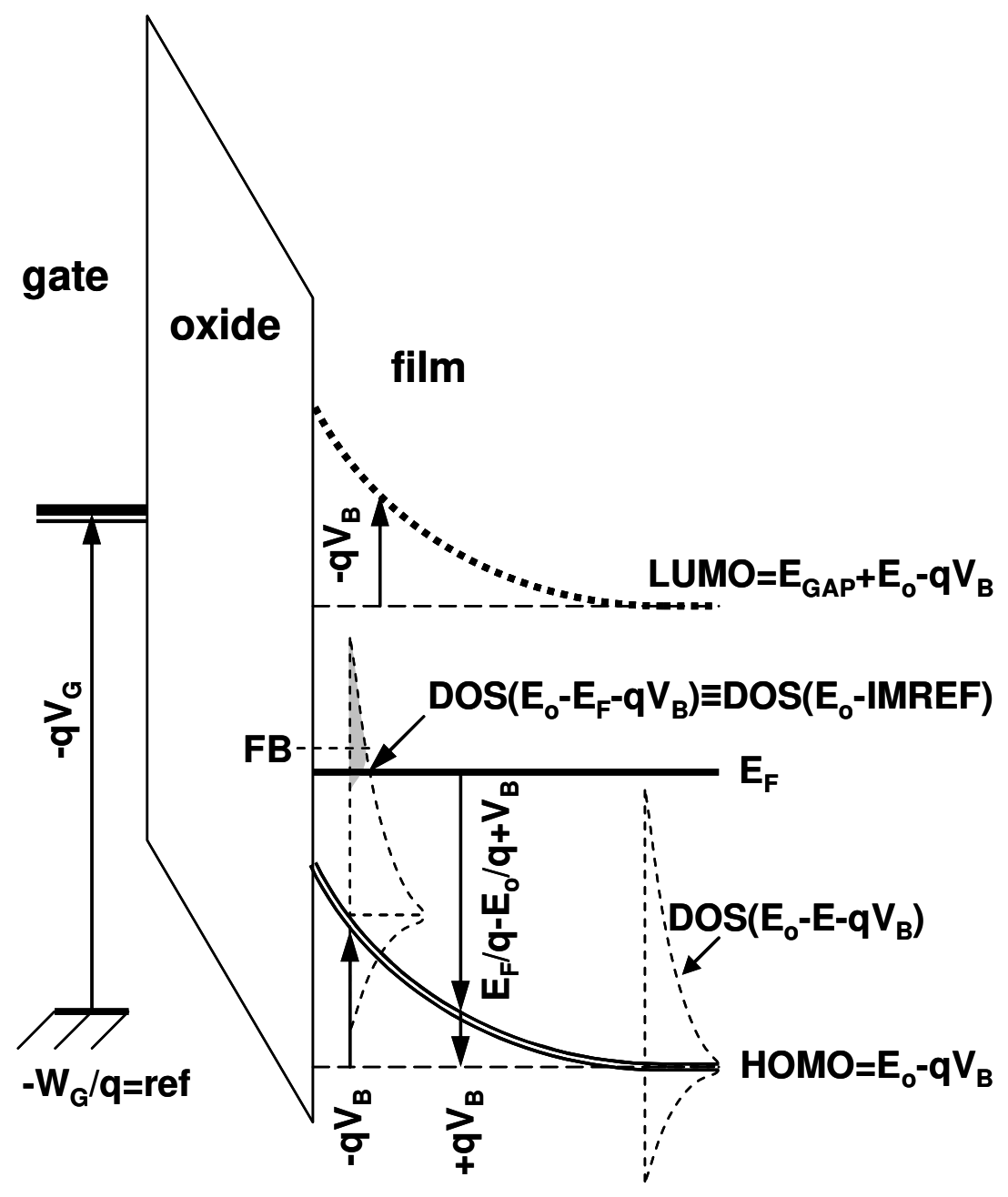

Figure 3. $\quad$ Energy diagram (not to scale) of p-type OTFT under negative gate bias $V_{\mathrm{G}}$ above threshold voltage $\mathrm{V}_{\mathrm{T}}$. HOMO (double line) and LUMO (dotted line) are bent up with $\left(-q \mathrm{~V}_{\mathrm{B}}\right)>0$ (vertical arrows). Note that $V_{G}$ and $V_{B}$ are negative voltages for p-type TFT, thus, they increase the electron potential energy. The DOS (dashed curves) is for HOMO (with energy level $\mathrm{E}_{\mathrm{o}}$ in the figure at no bias, $\mathrm{V}_{\mathrm{B}}=0$ ), following the bending voltage $\mathrm{V}_{\mathrm{B}}$. Gray-color shaded area in the left-hand DOS depicts the charge induced in the DOS tail by the gate bias. The centroid energy level FB of the induced charge can be different from Fermi level $E_{F}$. Consider $\mathrm{IMREF}=\left(\mathrm{E}_{\mathrm{F}}+\mathrm{qV}_{\mathrm{B}}\right)$ as a quasi-Fermi level under bending due to bias and exponential $\operatorname{DOS}^{k T_{o}} \propto \exp (H O M O-E)$, then DOS $\left(\mathrm{E}_{\mathrm{o}}-\mathrm{qV}_{\mathrm{B}}-\mathrm{E}_{\mathrm{F}} \pm \Delta \mathrm{E}\right)=\mathrm{DOS}\left(\mathrm{E}_{\mathrm{o}}-\mathrm{IMREF} \pm \Delta \mathrm{E}\right)$, where $\Delta \mathrm{E}$ is an energy span of interest and the bending $-\mathrm{qV}_{\mathrm{B}}$ is embedded in IMREF; see after eq. (23) for more explanations. 


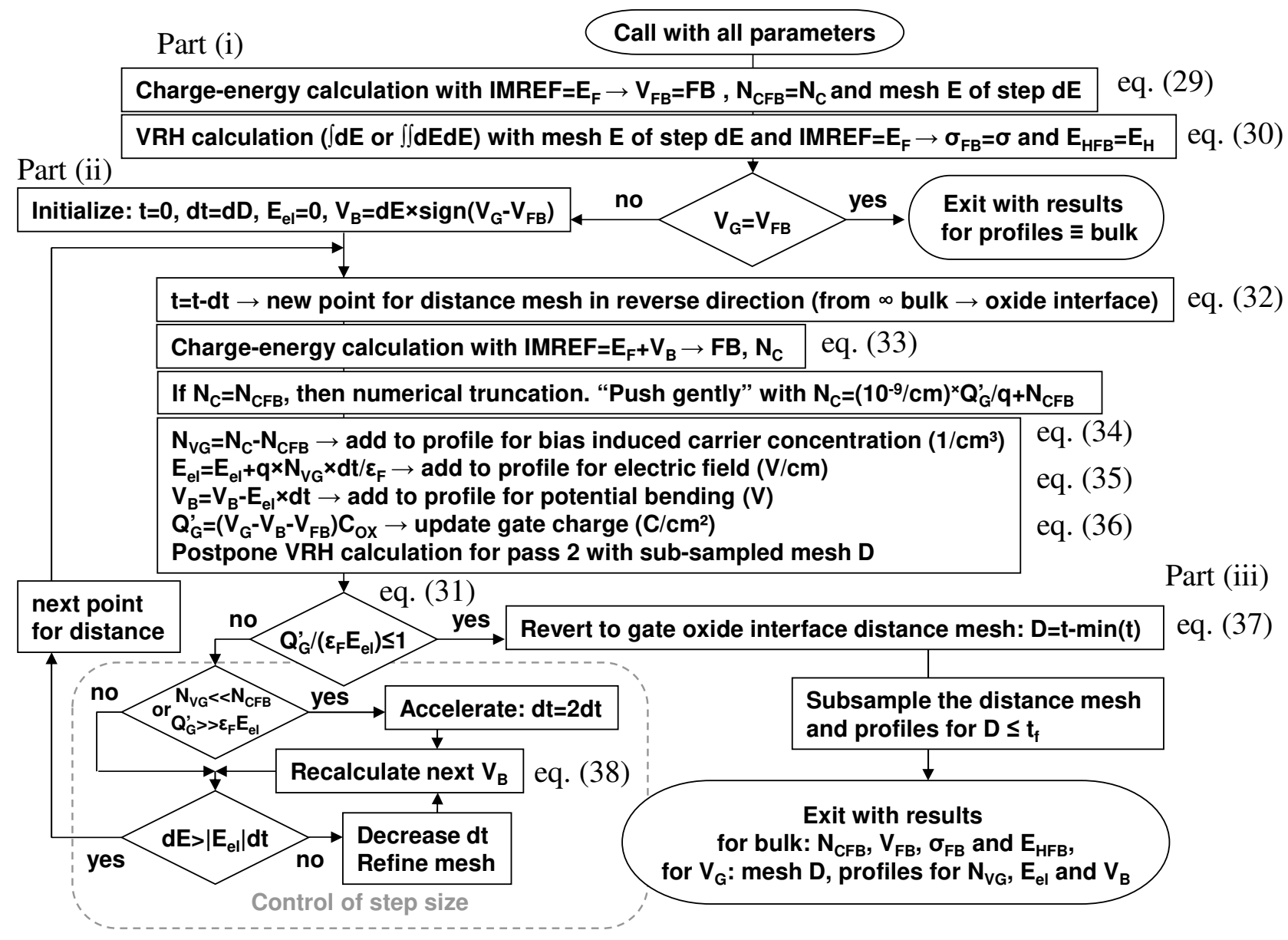

Figure 4. Flow of the algorithm of the charge-distance calculation module. First, the equilibrium charge $\mathrm{N}_{\mathrm{CFB}}$ and potential $\mathrm{V}_{\mathrm{FB}}$ in bulk material are calculated, along with $\mathrm{VRH}$ conductivity $\sigma_{\mathrm{FB}}$ and hopping energy $E_{H F B}$ for the bulk material. Then, at given gate bias voltage $V_{G}$, the potential bending $V_{B}$ and corresponding profiles for electrostatic quantities are calculated in an iterative loop with variable step dt in reverse of the film depth $(\mathrm{t}<0$, last $\mathrm{t}=$ min corresponds to dielectric-semiconductor film interface) until the gate charge $\mathrm{Q}_{\mathrm{G}}$ is balanced by the electric field $\mathrm{E}_{\mathrm{el}}\left(\mathrm{Q}_{\mathrm{G}} \leq \varepsilon_{\mathrm{F}} \mathrm{E}_{\mathrm{el}}\right)$. Finally, the reversed mesh $\mathrm{t}$ is reverted to mesh $\mathrm{D}=[\mathrm{t}-\min (\mathrm{t})]$ for film depth $\mathrm{D}$ from gate dielectric-semiconductor film interface $(\mathrm{D}=0)$ up to the film thickness $\left(\mathrm{D}=\mathrm{t}_{\mathrm{f}}\right)$, and the mesh $\mathrm{D}$ and profiles are sub-sampled logarithmically to reduce the subsequent VRH calculations. 


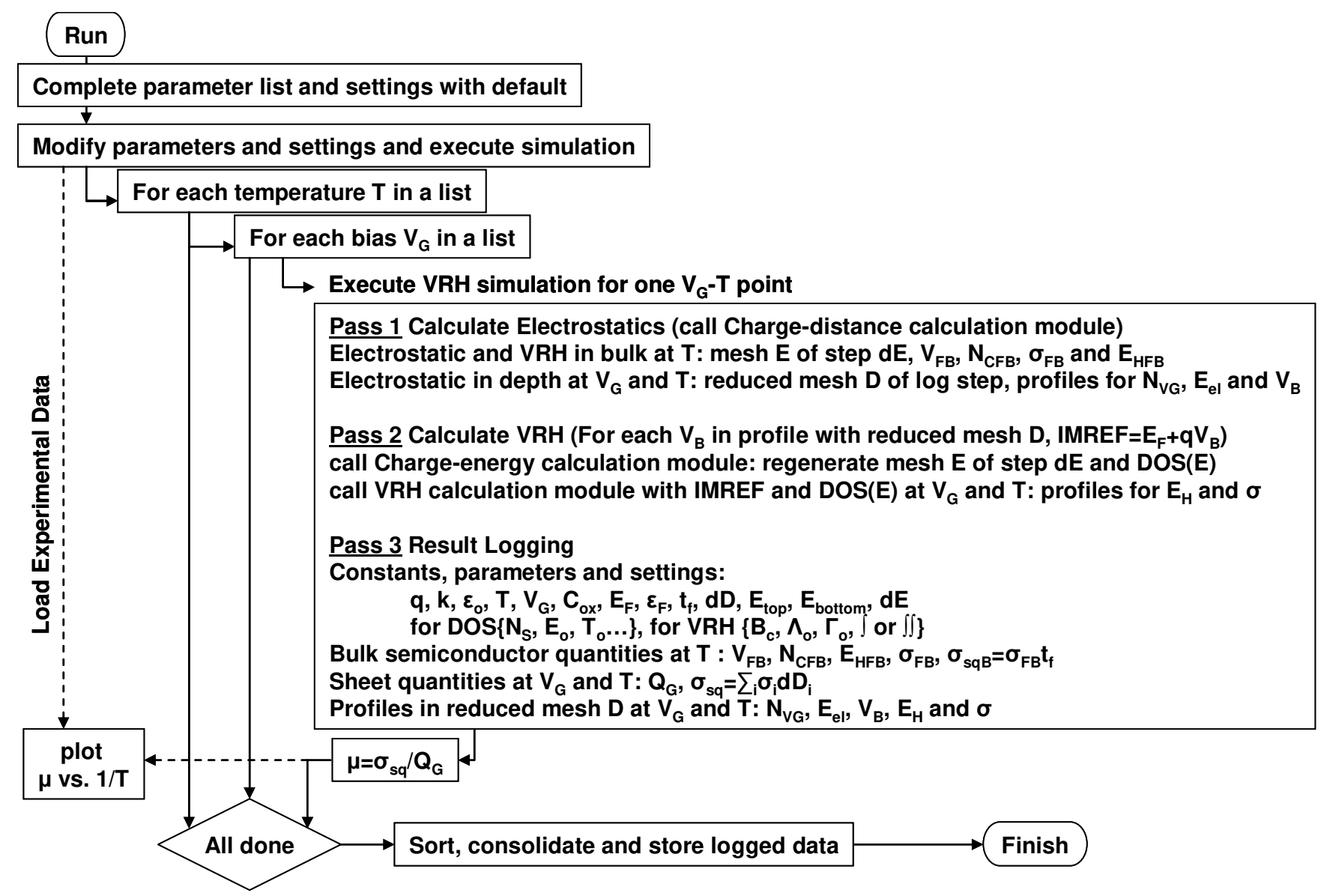

Figure 5. Overall flow of the VRH numerical simulator for the OTFT's conductivity. The simulation core is for one bias-temperature $\left(\mathrm{V}_{\mathrm{G}}-\mathrm{T}\right)$ point and comprises three passes for electrostatic and VRH calculations and logging of the simulation results. 

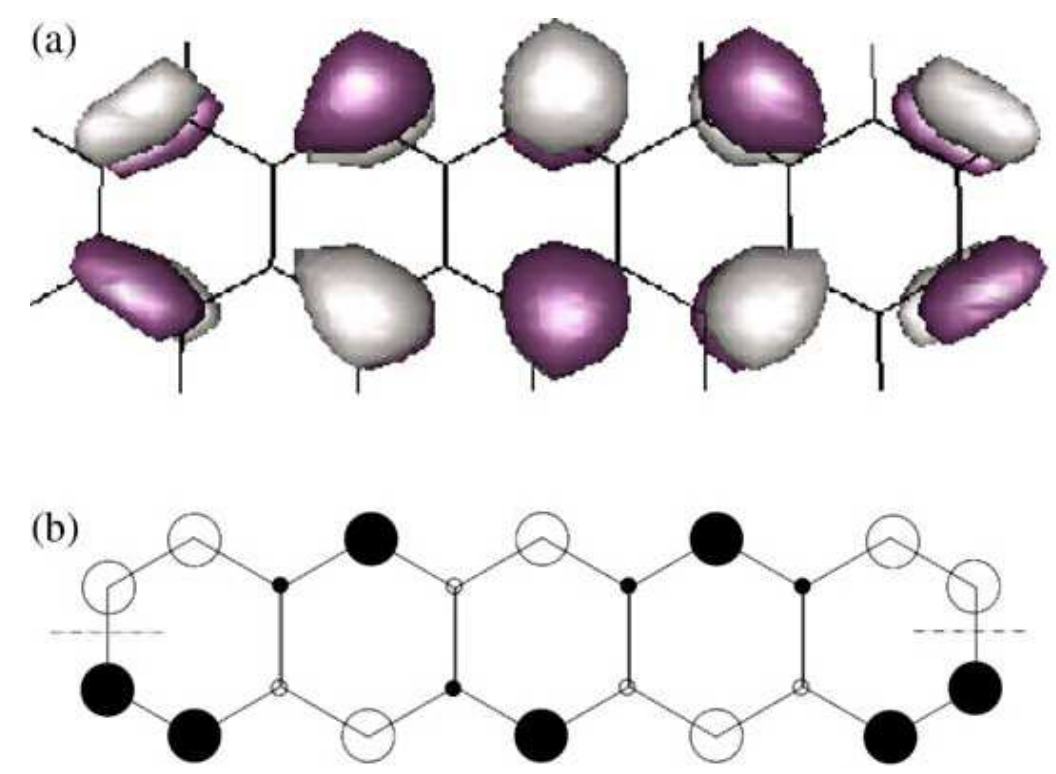

Figure 6. Wave functions for HOMO of isolated pentacene molecule (adapted from [32], with permission 3437410676840 from Elsevier). The dark color represents a positive sign, the light color a negative sign. (a) isosurface at $\pm 0.05 / \AA^{3 / 2}$; (b) coefficients of the wave function: large circles $0.12-0.3$, small circles 0.01-0.09. 


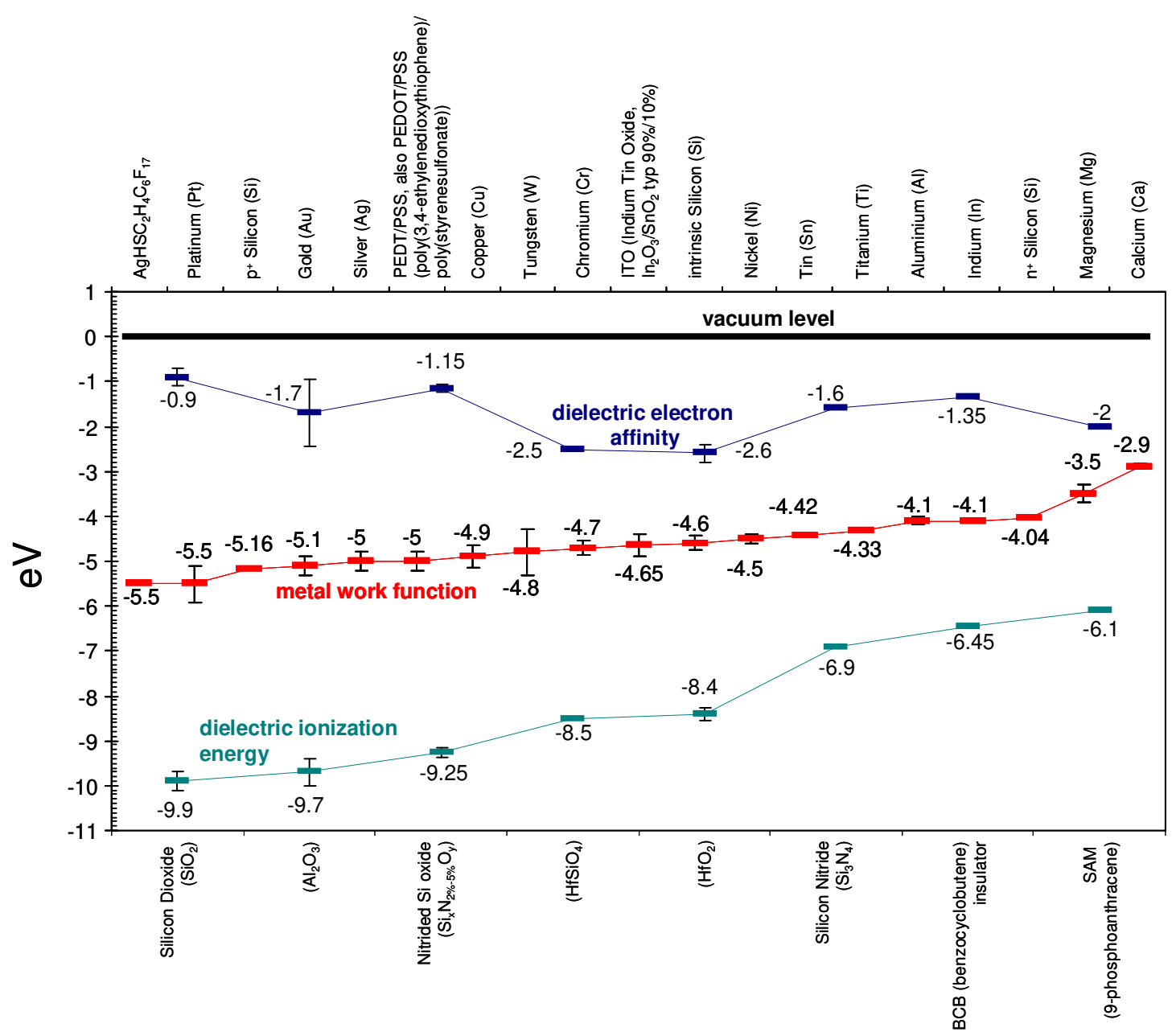

Figure 7. Metal work function (middle plot) of conductive materials (top axis) that might be used as gate electrodes in OTFT, and electron affinity (upper plot) and ionization energies (bottom plot) of insulating materials (bottom axis) that can be used as gate dielectrics in OTFT. The data correspond to columns "Recommended values" in Table I and Table II. All values are inverted in order to correspond to the vacuum reference level (the solid line on the top). 


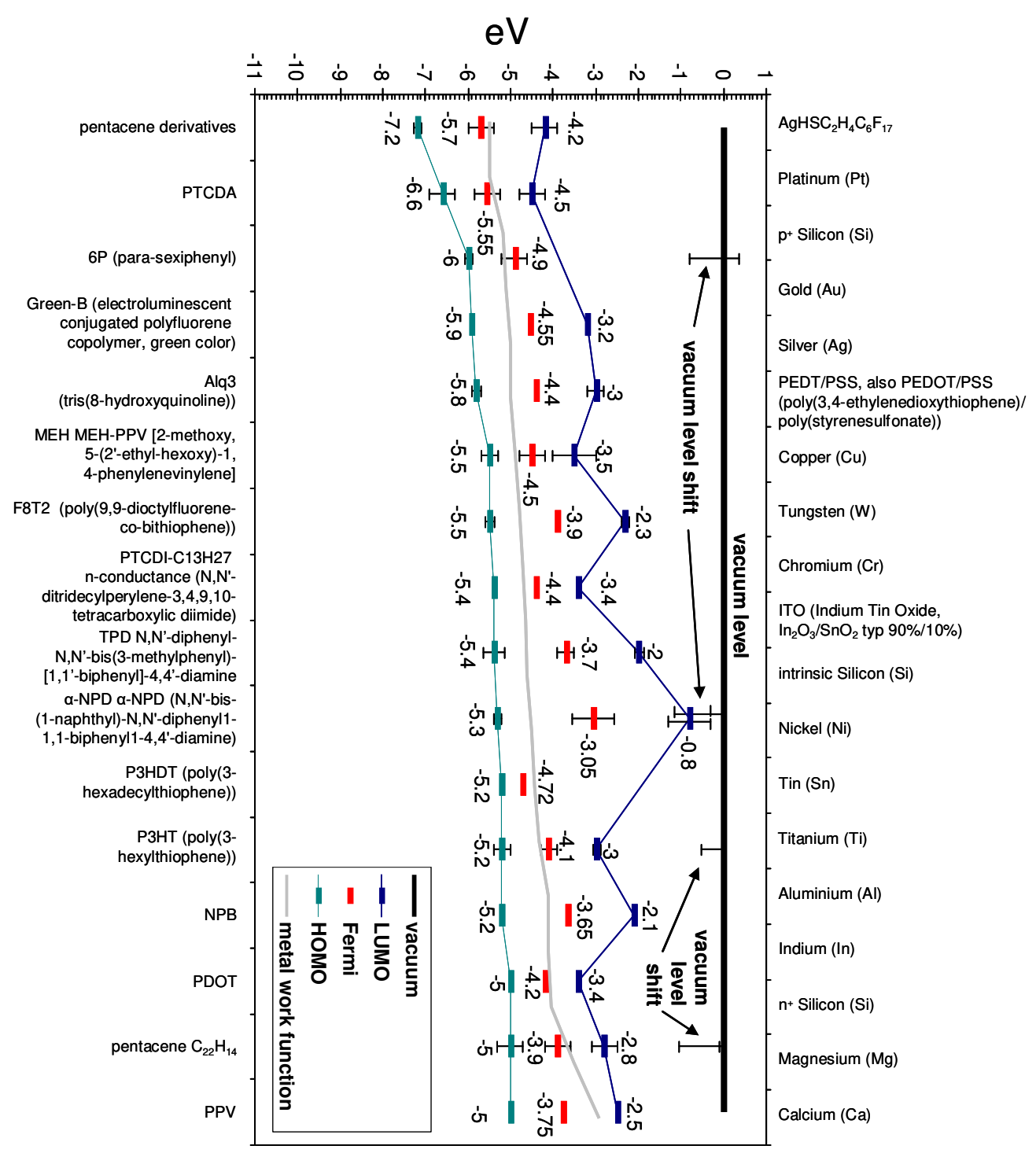




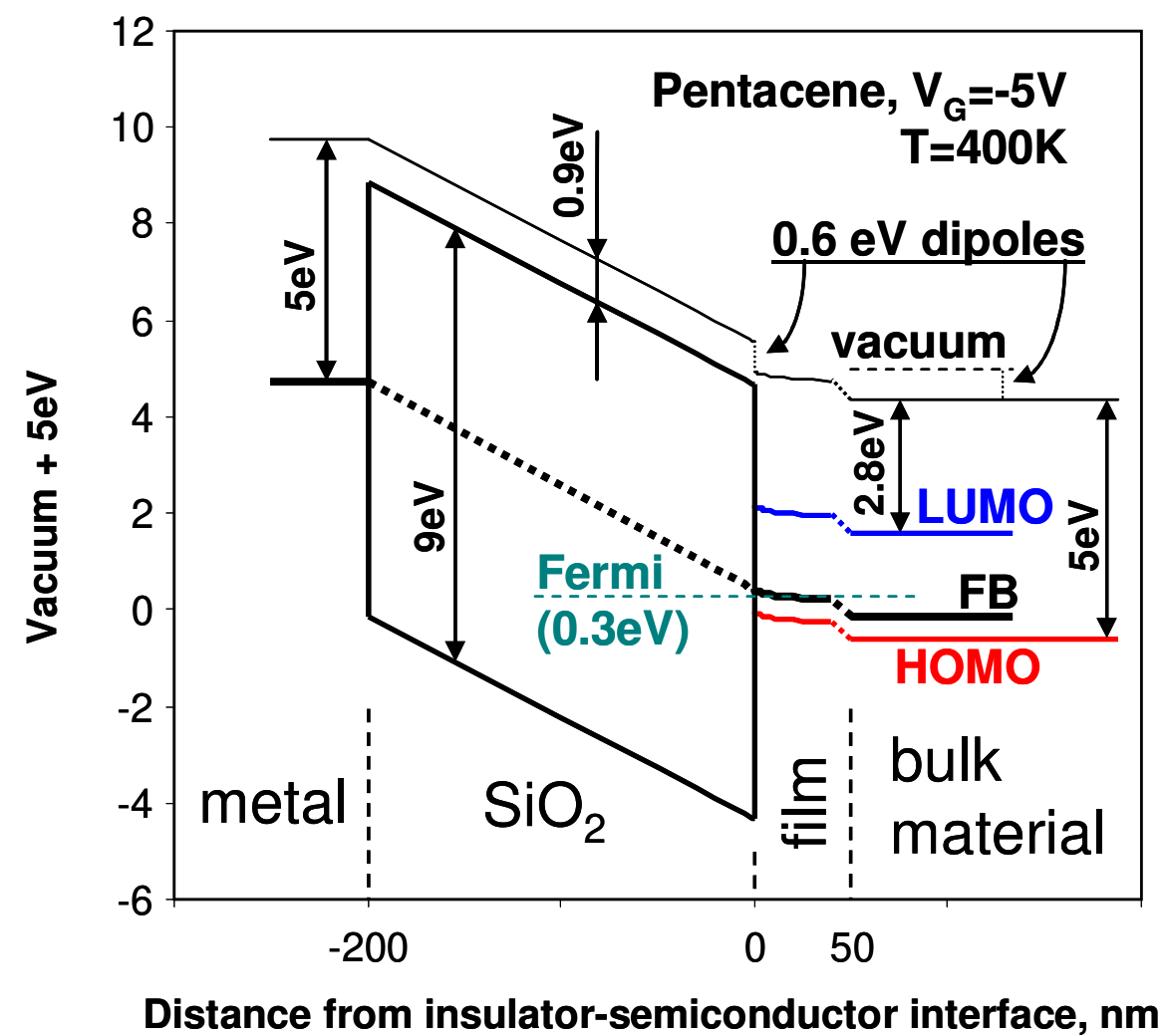

Figure 9. Energy diagram of a pentacene TFT with $200 \mathrm{~nm} \mathrm{SiO}_{2}$ gate dielectric and gate electrode corresponding to $\mathrm{p}^{+} \mathrm{Si}, \mathrm{Au}$ or $\mathrm{Ag}$, and at temperature of $\mathrm{T}=400 \mathrm{~K}$ and gate bias of $\mathrm{V}_{\mathrm{G}}=-5 \mathrm{~V}$. Note the dipole shift of $-0.6 \mathrm{eV}$, the difference between Fermi and flat-band (FB) levels, and the steps between the levels at the back of the film and the levels corresponding to bulk material. 


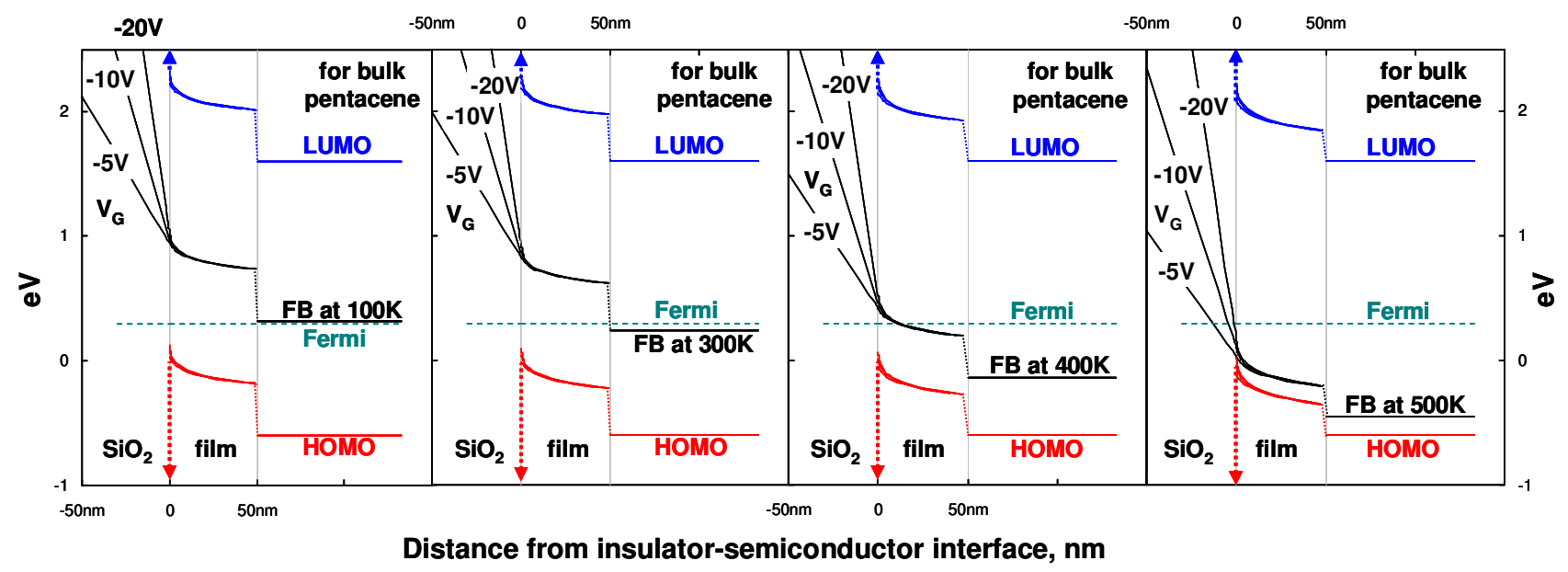

Figure 10. Close look at the energy diagrams of the pentacene OTFT from Figure 9 at three gate bias voltages, $\mathrm{V}_{\mathrm{G}}=\{-5 \mathrm{~V},-10 \mathrm{~V}$ and $-20 \mathrm{~V}\}$ and from left to right for four temperatures, $\mathrm{T}=\{100 \mathrm{~K}, 300 \mathrm{~K}$, $400 \mathrm{~K}=\mathrm{T}_{\mathrm{o}}$ and $500 \mathrm{~K}$. Note that the levels in the back of film do not reach the LUMO, FB and HOMO levels of the bulk material, the difference decreases when the temperature increases, accompanied with a shift of the flat-band level FB from the Fermi level at low temperature to the HOMO level at high temperature. 


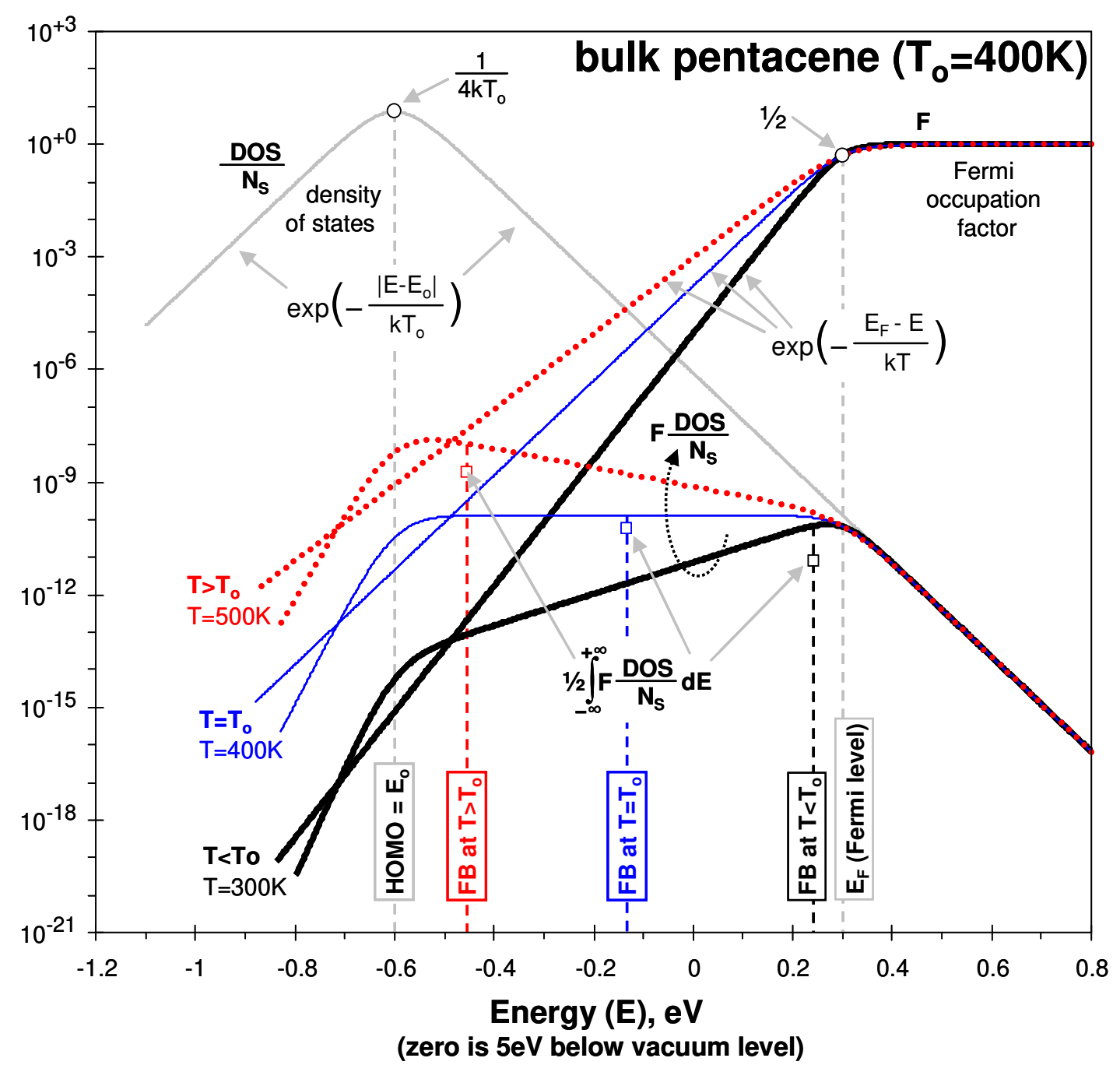

Figure 11. Evolution of the DOS occupancy with temperature, resulting in a shift of the flat-band (FB) level from close to the Fermi level at low temperatures $\left(\mathrm{T}<\mathrm{T}_{\mathrm{o}}\right)$ to close to the DOS centroid level $\left(\mathrm{E}_{\mathrm{o}}=\mathrm{HOMO}\right.$ for $\mathrm{p}$-type OTFT) at high temperatures $\left(\mathrm{T}>\mathrm{T}_{\mathrm{o}}\right)$. The normalized double-exponential DOS/NS with a characteristic temperature $T_{0}=400 \mathrm{~K}$ is shown with a gray line. The total concentration of states is $\mathrm{N}_{S}=\int \mathrm{DOS}(\mathrm{E}) \mathrm{dE}$ for $(-\infty<\mathrm{E}<+\infty)$. Thick, thin and dotted lines denote a low temperature $\left(\mathrm{T}=300 \mathrm{~K}<\mathrm{T}_{\mathrm{o}}\right)$, through $\mathrm{T}=\mathrm{T}_{\mathrm{o}}=400 \mathrm{~K}$, to a high temperature $\left(\mathrm{T}=500 \mathrm{~K}>\mathrm{T}_{\mathrm{o}}\right.$ ), respectively, for the majority carrier (holes in this case) Fermi occupation factor $(\mathrm{F})$ and for the normalized DOS occupation (F $\times$ DOS/ $\left.\mathrm{N}_{\mathrm{S}}\right)$. The HOMO, Fermi and flat-band levels are shown with vertical dashed lines. Circles illustrate the characteristic values for $\operatorname{DOS}\left(E_{0}\right)$ and $F\left(E_{F}\right)$. Squares pointed with arrows illustrate the normalized values for the half concentration of occupied states, $1 / 2 \int \mathrm{F} \times \mathrm{DOSdE} / \mathrm{N}_{\mathrm{S}}$ by $(-\infty<\mathrm{E}<+\infty)$, and correspond to the condition in eq. (26) for determination of the flat-band (FB) level. 


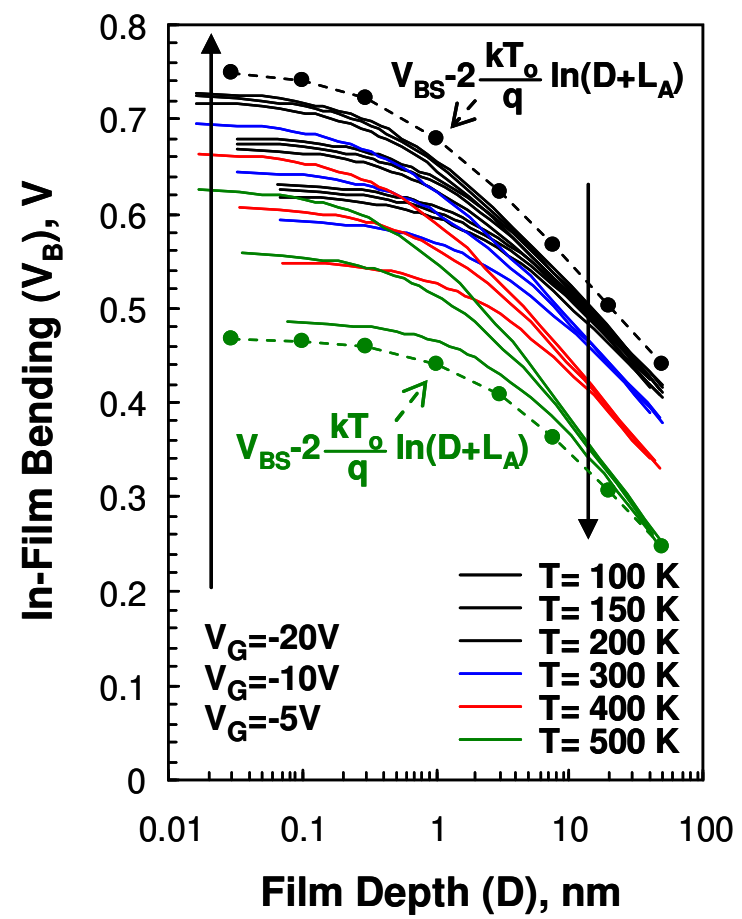

Figure 12. Simulated profiles of the potential bending $V_{B}$ (solid lines) in the OTFT's 50nm pentacene film for three gate bias voltages $V_{G}$ (triplets of curves that coincide on right at each temperature) and at several ambient temperatures $\mathrm{T}$ (each triplet of curves is for one temperature). The gate insulator is $200 \mathrm{~nm}$ $\mathrm{SiO}_{2}$. The characteristic temperature of the double-exponential DOS is $\mathrm{T}_{0}=400 \mathrm{~K}$ and the concentration of states is $\mathrm{N}_{S}=\int \mathrm{DOS}(\mathrm{E}) \mathrm{dE}=5 \times 10^{21} \mathrm{~cm}^{-3}$ for $(-\infty<\mathrm{E}<+\infty)$. The permittivity of the organic material is assumed $\varepsilon_{\mathrm{f}}=3 \varepsilon_{\mathrm{o}} \approx 2.66 \times 10^{-13} \mathrm{~F} / \mathrm{cm}$. The dashed lines with circles represent the logarithmic dependence of $\mathrm{V}_{\mathrm{B}}$ on the depth $\mathrm{D}$ in the film, calculated with eq. (48) for $\mathrm{V}_{\mathrm{G}}=-20 \mathrm{~V}$ (top dashed line) and $\mathrm{V}_{\mathrm{G}}=-5 \mathrm{~V}$ (bottom dashed line). The values for $\mathrm{V}_{\mathrm{BS}}$ are arbitrarily chosen so that the dashed lines are close to, but not overlapping the profiles of $\mathrm{V}_{\mathrm{B}}$. 


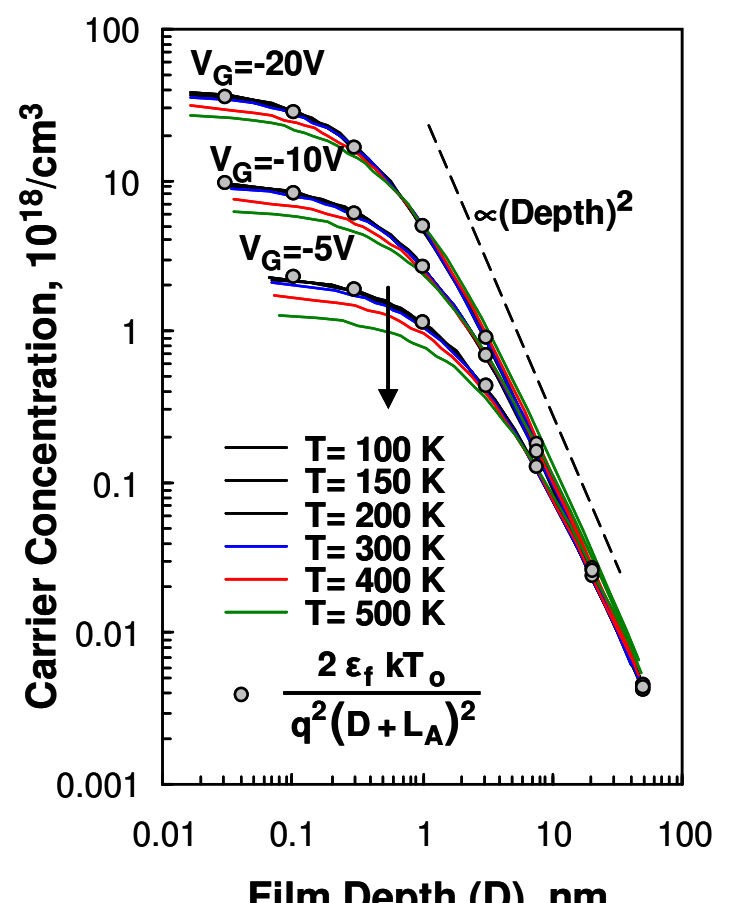

Film Depth (D), nm

Figure 13. Simulated profiles of carrier concentration $\mathrm{N}_{\mathrm{C}}$ (solid lines) in the OTFT's 50nm pentacene film at different gate bias voltages $\mathrm{V}_{\mathrm{G}}$ and ambient temperatures $\mathrm{T}$. Parameters are as in Figure 12. The circles are calculated with eq. (49). 


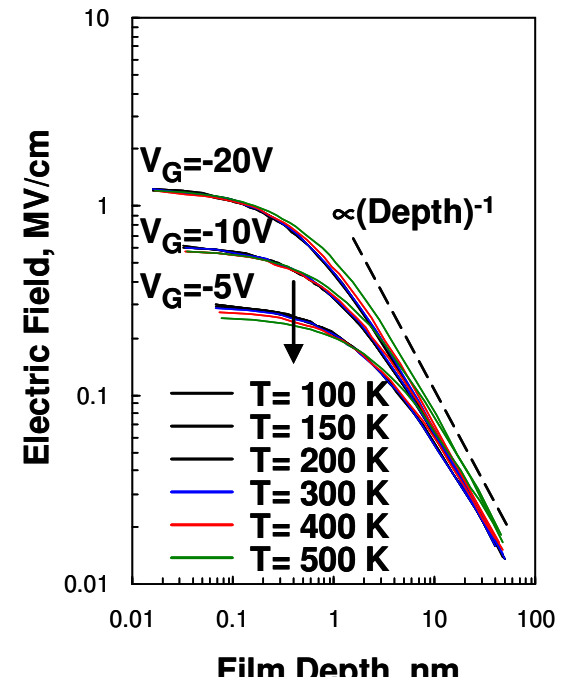

Film Depth, nm

(a)

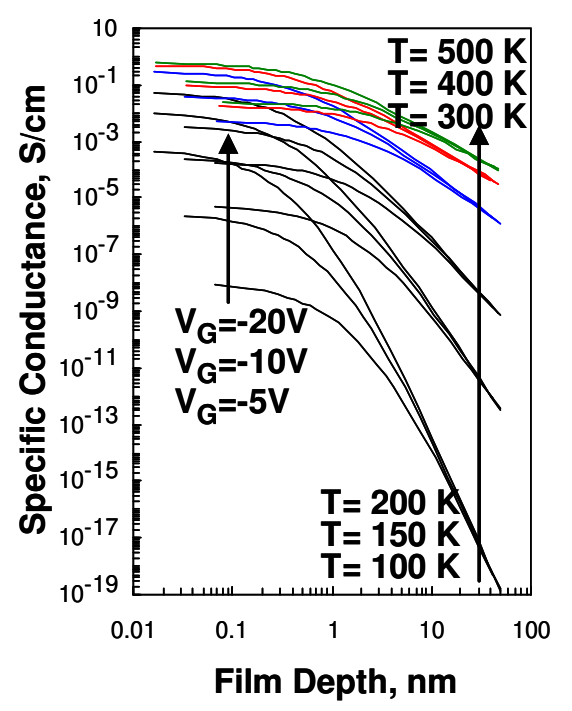

(c)

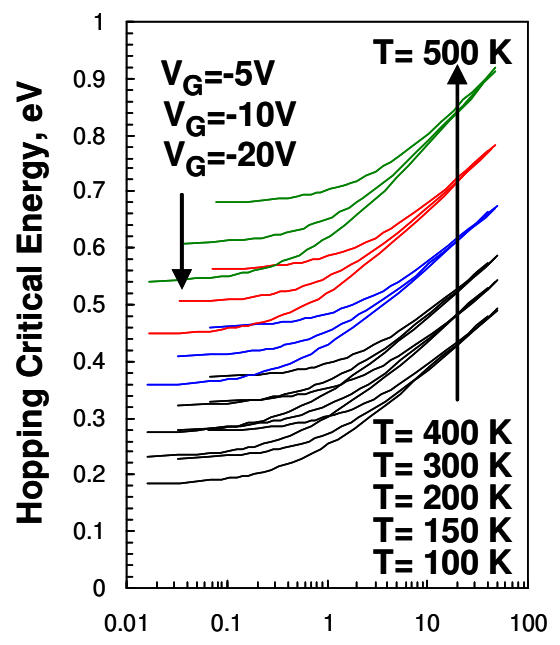

Film Depth, nm

(b)

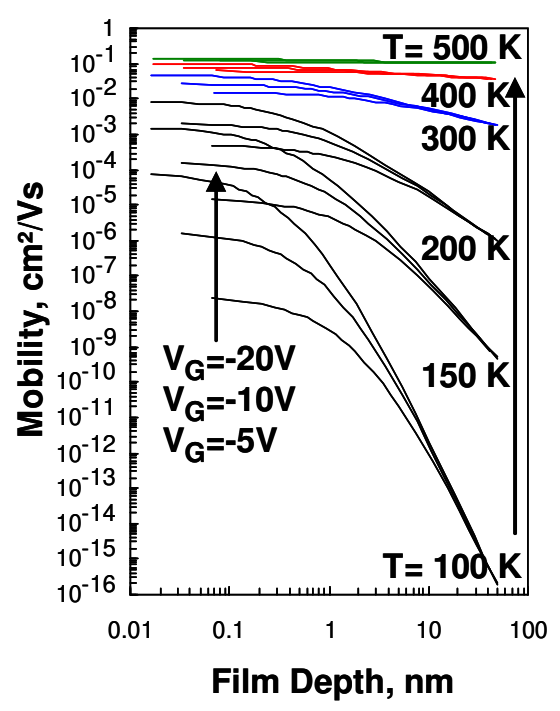

(d)

Figure 14. Profiles of the (a) electric field, (b) hopping critical energy, (c) conductance and (d) mobility in the OTFT's 50nm pentacene film at different gate bias voltages $\mathrm{V}_{\mathrm{G}}$ and temperatures $\mathrm{T}$. Parameters are as in Figure 12. 

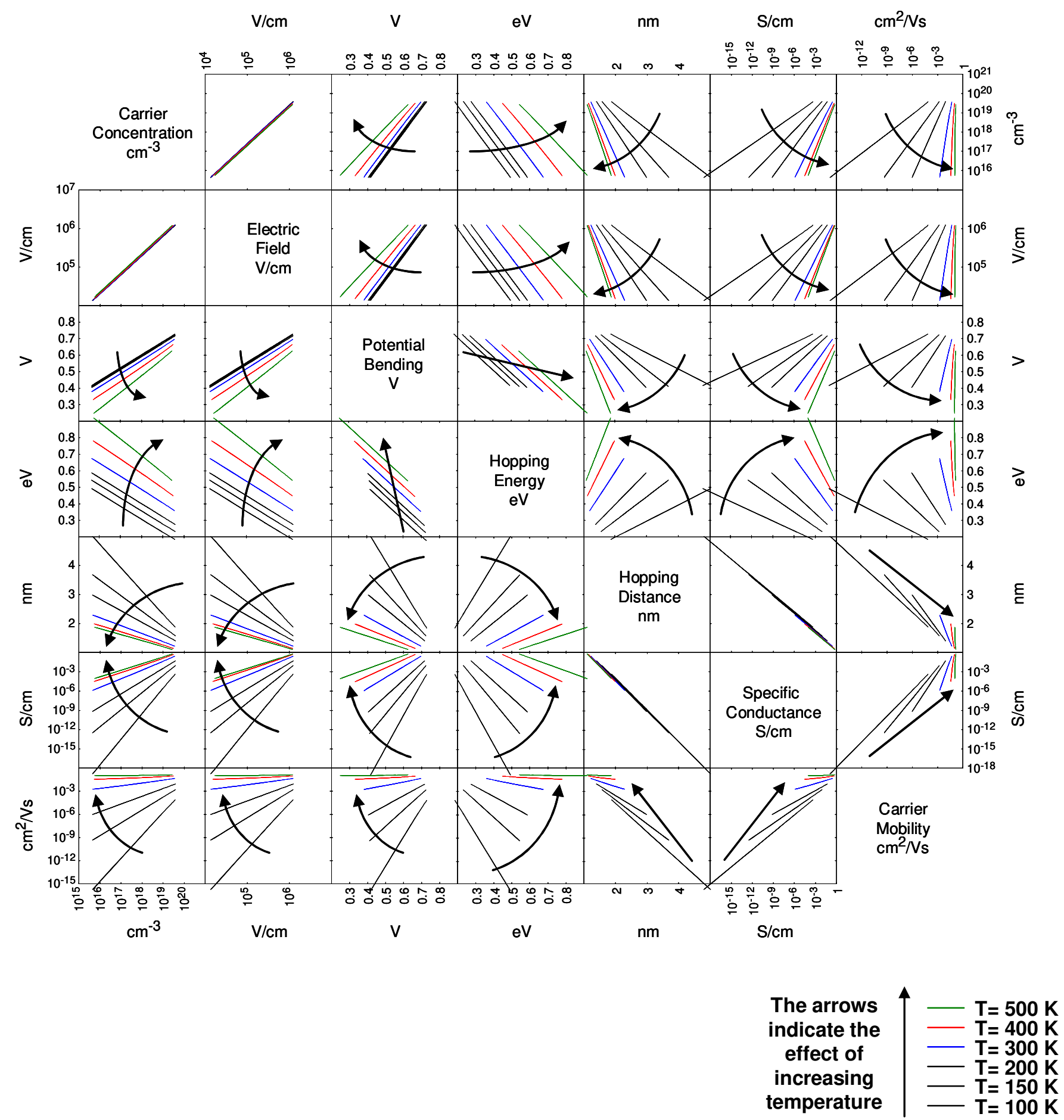

Figure 15. Correlations between several quantities in the $50 \mathrm{~nm}$ pentacene film of an OTFT at different gate bias voltages $\mathrm{V}_{\mathrm{G}}$ and ambient temperatures T. Parameters are as in Figure 12. Note that the correlations are bias-independent, since the lines at different $\mathrm{V}_{\mathrm{G}}=\{-5 \mathrm{~V},-10 \mathrm{~V},-20 \mathrm{~V}\}$ overlap, just spanning different intervals. The correlations are temperature-dependent, as indicated by arrows for the effect of increasing temperature $\mathrm{T}=\{100 \mathrm{~K}, 150 \mathrm{~K}, 200 \mathrm{~K}, 300 \mathrm{~K}, 400 \mathrm{~K}$ to $500 \mathrm{~K}\}$. 


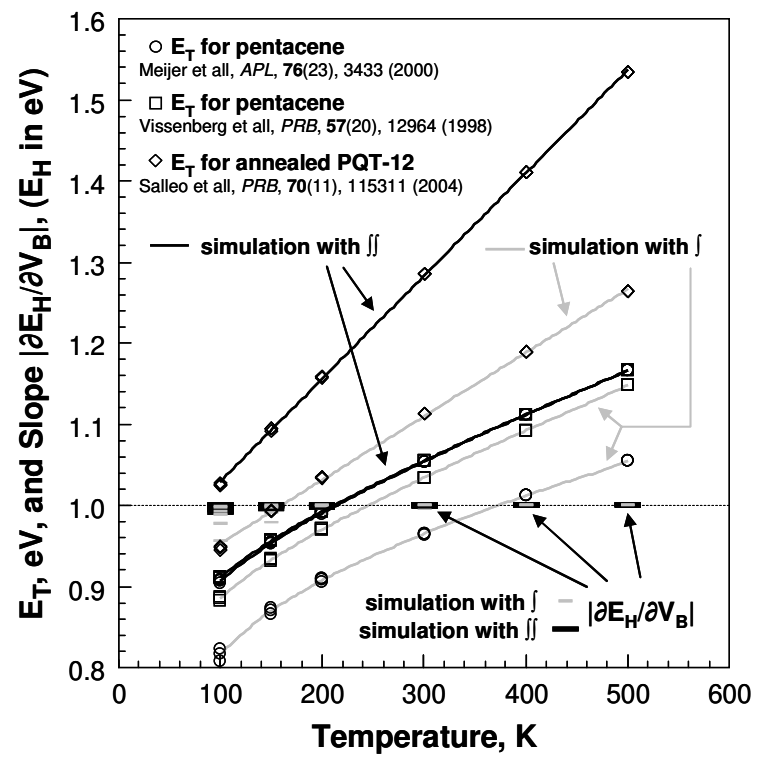

(a)

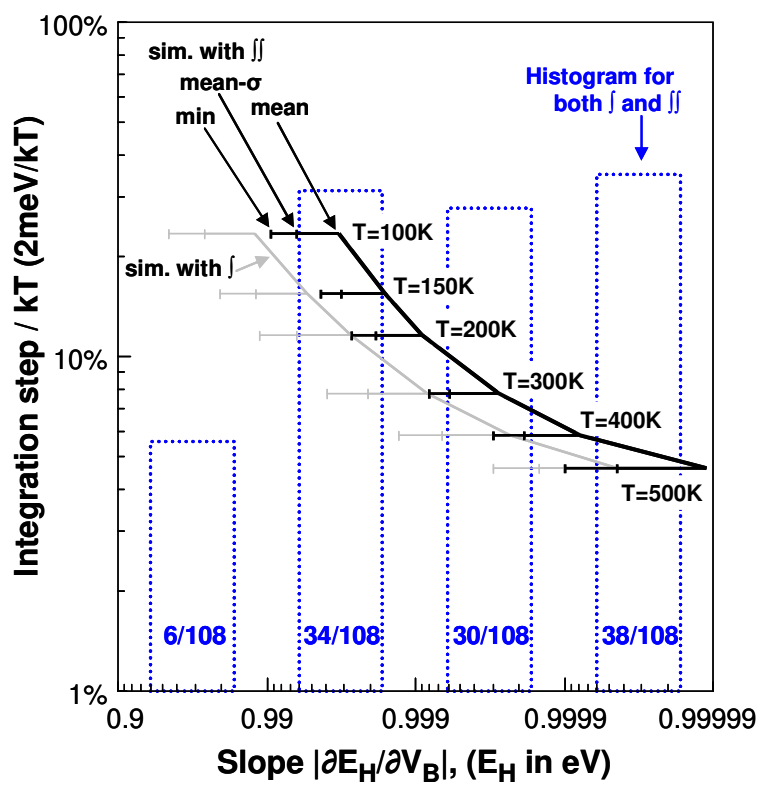

(b)

Figure 16. Analyses of the correlation $\left(\mathrm{E}_{\mathrm{H}}+\mathrm{q} \mathrm{V}_{\mathrm{B}} \times\left|\partial \mathrm{E}_{\mathrm{H}} / \partial \mathrm{V}_{\mathrm{B}}\right|\right)=\mathrm{E}_{\mathrm{T}}$, with $\left|\partial \mathrm{E}_{\mathrm{H}} / \partial \mathrm{V}_{\mathrm{B}}\right|$ obtained from the slope of the linear regression of $E_{H} v s . V_{B}$, between hopping critical energy $E_{H}$ and potential bending $V_{B}$, see eq. (50), at different temperatures $(\mathrm{T})$ and gate bias $\left(\mathrm{V}_{\mathrm{G}}\right)$ in three OTFTs by two simulation methods for the hopping conduction, single $\int \mathrm{dE}$ integration (gray color) and multiple $\iint \mathrm{dEdE}$ integrations (black color). (a) The values for $\mathrm{E}_{\mathrm{T}}$ are denoted with symbols $\bigcirc \square \diamond$ for two pentacene OTFTs and a PQT-12 OTFT, from $[22,60,73]$, respectively, and $\mathrm{E}_{\mathrm{T}}$ is bias-independent, since the symbols overlap (one exception: look at the three circles in the bottom-left). The values for the correlation slope $\left|\partial \mathrm{E}_{\mathrm{H}} / \partial \mathrm{V}_{\mathrm{B}}\right| \approx 1$ are denoted with dashes. (b) Statistics of the slope $\left|\partial \mathrm{E}_{\mathrm{H}} / \partial \mathrm{V}_{\mathrm{B}}\right|$ for deviations from unity. The curved lines (mean) represent average values for the slope $\left|\partial \mathrm{E}_{\mathrm{H}} / \partial \mathrm{V}_{\mathrm{B}}\right|$ vs. the energy step normalized to the thermal energy $\mathrm{kT}, \mathrm{dE} / \mathrm{kT}=2 \mathrm{meV} / \mathrm{kT}$. They are evaluated with numerical integrations with error bars for one standard deviation (mean $-\sigma$ ) and greatest reduction (min), showing that the deviation of the slope $\left|\partial \mathrm{E}_{\mathrm{H}} / \partial \mathrm{V}_{\mathrm{B}}\right|$ from unity is due to a coarse integration step at low temperatures $(\mathrm{dE} / \mathrm{kT}>10 \%$ at $\mathrm{T} \leq 200 \mathrm{~K})$. The histogram (dotted bars for 108 data points, blue color) of the slope $\left|\partial \mathrm{E}_{\mathrm{H}} / \partial \mathrm{V}_{\mathrm{B}}\right|$ values implies that $\left|\partial \mathrm{E}_{\mathrm{H}} / \partial \mathrm{V}_{\mathrm{B}}\right|=1 \pm 1 \%$ with $90 \%$ confidence. 


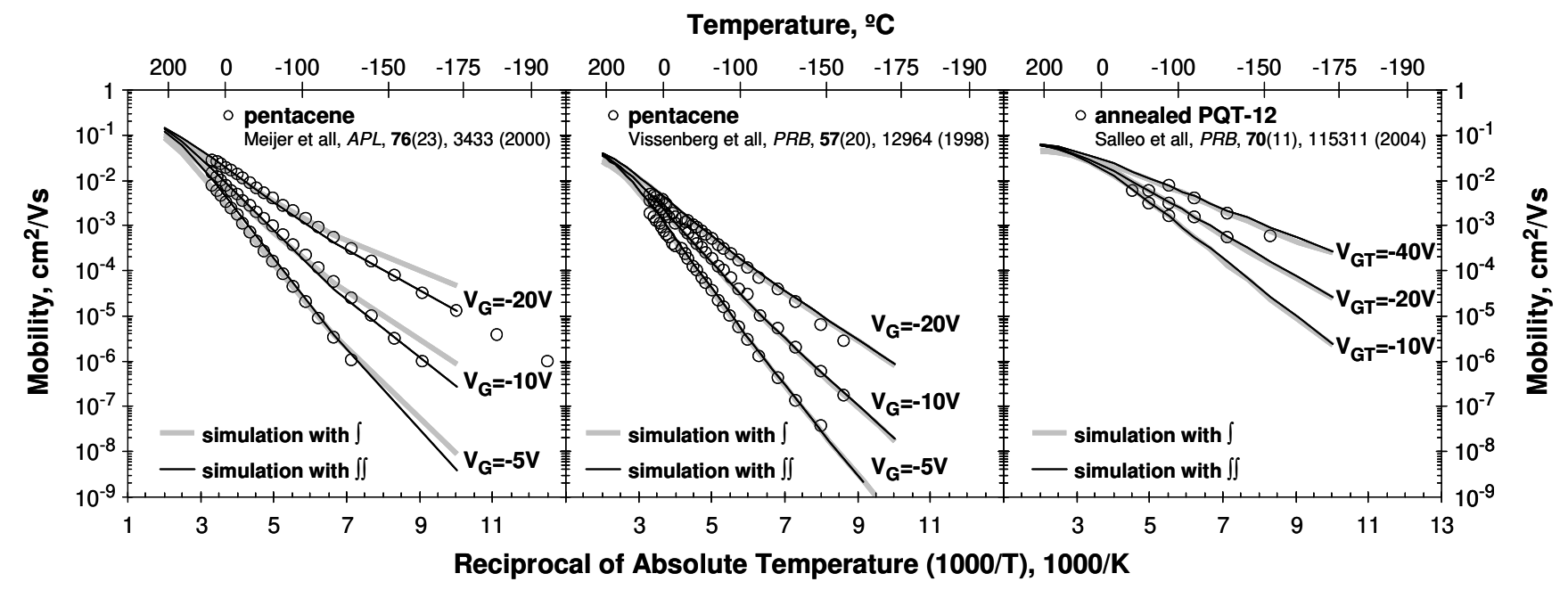

(a)

(b)

(c)

Figure 17. Temperature-bias dependence of mobility in three OTFTs. Experimental data (circles) in plots (a) from [73], (b) from [22] and (c) from [60] are for two pentacene OTFTs (at gate bias voltages $V_{G}$ ) and a PQT-12 OTFT (at gate overdrive voltages $\mathrm{V}_{\mathrm{GT}}=\mathrm{V}_{\mathrm{G}}-\mathrm{V}_{\mathrm{T}}$ ), respectively. The lines are data obtained by two simulation methods for the hopping conduction, single $\int \mathrm{dE}$ integration (gray-color thick lines) and multiple $\iint \mathrm{dEdE}$ integrations (black-color thin lines). The parameters used in the simulations are given in Table IV. The colors and plots (a), (b) and (c) correspond to the colors and symbols $\bigcirc \square \diamond$ in Figure 16(a). 


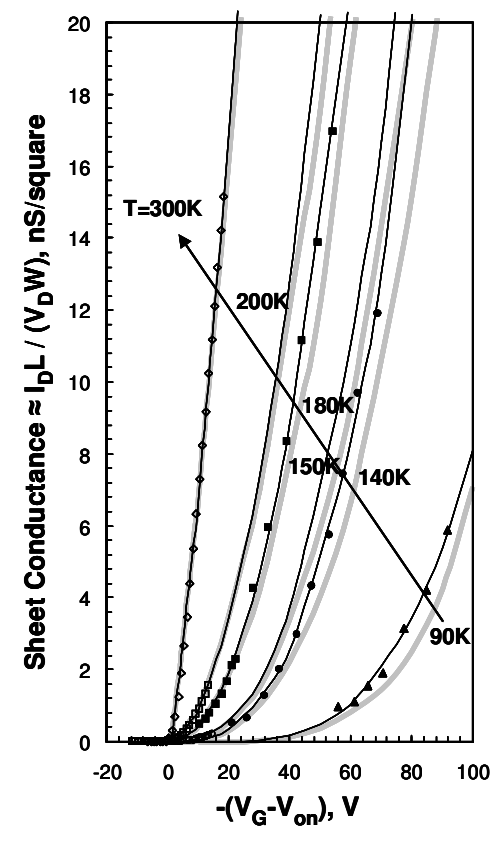

(a)

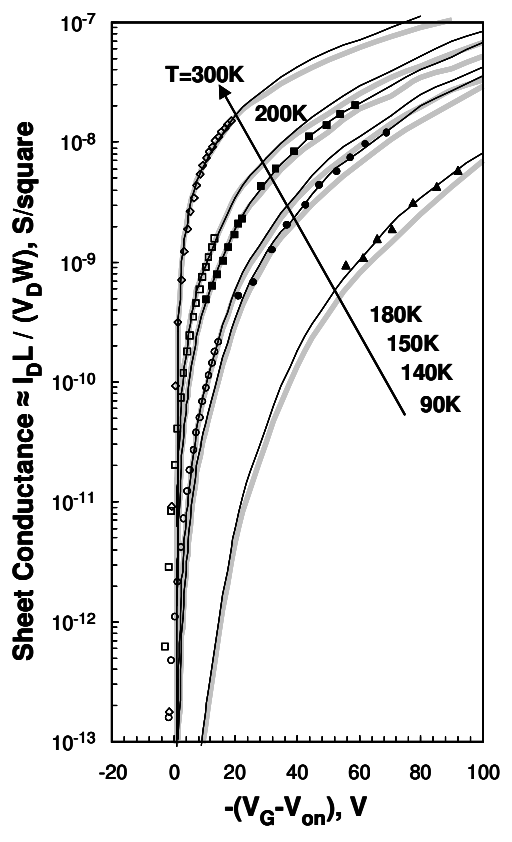

(b)

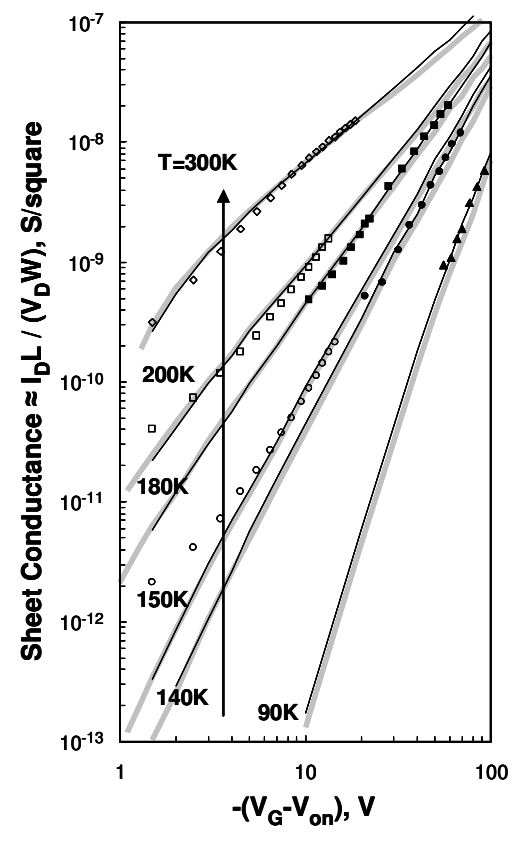

(c)

Figure 18. Linear (a), semi-logarithmic (b) and logarithmic (c) plots of the sheet conductance $\left(\sigma_{\mathrm{sq}}\right)$ of PQT-12 OTFTs vs. gate overdrive $\left(\mathrm{V}_{\mathrm{G}}-\mathrm{V}_{\mathrm{on}}\right)$, corresponding to the mobility in Figure 17c and diamond symbols $(\diamond)$ in Figure 16a. The lines are data obtained by two simulation methods for the hopping conduction, single $\int \mathrm{dE}$ integration (gray-color thick lines) and multiple $\iint \mathrm{dEdE}$ integrations (black-color thin lines). The symbols are recalculated using $\sigma_{\mathrm{sq}} \approx\left(\mathrm{I}_{\mathrm{D}} / \mathrm{V}_{\mathrm{D}}\right) \times(\mathrm{L} / \mathrm{W})$, eq. (53) from the experimental data for the drain current $I_{D}$ at low drain voltage $V_{D}=-1 \mathrm{~V}$ reported in [60], respectively, in Fig. 2a (open symbols at lower $\left|V_{G}-V_{o n}\right|$ ) and Fig. 8a (filled symbols at higher $\left|V_{G}-V_{o n}\right|$ ). 


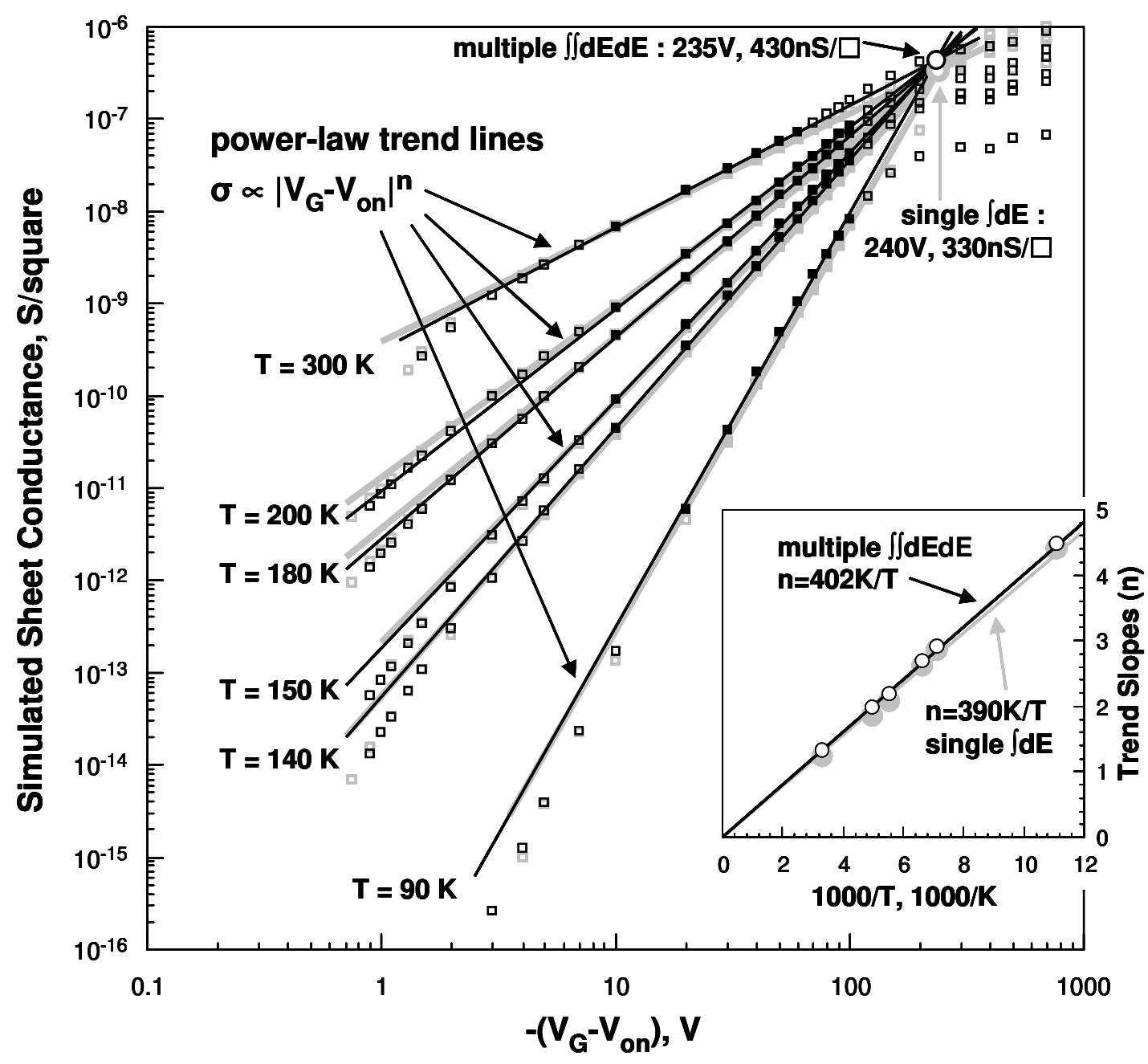

Figure 19. Close look at the numerical simulation results for the PQT-12 OTFT sheet conductance $\left(\sigma_{\mathrm{sq}}\right)$ vs. gate overdrive $\left(\mathrm{V}_{\mathrm{G}}-\mathrm{V}_{\mathrm{on}}\right)$ at different temperatures. The data from the simulation of $\sigma_{\mathrm{sq}}$ are shown with square symbols, corresponding to Figure 18c. Power-law trend lines $\sigma_{\mathrm{sq}} \propto\left|\mathrm{V}_{\mathrm{G}}-\mathrm{V}_{\mathrm{on}}\right|^{\mathrm{n}}$ are fitted to the data denoted with filled squares, and the trend lines intersect in points denoted with large circles at values of $\left|\mathrm{V}_{\mathrm{Gx}}-\mathrm{V}_{\mathrm{on}}\right|$ and $\sigma_{\mathrm{sqx}}$, as indicated. The slopes of the trend lines are the exponential factor (n) in the power law, and the values for $\mathrm{n}$ are shown in the inset as function of the reciprocal of the temperature 1/T, demonstrating the $1 / \mathrm{T}$ dependence of $\mathrm{n}$, and thus, of the thermal activation in eq. (56). Note also the deviations from the power law at low and high gate biases. Gray color corresponds to VRH simulation with single $\int \mathrm{dE}$ integration. Black color corresponds to VRH simulation with multiple $\iint \mathrm{dEdE}$ integrations. 
Reciprocal of Absolute Temperature (1000/T), 1000/K

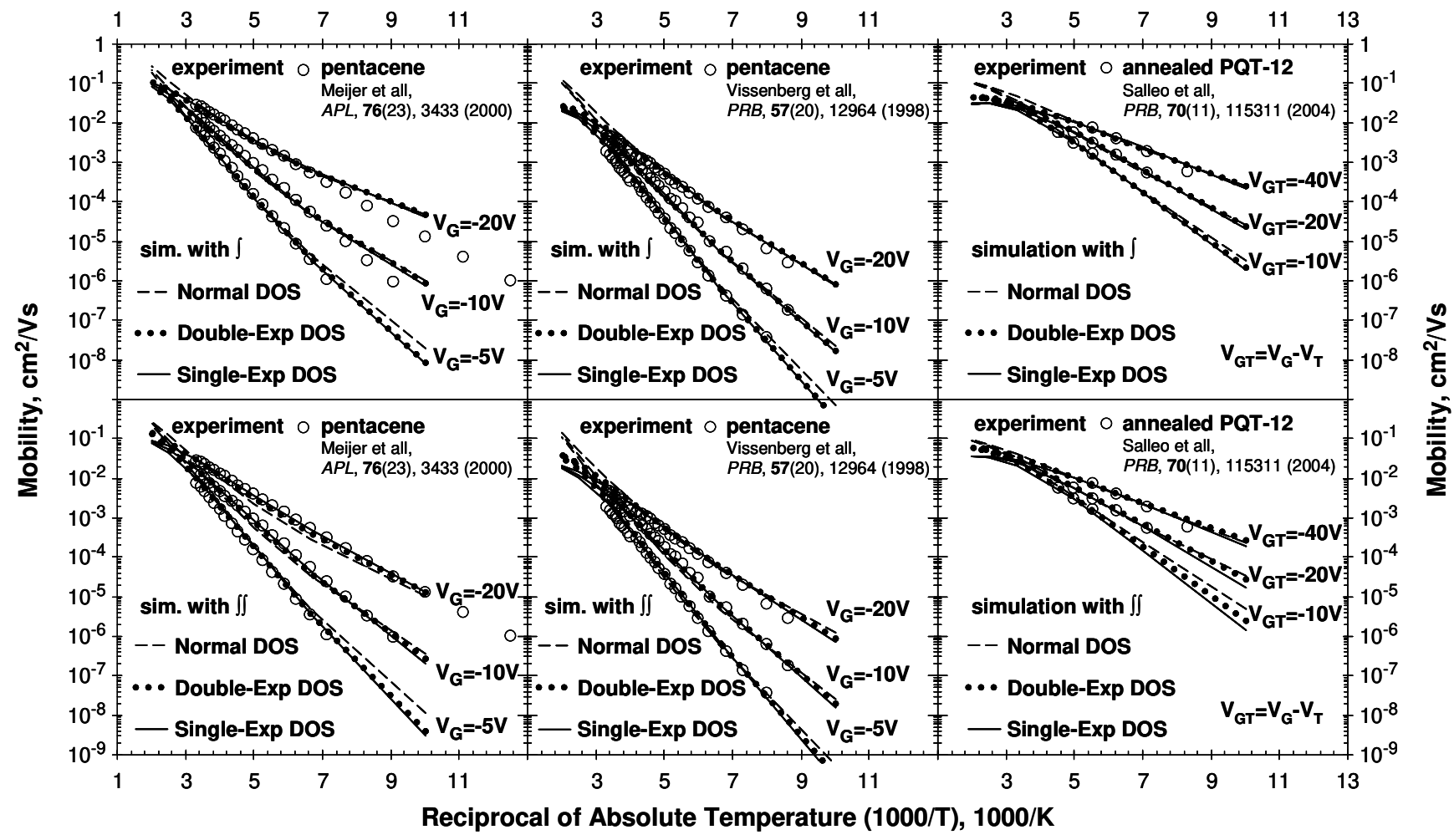

Figure 20. Temperature-bias dependence of the mobility in OTFTs, assuming different types of DOS and using VRH simulations with single $\int \mathrm{dE}$ integration (upper plots) and multiple $\iint \mathrm{dEdE}$ integrations (bottom plots). Dashed lines are for normally distributed DOS, eq. (58). Dotted lines are for double-sided exponential DOS $\mathrm{DE}$, eq. (45). Solid lines are for single-sided exponential DOS $\mathrm{SE}$, eq. (22). Experimental data are shown with open circles $(\bigcirc)$, and are the same as in Figure 17. 

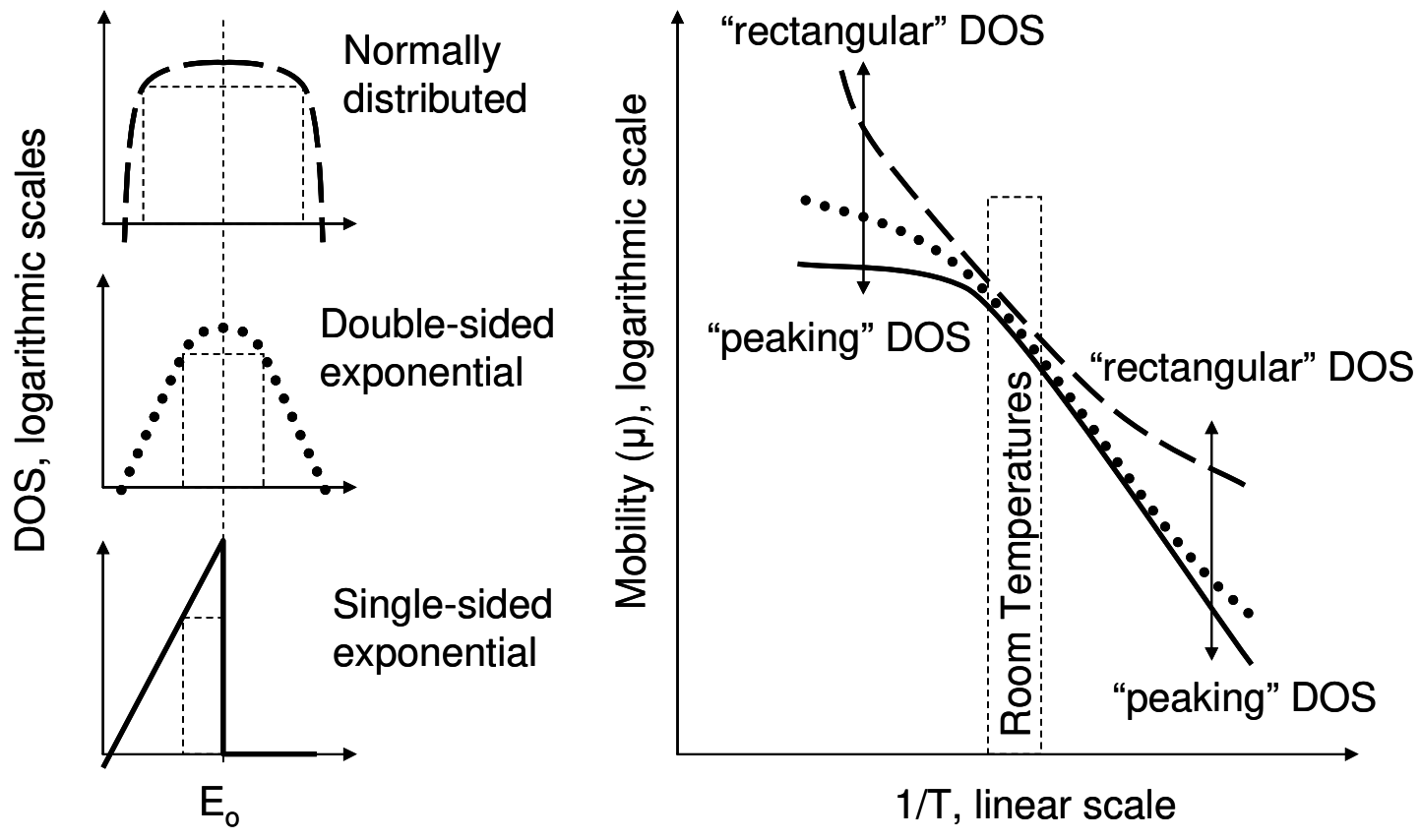

Figure 21. Schematic representation of the effect of DOS "rectangularity" on the behavior of the thermal activation of VRH mobility. The "rectangularity" of normally distributed DOS $\mathrm{ND}_{\mathrm{N}}$ is the highest, compared with the double-sided exponential DOS $\mathrm{DE}$ and the "peaking" single-sided exponential DOS $\mathrm{SE}$. For p-type OTFTs, flip horizontally the single-sided exponential distribution. 


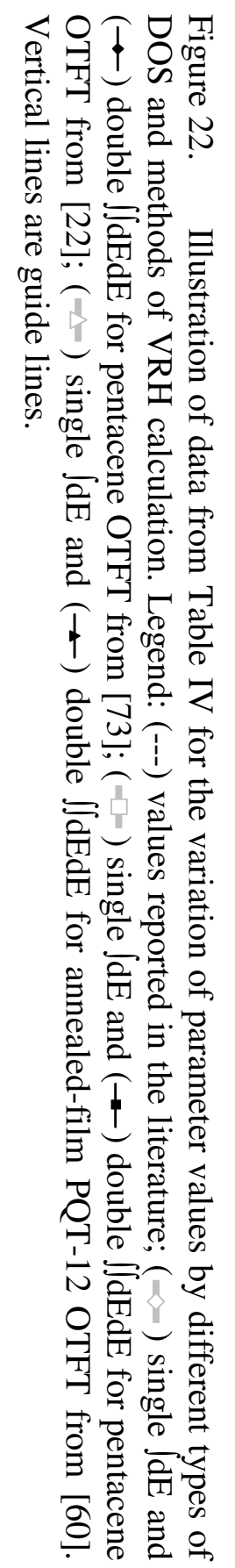

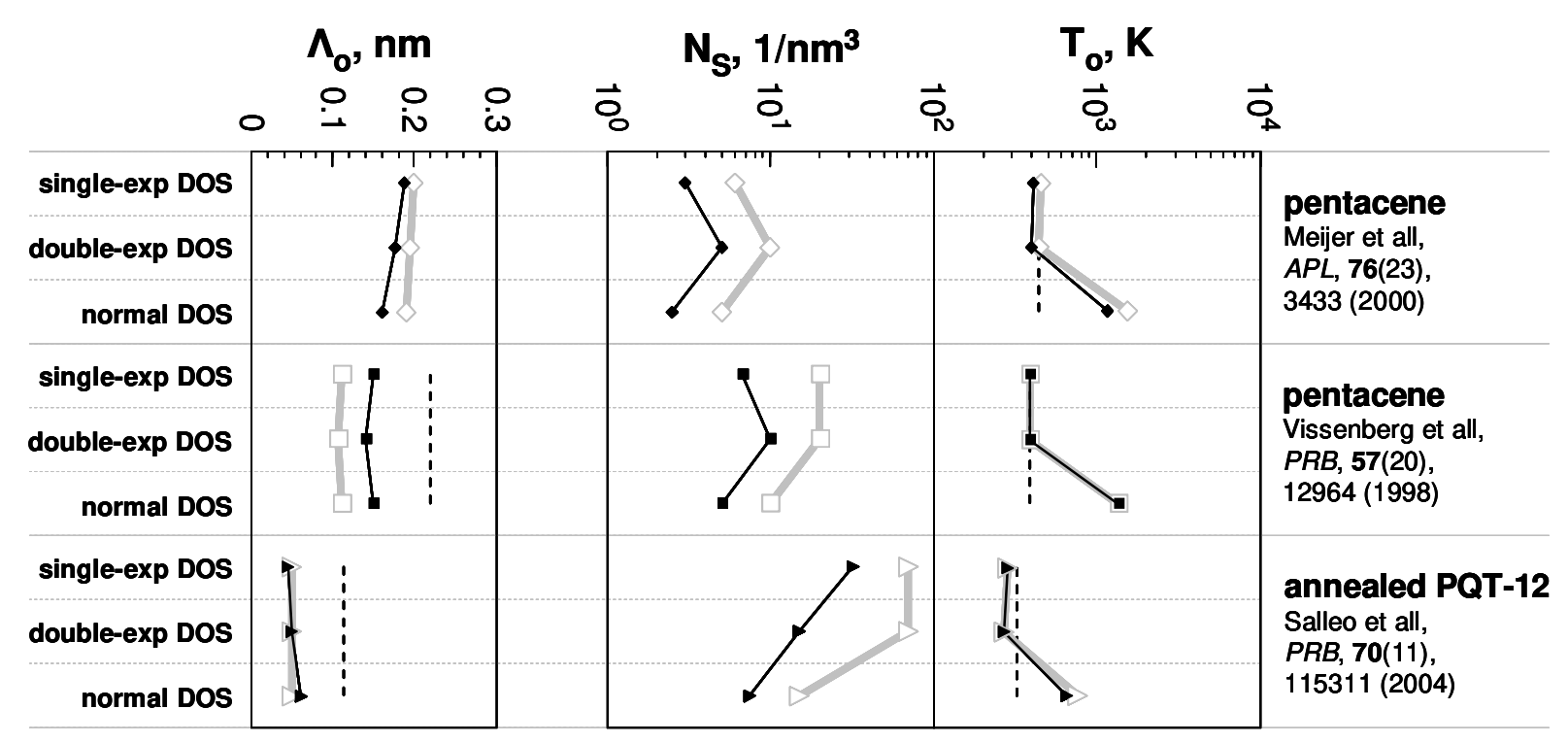

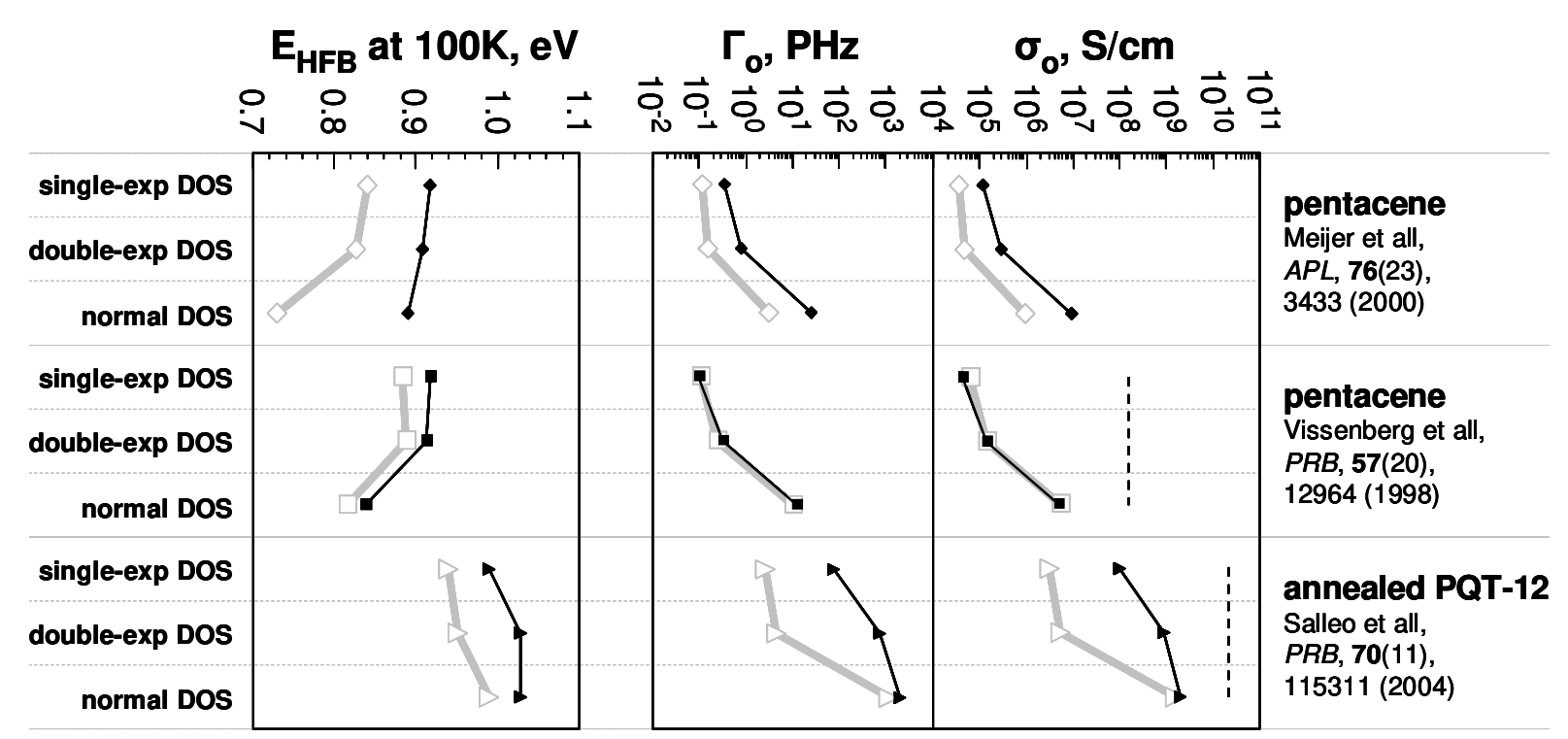



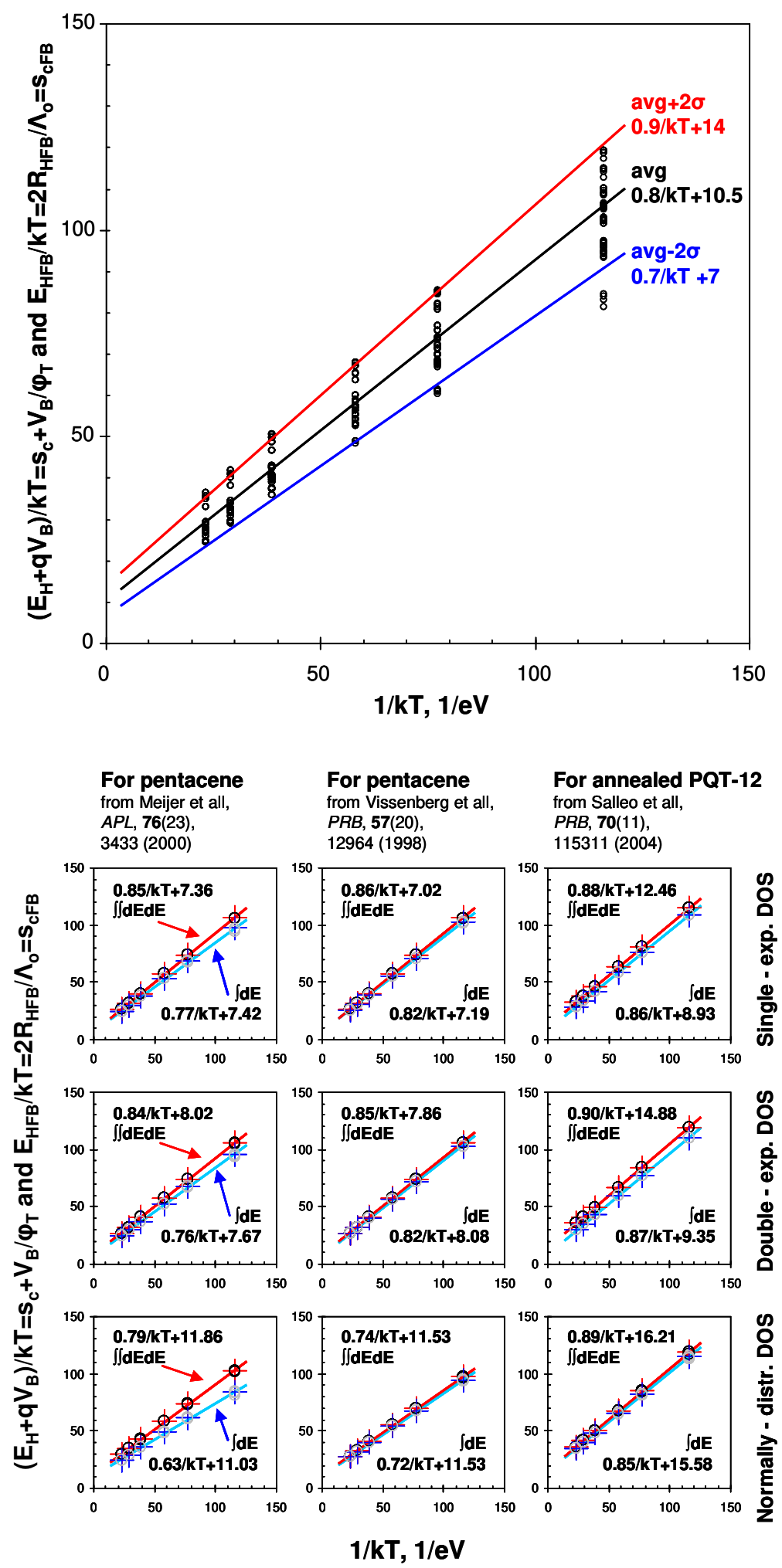

Figure 23. Apparent 1/T dependence for the hopping critical factor $\mathrm{S}_{\mathrm{cFB}}=\mathrm{S}_{\mathrm{c}}+\mathrm{V}_{\mathrm{B}} / \varphi_{\mathrm{T}}=10 \pm 4+(0.8 \pm 0.1) \mathrm{eV} / \mathrm{kT}$, see eq. (60), observed in the top plot, in which all data points are collected from the other plots below. The crosses in the latter plots are for $\mathrm{s}_{\mathrm{cFB}}=\mathrm{E}_{\mathrm{HFB}} / \mathrm{kT}$ in bulk semiconductor $\left(\mathrm{V}_{\mathrm{B}}=0\right)$, and the crosses match the overlapping circles for $\left(\mathrm{E}_{\mathrm{H}}+\mathrm{q} \mathrm{V}_{\mathrm{B}}\right) / \mathrm{kT}$ at different bending voltages $\mathrm{V}_{\mathrm{B}}$ corresponding to different gate bias voltages $\mathrm{V}_{\mathrm{G}}$. 

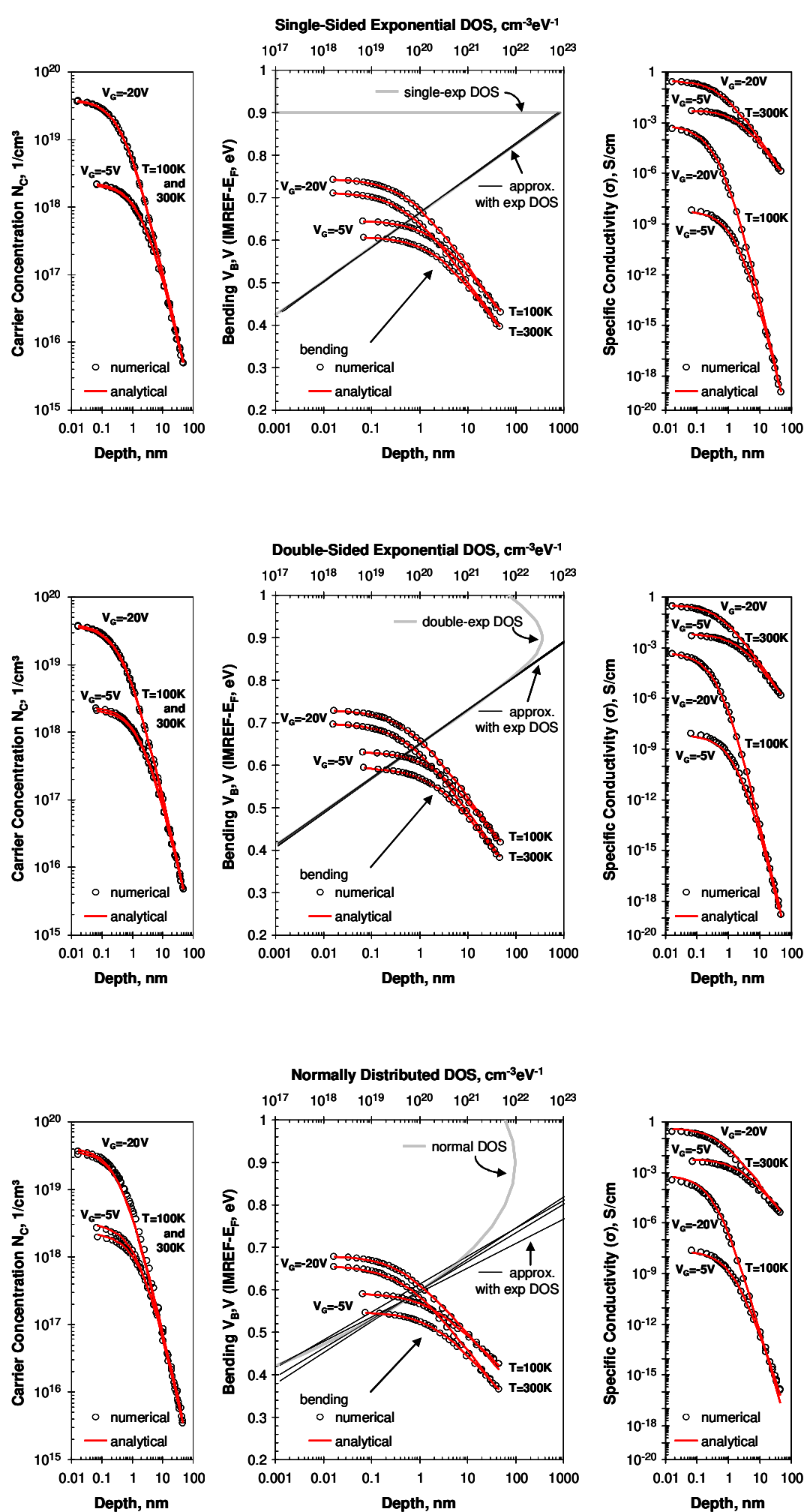

Figure 24. Comparison between numerical $(\bigcirc)$ and analytical (lines, red color) calculations of profiles for single-sided exponential DOS (top plots), double-sided exponential DOS (middle) and normally distributed DOS (bottom plots). Thick gray-color lines depict the DOS as function of bending $\left(\mathrm{qV}_{\mathrm{B}}=\mathrm{IMREF}-\mathrm{E}_{\mathrm{F}}\right)$. Thin black lines illustrate exponential approximation for the DOS by the analytical calculations. 


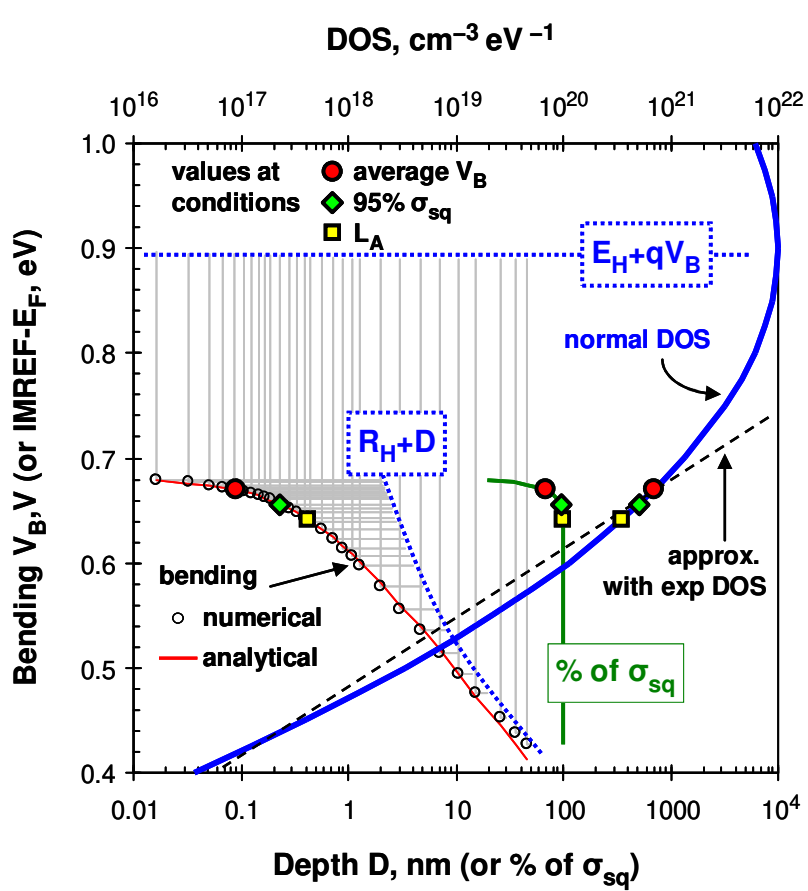

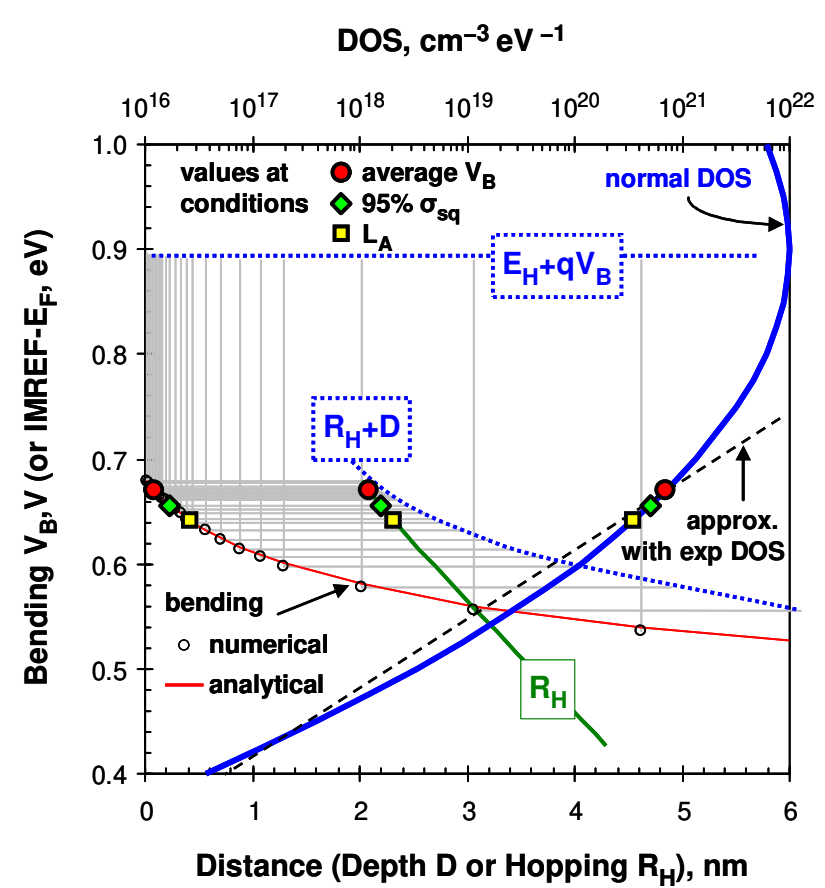

Figure 25. Illustration of several quantities related to VRH in a pentacene OTFT at temperature T=100K and gate bias $\mathrm{V}_{\mathrm{G}}=-20 \mathrm{~V}$ (see the text for correspondence to other figures). Note that all the quantities (except for the vertical error bars for hopping energy $E_{H}$ ) are given as function of potential bending $V_{B}$ in the vertical axes of the plots. The two plots show the same data at logarithmic (left-hand plot) and linear (right-hand plot) scales for distance (bottom horizontal axes), except for the data depicted with lines (green color) for part of the sheet conductance $\left(\%\right.$ of $\left.\sigma_{\mathrm{sq}}\right)$ in the left-hand plot and hopping distance $\mathrm{R}_{\mathrm{H}}$ in the righthand plot. The small open circles $(\circ)$ are the profile for the bending voltage $V_{B}(D)$ after numerical calculations and the thin line (red color) through the circles is the analytical approximation $V_{\mathrm{B} \text {,an }}$ (D) of the potential bending profile. The gray-color error bars begin at the small open circles $(\circ)$ and their length show the results of the following numerical calculations. Vertical error bars: The length of the vertical error bars corresponds to the hopping energy $\mathrm{E}_{\mathrm{H}}$; the upper ends of these error bars reach a constant level $\left(\mathrm{E}_{\mathrm{H}}+\mathrm{q} \mathrm{V}_{\mathrm{B}}\right)=\mathrm{E}_{\mathrm{HFB}}$ denoted with a dotted blue horizontal line in both figures. Horizontal error bars: The length of the horizontal error bars corresponds to the hopping distance $\mathrm{R}_{\mathrm{H}}$; the right-hand end of these error bars is the depth $\left(R_{H}+D\right)$ to which the VRH carriers reach in the organic film; $\left(R_{H}+D\right)$ is denoted with a dotted blue curve in each figure. The normally distributed DOS (used in the numerical calculations) is shown with a thick solid curve in each figure. The DOS values in $1 /\left(\mathrm{cm}^{3} \mathrm{eV}\right)$ are represented on the logarithmic horizontal axes on top of the plots vs. the vertical linear axes for energy in $\mathrm{eV}$. The thin black dashed lines indicate the approximation with exponential DOS in analytical calculations. The slope of the exponential DOS approximation is $\partial \ln (\mathrm{DOS}) / \partial \mathrm{E}=1 / \varphi_{\mathrm{B}, \text { an }}$, in which the value of $\varphi_{\mathrm{B} \text {,an }}$ was deduced after fitting the potential bending with the analytical expression $V_{B}(D) \approx V_{B, a n}(D)=\left[V_{B S}-2 \varphi B\right.$,an $\left.\times \ln \left(1+D / L_{A, a n}\right)\right]$ of eq. (48). The magnitude of the exponential DOS approximation is equal to the magnitude of the normally distributed DOS at the condition $\operatorname{avg} \mathrm{V}_{\mathrm{B}}$ for average $\mathrm{V}_{\mathrm{B}}$. The value of $\operatorname{avg} \mathrm{V}_{\mathrm{B}}$ is calculated with eq. (70), weighting $V_{B}$ by the conductivity, as explained in the text when describing eq. (70). The values for DOS and other quantities represented in the figures corresponding to this condition of average $V_{B}$ are denoted with large circles $(\bigcirc$, filled in red color). The values of the same quantities evaluated at other conditions are depicted by large diamonds ( $\diamond$, filled in green color) for the depth of the channel at which the sheet conductance is $95 \%$ of $\sigma_{\mathrm{sq}}$, and by large squares $\left(\square\right.$, filled in yellow color) for a depth equal to $\mathrm{L}_{\mathrm{A}}$, respectively. 


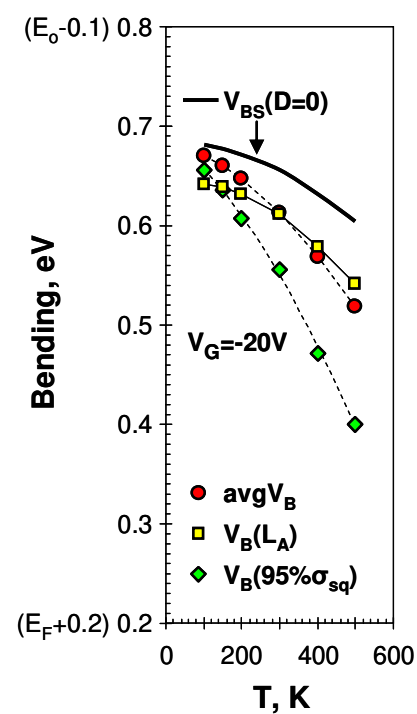

(a)

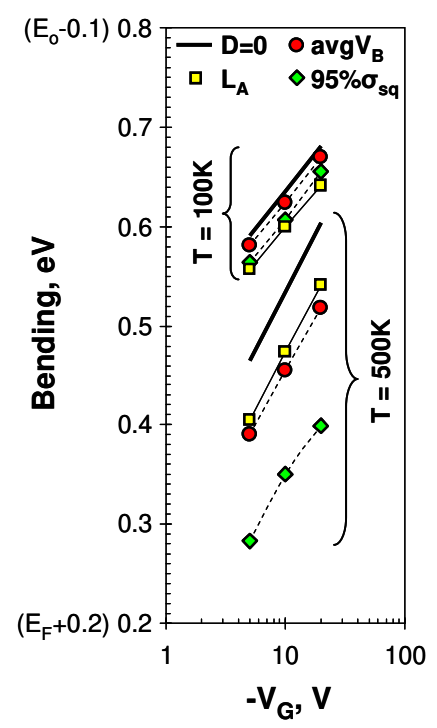

(b)

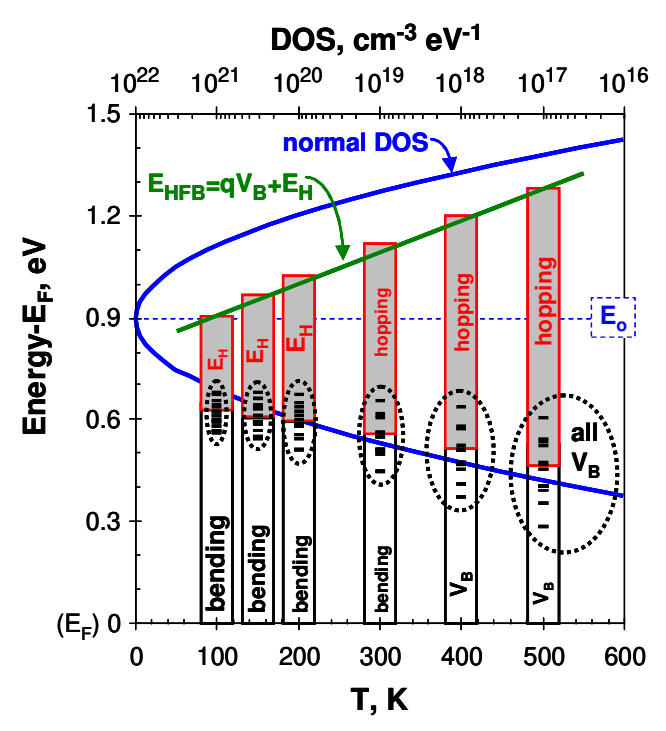

(c)

Figure 26. Comparison between bending $\left(\mathrm{qV}_{\mathrm{B}}\right.$, at various conditions for extraction of the values for $\mathrm{V}_{\mathrm{B}}$ ), DOS and hopping energy $\left(\mathrm{E}_{\mathrm{H}}\right)$ for a pentacene OTFT, after fitting of experimental data for mobility from [73] and by assumption for normally distributed DOS. Note that the polarities of bending and energy are inverted, since the pentacene OTFT is a p-type device. (a) The bending decreases at high temperature, as shown for given gate bias voltage $\mathrm{V}_{\mathrm{G}}=-20 \mathrm{~V}$. (b) The bending increases as the logarithm of gate bias voltage $\mathrm{V}_{\mathrm{G}}=\{-5 \mathrm{~V},-10 \mathrm{~V},-20 \mathrm{~V}\}$, as shown for low $(\mathrm{T}=100 \mathrm{~K})$ and high $(\mathrm{T}=500 \mathrm{~K})$ temperatures. The conditions for extraction of the values for $\mathrm{V}_{\mathrm{B}}$ and notations in (a) and (b) are: solid lines (-) for the semiconductor-gate dielectric interface (depth $\left.\mathrm{D}=0, \mathrm{~V}_{\mathrm{B}}(0) \equiv \mathrm{V}_{\mathrm{BS}}\right)$; circles $\left(\bigcirc\right.$, filled in red color) for the average bending avg $\mathrm{V}_{\mathrm{B}}-$ see eq. (70); diamonds ( $\diamond$, filled in green color) for the bending $\mathrm{V}_{\mathrm{B}}\left(95 \% \sigma_{\mathrm{sq}}\right)$ at depth $\mathrm{D}=\mathrm{D}_{95 \% \sigma s q}$, corresponding to sheet conductance $95 \%$ of $\sigma_{\mathrm{sq}}-$ see eq. (69); and the squares ( $\square$, filled in yellow color) are for the bending $\mathrm{V}_{\mathrm{B}}\left(\mathrm{L}_{\mathrm{A}}\right)$ at depth $\mathrm{D}=\mathrm{L}_{\mathrm{A}}$, where $\mathrm{L}_{\mathrm{A}}=\mathrm{L}_{\mathrm{A}}$,an is obtained after fitting the charge profile $\mathrm{N}_{C}(\mathrm{D})$ from numerical calculations with $N_{C}(D) \propto\left(D+L_{A}, a n\right)^{-2}$, see eq. (49). (c) The values for bending for all cases of biasing and conditions for extraction are aggregated and shown with small dashes surrounded with dotted ellipses, illustrating also with blank bars the trend of decrease of the bending with temperature. However, the hopping energy $E_{H}$ (upper portion of the bars, filled in gray color) increases with temperature, so that the $\operatorname{sum}\left(\mathrm{E}_{\mathrm{H}}+\mathrm{q} \mathrm{V}_{\mathrm{B}}\right)=\mathrm{E}_{\mathrm{HFB}}$ (straight line, green color) increases with temperature; and $\mathrm{E}_{\mathrm{HBF}}$ is well above the DOS centroid $E_{0}$, where $E_{o}$ corresponds to HOMO level of pentacene (reminder - to show magnitudes in the plots, the polarity of the energy is inverted for the p-type pentacene OTFT, so that $E_{0}$ of HOMO is "above" the Fermi level $\mathrm{E}_{\mathrm{F}}$ ). 

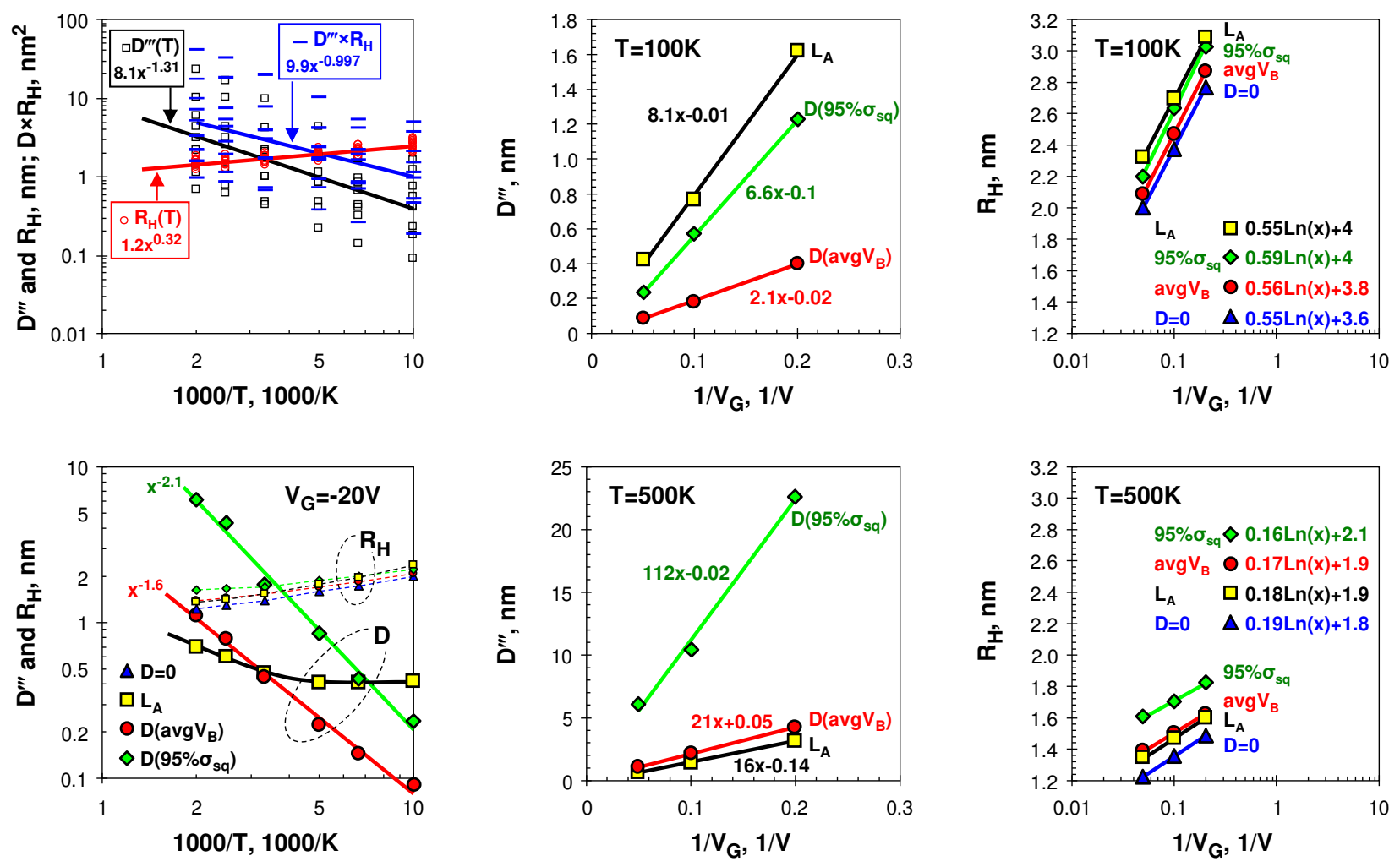

Figure 27. Comparison between characteristic electrostatic and hopping distances for a pentacene OTFT, after fitting of experimental data for mobility from [73] and assuming a normally distributed DOS. The bending and energies are shown in Figure 26. The top-left plot is aggregated data for the set of the three characteristic electrostatic distances $\mathrm{D}^{\prime \prime \prime}=\left\{\mathrm{L}_{\mathrm{A}}, \mathrm{D}_{95 \% \sigma s q}, \mathrm{D}_{\mathrm{avgVB}}\right\}\left(\square\right.$, black color), hopping distances $\mathrm{R}_{\mathrm{H}}(\mathrm{O}$, red color) and their product $\mathrm{D}^{\prime \prime \prime} \times \mathrm{R}_{\mathrm{H}}(-$, blue-color dashes) for all cases of biasing and conditions for value extraction, as per the caption of Figure 26(c). The bottom-left plot details the temperature dependences at gate bias voltage $\mathrm{V}_{\mathrm{G}}=-20 \mathrm{~V}$, as in Figure 26(a), with solid lines denoting data for the set of characteristic distances $\mathrm{D}^{\prime \prime \prime}$ (large symbols) and dashed lines denoting data for hopping distances $\mathrm{R}_{\mathrm{H}}$ (small symbols), and also indicated with ellipses labeled with $\mathrm{D}^{\prime \prime \prime}$ and $\mathrm{R}_{\mathrm{H}}$, respectively. The remaining plots, as in Figure 26(b), detail the bias dependences at low and high temperatures $(\mathrm{T}=100 \mathrm{~K}$ and $\mathrm{T}=500 \mathrm{~K}$, top and bottom plots, respectively) for $\mathrm{D}$ and $\mathrm{R}_{\mathrm{H}}$ (middle and right-hand plots, respectively). Following the scheme of symbols in Figure 26(a) and (b), and except for the top-left plot with aggregated data, the conditions for value extraction are denoted with triangles $(\boldsymbol{\Lambda}$, filled in blue color) for the semiconductor-gate dielectric interface (depth $\mathrm{D}=0)$ ), circles $\left(\mathrm{O}\right.$, filled in red color) at condition for average bending avg $\mathrm{V}_{\mathrm{B}}-$ see eq. (70), diamonds ( $\diamond$, filled in green color) at depth $D_{95 \% \sigma s q}$ corresponding to sheet conductance $95 \%$ of $\sigma_{\mathrm{sq}}-$ see eq. (69), and squares $\left(\square\right.$, filled in yellow color) are for depth $\mathrm{D}=\mathrm{L}_{\mathrm{A}}$, where $\mathrm{L}_{\mathrm{A}}=\mathrm{L}_{\mathrm{A}}$,an is obtained after fitting the charge profile $\mathrm{N}_{C}(D)$ from numerical calculations with $\mathrm{N}_{C}(\mathrm{D}) \propto\left(\mathrm{D}+\mathrm{L}_{\mathrm{A}, \text { an }}\right)^{-2}$, see eq. (49). 

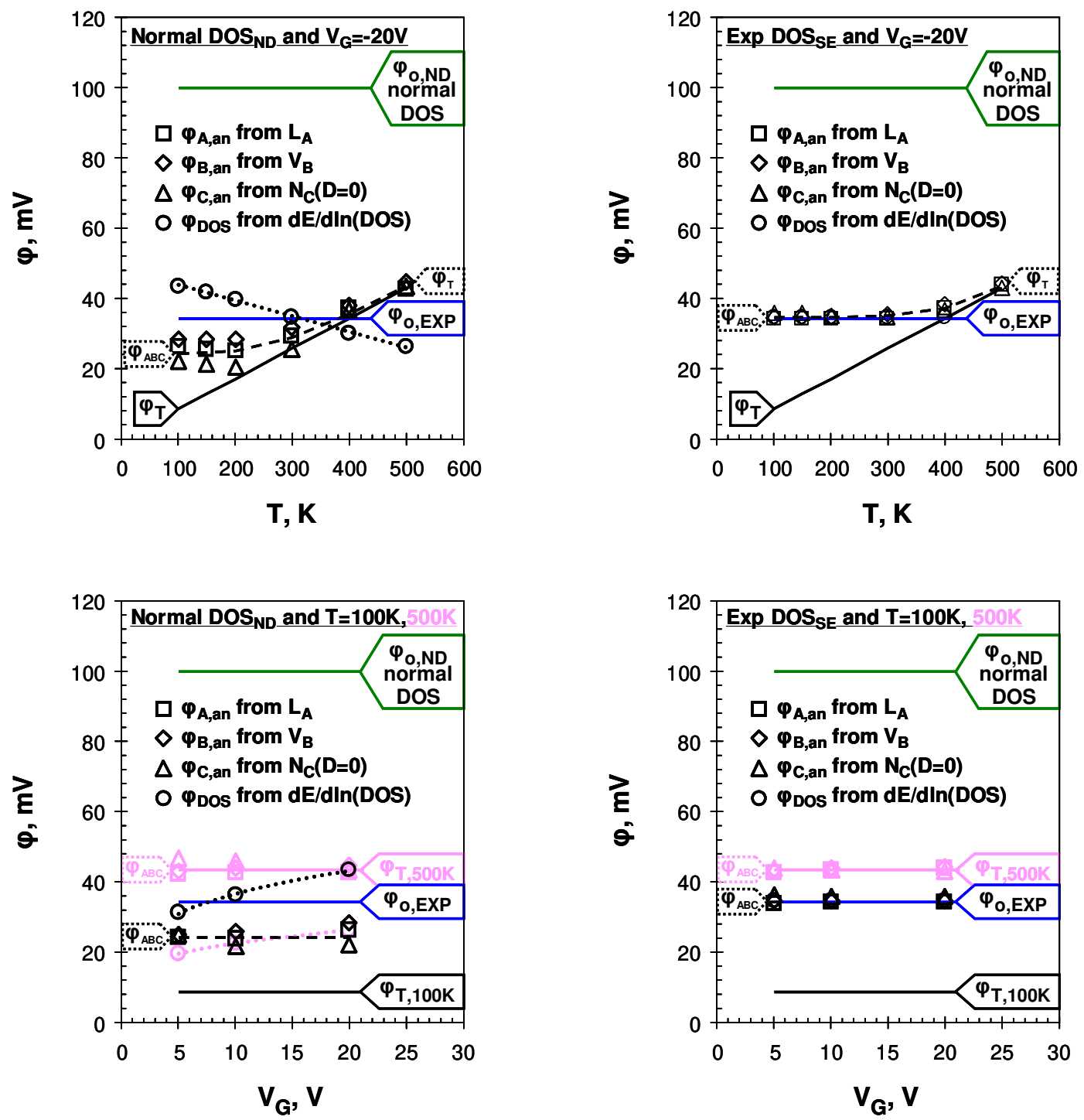

Figure 28. Comparison between characteristic "thermal-like" voltages ( $\varphi$ 's) after fitting of experimental data for mobility from [73] and assuming a normally distributed DOS (on the left) and single-sided exponential DOS (on the right), as function of absolute temperature $\mathrm{T}$ (upper plots, for $\mathrm{V}_{\mathrm{G}}=-20 \mathrm{~V}$ ) and gate bias voltage $\mathrm{V}_{\mathrm{G}}$ (bottom plots for low $\mathrm{T}=100 \mathrm{~K}$ and high $\mathrm{T}=500 \mathrm{~K}$ ). The polarity of $\mathrm{V}_{\mathrm{G}}$ is inverted, since the pentacene OTFT is $\mathrm{p}$ type transistor. The DOS widths are $\varphi_{\mathrm{o}, \mathrm{ND}}=100 \mathrm{mV}$ for the normally distributed DOS $\mathrm{ND}_{\mathrm{ND}}$ (horizontal solid lines in all plots, green color) and $\varphi_{\mathrm{o}, \mathrm{EXP}}=34.9 \mathrm{mV}$ for the exponential $\mathrm{DOS}_{\mathrm{SE}}$ (horizontal solid lines in all plots, blue color). The thermal voltage $\varphi_{\mathrm{T}}=\mathrm{kT} / \mathrm{q}$ (solid lines in all plots) is proportional to the absolute temperature $\mathrm{T}$, as shown with raising straight lines in the upper plots, and $\varphi_{\mathrm{T}}$ is bias independent (horizontal lines in bottom plots). The values of the characteristic voltages $\varphi_{\mathrm{A} \text {,an }}(\square), \varphi_{\mathrm{B} \text {,an }}(\diamond)$ and $\varphi_{\mathrm{C} \text {,an }}(\triangle)$ are obtained after fitting profiles from the numerical calculations with the analytical approximations for $\mathrm{L}_{\mathrm{A}}, \mathrm{V}_{\mathrm{B}}$ and $\mathrm{N}_{\mathrm{C}}(\mathrm{D}=0)$ at the gate dielectricsemiconductor interface ( $\mathrm{D}=0$ ), eqs. (47), (48) and (49), respectively, and the symbols are around the dashed lines (---), which depict the empirical relation for "dominance of the larger", eq. (79) with $n=5$ for the normally distributed DOS, and $n=10$ for the exponential DOS. The values $\varphi_{\text {DOS }}(\bigcirc)$ for the reciprocal of DOS slope are at the condition for average bending ( $\mathrm{IMREF}=\mathrm{E}_{\mathrm{F}}+\mathrm{q} \times a v g \mathrm{~V}_{\mathrm{B}}$ ), and dotted lines (...) connect the circles with linear trend in the upper-left plot and power-law trend in the bottom-left plot. The high temperature $\mathrm{T}=500 \mathrm{~K}$ in the bottom plots is depicted with light color (pink), and the black color corresponds to low temperature $\mathrm{T}=100 \mathrm{~K}$. 


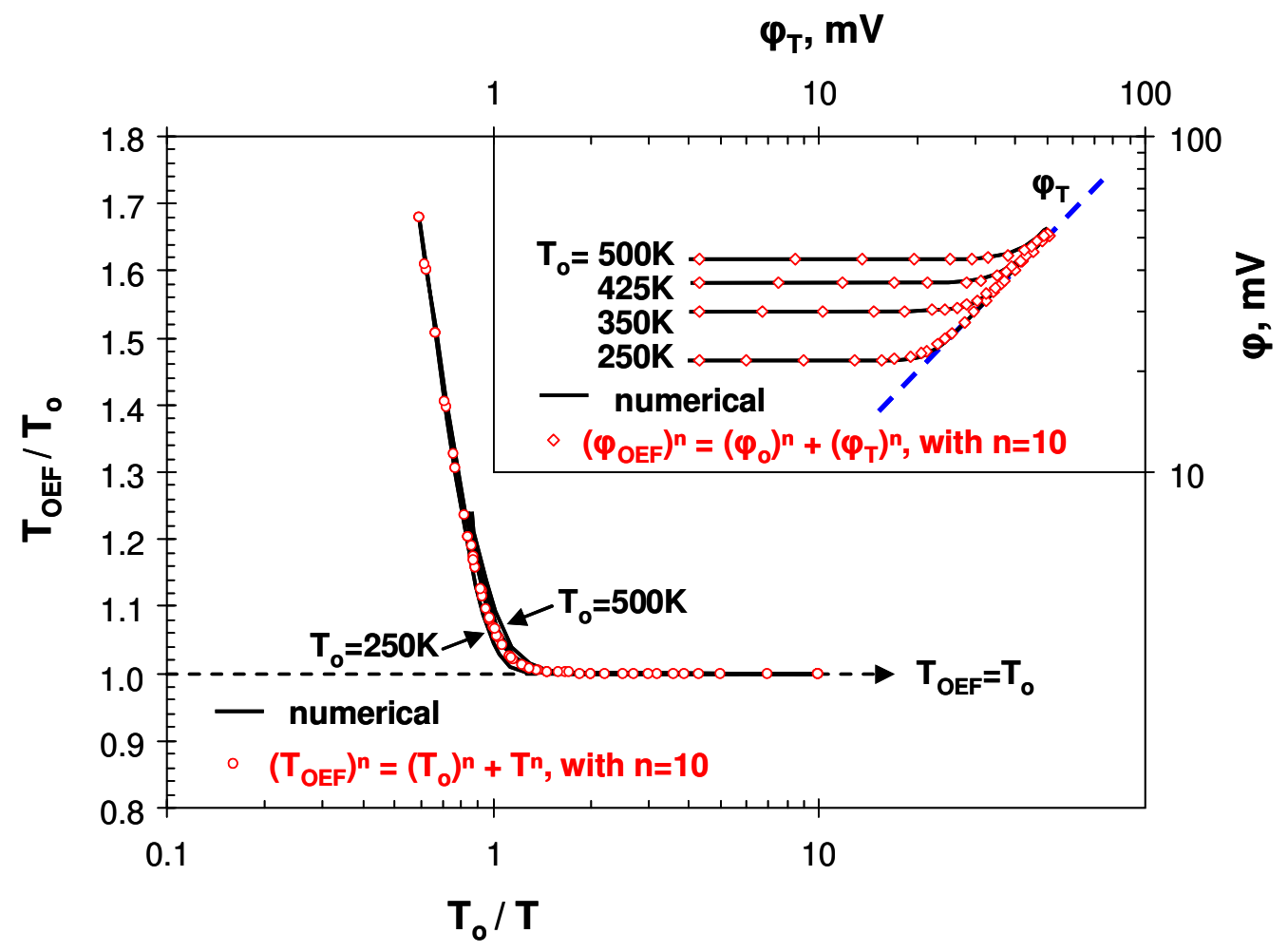

Figure 29. Overlap between (solid lines) the numerical simulations reported in the appendix of [11] for $\mathrm{T}_{\mathrm{o}}=\{250 \mathrm{~K}, 350 \mathrm{~K}, 425 \mathrm{~K}$ and $500 \mathrm{~K}\}$ and (symbols) the analytical expression of eq. (79), illustrated with data for the effective characteristic temperature $\mathrm{T}_{\mathrm{OEF}}$ (main plot $\mathrm{T}_{\mathrm{OEF}} / \mathrm{T}_{\mathrm{o}} \mathrm{vs} . \mathrm{T}_{\mathrm{o}} / \mathrm{T}$ ) and the effective characteristic voltage $\varphi_{\mathrm{OEF}}=\mathrm{kT} T_{\mathrm{OEF}} / \mathrm{q}$ (inset, $\varphi_{\mathrm{OEF}}=\mathrm{kT} \mathrm{OEF}_{\mathrm{O}} / \mathrm{q}$ vs. $\varphi_{\mathrm{T}}=\mathrm{kT} / \mathrm{q}$ ). The value of the exponential factor $\mathrm{n}=10$ is taken as deduced for exponential DOS in Figure 28. T $\mathrm{T}_{\mathrm{OEF}}$ and $\varphi_{\mathrm{OEF}}$ replace $\mathrm{T}_{\mathrm{o}}$ and $\varphi_{\mathrm{o}} \equiv \mathrm{k} \mathrm{T}_{\mathrm{o}} / \mathrm{q}$, respectively, in analytical approximations based on exponential DOS, when the absolute temperature $\mathrm{T}$ is elevated close or above the characteristic temperature $\mathrm{T}_{\mathrm{o}}$ of the exponential DOS, e.g., $\mathrm{T}>\mathrm{T}_{\mathrm{o}} / 2$. The dashed line in the main plot denotes $\mathrm{T}_{\mathrm{OEF}}=\mathrm{T}_{\mathrm{o}}$ at low temperatures, e.g., $\mathrm{T}<\mathrm{T}_{\mathrm{o}} / 2$. The dashed line (blue color) in the inset denotes $\varphi_{\mathrm{OEF}}=\varphi_{\mathrm{T}}$ at high temperatures, e.g., $\mathrm{T}>2 \mathrm{~T}_{\mathrm{o}}$. 


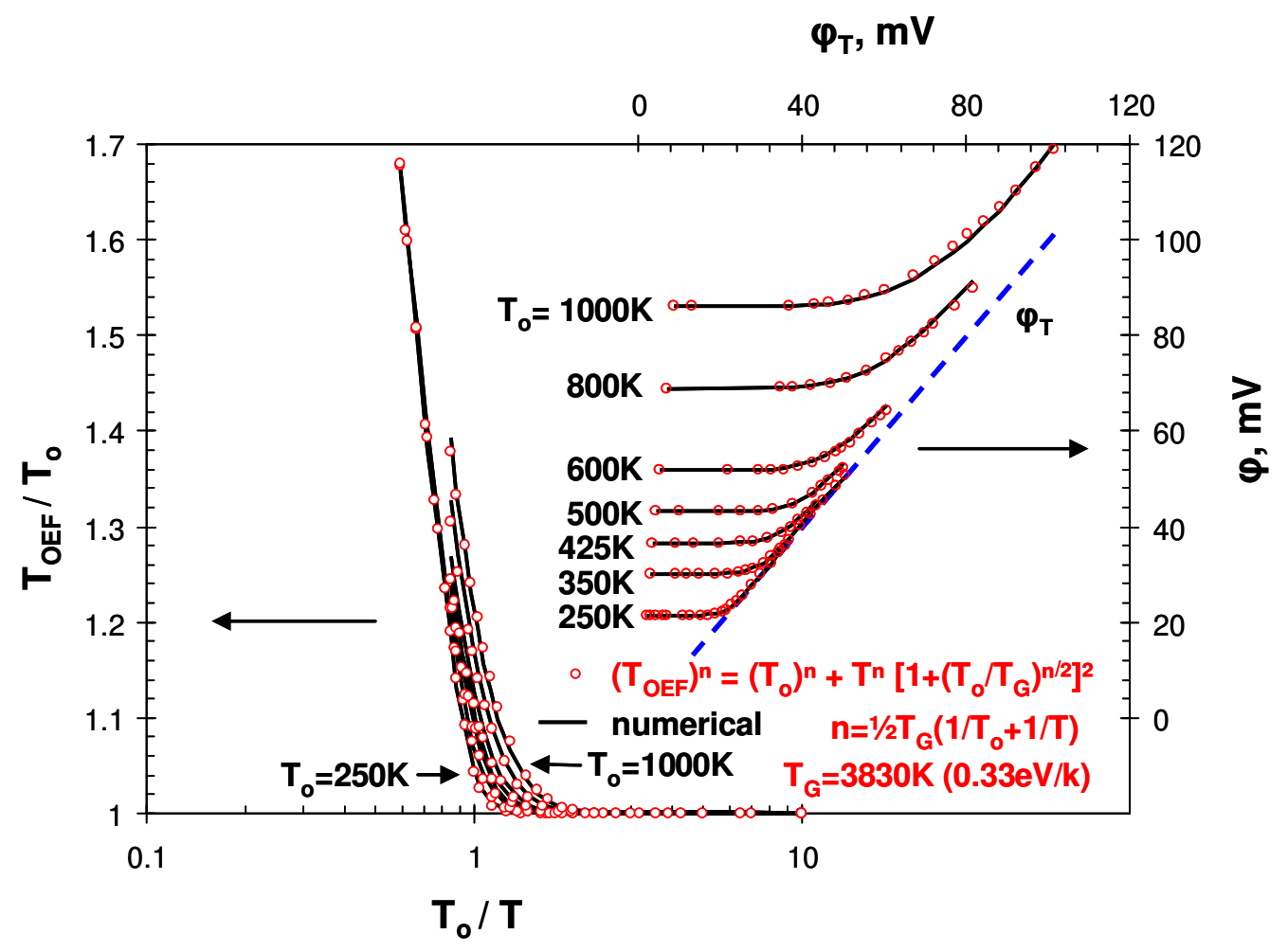

Figure 30. Better fit of (symbols) the analytical expression of eq. (80) for the "dominance of the larger" rule to (solid lines) the numerical simulations reported in the appendix of [11] for the temperature variation of the effective characteristic temperature $\mathrm{T}_{\mathrm{OEF}}$ of the exponential DOS. 

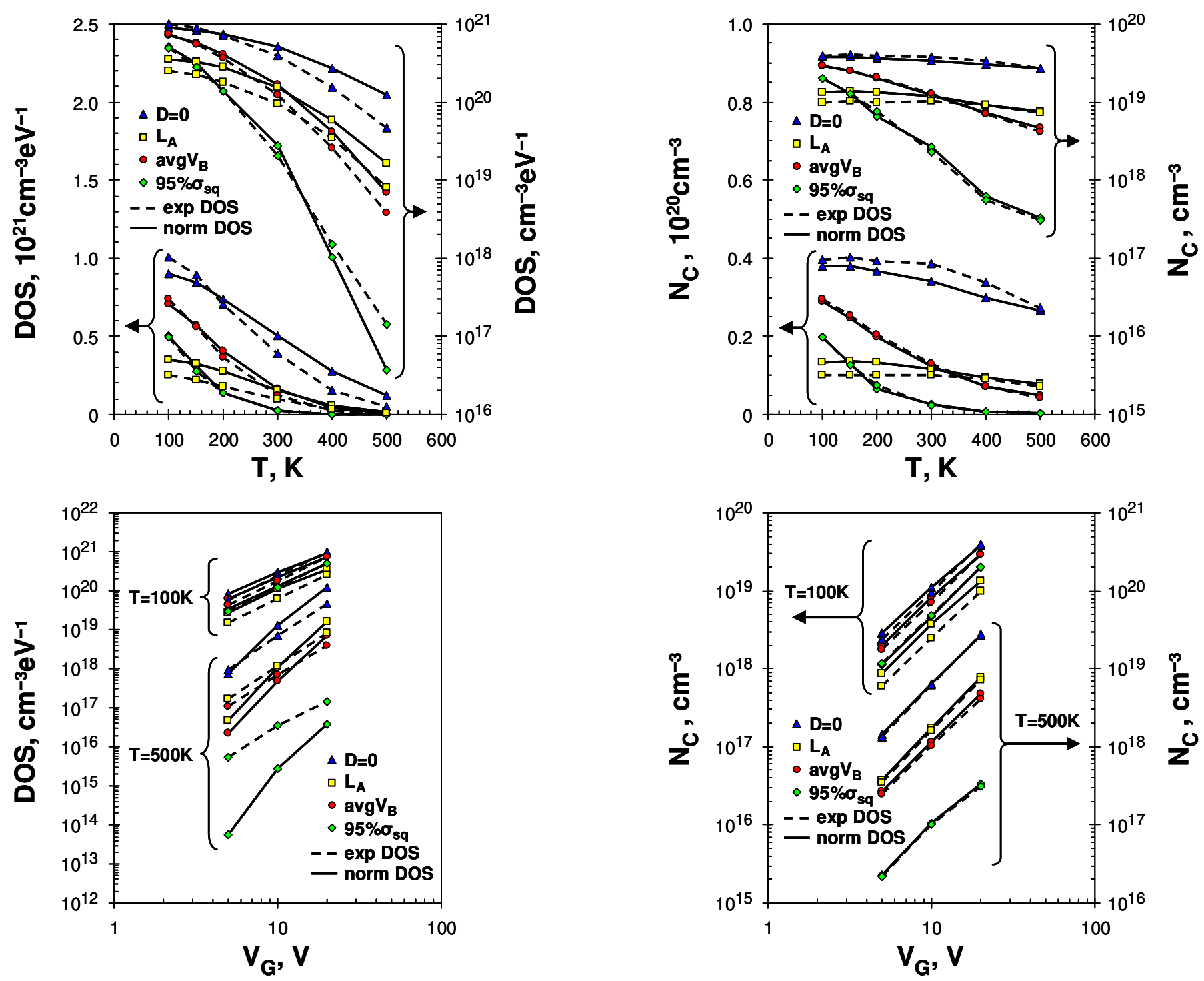

Figure 31. Comparison between characteristic values for DOS (left-hand plots) and carrier concentration $\mathrm{N}_{\mathrm{C}}$ (right-hand plots) after fitting of experimental data for mobility from [73] for a pentacene OTFT and assuming a normally distributed DOS (solid lines) and a single-sided exponential DOS (dashed lines), as function of absolute temperature $\mathrm{T}$ (upper plots, for $\mathrm{V}_{\mathrm{G}}=-20 \mathrm{~V}$, same data shown in linear and logarithmic scales) and gate bias voltage $\mathrm{V}_{\mathrm{G}}$ (bottom plots for low $\mathrm{T}=100 \mathrm{~K}$ and high $\mathrm{T}=500 \mathrm{~K}$ ). The polarity of $\mathrm{V}_{\mathrm{G}}$ is inverted, since the pentacene OTFT is p-type transistor. The bottom-right plot $\mathrm{N}_{\mathrm{C}}$ vs. $\mathrm{V}_{\mathrm{G}}$ uses two vertical axes shifted one decade from each other for the two temperatures, because data overlap, since $\mathrm{N}_{C}(\mathrm{D}=0)$ and $\mathrm{N}_{\mathrm{C}}\left(\mathrm{L}_{\mathrm{A}}\right)$ are almost independent of the temperature, as seen in the upper-right plot $\mathrm{N}_{\mathrm{C}}$ vs. T. As in Figure 28, the symbols denote the conditions at which the values are extracted, in particular, triangles $(\triangle$, filled in blue color) for the semiconductor-gate dielectric interface (depth $D=0)$, circles $(O$, filled in red color) at condition for average bending avg $\mathrm{V}_{\mathrm{B}}-$ see eq. (70), diamonds ( $\diamond$, filled in green color) at depth D95\% бsq corresponding to sheet conductance $95 \%$ of $\sigma_{\mathrm{sq}}-$ see eq. (69), and squares ( $\square$, filled in yellow color) are for depth $\mathrm{D}=\mathrm{L}_{\mathrm{A}}$, where $\mathrm{L}_{\mathrm{A}}=\mathrm{L}_{\mathrm{A}}$,an is obtained after fitting the charge profile $\mathrm{N}_{\mathrm{C}}(\mathrm{D})$ from numerical calculations with $\mathrm{N}_{C}(\mathrm{D}) \propto\left(\mathrm{D}+\mathrm{L}_{\mathrm{A}, \text { an }}\right)^{-2}$, see eq. (49). 


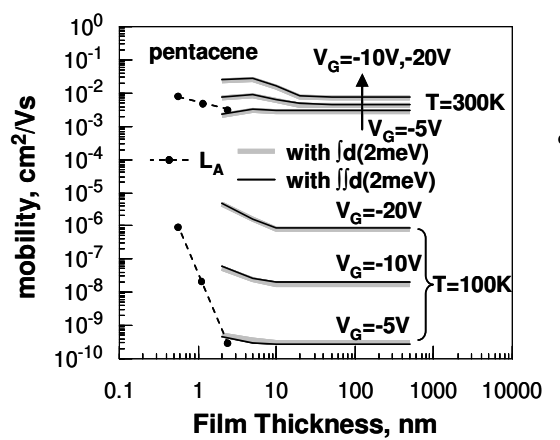

(a)

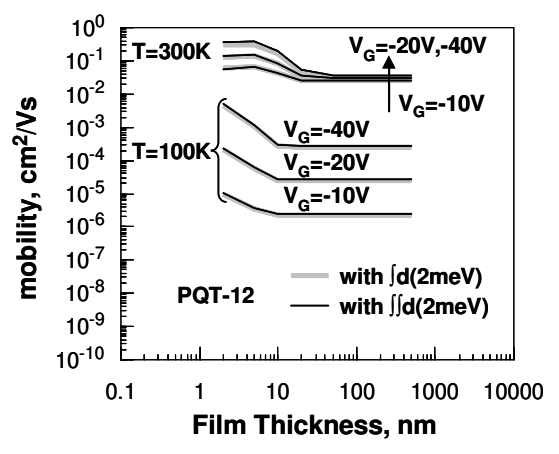

(b)

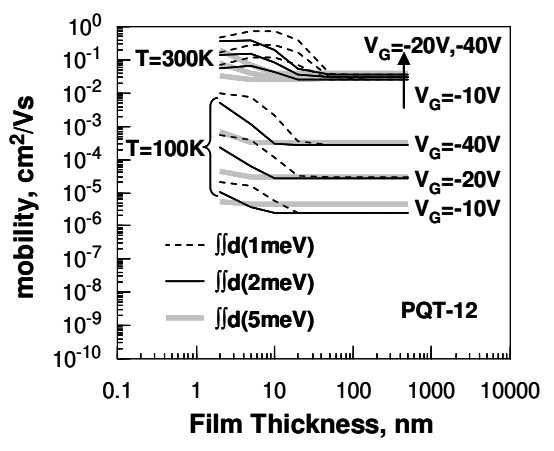

(c)

Figure 32. Film thickness dependence of the VRH film mobility in OTFTs at low $(\mathrm{T}=100 \mathrm{~K})$ and room $(\mathrm{T}=300 \mathrm{~K})$ temperatures, and at gate bias voltages $\mathrm{V}_{\mathrm{G}}$, as indicated in the plots, by VRH calculations of single $\left(\int \mathrm{dE}\right)$ and multiple $\left(\iint \mathrm{dEdE}\right)$ integrations with energy step $\mathrm{dE}=(1 \mathrm{meV}),(2 \mathrm{meV})$ or $(5 \mathrm{meV})$, as also indicated in the plots with different labels, type and colors of lines. Double-exponential DOS assumed in the simulations with device and simulation parameters as listed in Table IV, except for film thickness $t_{\mathrm{f}}$ (horizontal axis of the plots) and the integration step $\mathrm{dE}$, which have been varied. (a) pentacene OTFT with parameter values obtained after the fitting shown in Figure 17(b) of the experimental data for mobility reported in [22] for one film thickness. The two dashed lines denote the electrostatic length $\mathrm{L}_{\mathrm{A}}$ for the two temperatures, connecting symbols corresponding to the three gate bias voltages. (b) PQT-12 annealed-film OTFT with parameter values obtained after the fitting shown in Figure 17(c) of the experimental data reported in [60] for one film thickness, and by the two methods for VRH calculation with single $\int \mathrm{dE}$ and multiple $\iint \mathrm{dEdE}$ integrations. (c) same as (b), but at different energy integration steps $\mathrm{dE}$ and only by the method of multiple $\iint \mathrm{dEdE}$ integrations. 


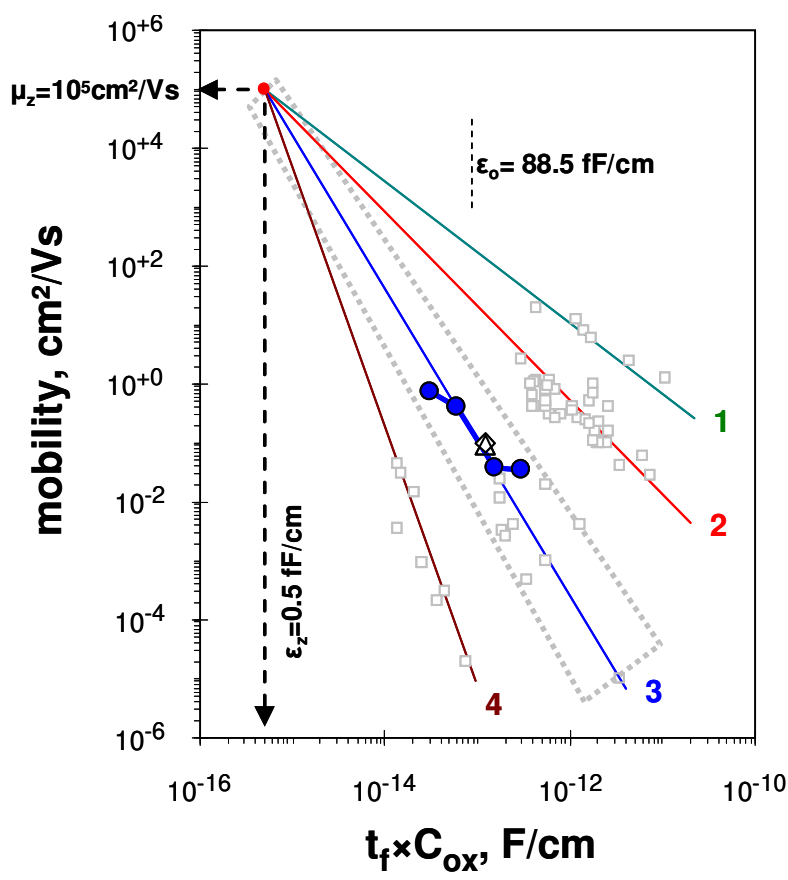

Figure 33. Film thickness dependence of mobility from numerical VRH calculation is coinciding with the trend for mobility in OTFTs [82]. Filled circles (, blue color) correspond to the top dashed line in Figure 32 (c) for the virtual PQT-12 OTFTs of film thicknesses $t_{\mathrm{f}}=\{10 \mathrm{~nm}, 20 \mathrm{~nm}, 50 \mathrm{~nm}$ and $100 \mathrm{~nm}\}$ at room temperature $\mathrm{T}=300 \mathrm{~K}$ and after $\mathrm{VRH}$ calculation by multiple $\iint \mathrm{dEdE}$ integrations with integration step $\mathrm{dE}=1 \mathrm{meV}$. Open triangle $(\triangle)$ and diamond $(\diamond)$ are data from experiments with PQT OTFTs of film thicknesses $t_{f}=35-40 \mathrm{~nm}$, reported in $[60,77]$, respectively. Small open squares $(\square$, gray color) are data collected in [88] for OTFTs with other organic semiconductors, coinciding with the trend lines to rubrene (1), pentacene (2), solution-processed polymers (3) and other "low-mobility" materials (4). The trend lines are according to eq. (82). The dotted polygon denotes the normally observed range for variation around material line (3), and the polygon corresponds to the margins for $\mu_{z}, \varepsilon_{z}$ and $S_{\mu}$ of solution-processed polymeric semiconductors, given also by eq. (82). 


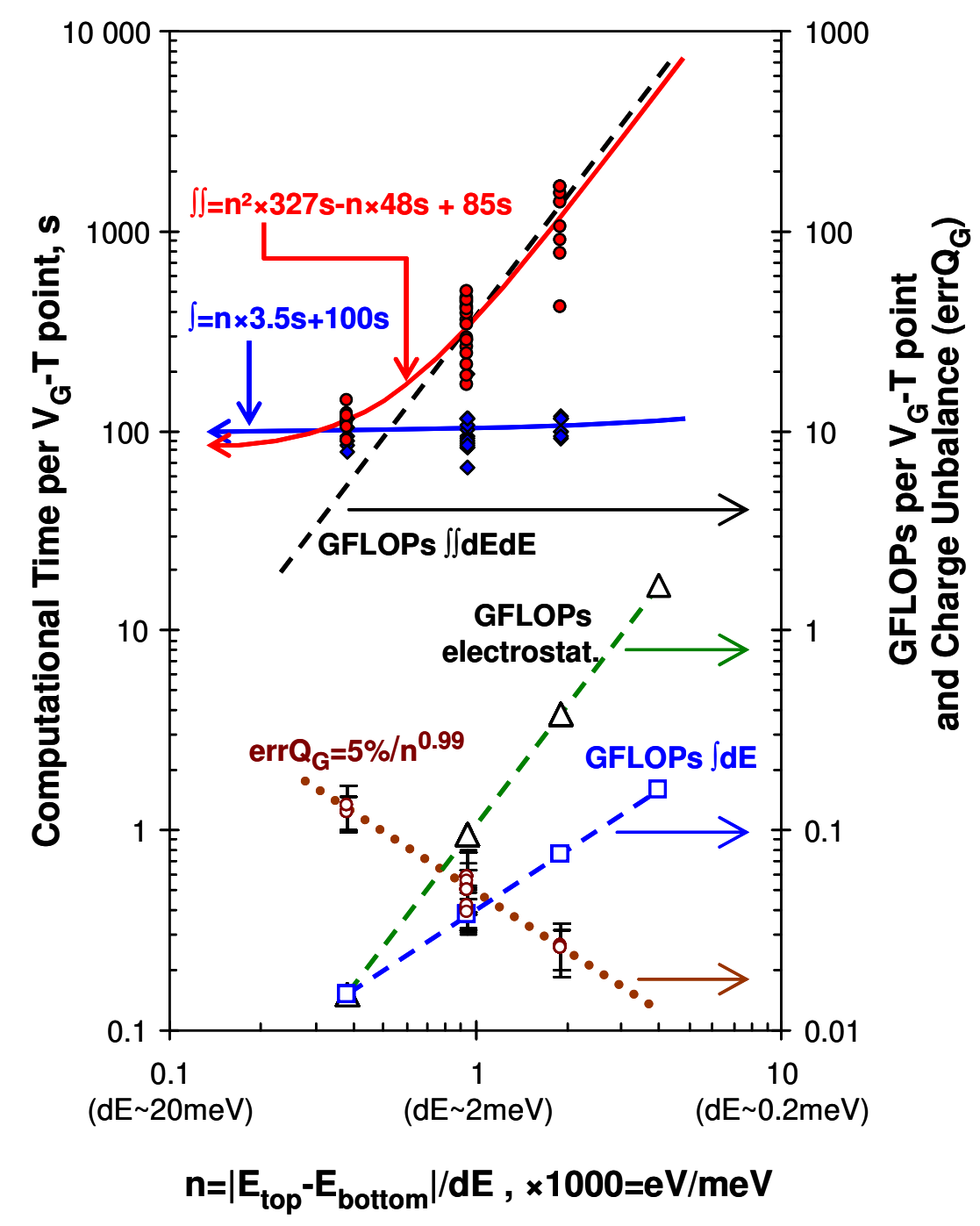

Figure 34. Computational demand of one-dimensional VRH numerical calculation for one biastemperature point $\left(\mathrm{V}_{\mathrm{G}}-\mathrm{T}\right)$. Circles $\left(\boldsymbol{O}\right.$, red color) for VRH calculation with multiple $\iint \mathrm{dEdE}$ integrations and diamonds $\left(\diamond\right.$, blue color) for VRH calculation with single $\int \mathrm{dE}$ integration, with quadratic and linear trend lines, respectively, correspond to the left-hand axis for computational time (in seconds) when using parallel computing of 18 or more $\left(\mathrm{V}_{\mathrm{G}}-\mathrm{T}\right)$ points. Dashed lines correspond to the right-hand axis, as indicated by right-pointing arrows, denoting computational volume in GFLOPs for VRH calculation with multiple $\iint \mathrm{dEdE}$ integrations (no symbols), VRH calculation with single $\int \mathrm{dE}$ integration (squares $\square$ ) and electrostatic calculation (triangles $\triangle$ ). Dotted line ( $\cdots$, brown color) with small circles $(O)$ depicts the average mismatch (errQ $\left.\mathrm{Q}_{\mathrm{G}}\right)$ by balancing the film charge with the gate charge $\left(\mathrm{Q}_{\mathrm{G}}=\mathrm{C}_{\mathrm{OX}}\left|\mathrm{V}_{\mathrm{G}}-\mathrm{V}_{\mathrm{FB}}\right|\right)$ during the electrostatic calculations, and the vertical error bars are of size $\left(\sim 1 / 3 \mathrm{errQ}_{\mathrm{G}}\right)$ for the standard deviation of errQ $\mathrm{Q}_{\mathrm{G}}$. All quantities are given as function of the horizontal axis for number (n) of points in the energy mesh $\mathrm{E}=\left\{\mathrm{E}_{\text {bottom }},\left(\mathrm{E}_{\text {bottom }}+\mathrm{dE}\right),\left(\mathrm{E}_{\text {bottom }}+2 \mathrm{dE}\right), \ldots, \mathrm{E}_{\mathrm{top}}\right\}$, as explained in previous sections, see between eqs. (16) and (18), for example. Since $\left|E_{\text {top }}-E_{b o t t o m}\right| \sim 2 \mathrm{eV}$, then an energy integration step of size $\mathrm{dE}=2 \mathrm{meV}$ corresponds to $\mathrm{n} \approx 1000$. Note that $(\mathrm{n})$ is in unit "thousands of points" in the expressions of the trend lines. 


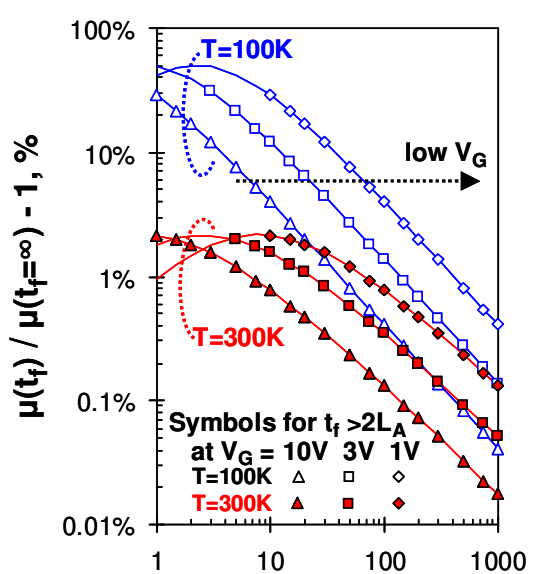

Film Thickness $\left(\mathrm{t}_{\mathrm{f}}\right), \mathrm{nm}$

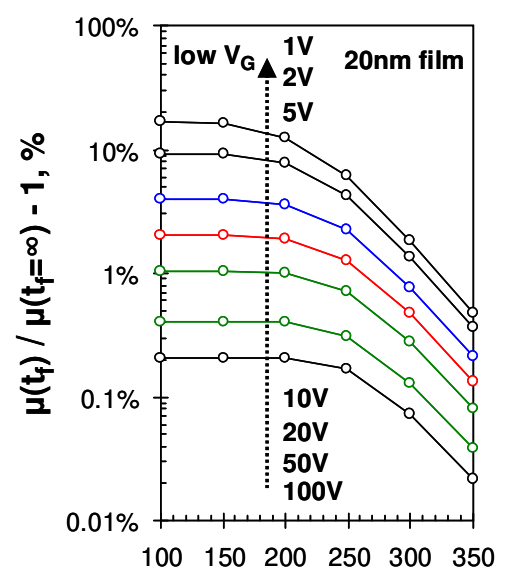

Temperature, $\mathrm{K}$

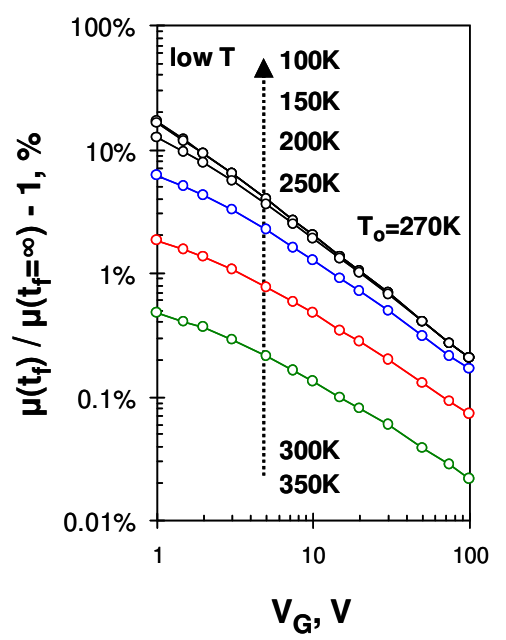

(c)

(a)

(b)

Figure 35. Relative variations of mobility due to finite thickness $\mathrm{t}_{\mathrm{f}}<\infty$ of the semiconducting film in the OTFT. (a) as function of film thickness $t_{\mathrm{f}}$ at a low temperature $(\mathrm{T}=100 \mathrm{~K}$, open symbols, blue color) and room temperature ( $\mathrm{T}=300 \mathrm{~K}$, filled symbols, red color). (b) as function of temperature, and (c) as function of gate bias voltage $\mathrm{V}_{\mathrm{G}}$. The data are after calculation with the approximate eq. (98) and the symbols are when the condition $t_{\mathrm{f}}>2 \mathrm{~L}_{\mathrm{A}}$ is satisfied in this equation. The trend in the plots is that the effective mobility relatively increases (as compared to the mobility $\mu_{\mathrm{tf}=\infty}$ of infinite-thick film OTFT), when the film thickness $\left(\mathrm{t}_{\mathrm{f}}\right)$, the temperature $(\mathrm{T})$ or the bias $\left(\mathrm{V}_{\mathrm{G}}\right)$ decrease. (The trends for the absolute magnitude of the mobility are different) The parameters used in the calculation correspond to Figure 32 (b) and (c) for the PQT-12 annealed-film OTFT, assuming an exponential DOS, except for film thicknesses, and the values of the parameters are: $\mathrm{COX}_{\mathrm{OX}}=30 \mathrm{nF} / \mathrm{cm}^{2}, \varepsilon_{\mathrm{f}}=3 \varepsilon_{0}=2.6 \times 10^{-13} \mathrm{~F} / \mathrm{cm}, \mathrm{T}_{\mathrm{o}}=270 \mathrm{~K}, \mathrm{~T}_{\mathrm{OEF}} \mathrm{F}^{\mathrm{n}}=\mathrm{T}_{\mathrm{o}}{ }^{\mathrm{n}}+\mathrm{T}^{\mathrm{n}}, \mathrm{n}=10$ for all plots and film thickness $\mathrm{t}_{\mathrm{f}}=20 \mathrm{~nm}$ in plots (b) and (c). 


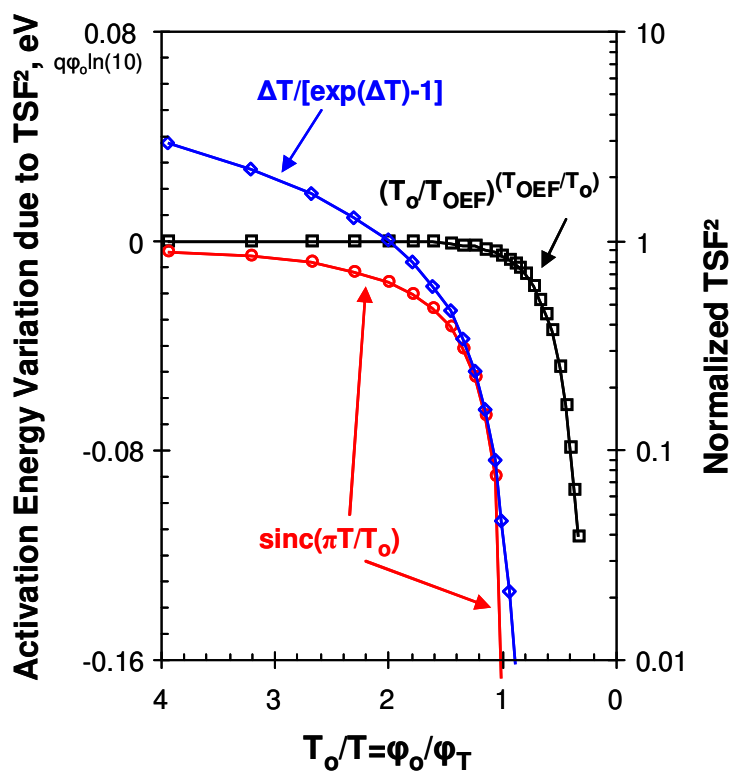

(a)

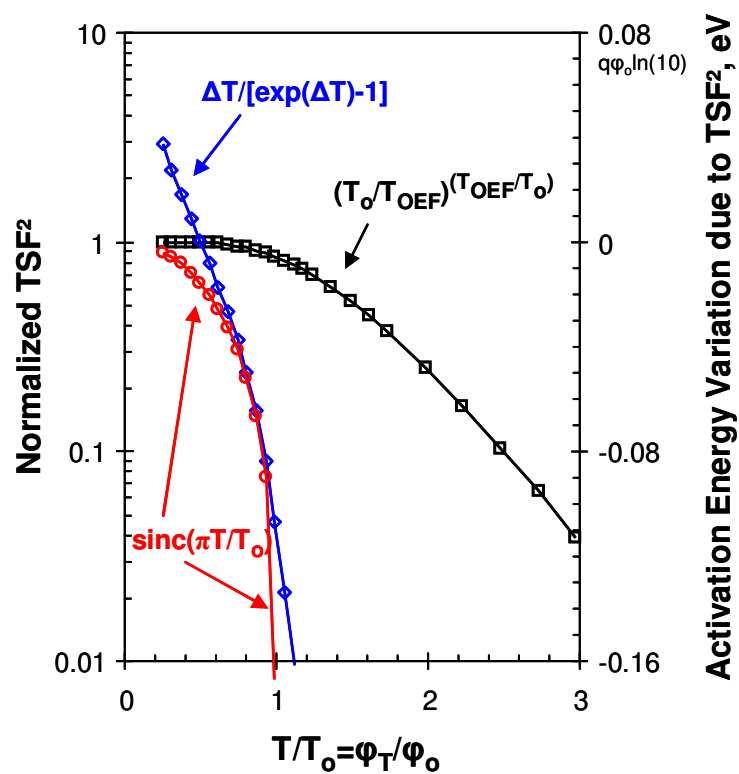

(b)

Figure 36. Comparison of the temperature shaping functions $\left(\mathrm{TSF}^{2}\right)$ normalized to their constant multipliers, discussed between eqs. (108) and (111). The two plots show the same data for the normalized $\mathrm{TSF}^{2}$ vs. inverse temperature, (a) on left; and proportional to temperature, (b) on right; in order to magnify at low and high temperatures, respectively. The horizontal axis of plot (a) is flipped so that the low temperature is on the left-hand side and the high temperature is on the right-hand side in both plots. Circles $\left(\bigcirc\right.$, red color), diamonds $\left(\diamond\right.$, blue color) and squares $\left(\square\right.$, black color) denote the three TSF $^{2}$ of type $\operatorname{sinc}\left(\pi \mathrm{T} / \mathrm{T}_{\mathrm{o}}\right), \Delta \mathrm{T} /[\exp (\Delta \mathrm{T})-1]$, and the power-law $\mathrm{TSF}^{2} \propto\left(\mathrm{T}_{\mathrm{o}} / \mathrm{T}_{\mathrm{OEF}}\right)^{\left(\mathrm{ToEF}_{\mathrm{T}}\right)}$, respectively, the latter $\mathrm{TSF}^{2}$ defined by eq. (110) and the former two $\mathrm{TSF}^{2}$ in [6]. The significance of the normalized $\mathrm{TSF}^{2}$ is that it describes the deviation of the thermal activation of $\mu_{\mathrm{o}}$ from Arrhenius law via temperature variation $\Delta \mathrm{E}_{\mathrm{A}}(\mathrm{T})$ of the activation energy $\mathrm{E}_{\mathrm{A}}$, since $\mu_{0} \propto\left(\mathrm{TSF}^{2}\right)^{\mathrm{kT}_{0} / \mathrm{kT}}$ from eq. (108), then $\ln \left(\mu_{\mathrm{o}}\right)=\mathrm{E}_{\mathrm{A} 0} / \mathrm{kT}+\Delta \mathrm{E}_{\mathrm{A}} / \mathrm{kT}$, where $\mathrm{E}_{\mathrm{A} 0}$ is temperature independent and the activation energy temperature variation becomes a logarithmic function of the variation of $\mathrm{TSF}^{2}$, as $\Delta \mathrm{E}_{\mathrm{A}}=\mathrm{kT} \mathrm{T}_{\mathrm{o}} \times \ln \left(\mathrm{TSF}^{2}\right)=\mathrm{q} \varphi_{\mathrm{o}} \times \ln \left(\mathrm{TSF}^{2}\right)$, thus, at rate $\mathrm{q} \varphi_{\mathrm{o}}$, which is reflected in the complementary vertical axes on left in plot (a) and on right in plot (b). It was chosen $\mathrm{T}_{0}=403.2567 \mathrm{~K}$ in the calculations, in order to have round number for $\mathrm{q} \varphi_{\mathrm{o}} \times \ln (10) \approx 34.75 \mathrm{meV} \times 2.3 \approx 0.08 \mathrm{eV}$, which synchronizes the linear axis for $\Delta \mathrm{E}_{\mathrm{A}}$ with the logarithmic axis for the normalized $\mathrm{TSF}^{2}$. 


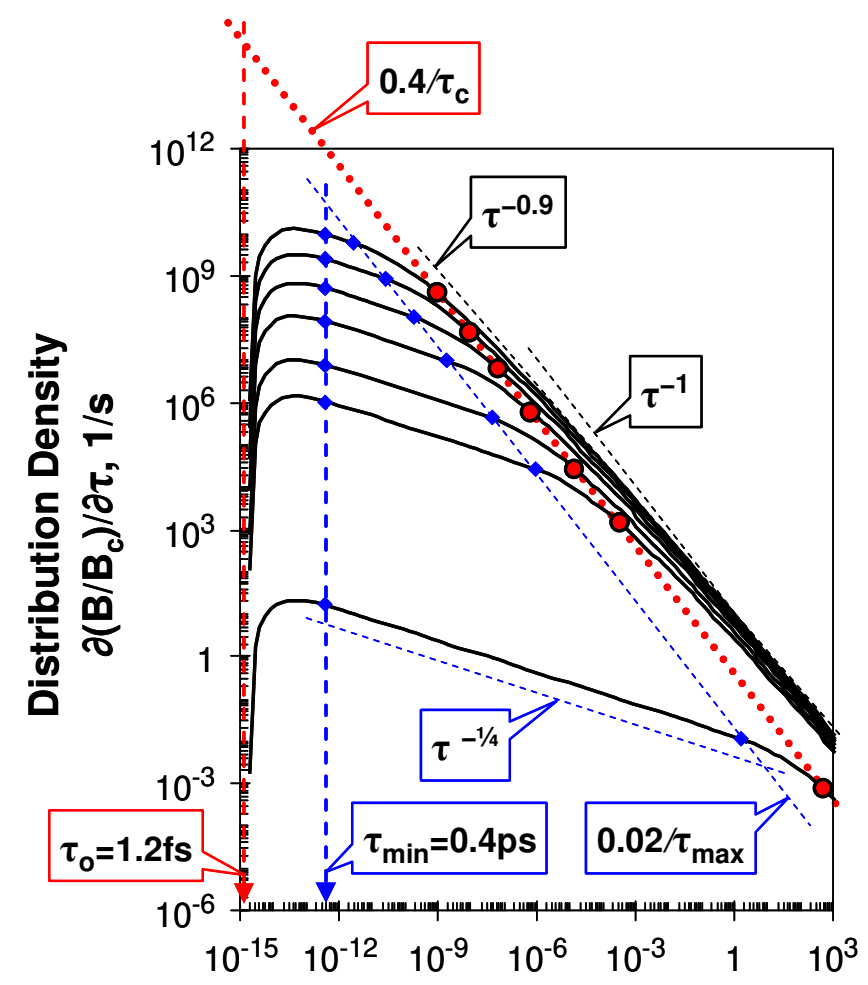

\section{Hopping Time $(\tau)$, s}

Figure 37. Distributions of hopping time (solid black lines, being the same as the normalized distributions of hopping bonds) at several characteristic depths D in the semiconducting film of an OTFT at temperature $T=300 \mathrm{~K}$ and gate bias voltage $V_{G}=-20 \mathrm{~V}$, after $\mathrm{VRH}$ calculations with multiple $\iint \mathrm{dEdE}$ integrations. The parameters correspond to the pentacene OTFT from [73] in Table IV. The characteristic depths from top to bottom are $\mathrm{D}=0$ (gate dielectric - semiconductor interface, electric field $\mathrm{E}_{\mathrm{el}} \approx 1.2 \mathrm{MV} / \mathrm{cm}$ ), $\mathrm{L}_{\mathrm{A}}=0.55 \mathrm{~nm}$ (electrostatic depth for $\mathrm{Q}_{\mathrm{F}}=1 / 2 \mathrm{Q}_{\mathrm{G}}, \mathrm{E}_{\mathrm{el}} \approx 0.64 \mathrm{MV} / \mathrm{cm}$ ), $\mathrm{D}_{95 \% \mathrm{\sigma}}=1.87 \mathrm{~nm}$ (depth for $95 \%$ sheet conductance $\left.\quad \sigma_{\mathrm{sq}}, \quad \mathrm{E}_{\mathrm{el}} \approx 0.3 \mathrm{MV} / \mathrm{cm}\right), \quad \mathrm{D}=5 \mathrm{~nm} \quad\left(10 \% \times \mathrm{t}_{\mathrm{f}}, \quad \mathrm{E}_{\mathrm{el}} \approx 0.12 \mathrm{MV} / \mathrm{cm}\right), \quad \mathrm{D}=17.5 \mathrm{~nm} \quad\left(35 \% \times \mathrm{t}_{\mathrm{f}}\right.$, $\mathrm{E}_{\mathrm{el}} \approx 0.04 \mathrm{MV} / \mathrm{cm}$ ), $\mathrm{D}=\mathrm{t}_{\mathrm{f}}=50 \mathrm{~nm}$ (semiconducting film back, $\mathrm{E}_{\mathrm{el}} \approx 14 \mathrm{kV} / \mathrm{cm}$ ) and $\mathrm{D}=\infty$ (bulk material, $\mathrm{E}_{\mathrm{el}}=0$ ). Circles $\left(\bigcirc\right.$, red color, aligned to the dotted trend line $\left.0.4 / \tau_{\mathrm{c}}\right)$ denote the critical hopping time $\tau_{\mathrm{c}}=1 / \Gamma_{\mathrm{c}}$ for each depth D. The distributions are of type $\tau^{\mathrm{n}}$ with two values for the characteristic slope $\mathrm{n}$. For the range of the "plateau", $\mathrm{n} \approx-1 / 4>-1$ between $\tau_{\min } \approx \tau_{0} \exp (6) \approx 0.4 \mathrm{ps}$ and $\tau_{\max } \approx \tau_{\mathrm{c}} \exp (-6)$, where $\tau_{\min }$ and $\tau_{\max }$ are denoted with small diamonds $\left(\bullet\right.$, blue color). The values corresponding to $\tau_{\max }$ are aligned on the trend line $0.02 / \tau_{\max }$. For the range $\tau>\tau_{\mathrm{c}} \exp (6)$, the distribution densities gradually tend to $1 / \tau$ distribution, with $(-0.9>n>-1)$, as depicted with the two dashed lines on the right-hand side and denoted with $\tau^{-0.9}$ and $\tau^{-1}$. 


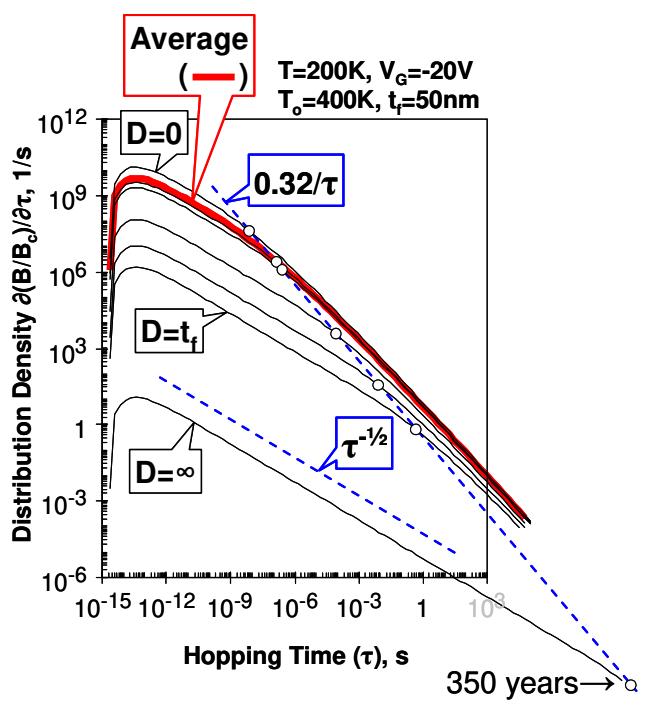

(a)

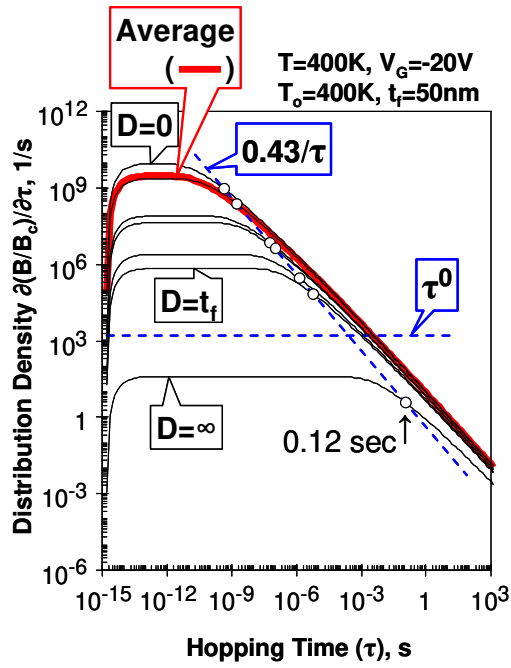

(c)

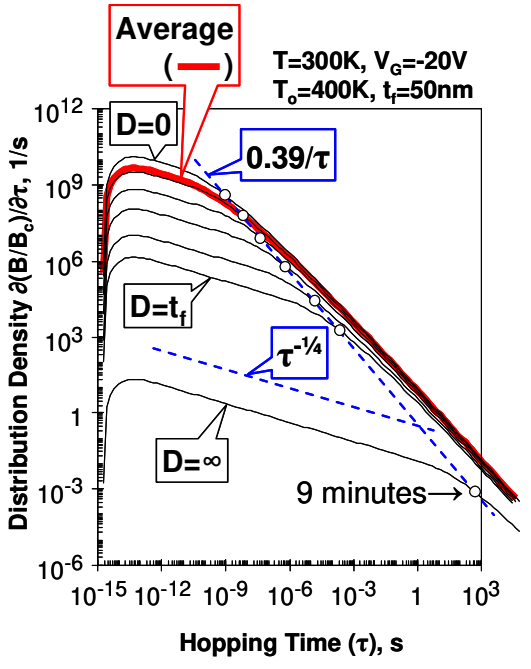

(b)

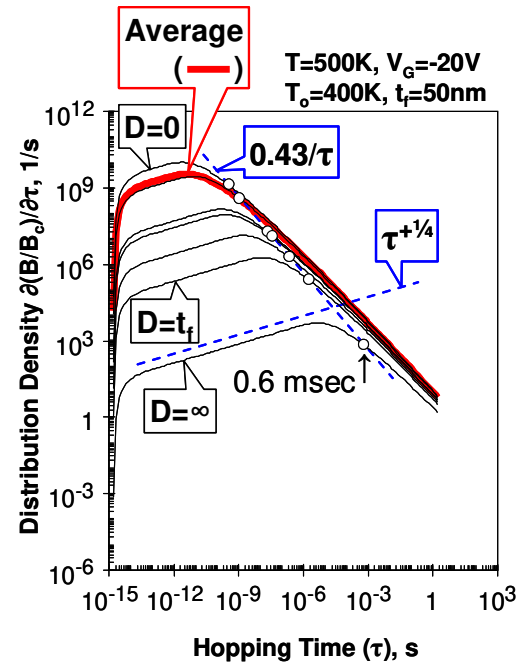

(d)

Figure 38. Temperature evolution of the hopping time distributions (thin black lines, being the same as the normalized distributions of hopping bonds), increasing the temperature from (a) to (d). The device, the gate bias $V_{G}=-20 \mathrm{~V}$ and the depths in the film are the same as in Figure 37 (e.g., labels $\mathrm{D}=\infty, \mathrm{D}=\mathrm{t}_{\mathrm{f}}$ and $\mathrm{D}=0$ from bottom to top denote a bulk material, the back of the semiconductor film and the interface with the gate dielectric, respectively). Thick solid lines (red color) labeled with "Average" are after weighed averaging with the charge profile $\mathrm{N}_{C}(\mathrm{D})$, according to eq. (131), and coincide well (but slightly above) with the distributions at the electrostatic depths $\mathrm{L}_{\mathrm{A}}$. Open circles $(O)$ denote the critical hopping time $\tau_{\mathrm{c}}=1 / \Gamma_{\mathrm{c}}$ for each depth $\mathrm{D}$, and are always aligned around $b / \tau$ function (dashed trend lines, blue color), with $b=\{0.32,0.39,0.43$ and 0.43$\}$ being a logarithmic function of the temperature $\mathrm{T}$, when $\mathrm{T}<\mathrm{T}_{\mathrm{o}}$, and constant at $\mathrm{T}>\mathrm{T}_{\mathrm{o}}$. The other straight dashed lines (also blue color) illustrate the slope of the $\tau^{\mathrm{n}}$ distributions, with $n=\{-1 / 2,-1 / 4,0$ and $+1 / 4\}=\left(\mathrm{T} / \mathrm{T}_{0}-1\right)$, according to eq. (127). Note that the relaxation time, assumed in the range of the critical hopping time $\tau_{c}$, decreases with the temperature, being $\tau_{c}=\{350$ years, 9 minutes, 0.12 seconds and 0.6 milliseconds $\}$ for bulk material at temperatures $\mathrm{T}=\{200 \mathrm{~K}, 300 \mathrm{~K}, 400 \mathrm{~K}$ and $500 \mathrm{~K}\}$. The temperature variations of $\tau_{\mathrm{c}}$ are much smaller under gate bias, just 2-3 decades at $\mathrm{D}=0$ and $\mathrm{D}=\mathrm{L}_{\mathrm{A}}$ close to interface with the gate dielectric, and almost vanishing at high gate biasing (not shown). 


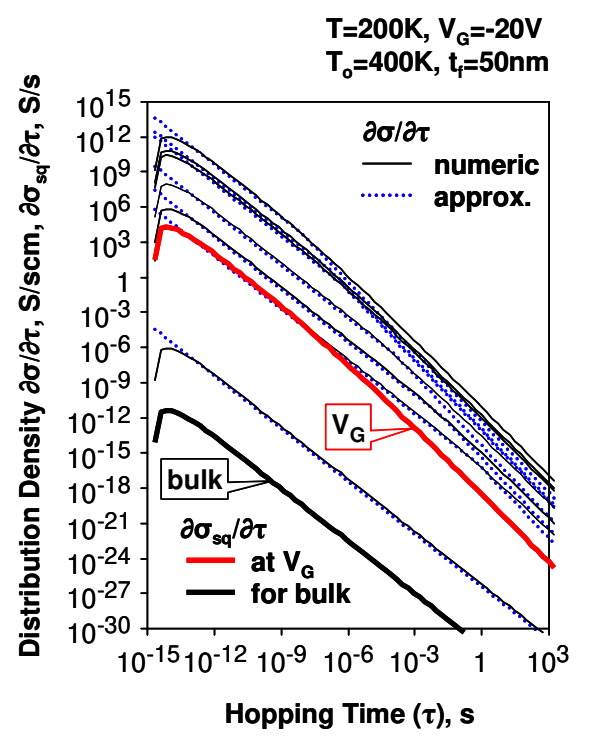

(a)

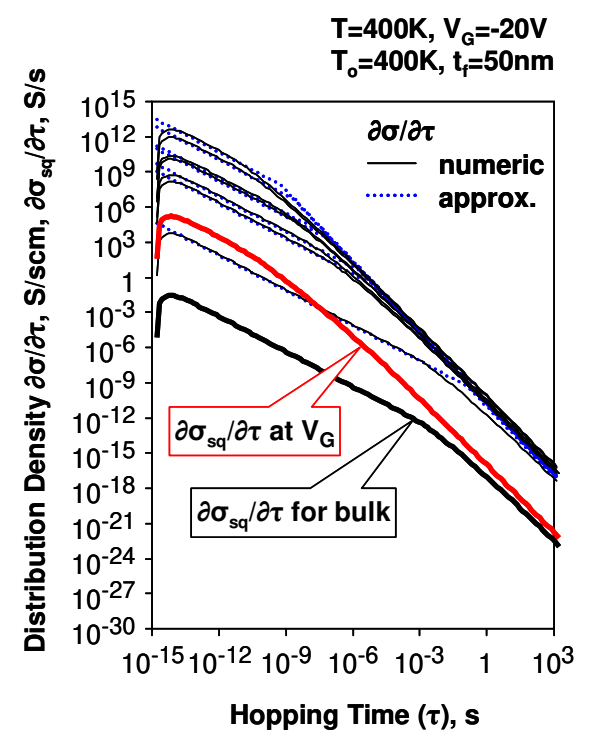

(c)

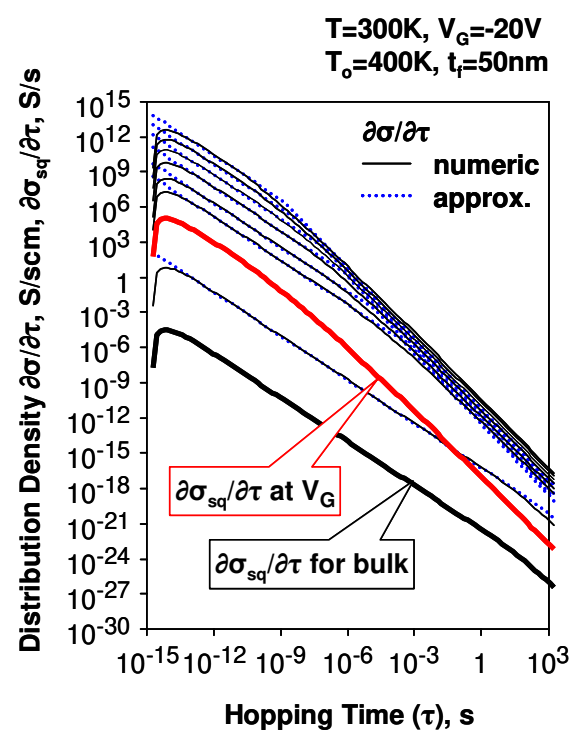

(b)

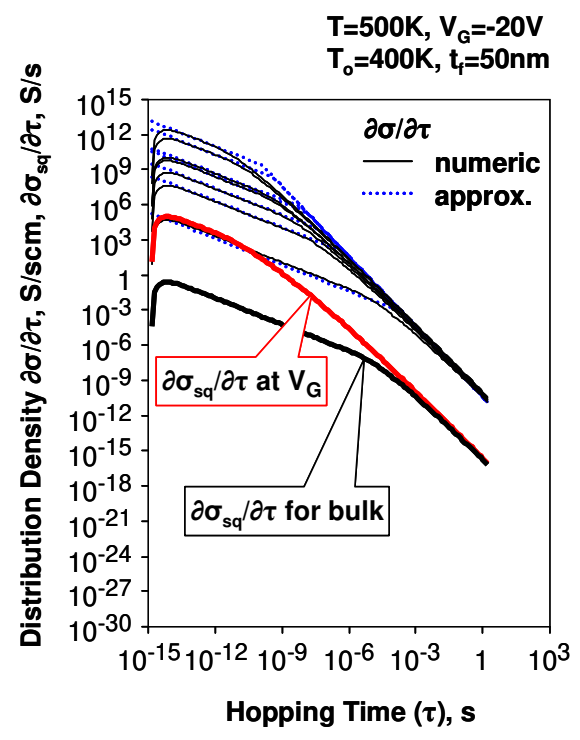

(d)

Figure 39. Temperature evolution of the hopping conductance distributions in a pentacene OTFT, increasing the temperature from (a) to (d). The device, the gate bias $\mathrm{V}_{\mathrm{G}}=-20 \mathrm{~V}$, the depths in the film and temperatures $\mathrm{T}=\{200 \mathrm{~K}, 300 \mathrm{~K}, 400 \mathrm{~K}$ and $500 \mathrm{~K}\}$ are the same as in Figure 38. Unlabeled thin black lines are for the distributions $\partial \sigma / \partial \tau$ of the specific conductance $\sigma(\mathrm{D})$ at different depths $\mathrm{D}$ in the semiconducting film from the gate dielectric interface on the top, down to the back of the film and bulk material in the bottom. Dotted lines (blue color) denote the approximations for $\partial \sigma / \partial \tau$ by eqs. (139) and (140) with normalization coefficients A calculated by eq. (142), using in these equations $\tau_{\mathrm{o}} \exp (3)$ instead of $\tau_{\mathrm{o}}$ for all temperatures, and $\tau_{\mathrm{c}} / 3$ instead of $\tau_{\mathrm{c}}$ for $\mathrm{T}=500 \mathrm{~K}$. The thick solid lines illustrate the distributions $\partial \sigma_{\mathrm{sq}} / \partial \tau$ of the sheet conductance $\sigma_{\mathrm{sq}}$. These thick lines are labeled. The upper thick lines (red color) are for $\partial \sigma_{\mathrm{sq}} / \partial \tau$ at $\mathrm{V}_{\mathrm{G}}=-20 \mathrm{~V}$, corresponding to the on-state of the OTFT, and the lower thick lines are for $\partial \sigma_{\mathrm{sq}} / \partial \tau$ of bulk pentacene of thickness equal to the thickness of the semiconductor film of the OTFT, corresponding to the off-state of the OTFT. 


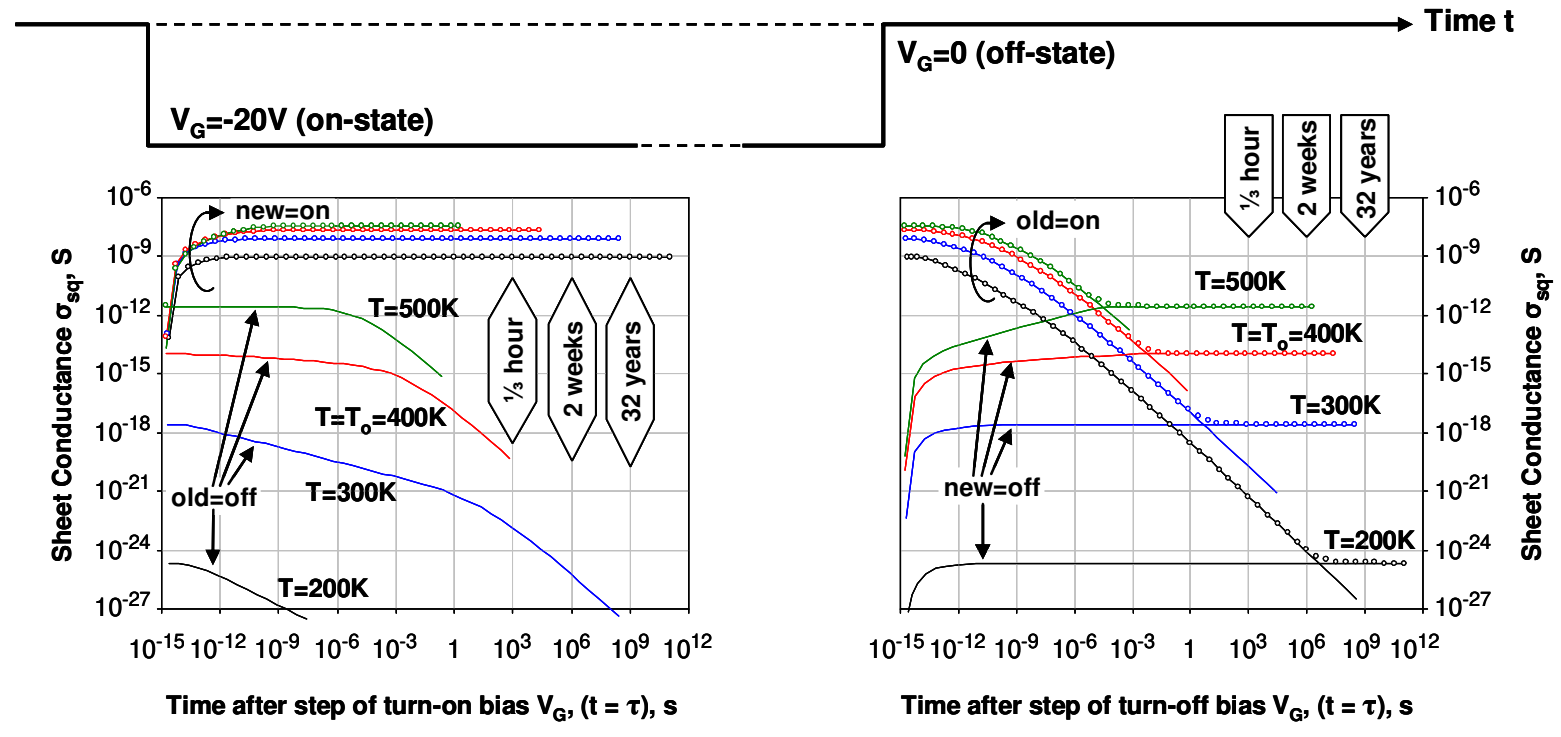

Figure 40. Transient VRH conductivity of a pentacene OTFT, as deduced by time demarcation between on- and off-states. The device, the temperatures $\mathrm{T}=\{200 \mathrm{~K}, 300 \mathrm{~K}, 400 \mathrm{~K}$ and $500 \mathrm{~K}\}$, the gate bias $\mathrm{V}_{\mathrm{G}}=-20 \mathrm{~V}$ for the on-state and $\mathrm{V}_{\mathrm{G}}=0$ (bulk semiconductor) for the off-state are the same as in Figure 39. The demarcation is between on- and off-states of the sheet conductivity $\sigma_{\mathrm{sq}}$, with corresponding distributions $\partial \sigma_{\mathrm{sq}} / \partial \tau$, as shown by the thick lines in Figure 39. Arrows point to the lines of the evolution of the off-state. Arcs surround the lines of the evolution of the on-state. Open circles $(\circ)$ illustrate the superposition of these evolutions. The steps of the gate bias voltage $\mathrm{V}_{\mathrm{G}}$ corresponding to transition from off- to on-state (on left) and from on- to off-state (on right) are depicted on the top of the plots for the sheet conductance $\sigma_{\mathrm{sq}}$. 


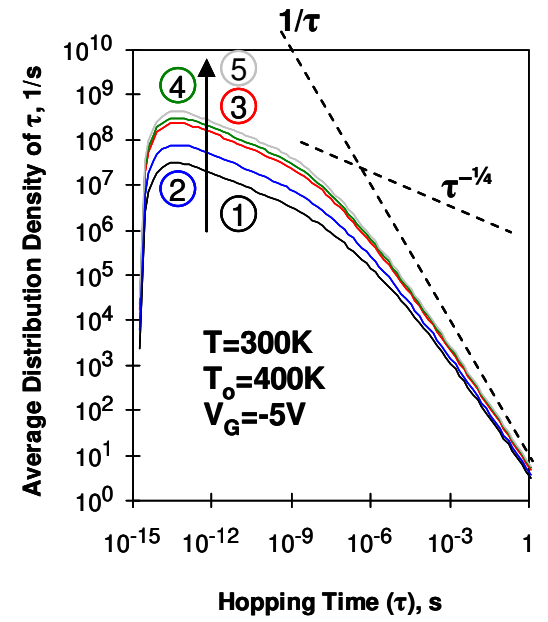

(a)

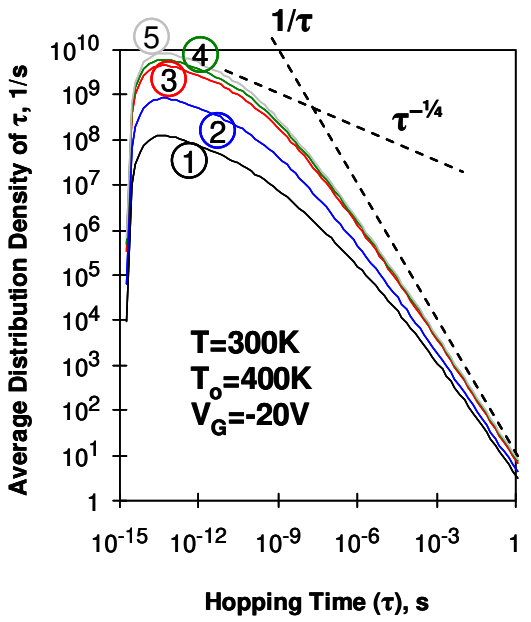

(b)

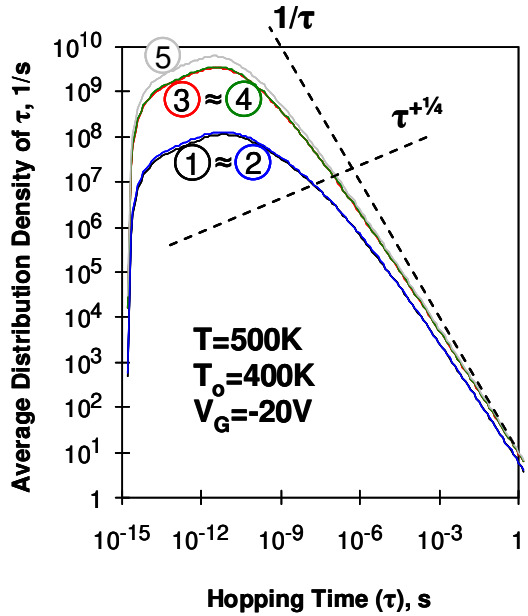

(c)

Figure 41. Average distributions $\partial\left(\mathrm{B} / \mathrm{B}_{\mathrm{c}}\right)_{\mathrm{AVG}} / \partial \tau$ of the hopping time $\tau$ in a pentacene OTFT under bias $\mathrm{V}_{\mathrm{G}} \neq 0$, calculated by the different hypotheses in eq. (147) for possible origin of the flicker low-frequency noise. The device and method of VRH calculation are the same as given in the caption of Figure 37. The reference plot is (b), and plot (a) is for low bias, while plot (c) is for high temperature. Labels (1) and blackcolor solid lines correspond to non-weighted averaging $(\mathrm{Y}=1)$ of hypothesis (1), thus, represent the distributions of $\tau$ itself. Labels (2) and blue-color solid lines correspond to hypothesis (2) for $\Delta \mu$ noise by using weighting function $\mathrm{Y}=\sigma / \mathrm{N}_{C} \propto \mu$ in eq. (147). Labels (3) and red-color solid lines correspond to hypothesis (3) for $\Delta \mathrm{N}$ noise by using weighting function $\mathrm{Y}=\mathrm{N}_{\mathrm{C}}$ in eq. (147). Labels (4) and green-color solid lines correspond to hypothesis (4) for conductance $\Delta \sigma$ noise by using weighting function $Y=\sigma$ in eq. (147). Labels (5) and gray-color solid lines correspond to hypothesis (5) for correlated conductance-carrier number $(\Delta \sigma-\Delta \mathrm{N})$ noise by using weighting function $\mathrm{Y}=\sigma \times \mathrm{N}_{\mathrm{C}}$ in eq. (147). Observe that the distributions tend to $1 / \tau$ distribution (dashed lines) at $\tau>10 \mu \mathrm{s}$, and the higher is $\tau$, the smaller are the differences between the distributions obtained by different hypotheses. In contrast, large differences exist at $\tau<10$ ns, especially when increasing the bias - compare plots (a) and (b). Also, the slopes of the distributions vary with temperature, being $\tau^{\mathrm{n}}$ functions at $\tau<1 \mathrm{~ns}$, c.f. eq. (123), with $\mathrm{n}=\left(\mathrm{T} / \mathrm{T}_{\mathrm{o}}-1\right)$ according to eq. (127) - compare dashed lines labeled with $\tau^{-1 / 4}$ and $\tau^{+1 / 4}$ in plots (b) and (c). Other observations are that the magnitudes of $\partial\left(\mathrm{B} / \mathrm{B}_{\mathrm{c}}\right)_{\mathrm{AVG}} / \partial \tau$ increase changing the hypothesis from (1) to (5), but (1) $\approx(2)$ and (3) (4) at high temperature, and (5) is just a little above (4) at all temperature and bias conditions. 


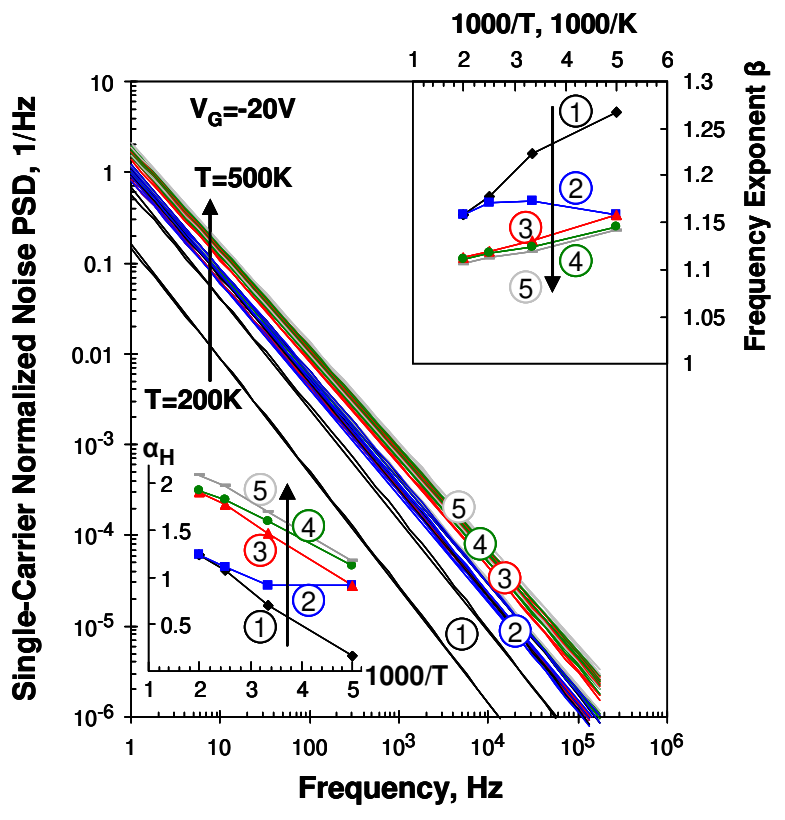

(a)

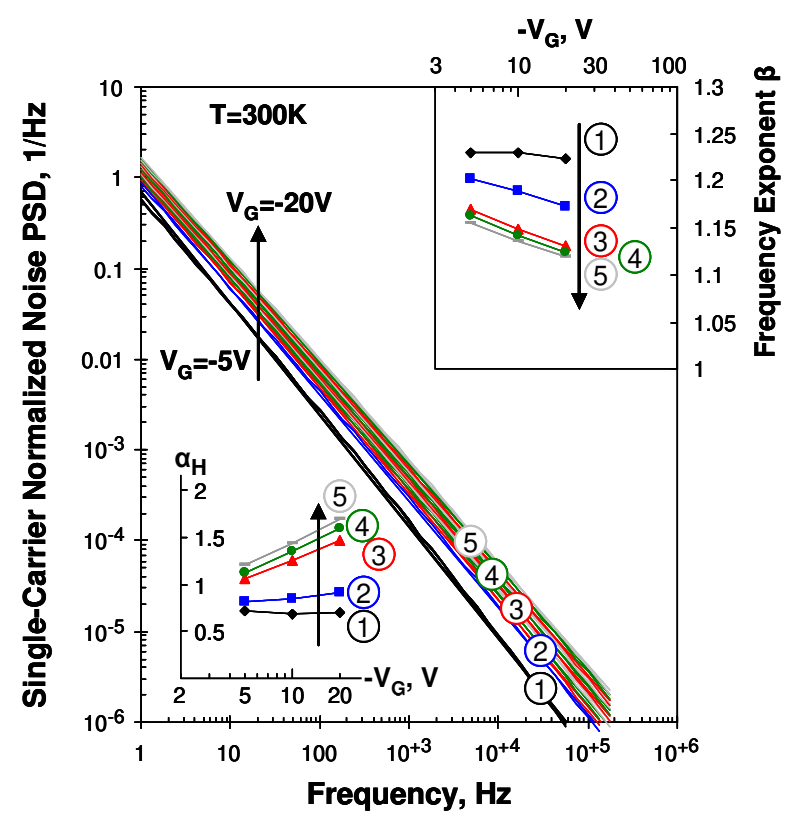

(b)

Figure 42. Single-carrier normalized noise $S_{n 1}$ by the different hypotheses (1) (2) (3) (4) (5) in eq. (147) for the origin of the LFN in an OTFT. (a) For different temperatures $\mathrm{T}=\{200 \mathrm{~K}, 300 \mathrm{~K}, 400 \mathrm{~K}, 500 \mathrm{~K}\}$ at gate bias voltage $\mathrm{V}_{\mathrm{G}}=-20 \mathrm{~V}$. (b) For different $\mathrm{V}_{\mathrm{G}}=\{-5 \mathrm{~V},-10 \mathrm{~V},-20 \mathrm{~V}\}$ at room temperature $\mathrm{T}=300 \mathrm{~K}$. The device and method of VRH calculation are the same as given in the caption of Figure 37. The insets show the values of the Hooge parameter $\alpha_{H}$ and the frequency exponent $\beta$ of the single-carrier flicker noise, after fitting the numerically calculated spectra $S_{\mathrm{n} 1}$ (main plots) with the approximation $\alpha_{\mathrm{H}} / \mathrm{f}^{\beta} \approx \mathrm{S}_{\mathrm{n} 1}$, see eq. (146). The insets in (a) are drawn vs. the reciprocal 1000/T of the temperature $\mathrm{T}$, but note that the axes are linear. The insets in (b) are drawn vs. the magnitude of gate bias voltage; showing that both $\alpha_{H}$ and $\beta$ are nearly logarithmic functions of the bias. 


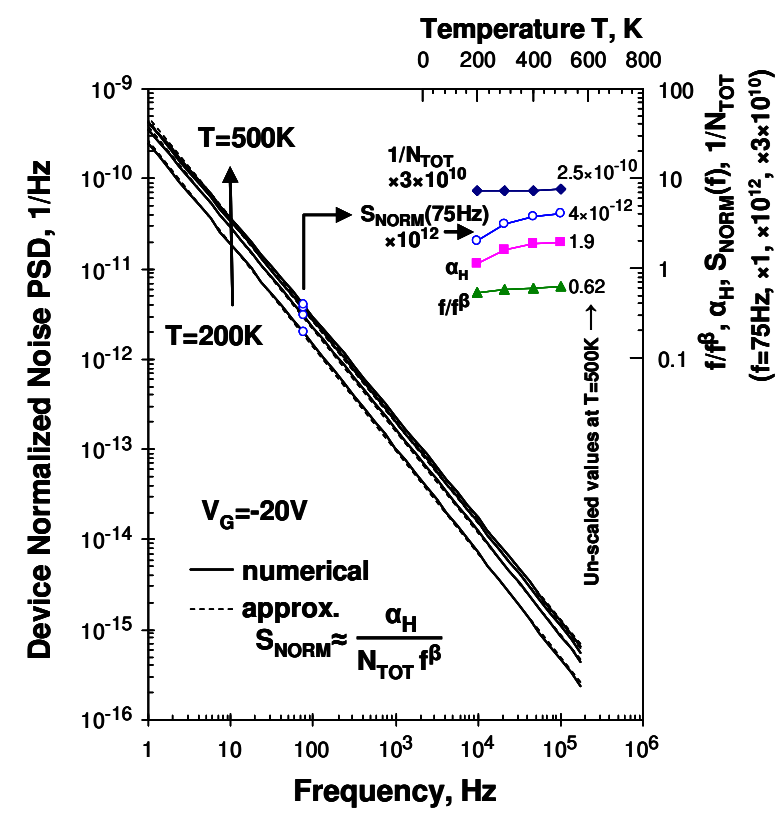

(a)

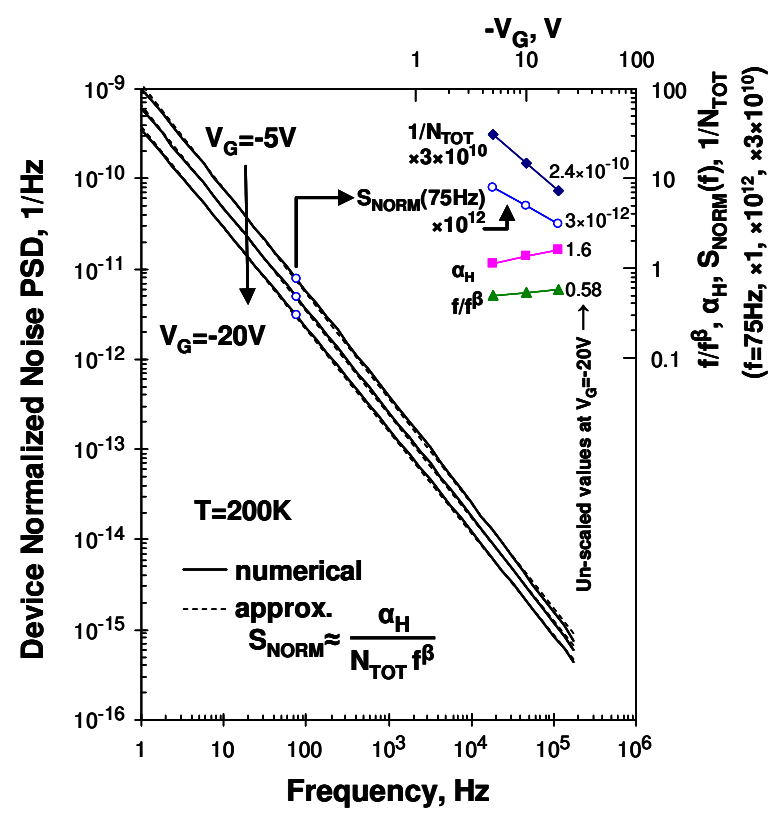

(b)

Figure 43. Device normalized noise $S_{\text {NORM }}$ by the hypothesis (4) in eq. (147) for $\Delta \sigma$ origin of the LFN. (a) For different temperatures $\mathrm{T}=\{200 \mathrm{~K}, 300 \mathrm{~K}, 400 \mathrm{~K}, 500 \mathrm{~K}\}$ at gate bias voltage $\mathrm{V}_{\mathrm{G}}=-20 \mathrm{~V}$. (b) For different $\mathrm{V}_{\mathrm{G}}=\{-5 \mathrm{~V},-10 \mathrm{~V},-20 \mathrm{~V}\}$ at room temperature $\mathrm{T}=300 \mathrm{~K}$. The device and method of VRH calculation are the same as given in the caption of Figure 37. The data also correspond to the subset (4) of the data in Figure 42 for the single-carrier normalized noise $S_{\mathrm{n} 1}$ and its approximation with $\alpha_{\mathrm{H}} / \mathrm{f}^{\beta} \approx \mathrm{S}_{\mathrm{n} 1}$. The solid lines in the main plots denote the numerical calculation $S_{N O R M}=S_{n 1} / N_{T O T}$, where $N_{T O T}$ is the total number of carriers. The dashed lines are after the approximation $S_{N O R M} \approx\left(\alpha_{H} / f^{\beta}\right) / \mathrm{N}_{\text {TOT }}$. The insets show the split of the different contributions for the increase of $\mathrm{S}_{\mathrm{NORM}}$ from the increase of the reciprocal $\left(1 / \mathrm{N}_{\mathrm{TOT}}\right)$, diamonds $(\bullet)$, from the increase of $\alpha_{\mathrm{H}}$, squares $(\boldsymbol{\nabla})$, and from the decrease of the frequency exponent $\beta$ of the flicker noise, triangles $(\boldsymbol{\Delta})$, so that $\mathrm{f} / \mathrm{f}^{\beta}$ is larger when $\beta$ is smaller. By this formulation of the split factors, the factors are with multiplicative contribution to $\mathrm{S}_{\mathrm{NORM}}$, and therefore, are plotted in logarithmic scales when examining the contributions from variations with temperature and bias. The data points shown in the insets are scaled versions of $\left(1 / \mathrm{N}_{\mathrm{TOT}}\right)$ and $\mathrm{S}_{\mathrm{NORM}}$, in order to bring the data together with $\alpha_{\mathrm{H}}$ and $\mathrm{f} / \mathrm{f}^{\beta}$ in plots with one vertical axis. The scaling multipliers are $3 \times 10^{10}$ for $\left(1 / \mathrm{N}_{\mathrm{TOT}}\right)$ and $10^{12}$ for $\mathrm{S}_{\mathrm{NORM}}$. To avoid misinterpretations of the use of the multipliers, the numerical data shown in the insets are the original unscaled values of the quantities for the right-most points at $\mathrm{T}=500 \mathrm{~K}$ and $\mathrm{V}_{\mathrm{G}}=-20 \mathrm{~V}$ in (a), and at $\mathrm{T}=300 \mathrm{~K}$ and $\mathrm{V}_{\mathrm{G}}=-20 \mathrm{~V}$ in $(\mathrm{b})$. The open circles $(\mathrm{O})$ denote $\mathrm{S}_{\mathrm{NORM}}$ (or its scaled version in the insets) at an arbitrary chosen frequency of $\mathrm{f}=75 \mathrm{~Hz}$. 


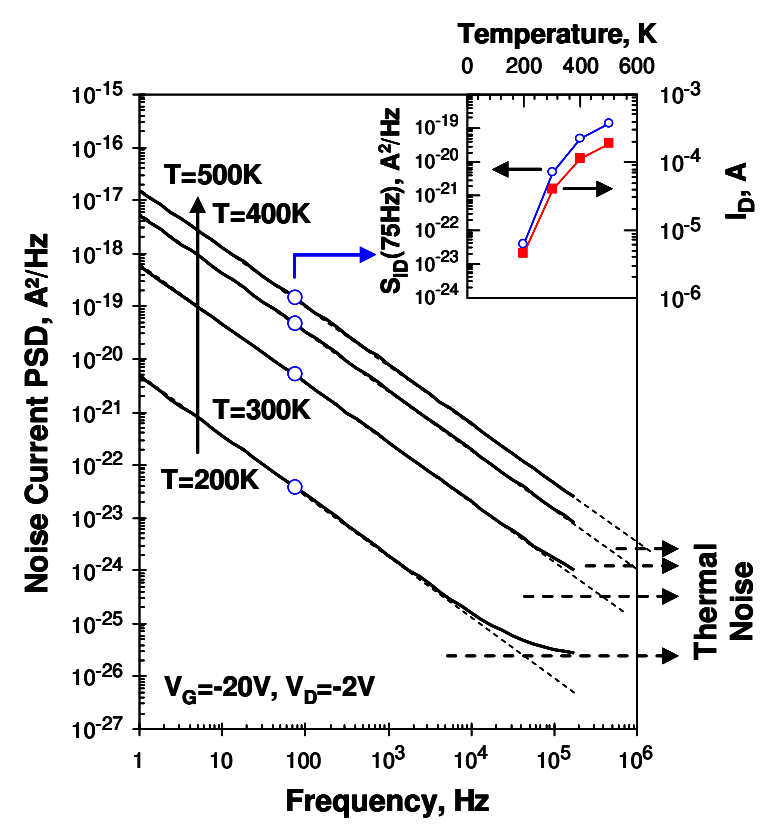

(a)

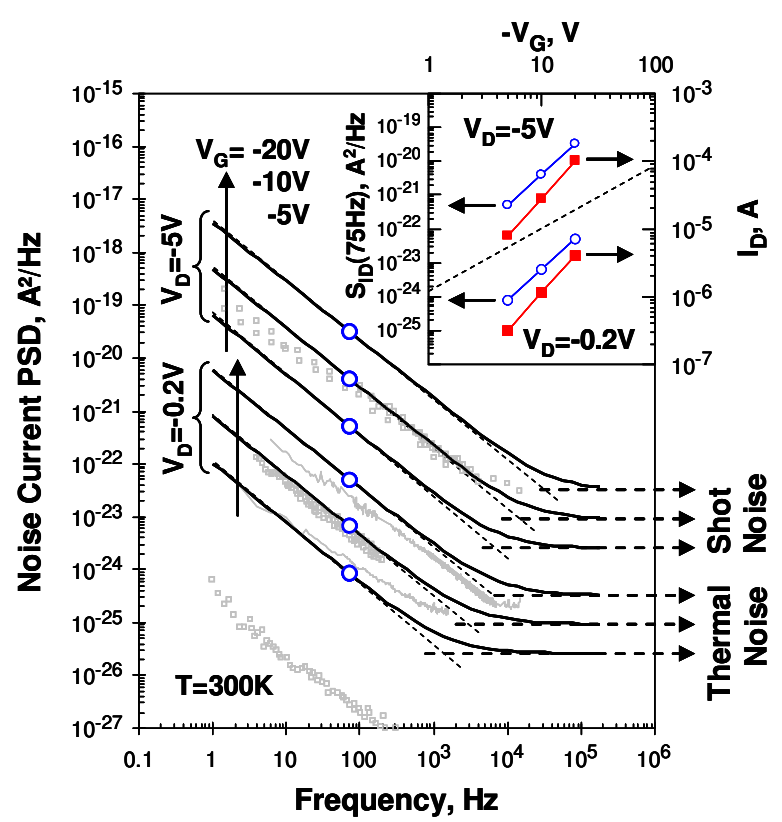

(b)

Figure 44. Power spectrum density of the OTFT channel noise current by the hypothesis (4) in eq. (147) for $\Delta \sigma$ origin of the LFN. (a) For different temperatures $\mathrm{T}=\{200 \mathrm{~K}, 300 \mathrm{~K}, 400 \mathrm{~K}, 500 \mathrm{~K}\}$ at gate bias voltage $\mathrm{V}_{\mathrm{G}}=-20 \mathrm{~V}$ and drain bias voltage $\mathrm{V}_{\mathrm{D}}=-2 \mathrm{~V}$. (b) For different $\mathrm{V}_{\mathrm{G}}=\{-5 \mathrm{~V},-10 \mathrm{~V},-20 \mathrm{~V}\}$ and $\mathrm{V}_{\mathrm{D}}=\{-0.2 \mathrm{~V}$, $-5 \mathrm{~V}\}$ at room temperature $\mathrm{T}=300 \mathrm{~K}$. Thermal noise $\left(4 \mathrm{kT} \sigma_{\mathrm{sq}} \mathrm{W} / \mathrm{L}\right)$ or shot noise $\left(2 \mathrm{q} \mathrm{I}_{\mathrm{D}}\right)$ is added, as indicated. The device and method of VRH calculation are the same as given in the caption of Figure 37. The solid lines in the main plots denote the numerical calculation $\mathrm{S}_{\mathrm{ID}}=\mathrm{I}^{2} \mathrm{~S}_{\mathrm{NORM}}=\mathrm{I}_{\mathrm{D}}{ }^{2} \mathrm{~S}_{\mathrm{n}} / \mathrm{N}_{\mathrm{TOT}}$. The tilted dashed lines represent the approximations with the de-normalized Hooge equation $\mathrm{S}_{\mathrm{ID}} \approx \mathrm{I}_{\mathrm{D}}{ }^{2} \alpha_{\mathrm{H}} /\left(\mathrm{N}_{\mathrm{TOT}} \mathrm{f}^{\beta}\right)$ for the flicker noise component, and the horizontal dashed lines depict the white noise due to the fundamental thermal or shot noise. The insets are arranged to examine the proportionality $\mathrm{S}_{\mathrm{ID}}(75 \mathrm{~Hz}) \propto \mathrm{I}_{\mathrm{D}}^{2}$ at an arbitrary chosen frequency of $f=75 \mathrm{~Hz}$ for $S_{\mathrm{ID}}$. The open circles $(\mathrm{O})$ on the solid lines and in the insets are $\mathrm{S}_{\mathrm{ID}}(75 \mathrm{~Hz})$, as obtained from the numerical calculation with the white noise added. The squares $(\boldsymbol{\square})$ in the insets are the corresponding values for the DC current $\mathrm{I}_{\mathrm{D}}$. For qualitative only comparisons, the gray symbols and lines in (b) are experimental data published in $[120,121,122,123,124,125]$ for several OTFTs of different sizes and fabrication approaches, since no data for noise are available for the particular pentacene sample, which mobility data is given in [73], and we have used the mobility data to determine the VRH parameters in Table IV. 
This Figure 45 is in Appendix 4

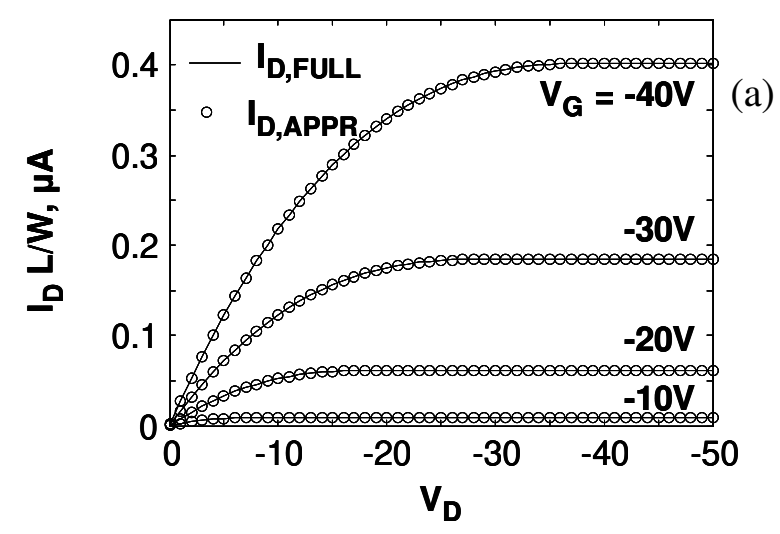

(c)

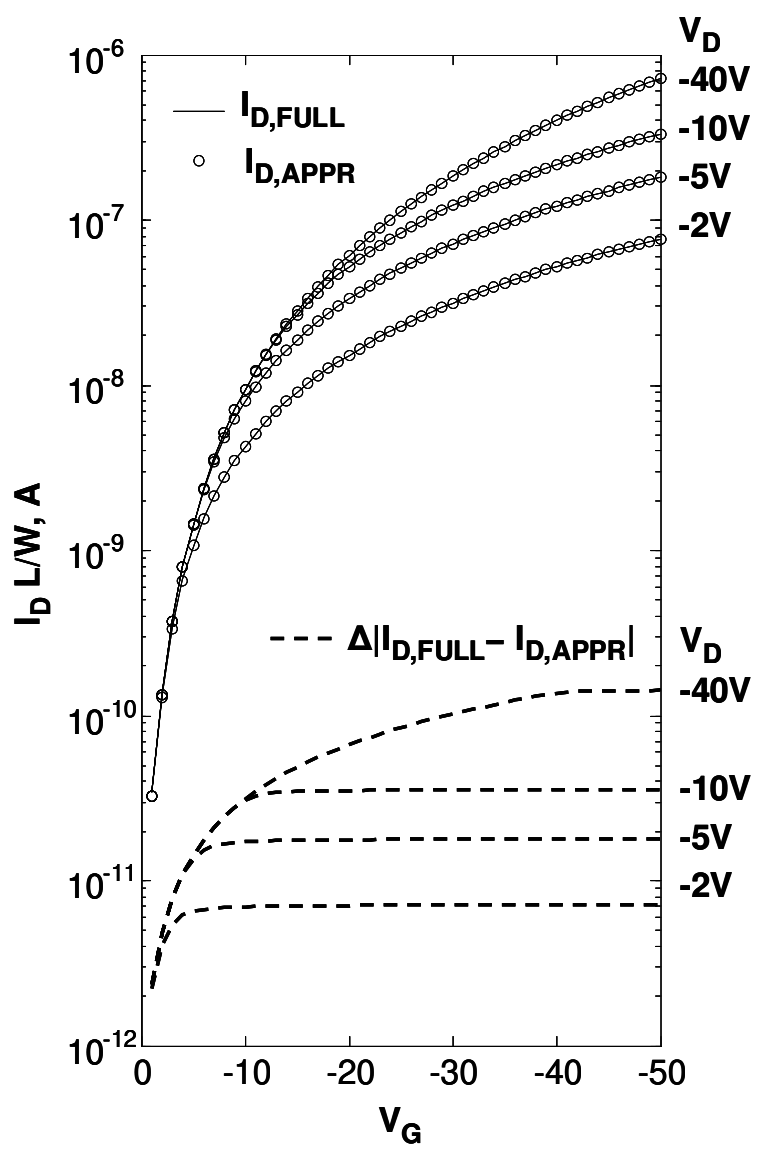

Figure 45. Comparison between the full solution $\mathrm{I}_{\mathrm{D}, \mathrm{FULL}}$ (solid lines), eq. (173) with hyper-geometric component, and the approximate solution ID,APPR, (circles $\bigcirc$ ), eq. (174) without hyper-geometric component, for the drain current $\mathrm{I}_{\mathrm{D}} \mathrm{L} / \mathrm{W}$ in square-shaped OTFT. The dashed lines in (b) and (c) denote the difference $\Delta_{I D}=\left|I_{D, F U L L}-I_{D, A P P R}\right|$. The trend is that $I_{D, F U L L} \approx I_{D, A P P R}$, since the circles overlap with the solid lines, and the difference $\Delta_{\mathrm{ID}}$ is a small fraction of a percent at normal gate bias $\left|\mathrm{V}_{\mathrm{G}}\right|>10 \mathrm{~V}$, but $\Delta_{\mathrm{ID}}$ raises to about $10 \% \mathrm{I}_{\mathrm{D}}$ in subthreshold regime, when the approximation with dominant gate charge $\mathrm{Q}_{\mathrm{F}} \approx \mathrm{Q}_{\mathrm{G}}$ is not precise. The parameters used in the calculations are $\mathrm{T}=300 \mathrm{~K}, \mathrm{~T}_{\mathrm{o}}=405 \mathrm{~K}, \mathrm{~T}_{\mathrm{OEF}}=406.33 \mathrm{~K}, \mathrm{C}_{\mathrm{OX}}=17.3 \mathrm{nF} / \mathrm{cm}^{2}$, $\varepsilon=3 \varepsilon 0=265 \mathrm{fF} / \mathrm{cm}, \mathrm{t}_{\mathrm{f}}=50 \mathrm{~nm}, \eta=0.0584(\mathrm{~S} / \mathrm{cm})^{\mathrm{T}} \mathrm{OEF} / \mathrm{T} / \mathrm{C}, \mathrm{V}_{\mathrm{SS}}=0.8686 \mathrm{~V}$, resulting in values for $\mathrm{b}=4.64 \mathrm{~V}^{-1}$ and $\mathrm{a}=3.6 \times 10^{-12}$ (complex unit) calculated by the definitions in eq. (101). 